\title{
ANALYSIS OF HOUSING DATA COLLECTED IN A LEAD-BASED \\ PAINT SURVEY IN PITTSBURGH, PENNSYLVANIA -- PART II
}

Douglas R. Shier

and

William G. Hall

Applied Mathematics Division Institute for Basic Standards National Bureau of Standards Washington, D.C. 20234

June 1977

Final

Prepared for:

Housing Technology Programs

Center for Building Technology

Institute of Applied Technology, NBS

Sponsored by:

Office of Policy Development and Research Department of Housing and Urban Development Washington, D.C. 20410

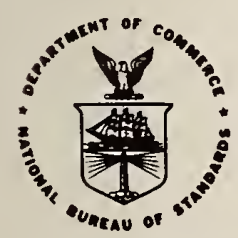

U.S. DEPARTMENT OF COMMERCE, Juanita M. Kreps, Secretary Dr. Sidney Harman, Under Secretary Jordan J. Baruch, Assistant Secretary for Science and Technology NATIONAL BUREAU OF STANDARDS, Ernest Ambler, Acting Director 



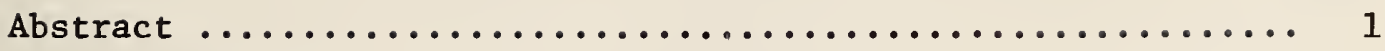

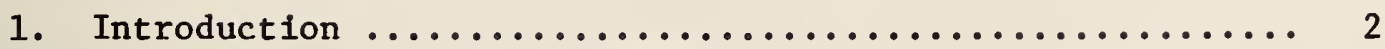

2. Description of the Tables $\ldots \ldots \ldots \ldots \ldots \ldots \ldots \ldots \ldots \ldots$. 4

2.1 Set A Tables (Housing Data)................. 4

2.2 Set B Tables (Housing Data).................. 8

2.3 Set $C$ Tables (Housing/Blood Data)............... 11

3. References $\ldots \ldots \ldots \ldots \ldots \ldots \ldots \ldots \ldots \ldots \ldots \ldots \ldots \ldots \ldots$

Appendix A: Set A Tables $\ldots \ldots \ldots \ldots \ldots \ldots \ldots \ldots \ldots \ldots \ldots, 14$

Appendix B: Set B Tables $\ldots \ldots \ldots \ldots \ldots \ldots \ldots \ldots \ldots \ldots, \ldots 7$

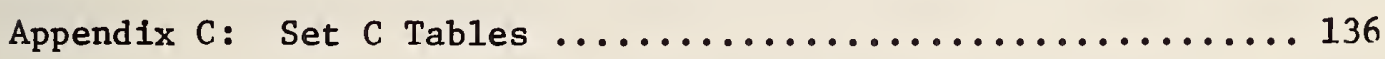

Appendix D: Data Collection Form for Housing Inspection .....185 



\section{ANALYSIS OF HOUSING DATA COLLECTED IN A LEAD-BASED \\ PAINT SURVEY IN PITTSBURGH, PENNSYLVANIA}

\section{Part II}

\section{Abstract}

This report represents Part II of a two-part document describing a lead-based paint survey conducted in Pittsburgh, Pennsylvanfa. Part I of this document (NBSIR 77-1250) presented details of the design, methodology and findings of this survey. The current report (Part II) presents and describes a series of tables summarizing the Pittsburgh survey data base, which includes measured lead levels of various surfaces in dwelling units and blood lead levels of children resident in those units. Most of the data analyses conducted in Part I derive from these tables. Thus, the tabulations given in this report provide not only the basis for those analyses but also a potential source for additional data analyses.

Key Works: Blood lead; children; data analysis; housing; lead paint; lead poisoning; surveys. 


\section{INTRODUCTION}

During 1974 and 1975, the National Bureau of Standards (NBS), under sponsorship of the Department of Housing and Urban Development (HUD), conducted, in cooperation with the Allegheny County Health Department, a large-scale survey of housing in Pittsburgh, Pennsylvania. This survey collected various data on the extent, magnitude and distribution of leadbearing paint surfaces within the dwelling units of the sampled area. Also, blood samples and socioeconomic information were collected from children under seven years of age resident in those units. In contrast to previous surveys, the Pittsburgh lead-based paint survey was a largescale, statistically designed effort aimed at characterizing the lead levels in dwelling units comprising an entire city, and not simply a highrisk area. Additional details concerning the design, methodology and findings of the survey are provided in [1]*, which constitutes Part I of this two-part document. A statistical study of the children's blood lead levels, together with detalls of the chemical analysis techniques employed, can be found in a previous NBS report [2].

The current report (Part II) presents and describes a series of tables that summarize the Pittsburgh survey data. In fact, the data analyses given in [1] are for the most part derived from these tables. In addition, the tables provide a potential source for further data analyses, based on other combinations of housing characteristics (described In Section 2) which may not have been explicitly treated in Part I.

The entire set of tabulations actually produced, using the Pitts*Figures in brackets indicate references listed in Section 3. 
burgh data base, are far too voluminous for inclusion in this report. Accordingly, a representative subset of those tables is provided here, and this set should suffice for the purposes of most analyses. Listings of additional tables (which follow the same formats as those described here) can be provided by NBS, upon request, to interested researchers. In Section 2, the classifications used in structuring the tables are defined and the data elements forming each table are discussed. The tables themselves (arranged into three sets of tables) are displayed in the Appendices. These tables provide supporting data tabulations on which the analyses and conclusions of Part I have been based. 


\section{DESCRIPTION OF THE TABLES}

The various tables provided in this report have been classified into three distinct sets: those designated as "Set A," "Set B," and "Set C." The tables in Sets A and B summarize the interior lead level measurements obtained in the survey according to the values of certain housing-related variables. (The data collection form on which these and other measurements were recorded is shown in Appendix D.) The tables in Set $C$ relate the interior lead level measurements in dwelling units to the blood lead levels found in children residing in those units, also grouped according to various housing variables. The contents and use of each set of tables will be discussed in this section.

\subsection{Set A Tables (Housing Data)}

Tables from this set are those from which most of the cumulative distributions of Part I have been derived. Each table of this set shows (by age of unit, occupancy class, room type, surface type and surface condition) the fractions of the dwelling units, rooms, and surfaces in which the highest lead level reading equals or exceeds certain specified levels. Since each table involves a number of variables and stratifications for such variables, this information will be described first. Subsequently, the interpretation and use of these tables will be discussed in some detail.

Tables have been calculated for every combination of values assumed by the variables listed below. Only a certain subset of these calculated tables are, however, presented in the Set A Tables. An asterisk in the 1ist below denotes those values of the variables for which a corresponding 
table has been included in Appendix A.

I. Age of Dwelling Unit

*Pre 1940

*1940-1959

*1960-1975

*ALL

II. Occupancy Class

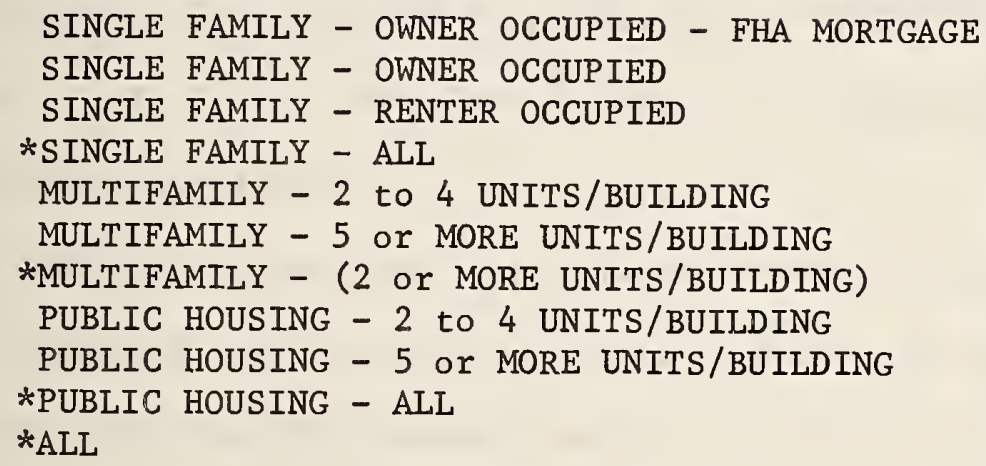

III. Room Type

*DRY (a11 rooms except kitchens and bathrooms)

*WET (kitchens and bathrooms)

*ALL

IV. Surface Type (interior)

*WALLS

*WINDOWS

*DOORS

V. Surface Condition

This designation does not appear in the data base for each surface, but rather is recorded by surface type (walls, trim) for each room. Note that inspectors were instructed to assign these condition codes on the basis of the worst condition present in the room.

*PEELING - denotes a surface on which the paint film itself is peeling, flaking, blistering or powdering.

* $\mathrm{BAD}$ - denotes a surface which has a bad substrate regardless of the condition of the surface; this category includes all 
imperfections except halrline cracks, nall holes, etc.

*EITHER - denotes a surface which is either PEELING or BAD or both.

*BOTH - denotes a surface that is both PEELING and BAD.

*ALL - denotes all surfaces, regardless of condition.

\section{Specified Lead Levels}

$$
\begin{array}{r}
* 0.0 \mathrm{mg} / \mathrm{cm}^{2} \\
* 0.5 \mathrm{mg} / \mathrm{cm}^{2} \\
* 1.0 \mathrm{mg} / \mathrm{cm}^{2} \\
1.5 \mathrm{mg} / \mathrm{cm}^{2} \\
* 2.0 \mathrm{mg} / \mathrm{cm}^{2} \\
2.5 \mathrm{mg} / \mathrm{cm}^{2} \\
3.0 \mathrm{mg} / \mathrm{cm}^{2} \\
4.0 \mathrm{mg} / \mathrm{cm}^{2} \\
* 5.0 \mathrm{mg} / \mathrm{cm}^{2} \\
* 7.5 \mathrm{mg} / \mathrm{cm}^{2} \\
10.0 \mathrm{mg} / \mathrm{cm}^{2}
\end{array}
$$

The tables in Set A provide (for defined values of the variables listed in I-V) the number and fraction of dwellings, rooms or surfaces for which the highest lead level equals or exceeds the specified lead level. Since it is possible to obtain negative lead level readings, such negative readings have been retained for the calculation of statistical quantities, such as the mean, median and standard deviation. However, any derived statistic which assumes a negative value has been set equal to zero for the purposes of the tables in this report. As a result, any table with specifled lead level $0.0 \mathrm{mg} / \mathrm{cm}^{2}$ actually provides a count of the number of dwellings, rooms, or surfaces according to the categories listed in $\mathrm{I}-\mathrm{V}$.

Each table of Set A contains as a heading a room type, a specified lead level, and an occupancy class. The three groups of columns refer to the three surface types, while the four groups of rows (ten lines each) 
refer to the age group of the dwelling unit. For each age group, the first line gives counts for the number of dwelling units in which at least one of the lead level readings equals or exceeds the specified lead level; these counts on the first line are further categorized by surface condition within surface type. The second line gives the fraction of the dwelling units in which at least one of the lead level readings equals or exceeds the specified level, also categorized by surface type and surface condition.

Lines 3-6 contain, for each column, one half the width of a confidence interval* (about the fraction in line 2) with confidence levels $99 \%, 95 \%, 90 \%$, and $80 \%$, respectively. For example, the third table of Set A shows that a $99 \%$ confidence interval, for the true fraction of all pre 1940 single-family dwelling units with at least one dry room wall reading equal to or exceeding $1.0 \mathrm{mg} / \mathrm{cm}^{2}$, is $.636 \pm .028$ (i.e., it extends from .608 to .664$)$. In repeated sampling, the true fraction should lie in a $99 \%$ confidence interval (calculated in this way) 99 times out of 100. Likewise, a 95\% confidence interval for the true fraction is given by $.636 \pm .021$, while an $80 \%$ confidence interval for the fraction is given by $.636 \pm .014$.

Lines 7 and 8 give the number and fraction of rooms (by surface type and condition) with at least one lead level reading equal to or exceeding :

*The calculation of these confidence intervals follows the procedure described in Appendix B of [3]. Despite the fact that the underlying statistical distribution of individual lead level readings is highly non-normal [1], the sample sizes given. in these tables are usually large enough (e.g., in excess of 50 observations) for the Central Limit Theorem [4] to hold. Since the confidence interval calculations rely on the application of this theorem, the indicated confidence intervals will be valid so long as the sample size is sufficiently large. 
the specified lead level. Since only a single lead level reading was taken for doors and windows within a room, the quantities appearing on these two lines for doors and windows also represent the number and fraction of lead level readings satisfying the stated conditions.

Lines 9 and 10 give the number and fraction of surfaces (by surface type and condition) with at least one lead level reading equal to or exceeding the specifled lead level. For doors and windows, it is assumed that all doors within a room have the same lead level as the measured door, and that all windows have the same lead levels as the measured window. In addition, the condition code for walls in a room is (con-. servatively) assumed to characterize all walls within the room, while the trim condition code is assumed to characterize all windows and doors within the room.

\subsection{Set B Tables (Housing Data)}

In contrast to the tables of Set A (which summarize the lead level readings by dwelling, room and surface), the tables of Set $B$ contain information aggregated on a room basis. In addition, these tables illustrate varlations in lead levels (a) within rooms, and (b) among rooms of the same type. The Set B tables (found In Appendix B) use variables having stratifications similar to those described in relation to Set $A$. The categorizing variables, and their associated values, are described below. As in Set $A$, the cases appearing in tables of this report are indicated by an aster1sk. The remaining cases have been calculated but do not appear here. 
I. Age of Dwelling Unit

*Pre 1940

*1940-1959

*1960-1975

*ALL

II. Occupancy Class

*SINGLE FAMILY

MULTIFAMILY

*ALL

III. Room Type

*DRY

*WET

ALL

IV. Surface Type (interior)

*WALLS

*TRIM (doors, windows, baseboards, cabinets, fireplaces)

*ALL

V. Surface Condition

*GOOD (surface tight and substrate good)

*BAD (surface peeling and/or substrate bad)

*ALL

VI. Specified Lead Intervals

$* 0.0-0.49 \mathrm{mg} / \mathrm{cm}^{2}$
$* 0.50-0.99 \mathrm{mg} / \mathrm{cm}^{2}$
$* 1.00-1.49 \mathrm{mg} / \mathrm{cm}^{2}$
$* 1.50-1.99 \mathrm{mg} / \mathrm{cm}^{2}$
$* 2.00-2.49 \mathrm{mg} / \mathrm{cm}^{2}$
$* 2.50-2.99 \mathrm{mg} / \mathrm{cm}_{2}^{2}$
$* 3.00-3.99 \mathrm{mg} / \mathrm{cm}_{2}^{2}$
$* 4.00-4.99 \mathrm{mg} / \mathrm{cm}^{2}$
$* 5.00-7.49 \mathrm{mg} / \mathrm{cm}^{2}$
$* 7.50-9.99 \mathrm{mg} / \mathrm{cm}^{2}$
$* 10.0 \mathrm{mg} / \mathrm{cm}^{2}$
$*$ ALL

Each table is identified by a lead level interval, an occupancy class, and a room type. The statistics given in the body of the table refer to those rooms in which the mean wall lead level lies in the 
specified lead level interval. In each table, the four groups of columns represent the four dwelling unit age categories, while the four groups of rows represent certain collections of surface types.

In the group of ten lines with the heading WALLS, the first line contains a count of the number of rooms (by age category and surface condition) with mean wall lead level in the specified interval. The second line converts this number into a percent of all rooms corresponding to that room type, occupancy class, age category, and room (wa11) condition.

Lines 3-4 contain, respectively, the mean of the room means for the wall lead levels and the standard deviation of these room means. In a similar way, lines 5-6 represent the mean and standard deviation of the within-room standard deviations for wall lead levels. The last four lines represent, respectively, the mean and standard deviation of the room medians, and the mean and standard deviation of the highest wall lead level in the room.

The TRIM and ALL groups of rows contain the same quantities as those given in lines 3-10 above for the WALLS group, except that these statistics are now based on the lead levels of the trim (TRIM), or on the walls plus ceilings plus trim (ALL). Again, these quantities have been calculated for all rooms in which the mean wall lead level lies within the specified lead interval.

The final group of lines $(T-W)$ contains statistics similar to those on lines $3-4$ and $7-10$ in the WALLS group, except that the calculations are now based on differences between trim and wall surfaces. More specifically, the two lines headed by MEAN give the mean and standard 
deviation of the mean trim minus the mean wall reading for each room. Similarly, the lines for MEDIAN are based on median trim minus median wall, while those for HIGH are based on high trim minus high wall. As before, such quantities are calculated for all rooms in which the mean wall lead level lies within the specified lead interval.

\subsection{Set C Tables (Housing/Blood Data)}

The tables of this set are quite similar to the Set B tables, except that the children's blood lead levels have been used to control the calculations of room-based statistics for lead levels. Thus all calculations for these tables are performed using those dwelling units containing children for whom blood lead levels had been determined. Apart from the blood lead level intervals described below, the remaining variables and their values remain the same as those listed in $I-V$, Set $B$.

VI. Specified Blood Lead Intervals

$$
\begin{aligned}
& \text { * } 0-4 \mu \mathrm{g} / 100 \mathrm{ml} \\
& \text { * } 5 \text { - } 9 \mathrm{\mu g} / 100 \mathrm{mI} \\
& * 10-14 \mu \mathrm{g} / 100 \mathrm{ml} \\
& \text { *15 - } 19 \mu \mathrm{g} / 100 \mathrm{ml} \\
& \text { *20 - } 24 \mu \mathrm{g} / 100 \mathrm{ml} \\
& \text { *25 - } 29 \mu \mathrm{g} / 100 \mathrm{ml} \\
& \text { *30 - } 34 \mathrm{\mu g} / 100 \mathrm{ml} \\
& \text { *35 - } 39 \mu \mathrm{g} / 100 \mathrm{ml} \\
& \text { *40 - } 44 \mu \mathrm{g} / 100 \mathrm{ml} \\
& \text { *45 - } 49 \mu \mathrm{g} / 100 \mathrm{ml} \\
& *_{\text {ALLL }} \geq 50 \mu \mathrm{g} / 100 \mathrm{ml}
\end{aligned}
$$

Each table is identified by a blood lead interval, occupancy class and room type; also given are the number and percent of children associated with that occupancy class with blood lead levels in the specified interval. The four groups of columns represent the four dwelling unit 
age categories. The IIne denoted by CHILDREN gives for each age category the number and percent of chlldren within that occupancy class having blood lead levels in the specified interval. As in the tables from Set B, the four groups of rows represent certaln collections of surface types. Apart from the differences noted above and the fact that a line does not appear for PERCENT (of rooms), the elements of this table correspond exactly to those defined in Set B. 


\section{REFERENCES}

[1] Shier, D. and Hall, W., "Analysis of Housing Data Collected in a Lead-Based Paint Survey in Pittsburgh Pennsylvania: Part I," NBSIR 77-1250, National Bureau of Standards, May 1977.

[2] Urban, W., "Statistical Analysis of Blood Lead Levels of Children Surveyed in Pittsburgh, Pennsylvania: Analytical Methodology and Summary Results," NBSIR 76-1024, National Bureau of Standards, Apri1 1976.

[3] Hall, W., and Slovic, L.T., "Survey Manual for Estimating the Incidence of Lead Paint in Housing," Technical Note 921, National Bureau of Standards, September 1976.

[4] Feller, W., An Introduction to Probability Theory and Its Applications, Vol. I, John Wiley and Sons, New York, 1968. 


\section{APPENDIX A}

Set "A" Tables

\section{Table of Contents}

Occupancy Class

SINGLE-FAMILY

MULTIFAMILY

PUBLIC

ALL
Room Type

DRY

WET

ALL

DRY

WET

ALL

DRY

WET

ALL

DRY

WET

ALL
Pages

15-20

21-26

27-32

33- 38

39-44

45-50

51-56

$57-62$

63-68

69-74

75-80

81-86 


\section{:}

귱ㅇㅇ $\rightarrow$ : ㅇํㅇ․

in?

ㅇ:

$\sim \circ M=\infty$

$\vec{N} \overrightarrow{0}+$

요유유:

ก

กำ

à

N N

$\stackrel{\infty}{\wedge}$

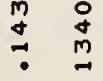

ก

茵等

in

$\stackrel{\sim}{N} \stackrel{M}{\sim} \stackrel{0}{\sim}: \frac{N}{0}: 0$

mo

$0 m$
응ㅇㅇㅇㅇ

n.

:ㅇํㅇ

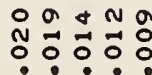

O $0 \rightarrow+0$

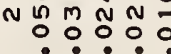

A $\infty$ a

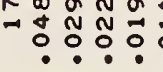

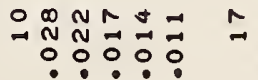

$\therefore: \frac{1}{2}:=$

$=$

m

$\stackrel{N}{N}$

เุ

$a$

N

กิ

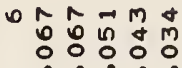

$\ln \left(\frac{10}{10}\right.$

$\stackrel{n}{m}$
궁 $\vec{n}:$

$\therefore$

$\stackrel{m}{n}$

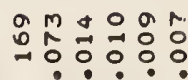

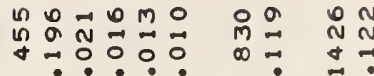

$m$
$m$
$m$
0

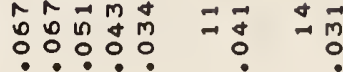

$\exists=$

$+$
N

$M \stackrel{N}{M} \overrightarrow{0} \overrightarrow{0} \overrightarrow{0} \quad \vec{n} \div \stackrel{M}{N} \div$

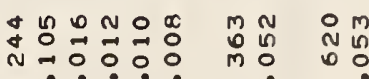

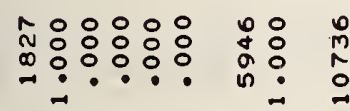

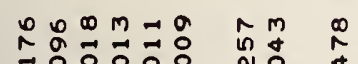

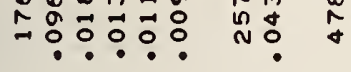

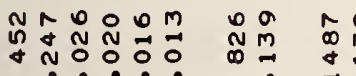

Mัd

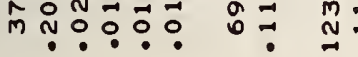

$\infty \rightarrow \infty m$

$\stackrel{n}{\infty} \pm \stackrel{n}{0}: \overrightarrow{0}: 0$

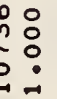

N N응유:

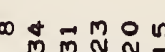

moOñ

(1)

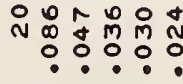

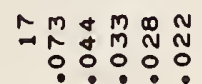
:ㅇ:ㅇ

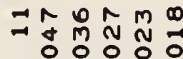

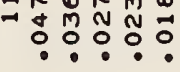

裙 离

$-1$

$-\frac{n}{0}$

$\stackrel{m}{N}$

$\stackrel{2}{0}$

$m$ n

m

in

in

?

$\stackrel{\infty}{M} \underset{N}{N}$
- 000000

응요유:

nOF+口

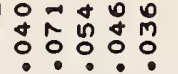

¿ :

$m \stackrel{0}{\mathrm{~N}}$

- $n$ n $m$ n

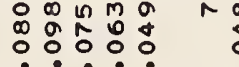

$\sin 0$

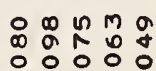

:ㅇㅇํ․

ล N

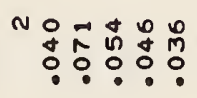

mo
ลี

० :

$=\stackrel{N}{n}$

$\infty 0 m \infty n$

\& N

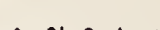

ำ $\sim$ N

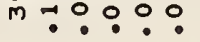

0

ก

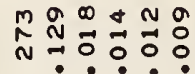$$
\text { m. }
$$

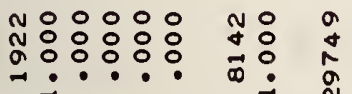

140

7

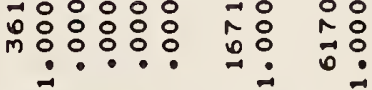

no

Do No:000

:ㅇ:ㅇㅇ

숑ㅇㅇㅇ

in

$-\exists=\infty$

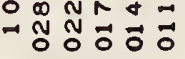

N $\overrightarrow{0}$

ำำกำ

$\rightarrow 2$

$+\frac{m}{8}$

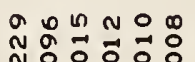

-

กำ:?

$m: \equiv$

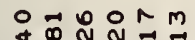

$\cong \hat{m} \quad \stackrel{N}{N}$

$m+m$ n

\begin{abstract}
om
\end{abstract}
$\min n$

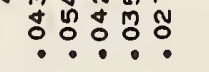

$\stackrel{0}{0}$

$\stackrel{\infty}{N} \stackrel{\infty}{0}$

and

$+\circ \circ M$

$\stackrel{\sim}{N} \stackrel{0}{\stackrel{N}{*}} \stackrel{\sim}{0} \stackrel{0}{\sim}$

$\vec{m}: \overrightarrow{0}: \stackrel{n}{\circ}$

实: ค:

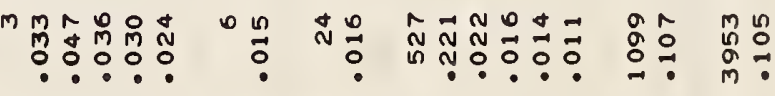

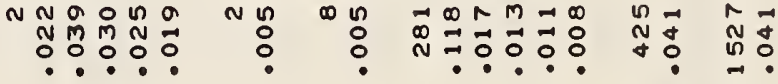
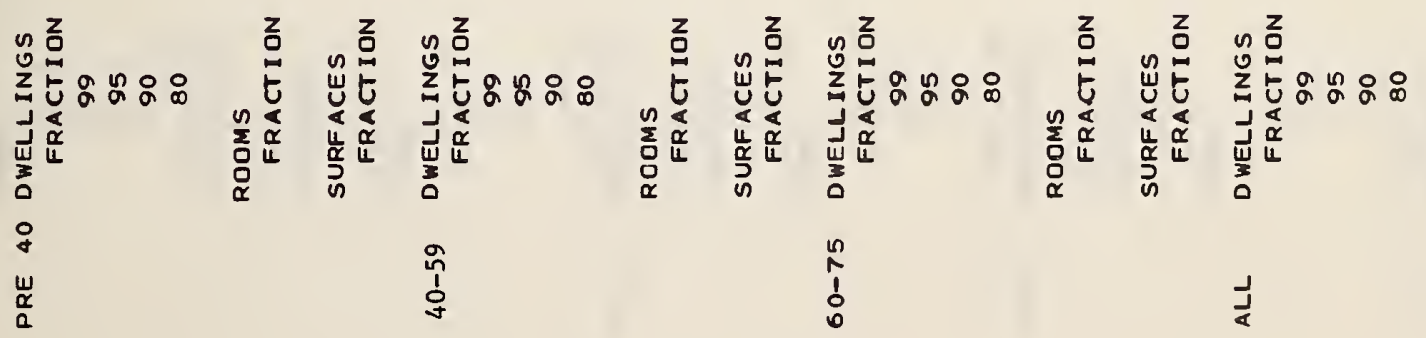

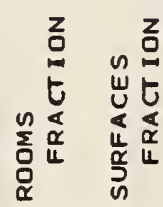


ำ

man mo

ำ i 0000

$\rightarrow \sim$ MN

일

I $\infty$ on $n$

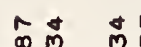

m.

-

Oก $M=0$

둥ㅇㅇㅇ

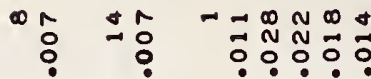

$\rightarrow$

$+\frac{0}{0}$

AMOD

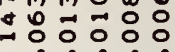

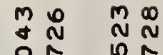

$\ln \infty \rightarrow \infty$

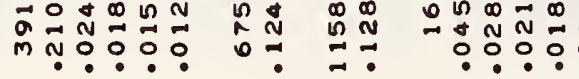

\section{$\stackrel{m}{n} \stackrel{0}{0}$}

?

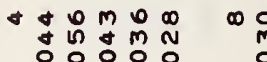

$0_{0}^{0}=$

a

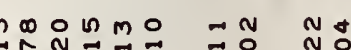

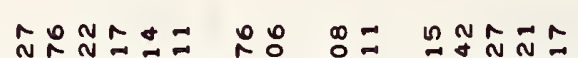

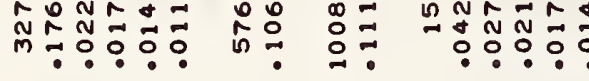

$\stackrel{N}{N} \stackrel{m}{*}$

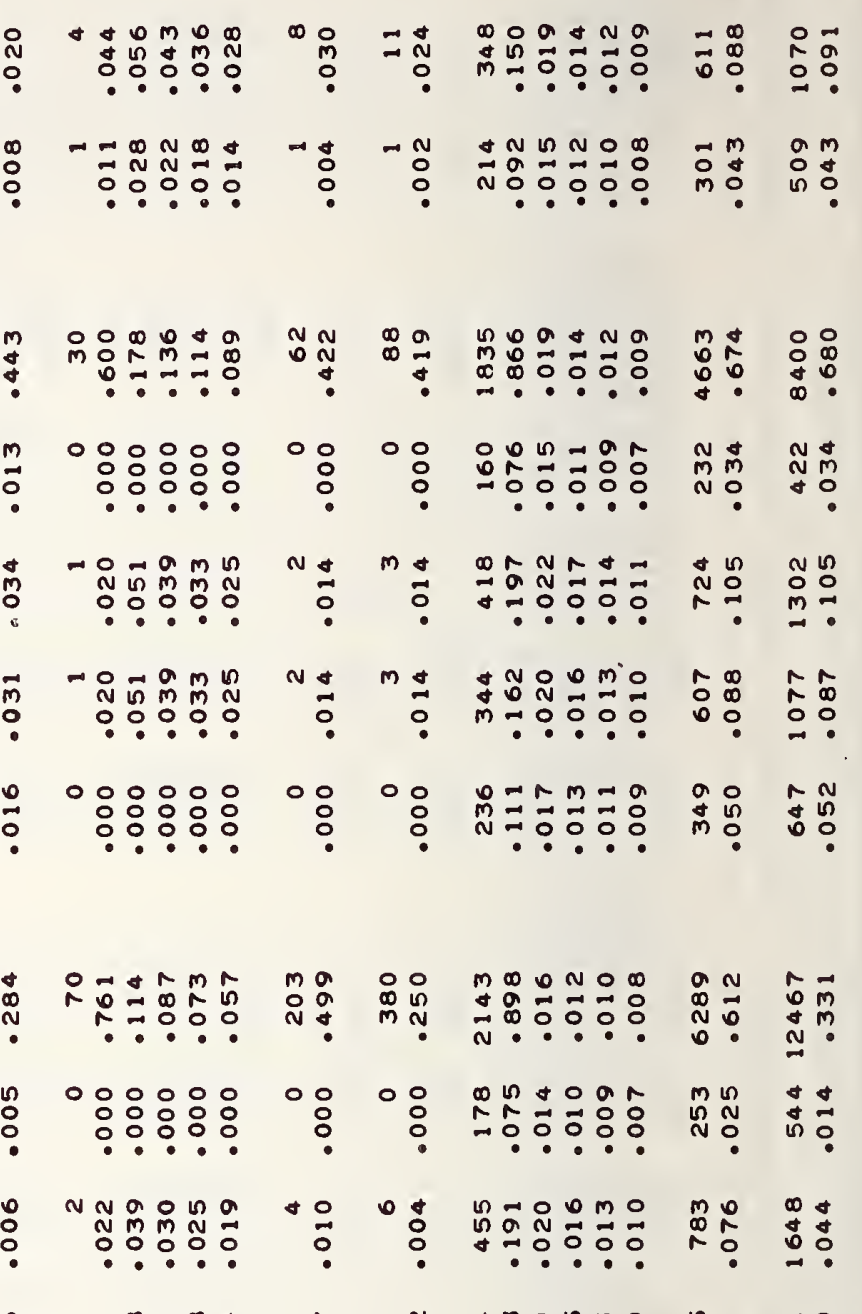

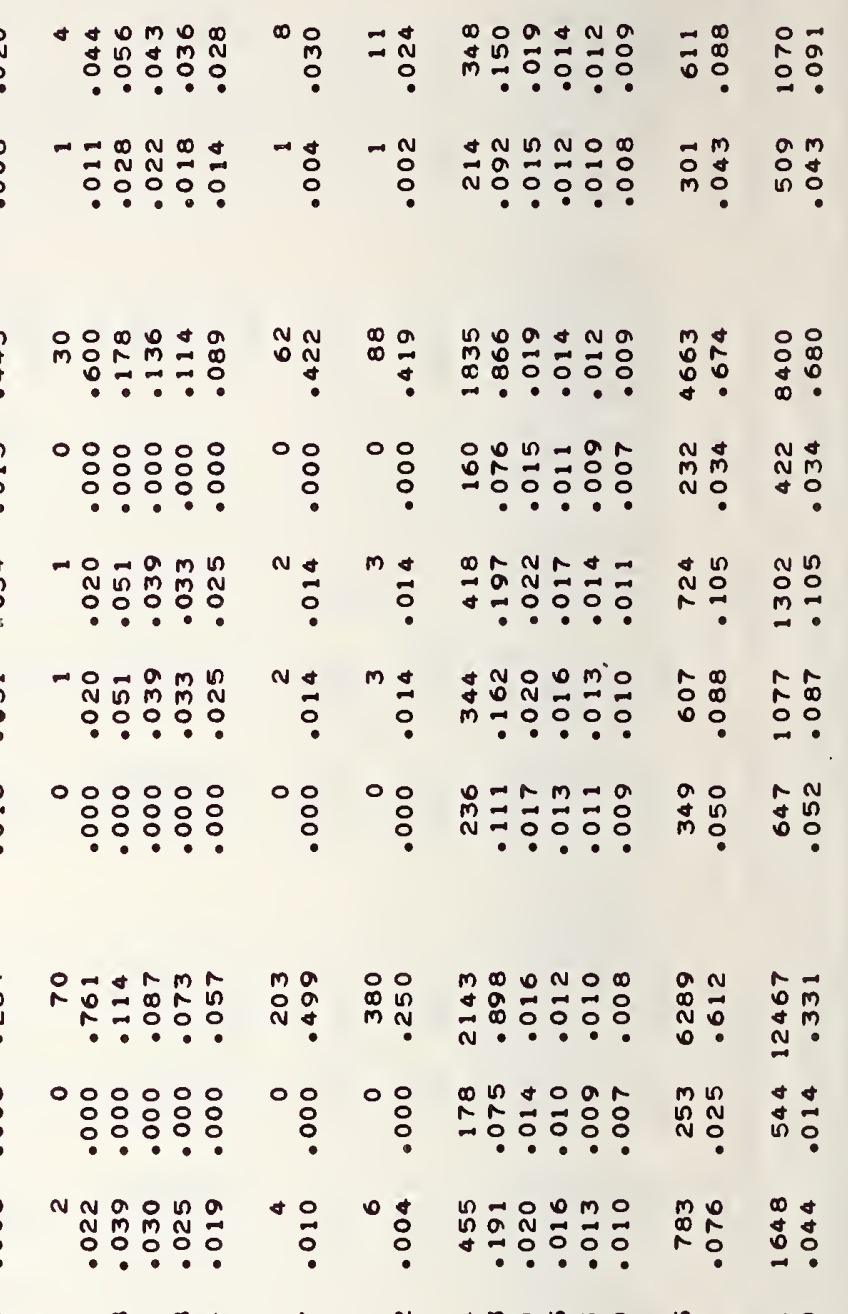

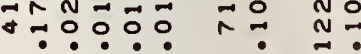

우요

$\stackrel{\infty}{\infty}$

in

in $\sim O O N=N a$

N $\div 0 \% 0$ $\bullet$ $a$

$\stackrel{0}{\circ} \div$

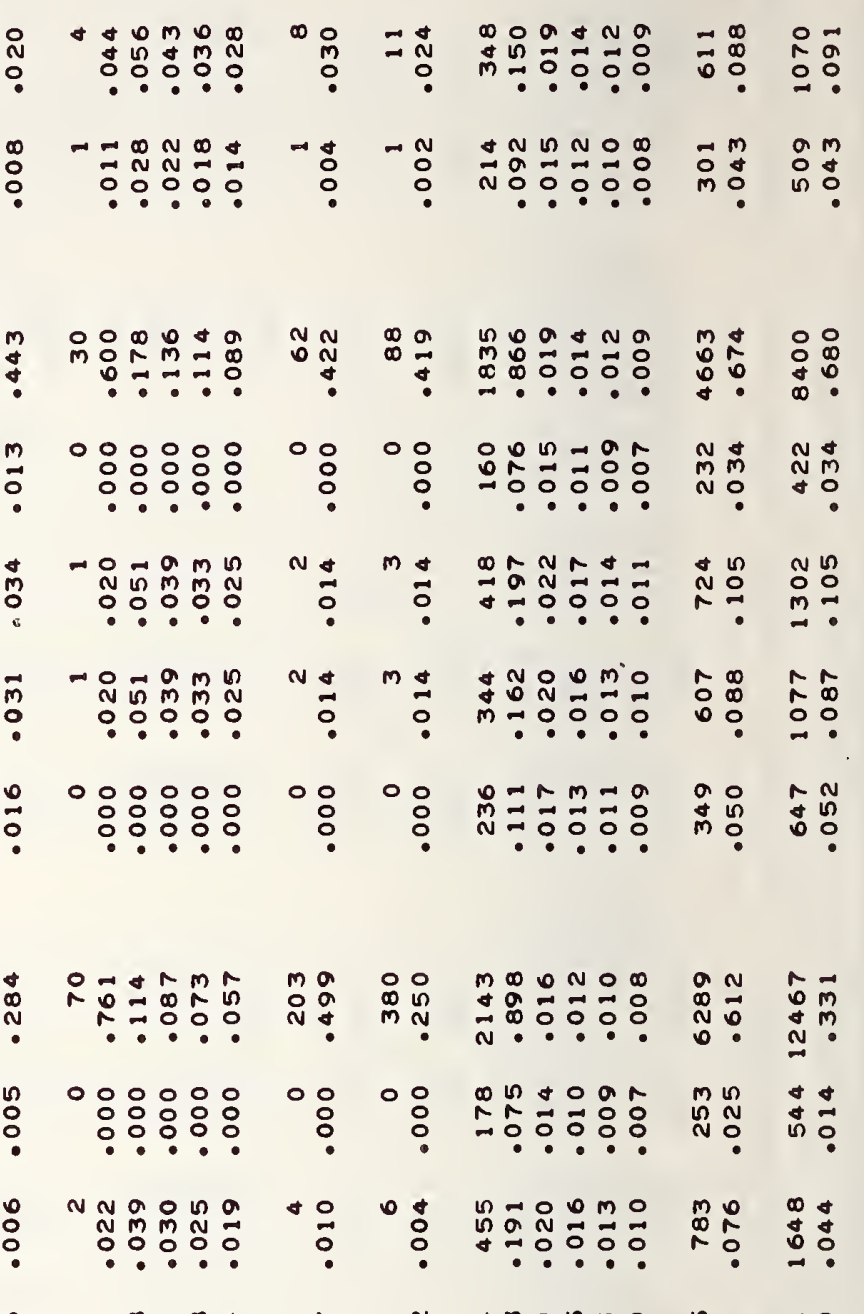

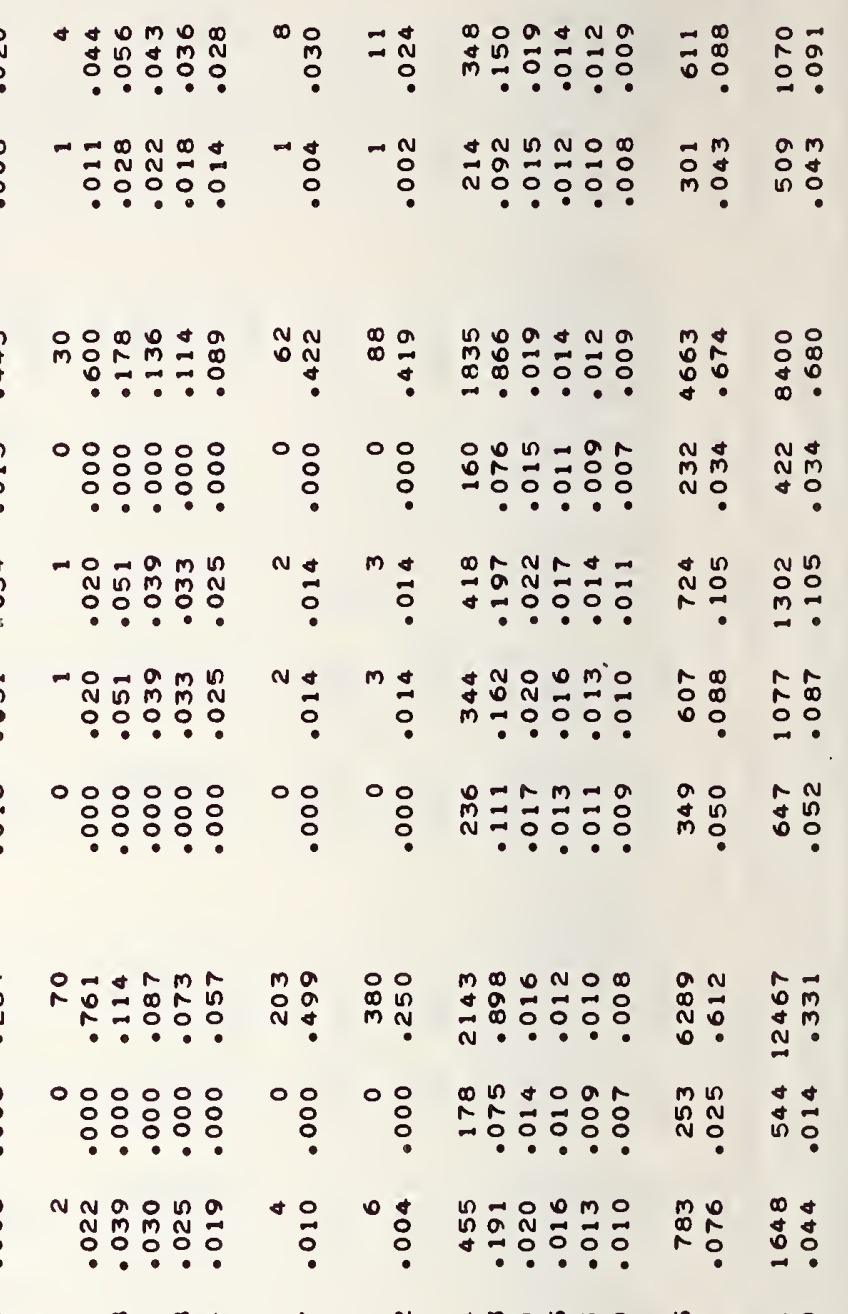

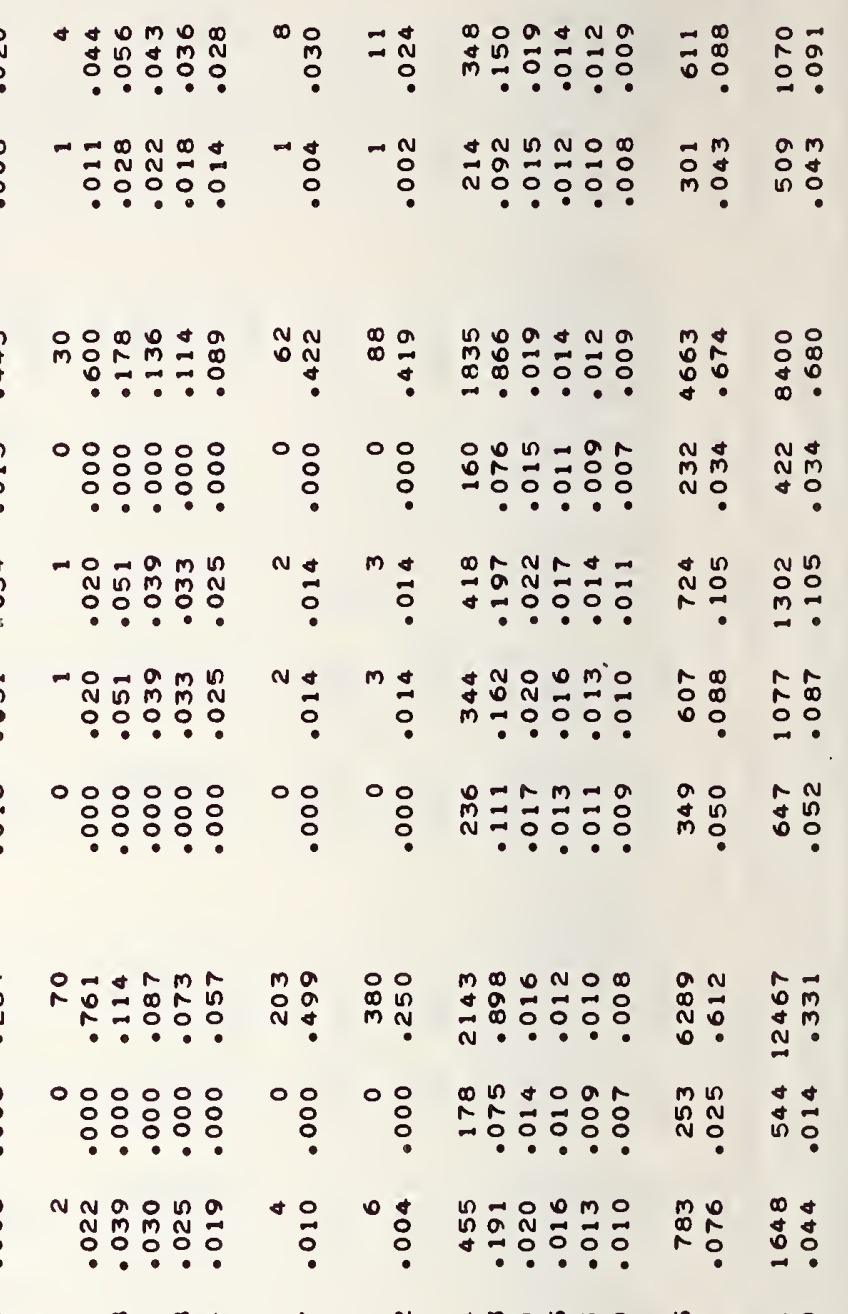

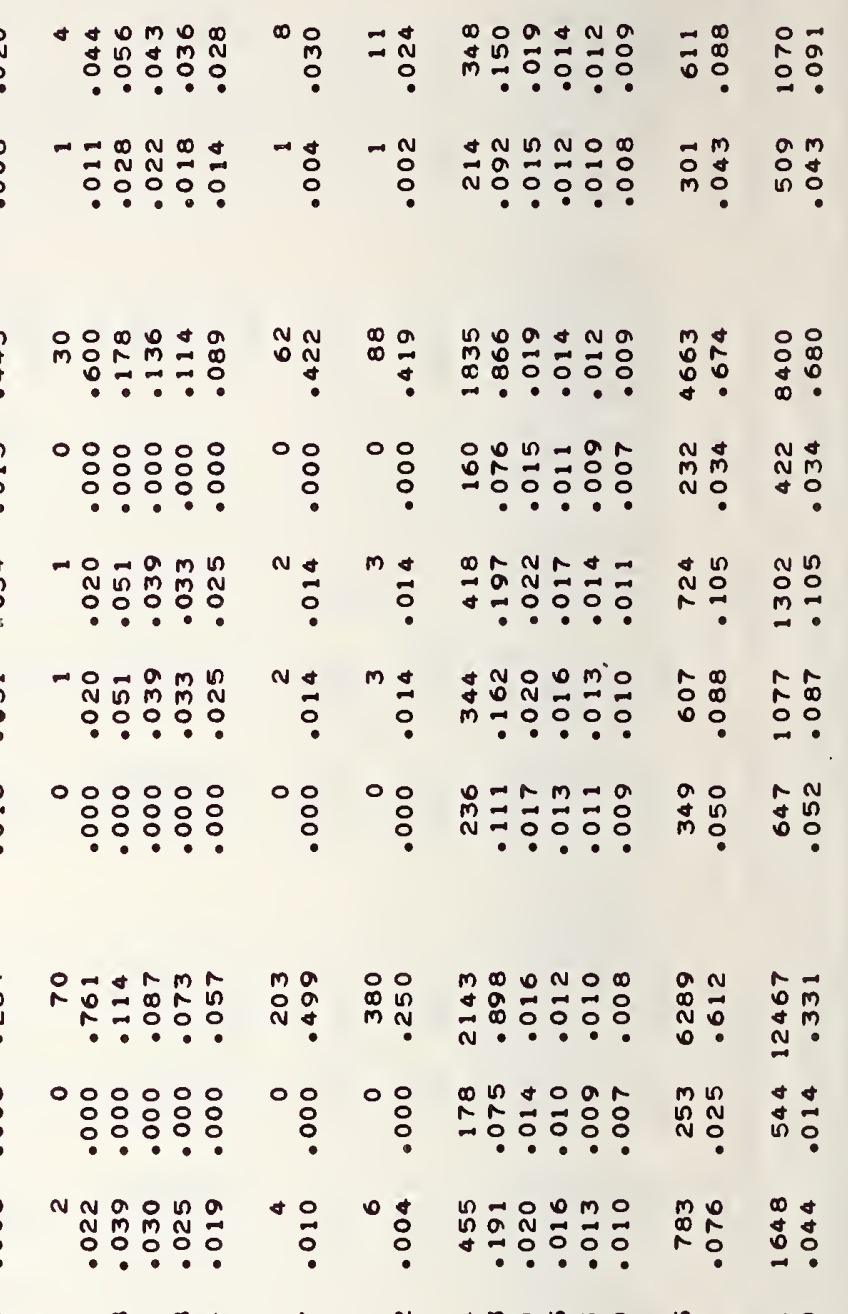

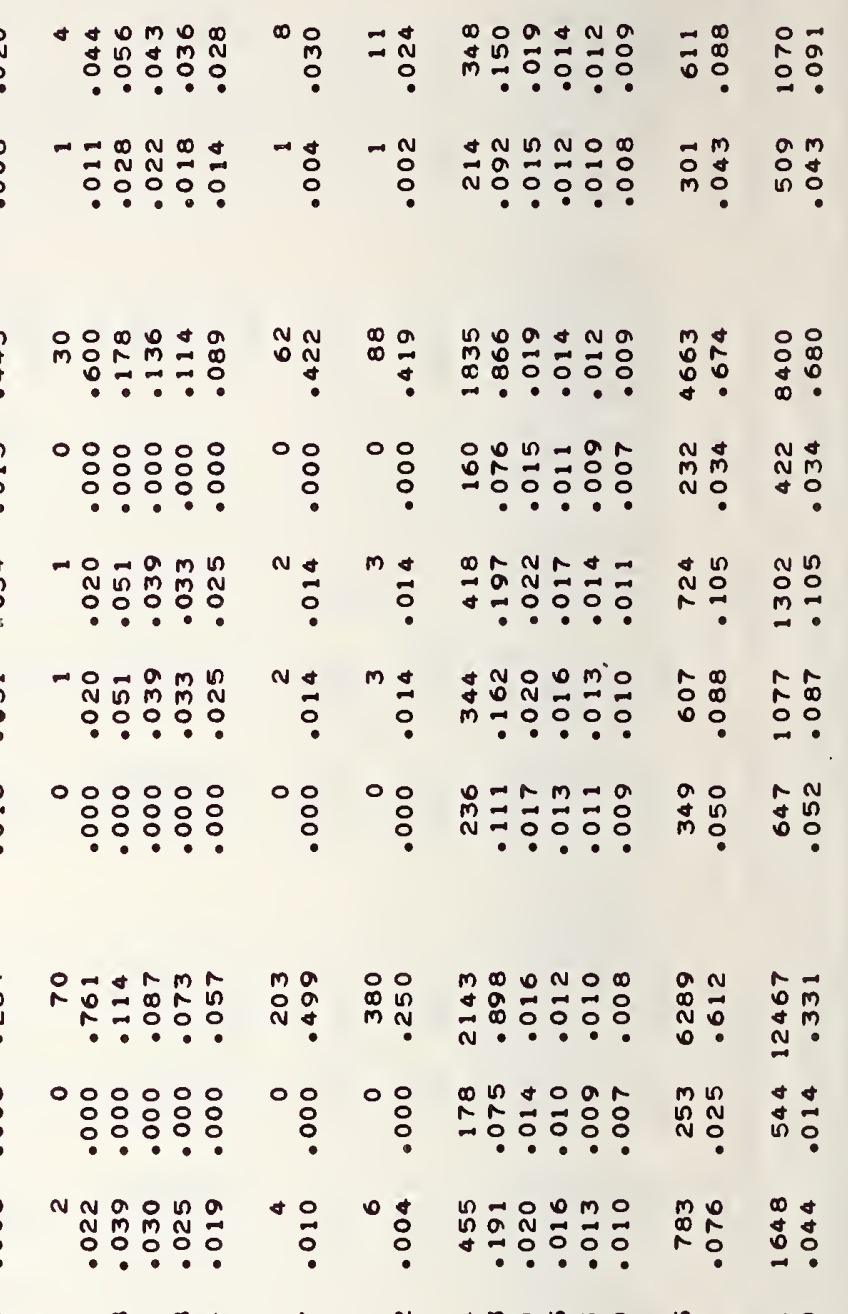

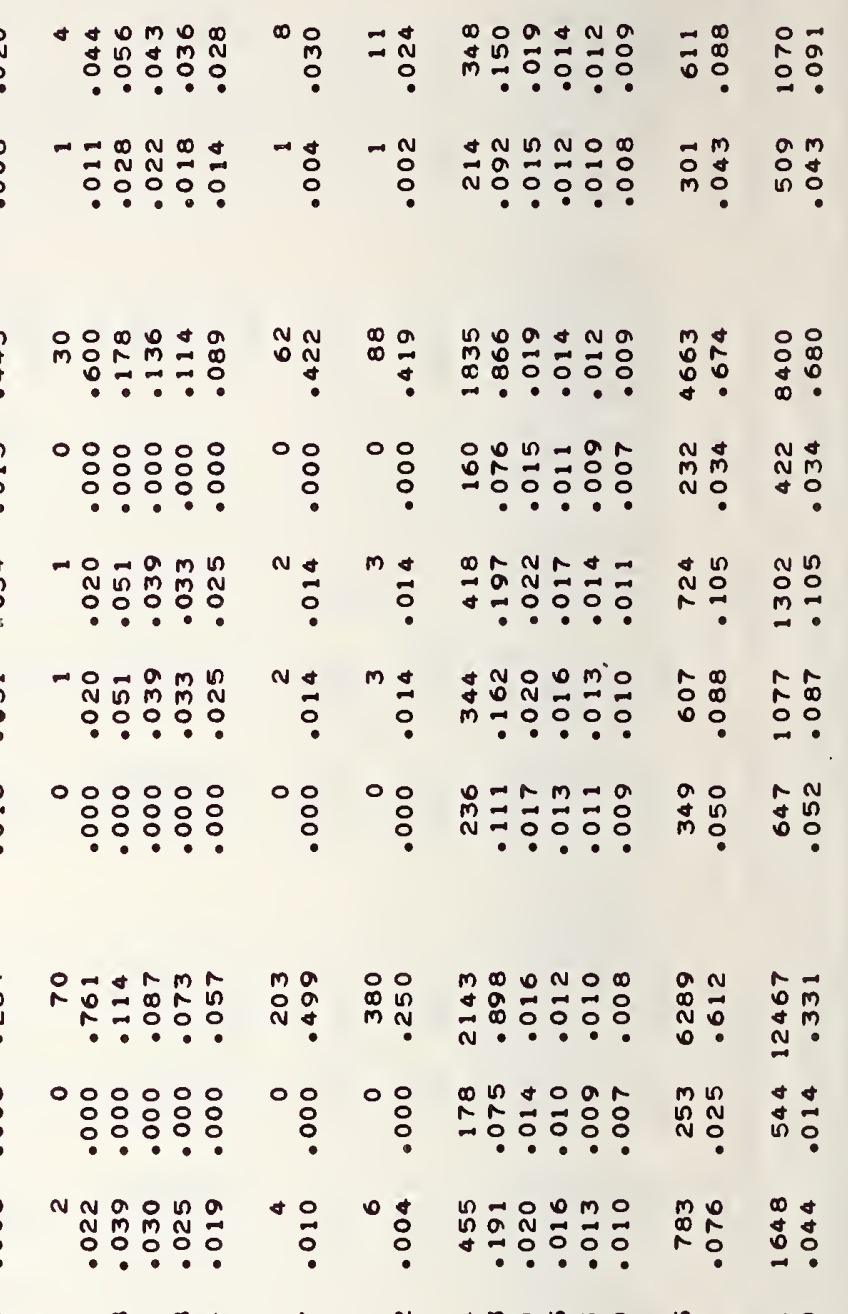

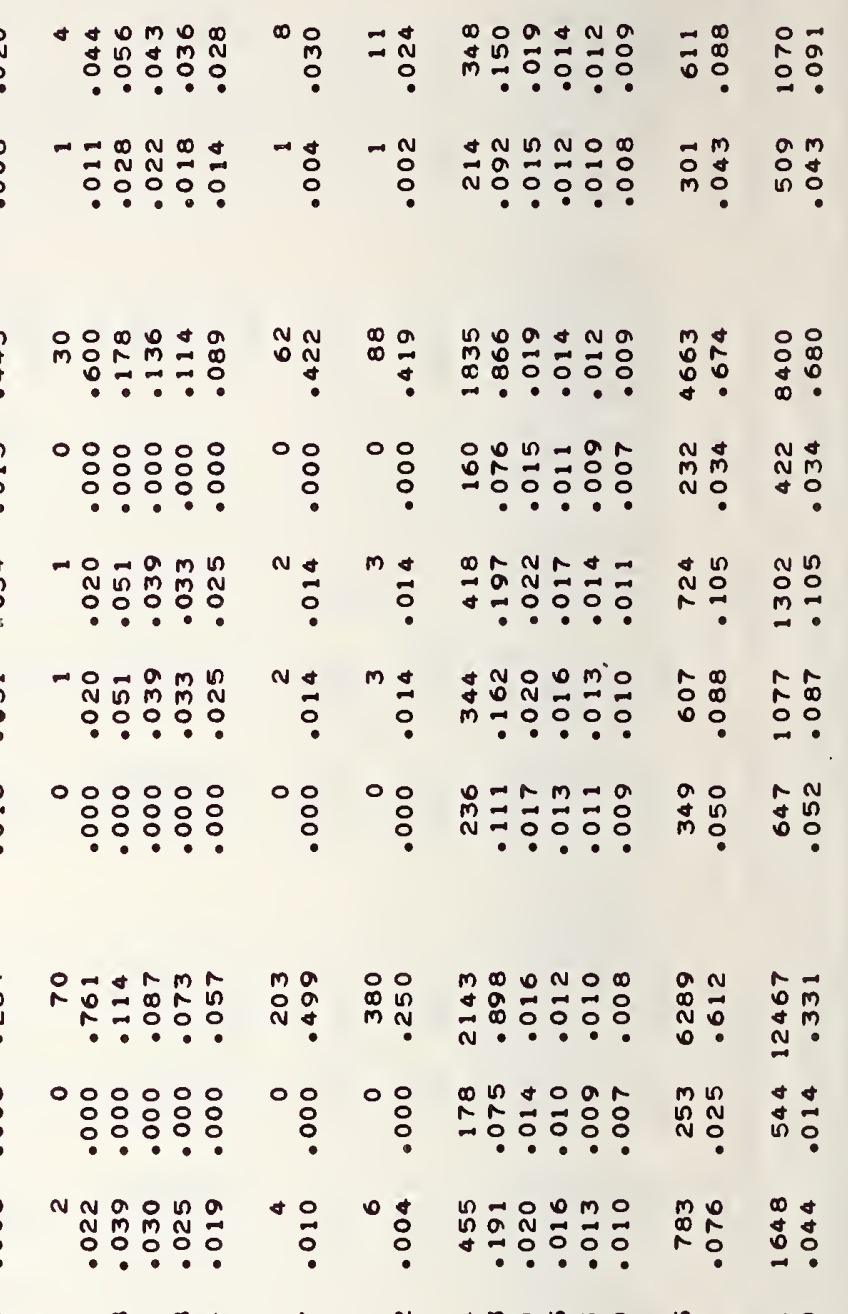

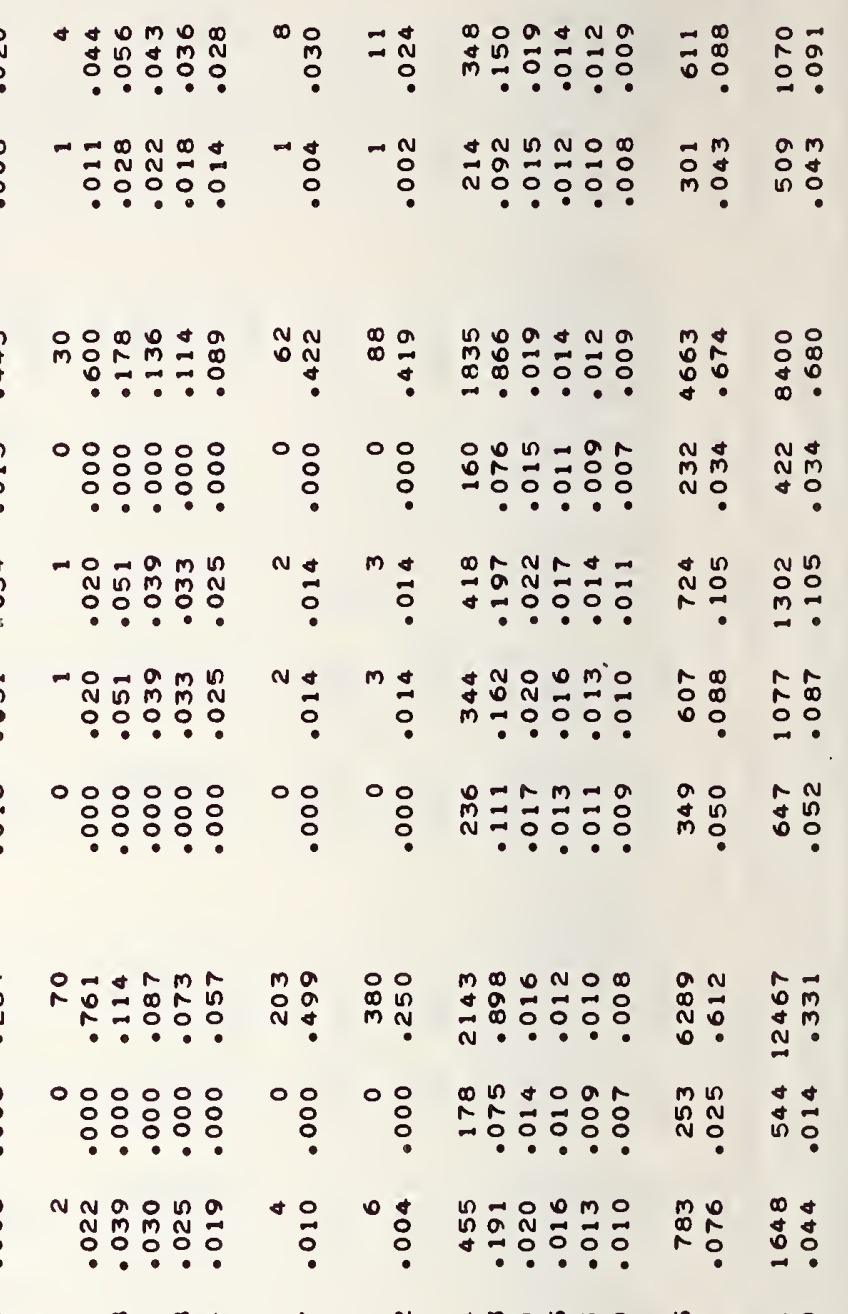

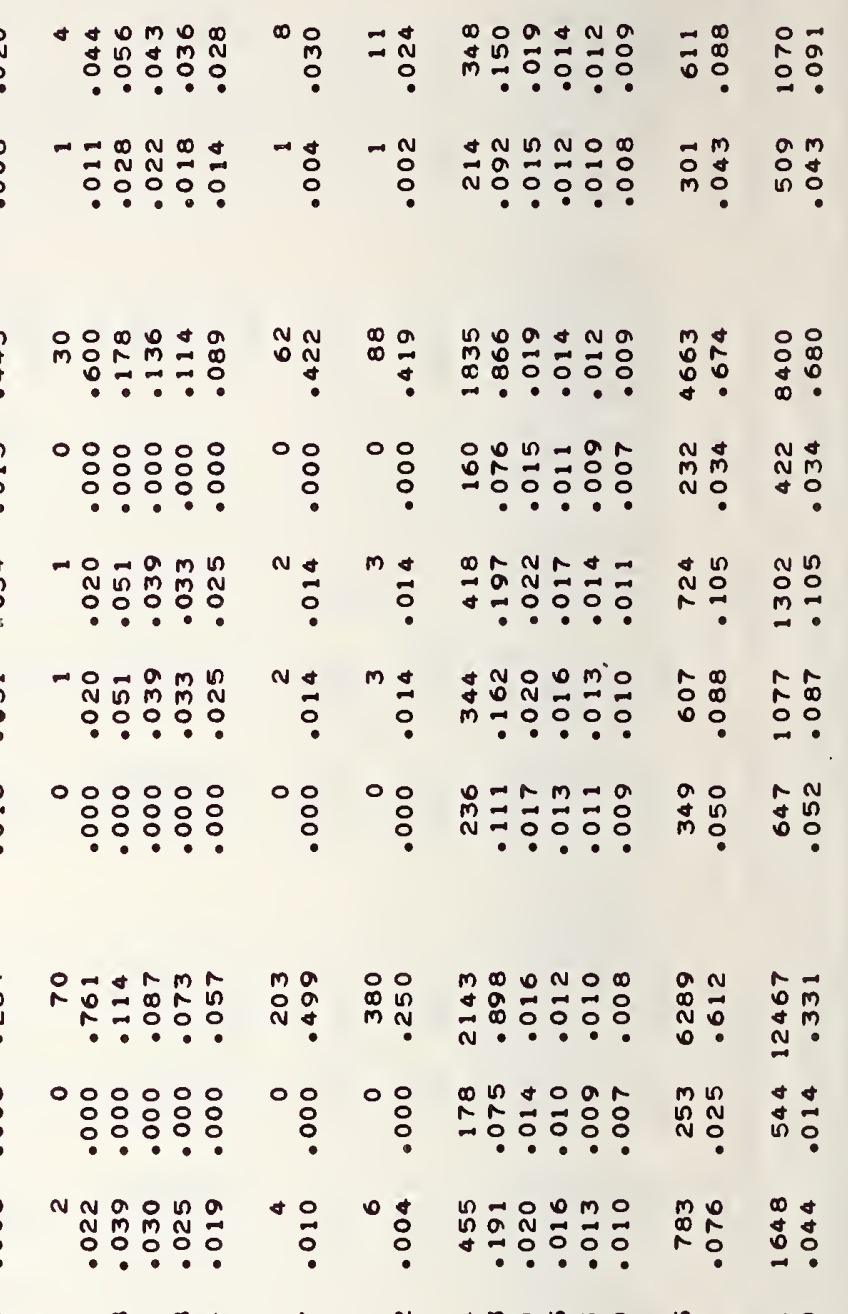

* $m g \infty+m a$

$0 \pi$

* 융ㅇㅇ

$\stackrel{\substack{m \\ \sim}}{q}$

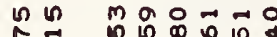
*

*

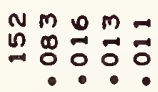

ล

品 n

?

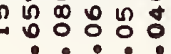

m

Nom

N N N N

等

$\infty \infty$ in a 0

要崖

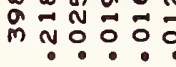

ำ $M=$

Mㄴ.

* ๙

*

*

*

n mo in $\mathrm{m}$

N N

0

$\underset{0}{N}$

尔

$\stackrel{n}{\sim} \hat{a}$

a d

ำ

$m \neq m \infty$

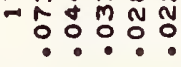

a.

$\frac{0 m}{0}=$

$\stackrel{\circ}{\circ}$

$\ln$

$\begin{array}{llll}0 & 0 & 0 & 0 \\ 0 & 9 & N & N \\ 0 & 0 & 0 & 0\end{array}$

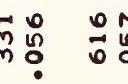

a a n n -0

$0 N$
$m$
$m$
0
ลก

$\stackrel{2}{\infty}$
$\stackrel{N}{N}$

$\div$

N

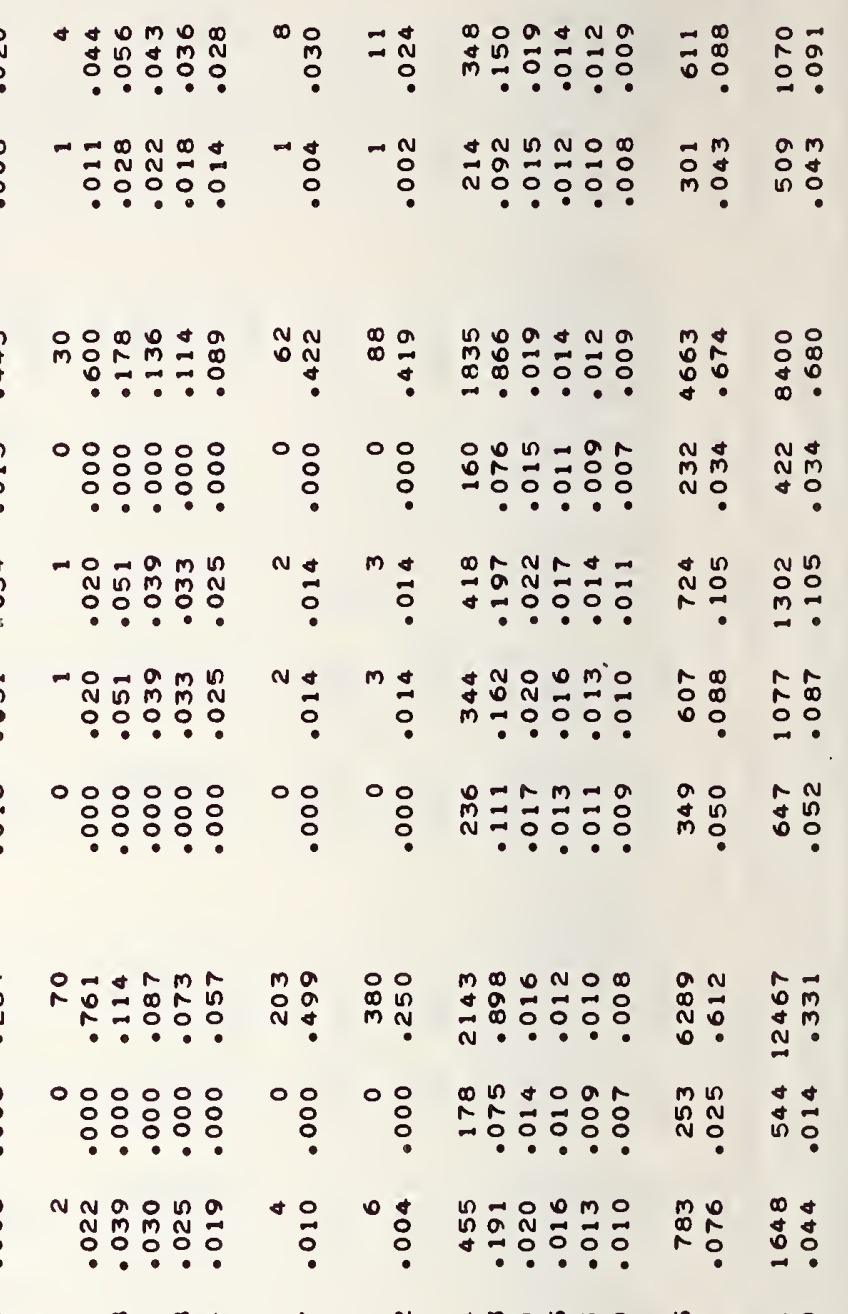

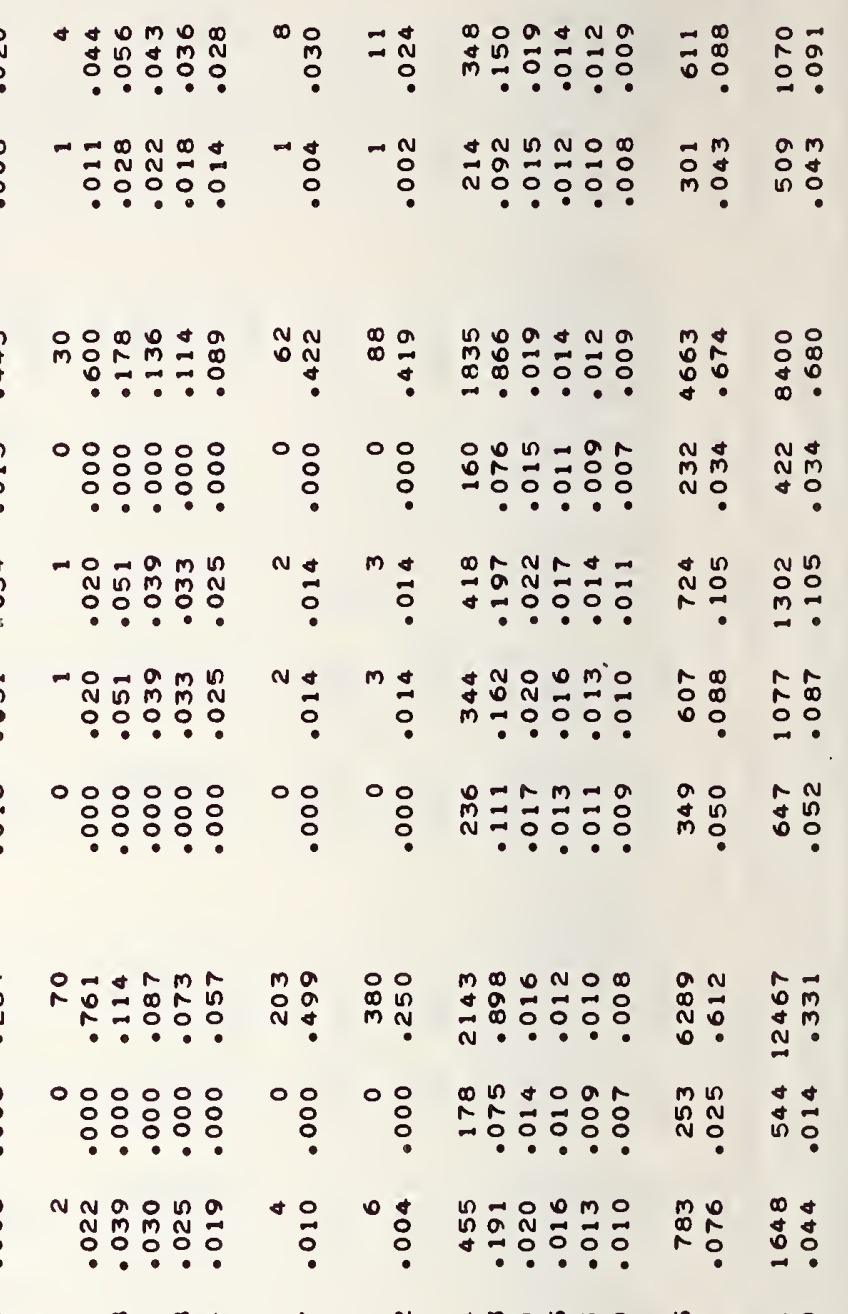

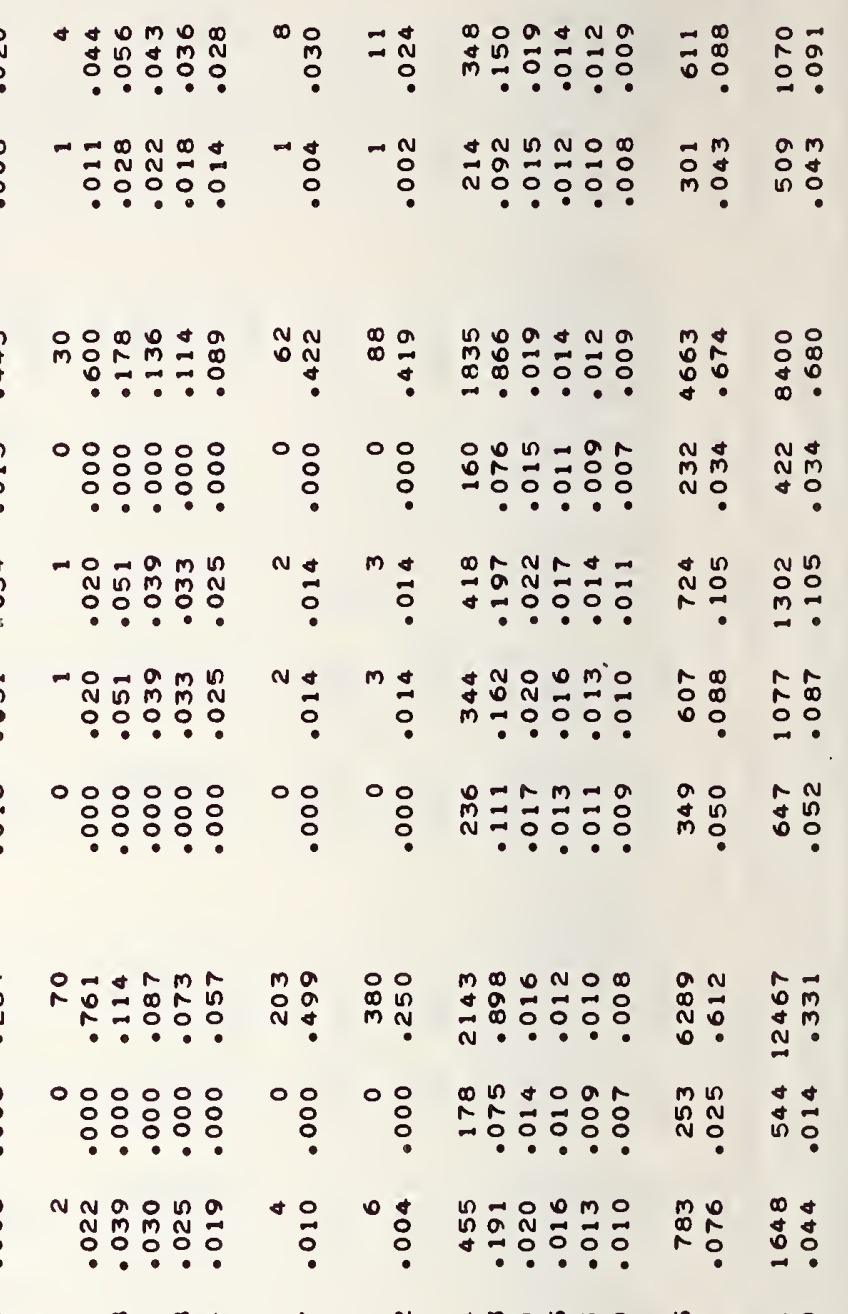

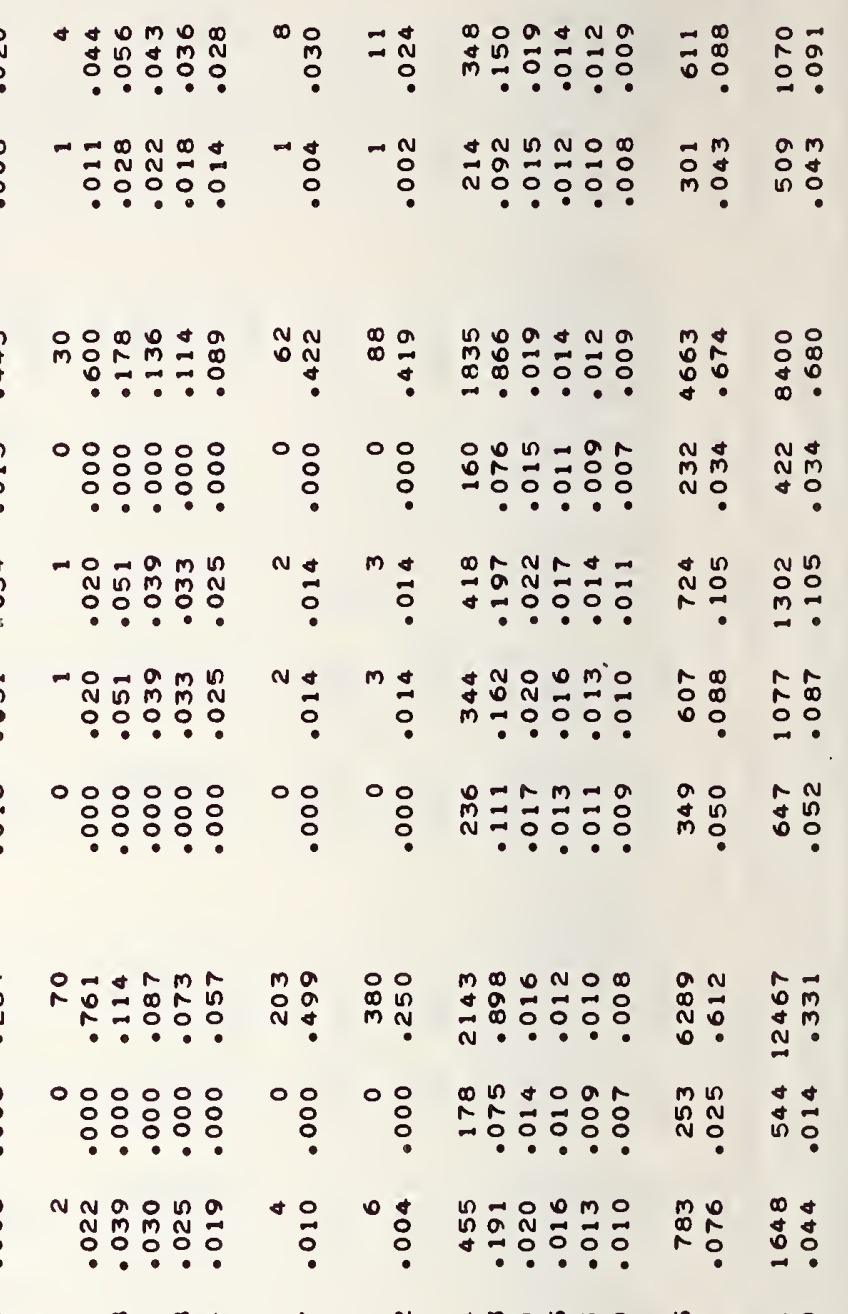

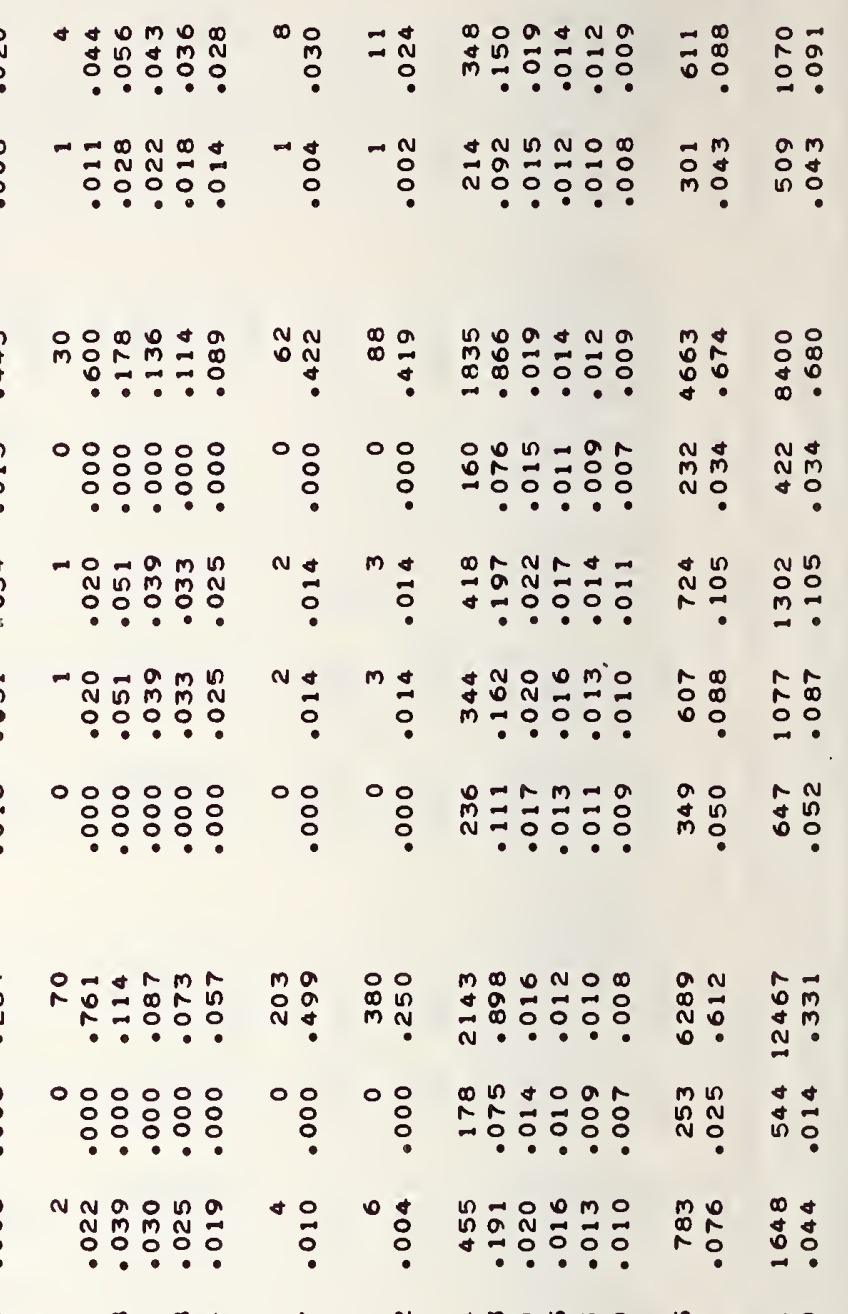

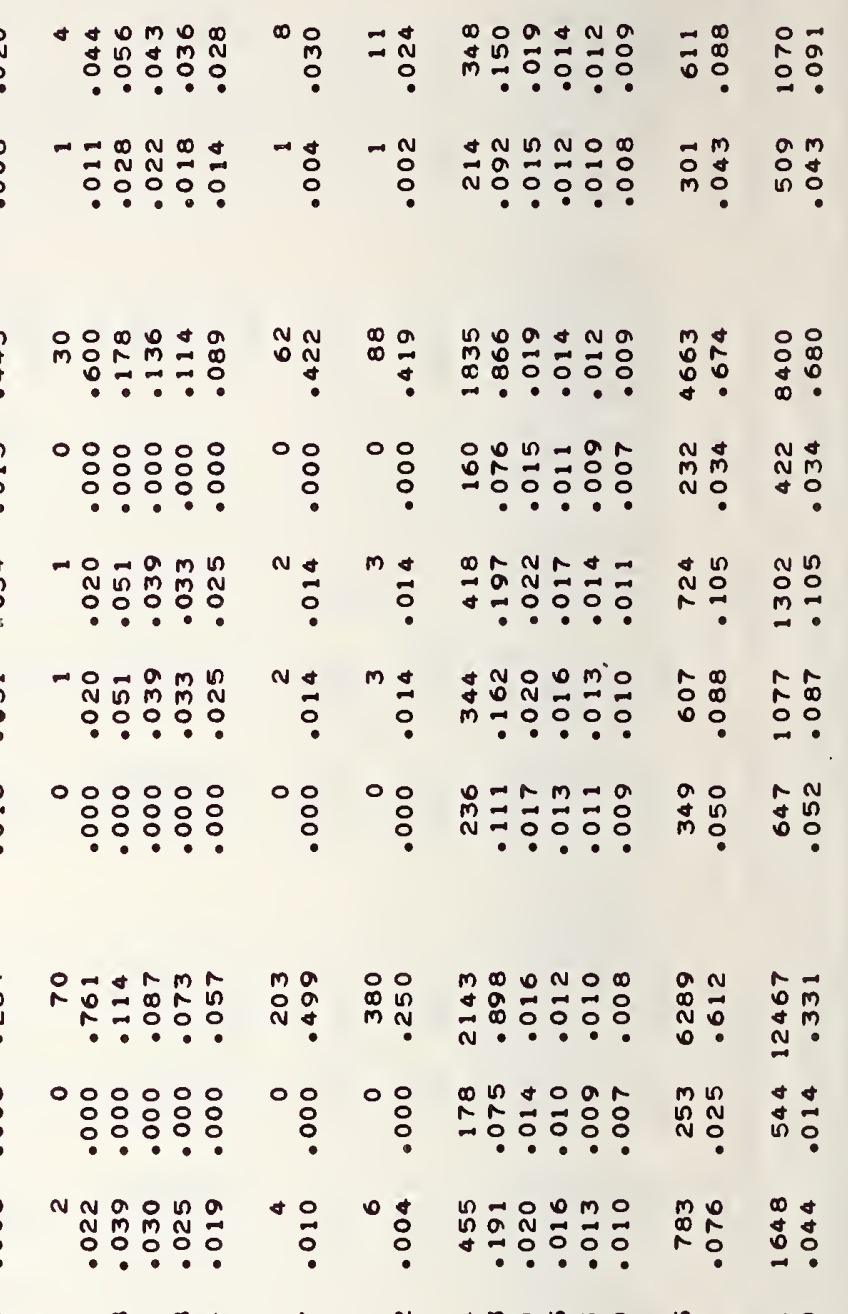

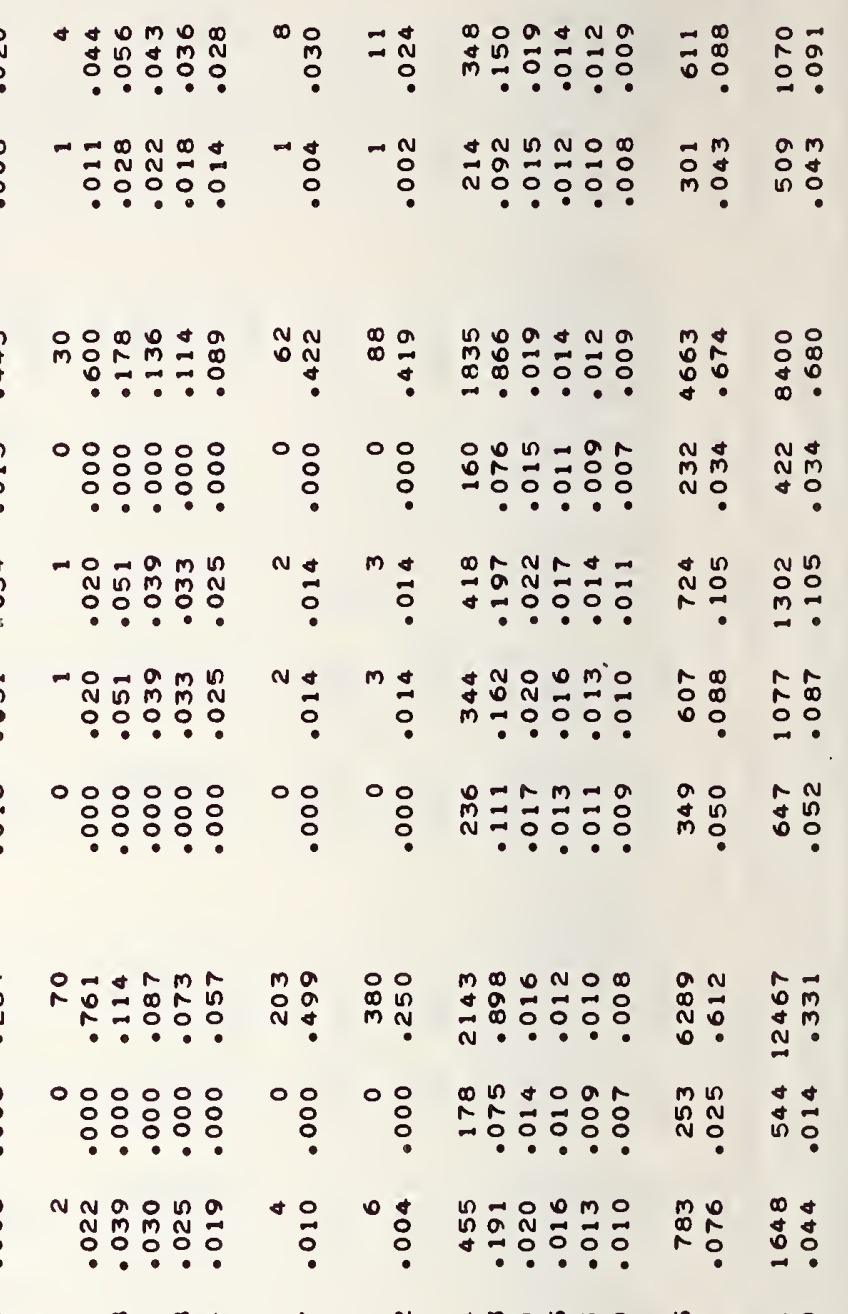

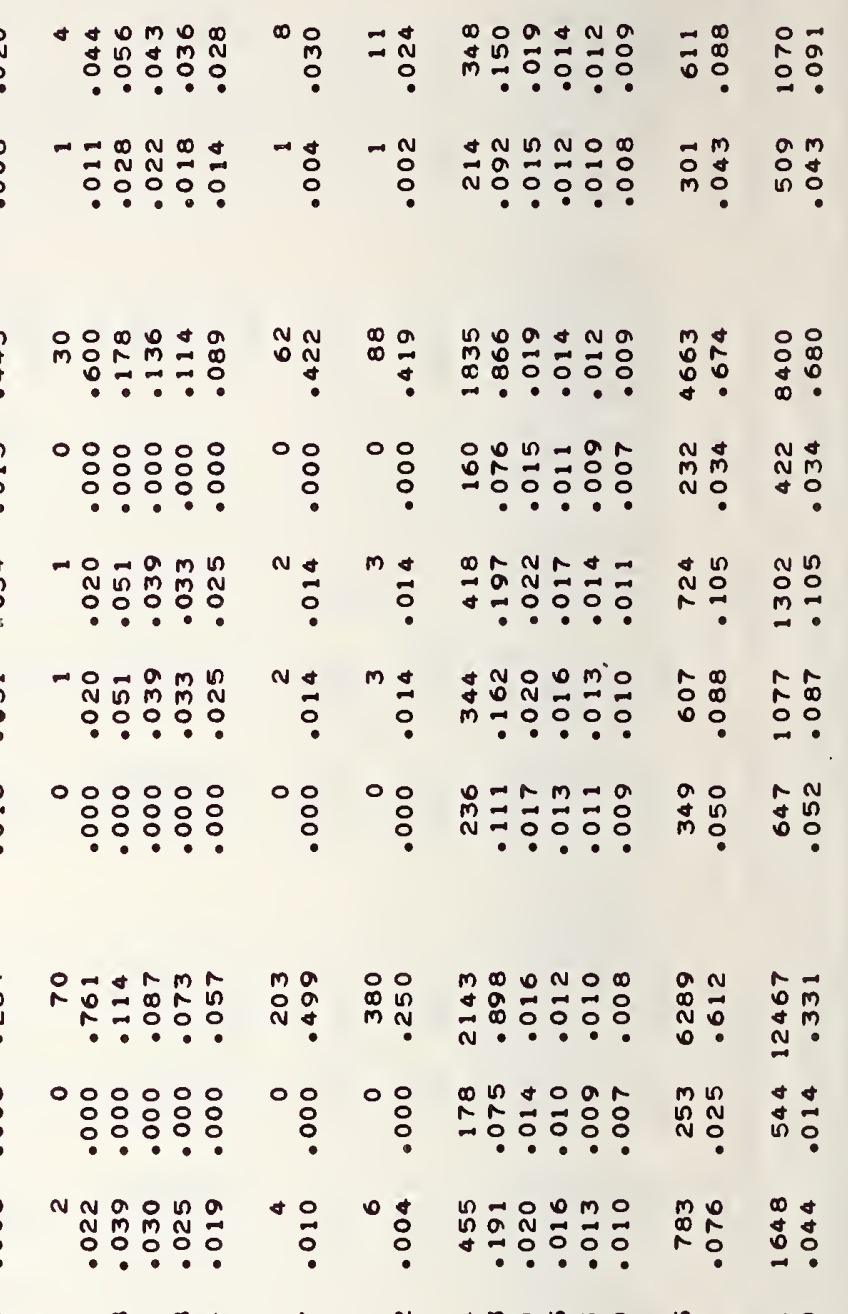

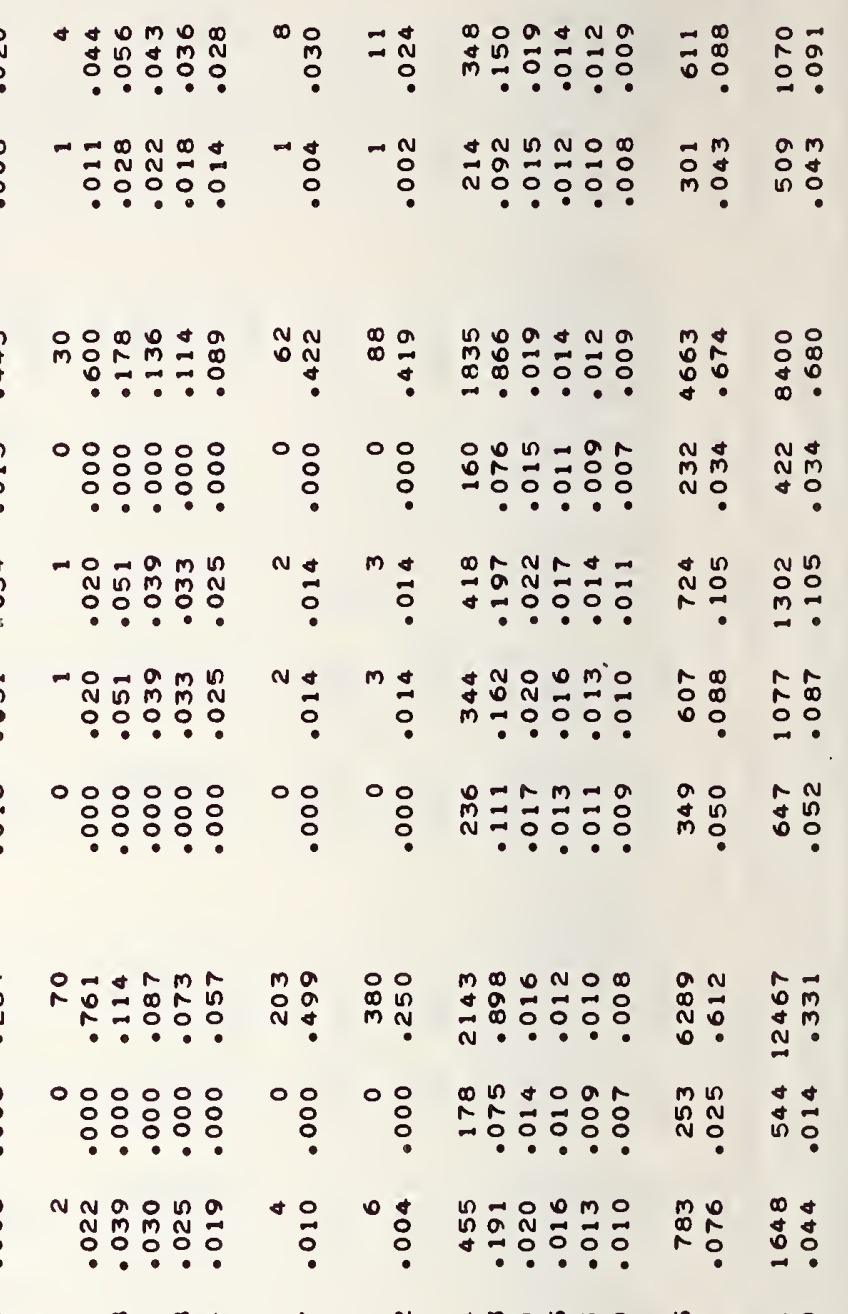

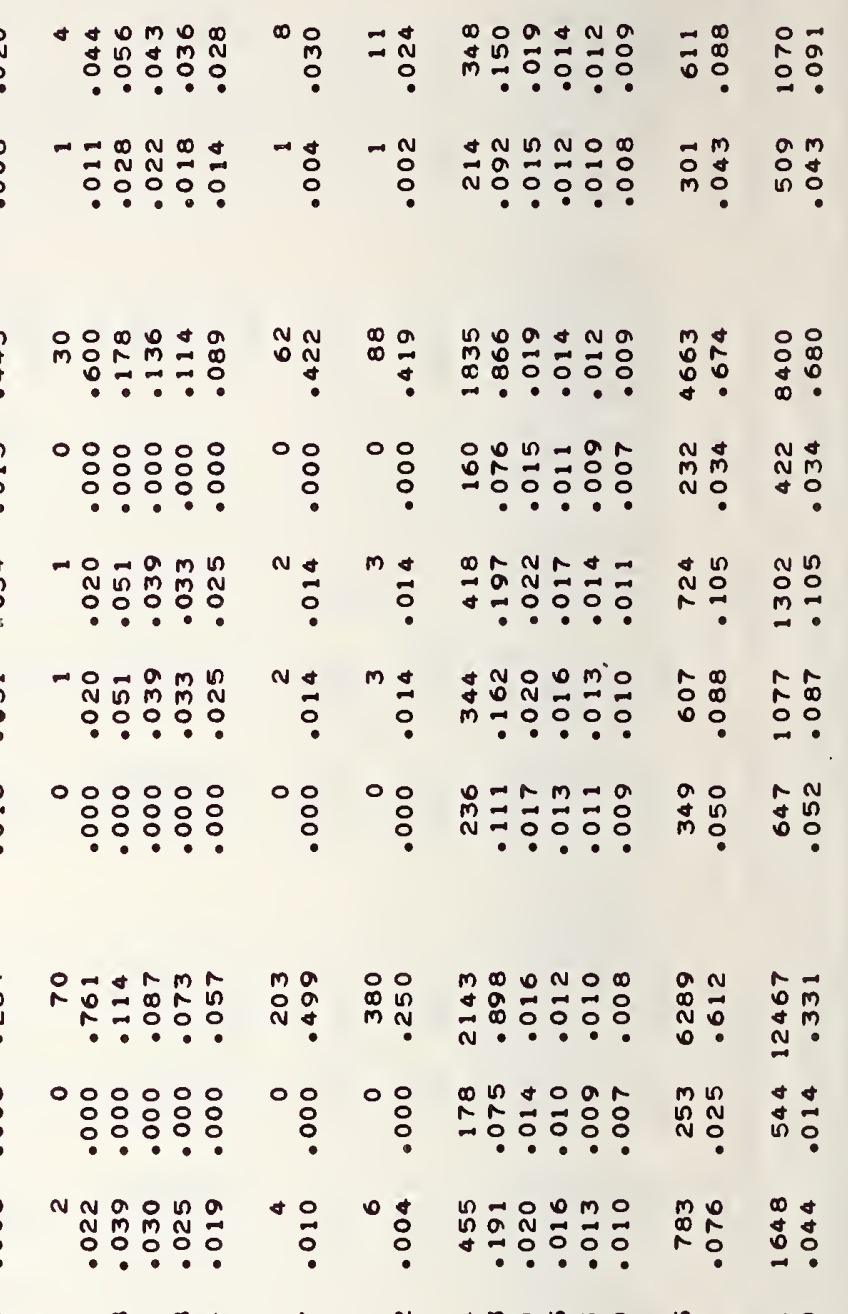

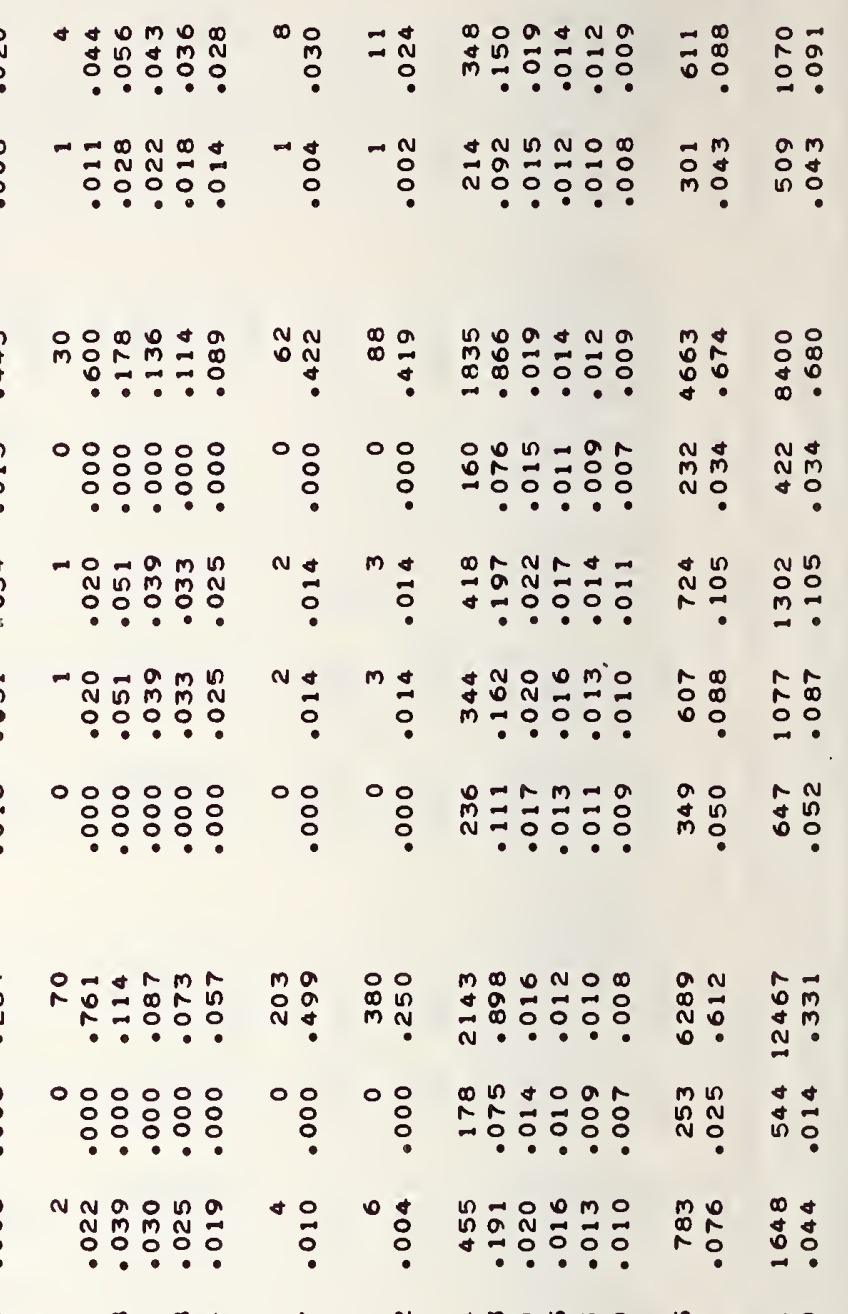

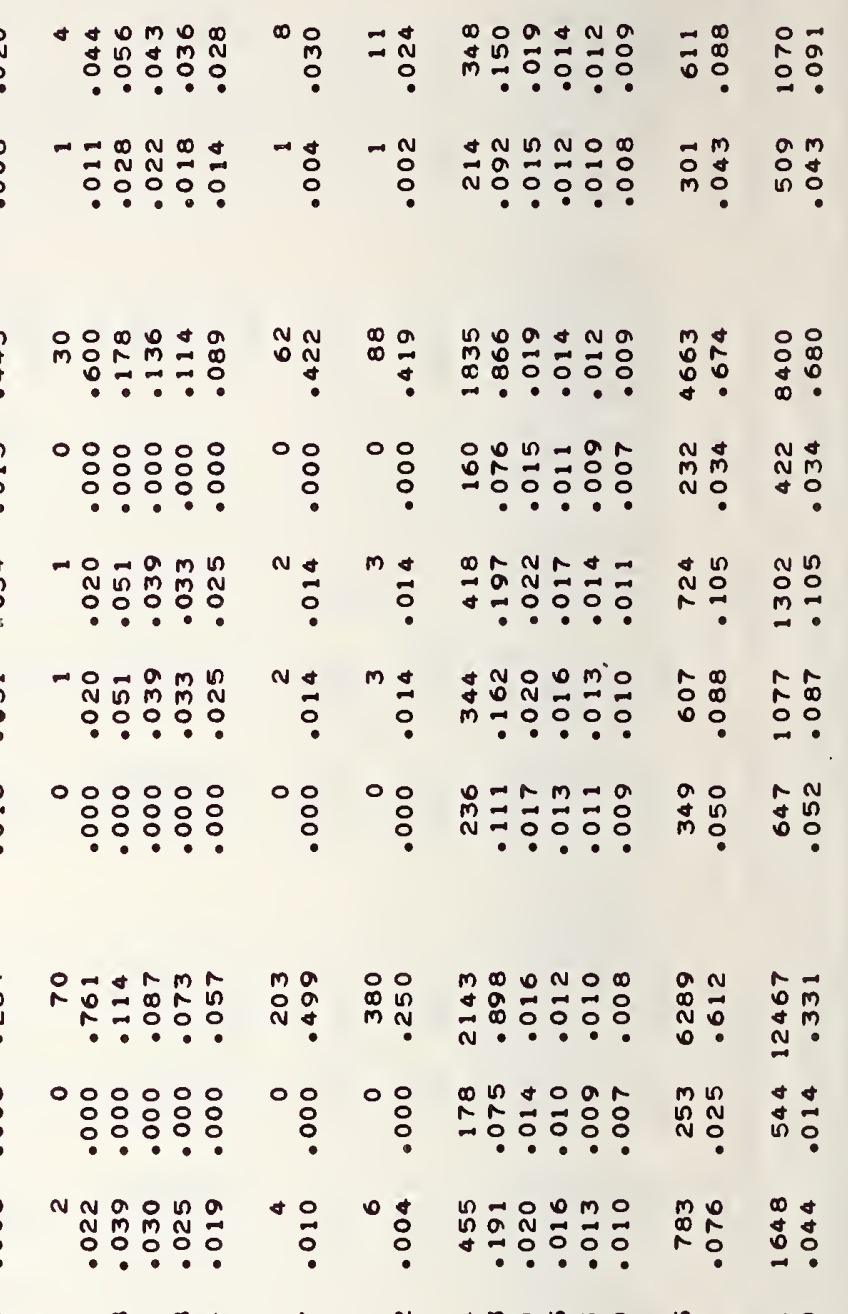

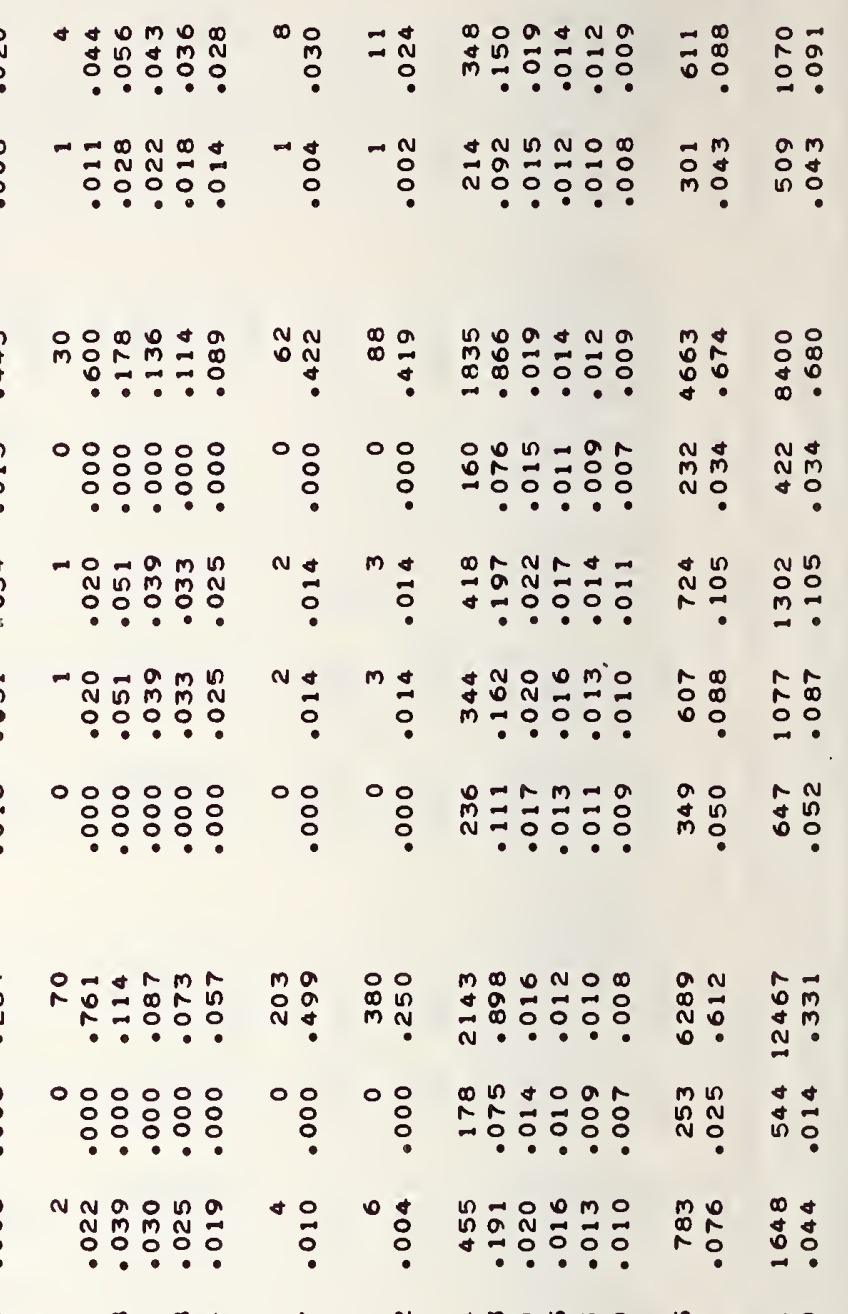

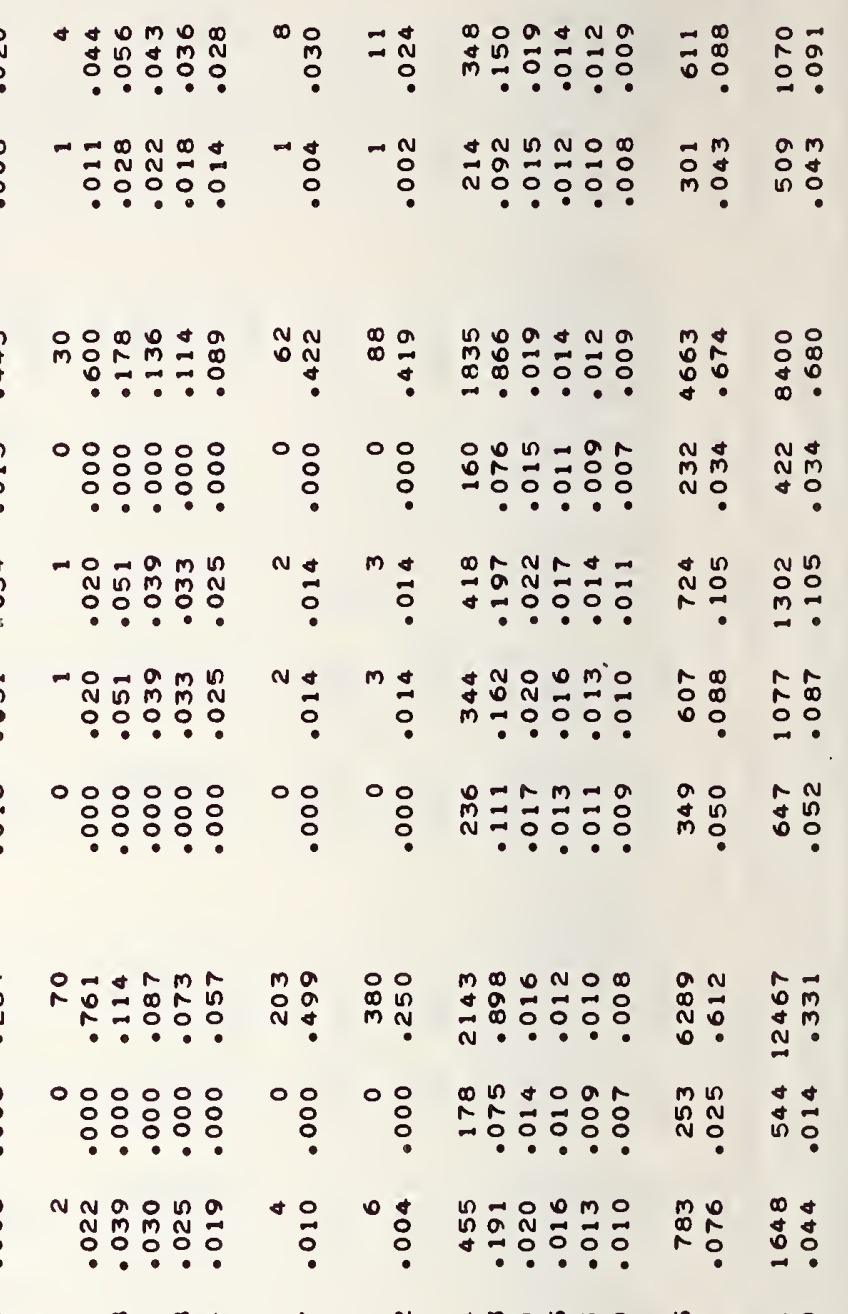

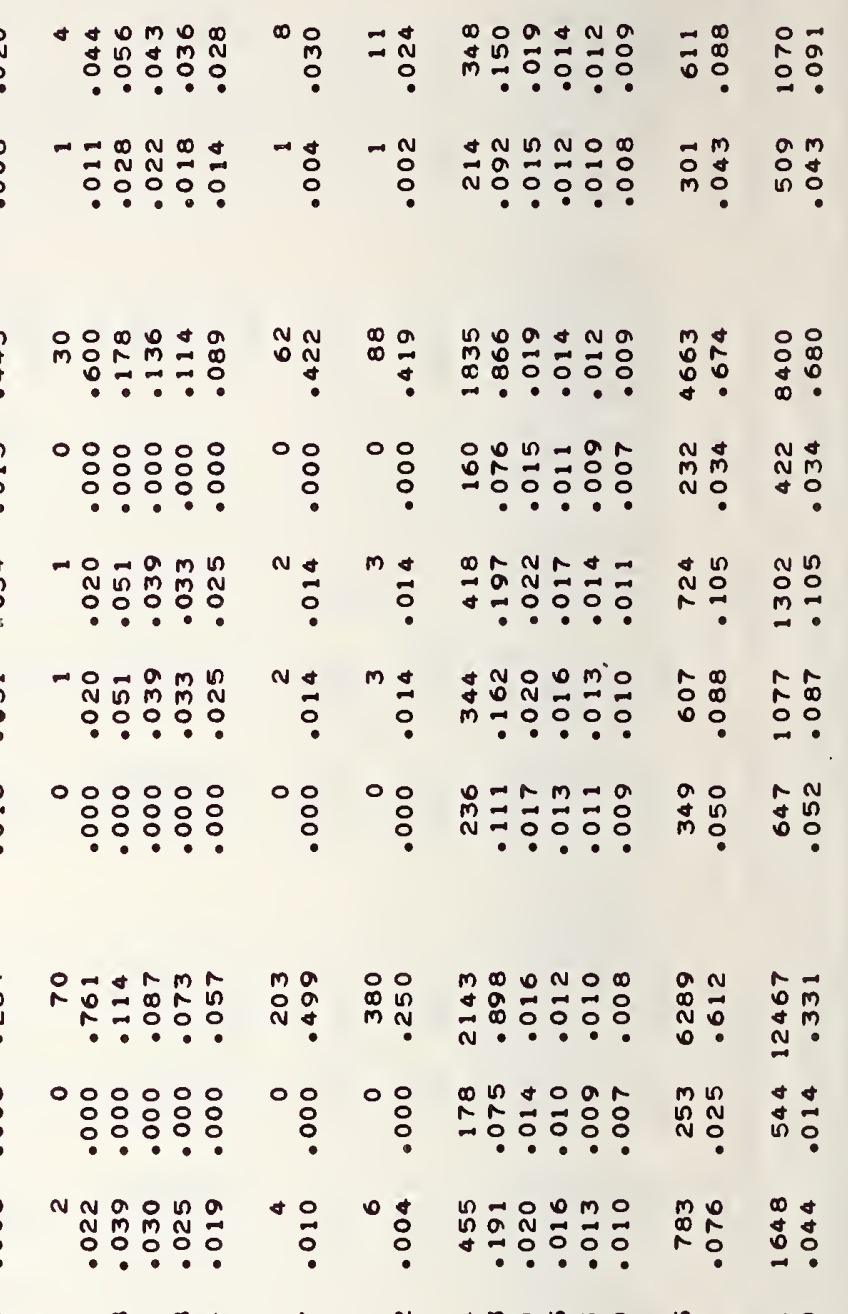

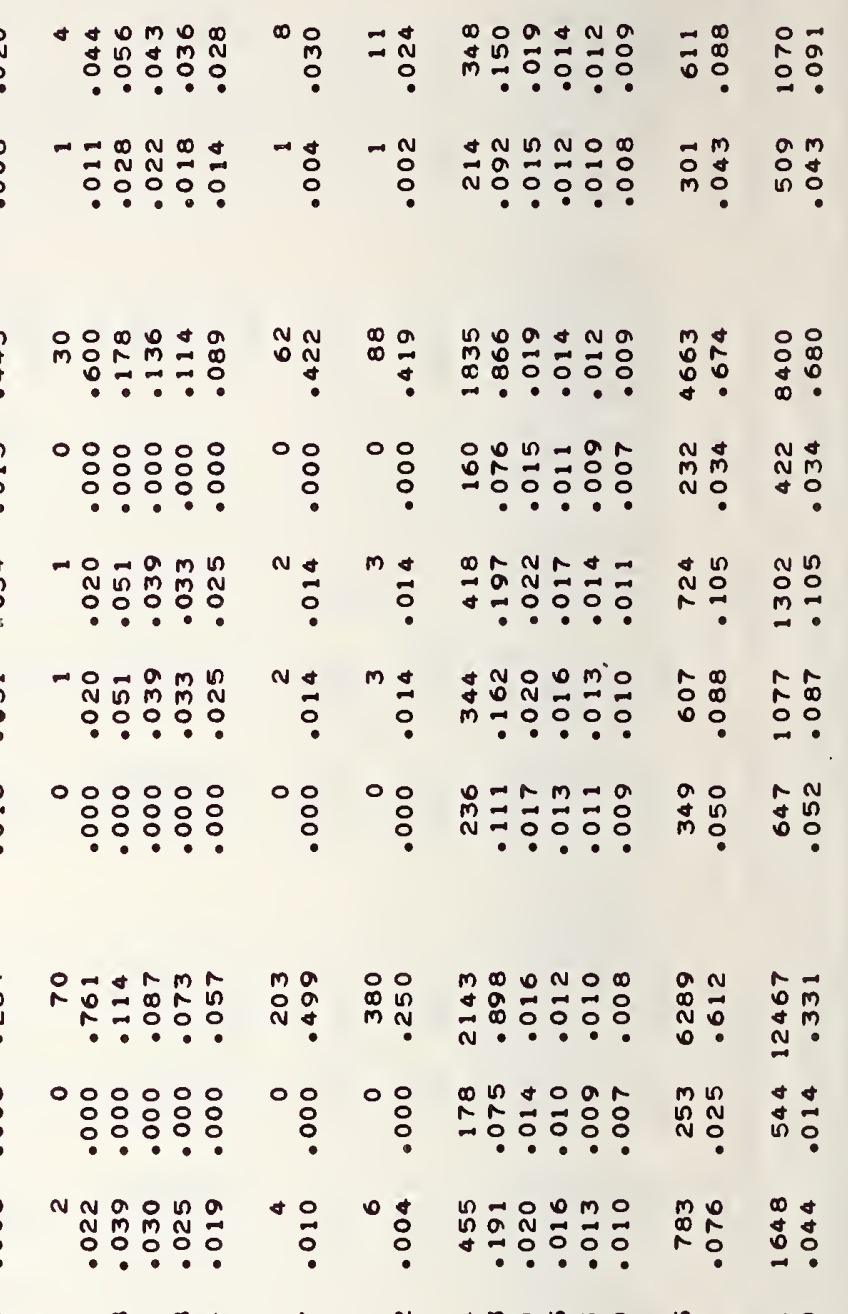

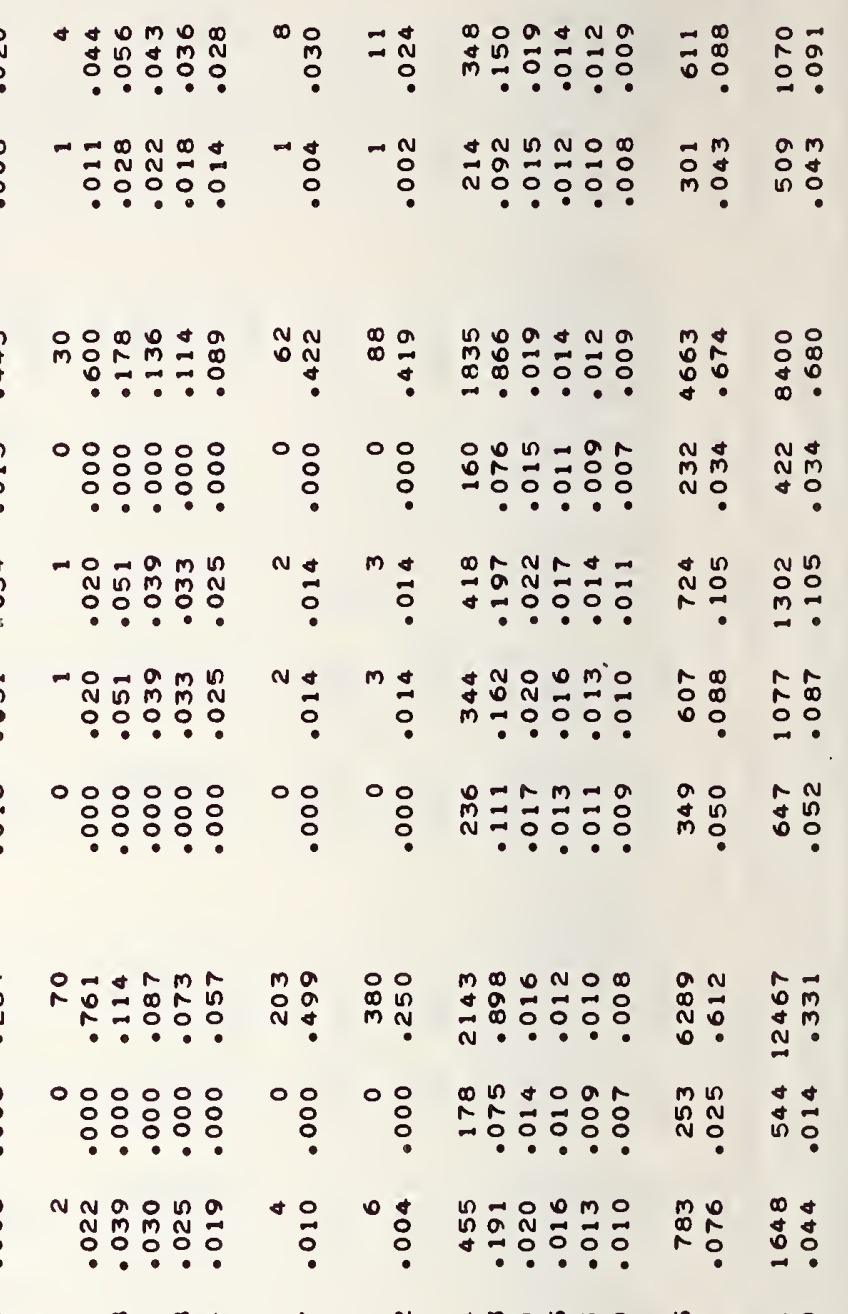

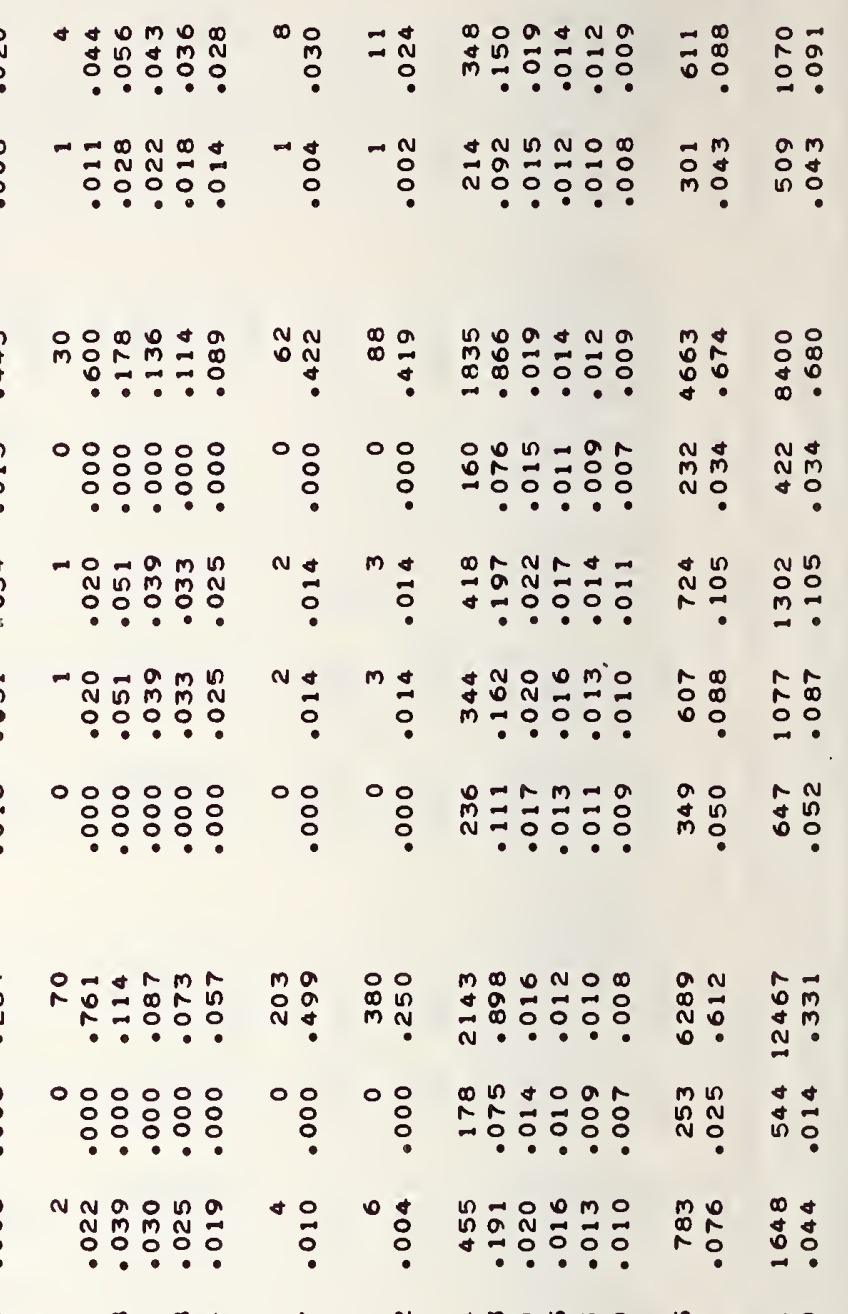

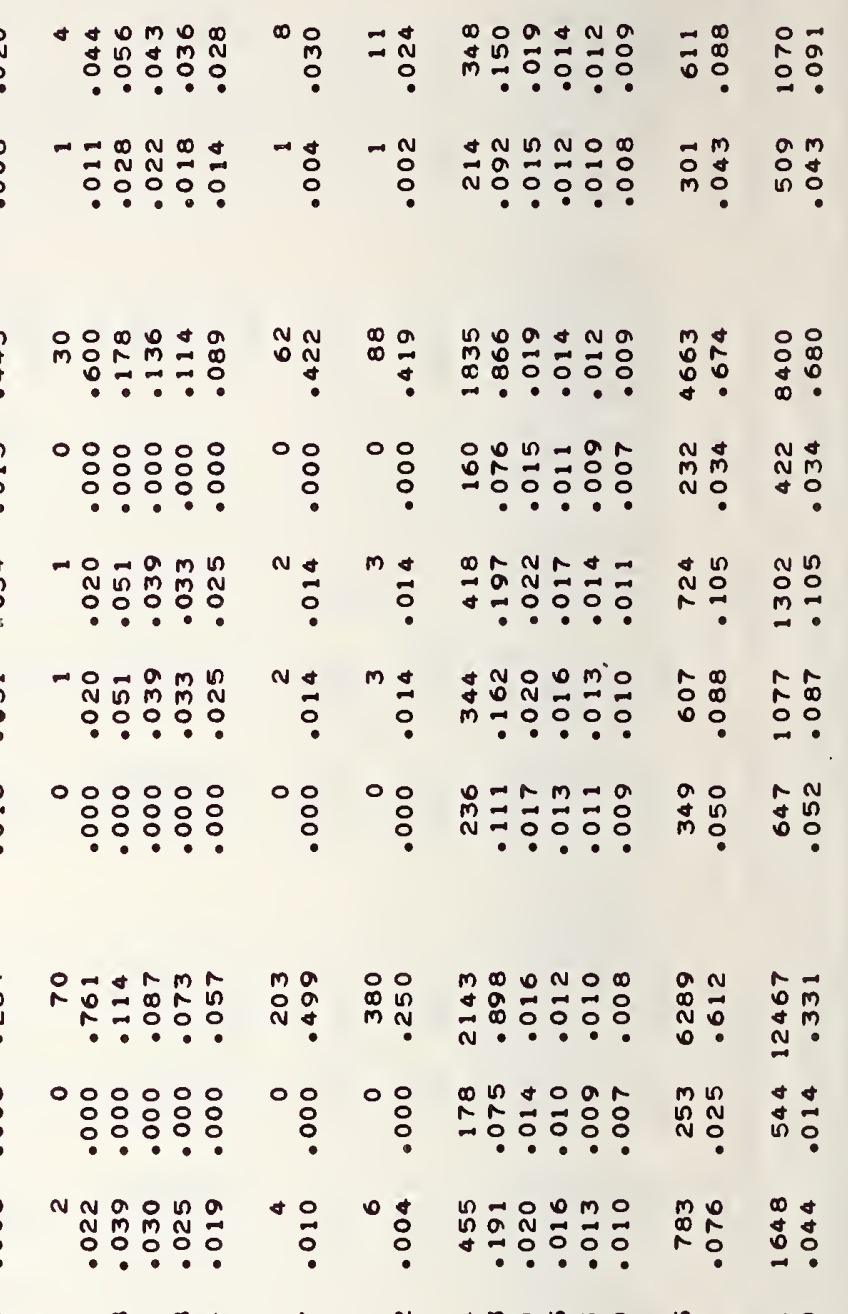

* * * * 我竞

웅

‡

* * * * * w

n n

N $=0.000$

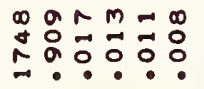

$00 m \rightarrow \infty$

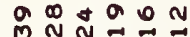

$0 \infty+\infty$ n

in a 0 in

in 40 in 0

in:

No m

mo:

$\stackrel{9}{N}$

in

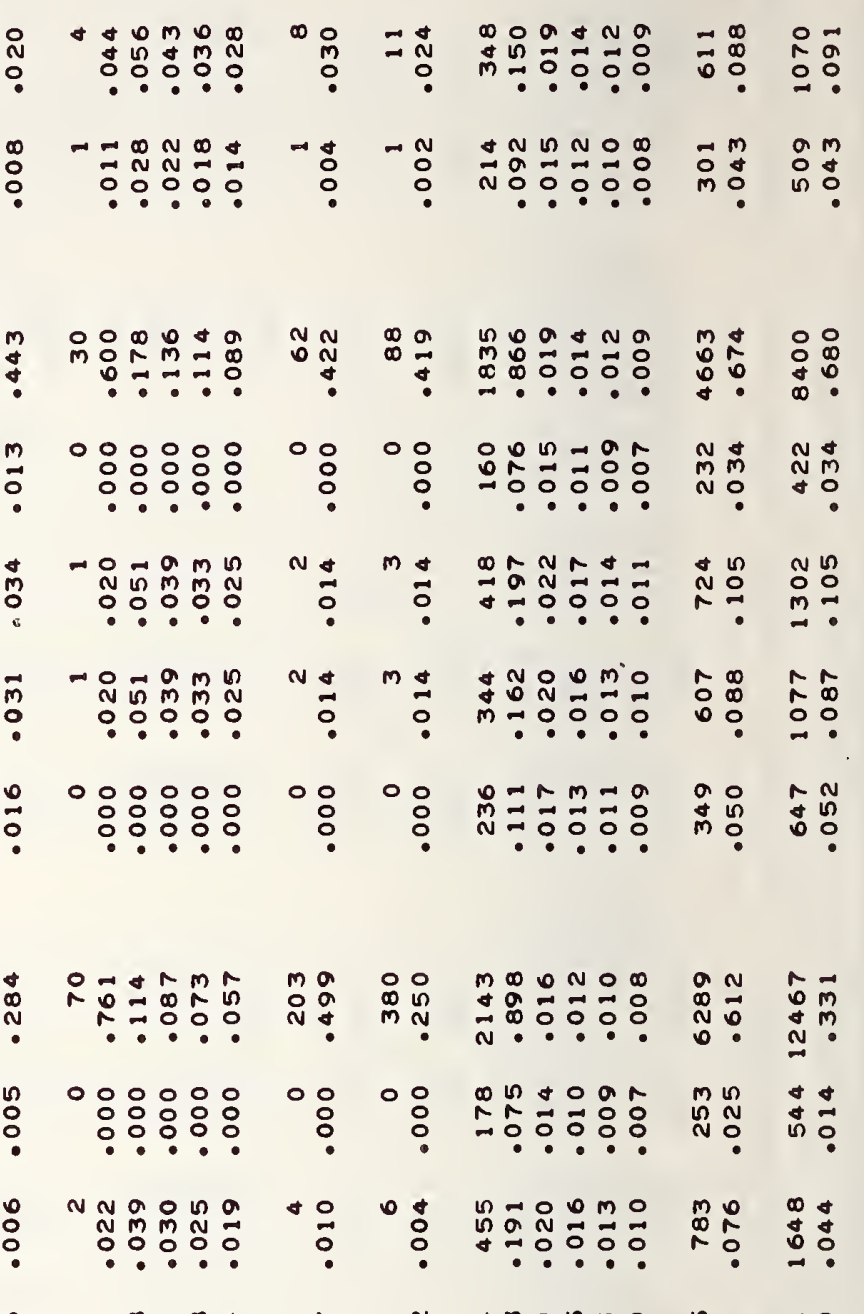

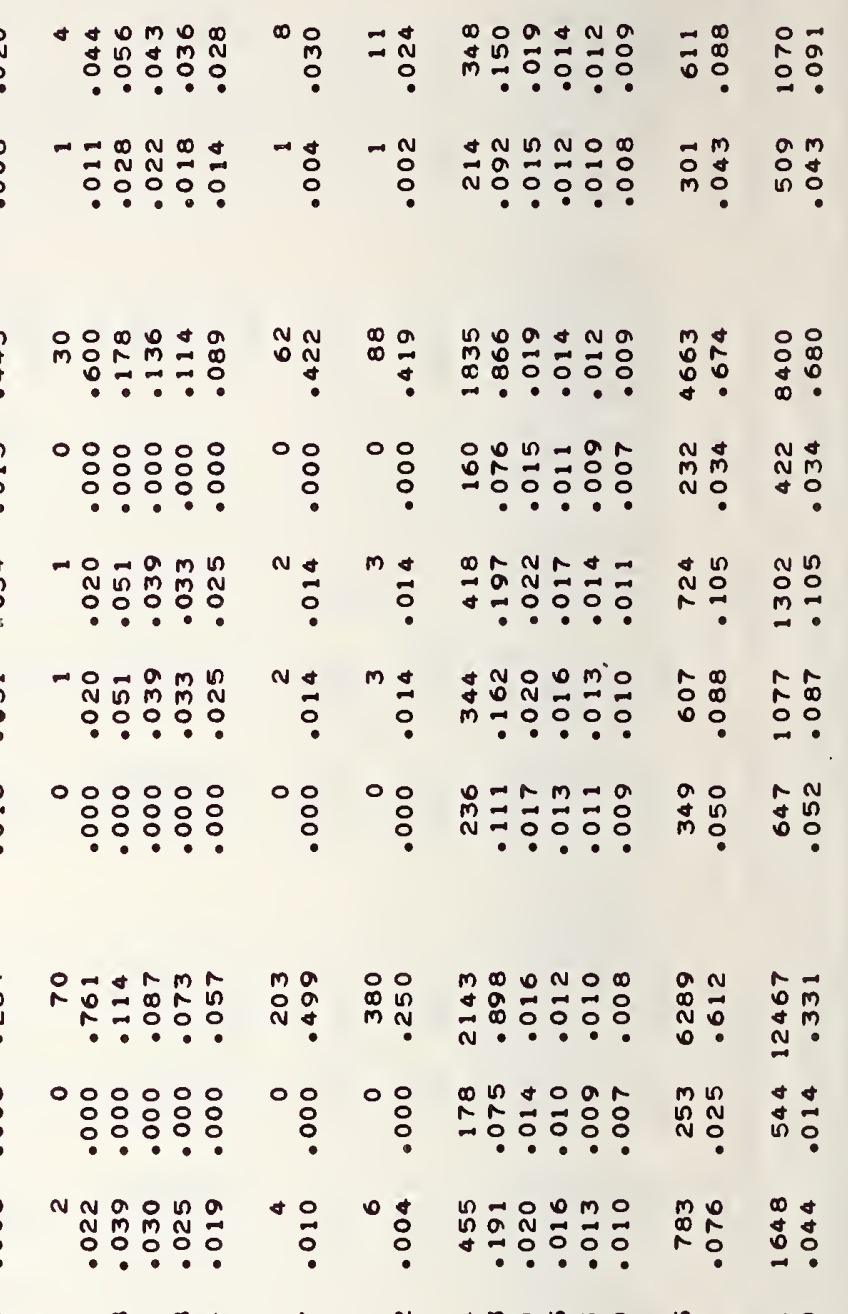

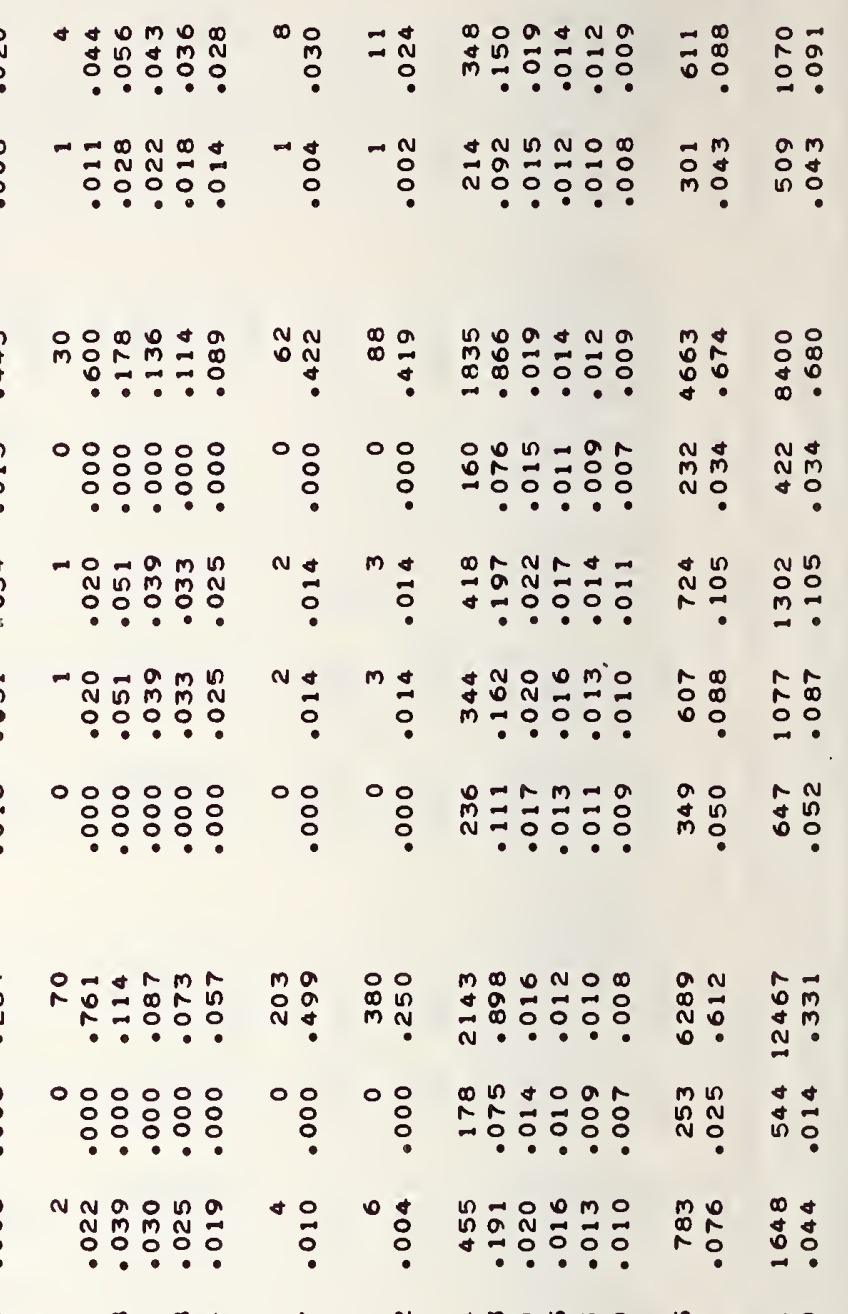

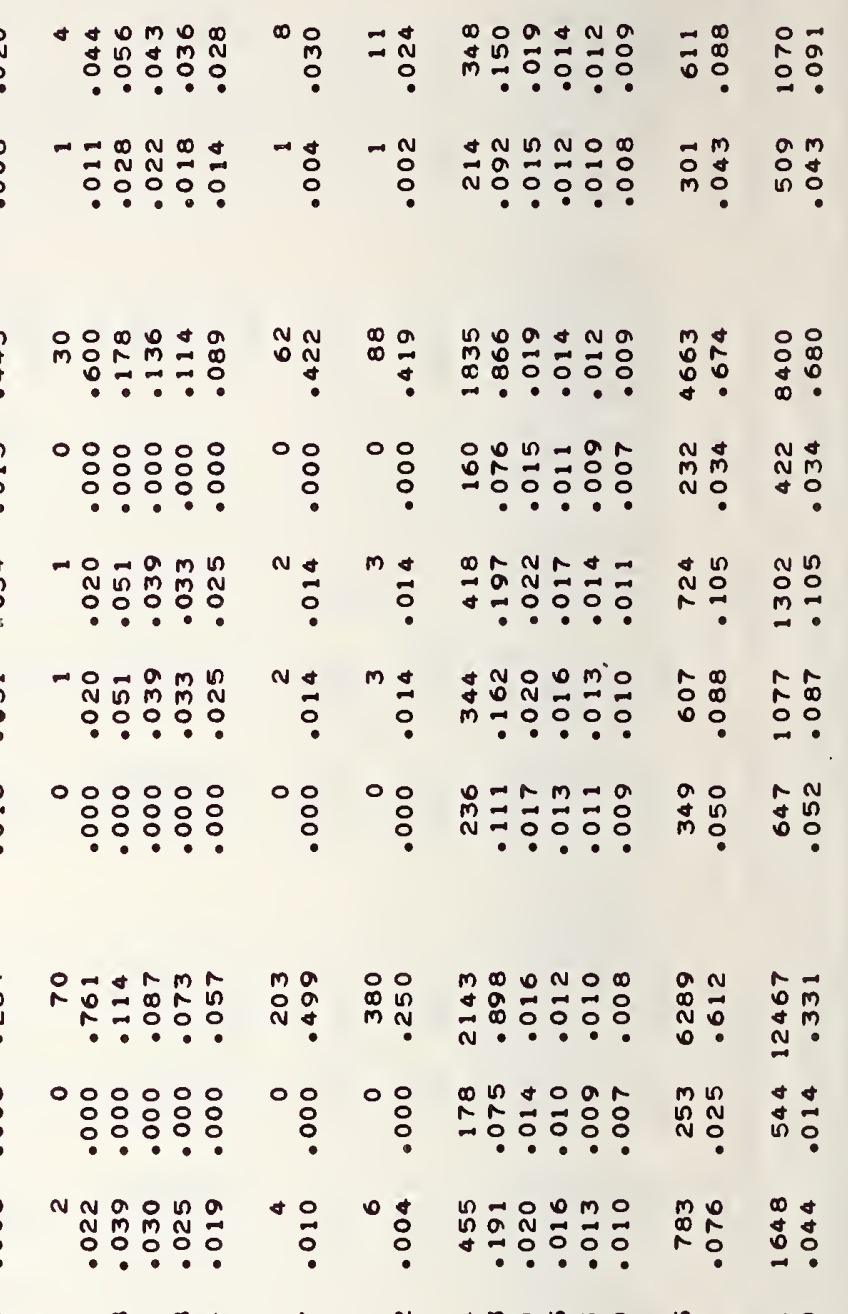

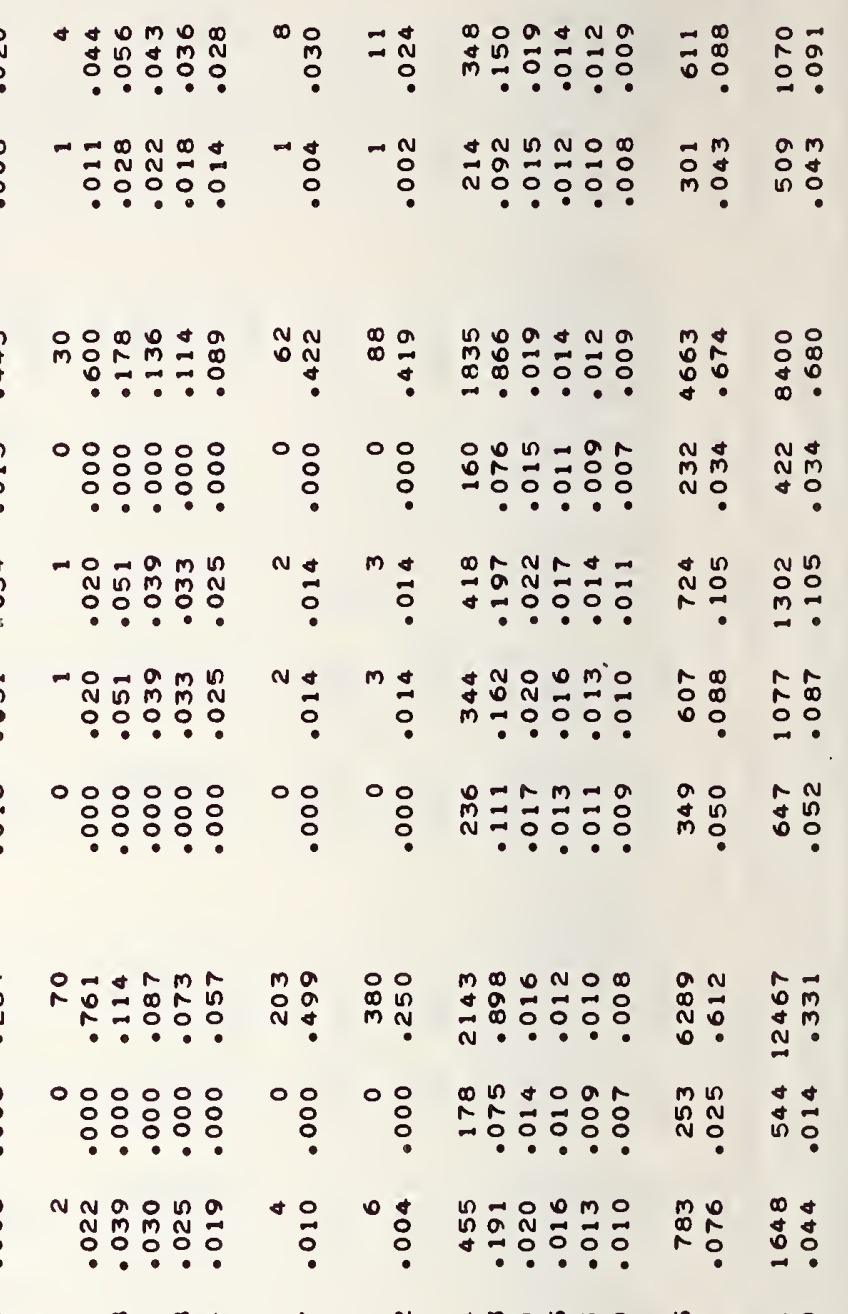

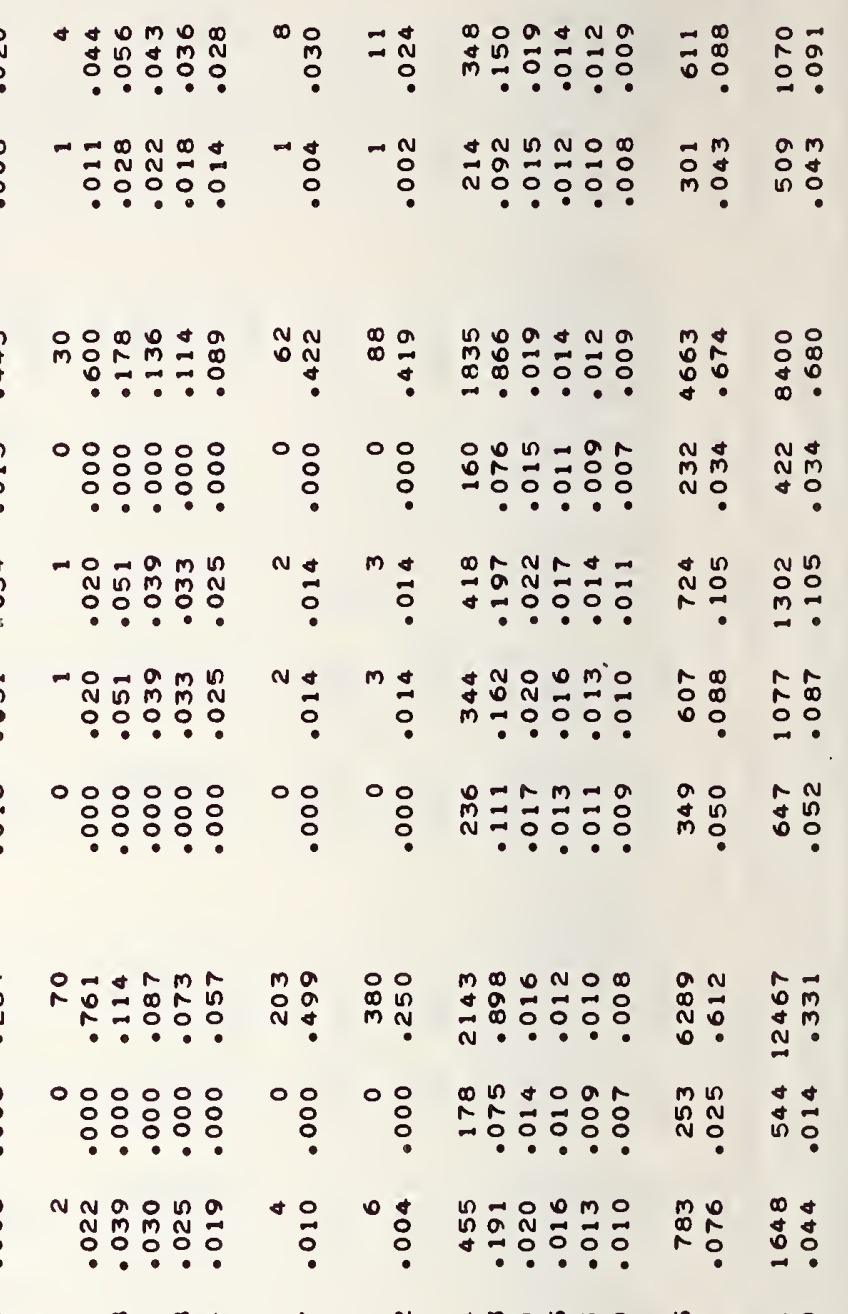

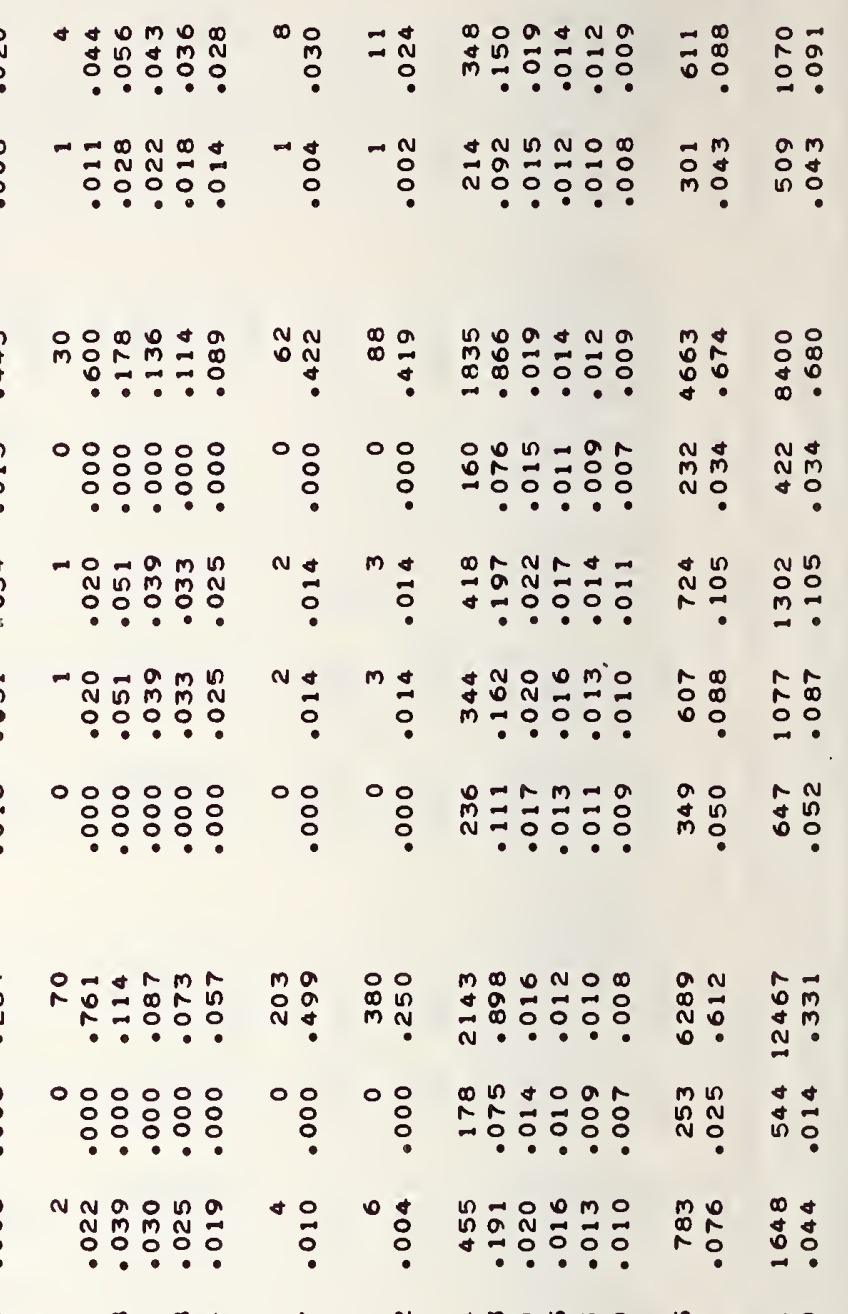

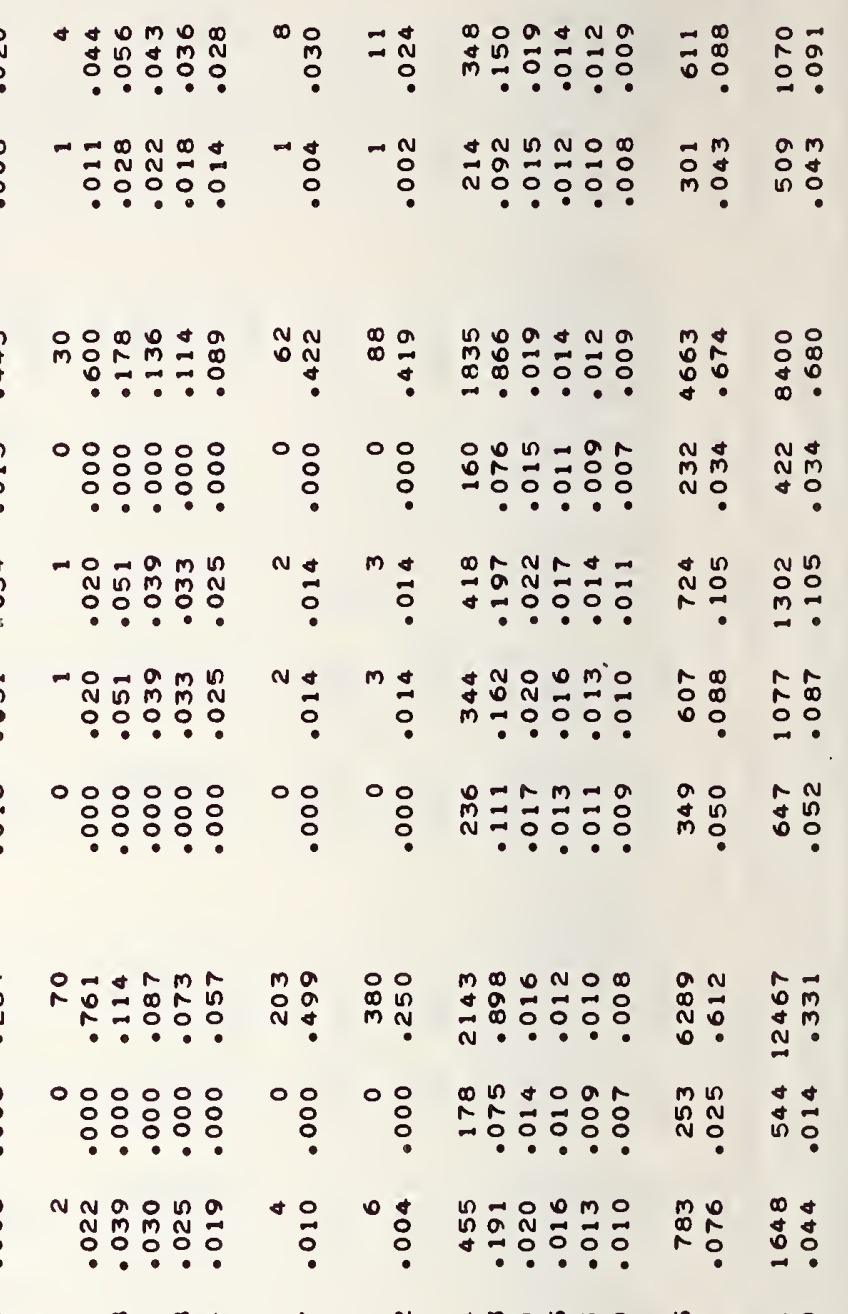

ma $m r$

a

rag a

0
0
0

$\$$

ㅇำㅇํㅇ

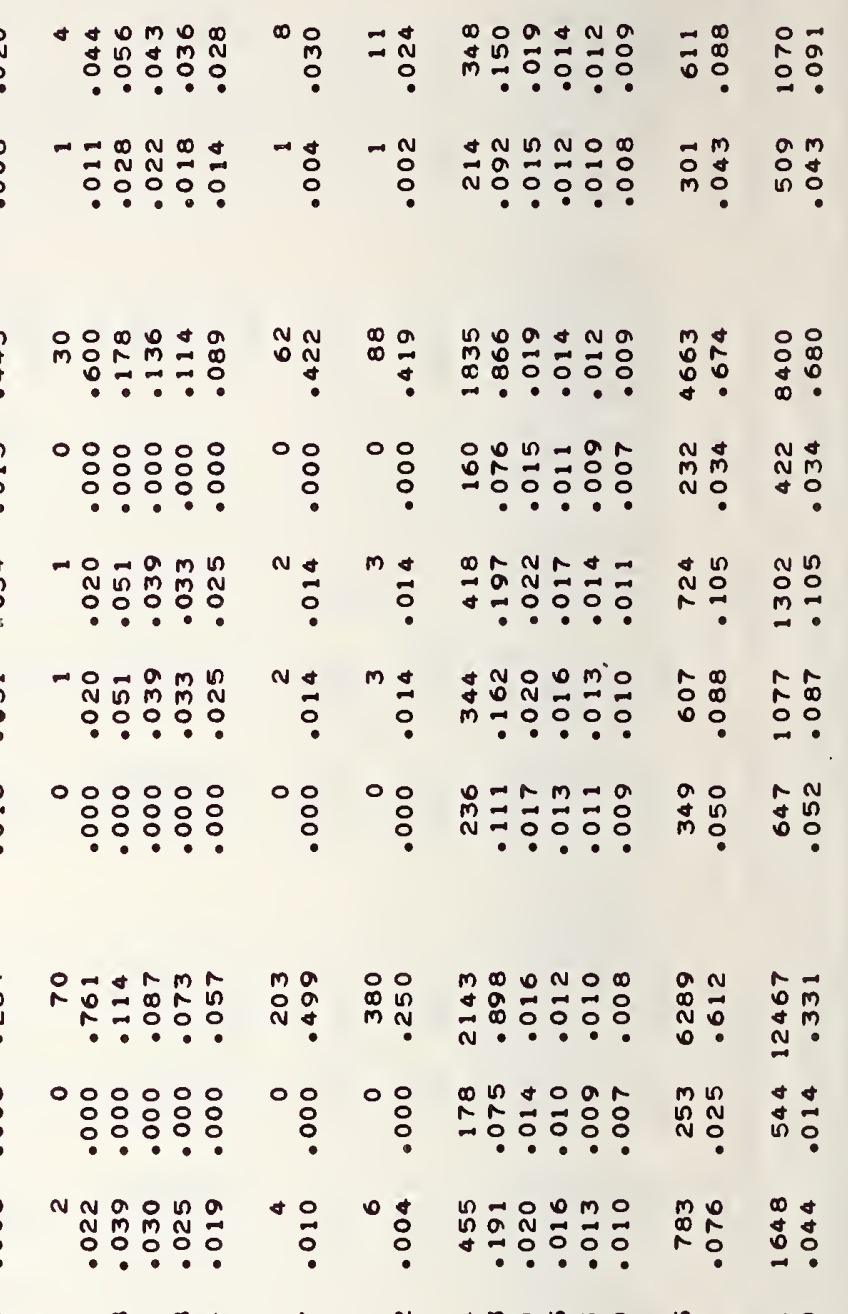

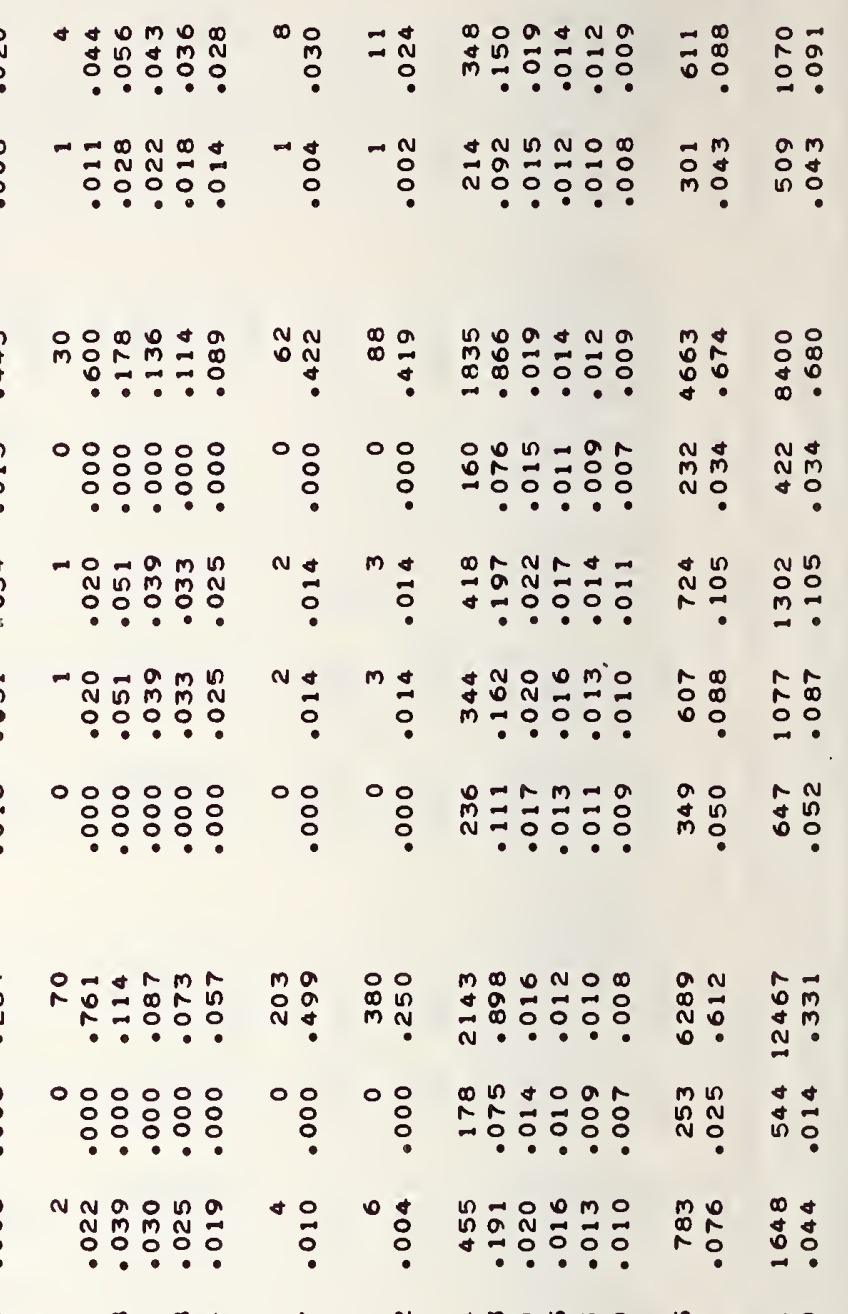

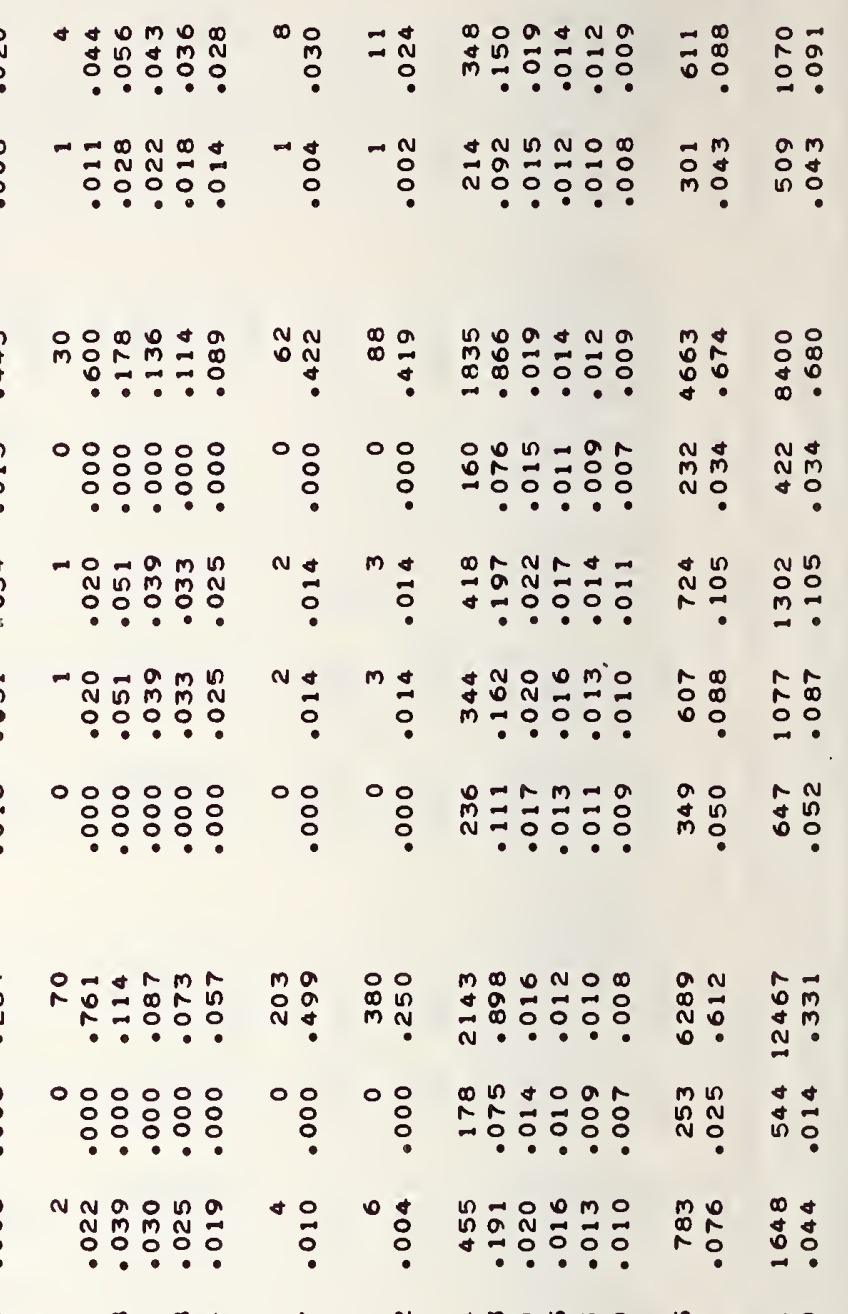

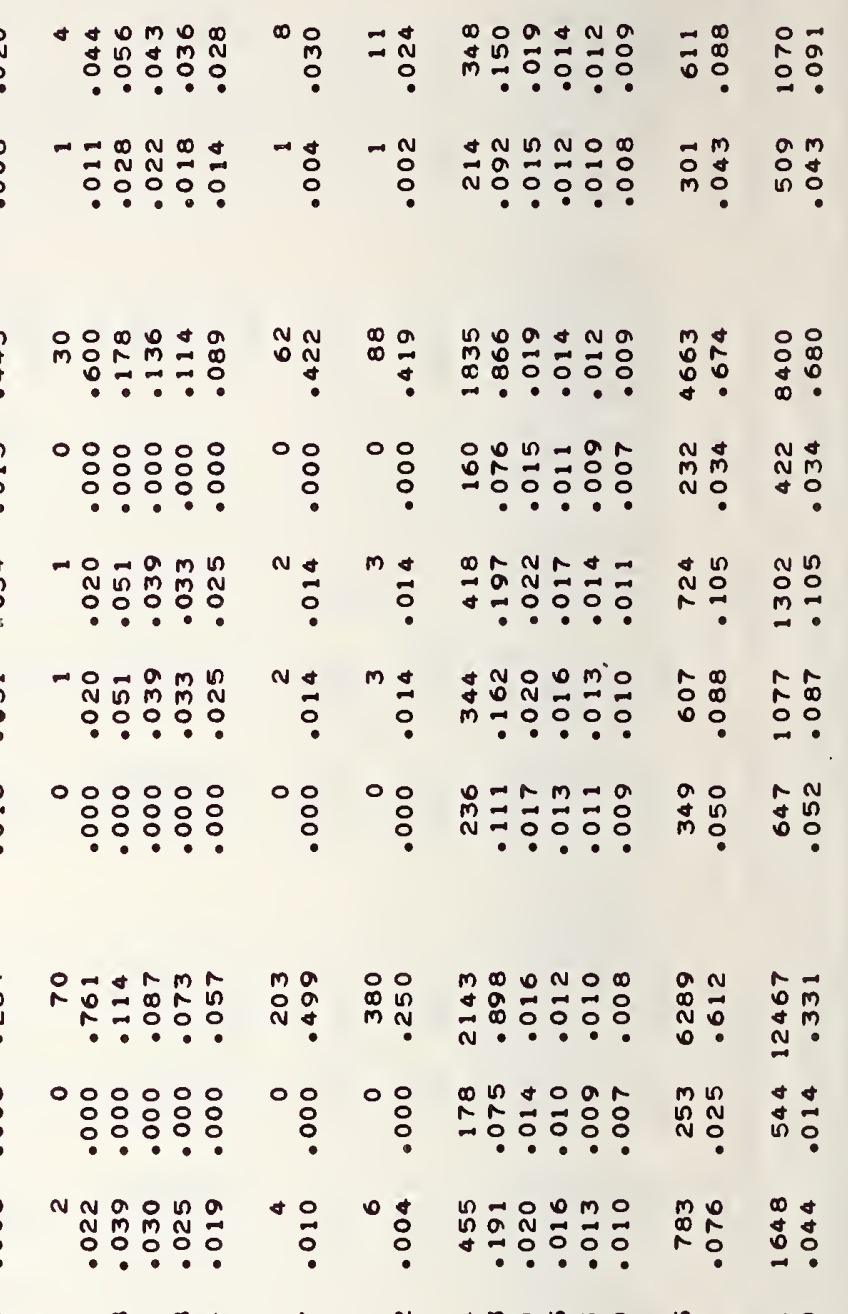

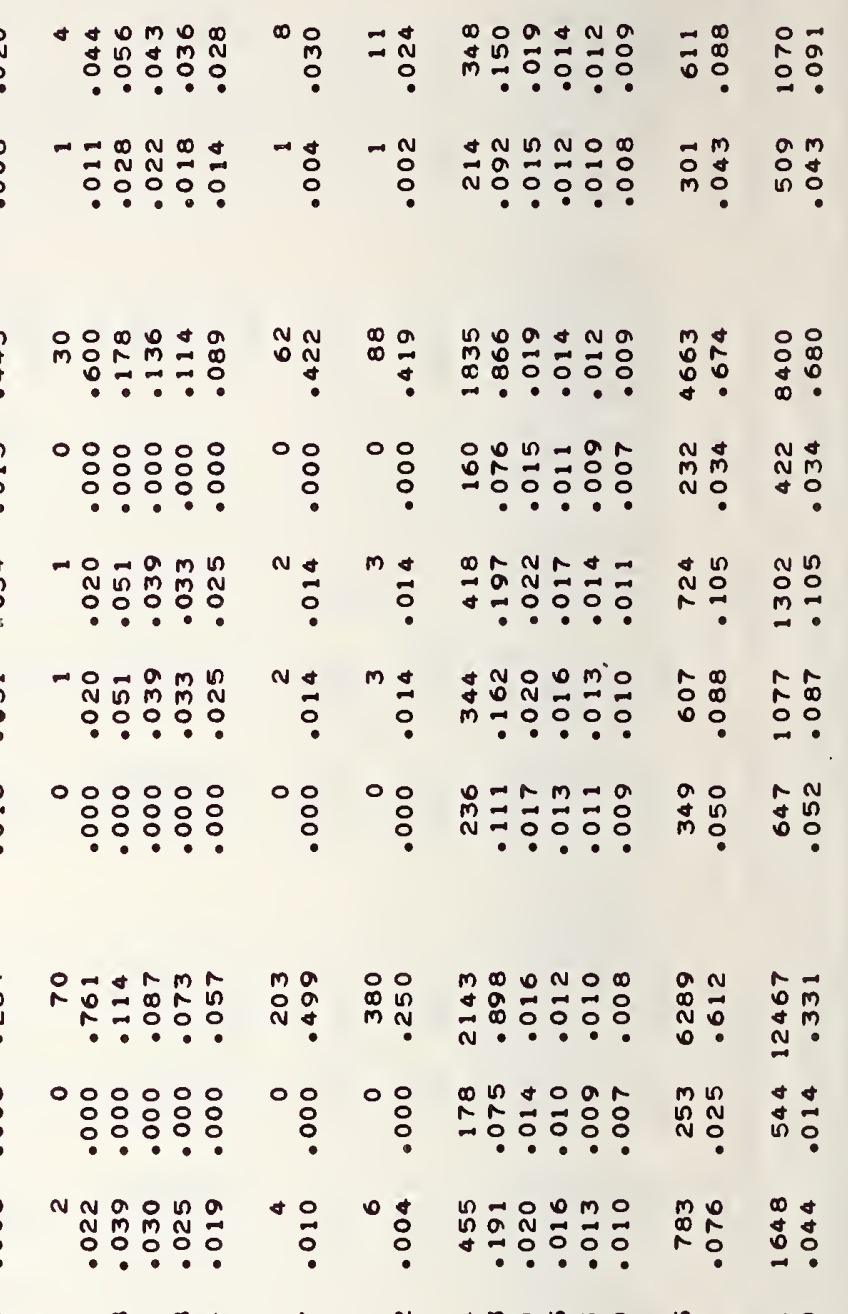

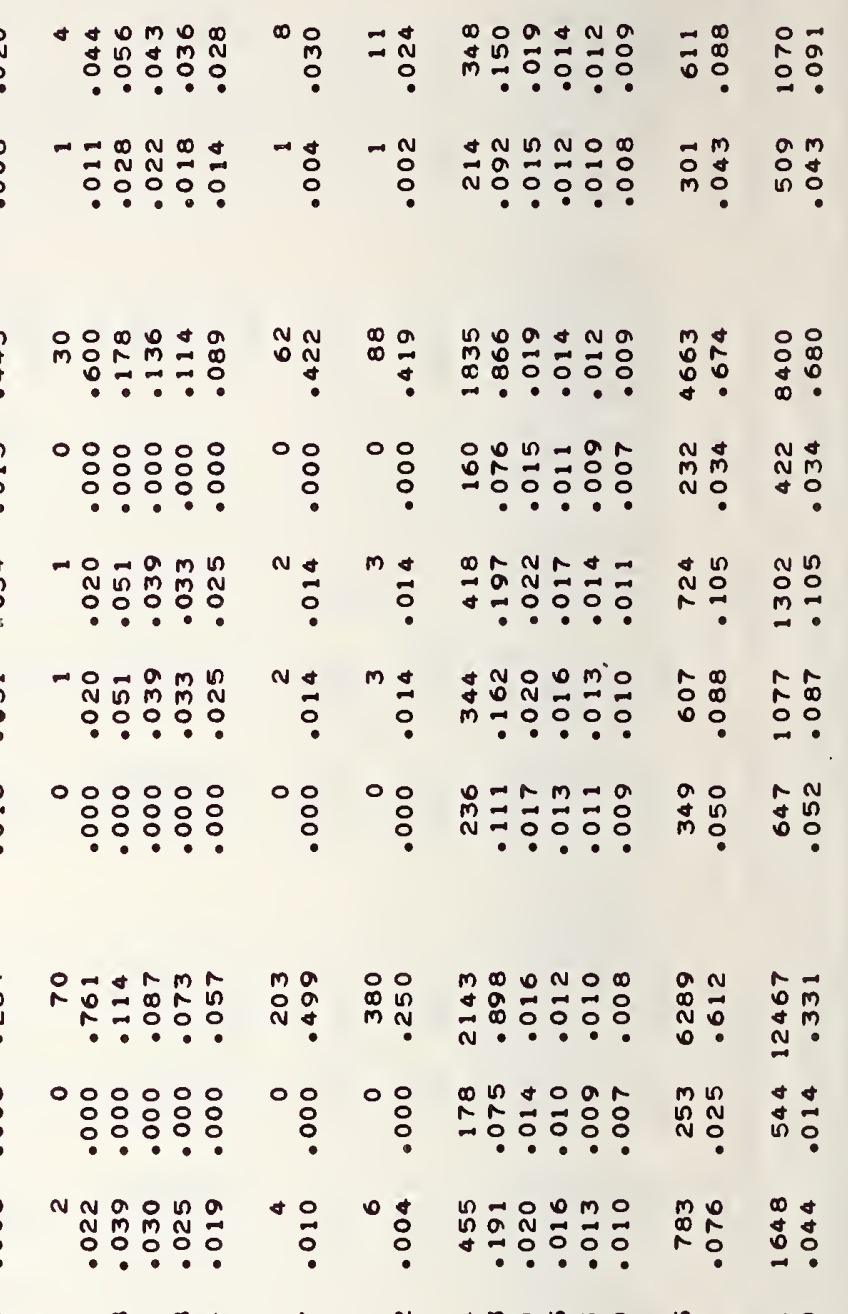

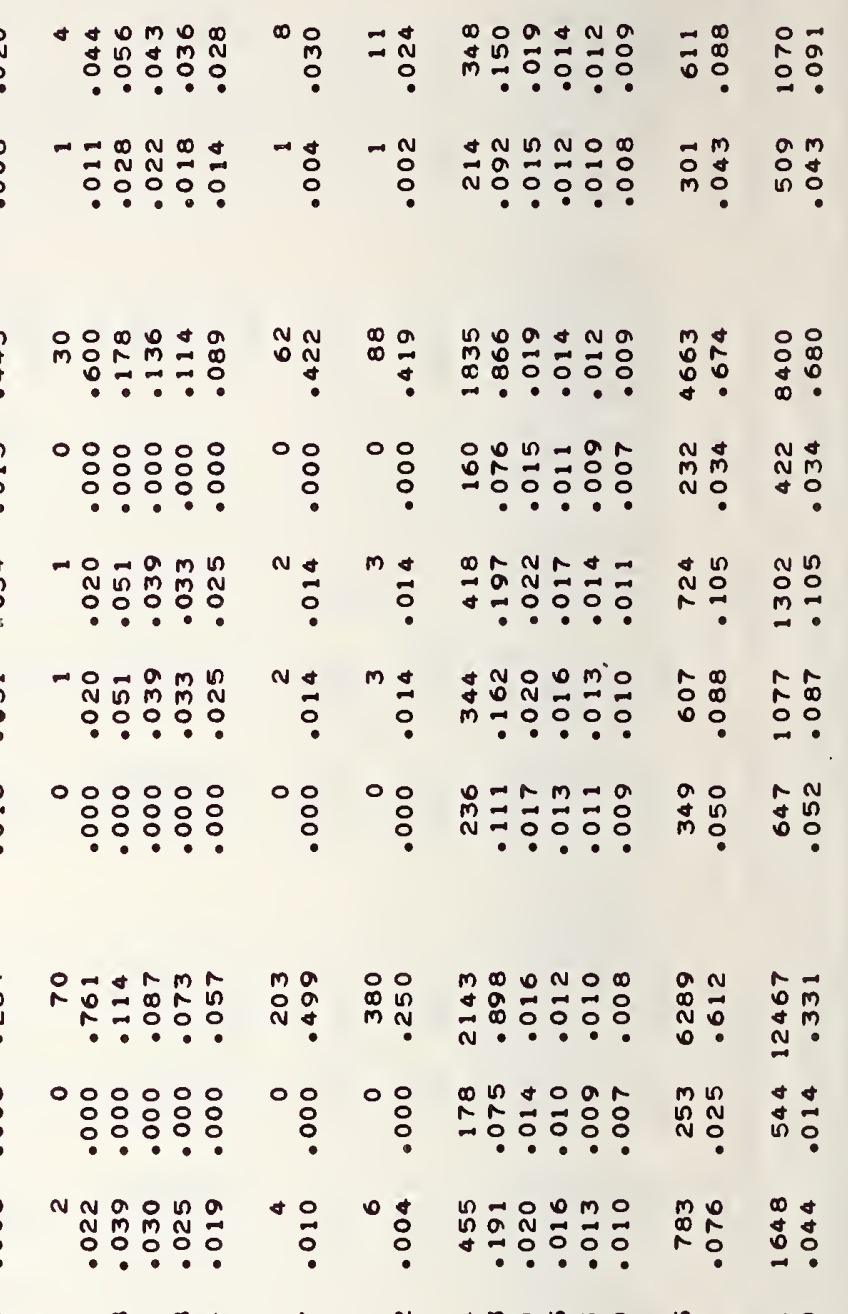

nกm

$\stackrel{N}{2} \stackrel{0}{\circ}$

i:

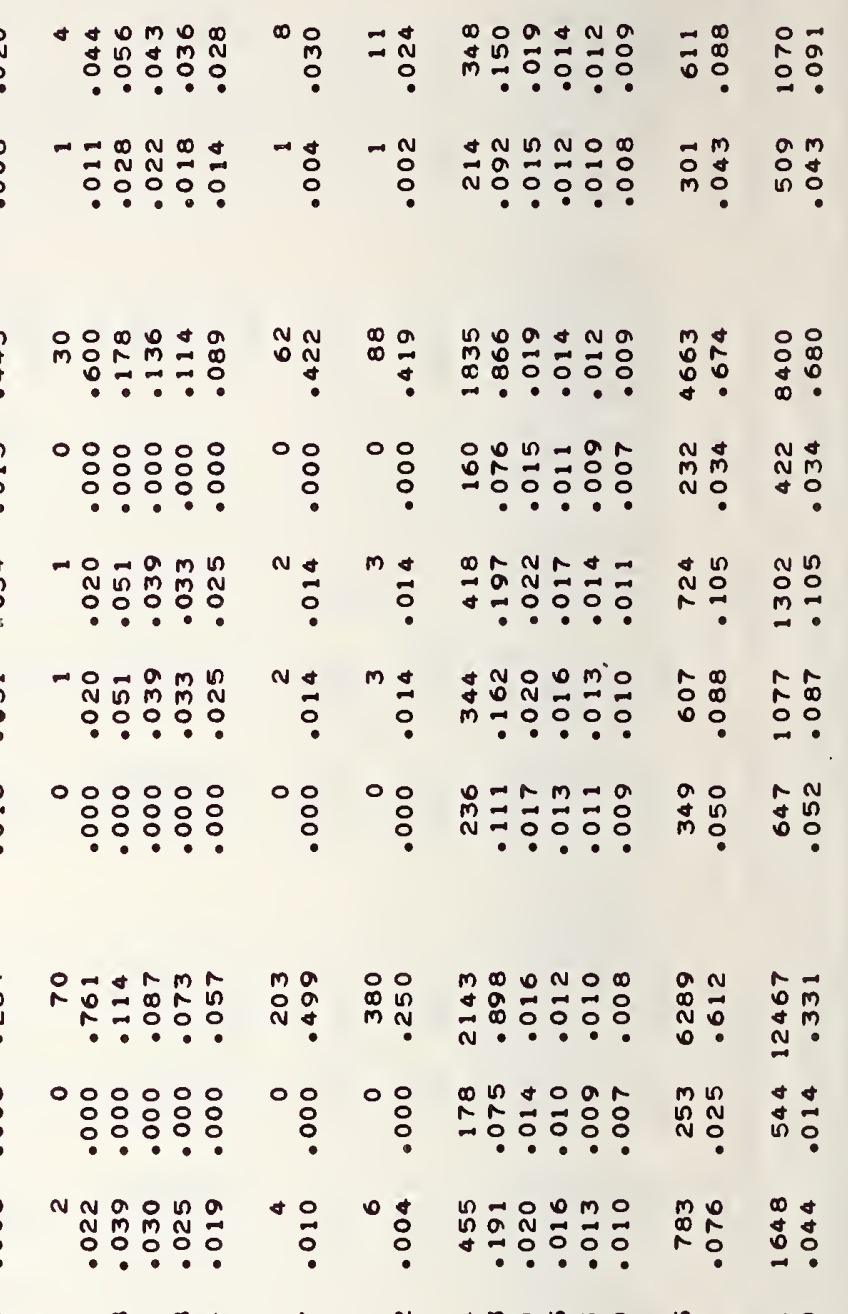

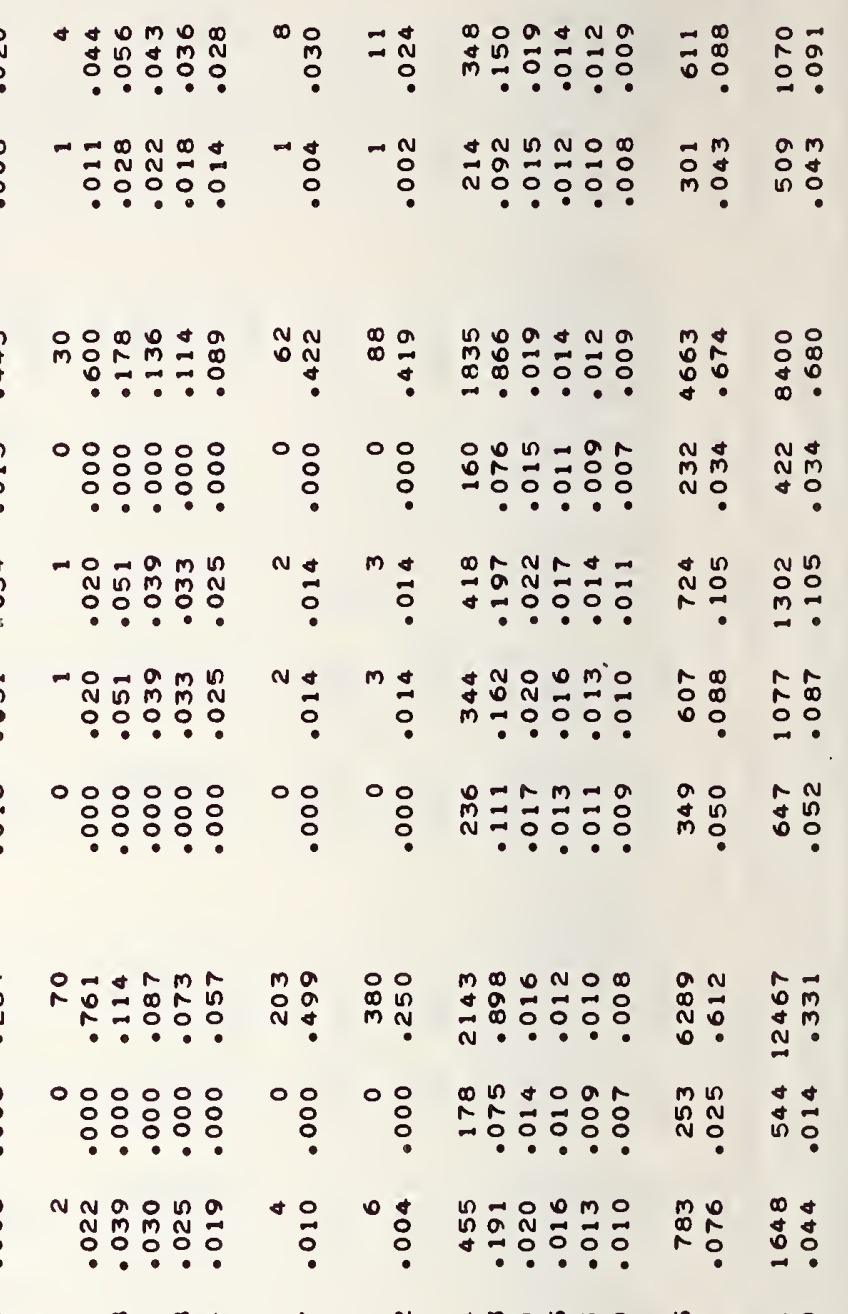

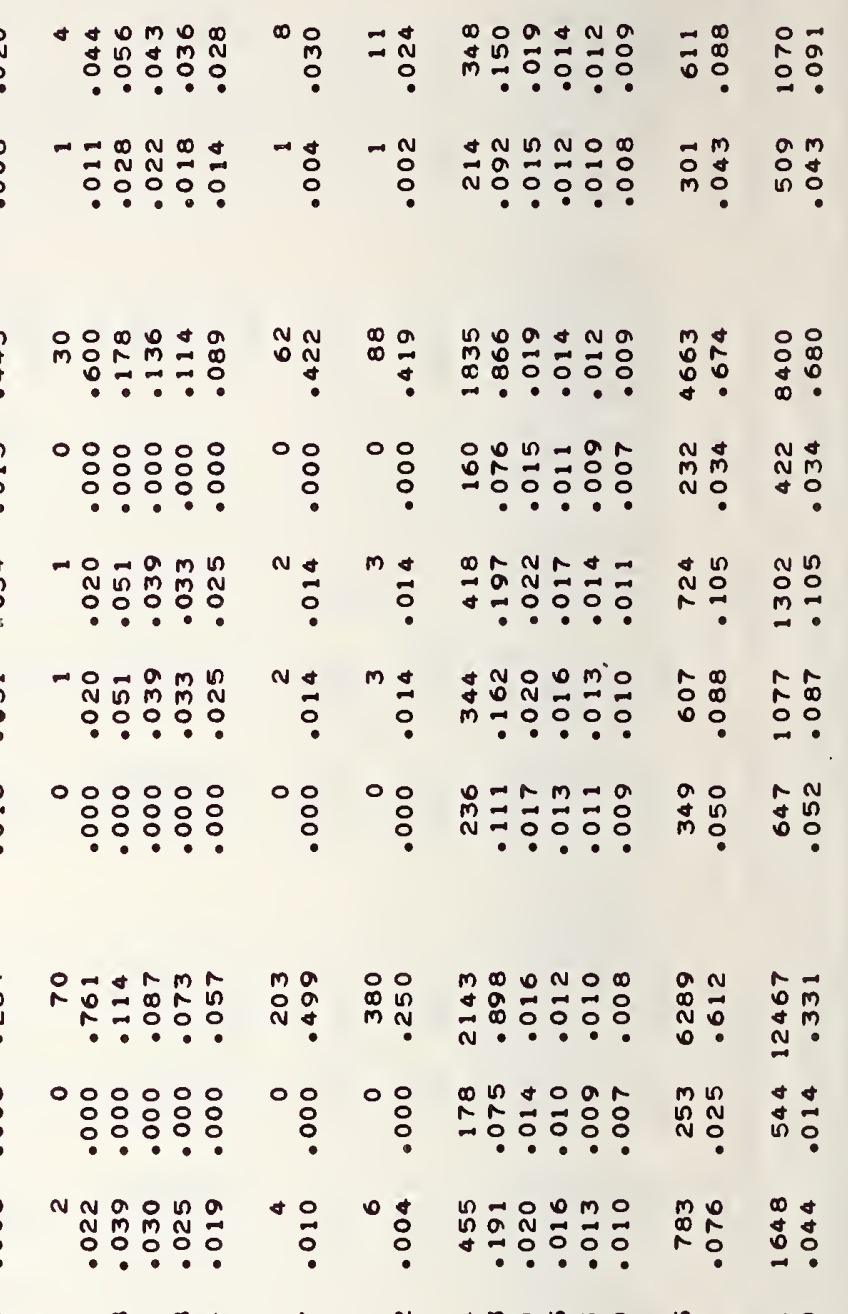

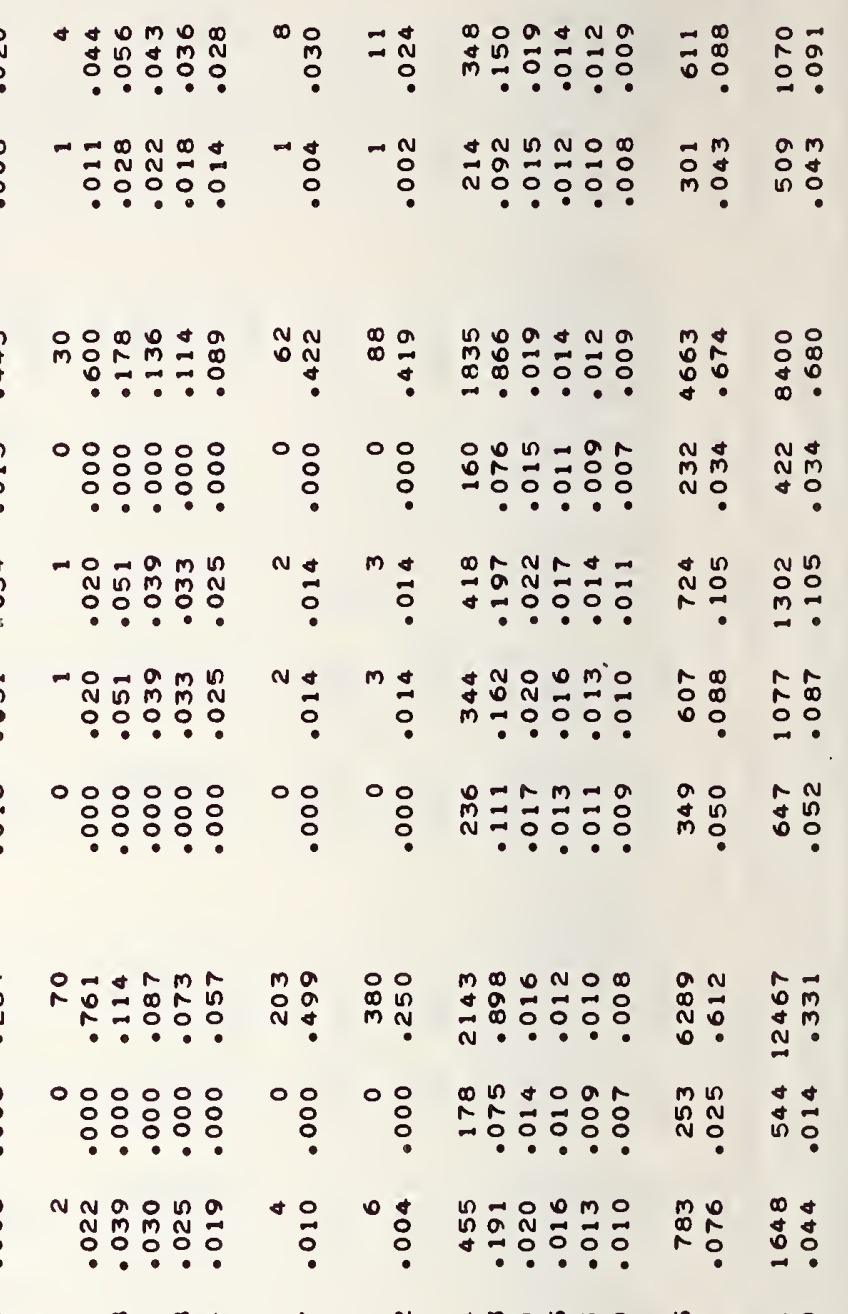

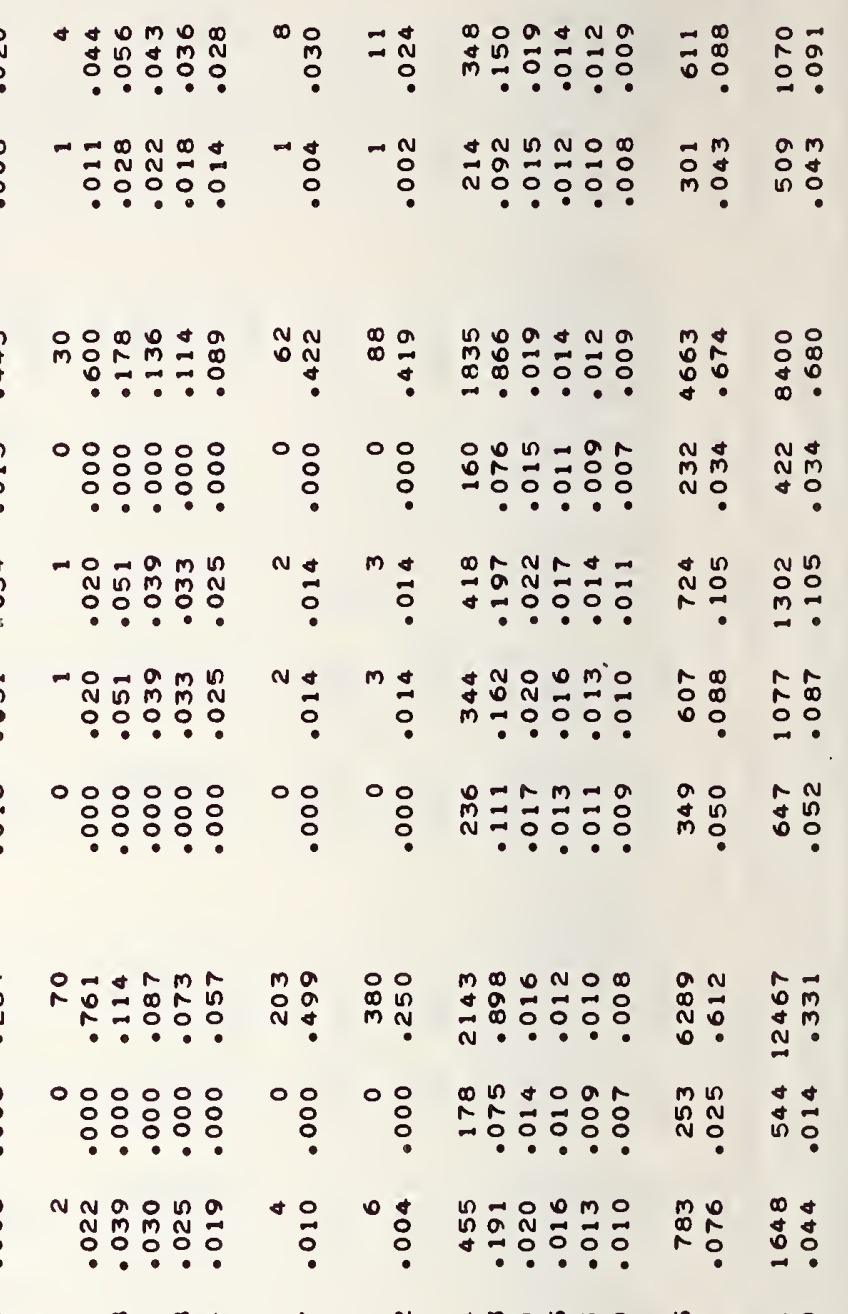

D

$\begin{array}{lll}0 & 0 \\ 0 & 0 & 0\end{array}$

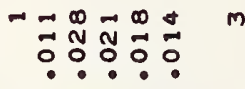

$m \cong \quad m$ 


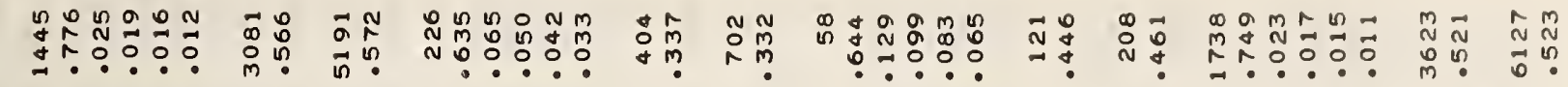

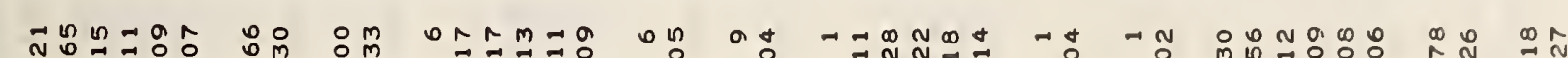

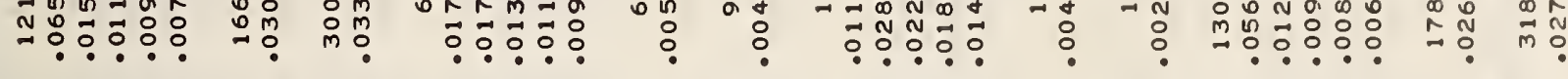

N

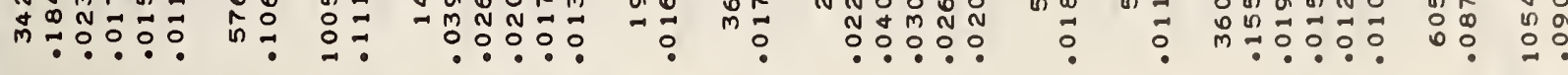

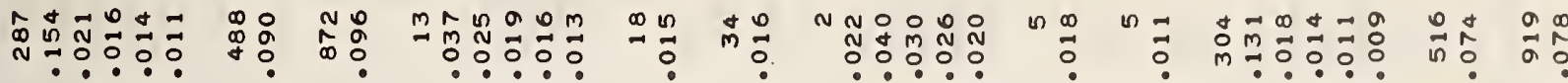

m

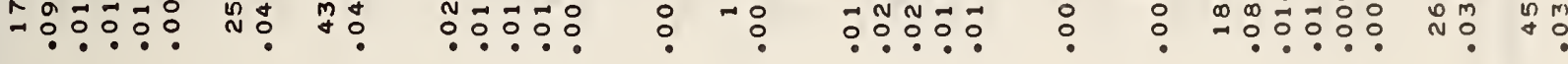

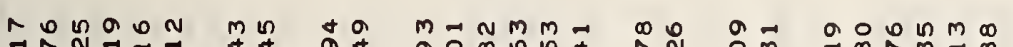

J.

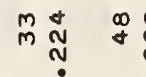

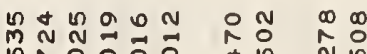

ก.

?은?

in ?

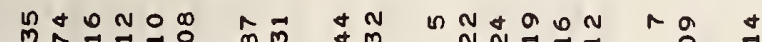

$\rightarrow \begin{array}{llll}0 & 0 & 0 & 0 \\ 0 & 0 & 0 & 0\end{array}$

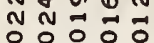

0 응ㅇㅇㅇㅛ

$\circ: 0 \%$

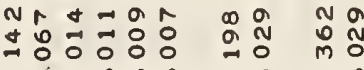

$a 0+\infty$ in

$\operatorname{mon}: 0$

- - .

융

n」m」

을 $\vec{n}$ 品

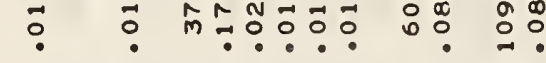

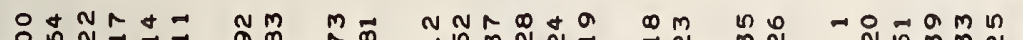

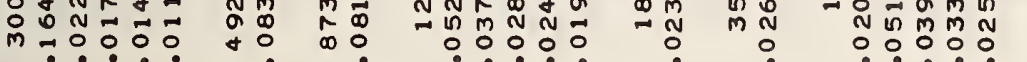

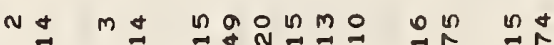

$\overrightarrow{0} \quad \overrightarrow{0} \div \overrightarrow{0}: \overrightarrow{0} 0$ मี

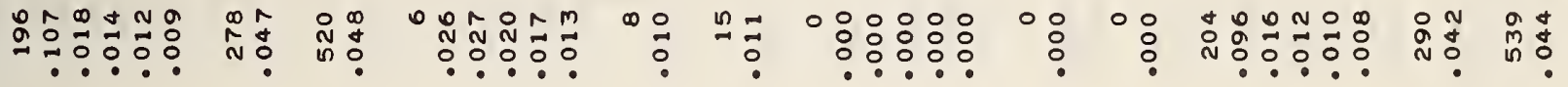

N

N

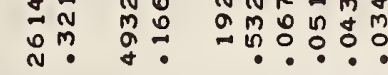

$\infty$ o

$m-0+$

\section{$\left(\frac{10}{0}\right.$}

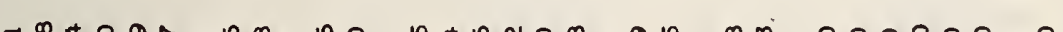

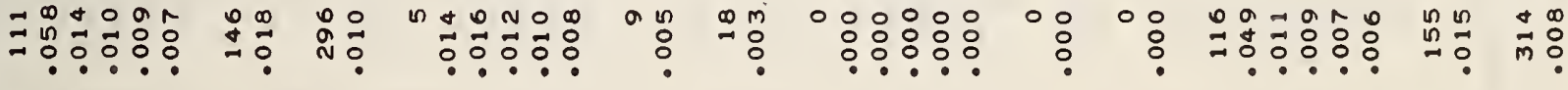

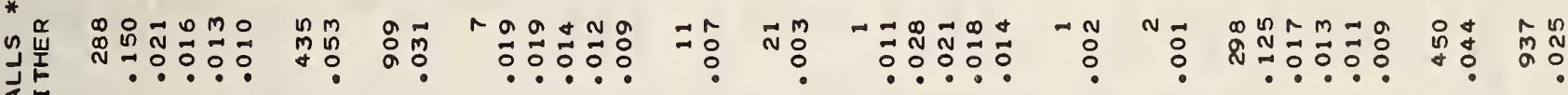

n $m$ 을

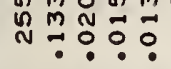

寉

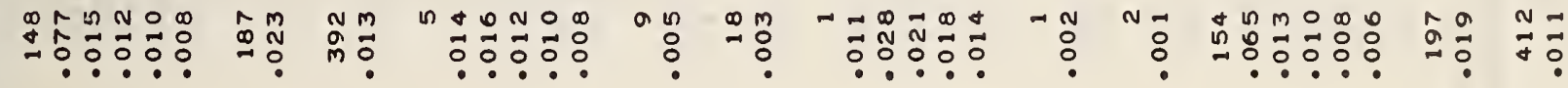

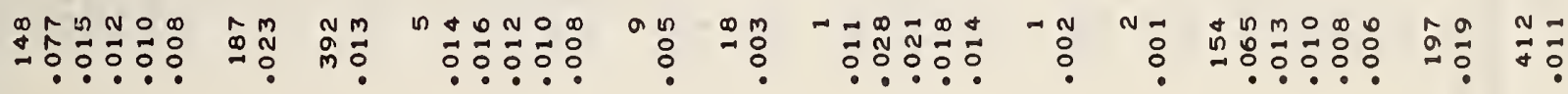

$\Rightarrow \stackrel{\mathrm{N}}{\mathrm{O}}$

$\circ \circ$

0 :

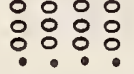

0 :

Do a

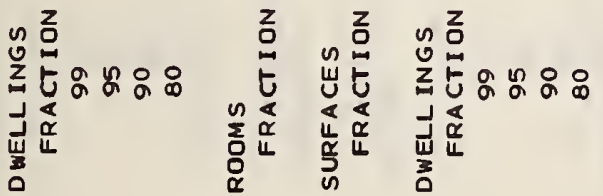

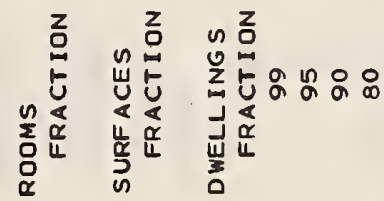

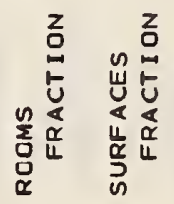

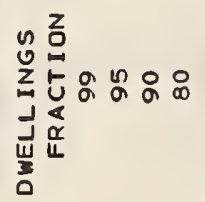

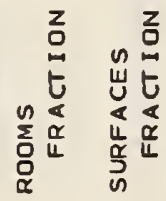
$\stackrel{\substack{\alpha \\ \alpha}}{a}$
in
n 


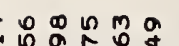

$\stackrel{\infty}{0} \underset{0}{0}$

na notorm

$\stackrel{m}{N} \stackrel{0}{N} \stackrel{0}{N}$

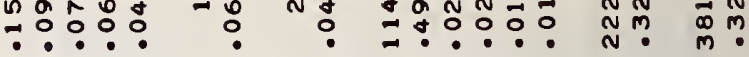

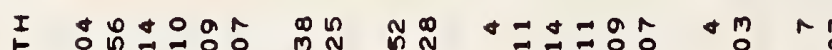

* I

n $\frac{\alpha}{\underline{W}}$

$m \sim ⿻ 0$

$\lim _{\infty}^{m} \hat{a}+\infty$

뭉ㅇㅇㅇ

$\exists \stackrel{\infty}{N} N \stackrel{\infty}{N}$

$-8$

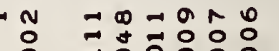

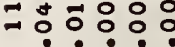

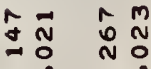

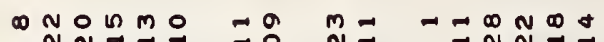

N

$N+\Delta+\infty m=9$

$-\infty \quad 0$ N:ㅇ:ㅇํ

O O O O.

$\vec{O}: \mathbb{O}: \overrightarrow{0}$

$m \rightarrow \circ$ n $m$

N N $N$

a no n mo

*

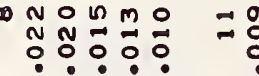

$\stackrel{m}{N}=$

$\neg \Xi \mathbb{N} N$

*

ก

ํำ

-

$+m$

N m

$\neg \equiv \underset{N}{\infty} N_{0}^{\infty}$ $\overrightarrow{0} \stackrel{N}{0}: \overrightarrow{0}:$

$\rightarrow$

@

กำ

$\rightarrow 0000$

잉 : mo

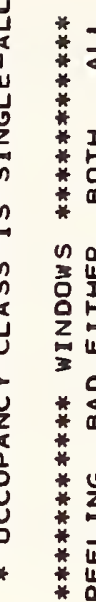

* J $\hat{~}$

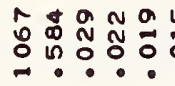

ลิ̀

n $⿻-a r$

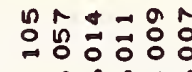

n

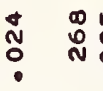

$\stackrel{n}{N}$

ำำㅇํㅇ

?

0
0
$\circ$

$\infty \infty n+a$

mก

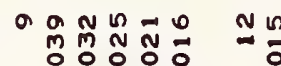

$\stackrel{\infty}{*}$

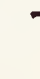

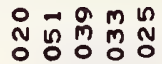

N $\because$ : ० :

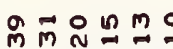

$\prod_{\infty}^{\infty}$

\& 9

m

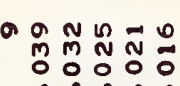

\section{$\cos$}

$\stackrel{\infty}{*}$

?

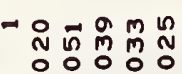

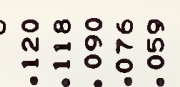

$=\stackrel{n}{0}$

$\stackrel{0}{=}$

ก

- $a_{\infty}^{\infty}-\infty$

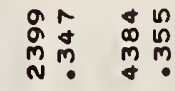

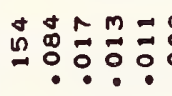

옹

$m m$

$\begin{array}{llll}m a n N o & +0 & \infty \\ 0 & 0 & 0 & 0\end{array}$

응ㅇㅇㅇㅇㅇㅇㅇ

$\circ \circ$

$\circ$

:

on nam

r

N $: m$

N $\mathbb{N}^{\infty} \mathrm{N}$

n\& m\& onanno

or $\sim$

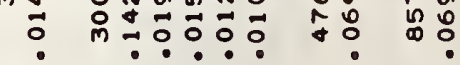

n $+m$

$\stackrel{5}{0}$

$\sim \infty \omega+0$.

- ${ }^{\infty} \circ \sim$

กํํํㅇํำ

$\circ:$

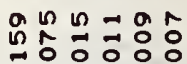

$\infty m \sim n$

$\stackrel{\infty}{N} \prod_{0}^{m} \hat{N} \stackrel{n}{m}$

$*$
$*$
$*$
$*$
$*$
$*$
$*$
$*$

n $\frac{\alpha}{\frac{1}{I}}$

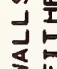

*

*

*

*

* 岩

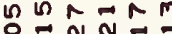

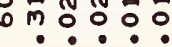

n NOM

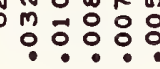

ํํㅁำ

웅유:

- m n N O

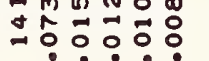

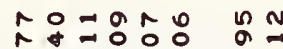

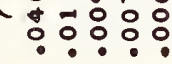

a.

解

ํ: $=$

ผู

$\stackrel{0}{\stackrel{D}{0}}$ (a)

ํํㅇํㅇ

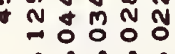
-...

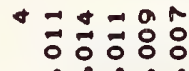
-

ํํํํํํํำ

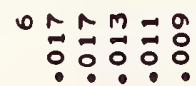

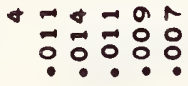

R̊

$\sim \stackrel{N}{0} \stackrel{0}{0}$

ำ

응ㅇㅇㅇㅇㅇㅇㅇㅇㅇ

. . .

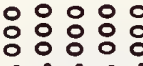

응ㅇㅇㅇㅇ

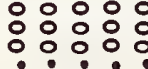

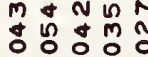

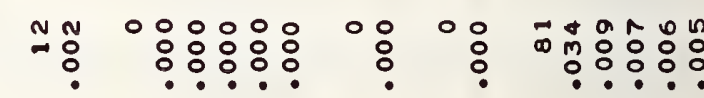

o.0.0

o

$\circ$

:

$\circ:$

ดั

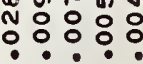

$\circ \therefore \quad 00 \%$

$\circ$
0
0

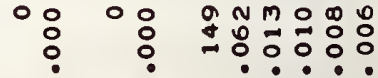

ลี

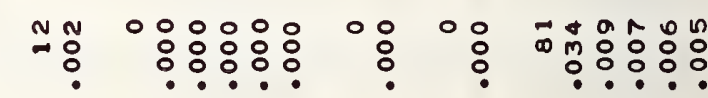

$\stackrel{m}{m} \stackrel{m}{m} \stackrel{m}{N}$

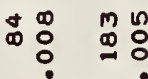

n $m \leftrightarrow$

M N

$\rightarrow \quad 0 m$

웅

$\stackrel{N}{\circ} \underset{0}{*} \stackrel{n}{\circ}$
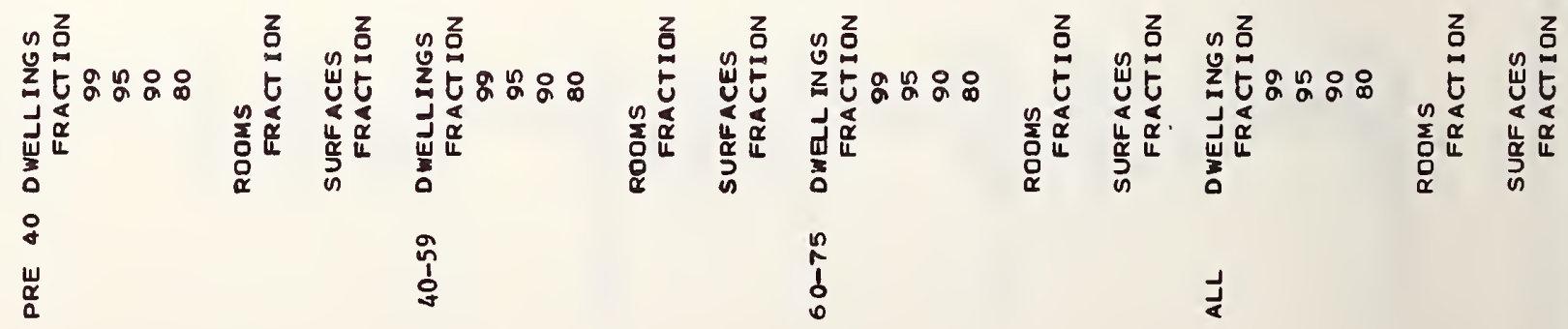


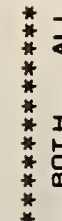

\section{*}

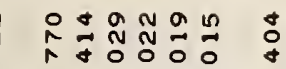
ำ

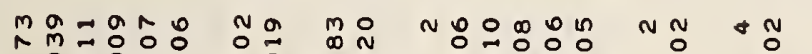

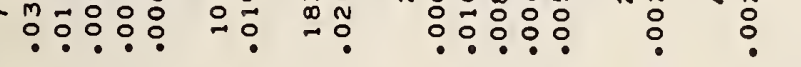

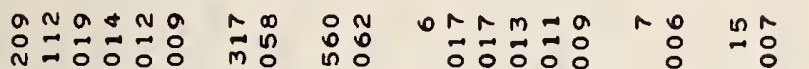

$\stackrel{\alpha}{\underline{I}}$

然

:

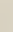

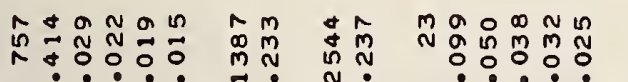

त)

$\stackrel{0}{\sim} \stackrel{m}{\sim}$

N

8

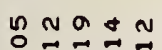

N $=\overrightarrow{0}: \overrightarrow{0}$

t n $n=9$

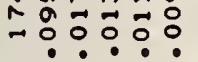

ํำ

$\circ \circ$

웅

N $N$ N

0

in $\stackrel{m}{\mathrm{~N}}$ in

?

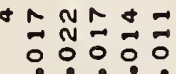

0

ํํำำ

?응ㅇㅇㅇㅇㅇ

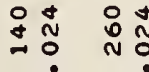

NAONOD

$\begin{array}{llll}9 & 0 & N & 0 \\ \circ & 0 & 0 & 0 \\ 0 & 0\end{array}$

N

응

응ㅇㅇㅇㅇ

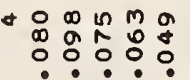

$+\hat{n} \sim m$

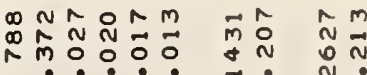

$\circ$
0
0

$\circ$

$\circ$

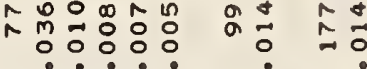

$\circ: 0: 0$
$\circ: 0: 0$
$\circ:$

00000

$\circ$

$m \notin N$ in

용요

$\circ$

8

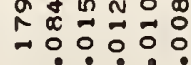

an $n \infty$

* ü

$\rightarrow \infty m \sim n$

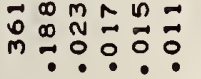

a)

等

mั

$\stackrel{\infty}{m}$

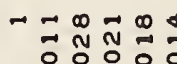

인

N

w

$\infty m a n N o$

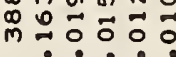

a 09

* I

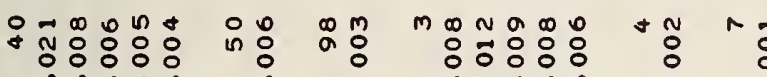

$\circ \circ \circ 000$

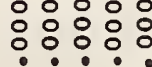

$\circ$

$m$ 的

a

.

$\therefore$

용요

in $n \mathrm{~m}$

古至

*

ڤึ

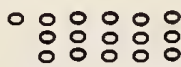

0

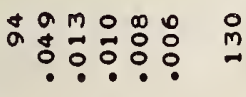

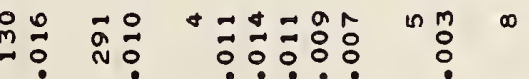

$\infty \div$

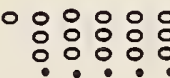

$\circ$

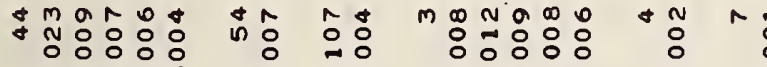

:

$\circ:$
$\circ$

$\circ$ 웅

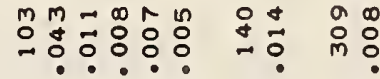

‥

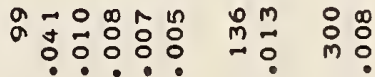

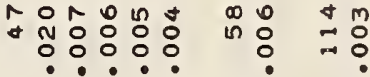

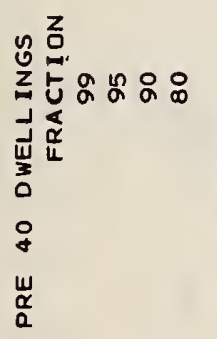

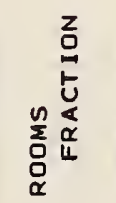
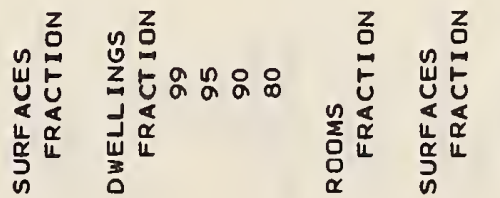

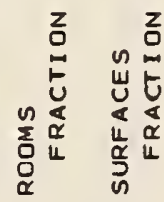

$n$
1
0
0

คิ 


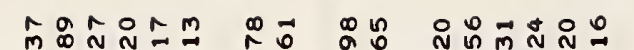

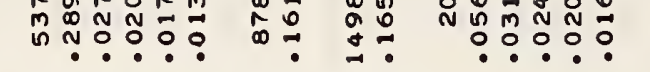

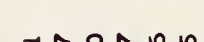
$\because \frac{0}{2} \stackrel{N}{0}$

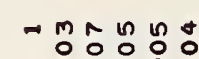

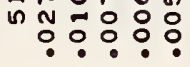

On 0 nO $=0$ *

吕点

nr n $=0$ * *

กิก

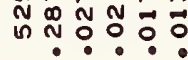

空

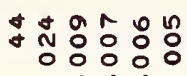
in: å

言

足:

*

*

*

*

ํำ

ํำคㅎํㅇㅇㅇ

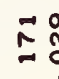

N. …

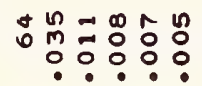

$\stackrel{4}{\sim}$

总

क

范 吕

\section{要:}

:ㅇ: :

m

$\because 0: 0:$

Ñ

$m \infty N O \infty$ :응유:

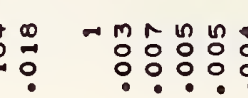

옹 용ㅇㅇㅇㅇㅇㅇ

$\underset{0}{m} \frac{0}{0} \frac{0}{0}$

$m a n N$

ta
$\stackrel{n}{N}$

\&

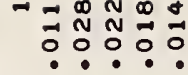

$-\overrightarrow{0}$

m

$m$

:

$m \stackrel{0}{\circ}$

a

ㅇㅇㅇㅇㅇㅇㅇ

응유

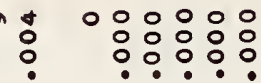

ㅇํㅁ

$\circ \circ 0 ㅇ ㅇ ㅇ$

응유:

$m:$

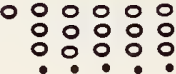

- 웅

$\circ$

$\circ \circ$

:

$\circ$

:

$\circ$
Uึ

กำกำ

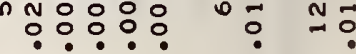

$a+m \circ \infty 0 \Delta-\infty N$

$\pm: 00:$ กั

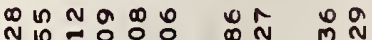

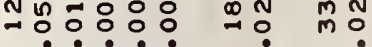

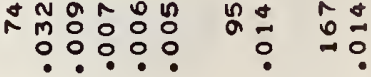

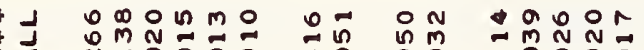

*

:

000000

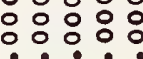

8

:

4

$0: 0: 0$ 는

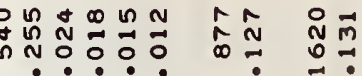

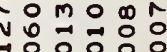

ง

ma 0

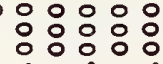

$\circ$

0 :

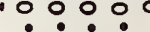

$m: 0$

$\circ::: ㅇ$

$\circ$ :

$\circ:$

nONamo

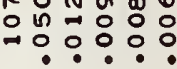

$\stackrel{2}{2}$

an

$\rightarrow$

N

응융ㅇㅇ

$\circ:$

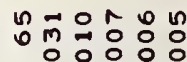

:ㅇํ유.

$\infty \stackrel{n}{0} \stackrel{n}{0}$

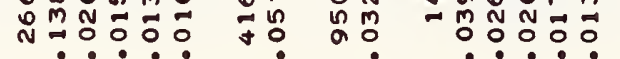

* *

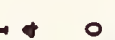

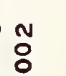

กับ

$\stackrel{m}{N}$

mo

000000 $\therefore: ㅇ: 0$

$\circ:$

$\circ:$

$N \infty \sim m \rightarrow \infty$

N $=0 \div 00$

$=m$

$\therefore \hat{2}$ mn

플

in $\stackrel{m}{\circ}$

$\therefore \stackrel{2}{0}$

$\cos$

(t)

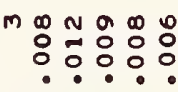

(1)

:

응ㅇㅇㅇㅇㅇㅇ

$\circ:$

$\circ$ :

ำำำำ

m:

mo 00

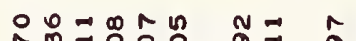

官 M

m 0

:

00000

응유:

$\circ$ :

$\circ$ :

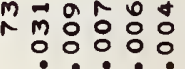

ลำำ

ํํㅇำ

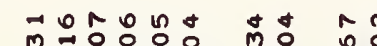

N $M{ }^{\infty} N O \infty$

\section{$m$}

$0 \div$

응융유

$\circ 8$

웅

mํำ *



$\frac{w}{a}$
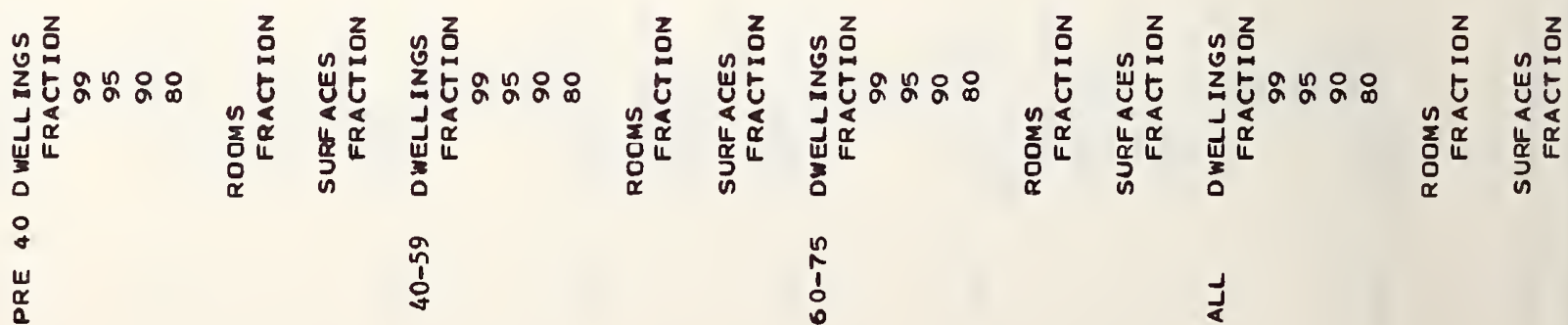

年 


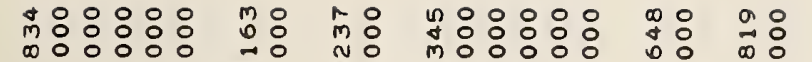

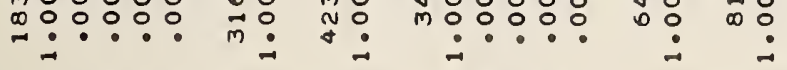

$\begin{array}{llllll}\alpha & 0 & 0 & 0 & 0 & 0 \\ 0 & 0 & 0 & 0 & 0\end{array}$

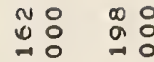

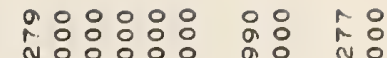

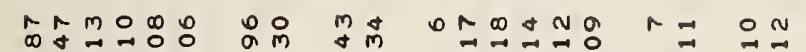

:으.

$\therefore$

n?:?

$\rightarrow=0 N \infty$

.00 .000

$-10 \rightarrow$

$0 n-\infty r n$

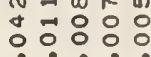

nONRAT

$\stackrel{0}{0} \stackrel{\infty}{0}$

O $m \infty D O N m$ $m \rightarrow$ : $: 0$

$\rightarrow$ in

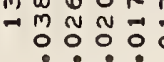

$\stackrel{0}{0} \stackrel{m}{\sim} \quad \stackrel{m}{\sim} \underset{\sim}{0}$

$\begin{array}{rllll}* & 0 & \\ 0 & 0 & m & 0 & 0 \\ 0 & 0 & 0 & 0 & 0\end{array}$

n $\vec{m} \propto$

mo 0

$m \neq \overrightarrow{0}=0$

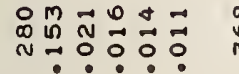

$\stackrel{m}{0} \stackrel{0}{0}=0$

NUn $00 M \quad \forall N \quad \vec{N}$

: ? :

* $40 \mathrm{~m}$

낭ำ

n

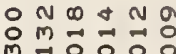

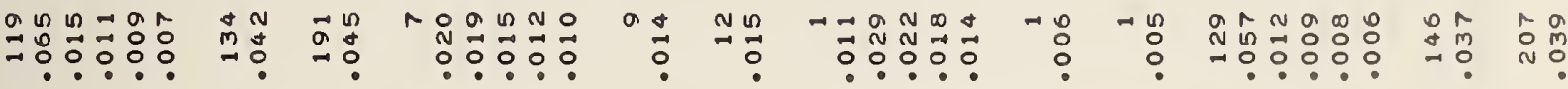

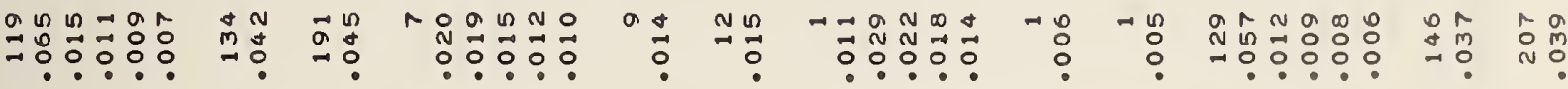

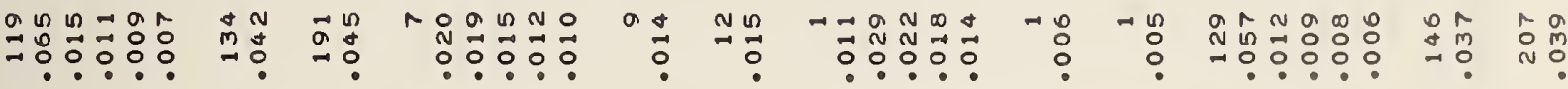

$\ln 0.00$

늉ㅇㅇㅇㅇㅇ

ㅇํㅇำ

12:잉

0 - 100000

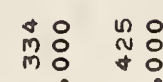

no: 000

40090

$\stackrel{8}{10}$

$\therefore \cdot \cdots \cdot$

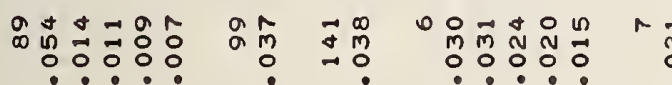

$\stackrel{2}{0}$

$\rightarrow \sim ⿻ m$

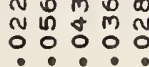

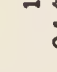

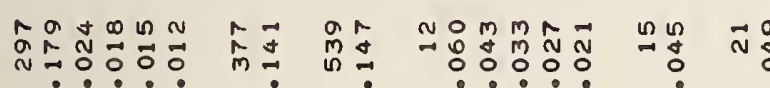

品品

s

。ำ

$m$

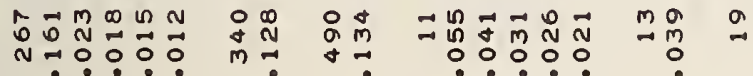

$\begin{array}{ccc}0 & 0 \\ 2 & 0 & 0 \\ 2 & 0 & 0\end{array}$

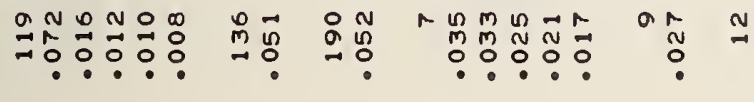

$\stackrel{\substack{N \\ \hdashline}}{\sim}$

- Nom

$\begin{array}{lll}0 & 0 \\ n & 0 & 0 \\ n & 0 & 0\end{array}$

?

8

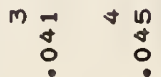

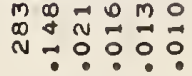

$m=\frac{\infty}{m} \stackrel{m}{m}$

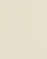

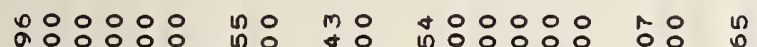



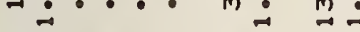

:- : :

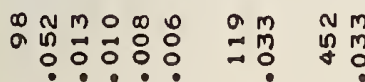

or $\infty m-a \quad a m$

$m \vec{m}$

no: 000

응ㅇㅇㅇ

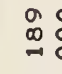

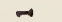

응ㅇㅇㅇ

응ㅇㅇㅇ용

0000

:

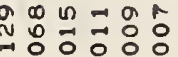

$\infty 0^{\infty}$

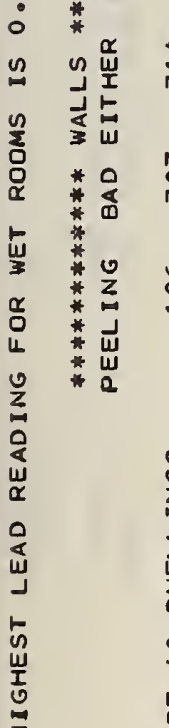

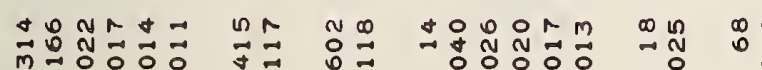

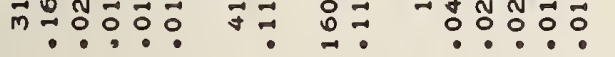

O

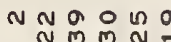

Nom

$+2$

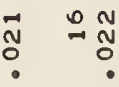

N

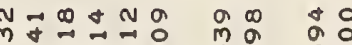

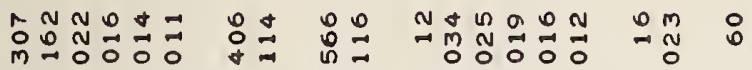

n n a o n

$+\frac{0}{n+\pi}$

$\prod_{m \rightarrow 0}^{m}=0$

v m

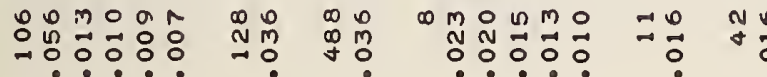

000000

용ㅇㅇㅇ

$\circ$

$\therefore$

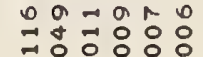

$m \infty$
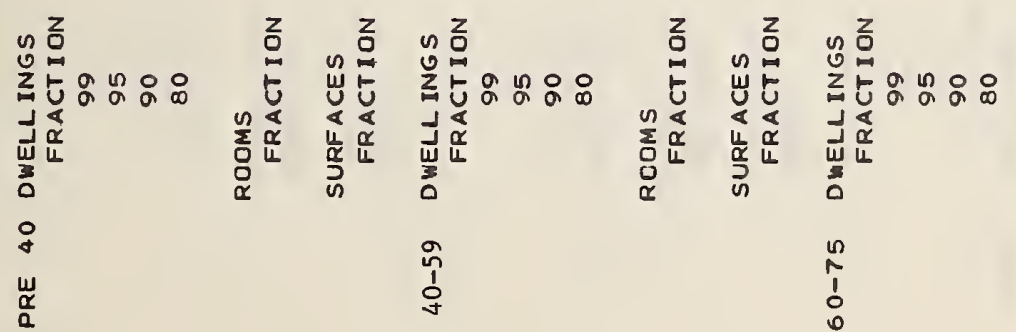

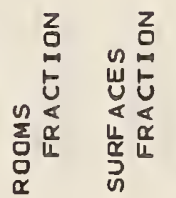

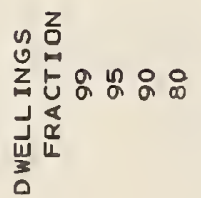

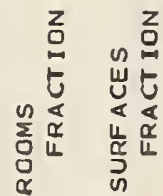

$\prod_{1}^{n}$ 


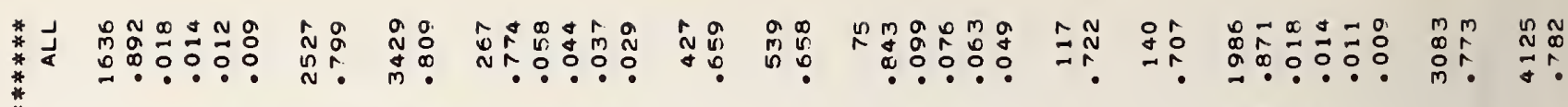

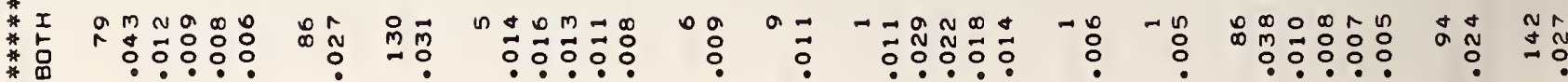

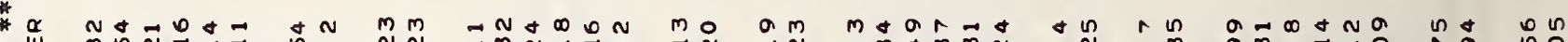

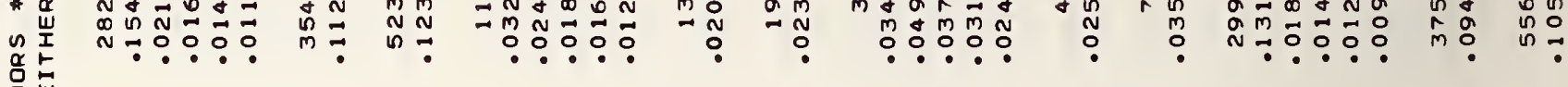
品 *

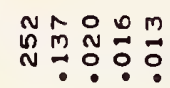

\begin{abstract}
$\infty$
\end{abstract}

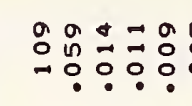

mํำ

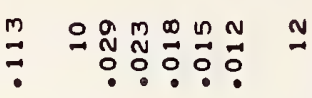

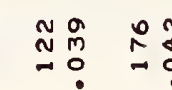

$\frac{0}{\square} \div$

N

$m \& a r \rightarrow+$

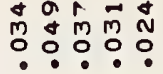

$\rightarrow$ n

$r$ in

$\begin{array}{lllll}\infty & \infty & m & -1 & 0 \\ 0 & = & \overrightarrow{0} & \overrightarrow{0} & 0\end{array}$

$\infty$ in 0 ט

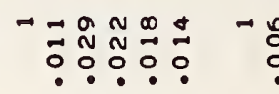

$\rightarrow$ in

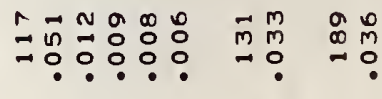

$\rightarrow \sim m N+$

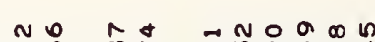

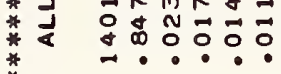

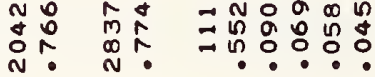

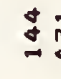

$\stackrel{8}{9}$

Nั่

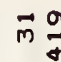

$+$

$m \stackrel{m}{m} \infty$ in $N$

ñ

$\stackrel{N}{N} \hat{N} \stackrel{m}{M}$

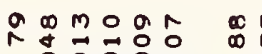

$\prod_{0}^{m} \stackrel{0}{m}$

in $\ln \infty \infty$

N $\begin{array}{lllll}N & N & N & 0 & 0 \\ 0 & 0 & 0 & 0 & 0\end{array}$

-

a.

- 00000

$\circ \circ$

$\circ$

$\stackrel{0}{0}$

$v \rightarrow r m$

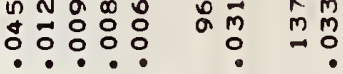

粢

N $m N n$

$\stackrel{M}{N} N$

กำ:

nOnNa

$\stackrel{*}{*} \stackrel{N}{*}$

a n r a t

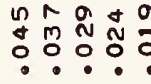

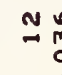

$=0$

- Nom $⿻$ N N

$\begin{array}{lllll}N & 0 & m & 0 & \alpha \\ N & 0 & 0 & m & N \\ 0 & 0 & 0 & 0 & 0\end{array}$

N

$m$

t mo $0 m o$

$N=m r$

Noํำ

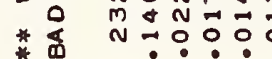

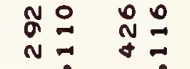

\&

*

*

* Z

a $00 N 0$

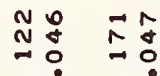

$\therefore: 000:$

$0 \circ \overrightarrow{0} \dot{0} \circ$ n

m m

.

o

No⿱ $)$

N

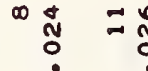

응ㅇㅇㅇㅇㅇㅇㅇ

$\circ$ :

8

$m \stackrel{m}{m}$

Mna n

N $\rightarrow 00000$

i

* 岩

*

$m \infty$ o 0 anam n

$\stackrel{m}{\vec{\imath}} \overrightarrow{0} \overrightarrow{0} \overrightarrow{0}:$

$\stackrel{\infty}{N} \stackrel{N}{N} \stackrel{N}{N}$

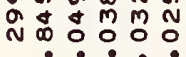

$N 0$

$\infty \quad 0 a \Rightarrow n N D N$

ar $0=$

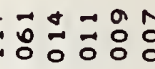

$\begin{array}{ll}N m & d \\ m & \infty\end{array}$

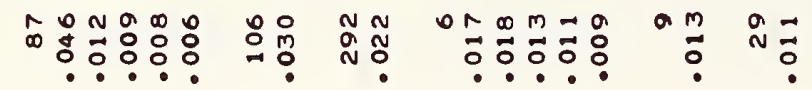

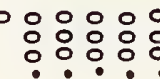

番

$0 \rightarrow \infty m 000$

$\stackrel{O}{\infty}_{\infty}^{\infty} \overrightarrow{\mathrm{N}} \stackrel{0}{-1}$

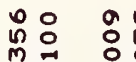

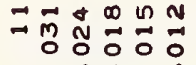

$\stackrel{n}{*}$

$\div 2$

$\stackrel{5}{0}$

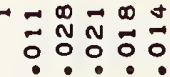

$m \& 00 m$

*o

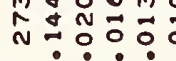

m

$\infty_{\infty}^{m}$

$\stackrel{m}{r}$

a ก

$\stackrel{\infty}{*} \underset{0}{0}$

o

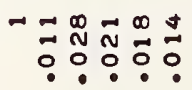

웅

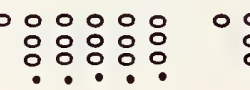

०:

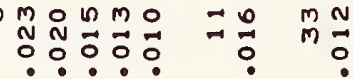

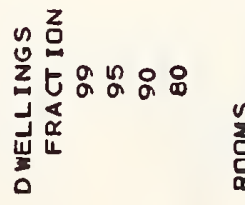

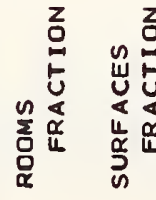

㫐告。

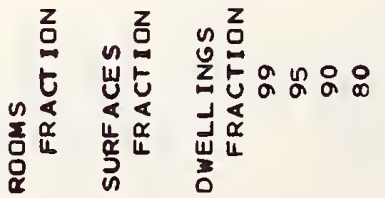
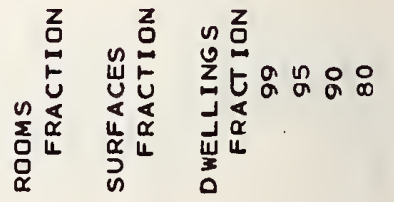

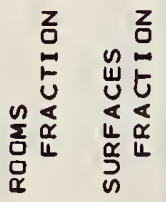

ڤิ

$\dot{m} \vec{m}$

$m \infty 0 N O \infty$

8

o t a

No $\infty 0$

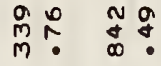

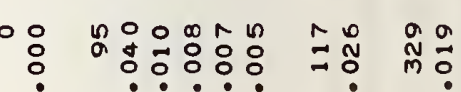

$\infty=\quad$ nnm $\Rightarrow$ n

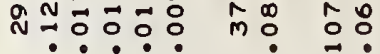

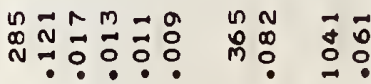

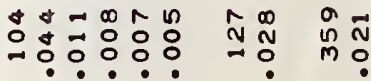

㟧

$n$
1
0 
ThN

งกำ?

mo

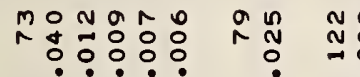

$0 N=0 M O$

Oำำㅇำ

N $: 0 \%$ :

$\vec{m} \stackrel{0}{n} m$

$\stackrel{m}{N} \stackrel{N}{0}: \overrightarrow{0}$

notod

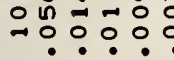

N

$\sim_{\infty}^{\infty} \cong$
*

$m-\infty-\infty$

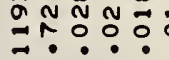

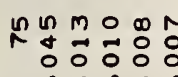

?ㅇ?ㅇ

$\cos 2$

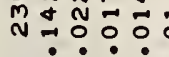

오ำ

กำ กี

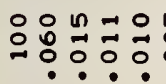

ต

ผ

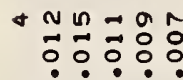

- om $m$ in

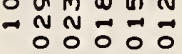

:? : ?

$\prod_{0}^{0}$

$\infty$

a ñ

ำ 000

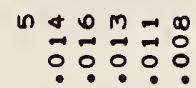

N

$\stackrel{0}{m} \underset{m}{0}$

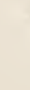

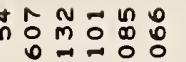

$\because \because$ ?

n $\begin{array}{r}n \\ \circ \\ \circ\end{array}$

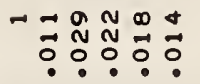

$\underset{0}{n} \cong$

$m \notin a r \vec{m}$

món $\begin{array}{ccc}m & \text { n } \\ 0 & 0 & 0\end{array}$

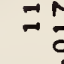

$\because$

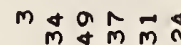

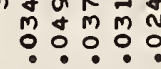

$\begin{array}{rr}0 & \infty \\ 0 & 0 \\ 0 & 0\end{array}$

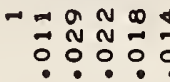

$\vec{\infty}:$

$\stackrel{\infty}{a} \underset{\alpha}{\infty}$

คิm

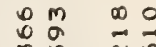

ก: : : $\quad \stackrel{m}{n}$ in $N$

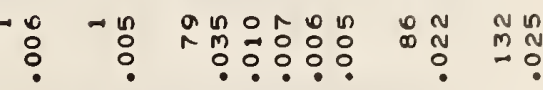

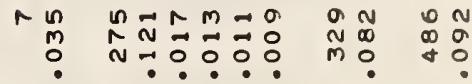

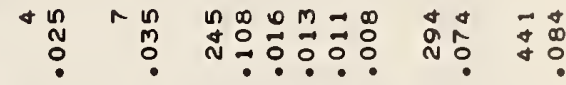

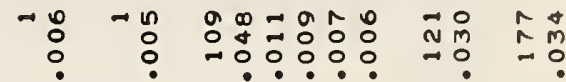

$\rightarrow \infty$ $\because N$

${ }_{\infty}^{m} \vec{m}=m$

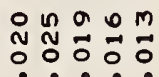

o

nีก

$\infty \circ$ Un $m$

o $\begin{gathered}m \\ 0\end{gathered}$

웅

$\stackrel{n}{2}$

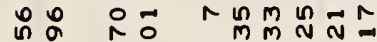

$m$
0
0

an

$\pm m$

N

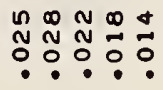

$\stackrel{\infty}{0}$

o.

a

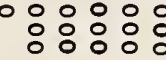

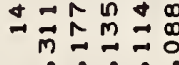

00000

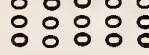

$\rightarrow N 0 M \circ D$

N

- NOMOD

N

-.? ?

ㅇ?ㅇㅇㅇ
$\stackrel{a}{N} \quad \vec{N}$

0 : 0

N

N $m$

N

N

$\circ:$

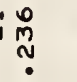

$\infty_{N}^{0} \stackrel{M}{N} \hat{N} \vec{N} \stackrel{\infty}{0}$

\section{$\cos 0$}

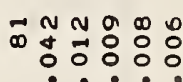

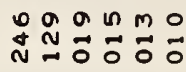

No

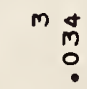

o n 0 \&

N $\Rightarrow \overrightarrow{0} \overrightarrow{0} \overrightarrow{0}$

or $a m$

0

Nomoar

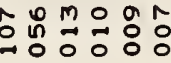

ㅇ 90

$\underset{\sim}{m} \stackrel{0}{0} d$
ํำ

MNOOOO

م. in ${ }_{\infty}^{\infty}$

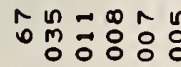

ก N

MN N $N$

$\circ$

ㄴ? 0 ?

$\stackrel{\infty}{N}$

ก $20 \pm 0$

N $=\overrightarrow{0} \overrightarrow{0} 0$

NA N N

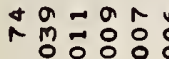

$\prod_{\infty}^{n}$

品

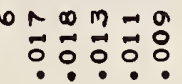

a

N

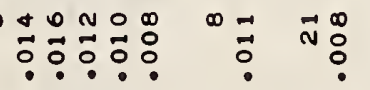

응ㅇㅇㅇㅇㅇㅇ

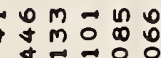

눈?

in

nู

nดษ 00

$\overrightarrow{0}: \begin{array}{llll}0 & \mathbb{1} & \overrightarrow{0} & \overrightarrow{0}\end{array}$

$\stackrel{n}{0} \rightarrow \stackrel{a}{n}$

旁药

: 0

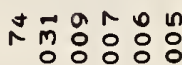

ก

$=-\infty-\infty$

$\vec{\sim} \stackrel{\infty}{\sim} \stackrel{\infty}{*}$

$N \underset{0}{\overrightarrow{0}} \quad \infty=$

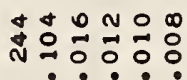

กัก

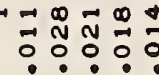

$N=\infty \overrightarrow{0}$

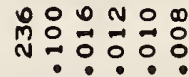

$\infty+N$ n

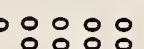

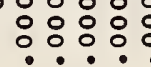

$\circ \circ$

n norom

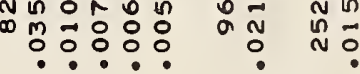

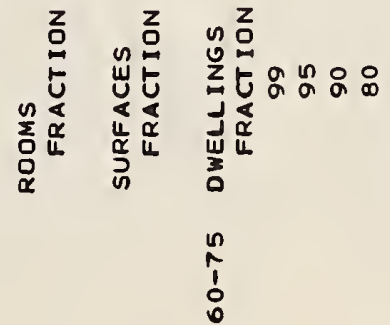

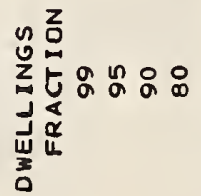

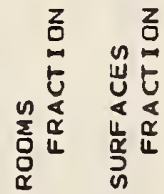




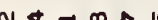

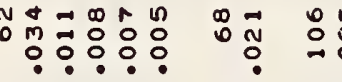

\section{in} $\begin{array}{llll}m & m & 0 & 0 \\ 0 & 1 & 0 \\ 0 & 0 & 0 & 0\end{array}$

ง

点是:

ON舟 N

ㅎํํํํํํำ

a

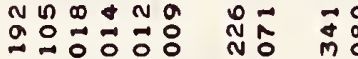

它品

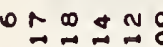

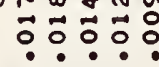

(1)

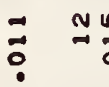

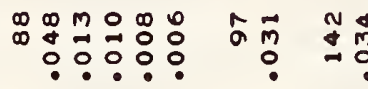

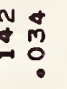

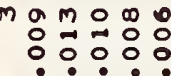

(1)

a oma 0

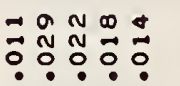

N NOFO

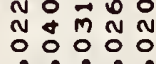

-. ?

N NOM

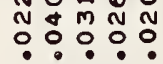

$0 \%$

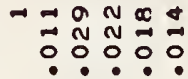

$\stackrel{\square}{\square} \stackrel{*}{\div}$

$\because \quad-0$

N $m$

:

N

N으유:

n

$+\because \quad-\because$

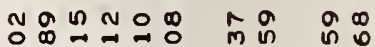

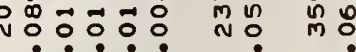

ตี

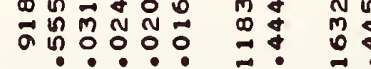

mid

Mำ

$m$

$\stackrel{\circ}{n}$

$\infty \mathbb{N}^{\infty} \infty$

\begin{tabular}{l}
0 \\
\hdashline \\
0
\end{tabular}

$\cong$

$\stackrel{n}{n}$

nก ㅇํㅇํำ

mm 옹

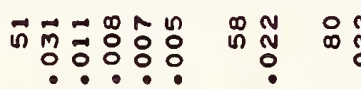

$m \cup N \sim$

ำำ

mo

응ㅇㅇㅇㅇㅇㅇ

$\circ 8$

$\circ:$

กี่ำำำ

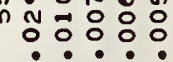

n ก $\infty N \infty$

N ${ }^{\infty} \underset{N}{N} \stackrel{N}{N}:$

$0 \stackrel{0}{0} \quad 0$

$\rightarrow$ N

w

ก

T $n \infty m-a$

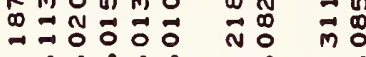

- $\bullet$

ㅇํㅇำ

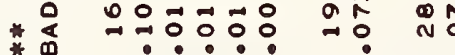

n $\mathbb{N}_{N}^{\infty} \underset{N}{N} \stackrel{\infty}{N}$

$0 \stackrel{0}{0}$

O: O O

:

$\vec{N}$

- NOMD舟

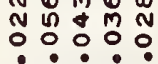

N

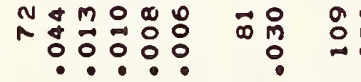

옹

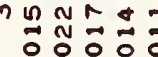

$m g+g$

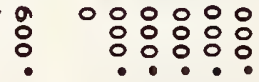

$\circ 8$

$m$
mo
0
0

$\rightarrow \overrightarrow{0} 0 \overrightarrow{0}:$

$\sin$

N

in

*

* d a a nan

*

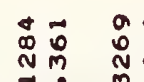

$\rightarrow+N m a \rightarrow n$

คำ?

$\rightarrow$ ?

-

N

* I

ค กำํำ กี

*

ㅇํㅇ

: $₫$

$n+0$ n

웅ㅎㅇ용

$\div \div$

응유유.

$\stackrel{0}{0}$

$\stackrel{\infty}{m}$ m

ก

$m=M=\infty$

$\Rightarrow$ 유유.

\& 00

0
0
0
0

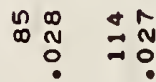

\section{n}

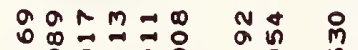

$\begin{array}{lll}m & 0 & m \\ N & N & M \\ 0 & 0 & 0\end{array}$

$\neq$

$\sim \frac{0}{0}$

$\stackrel{\leftrightarrow}{\mathrm{a}}=$

응ㅇㅇㅇㅇㅇㅇㅇ

$\circ:$

$\circ:$

$\Rightarrow$ : O O :

n $\vec{N}$ in …‥

$\prod_{0}^{m} 0 m=\infty$

$\cos$

omo n $m$ o

$\begin{array}{lll}m & \text { n } & m \\ N & N & 0 \\ 0 & 0 & 0\end{array}$

$\Rightarrow$

$: \stackrel{a}{*}=$

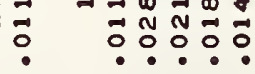

N

$\wedge$

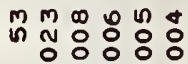

$\pm m \quad 0 N$

㝵

*

$\rightarrow$ ह

mºñ

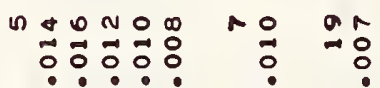

$\stackrel{7}{0}$

$\overrightarrow{\vec{D}} \stackrel{\infty}{N} \vec{N} \stackrel{\infty}{0} \stackrel{\overrightarrow{0}}{0}$

n

$\because \quad r$

$\stackrel{0}{\circ}$

$m_{00090}$

으의

$\therefore \stackrel{m}{\circ} \stackrel{0}{\circ}$ ㅇํㅇำ

? $\rightarrow$
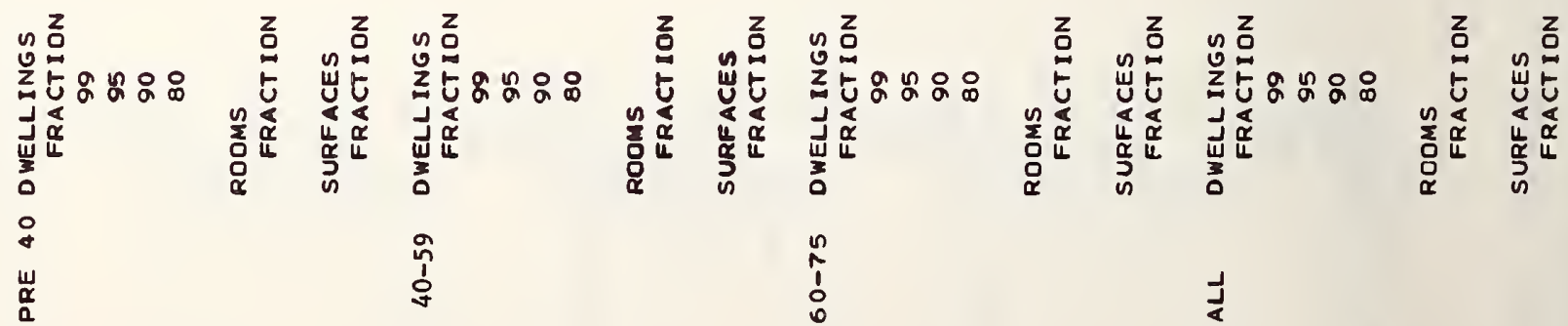


\section{onanad}

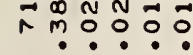

于

$\infty 0 \wedge m \rightarrow \infty$

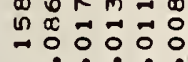

a 00 NO

Mำำㅇำ

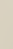

(1)

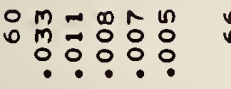

$\stackrel{0}{a} a$

워

$\hat{\wedge}$ i

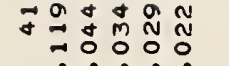

$\begin{array}{ll}n & 0 \\ 0 & 0 \\ 0 & 0\end{array}$

$m g m: \infty$
$: 00: 0$

no $0 m=\infty$

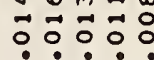

\section{$\infty a \infty$}

ำㅇํㅇㅇㅠ.

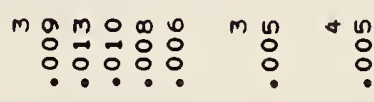

$\circ:$

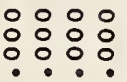

n $\triangle 0 m=\infty$

$n$
0
0
0
0
0

응ㅇㅇㅇㅇㅇㅇ

000000

0
0
$\circ$

응ㅇㅇㅇㅇㅇ

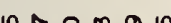

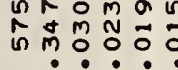

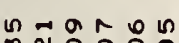

ำ ํํㅇㅇㅇㅇ융

(1)

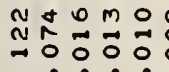

Anono

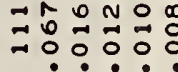

น

응유.
คํํำ N N $\because$ :은?

mำ ถึำ

mกN

อำำ.ำ

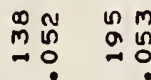

ก

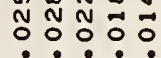

$\stackrel{0}{\sim} \underset{0}{0} \stackrel{0}{=}$

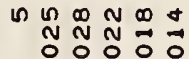

응ํํ

เ

$m ! N$
0
0
0

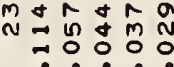

กิ

mo

$\infty$

$\infty$

mo

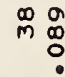

$+9$

$a$

$+$ $a$
+

응융요

ก.

- NOMDO

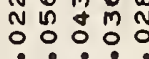

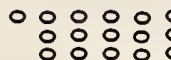

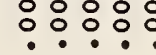

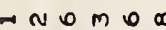

or

?

$\circ 8$

$\circ 0$

000

odnatar mo nn

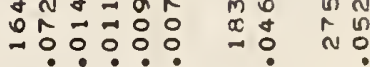

\section{0 na mono na a}

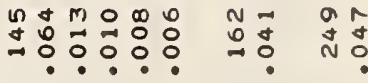

$\circ \circ$

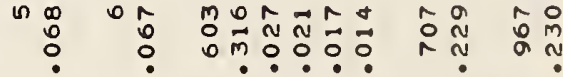

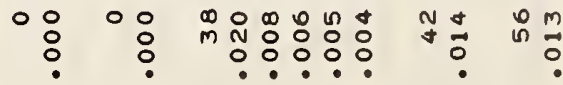

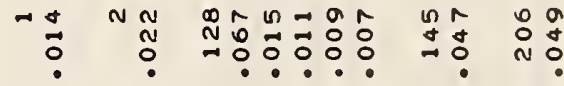

‥

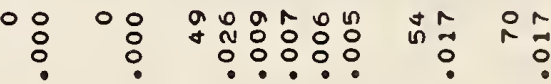

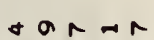

0.000 .000

mํํำ

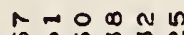

:

$\ln 0.000$

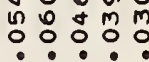

in

$\infty=$

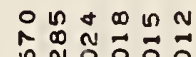

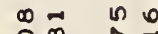

ำ

ำ:ㅇำ

$\rightarrow \frac{0}{2} \div$

-・.

$\therefore: ㅇ: 8$

0000

$\therefore$

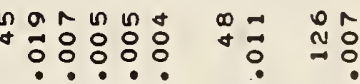

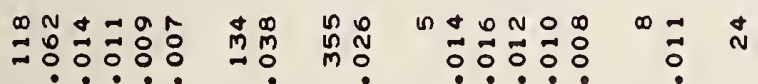

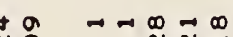

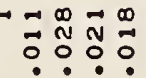

$\rightarrow$

nm

I $n$ in $\mathrm{m}$

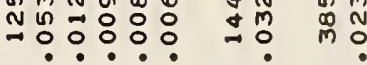

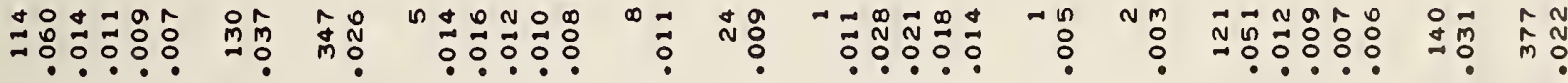

+m

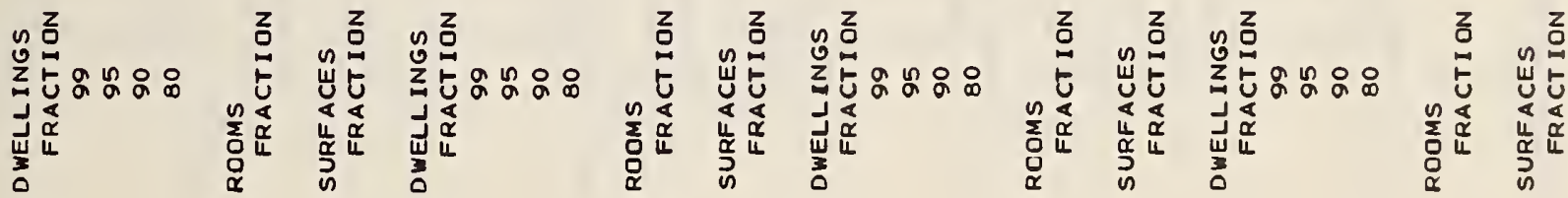
\&

$\stackrel{w}{\alpha}$

in

$\stackrel{n}{\grave{j}}$ 


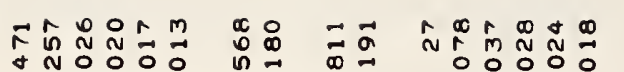

.

ก) $\div$

ㅇํㅇํำ

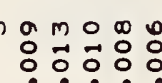

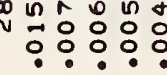

嵌

잉

*

*

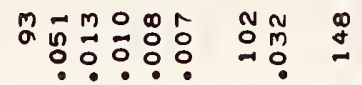

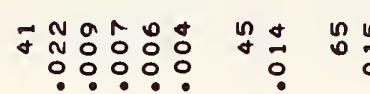

a moorm

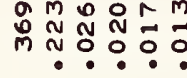

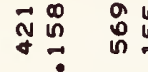

帒

a o n n

$\begin{array}{llll}a & m & 0 & 0 \\ 0 & 0 & 0 & 0 \\ 0 & 0 & 0\end{array}$

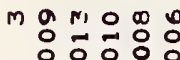

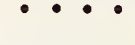

$m a m 0 \%:$
00000
000

ㅇํㅇㅇ․

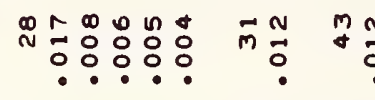

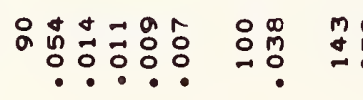

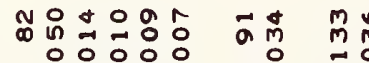

ㅇํㅇㅇํ

mกำํํㅇํㅇำ

ㅇㅇㅇㅇㅡ.

: $\rightarrow$ ก $m \circ \infty$

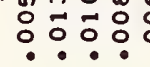

Nom $\infty$

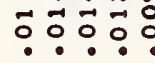

N

우융ㅇㅇㅇ

- $\begin{array}{r}m \\ 0 \\ 0\end{array}$

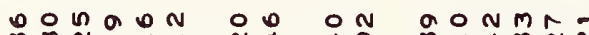

M MN

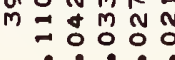

\section{$m$ m $N \circ \infty$}

웅ㅇㅇㅇㅇㅇ

N m

$\checkmark$

$\neg=$ $\overrightarrow{0}: N \quad \overrightarrow{0}$

$-\infty$
$\circ$

$r$ !

$\circ a \approx n \pm=$

ถก

$\begin{array}{lll}N & 0 & 0 \\ 0 & n & n \\ 0 & 0 & 0 \\ 0 & 0\end{array}$

m n

$\circ \circ: \circ: ㅇ$

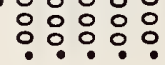

$\circ:$

$\circ$ 웅

$\vec{m}+000 \%$

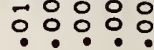

min n

m n

$+$

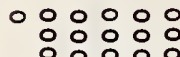

응ㅇㅇㅇㅇㅇㅇㅇㅇ

$\circ$ :

$\circ:$

$a \infty=0 \hat{n}$

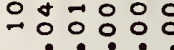

a o a

$=\stackrel{m}{0} \stackrel{m}{0}$

$m$ ก

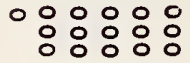

$\circ$ 응

$\circ$

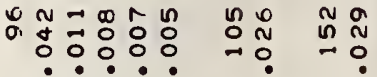

m

$\circ 0000$

0
$\circ$

$\circ$ :

$\circ$

a

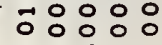

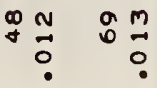

0
0
0

$\rightarrow N O M \circ \infty$

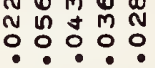

$\rightarrow+$

$\rightarrow \begin{array}{rr}m & -1 \\ 0 & 0\end{array}$

$m g \circ$

$m$

9 $\circ$

$-$
응ㅇㅇㅇㅇㅇㅇㅇㅇㅇ

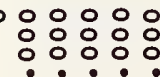
0
$\circ$ $\circ \circ$
$\circ$ n $N M \infty$ n $N$

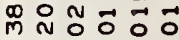
ำำำํำำ ㅇㅇㅇㅇ․

$2 \infty$

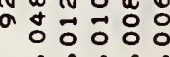

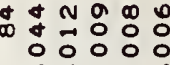
.. .

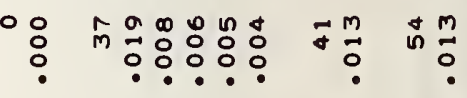

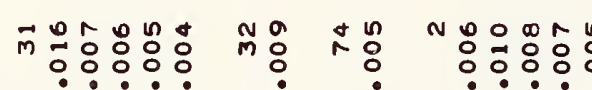

ㅇㅇㅇㅇㅇㅇㅇㅇ

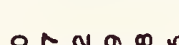

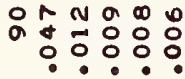

No mo

$\begin{array}{llll}\infty & N & 0 & 0 \\ \circ & 0 & 0 & 0\end{array}$

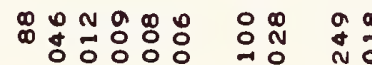

*

*

这

题

is

点

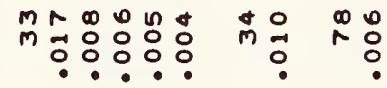

ồ

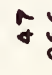

品

$m \circ \infty m$

n

n

:

$m \stackrel{\infty}{m} \stackrel{m}{\circ}$ $m M r$
$M$
0

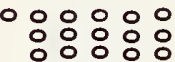

$\cdot \cdot \cdot$

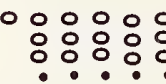

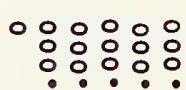

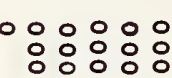

$m \frac{0}{0}$

$\circ \circ$

:

$\circ$

$\circ$

0 웅

$\circ \therefore$ a n 20 a

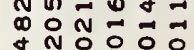

?.?

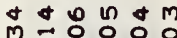

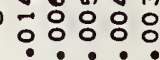

$\begin{array}{llll}0 & 0 & 0 \\ 0 & 0 & 0 & 0 \\ 0 & 0 & 0 \\ 0\end{array}$

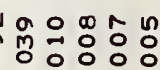

?.? ?

은
Da $N$ N

กิ

$0 \infty \infty$ in

?

$\infty \pm m \infty$

$\rightarrow$ No

$0+a 0$

용

m $\infty \circ$

m:
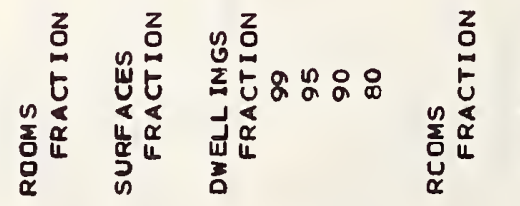

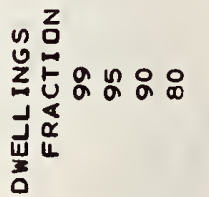

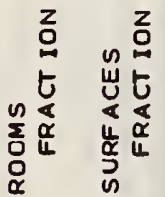


으융요

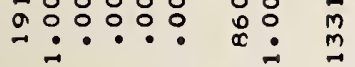

$0 m \infty+a$

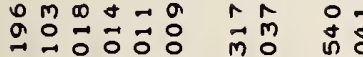

i. - i.

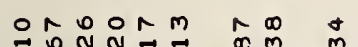

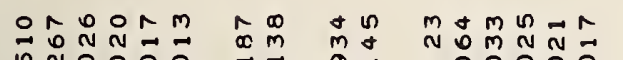

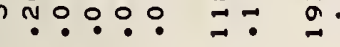

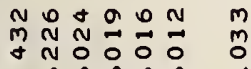

mo

is

n nOOMO

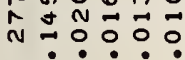

$\because \tilde{n} \hat{0}$

กิ

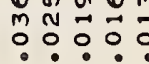

in $: \circ: \circ: \circ$

0.000 .0
20

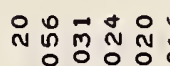

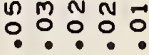

a

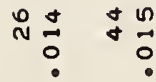
응요

+ $0 m 00$

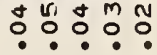

טヘ

○ 0 ○

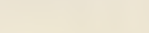

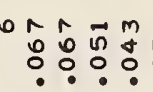

+ $0 m \infty$

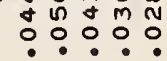

$m$

$n \pi$

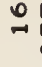

M N N

w

?

$\stackrel{0}{1}$

N

ô

nN $\frac{\pi}{0}$

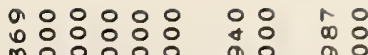

N

Nantor an a

$\vec{N} \stackrel{0}{0} \overrightarrow{0} \overrightarrow{0}:$ में ${ }^{m}$ n

$m a n T+a n$ in

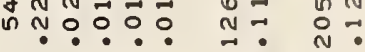

N n $\overrightarrow{0} 0 \mathrm{~m} 0 \mathrm{~N} \overrightarrow{0} \overrightarrow{0}$

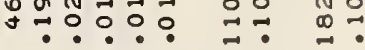

กำก

N
응ㅇㅇㅇㅇㅛ

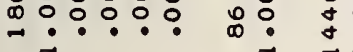

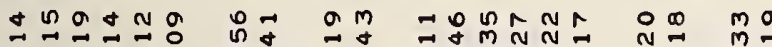

$\vec{N}=\overrightarrow{0} 000$ Mั

O NROAM mO D

MNMN

o

a. $0: 0.000$

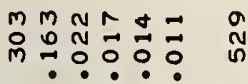

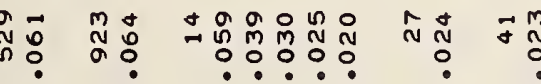

NO: 000

응ㅇㅇㅇㅇㅇ

$\rightarrow$

$m \infty m m m-$

เก

$\operatorname{sen} 2$

คิด ำ

$\operatorname{tinh} h r$

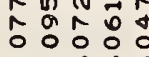

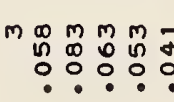

$\vec{N}:$

a

ก:

$r \stackrel{m}{M}$

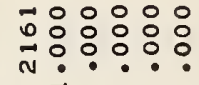

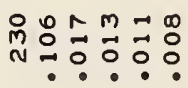

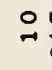

n?

in 0 i

กิก กับ

음

응

N⿴囗十心

+ กี:

$\stackrel{\infty}{\infty} \sim \stackrel{m}{N}$

na a in no

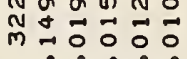

은

mo 00

mo

on $\stackrel{N}{N}:$

우웡응 운 $\stackrel{\infty}{-}$

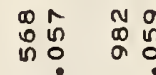

$+00000 \hat{2}$ N $\pi$ ㅇำ

NmA-Am

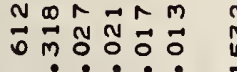

N $\vec{m} \quad \vec{m}$

M

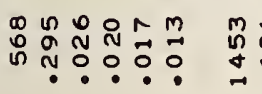

$\vec{m} \stackrel{\vec{N}}{0} \overrightarrow{0} \overrightarrow{0} \quad \overrightarrow{0}$

宊年

m: $m_{m}^{\infty}:$

$\begin{array}{ll}n & 0 \\ m & 0 \\ \infty & 0\end{array}$

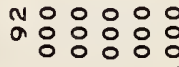

:? ?

용

N

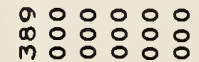

กำ no

$\therefore$. -

$\rightarrow$

ON $\rightarrow \vec{n} \vec{N}^{\infty}$

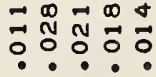

$\rightarrow n$

$+\frac{0}{2}$

N:- : *

¿- 尚:

o or $m=\infty$ om

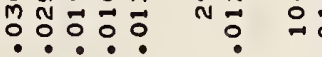

a or $m \infty$

:0:0?

กิ

Aman n

0
0
0

$=\underset{0}{0} \stackrel{+}{0}$

$\hat{N}=\overrightarrow{0} \overrightarrow{0} \overrightarrow{0}$

$+\begin{array}{lll}m & m \\ 0 & n & 0\end{array}$

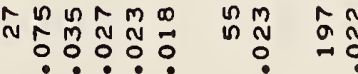

mMr O O

$\stackrel{\circ}{\circ}$

mo ? o

$\rightarrow 2$

$\stackrel{9}{\circ}$

N

คำกับ

$09+\infty$

ล

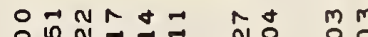

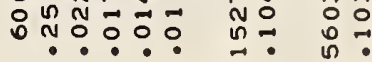

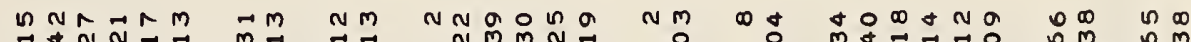

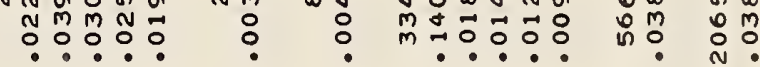
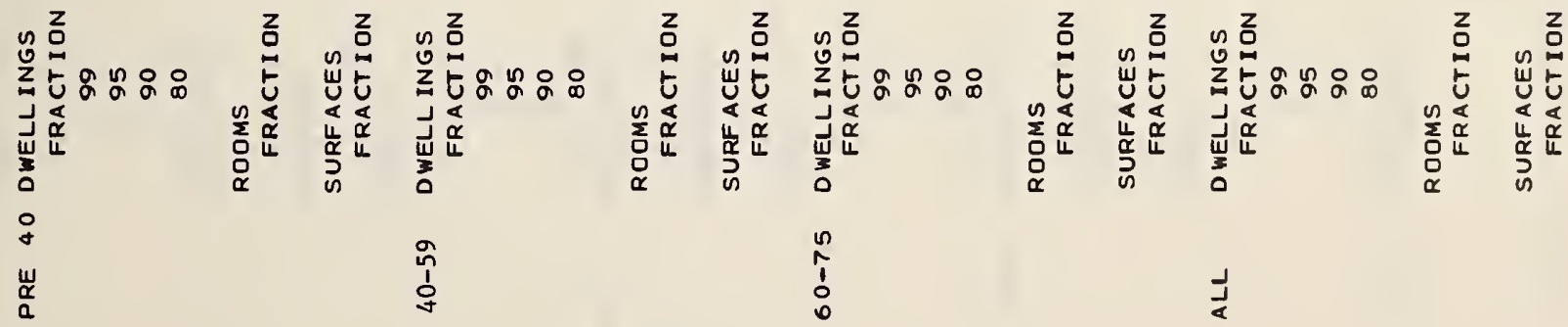


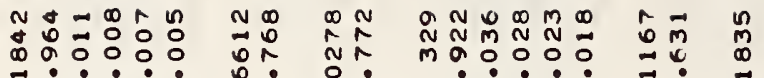

* \&

* I $R \vec{a} \tilde{a}=$

H.

号

次吉

$\overrightarrow{0} 0+\infty 0^{\infty} n$

*

\#

*

*

*

0 in mor

0 a

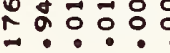

$\overbrace{\substack{n \\ 0}}^{\infty}$

\section{$n$}

?. ?

$\infty$ n

$\rightarrow$.

No 000

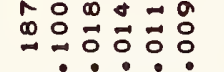

n

M. N

푸웅

ก M

$\stackrel{\infty}{\circ} \stackrel{0}{\sim}=$

$0 m+\infty$ n $N$

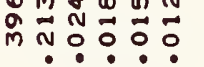

웅융ㅇㅇ

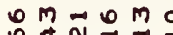

N 900000

$m$
0

冓

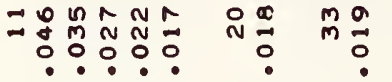

m

$\sin a t a n$

A으으.

舟品

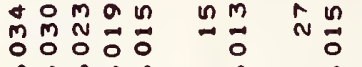
- $\bullet$

$\cdot$

in

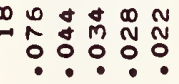

$\stackrel{0}{m} \underset{m}{m}$

$0 \stackrel{n}{0}$ $\because$ : : :

$\stackrel{\infty}{\circ}$

No

ng

ㅇำ 0 \%

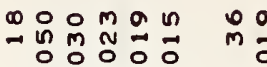

ma $\frac{1}{0}$

$-\infty a n \infty$

O

$\stackrel{\infty}{0} \overrightarrow{0}$

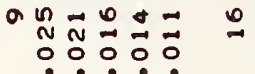

$\because 0 \quad \stackrel{0}{*}:$

? 0 ?
$+$

\& $0 \begin{array}{lll}m & 0 \\ 0 & 0 & 0\end{array}$

ํ.

$\begin{array}{llll}0 & 0 & 0 & 0 \\ N & 0 & 0 \\ N & 0 & 0 & 0 \\ N & 0 & 0 \\ N & 0 & 0\end{array}$

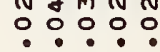

o 090

$00 n \frac{m}{a}$

$\infty N \rightarrow \propto \wedge n$

$2 m$

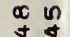

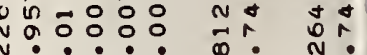

a

N.P.

n

$N M$

m D品

นna âa

$a$
0
0

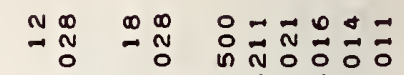

La $a$ in

0 a

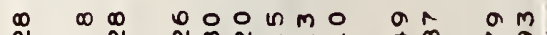
:

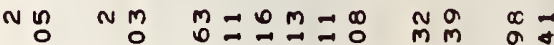

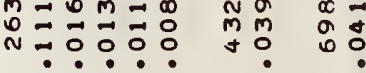

में

ma

$\vec{n}$

$\infty \infty$

+ $m \in \infty$

ค $\infty \stackrel{0}{\infty}$

\section{Doman nm to o o o o}

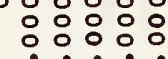

$\circ$

웅

a जे $\overrightarrow{0} 000$

0

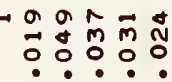

$+\frac{0}{0}$

$\stackrel{\infty}{\circ}$

O

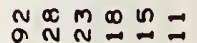

每: $0: 0$ :

Di n

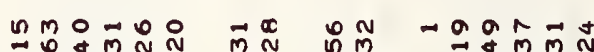

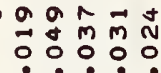

$+\infty$

1 은

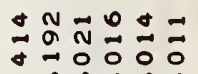

$m a$

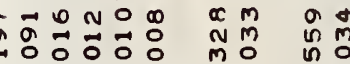

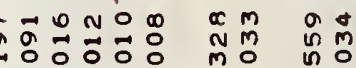

응ㅇㅇㅇㅇㅇㅇ $\circ \stackrel{\circ}{\circ}$

กิำ $\vec{\infty}$

冓

$\vec{m}_{\infty} \stackrel{0}{10}$
* * * *

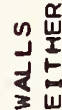

* $*$

* * Z * * on

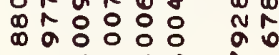
- - : $N$.

$n N \infty \circ n$ $\vec{N}=\overrightarrow{0} 00$

\&

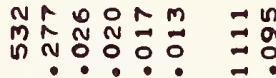

mon 0

o in

o

$n \infty \circ n m$

NM⿻上丨:

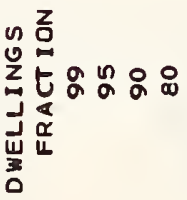

\&

$\underset{\alpha}{\frac{\pi}{a}}$

का

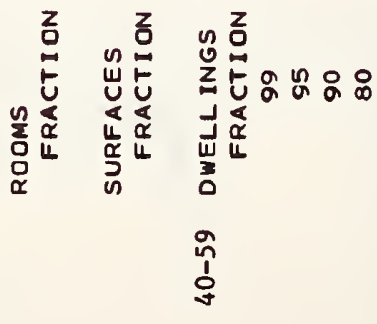

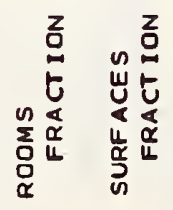
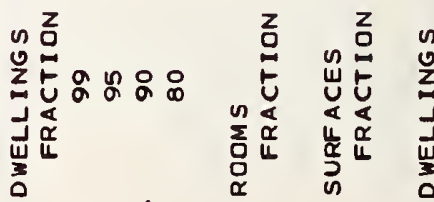

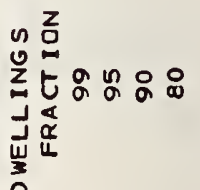

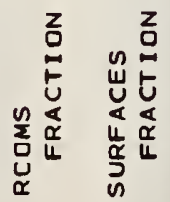

$0 \sim \quad n N$ $\begin{array}{llll}\infty & 0 & 0 & \infty \\ 0 & 0 & 0 & m \\ 0 & 0 & 0\end{array}$

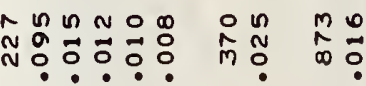

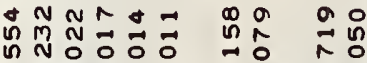

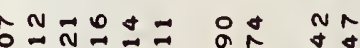

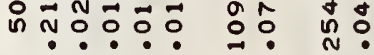

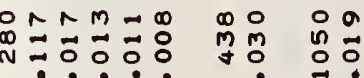

5
$w$
$\frac{1}{5}$
0
$I$

i 
$0 m \infty \& N a$

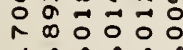

$\rightarrow$... ถ ํ. 各

$n \rightarrow 0 N O$

$\sim 0_{0}^{\infty} \overrightarrow{0} \overrightarrow{0} \quad \overrightarrow{0}:$

$\stackrel{n}{\mathrm{n}}$

in

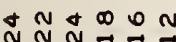

$\sim$ ก:?

$\infty \sim m \wedge n$ $\infty$

2
0
0
$d$
0

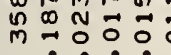

no

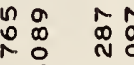

\& $2 \pi+a$ N $=\overrightarrow{0} \overrightarrow{0} \overrightarrow{0}$

$\hat{o}$

$\begin{array}{lll}m & 0 \\ 0 & 0 & 0 \\ 0 & 0 & 0\end{array}$

no $\infty \stackrel{\text { N }}{N} \cong m \circ$

융

คที่ำ.

-.

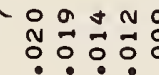

$\operatorname{nn}$ N

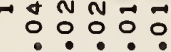

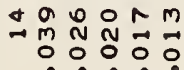
웅으

w

in

$m$

$\infty$

$0 \mathrm{~m}$

$\Rightarrow 0$

$\because 0$

$\vec{m} \overrightarrow{0} \quad \omega$ $\ln _{0}^{\infty}$

$m_{m}^{\infty} \hat{m} \vec{m}$

ㅇํㅇㅇ․

$m \stackrel{m}{m} \vec{m}$

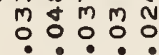

nNoOd no

0
n.
n
0
Nล⿵ n. ma

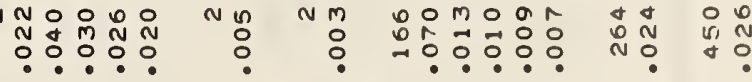

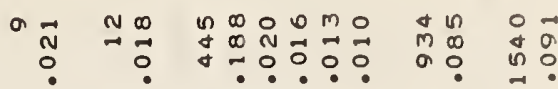

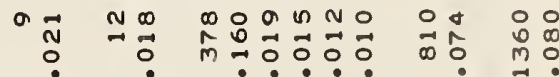

NUNM NOONON $N m$ N

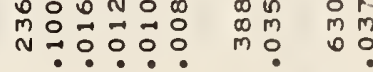

$0+\pi m^{0}$

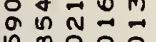
n $\infty$ : : :

$00 N 0 \quad \infty \infty m+m N$

$\ln (x)$

$0-\pi m \rightarrow$

$\stackrel{\sigma}{\sim} \overrightarrow{0} \overrightarrow{0} \overrightarrow{0} \overrightarrow{0}$

文?

- 40:

N?

\& $\because \because \exists$

กั

a

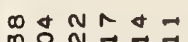

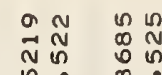

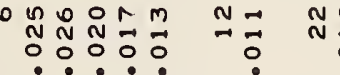

N $\frac{0}{N}$

응ㅇㅇㅇㅇㅇ

ㅇㅇㅇㅇㅇㅇ

$\circ$

$\circ \circ$

i

$m m$ n a o

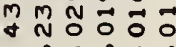

$\infty \Rightarrow \quad \infty=$

n $M O=0$

90

$\vec{n}+a g \hat{m} \vec{m}$

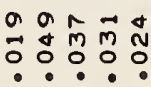

$+$

$\stackrel{\infty}{0} \quad 0$

$0 \infty m \infty$ in $N$

$\infty \sim \quad m$

กิ

$m \because 0: 0$

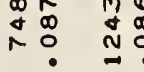

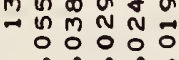

a g

$+\infty$

$0 \stackrel{0}{N}$

$\infty \infty \rightarrow \infty m 0$

$\because$ ? : ?

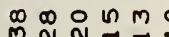

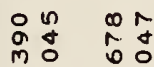

oroman

$\pm \frac{0}{N}$ ก

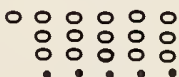

$\circ$

$\circ$ :

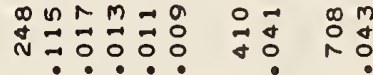

等

$m m-r \quad n+a n \infty \circ$

\&

od nUn-n

N

$\ln _{n}$

na in mo

$A+N N$

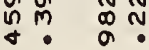

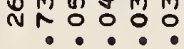

N N N

ทั?

$\rightarrow$

No

a. 0.000

m $m$ m

na n nOD

N $\stackrel{a}{a} \stackrel{\infty}{a}$

nNonmo

$\cong \hat{\circ} \quad$ m

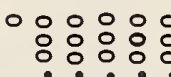

$\circ$

$\circ:$

$\sim \infty M \circ \infty N$

No no

no 0 o $\infty \mathrm{n}$

$n \rightarrow \infty \infty \quad N M+\infty n N$

do no nNaO na

ก:

$m$

2

- 응유.

虫 离

$+\vec{N}: 070$ -

ㅇํㅇำ

\%

$\vec{\sim} \stackrel{\infty}{N} \vec{N} \stackrel{\infty}{0}$

$\overrightarrow{0} 0$ :

N

$\infty$

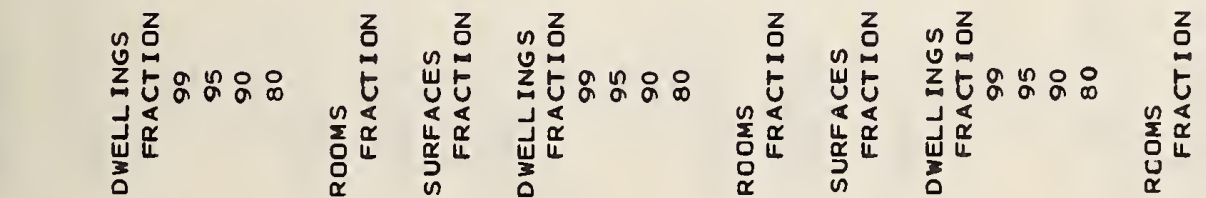

a

in

$\stackrel{n}{1}$

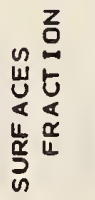

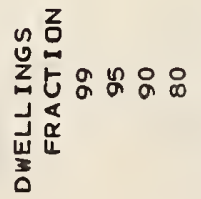

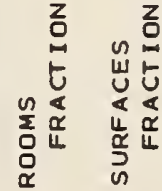

宸

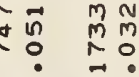

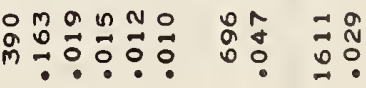

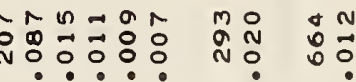


n 0

$0 \pi n=0$

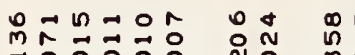
*

*

号

*

*

*

更

* 」

a $\infty$ R $m$

Mีำํำำำ

品

* I

$\hat{N} \stackrel{\infty}{0}: \begin{aligned} & n=0 \\ & 0\end{aligned}$

mo

呈䍃

$\stackrel{0}{0}$

ON M

空

$2 \quad$

七 *

$\begin{aligned} & \overrightarrow{0} \\ & 0 \\ & M\end{aligned} \stackrel{N}{0}: \overrightarrow{0}$

in

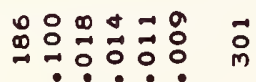

$\stackrel{0}{0} \stackrel{\infty}{0} \overrightarrow{0}=0$

:

虫品

* Ш⿱山⿰丨丨丷

$\frac{w}{\alpha}$

*

+

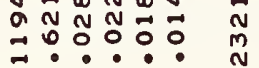

$\infty$ Na monONN

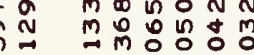

- Nmo

$N \cdot n$

$\because: 0$.

กั

on

an ma

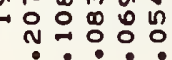

Non

in

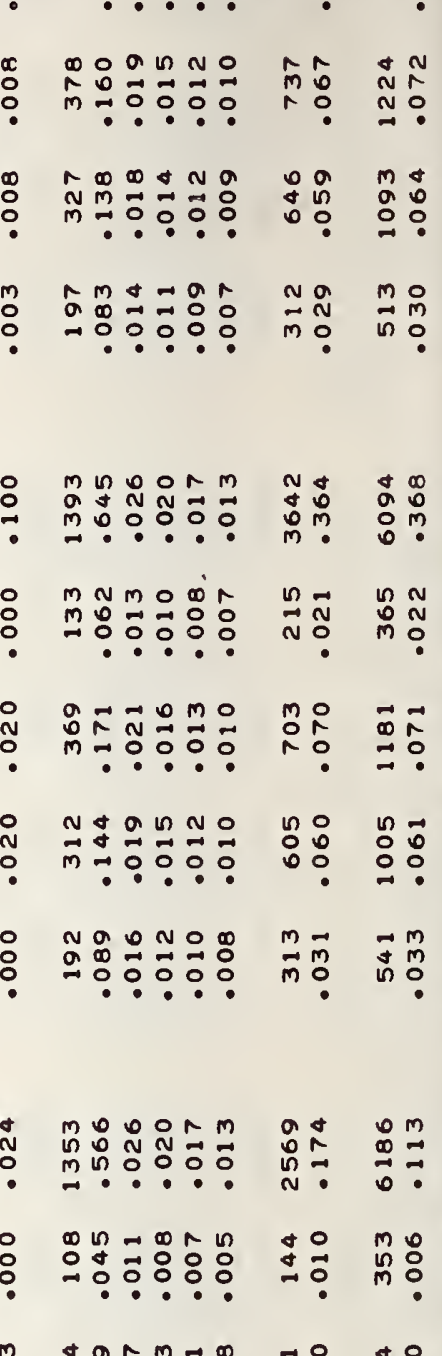

2

$\underset{\alpha}{\alpha}$

แ

*

*

*

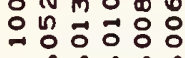

$\stackrel{a}{\sim}=\stackrel{\infty}{-1}$

\section{$\Rightarrow \rightarrow 0$ nmo}

N๋ำ

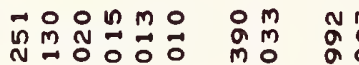

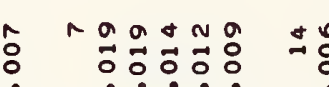

$\therefore$ m

m。

$\circ$ 웅ㅇㅇ

응ㅇㅇㅇㅇㅇㅇㅇㅇ

$\circ 0$

: 0

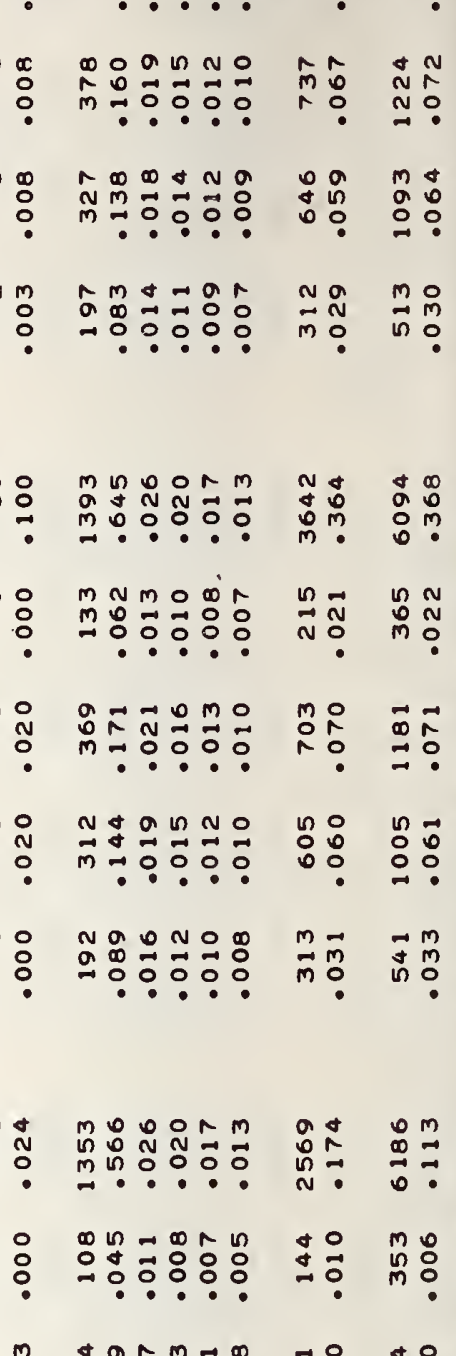

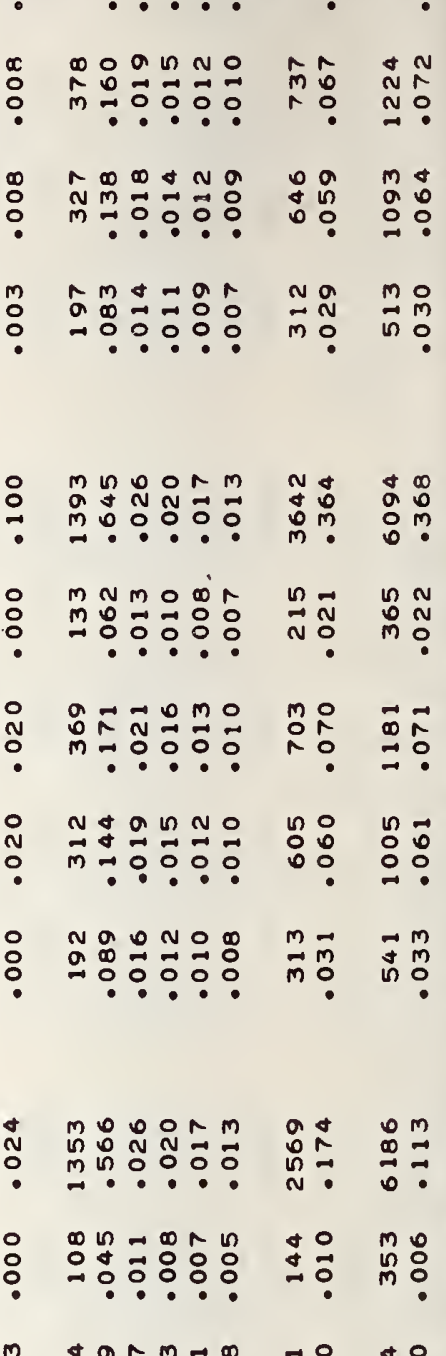

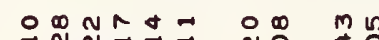

N

ㅇํำ Mำ

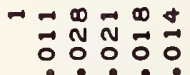

N

$\sim$

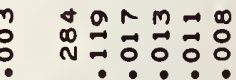

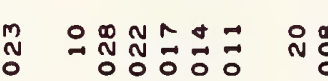

\%

!n

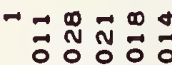

융ํㅇ

$N m$

$\sim$

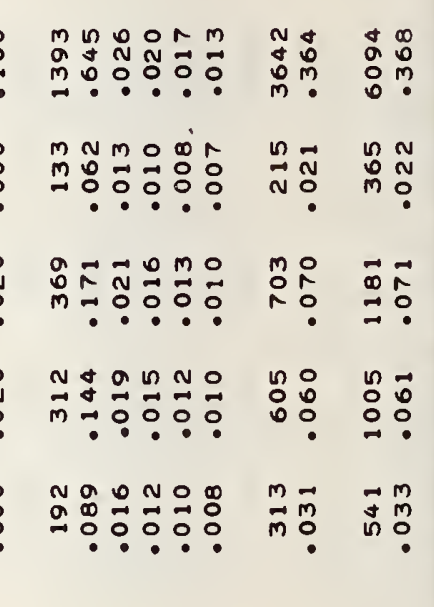

*

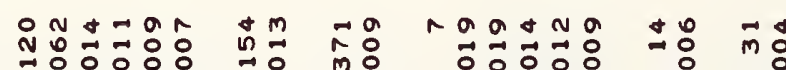

N: 000

*

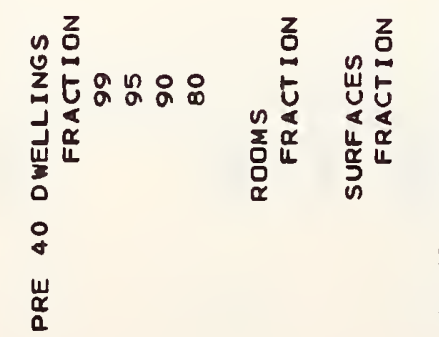

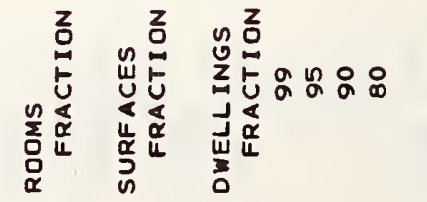
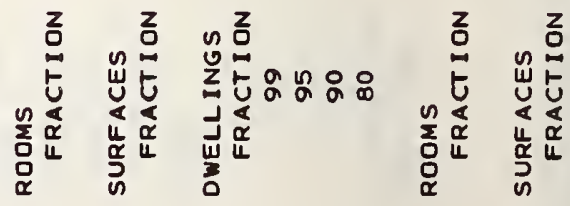

오ำ<smiles>[O][O]</smiles> 


\section{ํํำำกำ ลิำ}

ㄴ.?ㅇํㅇำ

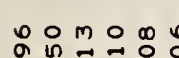

ํํㅇํํ유.

$N \infty-\infty m 0$

$\stackrel{N}{\sim} \underset{N}{0} \overrightarrow{0}: \overrightarrow{0}:$

ờ

o o a n $m 0$

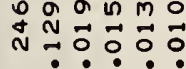

$m \circ n \rightarrow 0 n$

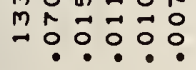

กำ

Na d $\rightarrow$ 는

to

mo
ที่⿺辶大

? ? :

두유.

$\begin{array}{lll}0 & 0 \\ N & 0 & 0 \\ N & 0 & 0 \\ N & 0 & 0\end{array}$

$\begin{array}{lll}1 & 0 \\ 0 & 0\end{array}$

ro요요

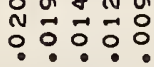

m $\stackrel{n}{N}+\overrightarrow{0}=0 \begin{aligned} & a \\ & 0\end{aligned}$

? : ० : ० : $m$ n
$\infty$
0

n $m$

N

$\rightarrow$

n $\mathrm{m}$

$\stackrel{4}{\circ}$

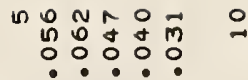

$\circ \circ$
0
$\circ$

$\circ \circ 000$

잉 000000

$\circ$

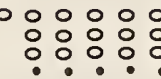

$\circ \circ: \circ: \circ:$

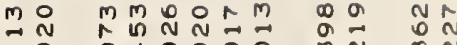
ㅇ․․․

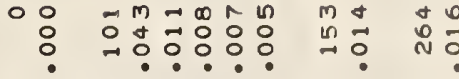

O O OnNm-a n

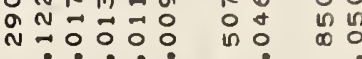

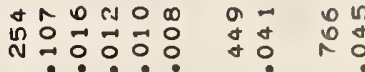

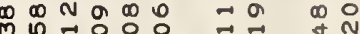
ก:ㅇ: N a o a ma in

O N N N aก?

$m \circ m \circ \infty$ 등

4

N $M$ N

o $\infty$ a n n

$\stackrel{N}{N} \overrightarrow{\overrightarrow{0}} \overrightarrow{0} \overrightarrow{0} \overrightarrow{0} \overrightarrow{0}$

$\infty a n=0$

$\stackrel{N}{\cong}: \overrightarrow{0} 00$ . $\begin{array}{ll}\overrightarrow{0} & a \\ 0 & 0 \\ \sim & 0 \\ \sim & m\end{array}$

$\prod_{\rightarrow \infty}^{n} \stackrel{n}{N}$

10

辛品

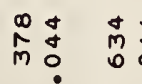

$\vec{a}$
Nִ

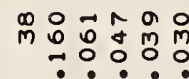

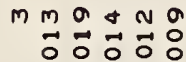

กำ

- $\bullet$ -

ㄴำ ํำำ

ก

$m M \frac{M}{0} \div 0 \%$ $\overrightarrow{0}$

8

$\stackrel{0}{0}$

$\overrightarrow{0} \quad N \overrightarrow{0}$

$\stackrel{N}{\overrightarrow{0}} \quad$ N $\overrightarrow{0}$

n)
욤ำ

\section{:}

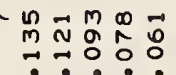

$\circ \circ$
$\circ$

a

-. ?

0
-9
0
0
0

응ㅇㅇㅇㅇㅇㅇ $a$

$\circ$

$\rightarrow$ 응

ñ

$-10$

$\circ \circ$ $\underset{\substack{m \\ \rightarrow}}{m}$

$\circ \circ$

:

N N

응

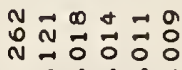

N $\quad \stackrel{\infty}{N} \circ \cong M=\infty$

a

$\circ \quad N \rightarrow M O \infty N$

$\underset{M}{N} \overrightarrow{0}: 000$

$\stackrel{\sim}{\circ}$ $\stackrel{0}{0}$

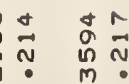

$\begin{array}{ll}\ddagger & m \\ \stackrel{m}{N} & \overrightarrow{0} \\ 0 & 0\end{array}$

$\begin{array}{llll}0 & 0 & n & 0 \\ 0 & 0 & 0 & 0\end{array}$

a no

0 ?

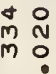

n $\infty$

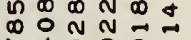

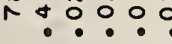

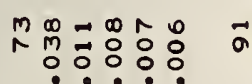

$\wedge \sim \sim m \rightarrow a$

$\stackrel{0}{\rightarrow} 0 \overrightarrow{0} 00$

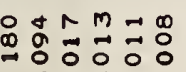

on wano

$\infty \frac{0}{0} 0.000$

$0+\infty$

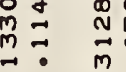

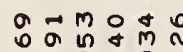

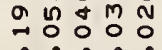

2

mo

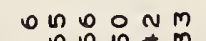

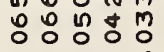

$\sim N$

응

$\begin{array}{llllll}n & m & 0 & 0 & 0 & N \\ 0 & 0 & N & 0 & 0 & 0 \\ \infty & m & 0 & 0 & 0 & 0\end{array}$

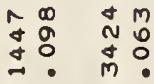

nナง

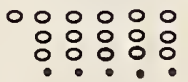

$\circ:$

$\circ$

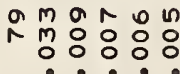

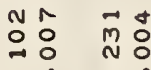

䑸

드유유.

Mn $N$

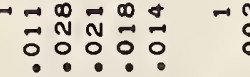

N

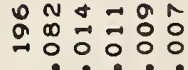

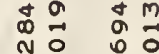

O $N \infty$

N

a $\vec{N}$

$\stackrel{\circ}{\circ} \stackrel{M}{0}=\frac{9}{0}$

$\rightarrow$

N

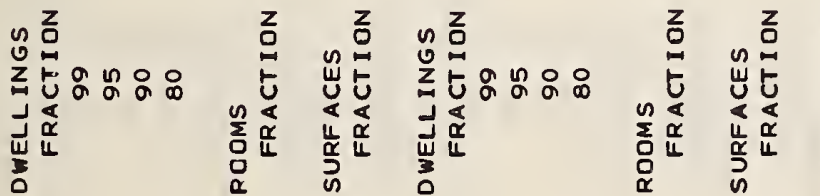

(3)

查

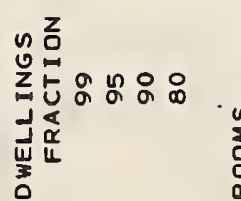

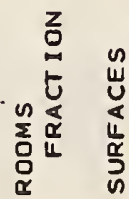

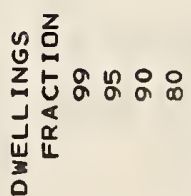

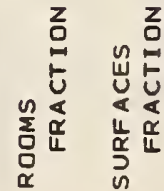

$n$
1
$o$
0

n $\begin{aligned} & 0 \\ & 0\end{aligned}$

- O OON

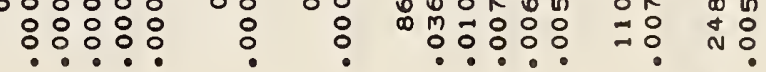

$\frac{\alpha}{a}$ 


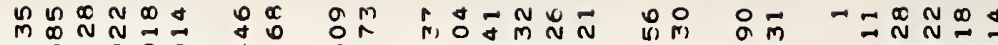

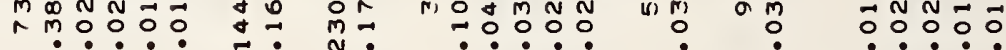
. $\bullet$ ?

응요

\section{à}

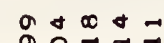

*

*

nm $m$

$\lim _{\substack{m \\ 0}}^{n}$ $(10)$

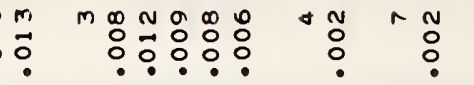

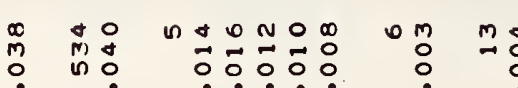

$\stackrel{N}{M}$

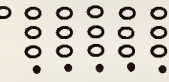

000000

옹ㅇㅇㅇㅇㅇ

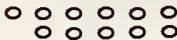

응ㅇㅇㅇㅇㅇㅇ

$\circ \circ \circ \circ \circ \circ$

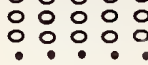

$\because \underset{m}{\ln } \overrightarrow{0} 00$ a $\div \overrightarrow{0}: \overrightarrow{0}$

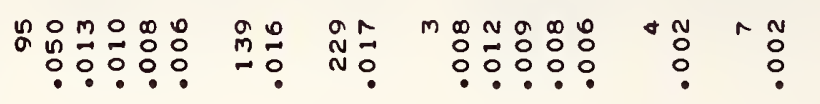

*

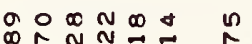

虽嵒

$=$

$\begin{array}{llll}0 & 0 \\ 0 & 0 & 0 \\ 0 & 0 & 0\end{array}$

*

*

*

*

$\frac{\sigma}{\frac{0}{2}}$

$\stackrel{\alpha}{0}$

$\stackrel{n}{n}$

n

我

品

द)

움 *

I naor no n

*

番

*

*

* Z

*

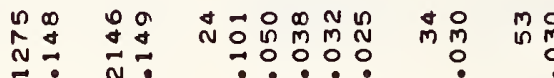

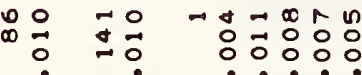

\section{-}

กิ

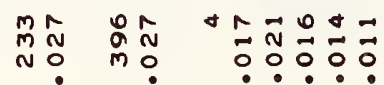

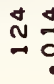

in

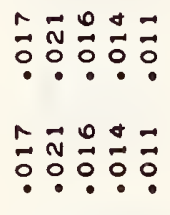

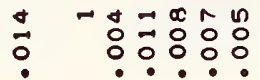

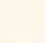

용응

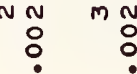

o o r ron nam

$\circ \dot{m} \div \circ: 0$

$\overrightarrow{0}$

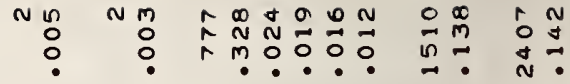

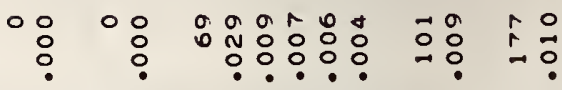

$\circ: 0$

+on-ar morn

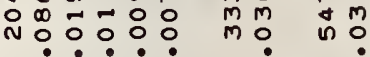

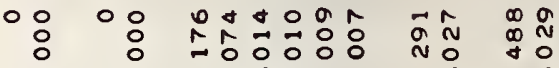

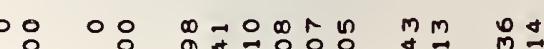

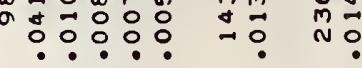
0 m. O?

N $\begin{gathered}m \\ 0 \\ 0\end{gathered}$ ㅇ?ㅇ

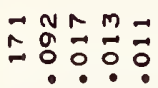

0 D 0 No

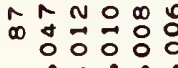

n
$N \infty N+$

m:

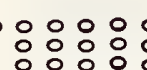

웅요

응ㅇㅇㅇㅇㅇㅇ

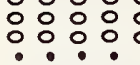

000000

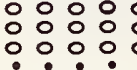

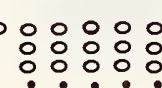

No

•

.

$\circ$

ํำ눙 $\infty_{-\infty}^{\infty}$

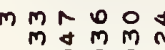

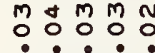

000000

응ㅇㅇㅇㅇㅇㅇㅇ

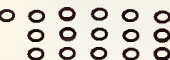

- $\cdot$

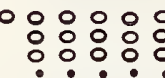

응응ㅇㅇㅇ응
0 0000
ก กูํํㅇㅇํㅇㅇㅇㅇ

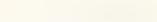

응유유.

non no

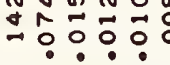

응ㅇㅇㅇㅇㅇ

0ㅇํㅇ ง

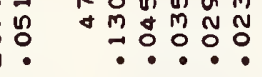

in

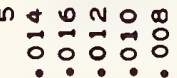

aำ

웅

$\log 20$

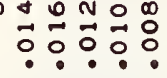

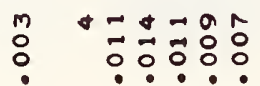
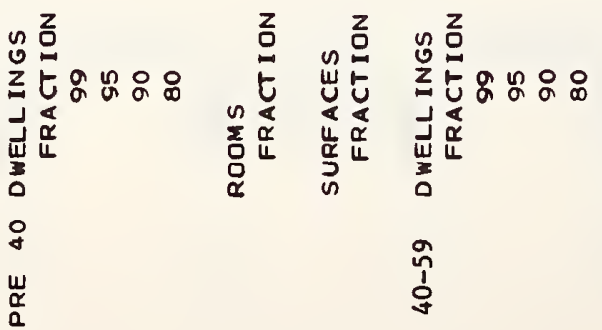

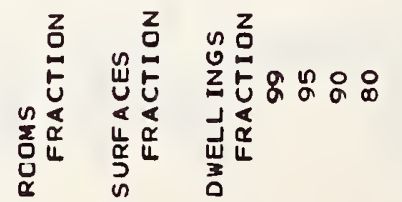

$a$
nิ
0

$n$
$\vdots$
0 mutn $a d m \infty \pi-$

$\therefore$ ㅇํำ

00000000

$\begin{array}{cccc}0 & 0 \\ 0 & 0 & 0 & 0 \\ 0 & 0 & 0 & 0 \\ 0 & 0 & 0\end{array}$

?. ?

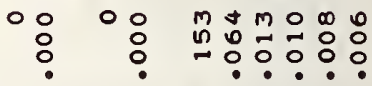

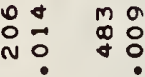

$\circ$ 윰

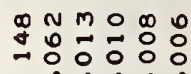

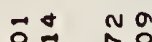

$\circ$

응 0

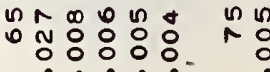

n?

$m$
0
0
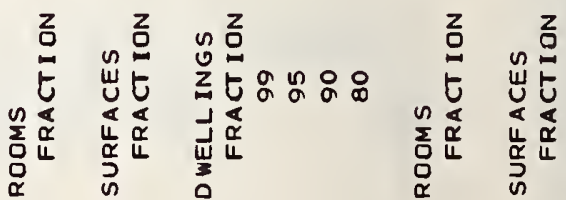
용ㅇㅇ in : $0: 0: 0$ $-$ $\begin{array}{llllll}0 & \infty & 0 & m & a & 1 \\ \text { in } & 0 & M & N & a & 0 \\ 0 & 0 & 0 & 0 & 0\end{array}$

$m \infty \wedge 0$

n

号产

응

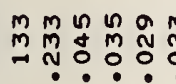

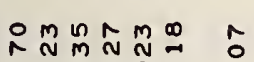
$\because: 0: 0$

000000

010002

ñ

or o om

\section{$\stackrel{\circ}{m}$}

$m$

$\min \rightarrow$

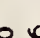

$: 00$

N

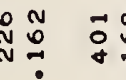

ถ

$\ddot{\infty}$
齐号

N

$\rightarrow$ ?

(9

$\underset{\infty}{\infty}$

ก

$\because: 0: 0$

๑

m:웅요 ㅇํㅇํㅇ

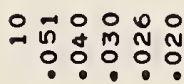

^⿻上丨

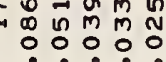

$\rightarrow$ N

mo

$+$

ํํำ

䈥

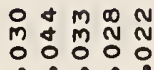

modmon
N
ㅇำ ํำ

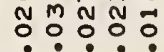

$+00 \ln n$ 0
0
0
0
N: iñ

n:

응ㅇㅇ웅

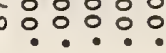

in:

O ONMD No N

MN N N

ำำ. n 0

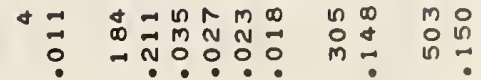

$m m m \infty \quad 0 \infty m n \pi r \quad n n a-$

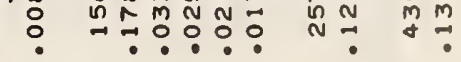

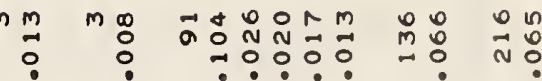

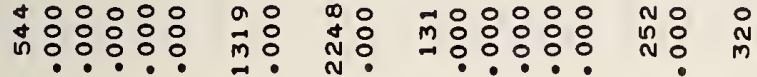

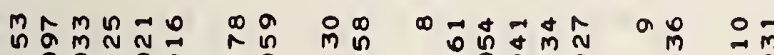

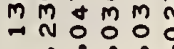

\section{a in a 0 in} 再:
: $\rightarrow$

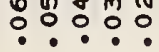

in

กั

M

Mi

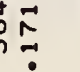

$\left(\frac{1}{2}\right.$

N

ONMON

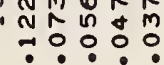

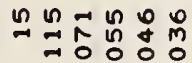

monn

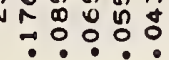

in

Nก

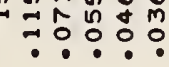

NN $m$

$\stackrel{\infty}{N}$

:

$\vec{m}$

.

N

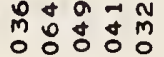

$\pi$

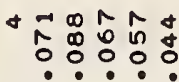

m品a

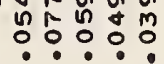

\begin{abstract}
ำ
\end{abstract}
每

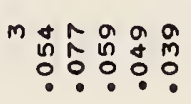

mo

m욤

ㅅํㅇㅇㅠ:융

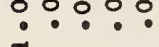

요 능유

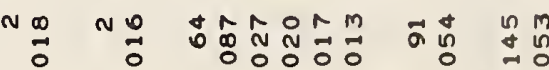

但

mN ma ํ.

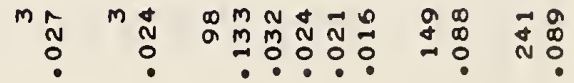

mo 000

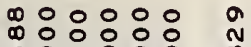

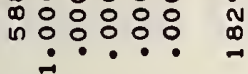

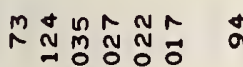
$\because: 0: 0$

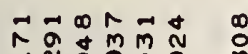

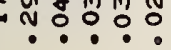

ำ

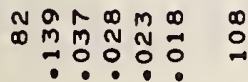

$\therefore 00$ no 0000 ก: N

के in m $\because \because$ :

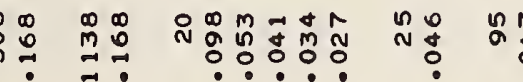
$\rightarrow 0_{-1}^{\infty}$ in

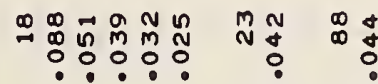

\section{a.}

它:

::ㅜㅇ

융ㅇㅇㅇㅇㅇ

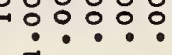

0 응요유

…ㄴ.?

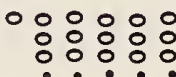

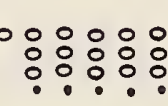

$\div: \because: 0$

$\therefore::: ㅇ$
ํํㅇ N

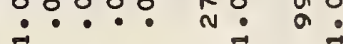

$\infty 0^{\infty} \infty n$

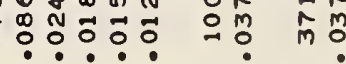

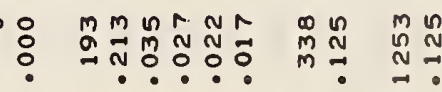
$0: 0$
0
0 $0000 \infty n a m$ m $m$

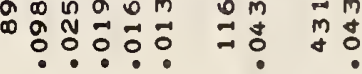
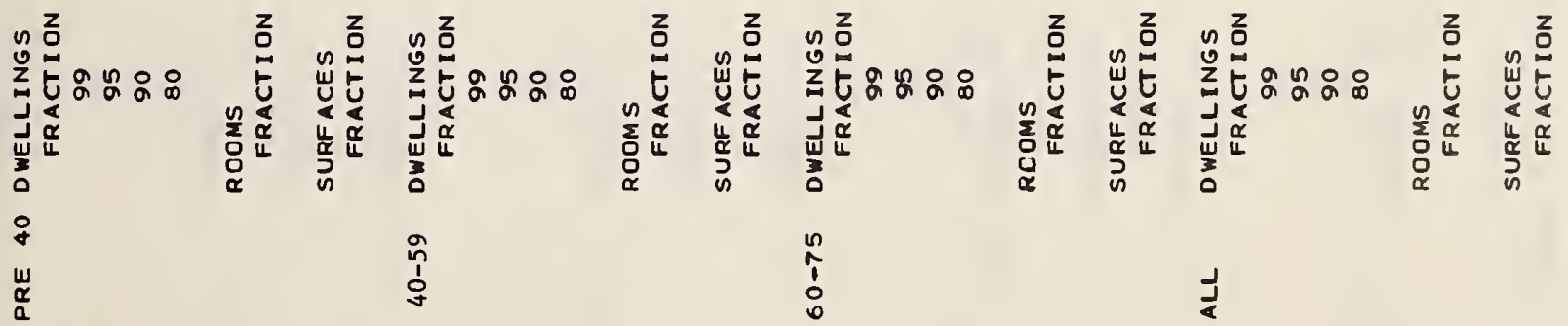
o-anan mi ma anmad

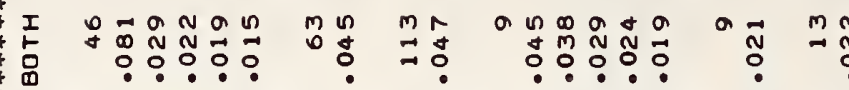

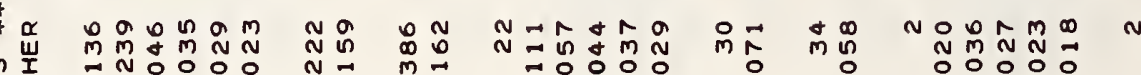

总

M

$\sim n m m \infty n$

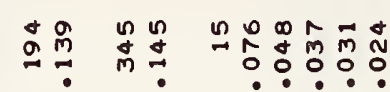

*

*

*

* -

$\stackrel{\infty}{m}$

m N

?

은?

?

N

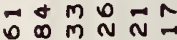

$\begin{array}{lllll}N & \vec{m} & 0 & n \\ \infty & M & N & N & 0 \\ 0 & 0 & 0 & 0\end{array}$

$\ln _{0}^{\infty} \pi$

$\sum_{\substack{0 \\ \infty}}^{\infty}$

.. .

$\rightarrow \stackrel{m}{N}$

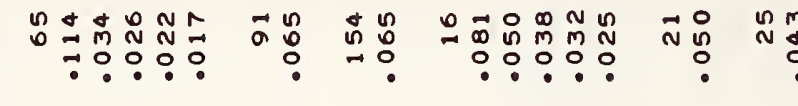

+0 n $90 m$

$\overrightarrow{0}$ 응 $\overrightarrow{0} \overrightarrow{0}$

7

$+m$

* $m=0$

MN.

ก) $N=$

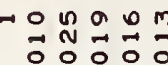
웅․

$-$

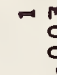

$m \backsim n a m \quad n \quad n$ ㅇํㅇำ

$\frac{3}{*}$

\section{$0+0 \circ \infty$} 0
0
0 onn n n o o d

n $x \rightarrow a$

$\rightarrow a \infty \hat{n}+$

吾

z出

‡N

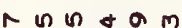

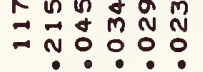

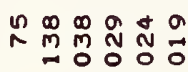

M M O N O

*

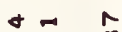

$0 \rightarrow$ m a moo $\begin{array}{llll}\infty & m & 0 & 0 \\ 0 & 0 & \infty & 0 \\ 0 & 0 & 0 & 0\end{array}$

$\stackrel{a}{N}$

\section{$N \quad \rightarrow$}

no 0 o 00 0
0
0
0 m $\rightarrow$ n n o m

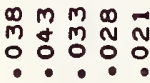
0

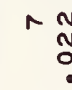
옹 N

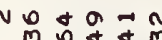

mㅇㅇㅇํㅇ mo 。

$N \cong m$

㘄

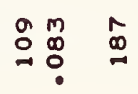

\section{$\stackrel{8}{\circ}$} ํ.

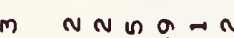

* 岀 $*$
$*$
$*$
$*$
$*$
$*$
$*$
$*$
$*$
$*$
$*$
$*$ $\begin{array}{ll}* & \\ * & 1 \\ * & \\ * & 0 \\ * & \end{array}$ 골 考点 * * * * a d n N N जิ ถิกัดิ 은?

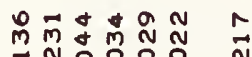
•.

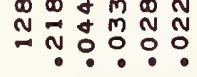

$\tilde{D}=M M_{M}^{n} \tilde{N}$ $\because$ O O O O $\infty \omega+M \infty N \quad n$

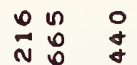
(⿻日土一

No

$=0$

$\stackrel{n}{N} \underset{\sim}{\sim} \stackrel{0}{\sim}$

$\begin{array}{ll}N & N \\ \infty & 0 \\ 0 & 0\end{array}$ $\infty=n \wedge \infty \infty$

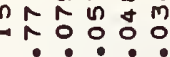
กำ

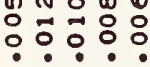

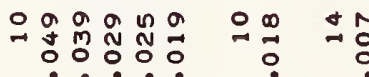

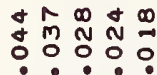
- $\bullet$.

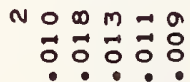
-

$\rightarrow \underset{0}{0} \rightarrow$

$a \stackrel{m}{0}$

N $\frac{0}{\circ}$
NN $\infty \infty \quad n-\infty=n=n$

N $\begin{aligned} & \text { M } \\ & M\end{aligned}$ $\circ$

\section{\%}

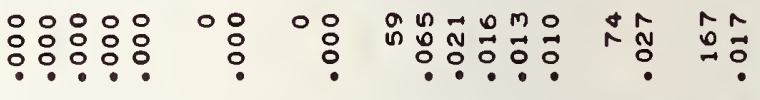
$\circ: \circ: \circ:$
$\circ:$

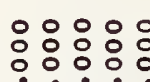
•. • •

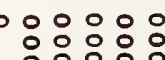
웅ㅇㅇㅇㅇㅇ

mo

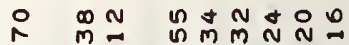

M N N

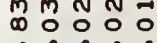

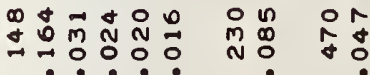

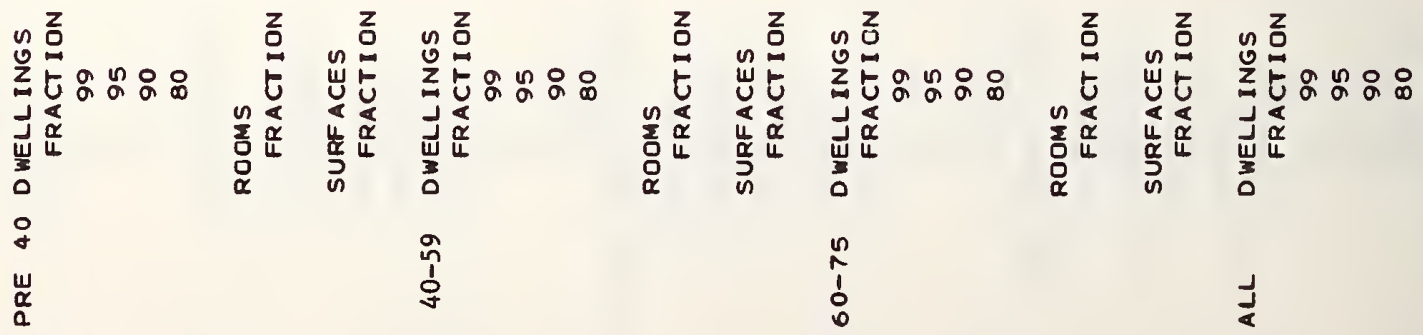




\section{H. \\ $\rightarrow N \& M m$

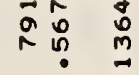

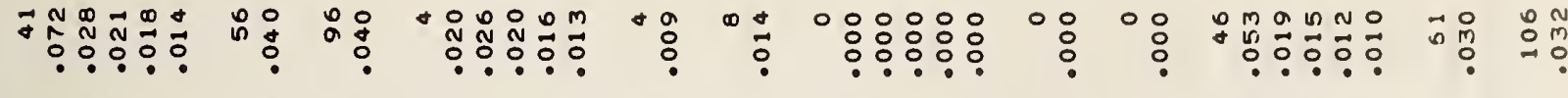 \\ - \\ $a=00000 \mathrm{~m}$ \\ : \\ 웅

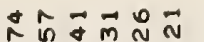 \\ ํํㅇㅇㅛ 웅

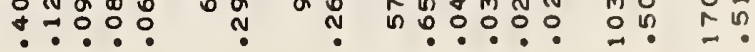 \\ * I

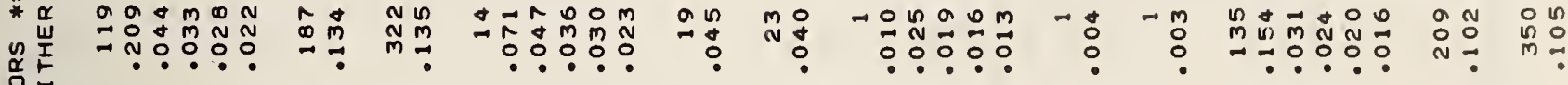 \\ $\stackrel{m}{M} \vec{\infty}=\stackrel{N}{N} \vec{N}$ \\ ?은? \\ :ㅇํㅇ

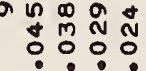

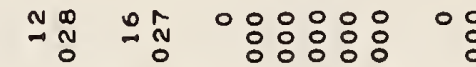 \\ $\circ \%$

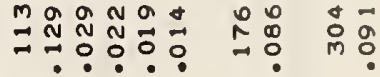 \\ กO \\ $\vec{\infty} \stackrel{\infty}{0} \stackrel{+}{\circ}$ \\ a n 000 \\ $\rightarrow 0 \quad 0$

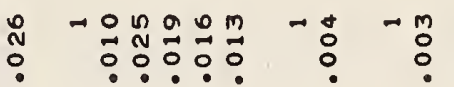

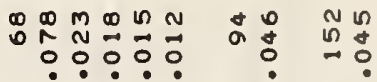

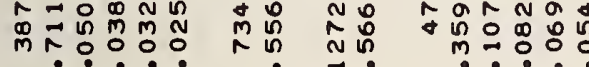

+oman on no t a a a n

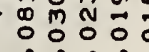

in

an $n$

$\sim N$

$\Rightarrow \overrightarrow{\mathrm{N}} 000$

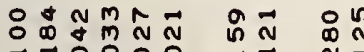

$\because$ : : : ?

$\stackrel{\infty}{\sim} \underset{M}{N} \stackrel{m}{N} \stackrel{\infty}{0}$ $\Rightarrow$ : O O min

a a mo

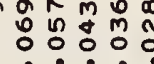

o o

\%

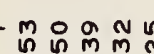

on $\because \begin{gathered}0 \\ 0 \\ 0\end{gathered}$

○ొ

옥

ำ

$+\frac{0}{0} \quad 10$

$\stackrel{0}{0}$

$-\infty$ nn $n$ a

: 0 M N

$a \stackrel{0}{m}=$

$-$

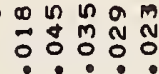

0

$\wedge$

$\rightarrow \infty \ln 6 \mathrm{~m}$

0
0
0
0
0

$\sim$

$a \stackrel{\infty}{2}$

$\begin{array}{rrrr}\infty & n & 0 & m \\ -1 & m & N & N \\ 0 & 0 & 0 & 0\end{array}$ $\pm \stackrel{-1}{N}$

$\rightarrow$

a $\rightarrow$

$\rightarrow \stackrel{0}{0}+$

$\rightarrow$

$\rightarrow$

$-\because$

$-\infty$

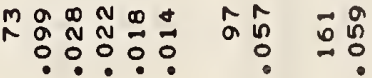

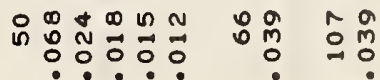

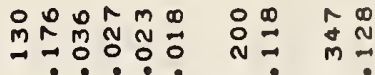

m $m$ L $\rightarrow$ : ? : $\rightarrow$ N

(4)

in $\Rightarrow \overrightarrow{0} m 0$ on $\infty$

mo: : : :

览

$\stackrel{m}{N} \stackrel{\infty}{-}$

$\hat{\sim} \infty$

m

no

N $0 m \sim 0 N 0$

$\rightarrow$

Mํํ요

$\because \underset{1}{0}: 0$

ก

$\mapsto \circ \%$ ?

N?

$\stackrel{\infty}{N}=0 \begin{array}{lll}n & 1 \\ 0 & 0 & 0\end{array}$

$+m$

in :

O ONNA

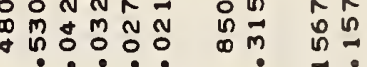

$\begin{array}{lll}0 & 0 & 0 \\ 0 & 0 & 0\end{array}$

$\rightarrow$ N $\rightarrow 0$

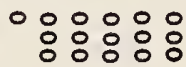

$\circ:$

$\circ$

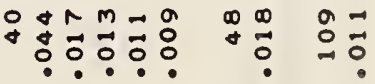

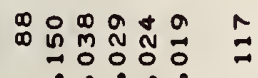

in

m n $\rightarrow 0+$

m

$\overrightarrow{0}: \overrightarrow{0} \overrightarrow{0}=$

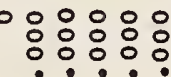

$\circ 8$

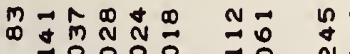

m n $\rightarrow 00$

$\overrightarrow{0}: \overrightarrow{0}: \overrightarrow{0}$

$m$

8

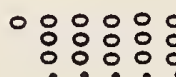

00

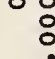

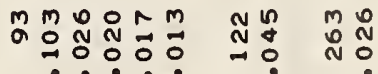

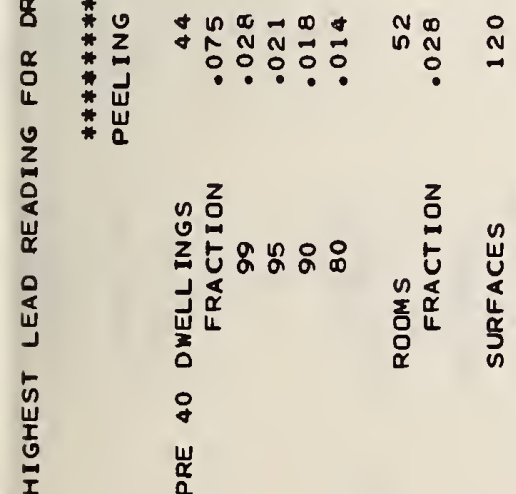

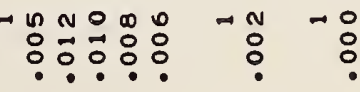

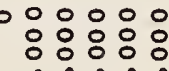

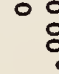

$\circ:$

แ

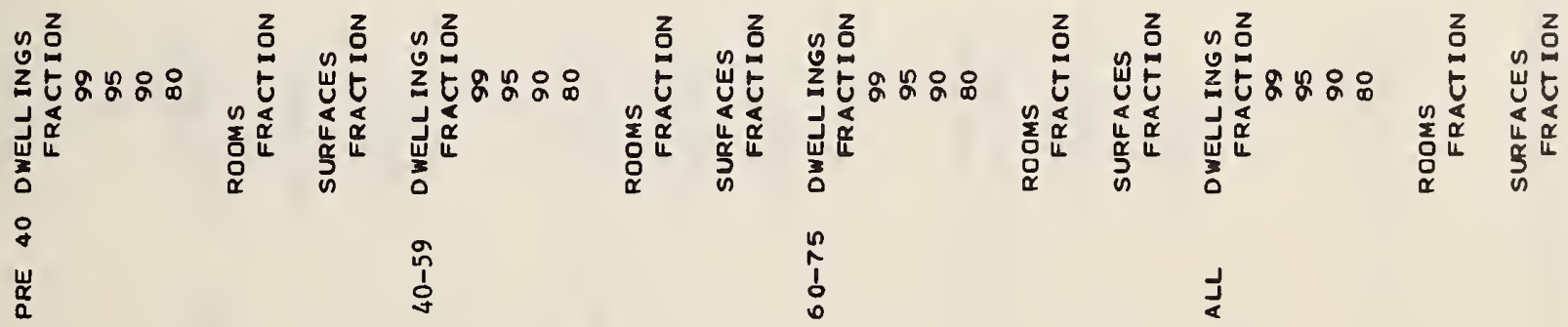




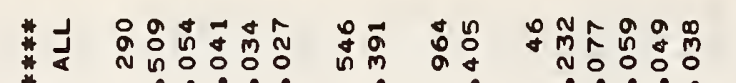
*

* I

-

*

总

0

*

*

*

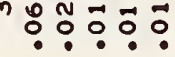

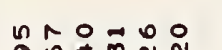

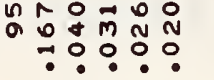

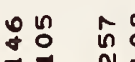

$\infty \quad 0=0000$

뭉ํำ

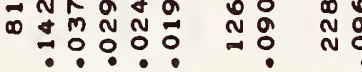

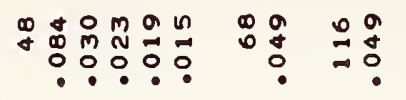

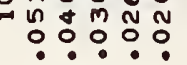

○品药운

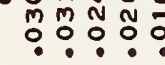

คํำ
- ntoda 00

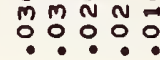
Mํํำ

N $\stackrel{n}{\circ}+$

응요유:

$=\underset{N}{0} \prod_{0}^{m}$

융유

ก

ㅇ.

$\circ \circ: 0 ㅇ ㅇ$

용유

0
0
0
0

$\pm \frac{0}{m}$

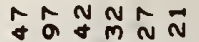

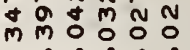

$\stackrel{0}{\circ}$

$0 m$

o $m$

N $N M=0$

m: $0 \div 00$

กี

a $\stackrel{\infty}{N}_{0}^{\infty}$

$\pi=\infty N \infty$

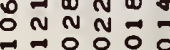

ถึกิก กัก

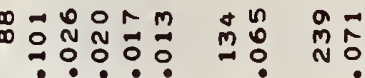

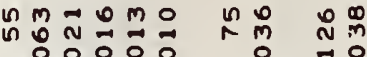
ㅇํㅇํำ

*

ํํㅇํㅇํำ

* I

m

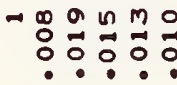

$m m \circ=r$

要

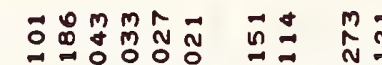

$\stackrel{n}{n} \stackrel{n}{*}$

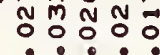

o

$\stackrel{0}{0} \div$

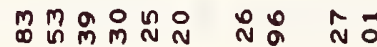

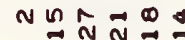

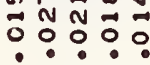

N

: $m:$

m:

융유:웅

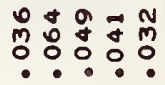

N

n

HONOOM

moํ.

ก $N \infty$

$\begin{array}{ll}N & \infty \\ \alpha & n \\ n\end{array}$ $\circ: \because:: \circ:$

$\circ:$

$\circ:$

m $m=0$

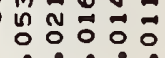

요응

00000000

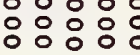

กทกิกับ

ㅇํㅇํำ

$N$
$\vdots$
0

응융ㅇㅇㅇㅇ

$\circ$

n $N m$ n $\rightarrow 0$

ำ

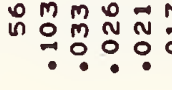

ำกำ กี

수음

$=00000$

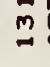

ก

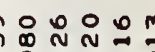

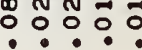

L

: $\stackrel{0}{0}$

*

은

مू

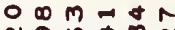

Nลํํㅇํำ

m

$\hat{n}$

$m \mathscr{N} \vec{N} \tilde{N} \vec{N}$

.

0

*

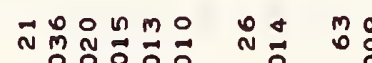

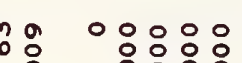

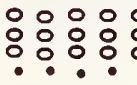

$\circ$

$\circ \circ:$

응요유:

$\circ:$

$\circ$

굴

Mำ N

$\stackrel{m}{\mathbb{N}}$

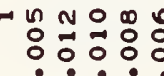

$\begin{array}{rr}- & -0 \\ -0 & 0\end{array}$

응ㅇㅇㅇㅇㅠ

$\circ:$

$\circ:$

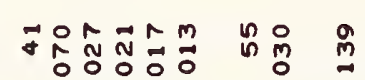

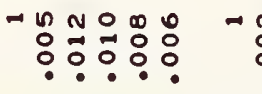

ก้

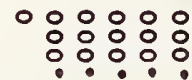

$\circ$

$\circ$

MNONONO $\stackrel{\infty}{m} \stackrel{0}{0}$

$\circ 0000$

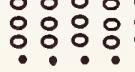

$\circ \circ \quad 0$ 웅

00000000

?ะ??ำ?

ڤำ

$\vdots$

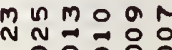

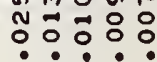

$\stackrel{\infty}{N} \stackrel{0}{0}$ ก ํํㅇ

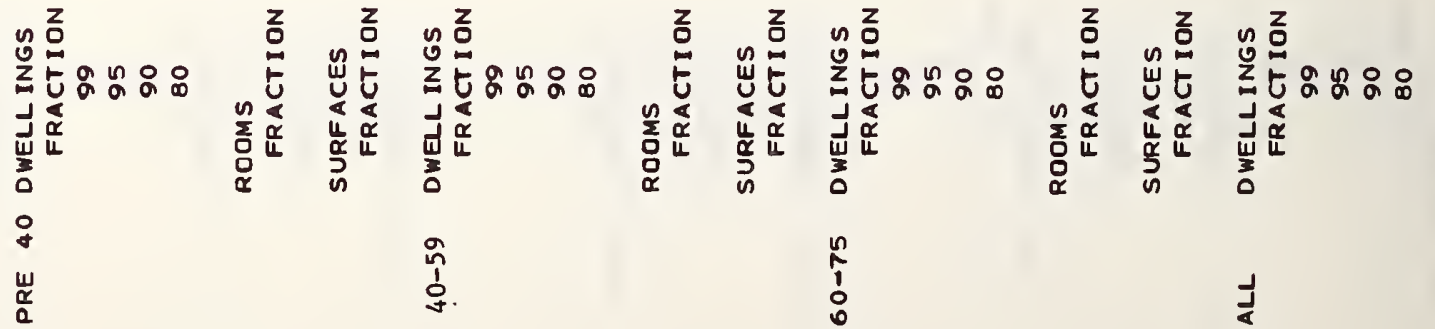

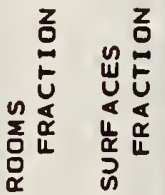


$a \infty^{0} \quad m \infty$ ?.

$\operatorname{lng} 0009$

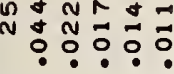

om $0 \infty m \infty$

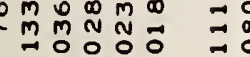

0

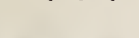

D

$\because 0 \% 00$

o

$\infty_{0}^{\infty}=$

*

ก $100 \wedge m$ $\begin{array}{lll}0 \\ 0 & N & 0 \\ 0 & 0 & 0\end{array}$
ถั่

20: a

ㅇํㅇํㅇ

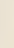

nn $\begin{array}{lll}m & 0 & 0 \\ 0 & 0 & 0 \\ 0 & 0 & 0\end{array}$

:? ?

- $\begin{array}{rrr}m & 0 & \infty \\ 0 & 0 & 0\end{array}$

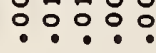

- n $m: 00$

- $\begin{gathered}m \\ 0 \\ \circ\end{gathered}$ $\stackrel{0}{1}$

$\rightarrow$

$+$

$+$

$\rightarrow$
ก

m $\frac{1}{0}$

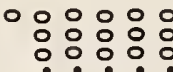

$m$

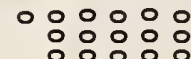

0000000

응ㅇㅇㅇㅇㅠ

0
0
$\circ$
○:

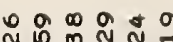

N N

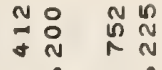

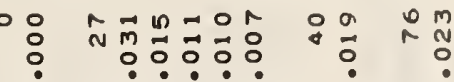

- o d a navam m n mo

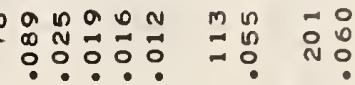

N $m$

$m$
0
0
0

añ

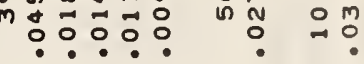

tada

ลี

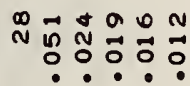

N $n$ mana

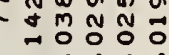

$+\infty n \sim m \infty$ $\begin{array}{lll}0 & 0 & N \\ 0 & 0 & 0\end{array}$

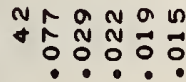

$m \circ \quad \infty$

ڤึ

$\stackrel{0}{m}$

in

$\stackrel{0}{\circ}$

$\stackrel{\infty}{\infty}$

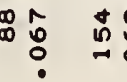

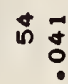

a.

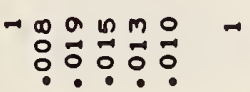

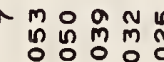

웅영

- 0 a n m

$\begin{array}{lll}0 & n & m \\ 0 & 0 & 0 \\ 0 & 0 & 0 \\ 0 & 0\end{array}$

N $\sim \hat{N} \vec{N} \infty$

-.?

$N$ ำ

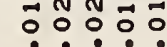

$-$

N

N

: $\quad$ :

(1)

N
응응ㅇㅇㅇㅇㅇㅇ

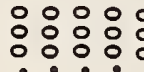

000000

응ㅇㅇㅇㅇㅇㅇ

ㅇㅇㅇㅇㅇㅇㅇ

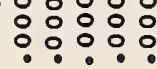

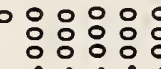

$\circ:$

8

$\circ \circ$

:

$\circ$ :

$\circ$

요 0
0 :

$\circ 8$

$\circ$ :

$\circ$ :

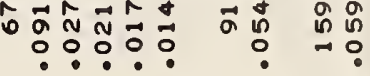

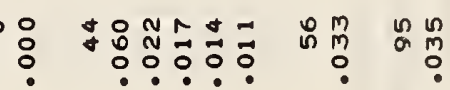

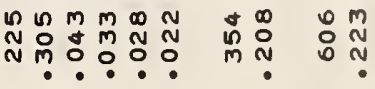

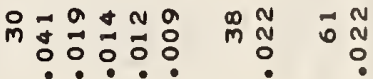

- agnan ad m= 윰ำ mâำ

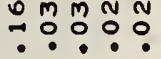
ํํㅇํㅇํㅇ

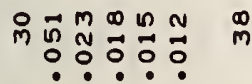

Nิ ㅇํ유.

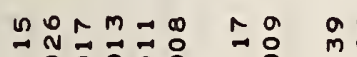

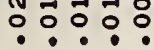

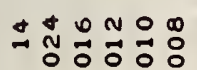

a 잉ㅇㅇㅇㅇ

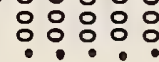

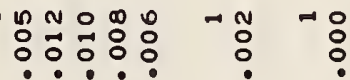

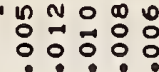

\section{$\circ 000$ 웅}

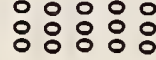

nก ลั mo

응ㅇㅇㅇㅇㅇ :. :

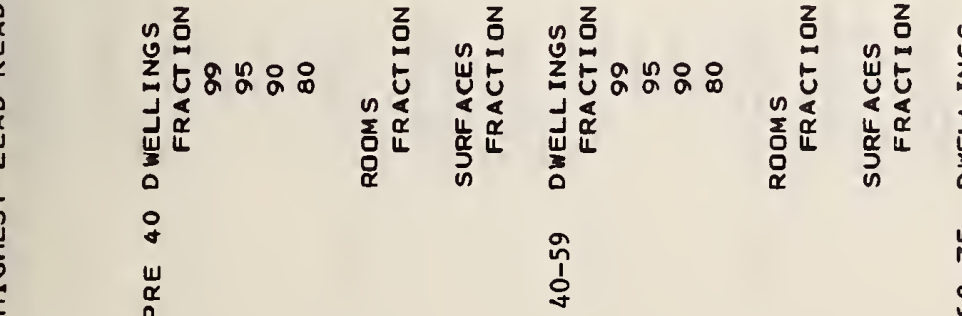

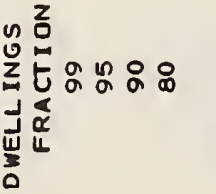

$n$
1
0
0

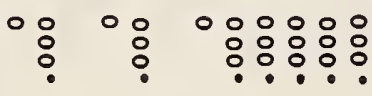
응ㅇㅇㅇㅇㅇㅇ

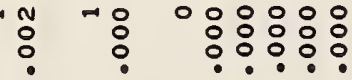

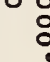

$\circ$

$\circ$ 응ㅇㅇㅇㅇㅇㅇㅇ
0

?

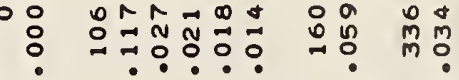

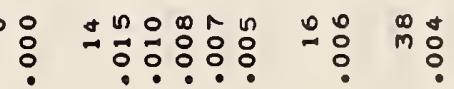

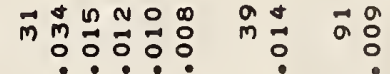

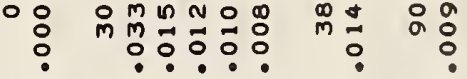
:

a
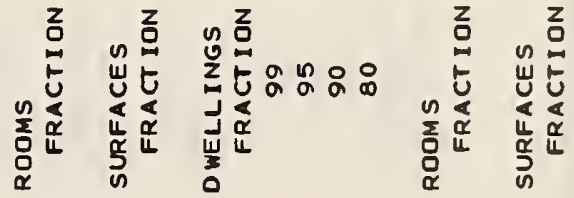
กีก์ -

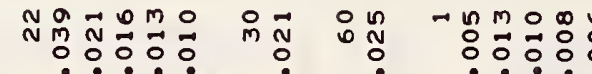

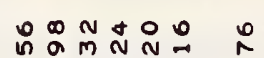

$\because \circ \%$ :

$0+a$

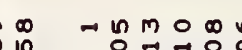

岳

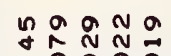

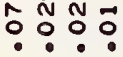

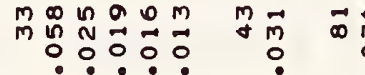

$\prod_{0}^{m} \underset{0}{0}=$

웅

$\because 00 \%$ :

-

要

*

$m \rightarrow a \infty N$

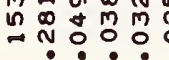

$\stackrel{n}{N}$

$\stackrel{n}{N} \underset{\sim}{m} \mathbb{m}_{m}^{m}$

$\stackrel{0}{\simeq}$

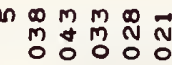

$\wedge \stackrel{\infty}{N}$

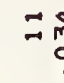

M $N$ N $N$ N

ก:

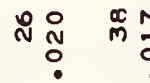

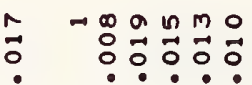

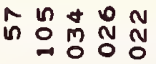

高

$z^{0}$

3

* 选

응 $\vec{m} \stackrel{\circ}{\circ}$

웅으

m n n 0

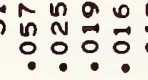

$\hat{m} \stackrel{\infty}{N} \quad \stackrel{N}{N} \underset{N}{0}$

n

mi

:

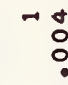

N:

응응ㅇㅇㅇㅇㅇ

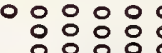

웅?

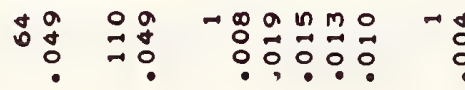

$\stackrel{\infty}{N}$

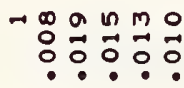

웅요

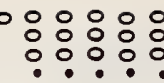

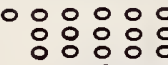

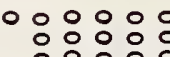

응ㅇㅇㅇ

응ㅇㅇㅇㅇㅇㅇㅇ

000000

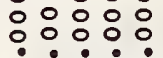

응ㅇㅇㅇㅇㅇㅇ

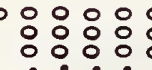

$\circ:$

$\circ 8$

ํํำ

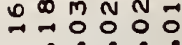

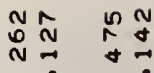

$\circ \stackrel{8}{\circ}$

$\circ$

$m o+\Rightarrow a r$

N

$\vec{m} \stackrel{n}{0} \frac{a}{0}$

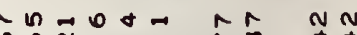

$\begin{array}{lllllll}0 & 0 & 0 & 0 & 0 & 0 & 0\end{array}$

omanno

in $\overrightarrow{0} \overrightarrow{0} \overrightarrow{0}:$

गु

舟 $M=\infty$

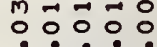

$+\vec{N} \quad 4 N$ ?

O־

-. : ?

$\because 0 \%$

$\overrightarrow{0} \quad 0$

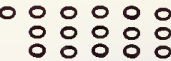

$\circ$

$\circ: 0 \%$

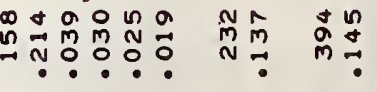

.

:

$\circ$

om $M \rightarrow m$

$\begin{array}{llll}m & m & m & 0 \\ 0 & 0 & 0\end{array}$

$\hat{N} \stackrel{0}{0} \div$

$\circ$ :

$\circ$

manno

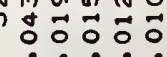

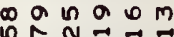

○ 잉 $\overrightarrow{0} \overrightarrow{0}$

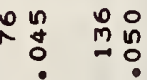

$0 \infty+\infty$ n $N$

n $m \quad N$

$\stackrel{m}{0}=0$

m $\underset{N}{N}$ ○

:

$+N M \infty n N$

: $0 \overrightarrow{0} \overrightarrow{0} \overrightarrow{0}$

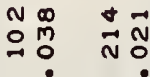

*

‡̛

*

*

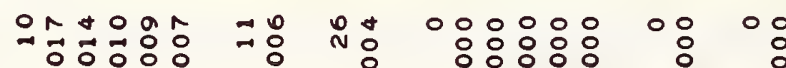

잉ㅇㅇ잉

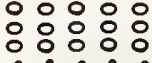

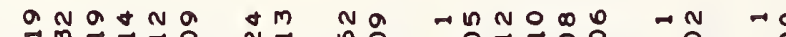

$\prod_{\substack{0 \\ 0}}^{0}: \frac{0}{0}$

No 00

$\therefore 0$

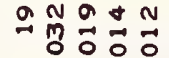

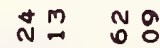

$\begin{array}{rlll}n & 0 \\ 0 & 0 & 0 & 0 \\ 0 & 0 & 0 \\ 0\end{array}$

O

$\stackrel{0}{\circ} \rightarrow$

응ㅇㅇㅇㅇㅇㅇ

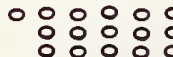

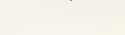

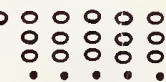

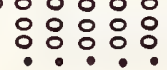

$\circ: 0 \%$

$\therefore \circ$

$\therefore$

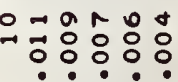

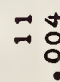

ํำ

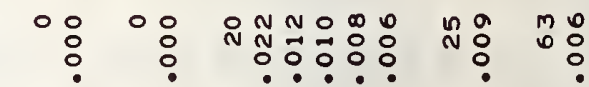
응
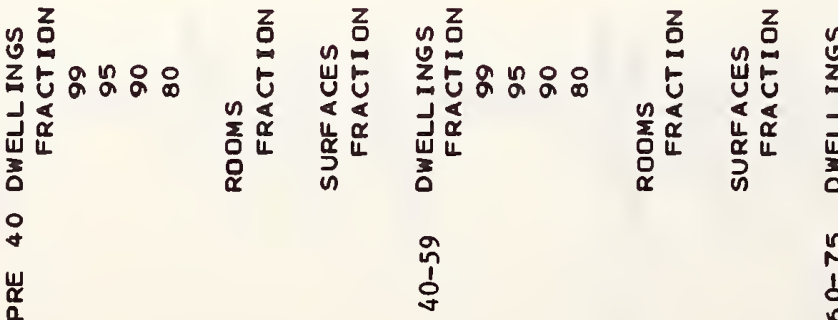

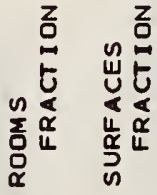




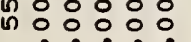

$\therefore$ 요요

응ㅇㅇㅇㅇ

\section{$\circ \circ$}

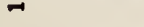

omomon

:ํ.:은?

$\ln \infty+\infty N$

$\rightarrow 0 \& M N N$

$\neg N 000$

No

$\because \because$ : ? O

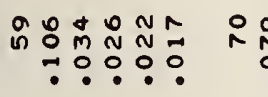

om $m$

in:

웅

$0_{\infty}^{\infty} \vec{n}$

$\rightarrow N$

0.0000
$0.00 \%$

ถี

용

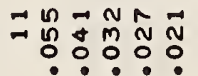

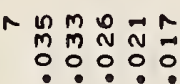

:유: a: a
*

$\circ 0000$

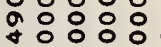
$\therefore$

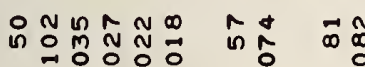

?. ?

- แ 0 n $N$ Un

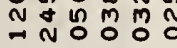

a n 0

in

$\because 0 \infty \ddot{m}$

$\therefore$ n.

$\underset{\sim}{+\infty} \stackrel{\infty}{\infty} \stackrel{0}{0}$

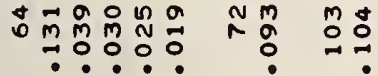

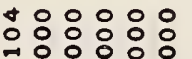
. . :

$+\infty \operatorname{mon}_{m}^{\infty} \vec{m}$

mot?

$m$ n $m+m$

$\because 00 \%$

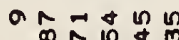

ㅇํㅇㅇำ

$\infty$ กิ in

๓ㅇํㄱ

$\sim m \infty m$

$\rightarrow$

Na $m$

$\rightarrow R \rightarrow$

$\circ: 0 ㅇ$
응ㅇㅇㅇㅇㅇ :ㅇํㅇㅇㅇ 응ㅇㅇㅇㅇㅇ

응융

No $0 \infty m \infty$

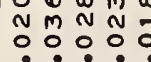

No $0 \infty m \infty$

:

$\circ$
0 $\stackrel{8}{\circ}$

$\underset{0}{0}:$

00

$\therefore$

$n+N n$

?.

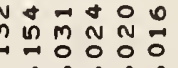

Non

N

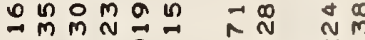

$\rightarrow \because 0: 0 \%$ I

$\circ 8$

โิ $\begin{array}{lllll}0 & 0 & 0 & 0 & 0\end{array}$

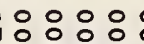

ㅇㅇㅇㅇㅇㅇㅇㅇ

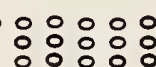

웅으

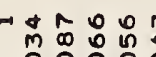

ํ.?ㄴ?

$\begin{array}{rlll}\rightarrow & 0 & 0 & m \\ m & 0 & 0 & 9 \\ 0 & 0 & 0 & 0 \\ 0 & 0 & 0\end{array}$

$\begin{aligned} \circ & 0\end{aligned}$
\&: No

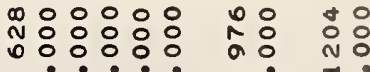

- -1

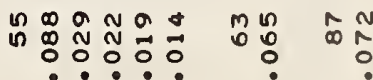

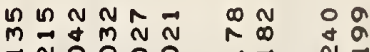

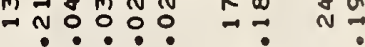

^⿻ㅇํ유요 $\Rightarrow \because 00 \%$ ก

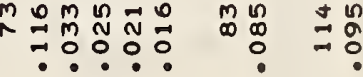

no 000

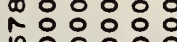
은?

No이의

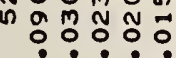

$0 m+\infty n$

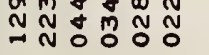

$\stackrel{\circ}{\sim} \quad \overrightarrow{0}$

$\infty$ :

舶:

요요

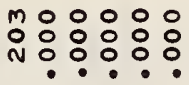

ò

$\stackrel{0}{0}$

웅요

NOO

$\overrightarrow{0} \overrightarrow{0} \overrightarrow{0} \overrightarrow{0}$ :

N

n $+0 \infty N$ n

$\begin{array}{llll}0 & 0 \\ 0 & 0 & 0 & 0 \\ 0 & 0 & 0 \\ 0 & 0\end{array}$

N

$m m+m$

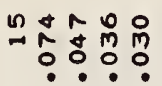

DON

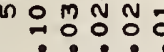

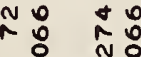

- 0 in 0 o $N$

ก
กㄴ ก 0000 : ^に $0: \circ: \circ:$

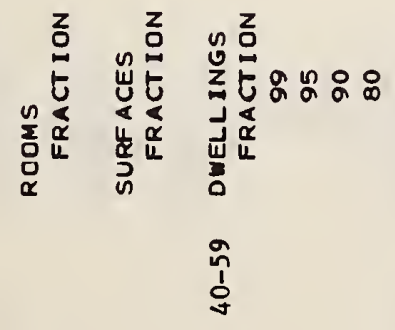

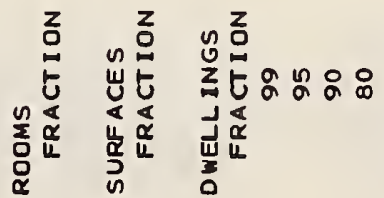
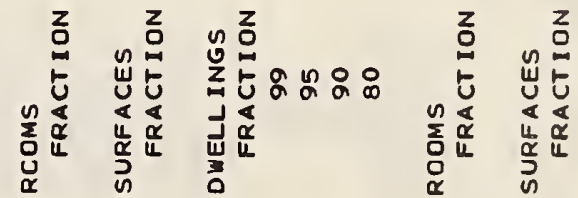<smiles>[O][Co]</smiles> 
nก NNA

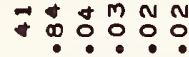

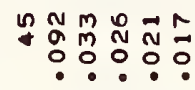

n

$\sum_{i=1}$

* *

$r \infty \infty r=4$

ำ ํํำ

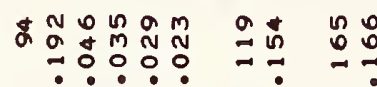

ํำ

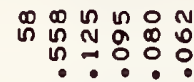

in: N

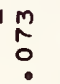

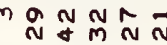

กำ

$m_{M N}^{m N}$

$\underset{0}{0}$

W $\hat{A} \hat{0}$ ถ

คํํำ ㅇํㅇ

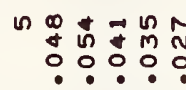

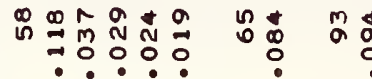

・

กำ
욤ำ

- $\bullet$ ? : $\begin{array}{lll}\circ & 0 & 0\end{array}$

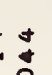

\section{$\sin 000$}

$\begin{array}{llll}0 & 0 & m & M \\ N & M & N & N \\ 0 & 0 & 0 & 0 \\ 0 & 0\end{array}$

N

$\stackrel{0}{\circ}$

in nNomon $\therefore \quad \pi \quad 0$ ก

o

nanat $\infty \Rightarrow \infty n$ ำกำ $\begin{array}{cccc}0 & 0 & 0 & 0 \\ 0 & 0 & 0 \\ 0 & 0 & 0\end{array}$ $\stackrel{n}{\circ}$ ㅁ.

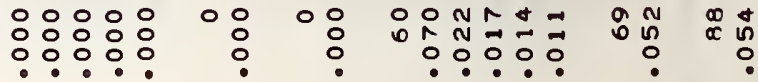

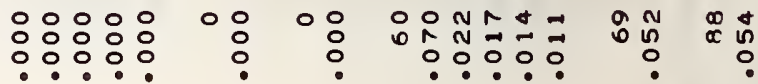

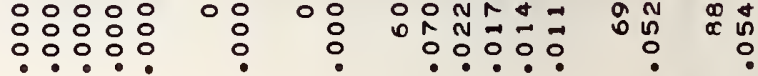

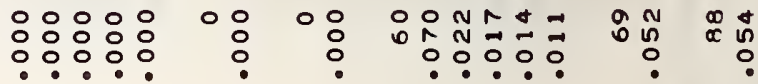

* $⿻$ omm $n \rightarrow 0$ \#

呂

n)$$
\text { . }
$$$$
\text { . }
$$

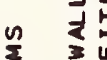$$
\text { อัด }
$$$$
\text { r }
$$$$
\text { 足 }
$$

䓀
*I

*

告

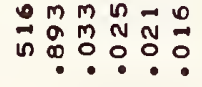

$\begin{array}{rllll}0 & 0 & 0 & 0 \\ 0 & 0 & 0 & 0 & 0 \\ 0 & N & 0 & 0\end{array}$

$\log \lim ^{2} n$

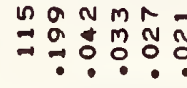

OONNN

0
$\Rightarrow$
0
0

$\Rightarrow \infty \circ m a$ n ⿰纟)

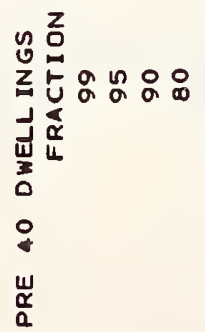

or n $\Rightarrow \quad+\infty \Rightarrow+\infty n$ $\infty \stackrel{N}{0} \quad$ N

$\infty m \wedge$

$\infty-n m \circ \infty$

응ㅎㅇㅇㅇㅇ

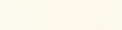

$\underset{N}{\operatorname{Na}} \rightarrow$

o

+ 90 \%

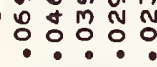

$\pm m$

ñ

$m \infty N$

ำ $\cong$ ำ
N

응응

m n N $\vec{\circ} \mathrm{N}: \overrightarrow{0}$ ro $m$ 의 $m$

?

$\infty m_{\text {ก }} \infty$

?

n $m_{0}^{m}$ n

0 $\stackrel{0}{\circ}$

5

$\stackrel{a}{0}$

웅

an

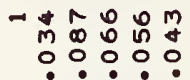

0
Nํำ

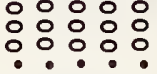

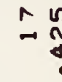

$\circ:$
$\stackrel{n}{N} \stackrel{\infty}{+}$

$\therefore$ i

$\therefore$ $\begin{array}{llll}0 & 0 \\ 0 & 0 & 0 & 0 \\ 0 & 0 & 0 & 0\end{array}$ 능으 n 0
0
0

ㄴำ $\stackrel{9}{\square} \div$ ำกำ

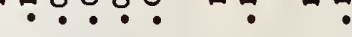

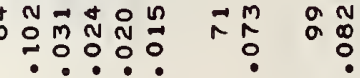

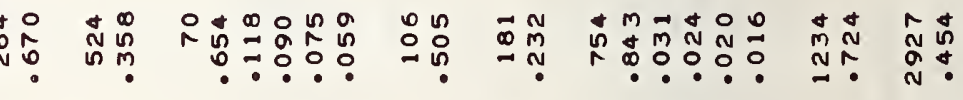

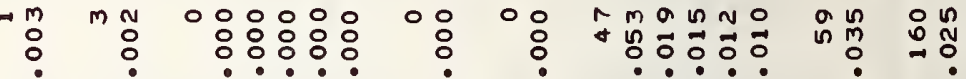

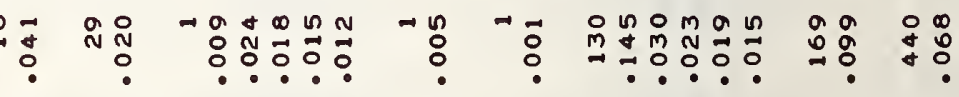

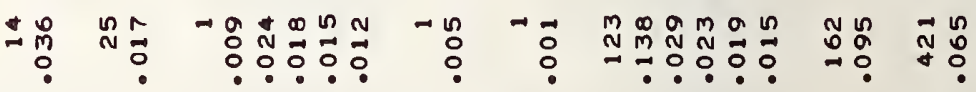

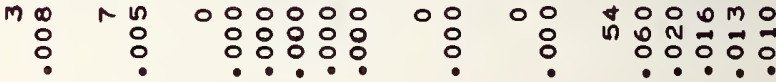

- a $a \infty$ $\stackrel{m}{0} \cong \stackrel{0}{0}$
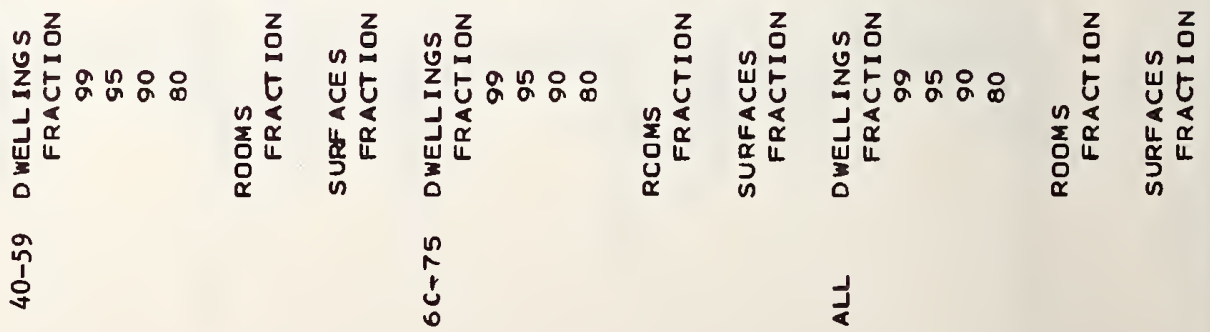
$\sin a n$

m.

品

m

$\begin{array}{llll} & N & N & 0 \\ 0 & 0 & 0 & 0\end{array}$

음

$\because 00 \%$

mog의

in mo m

$\cos 10$

$\stackrel{\infty}{+\infty} \vec{m} \stackrel{M}{N}$ 요

?:? 0
$\stackrel{N}{\circ}$

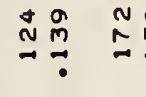

$\cong \stackrel{0}{N} \stackrel{n}{\sim}$

nิ

เึ

?

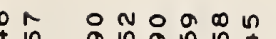

.

a

in

。

$\sim M_{M}^{M} \stackrel{0}{N} \vec{N}$

:은?

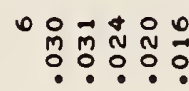

M ก ก N

$\overrightarrow{0} 0 \overrightarrow{0} \overrightarrow{0}$ $\stackrel{\sim}{\sim} \stackrel{N}{N}$

nOD na nN

?

:

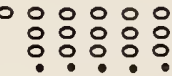

$a$

$a$

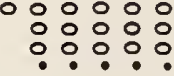

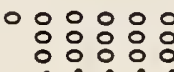

\%

$\infty$

$m \circ m:$

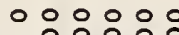

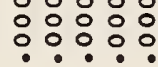

$a$
9
9

: 0

0

$\circ:$

:

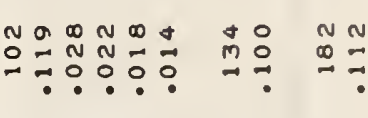

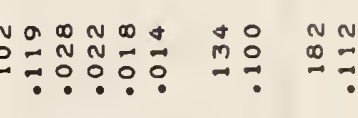

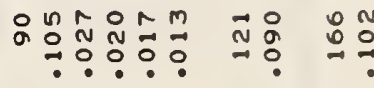

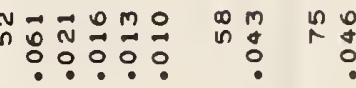

0
$:$

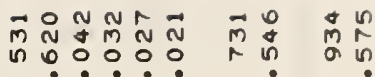

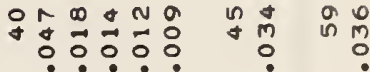

กิำ

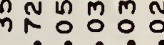

$\underset{\infty}{\infty}$

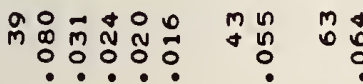

mot

Nan

$\rightarrow$ M N N

ลำ

-. .

$\circ \underset{\infty}{M} \stackrel{M}{M} \hat{M} \hat{N}$

$\because 0000$

$\circ \stackrel{0}{\circ}$

$8 \stackrel{2}{0} \underset{2}{2}$

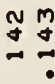

on $n$ n n

$\because 0000$

ถูก ร

요

mo

ON

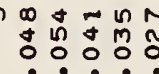

$+\infty \infty \mu+$

m品

ㅇํㅇํㅇ

$+2$

$i_{0}^{0}+2$

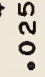

$m \underset{N}{O} \quad m$

$m \stackrel{a}{0}$

0

응ㅇㅇㅇㅇ

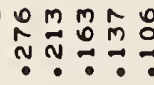

$\begin{array}{ll}m & 0 \\ 0 & 0\end{array} 0: 0 \%$

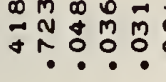

$\begin{array}{lll}1 & 0 & \infty \\ 0 & 0 & 0 \\ 0 & 0 & 0\end{array}$

$\stackrel{9}{m}$

On NOMNMN

a

$a+$

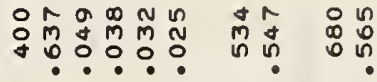

$\circ:$

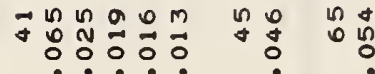

$\begin{array}{lllll}4 & 0 \\ 0 & 0 & 0 & 0 & 0 \\ 0 & 0 & 0 & 0\end{array}$

$n$
-10
$\circ 0$
$\circ$

$-\underset{0}{m}+$

- $0: 00 \%$

ก $\ln \infty-\infty+$

n $\frac{M}{0} \sim$

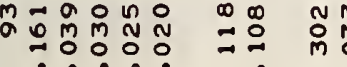

N

$\circ: \circ: \circ:$

8

:

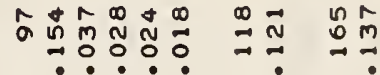
- ・. -

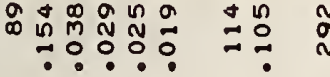

$\begin{array}{llll}\text { No } & 0 & 0 \\ \text { N } & 0 & 0 & 0 \\ 0 & 0 & 0\end{array}$

$\begin{array}{rrr}+0 & 0 \\ 0 & 0 & 0 \\ 0 & 0 & 0\end{array}$

$\circ 8$

$\circ 8$

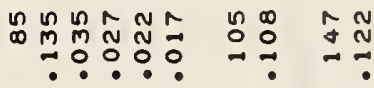

$\left.+\infty \mathbb{N}^{2}\right)$

in

No요 0

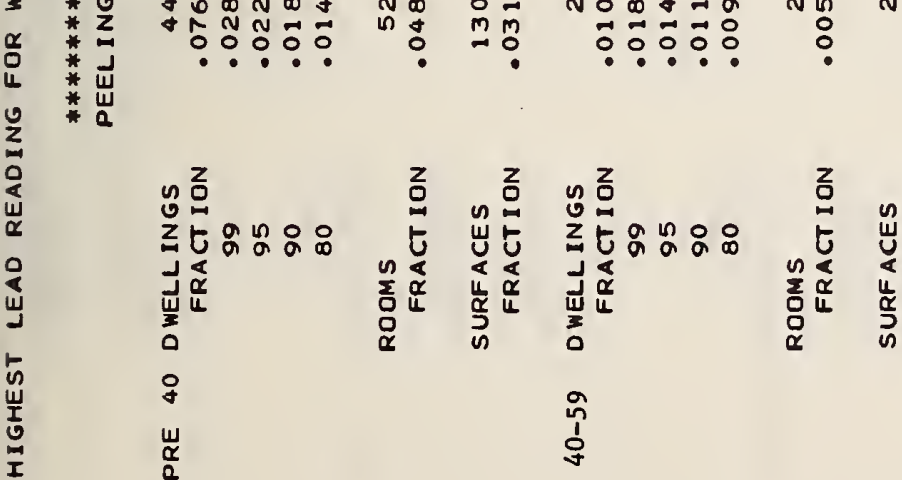

N $10 \quad 2$

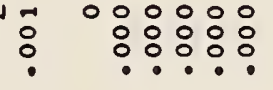

$0 \%$

$\circ:$

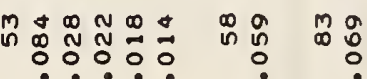




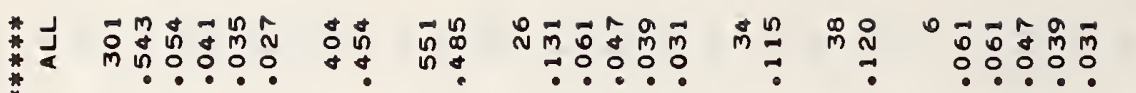

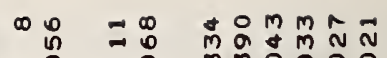

$\infty$ in

m

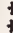

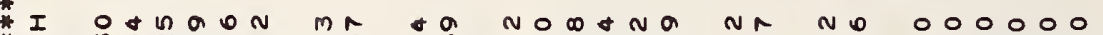

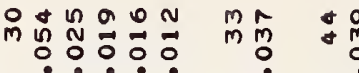

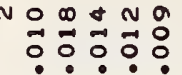

N No

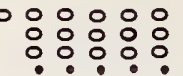

$\circ: 0$

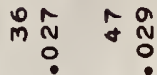

\#

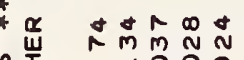

n

品

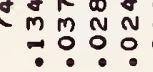

a

$\stackrel{0}{0}$

+ on 0 om oo

a.

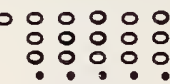

$\circ$

$\circ 0$

m $m_{0}=\frac{m}{0}=0$

0000

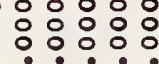

$\circ$

$\circ$

an in $a$ om

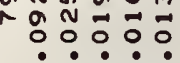

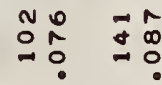

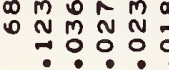

$\infty \stackrel{N}{2}$

은

N

o

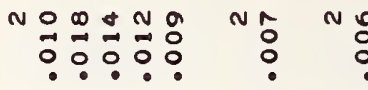

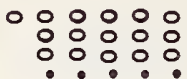

$\circ$

$\circ 8$

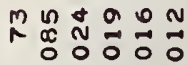

ณू $\vec{\sim} \vec{m} \vec{\infty}$

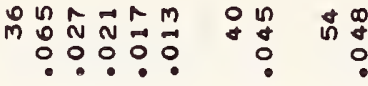

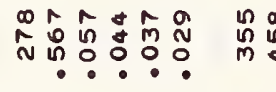

in

-

$\infty_{-1}^{\infty} \quad \infty m$

$\stackrel{m}{m}$

$m m n=m m$

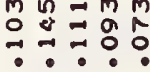

$m \stackrel{n}{n} m \stackrel{0}{\circ}$ 무요 $m$ in

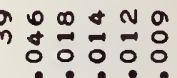

$-m \infty \infty$ in $\min _{0}^{\infty} \stackrel{\infty}{*}$

na non N N

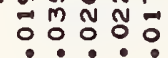

$\stackrel{n}{0}$

$N \frac{m}{0}$

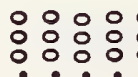

$\sum_{n=1}^{n}$

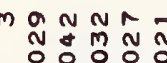

mo $m$

人ํํำ

유:

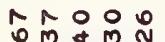

*

* 甾

$\because$ :? :

임

$\operatorname{monNa}$

ํํำ ํ.?

$m \circ \quad m o$

$m \frac{9}{0}$

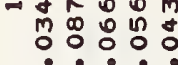

$\circ$

$\circ \%$

mmM $m=$

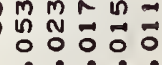

*

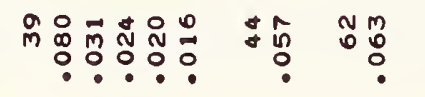

nanonh n n

$\begin{array}{lllll}0 & M & N & N & 0 \\ 0 & 0 & 0 & 0 & 0\end{array}$

:

N

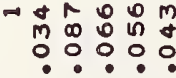

$-$

$\rightarrow$

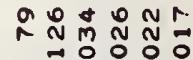

$\because 0 \%$ ?

må

*

*

$m \& m=* n$

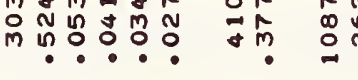

*

nู

*

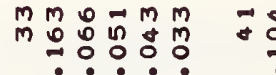

an

n $\sim$ n $\rightarrow$ R

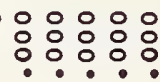

$\rightarrow$

$\rightarrow$

$=m N n=0$

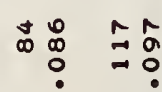

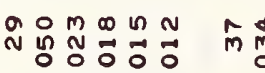

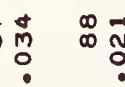

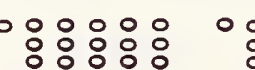

응응

$\circ$ 응

ㅇํㅇํㅇ․․

$\circ$ :

$\therefore \circ:$

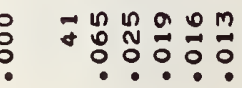

\&ั่

:

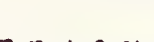

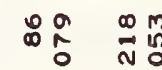

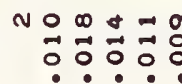

$\because$ ? O O

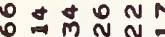

点

$\hat{\circ} \quad$ ำ

N우웡

8

\%

잉ㅇㅇㅇㅇㅇ

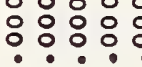

$\infty$

$\infty$
$m$
0

a $N$ n $N \vec{n}$

O요요

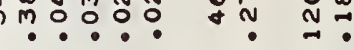

*

$\vec{m}+4 \infty$ in $⿻$

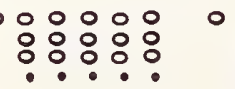

00000000

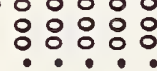

$\circ$ 웅

$\circ \circ$

nก

ก N

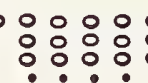

$\circ$

$0 \%$

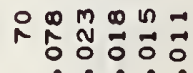

$\begin{array}{lll}\infty & N \\ \infty & \text { N } & \text { N } \\ 0\end{array}$

ㅇํㅇㅇำ

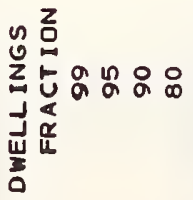
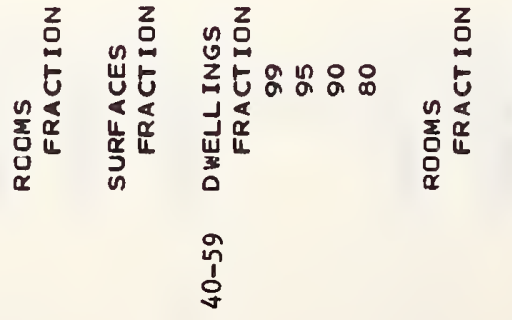

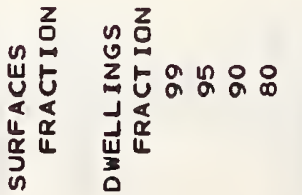
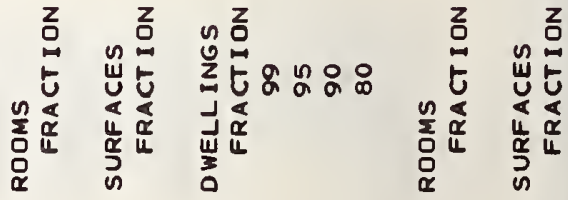

㟧

$\stackrel{n}{0}$ 


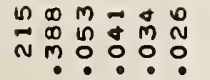

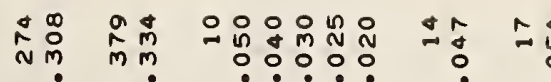

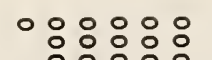

0

0

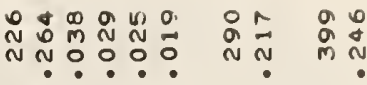

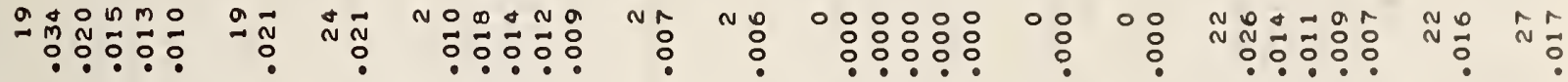

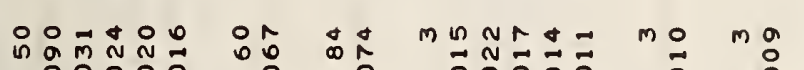

i:0:0

$\overrightarrow{0}: \overrightarrow{0}: \overrightarrow{0}:$ :

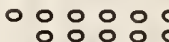

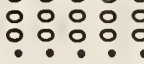

$\circ \div$

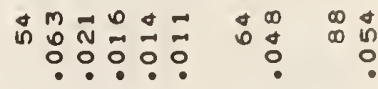

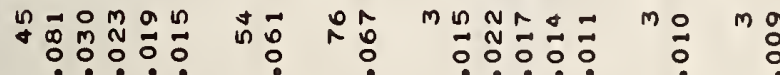

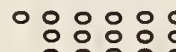

$\circ:$

$\circ:$

aro 0

$\begin{array}{lll}\infty & m & 0 \\ 0 & 0 & q \\ 0 & 0\end{array}$

$\checkmark m N R+$

N

$\stackrel{n}{\sim} \underset{\infty}{N} \quad N$

nom na n n

: N

000000

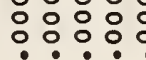

0

$\circ$

n $n N \circ$ n

no $0 m$

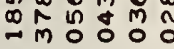

$\stackrel{\leftrightarrow}{N}$

$\stackrel{a}{\infty}$

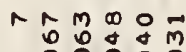

$\infty$

$\infty$ 응

N

ำกำ

N

ㅇํㅇํㅇㅇㅇ

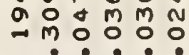

mㅇ 90

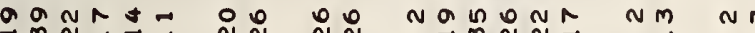

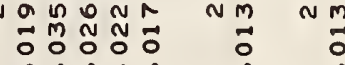

000000

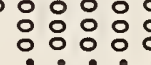

$\circ$

$\circ$ :

$\rightarrow m \infty \circ \infty$

$\prod_{0}^{m} \overrightarrow{0}: \overrightarrow{0}:$

$\stackrel{N}{N} N^{m}$

? ㅇํㅇ

nค \&

na $\ln$ on

$\begin{array}{lll}0 & 0 & N \\ M & N & N \\ 0 & 0 & 0\end{array}$

NM N

+ 0 o

ก

Nan on

ํ.

N

\%

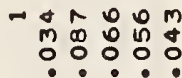

$\rightarrow$

$\rightarrow \stackrel{d}{N}$

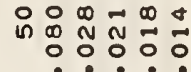

路

$\begin{array}{cccc}m & m & m & n \\ 0 & m & n & N \\ 0 & m & N & N \\ 0 & 0 & 0 & 0\end{array}$

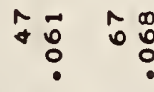

NanONA NM

m

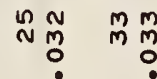

ํ.

-

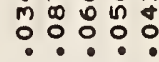

$\rightarrow$

$\rightarrow$

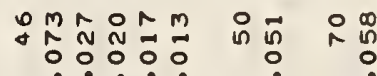

○? 0

๑):

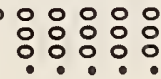

$\circ$

$\circ:$

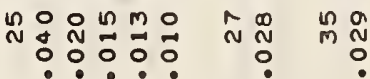

$a+a d n$

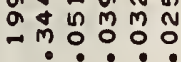

离

in

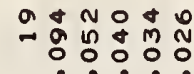

$\stackrel{m}{\sim}$

in

응ㅇㅇㅇㅇㅇㅇ

:ㅇㅇㅇㅇㅇ

$\circ$

융

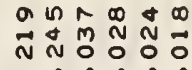

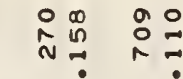

ลี่

in

in

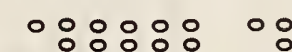

$\circ 8$

$\circ \circ \circ \circ$

웅ㅇㅇㅇㅇ

$\circ$

$\circ:$

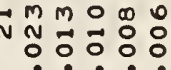

in เ $\wedge a$

NoOMO

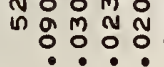

$\stackrel{N}{0}$

$\overbrace{n}^{\infty}$

$\rightarrow$ in $m \circ 0$

$\rightarrow m$

N $\overrightarrow{0}$

응ㅇㅇㅇㅇㅇ

옹ㅇㅇㅇㅇㅇㅇ

$\circ$

$\circ:$

m $\begin{array}{llll}m & \text { n } & m & 0 \\ \text { in } & 0 & -1 & 0\end{array}$

คำ

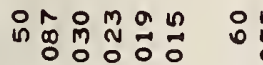

: : :

moำ

: 0

$\circ 0$
$\circ:$

:

(1)

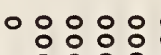

$\circ: 0 \%$
$\circ: 0$

$\circ$ :

$\circ \%$

ถิก

⿰ก

0000000

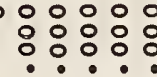

$\circ$

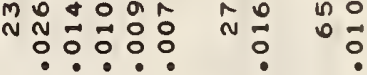

年

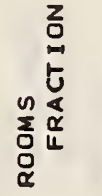

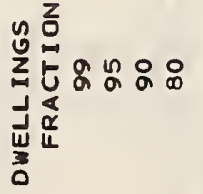

$\stackrel{n}{n}$
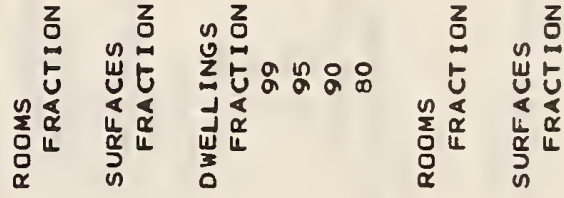


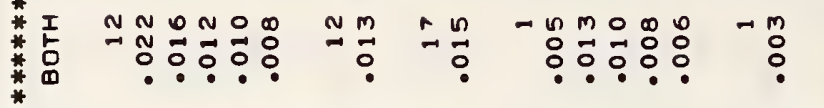

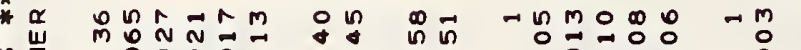

$\stackrel{\sim}{0}$

:

*

*

*

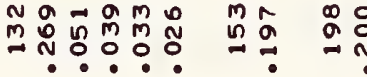

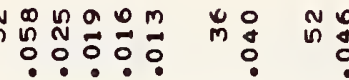

:

-

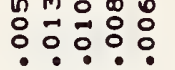

$+$

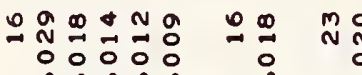

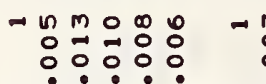

a N $0 \circ: 000$

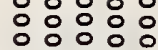

$\circ$ :

$\circ:$

natann

ODMONO

象昂

은은

-

응ㅇ:융

$\circ:$

$\circ \%$

m

$\circ: ㅇ ㅡ ㅇ ㅇ$

$\therefore: ㅇ ㅇ ㅇ$

$\circ: ㅇ: ㅇ ㅛ$

응유:

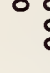

$\circ \circ 000$

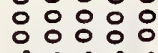

$\circ:$ $m \infty \neq-a$

$\vec{m}$

în

m

O nO

$\pm \frac{m}{0} \stackrel{0}{*}$

$\because: 0: 0$

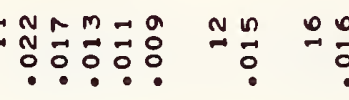

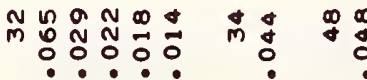

ลิดกำ

:?: :

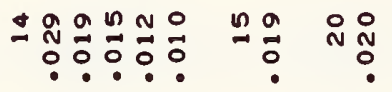

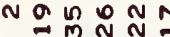

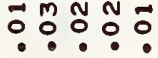

a $\operatorname{~} 0 N A$

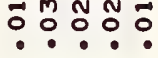

NOUDNR NM

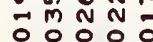

-

:

:

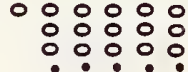

N

N

응ㅇ:융

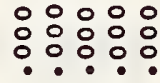

융ㅇㅇㅇ

응

$\circ$

$\stackrel{n}{\sim}$

$\rightarrow \frac{0}{0}$

Mิ N N $\mathcal{N} \tilde{N}$

西

$\because \frac{0}{0} \stackrel{0}{\circ}$

: $\because$ ก

$\stackrel{4}{\circ} \stackrel{\infty}{0}$

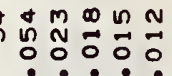

mำ

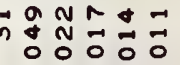

:ㅇํㅇ

$m$
$m$
$m$
$m$
0

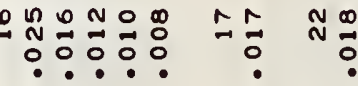

$\stackrel{u}{w} *$

*

**

n * * *

告 $m$ N

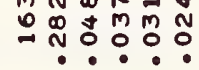

웅요

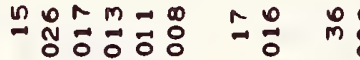

\section{or nomm}

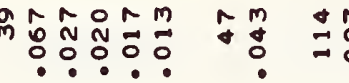

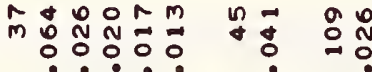

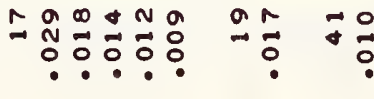

$+\frac{0}{0}$ $\therefore:: ㅇ ㅛ$

D- Nn 00000

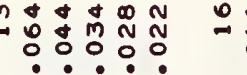

$\circ \circ \therefore ㅇ ㅇ ㅇ$

:ㅇ:ㅇ:

000000

$\therefore:: ㅇ ㅛ$

영ㅇㅇㅇㅇㅇㅇ

… $\circ \circ 0$

$\circ$

$\circ \circ$

$: \circ$
$0: 0$
0 $\therefore: ㅇ:$

$\circ: \therefore::::$

응ㅇㅇㅇㅇㅇㅇ

000 $\circ:$

$\circ$ :

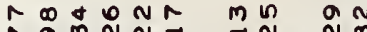

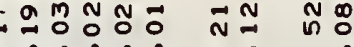

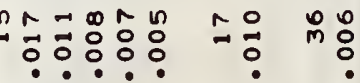

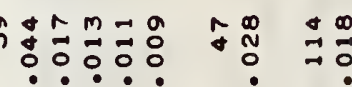

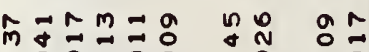

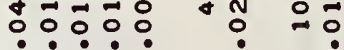
anar:

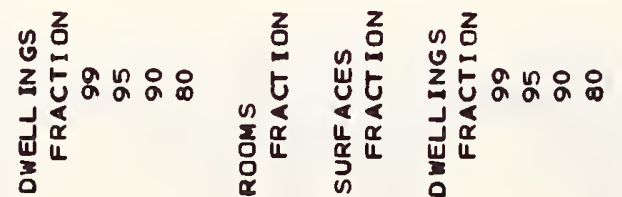

in

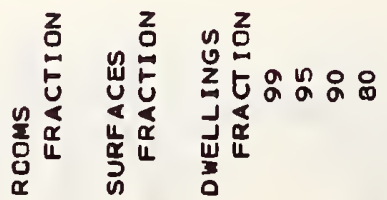

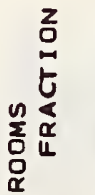

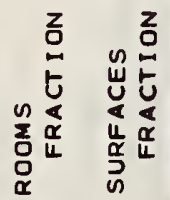


no:

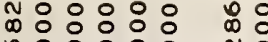

:ㅇ:? N

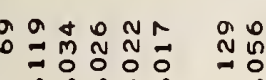

$\wedge \rightarrow$

$\stackrel{M}{N} \stackrel{\infty}{m} \vec{m}$

(1)

政

ง $\frac{4}{1}$

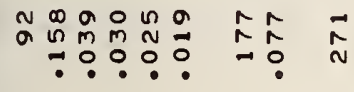

in :

인

กิ

$\vec{a}$

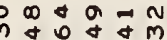

$\because \because 0$ :

$\sim \infty 0 M \circ \infty$

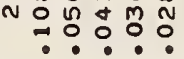

กัด蛙药

은?

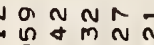

$\because \because 0 \%$ m:

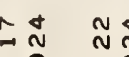

in

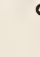

\section{N}

:

mi
음융용

$-1$

ก

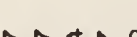

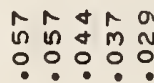

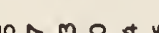

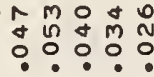

$M \stackrel{N}{N} \vec{O} \stackrel{N}{N} \vec{N}$

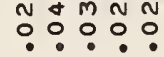

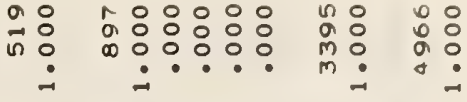

n耳 ๓

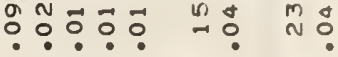

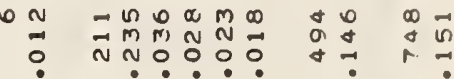

no a요

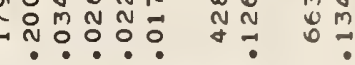

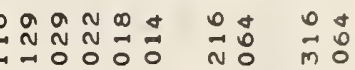
$\rightarrow$ ํํำ
1000

in: 응: $\dot{-}$

$m \circ \infty \infty m$

$\because 0.0$

a.

I N

g̊ :

ì

m̊t $=$

$\overrightarrow{\text { N }}$ :

뜽ㅇㅇㅇㅇㅇㅇㅇ

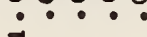

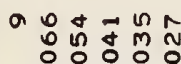

ㅇํㅇำ

$\stackrel{2}{0} \stackrel{m}{\circ}$

$\int_{0}^{+}$

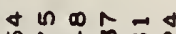

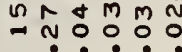

n n 20

ํํㅇㅇํํํํำ

70000

ถู

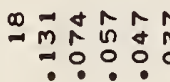

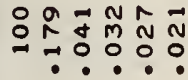

å o

má

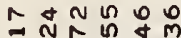

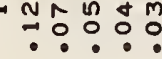

mi

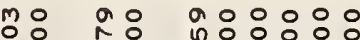

n응ㅇㅇㅇ응

in:

:

nO००००

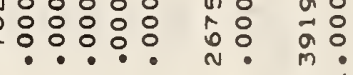

nN Unana

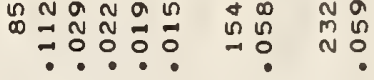

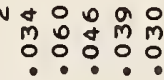

啇

n $m$ in

n n n n n

$\infty \sim \infty m$

d

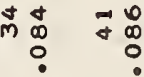

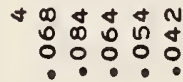

$+\underset{N}{\infty}$

$+$

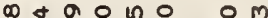

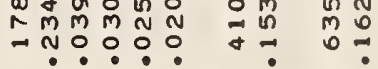

点 min m

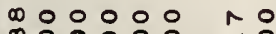
กั

aี :

0
$\dot{0}$
0
0

no 000

$m$

- 0 人 0 0

7.

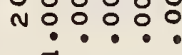

aํ.

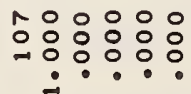

in:

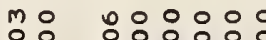

$\therefore: \div: 0: 0$

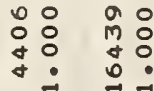

aำํํำ

용ํำ

离

n

ก

$\stackrel{\infty}{\rightarrow}$ ?

$\circ \circ \circ \circ \circ$

영유융

n๑n-n

$a \infty N \infty$

:ㅇํㅇ

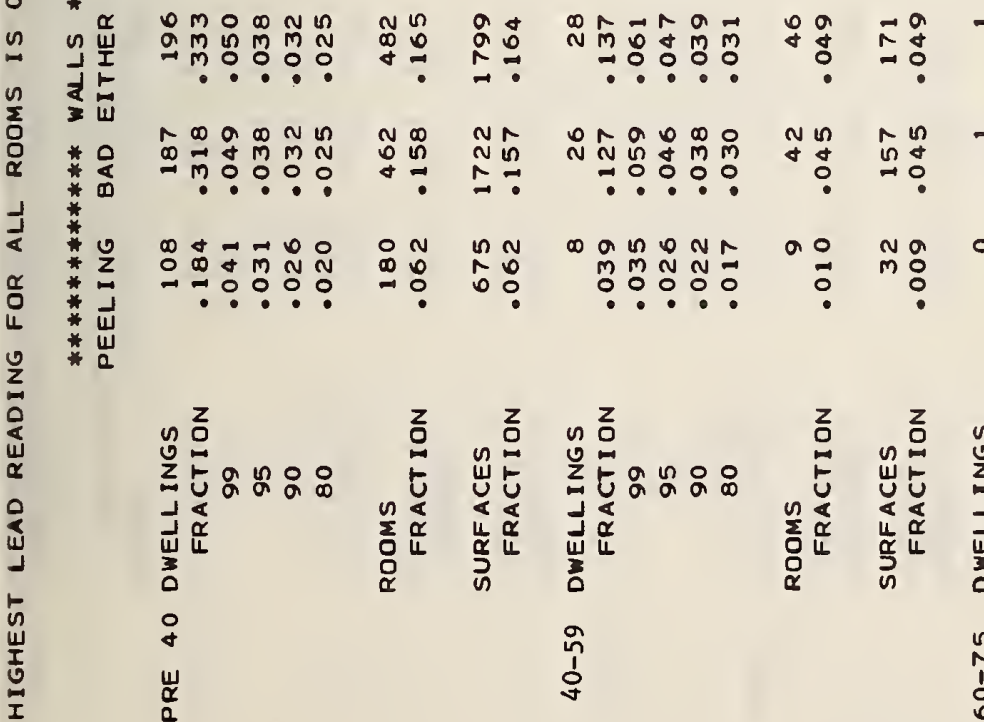

$\begin{array}{llll} & \\ 0\end{array}$

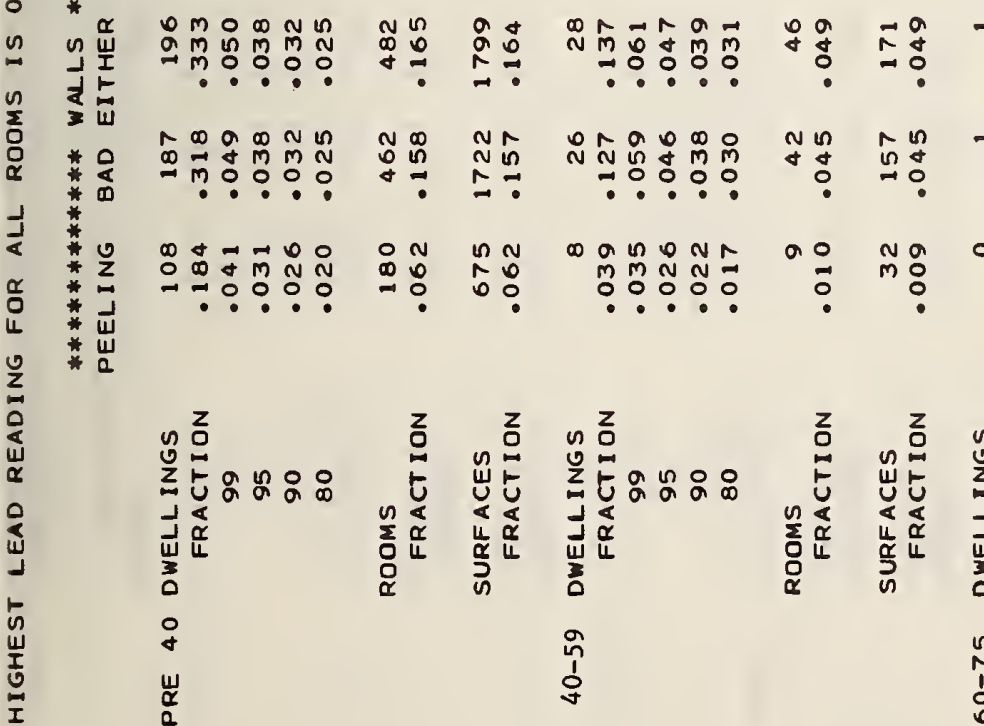

-

-

- $\begin{gathered}0 \\ -0 \\ 0\end{gathered}$

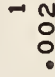

m

$\because \approx N$ N

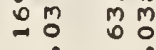

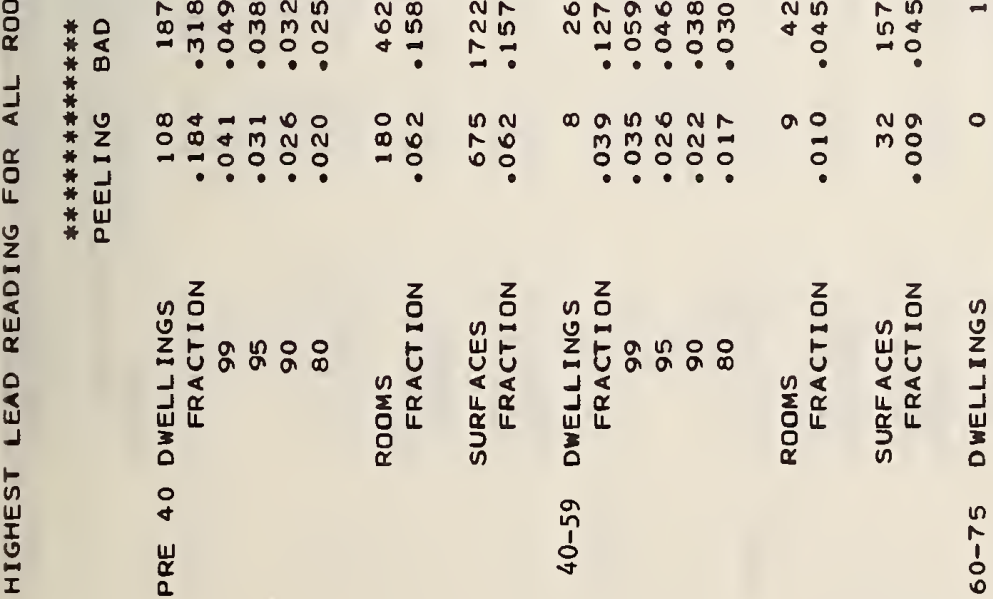

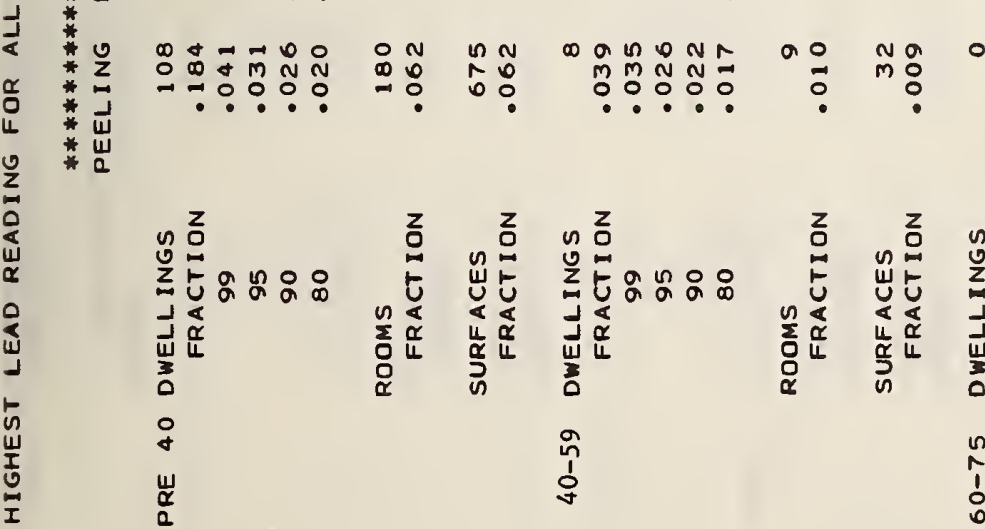

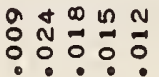

응ㅇㅇㅇㅇㅇㅇㅇㅇ
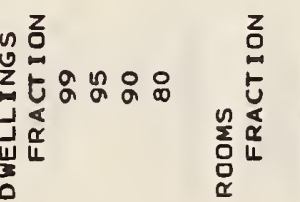

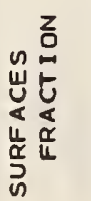

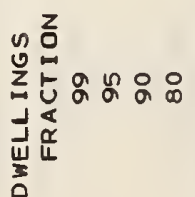

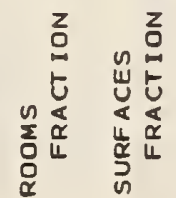


a $m a$ a $N$ N $N$

.

*

*

*

* $\alpha \quad \sim \infty \infty ⿻$

in

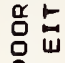

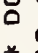

$*$

*

*

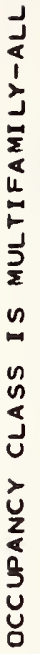

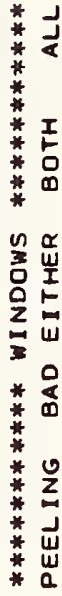

$0-\pi+a n$

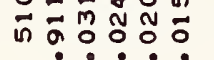

N

ח̃

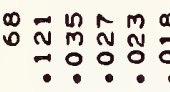

N

墭

aำํํำ

in :

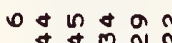

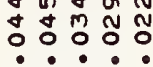

$\infty \circ 000$

*

*

,

*

*

*

*

*

*

$F=\infty+\infty$

$\infty=N$

- aranan

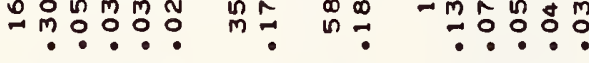

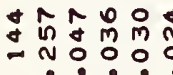

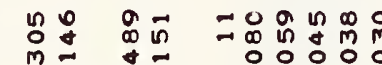

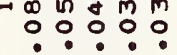

$+x \circ-0$

-

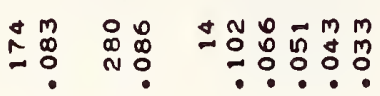

กั

a

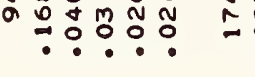

in $0 \overrightarrow{0}: \overrightarrow{0}:$

No nn mmnNon

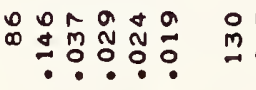

$\rightarrow \infty 0000$

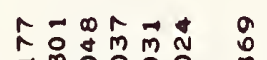

o

里

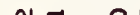

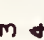

- b.

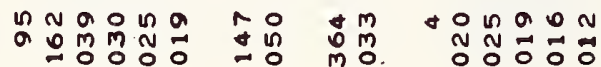

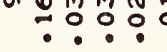

$\rightarrow$ m

ํํำ

$\circ$

ma g a

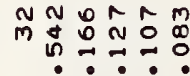

กั

in กิ

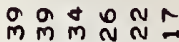

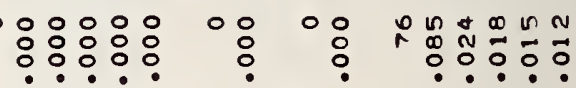

$\stackrel{0}{N}$

åd

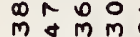

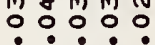

$=0$

+ 0 n $m$ o od ma

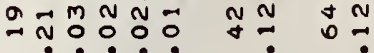

ํ.ดำ

$m: m:$

MNMM田 Mn 음

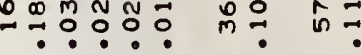

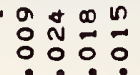

$\rightarrow$

$-$

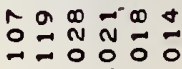

$m a 0$ o

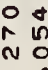

an N

พิ๊

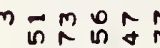

M m m m

으으.

$\rightarrow$

$-$

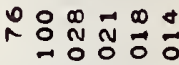

$\because \circ: 0$ 은

mo

옹

ถำำำ.

m

$m \infty$

जี

mr No

$\sim \stackrel{\infty}{\sim}$

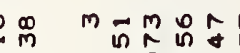

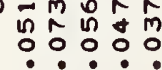

m

$m$

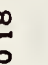

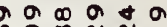

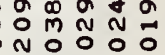

:

$\stackrel{\infty}{\stackrel{m}{*}} \stackrel{n}{n}$

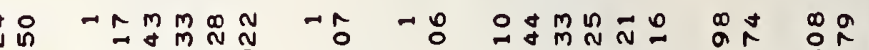

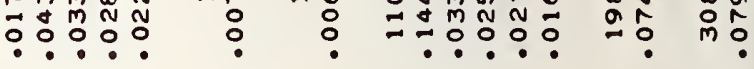

舟 N

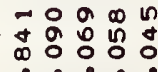

$\stackrel{a}{a}$

a d

in $\stackrel{\infty}{m}$ 운 0

mo: 000

in

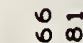
. $=$

-.・.

.

a o n a om

$\therefore: ㅇ: \div$

$\circ: \circ$

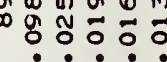

$m$

กิ ก

00

$-a+\infty$ in

ํํำำ

$\rightarrow$

ก

ดㅇำ $n^{m}$

añ

à

ㅇ in

$\stackrel{\infty}{m=}$

$-2+\infty n$

ำ

$-$

$-$

은은ํำ

$\mathfrak{m}_{\substack{a \\ 0}}^{0}$

mo

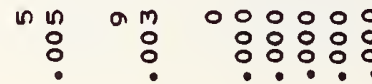

$\circ 8$

$\begin{array}{ll}\circ & 0 \\ : & :\end{array}$

$\begin{array}{lllll}0 & 0 & 0 & N & M \\ 0 & N & N & 0 & 0\end{array}$

in

no

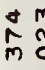
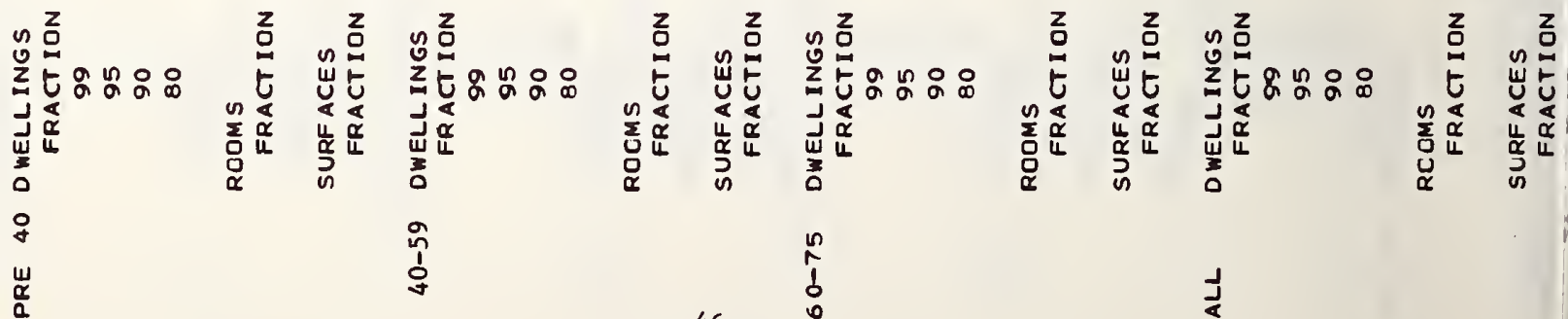

? 
ํํำ:0:

$0-0$ n. $0 \mathrm{~m}$

$\sqrt{0.00}$

?ㅇ?

$\infty d 0 \infty m$ Mn in $-1+4$ \&

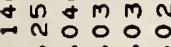

ก

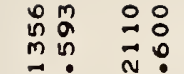

$\begin{array}{lllll}7 & 0 & m & m & -1 \\ 0 & 0 & 0 & 1 & 0 \\ 0 & 0 & 0 & 0 & 0\end{array}$

\section{a}

in

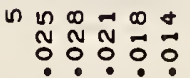

$m$

a

(

0舟的

ㅇํㅇํ․

ก

มี ก

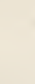

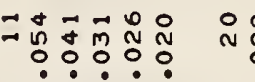

m

n̊

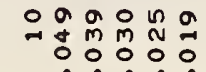
$\pm \frac{0}{0} \quad$

$0+$

0,0
0
0

$\stackrel{0}{*}$

$a_{0}^{0}$

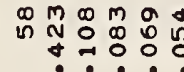

$\stackrel{\infty}{\circ}$

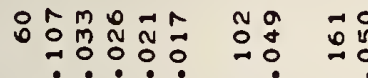

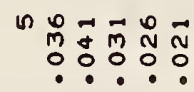

0

r

\& $\overrightarrow{0}$ g

资

*

กิ

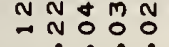

$\rightarrow \ln a+a$

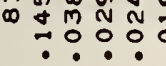

am $a$

$\cong \infty N \sim O \vec{m}$

-

+ $\stackrel{n}{m}$

$\because$

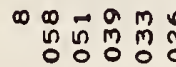

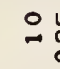

$\stackrel{a}{n}$

N

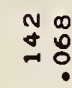

$\stackrel{\infty}{N} \stackrel{0}{N}$

a $\begin{aligned} & 0 \\ & 0 \\ & 0\end{aligned}$

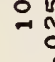

$\stackrel{n}{N}$

$\rightarrow$ ก

$\Rightarrow \stackrel{M}{N}$

NHODo

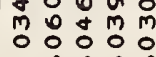

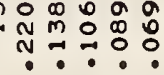

$\stackrel{m}{N}$

ํำ

Nod $m M N \vec{N}$

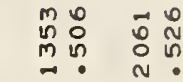

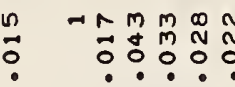

$\rightarrow$

$-1$

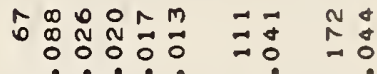

N

mo

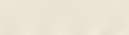

$\begin{array}{ccc}m & m \\ 0 & m & N \\ 0 & m & N \\ 0 & 0 & 0 \\ 0 & 0\end{array}$

N

N N

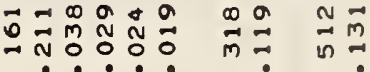

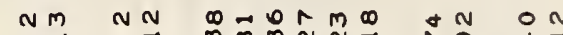

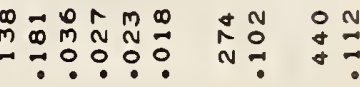

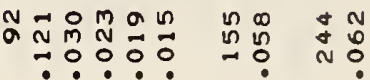

An a

in

กับ

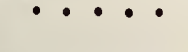

여ำ

$\because$ ? O O

a $\mathrm{m}$

$m$

- O n n a m

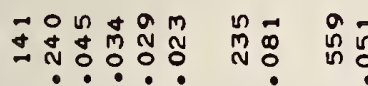

NOAO $0 \mathrm{~m}$

$\pi 0^{\circ}: 000$

ì

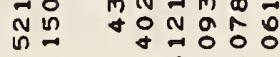

$\infty$

$\stackrel{n}{a}$

N

กำ

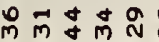

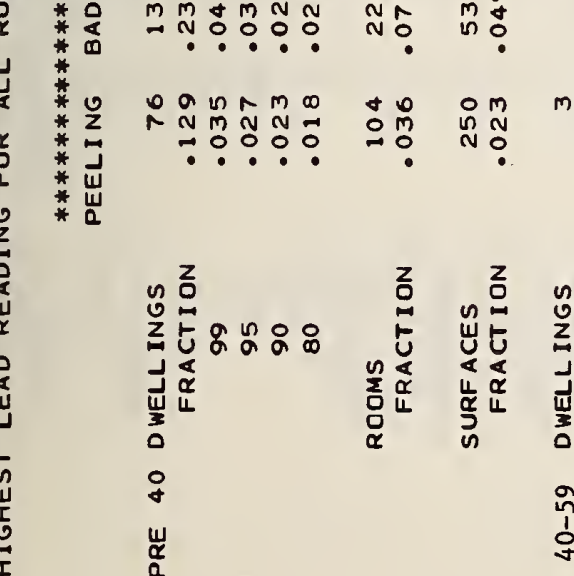

NN

$N \circ \infty m=0$

a

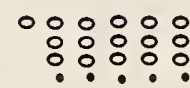

$\circ 8$

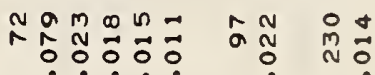

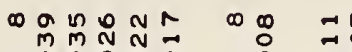

$\begin{array}{lll}0 & n & N \\ M & N & N \\ 0 & N & N \\ 0 & 0 & 0 \\ 0 & 0\end{array}$

$\begin{array}{llll}0 & 0 & 0 & 0 \\ \circ & 0 & 0 \\ 0 & 0 & 0 & 0\end{array}$

$\circ 8$

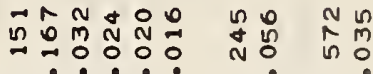

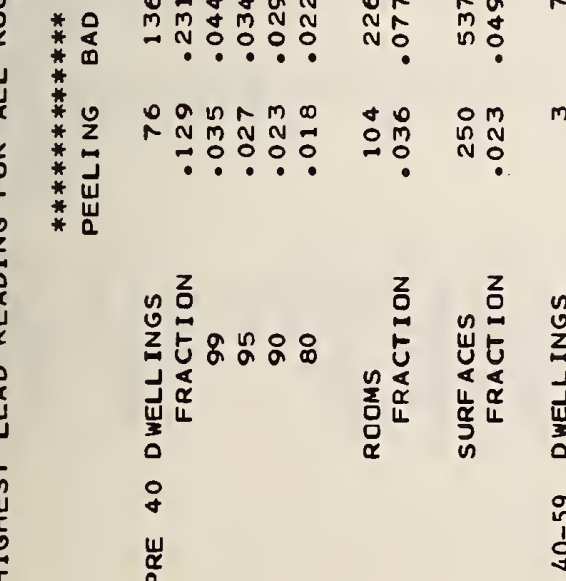

$\begin{array}{llll}+ & N & n & -1 \\ M & M & N & 0 \\ 0 & 0 & 0\end{array}$

$r$

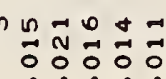

$m \mathrm{~m}$

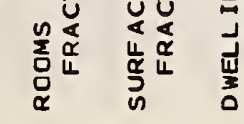
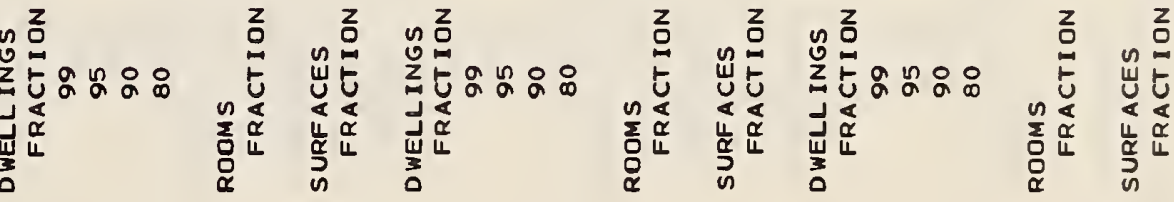

品

$\stackrel{n}{1}$ 
$\hat{\infty} \vec{N}$

$\because \underset{1}{-1}$

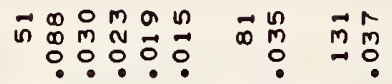

\section{우요 $m$}

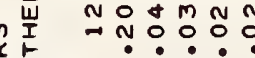

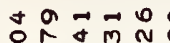

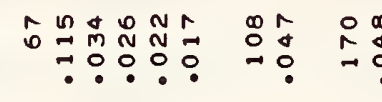

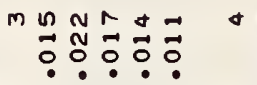

$\begin{array}{ll}11 & 0\end{array}$

$\vec{a}=$

NON N

:ㅇํㅇ

$\pm d \quad$ in

No

$\infty \min _{M} N N$

$\begin{array}{ccc}m & N & \\ 0 & 0 & 0 \\ 0 & 0 & 0\end{array}$

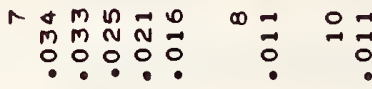

웅

$=\underset{0}{0} \pi$

\section{$\sim \vec{x}$}

옹

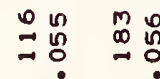

a

$\underset{\infty}{N} \underset{0}{m} \stackrel{m}{m} \underset{0}{m}$

京:

$\infty$ o

ㄴ? : ON N N

or $\infty$

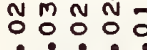

$m N N \backsim \vec{N}$

$\begin{array}{llll}N & M & N & -1 \\ 0 & 0 & 0 & 0\end{array}$

$M N N \backsim \vec{N}$

$\begin{array}{lllll}N & M & N & N & 0 \\ 0 & 0 & 0 & 0 & 0\end{array}$
O

ma

m.

0 in $\infty$

(1)

n

?

$+$

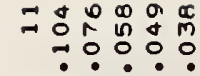

$\stackrel{\infty}{\rightarrow}$

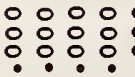

$0:$
$:$ $\circ:$
$:$

1 0 이응
Nmmra

o d o 000

:

moman

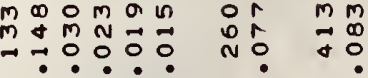
옹ㅇㅇㅇㅇㅇ 응ㅇㅇㅇㅇㅇㅇ

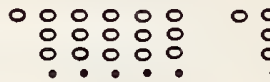

:

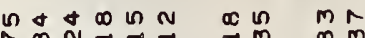

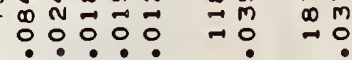

* *

I

资政

*

*

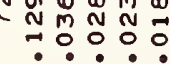

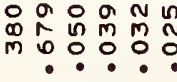

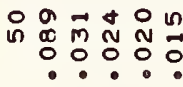

$\stackrel{0}{N} \stackrel{n}{n}$ in

A nO:

- 0 N N

0 a d m n

$\rightarrow$ : ? : :
$*$
$*$
$*$
$*$
$*$
$*$
$*$
$*$
$*$
$*$
$*$
$*$
$*$

กำ

? : : ०

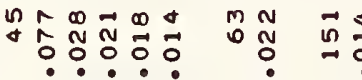

?ㄴ?

ก naㅇ no

幽耑

a ำ

$\overrightarrow{0}$ in 0

*

*

*

* $\because$ : 0 :

a man a n

+ $m a$

$\infty N \quad n$

$\stackrel{n}{\infty}$

$\overrightarrow{0}$

郶

$\stackrel{\infty}{m}$

a

$\hat{n} \stackrel{m}{m} \stackrel{m}{0} \frac{n}{0}$

\section{n}

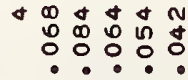

$\circ$

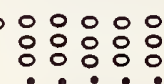

$\stackrel{1}{0}$

$\rightarrow r m m n$

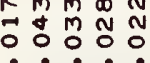

$\frac{m}{0}$

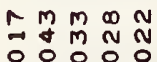

? ? ? ?

응ㅇㅇㅇㅇㅇㅇㅇ n $\begin{gathered}m \\ m \\ 0\end{gathered}$

no

$\circ 8$

$\rightarrow$

응 $\rightarrow$

$+1$

:

0 :

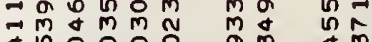
ก?: 0? 0 m $m \circ 40 n N \quad r m$ 웅 $\begin{array}{llllll}N & \overrightarrow{0} & \overrightarrow{0} & 0 & m & m \\ 0 & 0 & 0\end{array}$

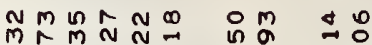
$\rightarrow$ 유 N

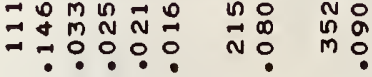
음에

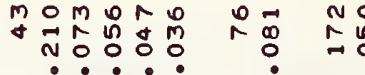

0

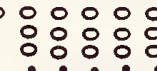

$m \leq \vec{n}: \overrightarrow{0}=\overrightarrow{0}$

$m \backsim \vec{n} 0 \div$

$\overrightarrow{0}: \overrightarrow{0}: \overrightarrow{0}$

$\circ$ 잉ㅇㅇ웅

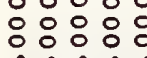

$m \underset{m}{m}+$

$m \stackrel{m}{0}+$

$\circ: \circ$

\section{$\begin{array}{rrr}0 & 0 & 0 \\ 0 & \circ & 0\end{array}$}

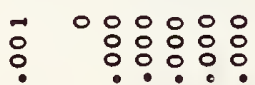

:

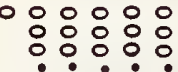

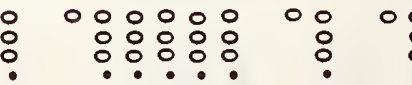

DONNN

a d o o o

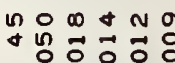

유요

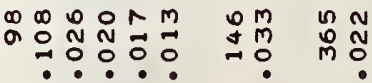

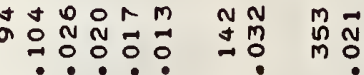

a.

읭 $\overrightarrow{0} \overrightarrow{0} \overrightarrow{0}$

กํ.

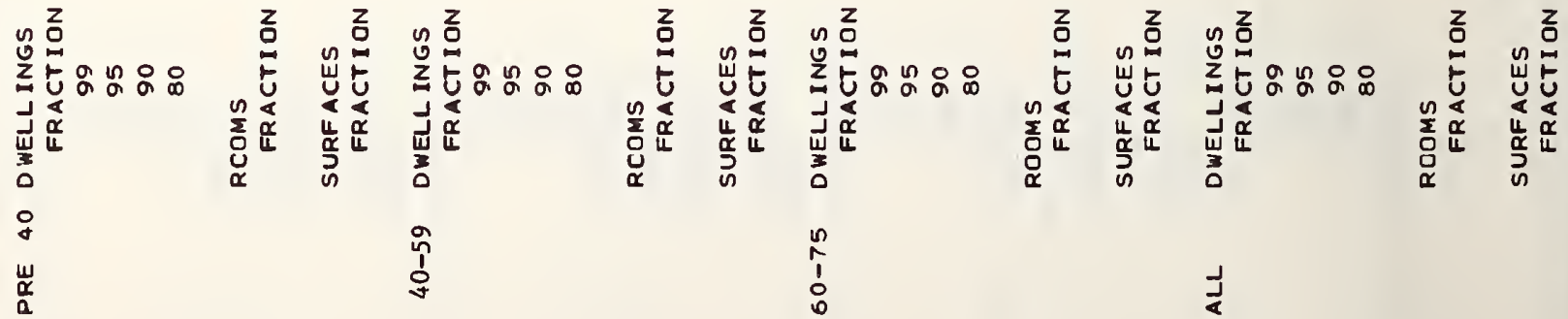




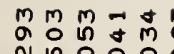

$m \&$

ก ?

กั $\stackrel{a}{m}=$

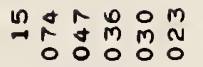

舫

ติ๊กำก

NO $\infty$ \& $\rightarrow$

$\overrightarrow{0} \overrightarrow{0} \overrightarrow{0} \overrightarrow{0}$

8

8

응ㅇㅇㅇ응

:

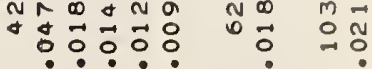

n

in

$m \ln _{n \rightarrow a} N=$

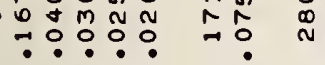

$\overrightarrow{0}: \overrightarrow{0}: \overrightarrow{0}$

0

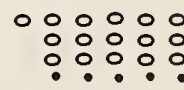

$\circ$

$\circ \circ$

$\vec{M} \hat{N} \vec{N} M$ N

$m m \sim \infty \&$

a

$m \Omega N A \pm I+\infty$

0

0 :유유 $\rightarrow 0000 \%$ ก

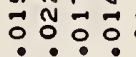

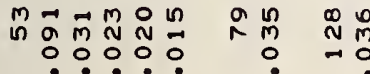

Nom 0

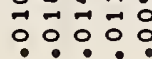

:

n)

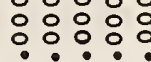

$\circ 8$

$\circ:$

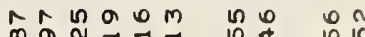

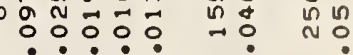

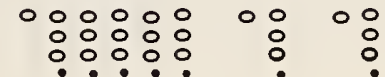

ถึกั๊

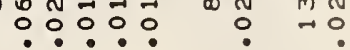

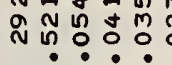

กิ๊

$\hat{n}$

음

$\stackrel{\infty}{\infty}$

i

Noㅇำ

N m

N N

ㅇํㅇํㅇ

m?

$\overrightarrow{0} \quad \overrightarrow{0}$

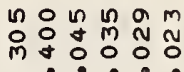

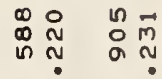

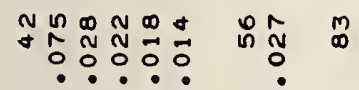

N I

nก

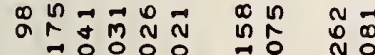

$m N N$ n

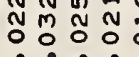

$m \stackrel{\circ}{\circ}$

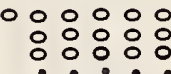

$\circ \stackrel{0}{\circ}$

$\circ:$

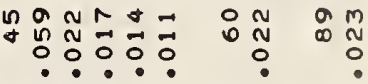

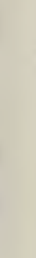

mơ

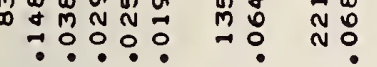

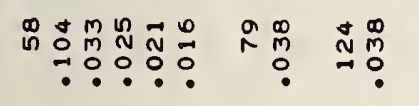

$m N w$ n -1

N $N$ N

:O: :

N 1 웅

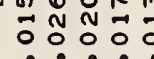

$+0$

no

$\begin{array}{rrrr} & \\ -M & m & \infty & N \\ 0 & M & N & N \\ 0 & 0 & 0\end{array}$

$\rightarrow \hat{0}$

$\rightarrow$

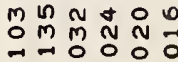

$\begin{array}{lll}\rightarrow & 0 & 0 \\ 0 & n & 0\end{array}$

no $\rightarrow$ M $m$ m

$\begin{array}{llll}M & m & N \\ 0 & m & N \\ 0 & 0 & 0 & 0\end{array}$

$\rightarrow \stackrel{0}{0}$

$\rightarrow$

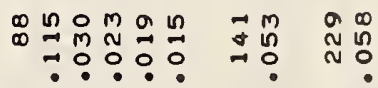

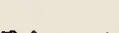

$+\infty$

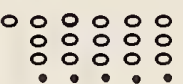

$\circ:$

$\circ:$

$\rightarrow 0$ in 0

$m_{\infty}^{m} \vec{m} \underset{m}{0} m_{0}^{m}$

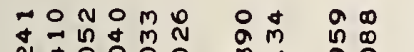
N.?o:?

$\operatorname{lng} 20000$

m.

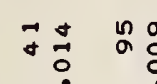

กั

$0 \infty m \rightarrow+r$

ผ

$\begin{array}{lll}m & 0 \\ 0 & 0 & 0 \\ 0 & 0\end{array}$

$\circ \circ$
0

$\circ \circ$

$\circ 0$

Na co a in

กับำำ.

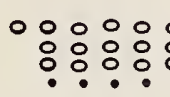

00

00000000

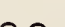

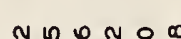

$\rightarrow a$ n 0

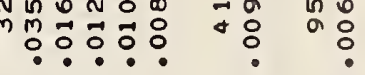

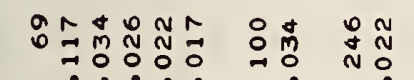

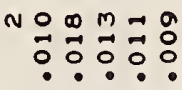

N

:

응ㅇㅇㅇㅇㅇ

$\circ:$

$\circ:$

$\approx \infty M N \ln$

Nom a n

DNMINA $\cap M N N \quad N O \infty M=$

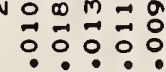

N $N$

:

응ㅇㅇㅇㅇㅇ

$\circ:$

00

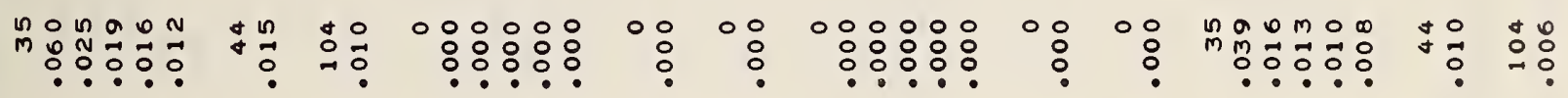

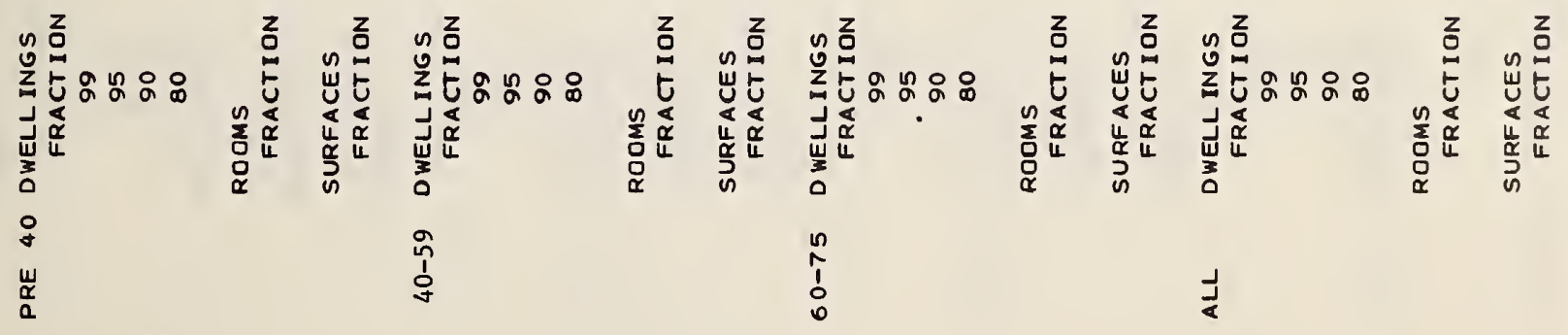


am nomo

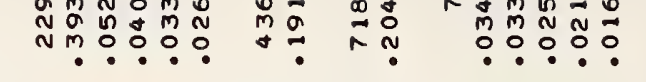

min

$\stackrel{\infty}{\infty} \hat{\sim}$

กับ

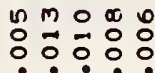

ํํำกัN $\stackrel{0}{=} \overrightarrow{0}$

i

ㅁำ

* 宁

*

*

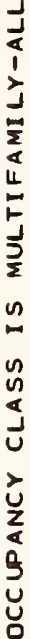

旁

*

*

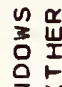

之!

*

*

*

*

* $\because \therefore:$

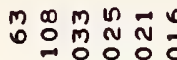

$\because 0: 00$

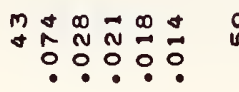

กิ

品

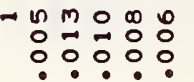

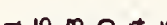

ㄱำ

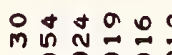

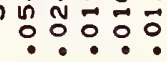

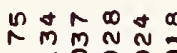

$\because$ :웅

กำำกำ

$\because$ ㅇํㅇ

$\rightarrow \stackrel{M}{N} \stackrel{\infty}{N} \underset{N}{N} \stackrel{\infty}{0}$

ํ.:ํ.:ㅇ.

เก N

分京

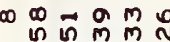

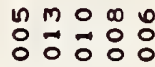

$\therefore \therefore$.

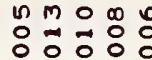

8

N

冝告

:

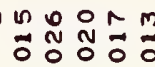

ํํㅇ ํํㅇ

号

N กํำำ?

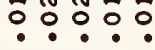

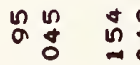

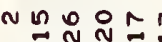

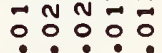

\%

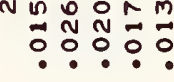

$m$

ก $\quad+\infty$

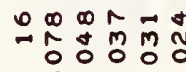

*

\% 0 o

ampon

$N$ 응

N $\underset{N}{N} \quad \exists \cong$

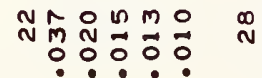

*

Noㄴ.

․․․․

- : : : : : :

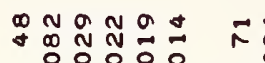

ต

安占

ㅇำ

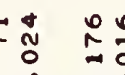

0

$\stackrel{0}{0}: 00: 0$

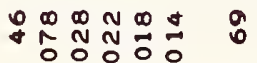

*

**

*

H H M O O n 0000000000

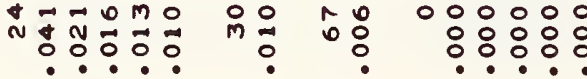

\%

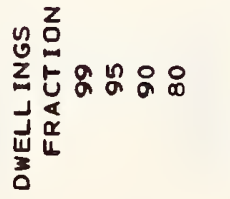

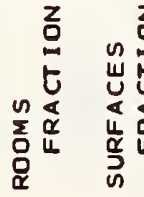

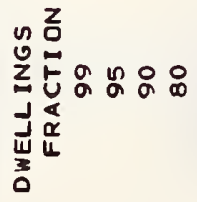

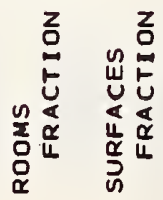
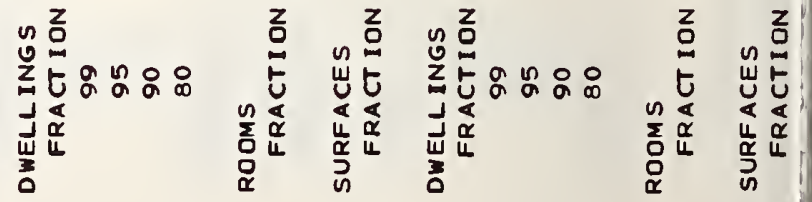

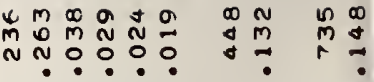

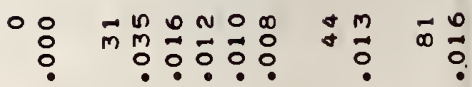

$\hbar \infty+\infty n n$ n $n$

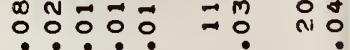

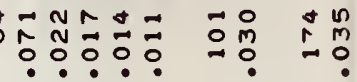

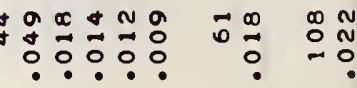

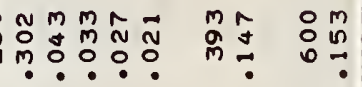

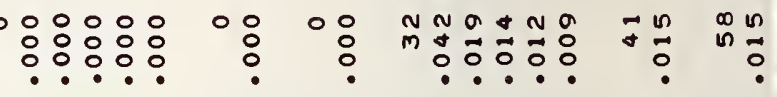

ㅇㅇㅇㅇㅇㅇำ

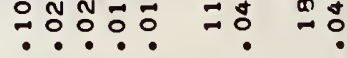

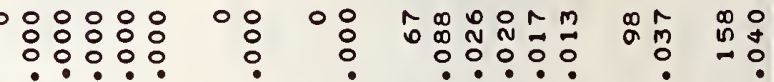

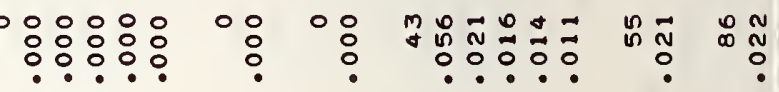

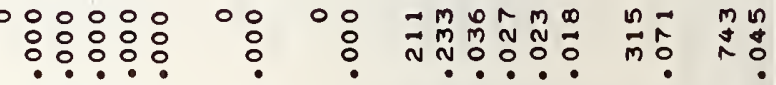

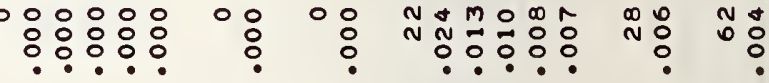

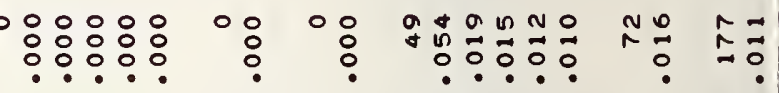

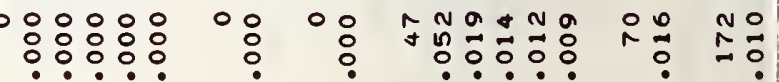

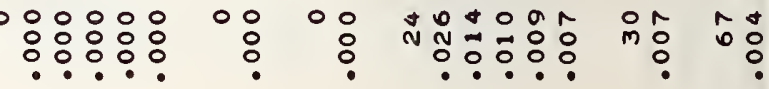

î̀

$n$
0
0 


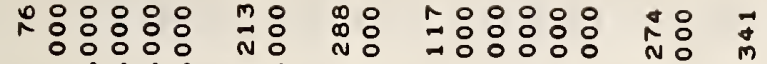

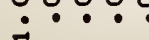

ก $0 \mathrm{~N}$ in 0

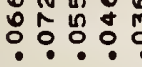

$m m+n$ on

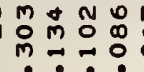

$+\rightarrow m \infty$

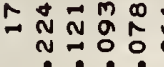

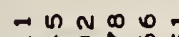

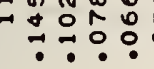
눙ㅇํㅇ $\rightarrow \rightarrow$

$\infty \stackrel{\infty}{N}$

$2 m \pi+n$

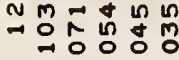

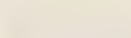

N

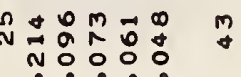

in in

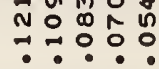
N $m$

an 0 o n

음

g엉

$\stackrel{7}{\square}$

๑以ํำ 은 ํํㅇㅇํㅇ $a$

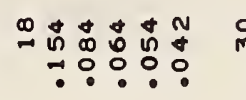

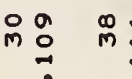

$\stackrel{\infty}{m}=$
⿰ก)

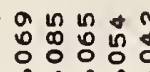

ํํㅇㅇㅇㅇㅇㅇㅇㅇ

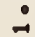

m

ñ

n

$\Rightarrow \begin{aligned} & 0 \\ & \sim \\ & 0\end{aligned}$

$\infty$
$\infty$
0
0

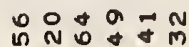
ㄴ․ㅇำ

$ㅇ$

$\rightarrow$ in

$m$

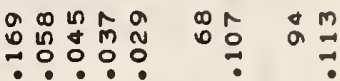

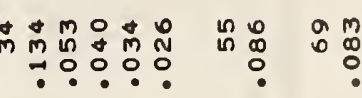

*

$\infty$ 웅ㅇㅇ :ㅇ:ㅇㅇㅇㅇ

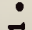

ด 舟

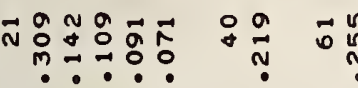

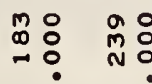

훙ㅇㅇㅇㅇㅇㅇㅇ

$-$

워ำ

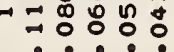

N온요ํ

N

ำ

$\stackrel{m}{*} \div \because$

ำ

$\infty$

ON+ONN

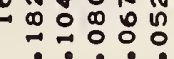

$\Rightarrow N \pm N$ $\because \begin{aligned} & N \\ & 0 \\ & 0\end{aligned}$

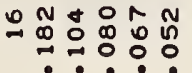

$\rightarrow$ N

$\rightarrow$

$0 m$

ก

* 0

$\underset{m}{m}$

N

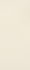

씅ㅇㅇㅇㅇㅇㅇㅇㅇ ㅇํㅇ ?

$\rightarrow$

웅ㅇํㅇํㅇ

ㅇํㅇㅇㅇㅇㅇํㅇ

$m+0 \mathrm{~N} N$

$M$
$M$
0
$\because$
$\because$

ㅇํำ

$\because \because \div 0$ ?

$m$
0
0
ำ: 용

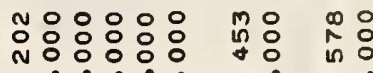

$\therefore$

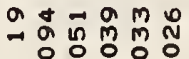

‥?

\section{- a}

$\stackrel{N}{N}$

in

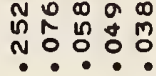

a $m$

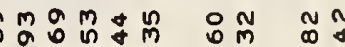

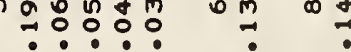

の $\infty \stackrel{m}{m}$

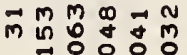

응 ถ
No 2 a

$\underset{\infty}{\infty} \stackrel{\infty}{\sim} \underset{\sim}{\infty}$ -. ?

n0.0ㅇ

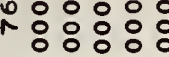
ㄴ?

manmo mㅇํㅇํㅇ

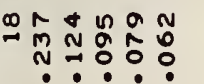

$\infty \hat{n}$ 电

m

nก:

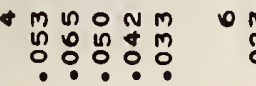

$\begin{array}{ll}0 & 0 \\ 0 & 0 \\ 0 & 0\end{array}$

å :

웅ㅇㅇㅇㅇ

न: : : : : :

용

:

ํㅡㅇㅇㅇㅇ웅

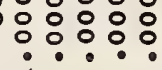

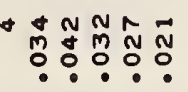

or

n $\stackrel{\text { ก }}{\stackrel{\text { N }}{0}}$

M

No $\infty$

$\stackrel{\infty}{\rightarrow}$

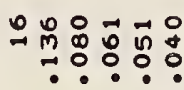

กั่

nN N D O M

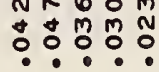

$\stackrel{9}{0} \rightarrow$

ำ $\stackrel{N}{\sim}$

?

nก

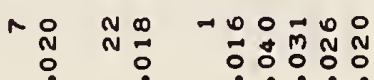

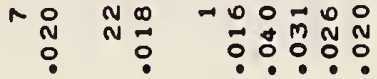

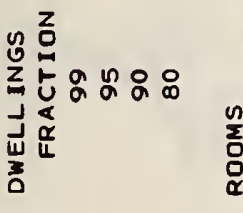

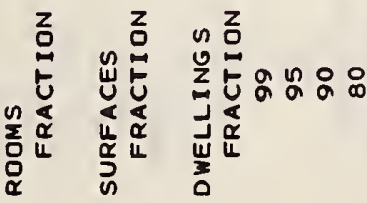

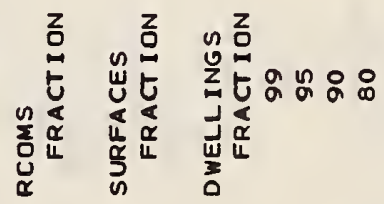

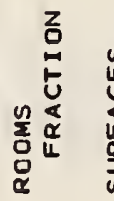

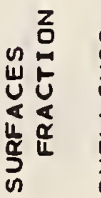

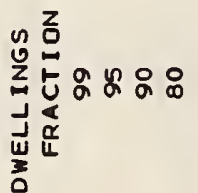

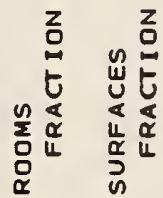
on
$n$
1
$\vdots$
0 


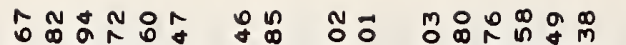

*

0

*

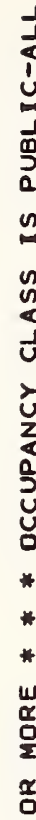

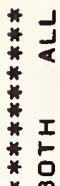

$\cong *$ 品

$>\quad 3$

2

5

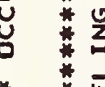

* * *

?

$n$

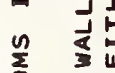

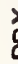

跣

,

言站

M̊̊

$\because \cdots:$.

-

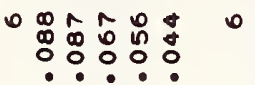

$\stackrel{m}{m} a$

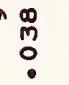

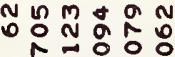

ㄴ?

○

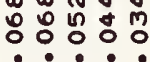

ำm

$\prod_{n}^{\pi}$ ñ

?

ำ

$\bullet \div$

N

$\because \because 0 \%$ 。

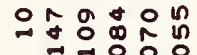

$\because \div$ ? ㅇํㅇ

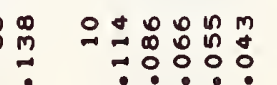

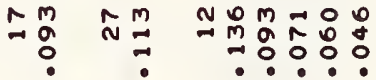

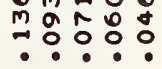

* J N N

*

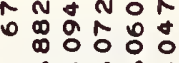

*

*

*

manmon n

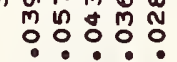

*

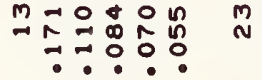

$m \rightarrow 0+0$ 요 $N M$

*

*

$\neg \cong \cong ั 0$

*

- M nONm กิ

No 0

N

m $\cos (n)$

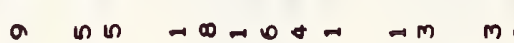

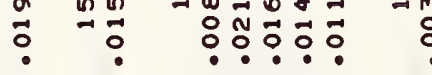

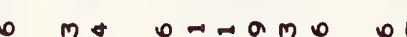

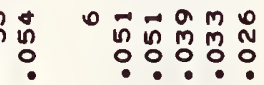

莒

ก $N$ กㅇํำ

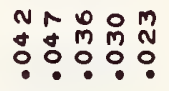

$0 \mathrm{~m} N$

$\stackrel{0}{2}$

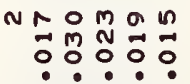

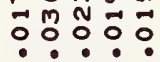

*

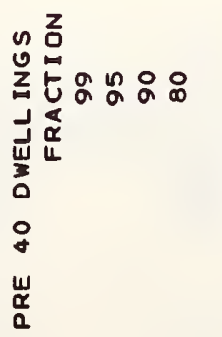

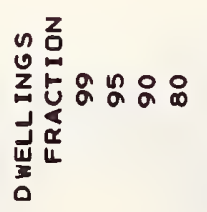

กิ

$\underset{7}{N}$

$\underset{\sim}{\stackrel{0}{0}}$

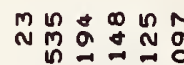

$\sim$

$\infty$

- $\begin{array}{rlll}m & 0 & n & 0 \\ 0 & 0 & 0 & 0 \\ 0 & 0 & 0 & 0 \\ 0\end{array}$

$\rightarrow$

战

$\stackrel{m}{N} \stackrel{n}{m} \underset{\cdots}{m}$

$M$

$\stackrel{0}{0} \stackrel{N}{0}$

$\stackrel{N}{0}$

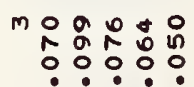

$\cong$

$\stackrel{n}{\circ}$

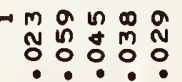

m

$\stackrel{N}{*}$

罚

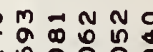

ด.

$m_{n}^{m} m \stackrel{m}{m}$

\&Mm

\begin{tabular}{llll}
\hline & $M$ & $N$ & $N$ \\
0 & $m$ & $N$ & $N$ \\
0 & 0 & 0 & 0
\end{tabular}

$\stackrel{\vec{m}}{0} \stackrel{\substack{m \\ 0}}{\vec{m}}$

$m \cup-m$

ํํㅇํำ

in $\stackrel{0}{\sim}$

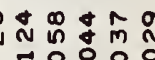

$\because$ : 0 ?

$\stackrel{\infty}{m}$

$m \neq 0 m \infty \infty$

N $m_{m}^{m} \stackrel{m}{n} \stackrel{\infty}{0}$
N

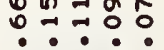
응ㅇㅇㅇㅇㅇㅇ $-0 \circ-100 m$ ㅇํㅇํํ유

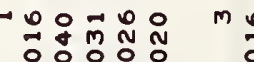
ㅇํㅇㅇํㅇ

00000000 응ㅇㅇㅇㅇㅇㅇㅇㅇ

$\mathfrak{g}_{i=1}^{\infty}=$

- 0 no 00 N

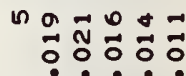
$\vec{D}+N \hat{N}$ N

$\stackrel{0}{\circ} \stackrel{a}{\square}$

$m$
$\substack{\infty \\ 0 \\ 0}$
0 : 
N

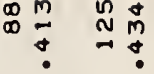

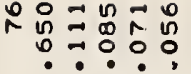

mo

$\stackrel{n}{\sim} \underset{n}{n}$

NONDNM

กิ๊

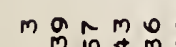

m:

$m \div n \pi$

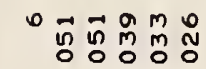

$0 \mathrm{~N}$

$0=a+0 a$

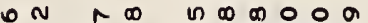

8

$ㅇ$

: $: \div \div:$

म.

. $m$.

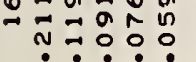

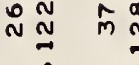

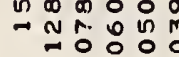

N

$\cong$

$\div: \div:$

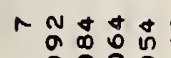

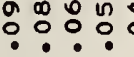

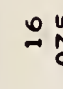

N

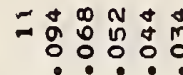

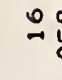

$m$

$$
\text { 옴 }
$$

N

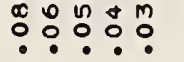

すั

0

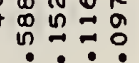

n?

ํํำ

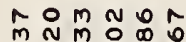

ज)

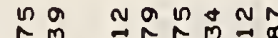

กำ

$-2$

- n

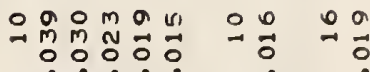

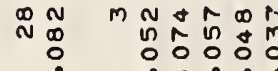

0

ㅁำ

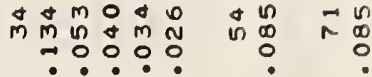

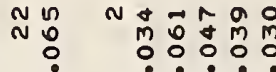

in

ก

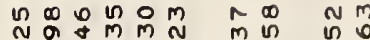

N

NHARO

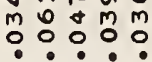

N

No

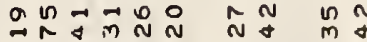

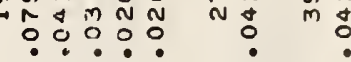

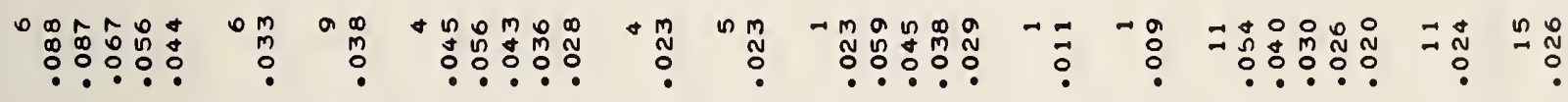

$m=\pi m \infty D N N D$ NOMON

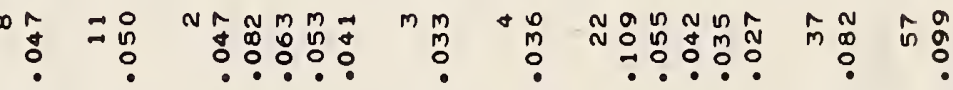

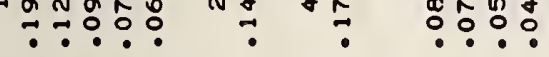

$=n \pm n m$

$\because \div: ?$

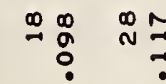

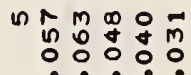

$\circ \mathrm{m}$

a

NNNMM

ํํํㅇํㅇ

$m m$

$+\infty$

$\infty a 0$ a

숭 $\overrightarrow{0}$

川

$\begin{array}{rlll}0 & 0 & 0 \\ 0 & 0 & 0 \\ 0 & 0 & 0 \\ 0 & 0 & 0 \\ 0 & 0 & 0\end{array}$

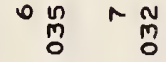

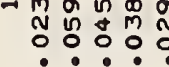

$-$

$\overline{0}-$

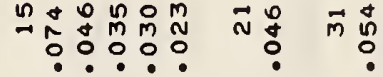

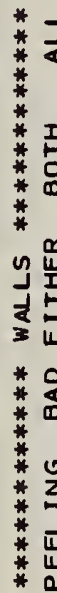

$m 0 \% 0 m a$

+ 吕

i

nก $\rightarrow$ ก

manmon no

no 9

กี

$a n=\pi n m \infty$

$\stackrel{\substack{m \\ \hdashline}}{\infty}$

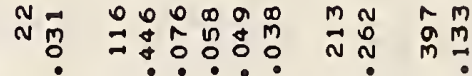

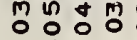

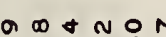
$\begin{array}{lll}0 & 0 \\ 0 & 0 & 0 \\ 0 & 0 & 0\end{array}$

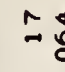

:

-

$m$

$\because \div: 0$ :

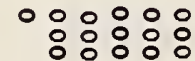

$\circ 8$

$\circ:$

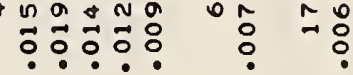

\section{……}

$0 \infty+N O$

$\frac{0}{0}$

$\stackrel{0}{0}$

m

$m \underset{N}{n} M_{0}^{M} \stackrel{\infty}{N} \stackrel{m}{N} \stackrel{\infty}{0}$

mo

0 ย

$\circ: \therefore::: 0$

$\circ \circ 0$

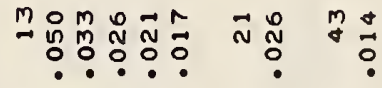

$+m^{n} n$

*

$0 m$
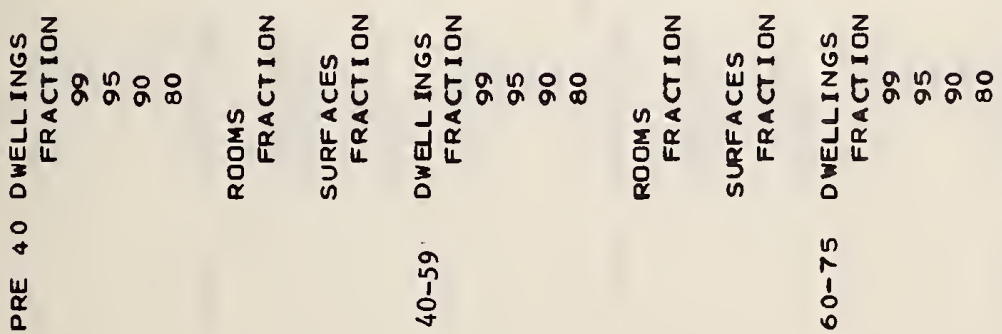

告

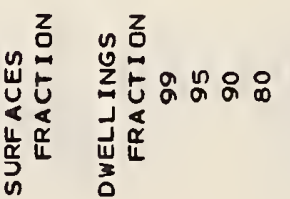

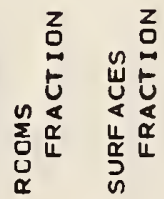

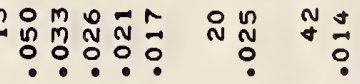

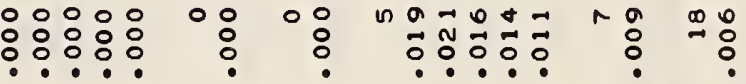

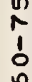




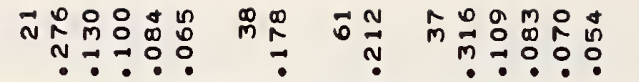

$m g \hat{m} 0^{\infty} m+n$

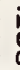

*

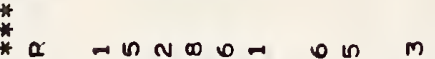

正至

$\begin{array}{rllll}0 & 0 & 0 & 0 \\ 0 & 0 & 0 \\ 0 & 0 & 0\end{array}$

政

*

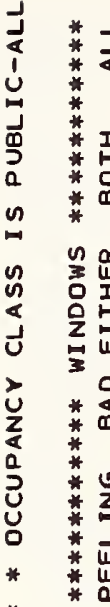

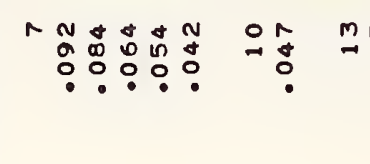

$\rightarrow m^{2}$

*

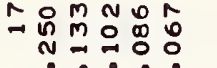

$\stackrel{\infty}{m}$ in

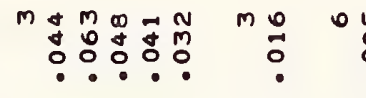

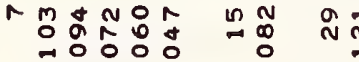

…

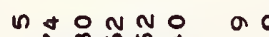

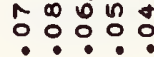

a

$\infty \quad \infty$

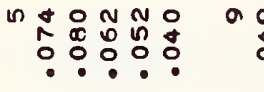

g

$\because \mathfrak{a}$

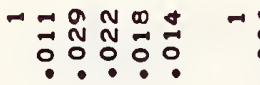

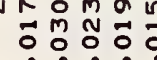

aก N

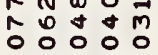

$\pi \rightarrow d m$

in

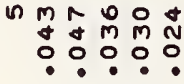

$\begin{array}{ll}0 & \infty \\ \sigma & 0\end{array}$

in NO $\because \rightarrow 000$

$\rightarrow$ a

옹

$\begin{array}{llll}\text { n. } & 0 \\ 0 & 0 & 0 & 0 \\ 0 & 0 & m & 0 \\ 0 & 0\end{array}$

a $\stackrel{n}{m} \stackrel{0}{0}$

Nô

$\frac{1}{0}$

$\sim m \infty N$

ㅇํำ

$\rightarrow$

$\rightarrow$ in

ก

$\because$ 응

$\because: \circ \stackrel{0}{\circ} \div$

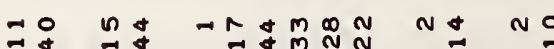

AMMMN

o 000000

$\stackrel{a}{N}$

织n $m$

$\begin{array}{llll}m & 0 & 0 & 0 \\ 0 & 0 & 0 & 0\end{array}$

$\stackrel{a}{9}$

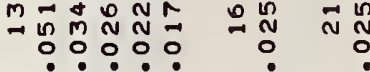

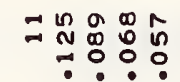

- $\rightarrow a n m$

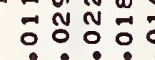

N $\begin{aligned} & m \\ & N\end{aligned}$

- : ?

N $\mathfrak{m}_{\mathrm{N}} \circ \vec{m} \stackrel{0}{0}$

Nom

$m$

$\stackrel{\infty}{0} \quad 0$

N

-

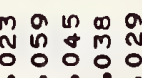

N 응ㅇㅇㅇㅇㅇㅇㅇ $\infty$ o

年: 。

o o o 0

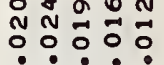

oma a

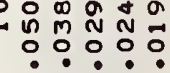

ㅇํㅇ

$00+0 N$

\%

$m$
0

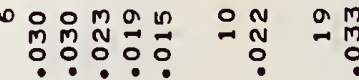

*

$0=0=0$

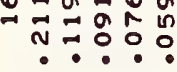

$\ddot{m} \stackrel{\infty}{\square}$

舟 $\underset{\infty}{N}$

오ำ

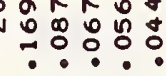

$\circ \stackrel{n}{\circ}$

?

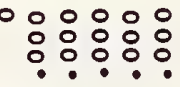

$\circ:$

*

*

N

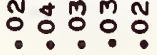

n)

0
0
0

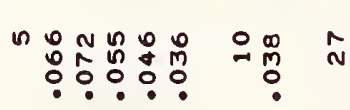

*

兴

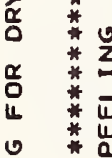

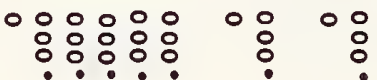

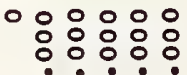

$\circ 8$

$\rightarrow \infty \vec{N} \stackrel{0}{*}+$

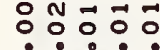

$\rightarrow$

$+$

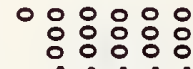

$-\infty_{0}^{\circ} \vec{n} \frac{0}{0}$

: ํ: 유:

$\rightarrow$

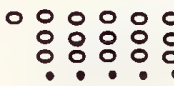

ㅇㅇㅇㅇㅇㅇ $\rightarrow$

:

응ㅇㅇㅇㅇㅇㅇ

$\begin{array}{ll}0 & 0 \\ 0 & :\end{array}$

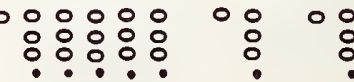

NOAOOM

Oำ 0 :

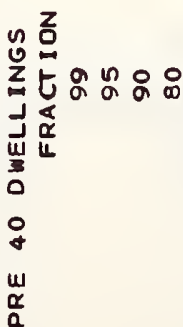

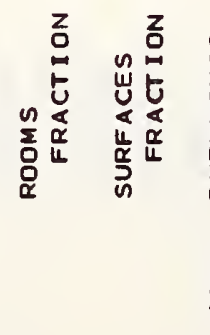

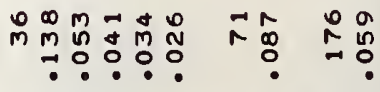

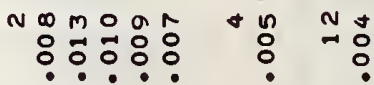

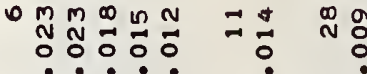

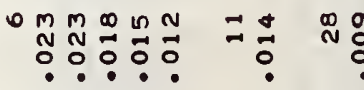

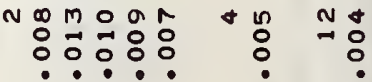

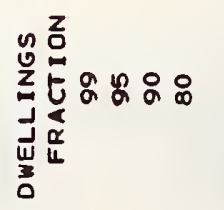

on

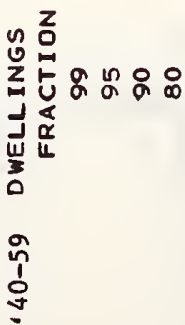

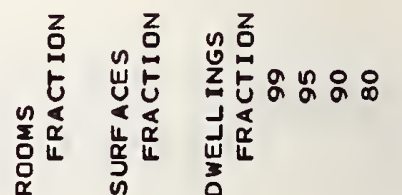

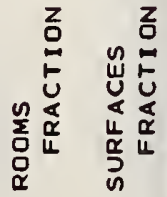




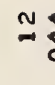

No

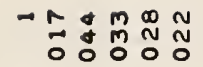

$\rightarrow$

- n

ㅇํㅇำ $N$

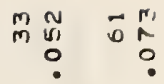

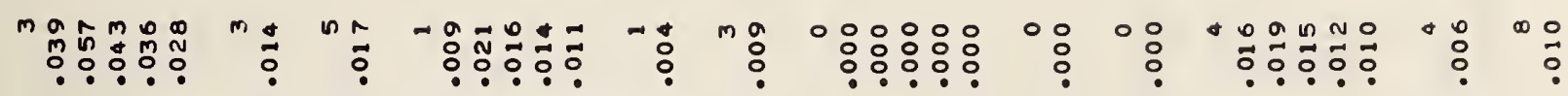

\# i $\quad$ in

NAOma in

느유.

$m \overrightarrow{0} \quad r$

웅ㅇㅇㅇㅇㅇㅇㅇㅇ

$\circ$

$\circ$

oa a o o

$\infty$

$m$ n

N

ำ ㅇํㅇํำ

$m=\wedge$

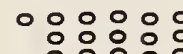

ㅇํㅇㅇํㅇ

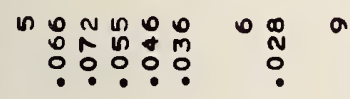

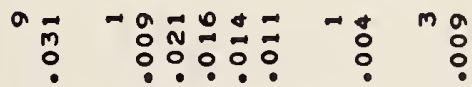

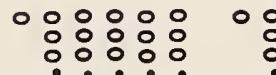

$\circ: \circ \%$

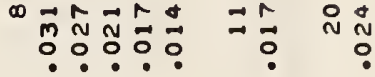

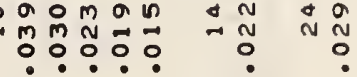

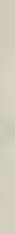

*

ONa。On a m $\because \div 0$ 。ำ

$\stackrel{0}{m} \quad m$

^ำ

$\stackrel{N}{N} \stackrel{m}{N}$

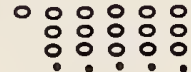

$\circ \stackrel{\circ}{\circ}$

0

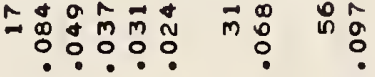

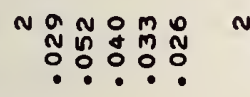

$N=m m$

$\rightarrow=0$ N

$\rightarrow$

No

000000

$\circ \%$

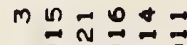

$\overrightarrow{0}: \overrightarrow{0} \overrightarrow{0} \overrightarrow{0}$

mค $\frac{9}{\circ}$ :

คMNํำ

$\simeq \stackrel{N}{N}$

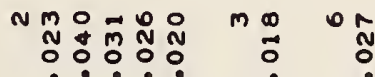

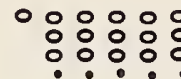

$0:$

$\circ 8$

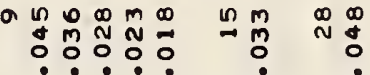

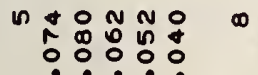

$\infty \pm$

N

$m \stackrel{\infty}{0}$

N

융ㅇㅇ:

$\circ$

$\circ$

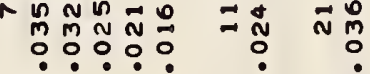

+

0

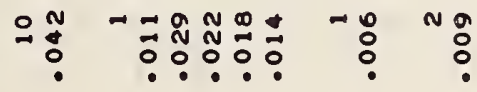

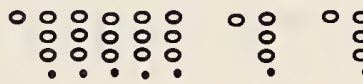

ก

กับ 0 ก

$\sim \stackrel{n}{\sim} \stackrel{\vec{N}}{0}$

.

$\operatorname{ann\infty } n$

$\pi+0 \%$

$\stackrel{a}{a} \dot{m}$

r 2 n

웅 웅

용ㅇㅇㅇㅇ

:

$\circ:$

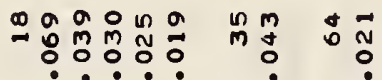

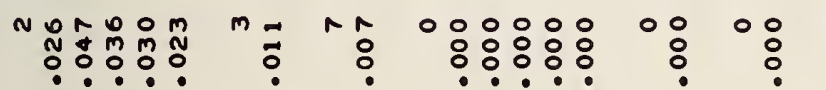

용:ㅇㅇㅇ

m

$0 m$

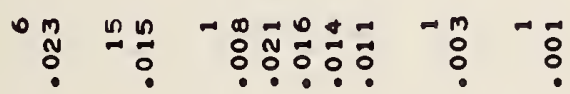

양ㅇㅇㅇㅇㅇㅇ

$\circ:$

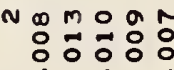

$m \& \sim N$

:

$m+\frac{1}{8}$

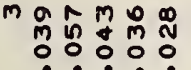

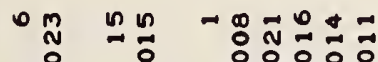

응ㅇㅇㅇㅇㅇ

o:

$\begin{array}{lll}0 & 0 \\ 0 & 0 & 0 \\ 0 & 0 & 0\end{array}$

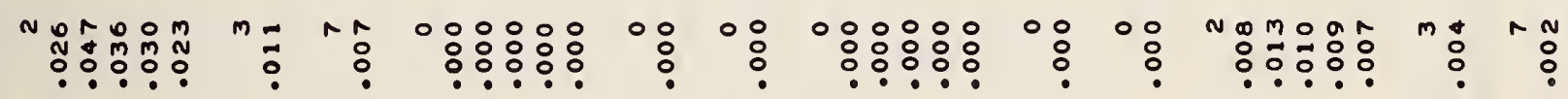

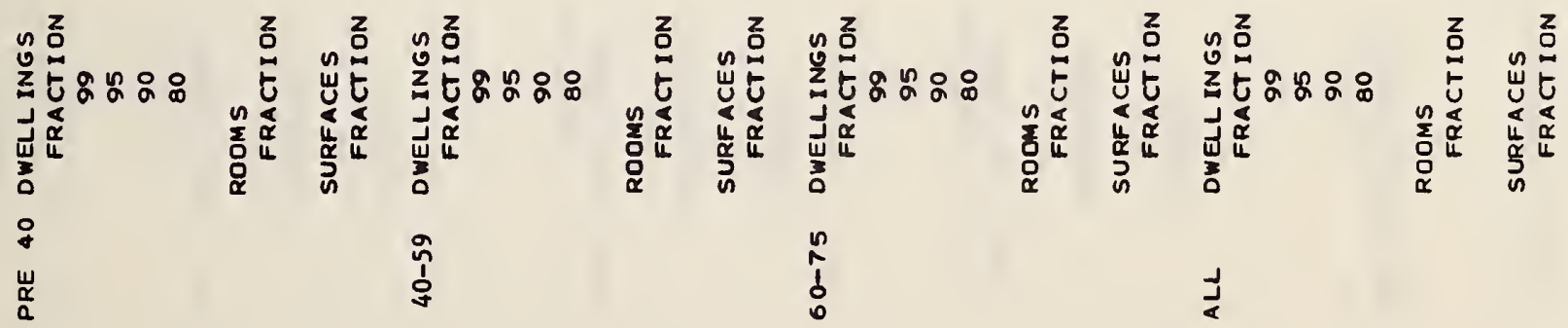


$\begin{array}{lllll}n & 0 & 0 & d \\ 0 & 0 & 0 & 0 & 0\end{array}$

$\wedge \stackrel{0}{N}$

N m

N

?ㅇ?

?

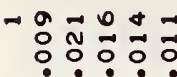

$\rightarrow$

m

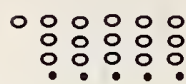

8

: 0 :

(n)

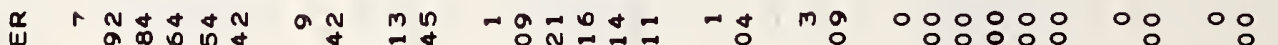

兄

*

*

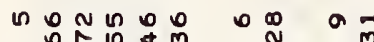

:

? : ? :

:

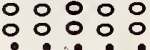

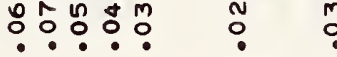

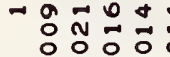

?

?

-.・.

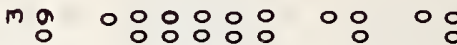

응요

○

:

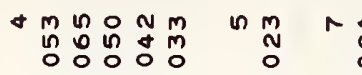

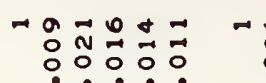

mo

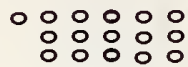

$\begin{array}{rr}0 & 0 \\ : & :\end{array}$

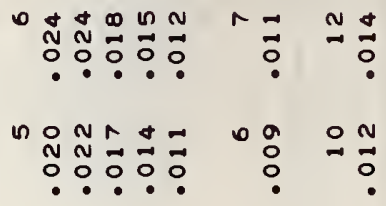

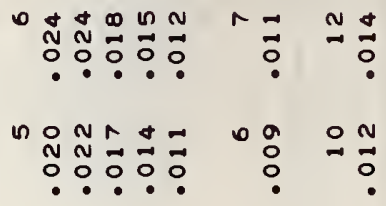

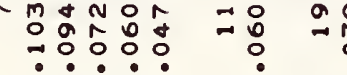

个ำ

$\circ$

$\stackrel{\infty}{\infty}$

:

$\circ \circ: 0$
$\circ:$

$\circ \circ$

$\circ \circ$

$+a \log 2$

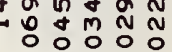

ลี

N

$\rightarrow=a$ N

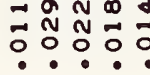

$-8$

n

$\circ \circ 0$ 응유

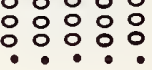

$\circ 8$

$\circ$ 웅

$m n \rightarrow 0+$

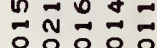

ㅇํㅇำ

mヘ

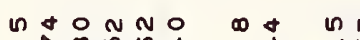

N $M$ ㅇํำ

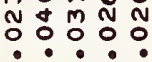

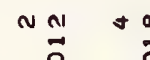

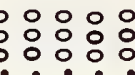

$\circ$ :

ก ก $N$ แn

m $\begin{array}{llll} & N & N & 0 \\ 0 & 0 & 0 & 0\end{array}$

$N$
N
0
0

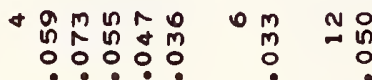

N $m \circ \div 0$

N

$N$

$+$

000000

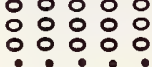

$\circ 8$

$0 \%$

o음ำ

m.

\begin{tabular}{c}
$\infty$ \\
$\stackrel{0}{0}$ \\
\hdashline
\end{tabular}

0
$m+m$
0
0

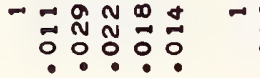

$\sim$

$\circ \circ$
$\circ$

$\circ \%$

$\circ:$

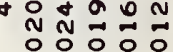

n)

* * *

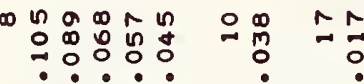

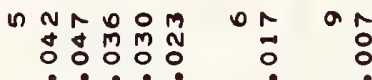

$\circ$
0

$\circ:$

$\circ$

:응

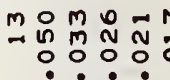

$\stackrel{0}{0} \stackrel{N}{\circ}:$

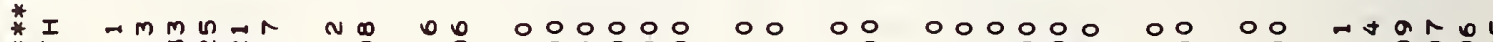

*

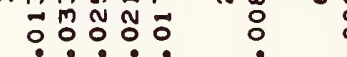

융ㅇㅇㅇㅇㅛ

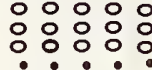

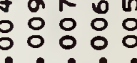

N N $D$ N

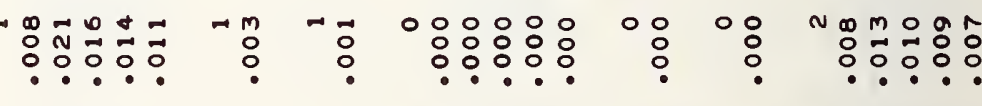

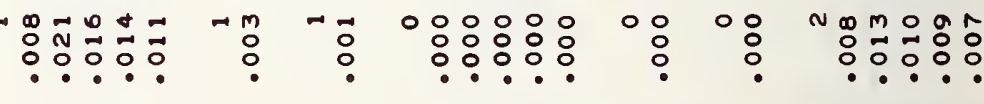

N⿴囗十心

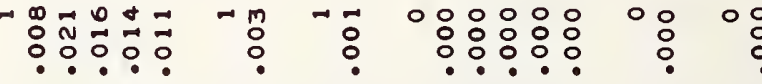

융

?

*

\section{nmmar ma}

$\circ$
$\circ$

0000

:

$\therefore: \circ: \circ:$

00

웅

-

$\begin{array}{rrr}N & 0 \\ 0 & 0 \\ 0\end{array}$

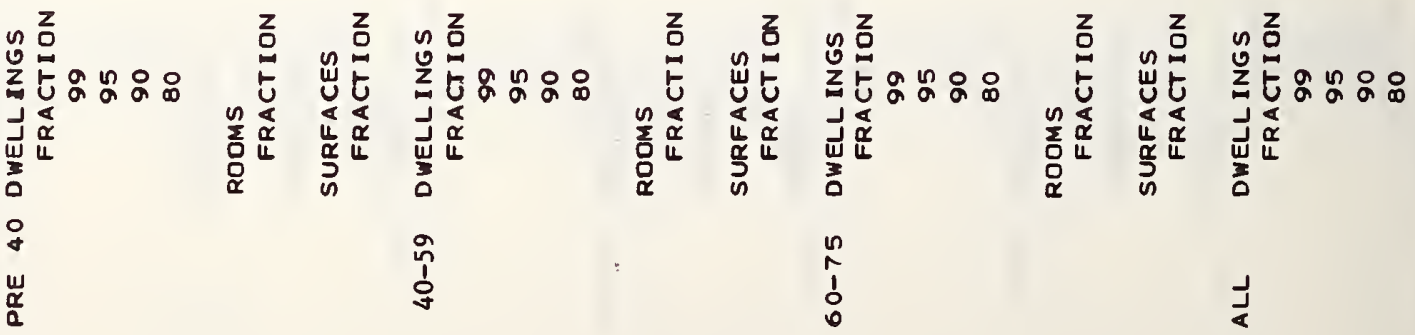


I $m=\infty$ in $N \stackrel{a}{N} m \stackrel{0}{N} m$

n marba $N$

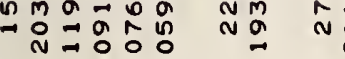

N $N$ m

$\begin{array}{lllll}0 & 0 & 0 & 0 & 0 \\ 0 & 0 & 0\end{array}$

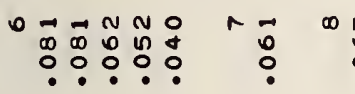

$\infty \underset{0}{m}$

x a a n wo

o n

$=$

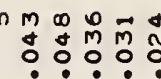

$\forall \rightarrow 0$ co 0

$\because$ ? 0 ?

\section{in a $m$ o}

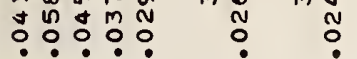

$(10)$

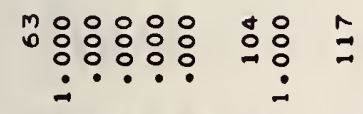

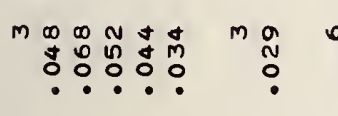

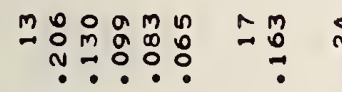

ก้

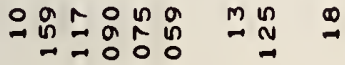

$\stackrel{\infty}{\rightarrow}$

ํํำํํำ

arma

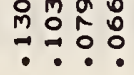

$\cos a$

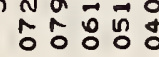

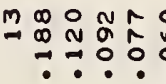

西 $\rightarrow \div$

ㅇํㅇ

둥ㅇㅇㅇㅇ 응응은

om

$0 \%$

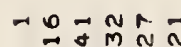

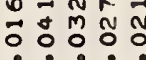

ㅇํㅁ तี

maot nn

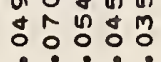

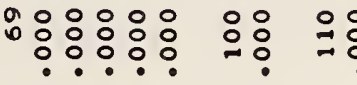

:

m응용융ㅇㅇ

m.

00 0 n -0000

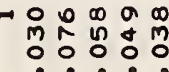

$\cong \stackrel{9}{-}=$

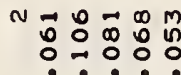

$=0$

$\stackrel{m}{\cdots}$

$N$
0
0
0

$\cong \stackrel{N}{\because} \cong$

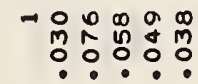

용

:

9
8
0

(1)

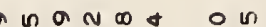

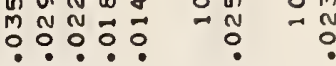

NUNOMONO $M$

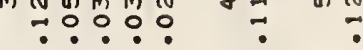

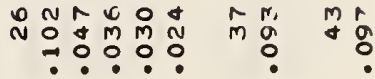

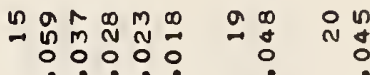

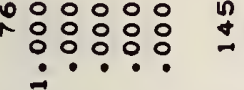 \\ 10 \\ in

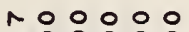 \\ 웅 \\ no 00000 \\ NAoman

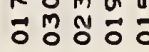

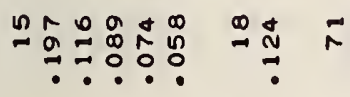

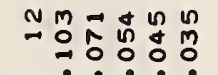 \\ $₫$

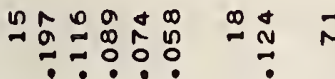 \\ An On

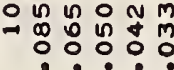

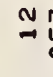 \\ - MnONm n

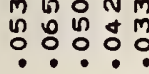 \\ n $\begin{gathered}\stackrel{m}{m} \\ 0\end{gathered}$ \\ ㅇํำ

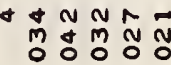 \\ $\because \frac{a}{0}$ \\ 응ㅇㅇㅇㅇㅇㅇㅇ}

aㅇ i

in:

$\infty \circ \circ \circ \circ$

iํㅇㅇㅇㅇ

ำ:ㄴ.

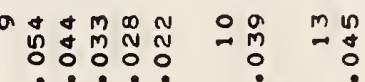

西

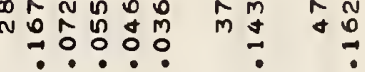

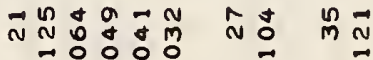

?ํำ ? ?

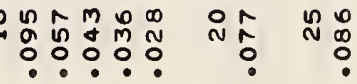
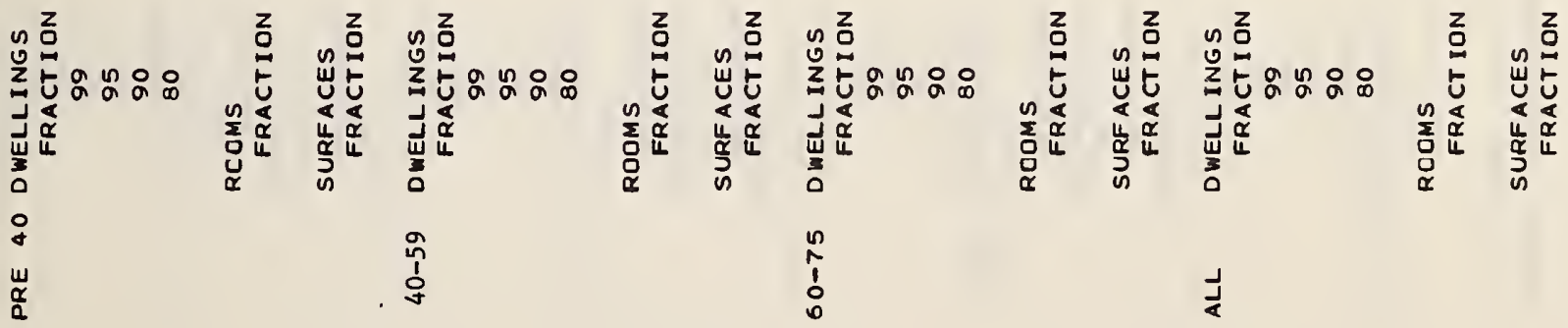

*

哇

$\circ$ * * *

J

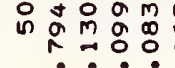

N m.?

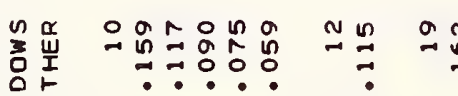

$\infty N N O$ $\because \because 00$

$+m_{0}^{\infty} 0$ ○ั: $a r$

$n$
$\infty$
0
0

$\begin{array}{lll}9 & m & 0 \\ 0 & 0 & 0 \\ 0 & 0 & 0\end{array}$

a

nn $\begin{array}{lll}\infty & 0 & n \\ 0 & 0 & \infty \\ 0 & 0 & 0\end{array}$

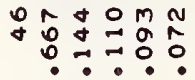

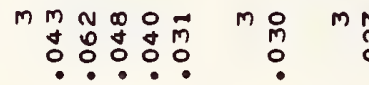

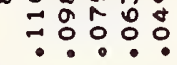

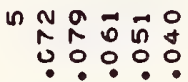
$\begin{array}{rrrr}0 & 0 & 0 & n \\ 0 & 0 & 0 \\ 0 & 0 & 0 & 0 \\ 0 & 0 & 0\end{array}$

둥

in ro in $m \stackrel{N}{N}$

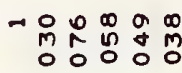

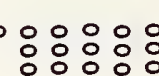

ma

\section{$=-9$}

$\wedge \infty$ in a $0 \mathrm{~m}$ N

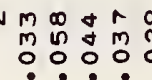

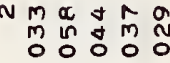
$\begin{array}{llll}0 & 0 \\ 0 & 0 & 0 & 0 \\ 0 & 0 & 0\end{array}$ $m$
$m$
$m$

$m$

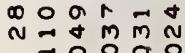
$\because 0 \%$ ?

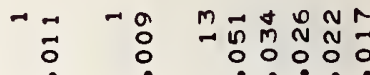

ma

$+\begin{array}{r}0 \\ 0 \\ 0\end{array}$ N $\stackrel{0}{n} \sim m$

\section{*} *

* I marmo * * บ

吉志

* * * * *
- $\infty+40$ ํํํㅇำ $\begin{array}{llll}0 & m & 0 \\ m & n & 0 \\ 0 & m & n \\ 0 & 0 & 0\end{array}$

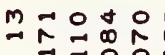
$\because \because 00$ :

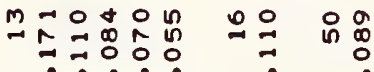
ma

\section{क व}

: $\rightarrow$

00

$\because \quad$ 용

$\stackrel{0}{0}$

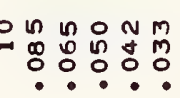

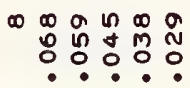
$\stackrel{N}{0}$

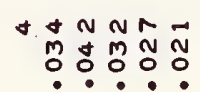

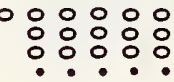

o 00000

$\begin{array}{llll}0 & 0 \\ m & 0 & 0 & 0 \\ 0 & 0 & 0 \\ 0 & 0 & 0\end{array}$

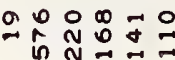

งั $\circ$

:웅

w $m$

N

$\circ$

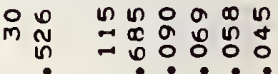

nom $m=0$

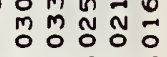

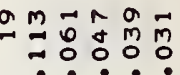

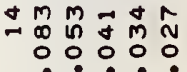
0

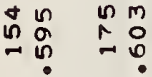

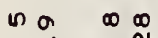
$\stackrel{\infty}{N}$ $N_{n}^{0}$ $=$ N $\stackrel{M}{N}$ 0
$N 0$
$N 0$

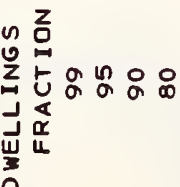

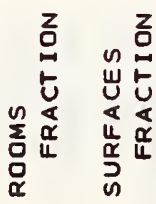

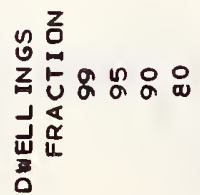

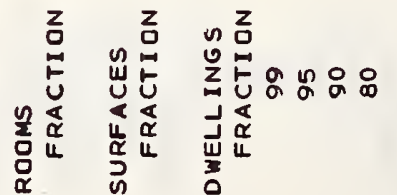

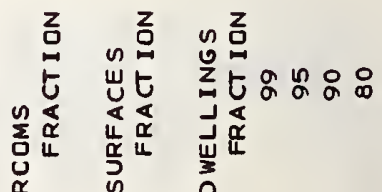
察
in
in

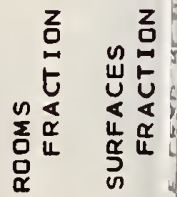




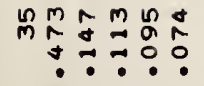

8

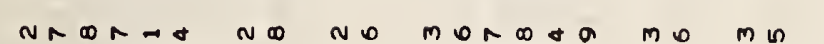

Ñ

N

o

N

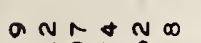

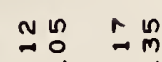

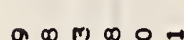

$\rightarrow 0 \begin{array}{llll}0 & 0 & 0 & 0\end{array}$

$\begin{array}{llllll}\infty & m & 0 & 0 & 1 \\ 0 & 0 & 0 & 0 & m & 0 \\ 0 & 0 & 0 & 0\end{array}$

:

$\overrightarrow{0}$

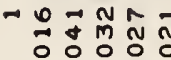

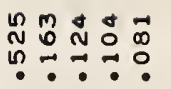

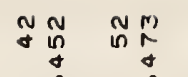

$0 \times \infty$ o 0 o

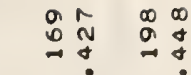

唱出

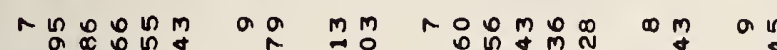

a

+

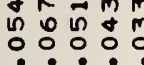
in

n̊ ㅇํㅇㅇํㅇ

。

o

N $\begin{gathered}m \\ m \\ 0\end{gathered}$

$\sim m \infty+m$

mon

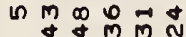

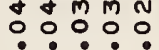

ก ก

ก)

$\rightarrow 0 \rightarrow N \hat{N}$

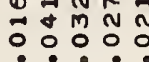

$\bullet$

.

? 0 ?

-

.
ำ?

$\operatorname{Nom} 0$

N

$m !$
$\div \div$

m임

in

om+ba

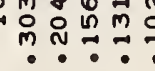

$m$

กำ

Noำ

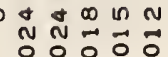

in $\log$

?

เ. ・.

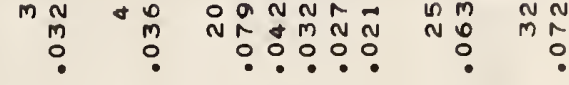

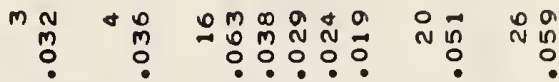

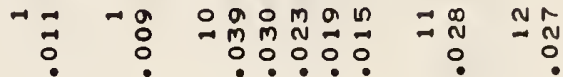

m.

a m N $O N 0$ $\underset{0}{7}=0.0$

$\operatorname{An} \rightarrow$ n $\Rightarrow 0 \hat{0} 00$ $\because \div$ ?

$+m \infty \circ \circ 0$ $\begin{array}{lll}m & \infty & 0 \\ 0 & 0 & 0 \\ 0 & 0 & 0\end{array}$ $=\stackrel{0}{\because} \stackrel{\infty}{\circ}$

$\infty \hat{\imath} \quad m \Xi$

n $\begin{gathered}\infty \\ 0 \\ 0\end{gathered}$

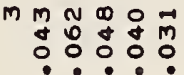

กำำ

+

กิำ
N

N

$+0$

!

mo

MN

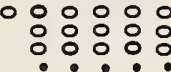

- 우요요

ma no

$\begin{array}{rlll}0 & 0 \\ m & 0 & 9 \\ 0 & 0 \\ 0 & 0 & 0 \\ 0 & 0 & 0\end{array}$

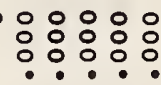

ㅇํㅇ?

N

$\circ 8$

: $\circ$

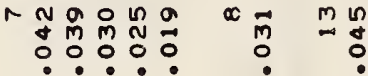

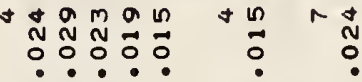

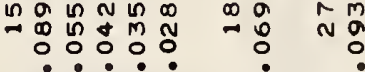

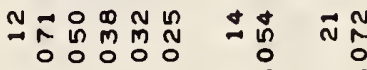

ㅇํㅇ ?
$*$
$*$
$*$
$*$
$*$
$*$
$*$
$*$
$*$
$*$

$20 \%$ $\because \div \div 8$

$m a n m 0$

$a r m$
$m$
$m$
0

$\operatorname{tin} 2000$

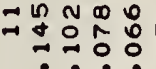
-. ?

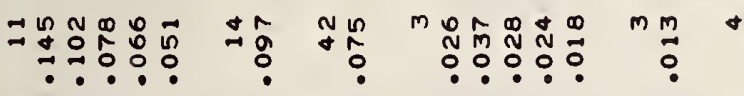

marmon $+\infty=0$ nRoman na nN in o 0
$\infty$
$N$
$N$
: $\underset{N}{m}$

$m m_{\infty}^{\infty} \infty d$

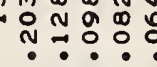

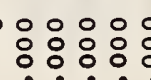

09 응 $\begin{array}{llll}0 & 0 & 0 & 0 \\ m & m & N & N \\ 0 & 0 & 0 & 0\end{array}$

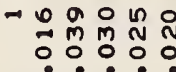

1000

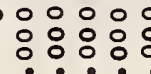

$\stackrel{0}{\circ}$

$\circ$

N

N

$\circ$

웅

: 9

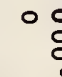

$\rightarrow 0$

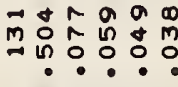

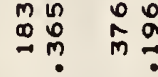

+ $\frac{0}{0} 0000$

$\stackrel{0}{N}$

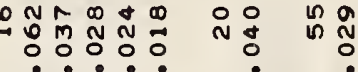

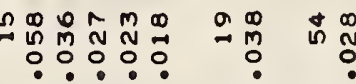

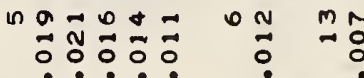

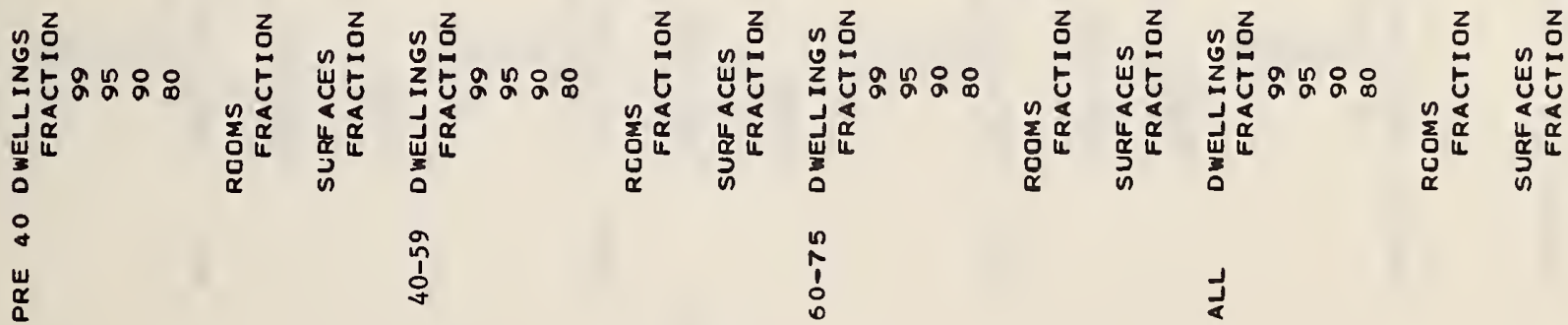


$\stackrel{m}{\stackrel{n}{*}}$

ลั

$0 \infty 0 \infty+\infty$ $+\approx=0$

$\sim a$

0

ถ゚さ

告

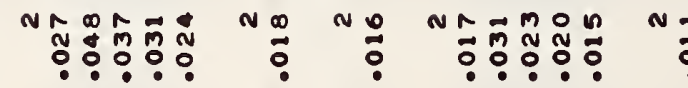

w

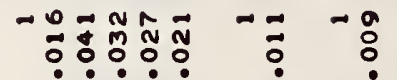

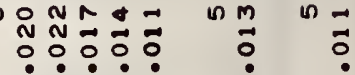

n 00 n

a $n$

- mm

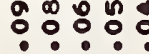

mo

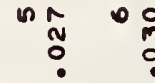

N $\begin{gathered}m \\ m \\ 0\end{gathered}$

N

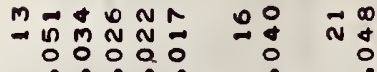

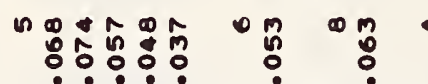

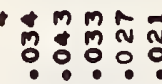

n

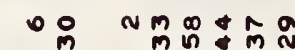

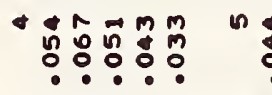

:

Na

:

$0=\underset{M}{N} \hat{N} \stackrel{N}{0}$

N

$\Rightarrow M N \stackrel{0}{2} 0$

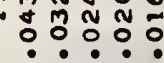

$m$
$\rightarrow \substack{m \\ 0}$
0

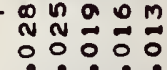

$\infty \underset{N}{0}$

a 은

?..? ?

NNMํำ

$c+00+x$

$\div 0$

\section{n}

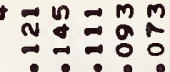

n

$\sim \stackrel{m}{\sim}$

옥

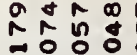

4.

\section{0}

Nomam

No

응ㅇㅇㅇㅇㅇㅇ

ㅇํㅇ응

N

:융ㅇ:유

$\circ$ :

ㅇ:

N

$\sim \overrightarrow{0} \mathrm{~m}$

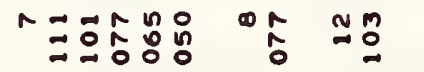

m $m$ No.

mo

$+$

$\begin{array}{llll}0 & 0 & 0 \\ m & 0 & 0 \\ 0 & 0 & 0 \\ 0 & 0\end{array}$

N

$m$

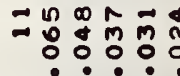

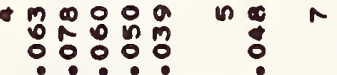

m 0.0

$m \underset{m}{m}$

$\begin{array}{lllll}0 & 0 & 0 & \infty \\ m & 0 & 0 & 0 & 0 \\ 0 & 0 & 0 & 0\end{array}$

N

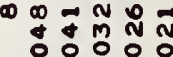

: \%?:

\section{$m \infty n N \$ m a$ n}

:

N

N

$\stackrel{\infty}{\circ}$

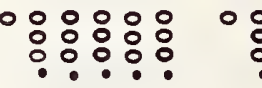

$\circ:$

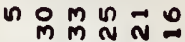

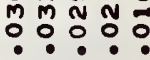

no

\section{.}

?

这

$*$

*

㟧

品

㟧

0000

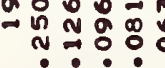

Na

m

No:

mi

0:

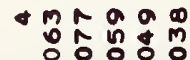

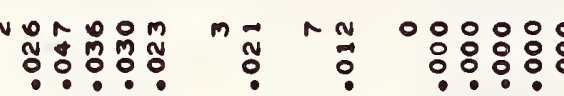

$\circ$

$\circ$

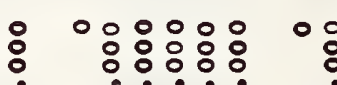

$\ddot{m} \stackrel{m}{\circ}$

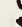

00000

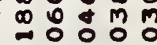

$\begin{array}{lll}n & 0 & \infty \\ 0 & \infty \\ 0 & 0 & 0 \\ 0\end{array}$

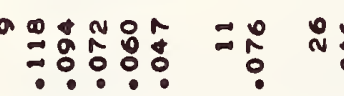

$\Rightarrow \stackrel{a}{\circ} \stackrel{0}{0}:$

$\rightarrow$

N

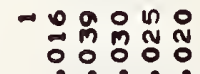

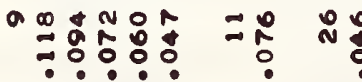

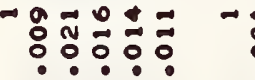

N

- ogono

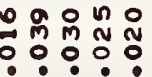

:

N $m \circ 9$

:

mon

Non

ㅇํㅇํำ

응ㅇㅇㅇㅇㅇㅇㅇ

a: 0

:

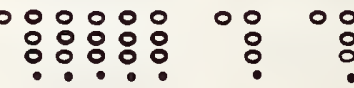

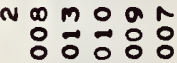

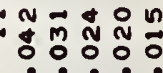

$\stackrel{n}{\circ}$

?

$\Rightarrow N A$

\%

$-0$

$\infty \quad 10$

욜

岃

过

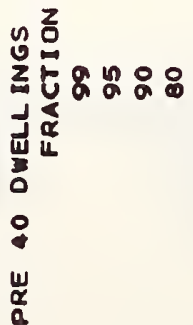

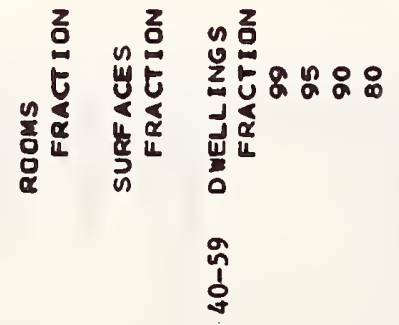

z
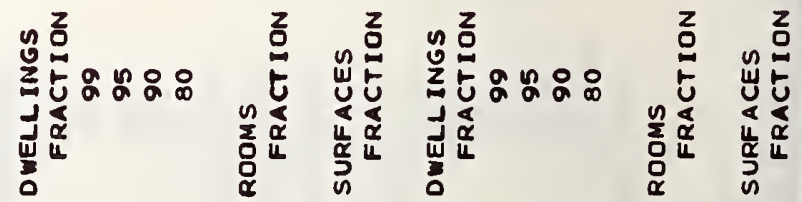


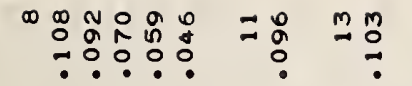

wa o n $\infty$ $\begin{array}{llll}0 & 0 & 0 \\ 0 & 0 & 0 \\ 0 & 0 & m \\ 0 & 0 & 0\end{array}$

$\stackrel{2}{2} 0$

$\stackrel{n}{n}$

ㅇㅇㅇㅇㅇㅇㅇㅇ

$\circ:$

$\circ:$

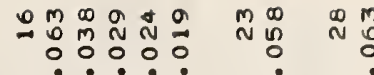

ㅁํํำ:

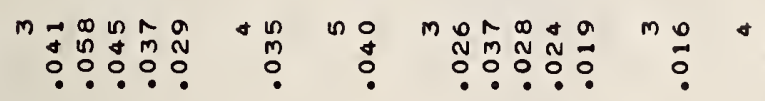

용

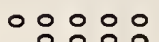

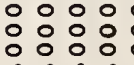

10

$m=\infty \backsim r$

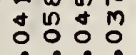

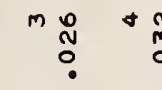

$m \stackrel{0}{0} \stackrel{0}{0}$

$\circ \circ 000$

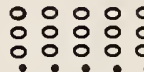

$\circ$

8

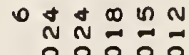

:ㅇ:으.

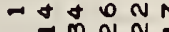

NDNON N N

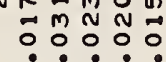

웅

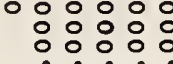

0

0

$m \sim n m-\infty$

$\overrightarrow{0}=\overrightarrow{0}=0$

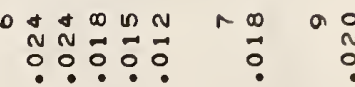
:. :

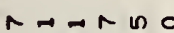
चำด $\vec{\bullet}:$ 응

○ 0000 응ㅇㅇㅇㅇㅇㅇ

$+m \infty 000$ ○ิ

N NOOMO

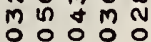
? ? ?

N N $O M \circ \infty$

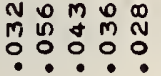

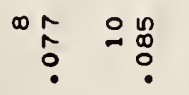

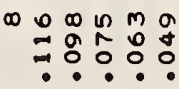

$\infty$
$m$
0

N $\overrightarrow{0} \stackrel{m}{*}$

N $\begin{gathered}a \\ \overrightarrow{0}\end{gathered}$
N

○०००?

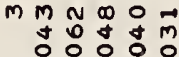

a

no N

o

$+$

-. . .

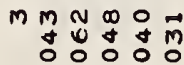

० \% :

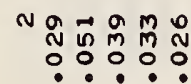

no

잉
N $\begin{array}{rrrrr}0 & 0 & 0 & 0 \\ m & 0 & 0 & 0 \\ 0 & 0 & 0 & m \\ 0 & 0 & 0 & 0\end{array}$

000000

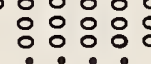

- 00 on $\infty$

m气 。

-00 m

mo? ?

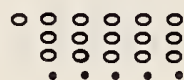

$\begin{array}{rr}0 & N \\ -0 & m \\ 0 & 0\end{array}$

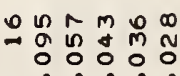

N N $\rightarrow$

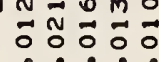

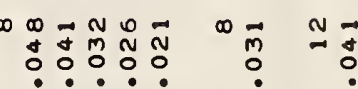

- o o m om a

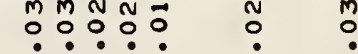

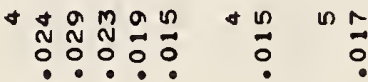

$+\log 20$ $\because \approx 0$ ? ?

$\stackrel{0}{0}$

m $\overrightarrow{0}$

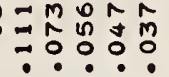

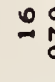

$\begin{array}{lll}a & 0 \\ 0 & 0\end{array}$

N $\bar{m}$ in $N$

N

$+9$

$\stackrel{N}{N} \infty \vec{m}$

N

$\begin{array}{ll}40 \\ m & 0 \\ 0 & 0\end{array}$

nor OOM ํํำ

N

N

$\circ \circ$

00000

N

N $\frac{0}{\circ}$

$\cos \theta+2$

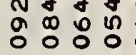

$\sim m$

- $\stackrel{0}{a} \vec{n} \stackrel{0}{0}=-d$

N N

$\therefore \circ: \circ:$

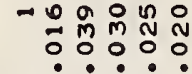

$-\infty$
0
0

N

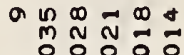

$\stackrel{2}{\circ} \mathrm{O}$

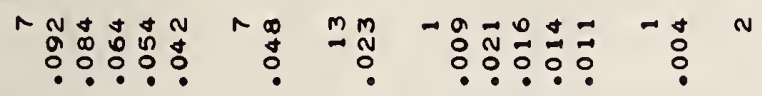

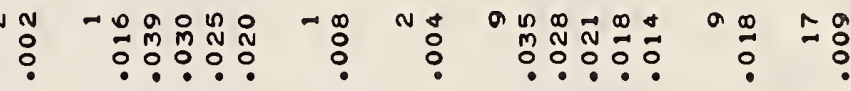

Non OOM

na na 00000000

000000000

$\begin{array}{lll}0 & 0 & 0\end{array}$

$\circ$

$\circ:$

Nm $m 09 \hat{0}$

웅ㅇㅇㅇㅇ

$\begin{array}{lll}N & 0 \\ \circ & 0\end{array}$
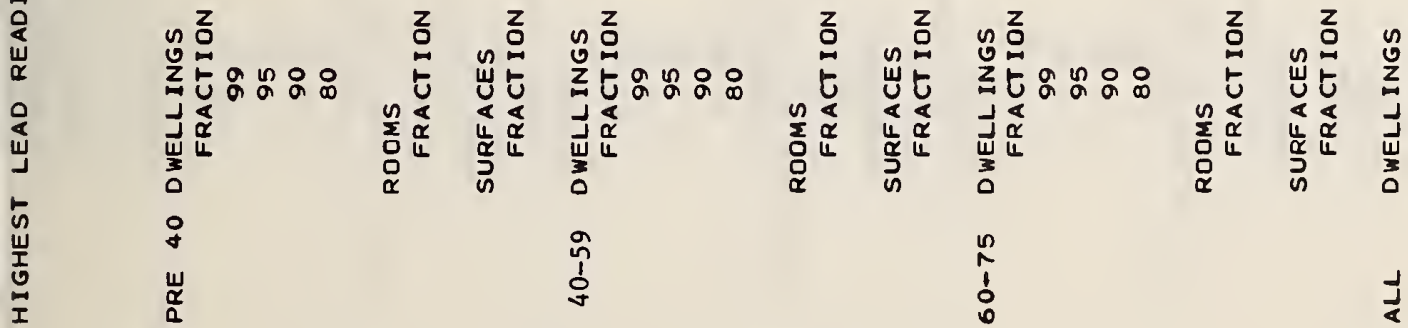
$\because \underset{N}{N} \stackrel{N}{0}=\overrightarrow{0}$

$-\begin{array}{rr}n \\ 0 \\ 0\end{array}$

$\circ: \circ: ㅇ ㅇ ㅇ$

$\circ:$

$\circ:$

hor $\cos$

융요

$\rightarrow$

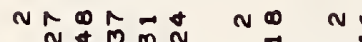

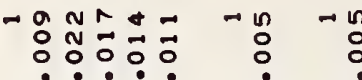

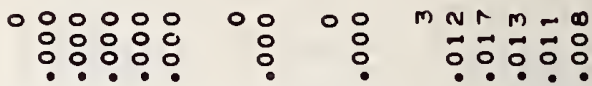

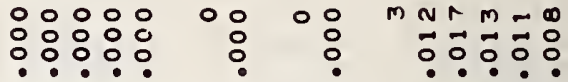

$m$

mi

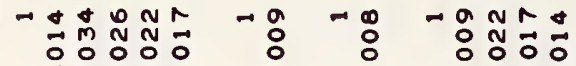

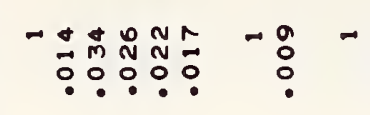

$\stackrel{\infty}{\circ}$

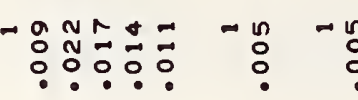

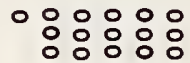

$0 \%$

N⿴囗十ㅇำ

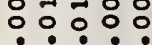

N

N in

웡ำ

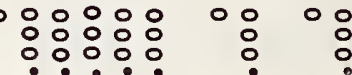

ㅇํㅇㅇㅇ

N 2

N in

?

*

害

范

*

*

*

*

0 in $x$

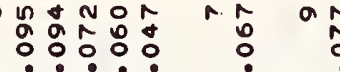

$\stackrel{n}{0}$

동ํำ

․․․․

윰:ㅇㅇㅇ

:

N

…․

+ $\begin{array}{ll}m \\ 0\end{array}$

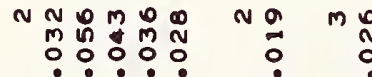

Noे

o $\overrightarrow{\text { in } m}$

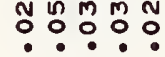

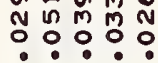

NNOMDONO MO NOTAMO

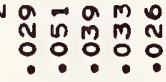

No

\%

$\stackrel{\infty}{\circ}$

응융유

응융용

$\circ:$

$\circ:$

MNNOM

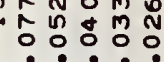

$\stackrel{n}{\sim} \stackrel{\infty}{n} \stackrel{\infty}{\sim} \stackrel{N}{0}$

NO ND $00 \% 0000000 N n-0 M 0$

$\rightarrow$ N

N

N N

응은

N $\underset{N}{N} \stackrel{N}{0}$

응응융융

0 :

$\circ 8$

000 m

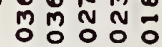

0

+40 a

$\circ 0000$

$+$

+ $a m a n$

ํํㅇํㅇํำ

:ㅇํำ

$\frac{n}{0} \quad n$

\section{* \\ * \\ *

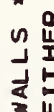

$\circ 0$

ㅇํ

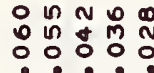

a

N

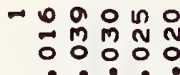

$-\stackrel{\infty}{\circ}$

N

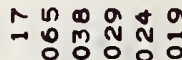

ㅇํㅇ \&

$-m m n n$

m ${ }^{m}$ ก N

$-\stackrel{\circ}{\circ}$

응ㅇㅇㅇ

\section{0}

\section{$\circ \circ$}

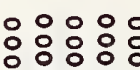

00

00

-

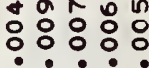

$\rightarrow+\overrightarrow{0}$

- MnONM

.

10

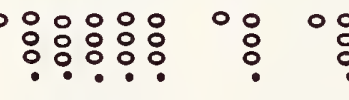

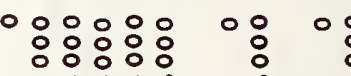

$+\frac{0}{2} 0.0$

뭉유

$\begin{array}{rr}\infty & r \\ 0 & 0 \\ 0 & 0\end{array}$

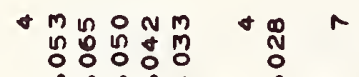

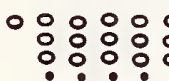

$\circ: \circ$

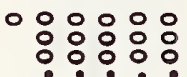

0 :

$\circ:$

$+\frac{0}{0} \div \div 0 \div 0$

$\infty$
0
0

$\rightarrow M \begin{aligned} & M \\ & \vec{M} \\ & 0\end{aligned}$

$\circ: \circ: 00$

응ㅇㅇㅇㅇㅇ

응

응ㅇㅇㅇㅇㅇㅇㅇㅇ

$\circ \circ$

$\circ$

-

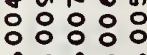

$-$ *

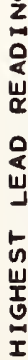
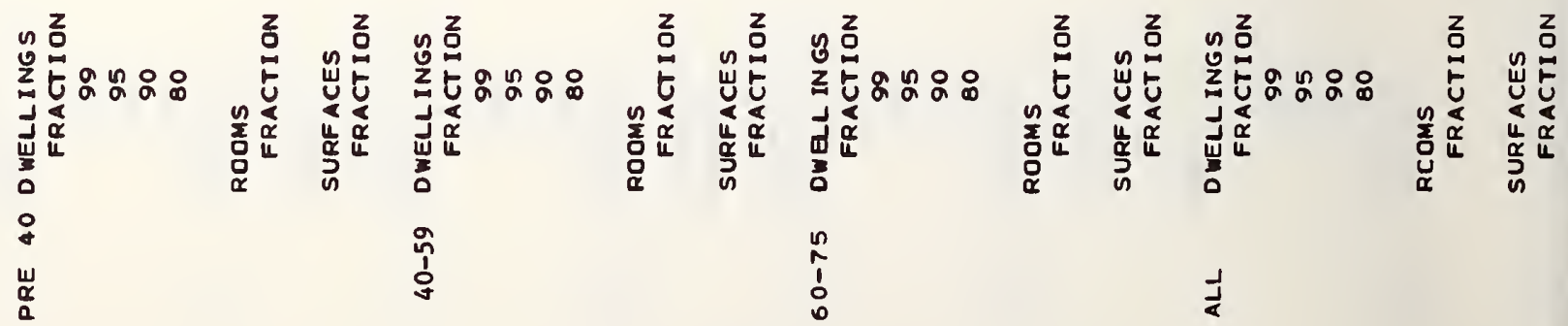
000000

r.:- $: \div: 0$

or

คㅇำ

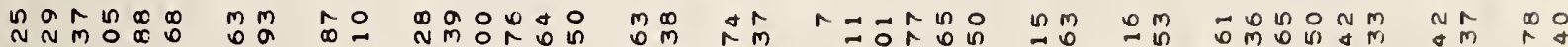

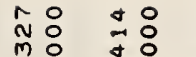

둥ㅇㅇㅇㅇㅛ

की

씀응ㅇㅇㅇ응

in

i:

․ㅇㅇㅇㅇㅇㅇ

40

mo

- : : :

-ㄴ.?요 N

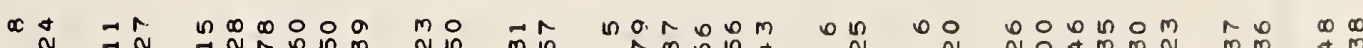

$\begin{array}{cccccc}0 & 0 \\ 0 & 0 & 0 & 0 & 0 & 0\end{array}$

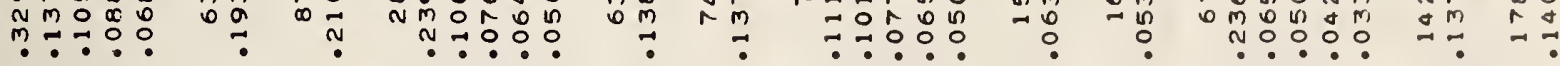

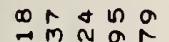

$\stackrel{m}{N} \because 0$

in $\infty$

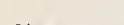

\section{in}

in

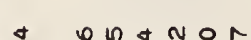

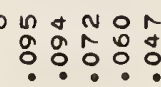

$\pm 0$

? 20

个

$m=0+0$

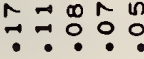

กั

$m$ mo

$\rightarrow 00$ a

- $0 \quad 0$

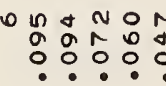

$r$

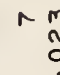

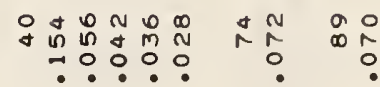

$\circ 0000$ $\begin{array}{llll}0 & 0 & 0 & 0 \\ 0 & 0 & 0 & 0 \\ 0 & 0 & 0\end{array}$

i 0000

ㅇํㅇำร

ก

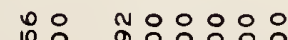

$\rightarrow 0$

$\vec{m}$

$\circ 0 \% 0 \%$

-. -

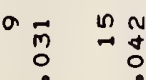

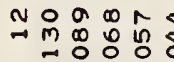

-

$a$

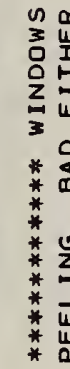

งัmoำ

m.?

$\stackrel{0}{a} 0$

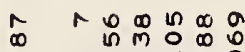

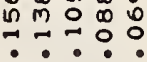

2

$\stackrel{\infty}{\infty}$

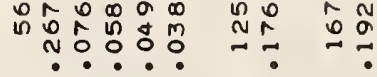

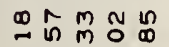

$\stackrel{\rightarrow}{\rightarrow} 0$

$a 00$

a 0 n 0 \% N

$\stackrel{n}{M} \stackrel{N}{\sim}$

$\underset{a}{n}$

omaamin

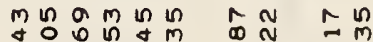
$\begin{array}{lll}m & \sim & 0 \\ \because & 0 & 0\end{array}$

$m \stackrel{\infty}{\rightarrow} \div$ $\because \exists$ ?

$\hat{N}$

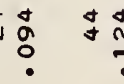

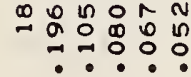

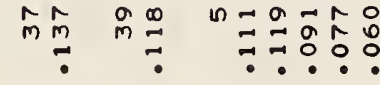

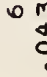

a.

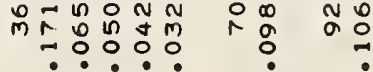

000000

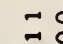

\section{$\circ 0$}

$\infty 00000$

mo ao 0000

$\stackrel{0}{10}$

$\circ \circ$

\section{0} :? : ? -

จ ตี

in :

ㅇํㅇㅇ․

m: $\rightarrow$ ?

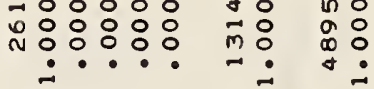

:ํํํำ

$\begin{array}{lll}0 & 0 \\ 0 & a & 0 \\ 0 & 0 & 0\end{array}$

००००。

$\infty \frac{1}{0}$

N

- 0 운

$\rightarrow$

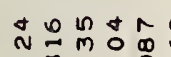

m?

$\vec{n}$

$\stackrel{m}{m} \stackrel{n}{n}$

$\sim 0=a \infty$

is n

- 0

요

$\underset{N}{N} \quad 0 N$

$\underset{0}{\infty} \underset{0}{n}$

$\because \pm$

$r+m a a_{\infty}=n$

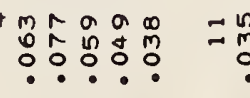

$=\begin{array}{ll}n & m \\ 0 & 9 \\ 0\end{array}$

售

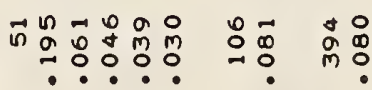

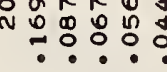

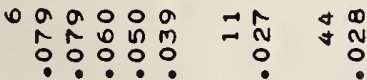

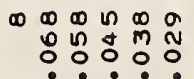

$\rightarrow \frac{9}{0}$

$\rightarrow$ ?

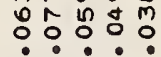

$m_{m}^{\infty} \frac{\infty}{0}$

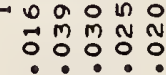

?

$m$

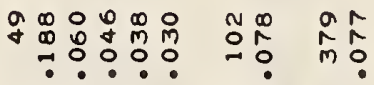

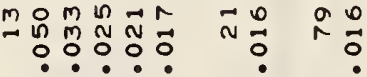

-. ? . -

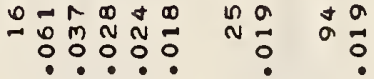

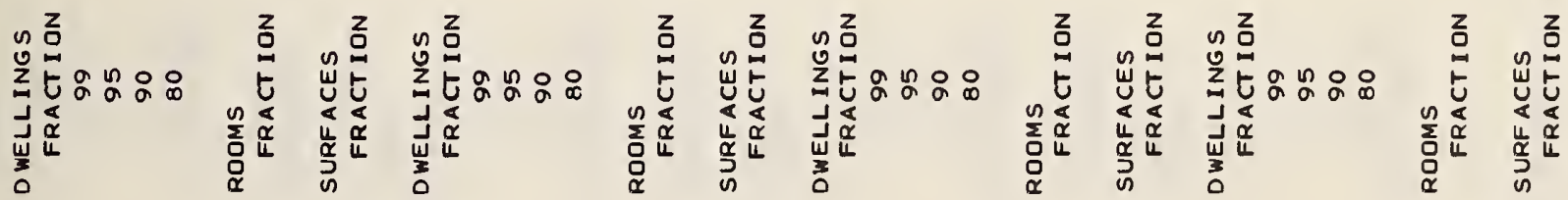

\&

in

$n$
1
0

J 
nNOONM $r \frac{0.000}{2}$

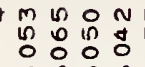

西

$\sim \underset{N}{N} \underset{M}{N}=0$

a

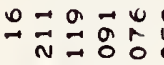

-..?

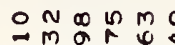

$\because 0: 0$ $m \infty$

$N \stackrel{N}{N}$

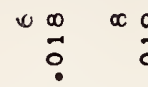

in

min $N$

$\stackrel{7}{a}$

m

品

$\stackrel{m}{\square}$

$\vec{\sim} \stackrel{0}{0}$
ㅇํㅇ

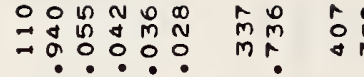

$000 \infty 0.0$ $\begin{array}{rrrr}0 & 0 \\ \cdots & 0 & 0 & 0 \\ 0 & 0 & 0 \\ 0 & 0 & 0 \\ 0 & 0\end{array}$

n m n 0 m.

궁요의

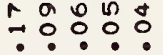

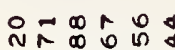
$\because \therefore: \therefore$.

\section{$=\stackrel{n}{0} \stackrel{d}{\circ}$}

or

응

min

mi

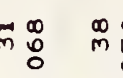

$N$ ก $N M \infty-$

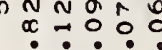
$\begin{array}{ccccc}N & & & \\ M & 0 & 0 & 0 & 0 \\ M & n & m & 0 & 0 \\ 0 & 0 & 0\end{array}$

$m$ $m$
$m$
0
0
0

\section{$\infty \infty N+$}

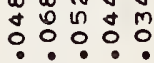

$m \propto \infty N+\sigma$

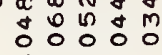

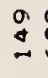

n.

$0 N$
$-\stackrel{n}{m}$
0
0

$r$
$m$
$\vdots$

$a \underset{m}{a} \circ m^{m}$

$m$$$
\text { र }
$$

w

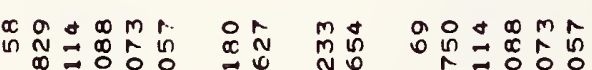
- $\bullet$ ?

○ 0 n $n \mathrm{~m}$

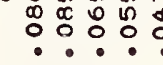
$\infty$

$\stackrel{\infty}{N} \stackrel{\circ}{?}$ m $\because \because 00$ ? $\alpha n r_{1} N \backsim 0$ $\because \because \div 0$

$\prod_{\rightarrow \infty}^{\infty} \propto 0$ $\because \approx 0$ :

*

\# *

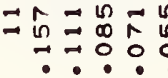

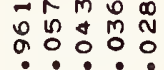

$\stackrel{d}{\infty}$

in $\vec{m}$ $\begin{array}{lll}0 & 0 \\ 0 & 0 \\ 0 & 0 & 0 \\ 0 & 0 & 0 \\ 0 & 0 & 0 \\ 0\end{array}$

$a$

里崖

$*$

*0

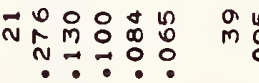

$\rightarrow 000+$

Nล⿻上丨

ㄴ? ? ?

non 0 :ㅇํㅇำ.

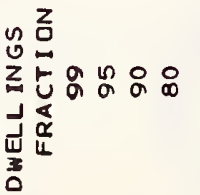

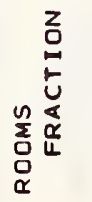

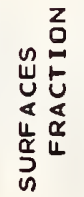
$\begin{array}{llll}0 & 0 & 0 & n \\ 0 & 0 & 0 & 0 \\ 0 & 0 & 0\end{array}$ $n m \infty 0$ $\begin{array}{ll}m & 0 \\ \rightarrow & 0\end{array}$

on $n$ $\because \frac{m}{m} \div$

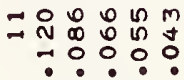

\&

$\underset{\alpha}{\alpha}$ $\stackrel{\infty}{m}$ $ㅇ$

\section{$\begin{array}{llll}0 & 0 \\ 0 & 0 & 0 & 0 \\ 0 & 0 & 0 & 0\end{array}$

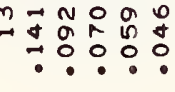

$\stackrel{\infty}{\rightarrow} 0$

$\stackrel{N}{N}$ $\stackrel{m}{0} 0$

a

옹

$0 \rightarrow$ no

\section{ñ}

m

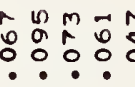

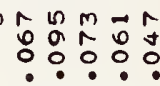

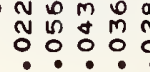

$\begin{array}{lll}0 & 0 \\ 0 & m & m \\ 0 & \square\end{array}$

$\begin{array}{llll}N & 0 & m & 0 \\ \text { N } & 0 & 0 & m \\ 0 & 0 & 0 \\ 0\end{array}$
웅

$$
-
$$

$r$
$\stackrel{0}{\circ}$

$0 m$

$\stackrel{m}{o}$

$\infty \stackrel{\infty}{*}$

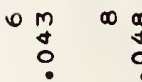

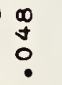

in $⿻$ $\because 0 \% 0 \%$

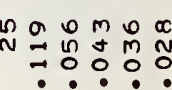

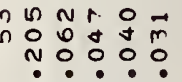

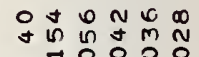

:ㅇ?을

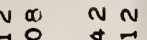
$\rightarrow \div$ Na 00 ? $\rightarrow$ ?

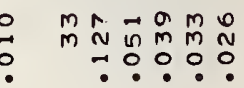

in $\mathrm{m}$ o m 
$m$ man

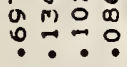

o m n o n m ㅇํㅇํำ.

$\infty \pi+\ln 2$

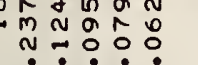

mo

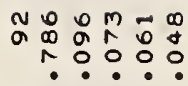

N

$\stackrel{n}{N}$

o

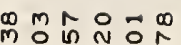

$\because \div \div \div 0$

이

$\infty \begin{array}{lllll}\infty & 0 & n & 0 & 0 \\ 0 & 0 & 0 & m & 0\end{array}$

a 오

$\stackrel{m}{\mathfrak{m}}$

$\begin{array}{lll}N & 0 & 0 \\ m & 0 \\ 0 & 0 & 0 \\ 0\end{array}$

$\stackrel{\infty}{m} \stackrel{0}{n}$

An NMm

N

$M=0 ㅇ$

$\because \because 0$ :

$a n+a$

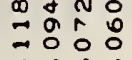

?응

ก

$\begin{array}{lll}0 & 0\end{array} \quad m-m \infty N$ $\begin{array}{llll}0 & 0 & 0 & 0 \\ 0 & 0 & 0\end{array}$

m

m

$m \infty \infty n a+$

。

$\begin{array}{lllll}N & 0 & & 0 \\ m & 0 & 0 & 0 & 0\end{array}$

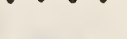

$\begin{array}{lllll}m & & \\ m & 0 & 0 & 0 & 0 \\ 0 & 0 & 0 & 0 & 0 \\ 0 & 0 & 0\end{array}$

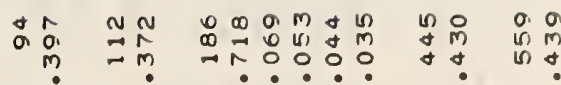

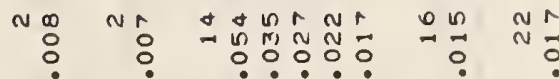

OD OM Dron

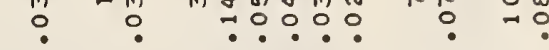

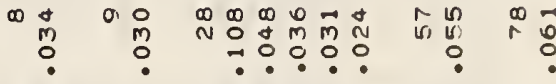

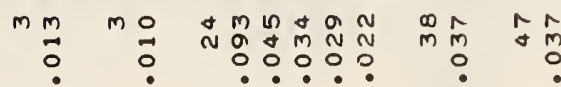

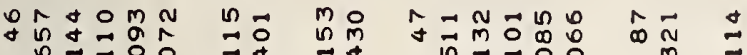

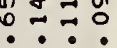

0 in $\ln$ in

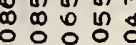

$\rightarrow$ a n

?ㄴ?

no $4 \ln 00$

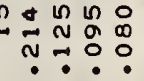

$\hat{m}$

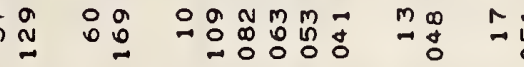

NA+ $\because \because$ ㅇํㅇ

$\stackrel{\circ}{N}$

- $\rightarrow$ n $\begin{array}{llll}0 & 0 \\ 0 & 0 & 0 & 0 \\ 0 & 0 & 0 \\ 0\end{array}$

$\therefore$

$\hat{m} \stackrel{\circ}{\circ} \stackrel{0}{\circ}$

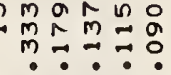

m

m to n $m$

\section{$n o$}

$\stackrel{0}{m} \stackrel{m}{m}$

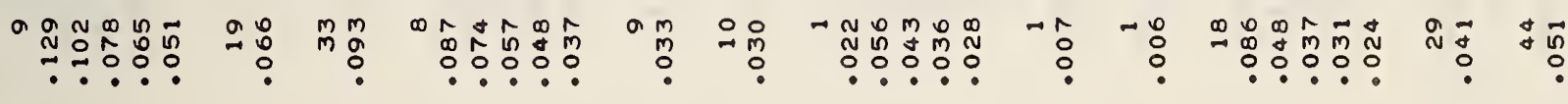

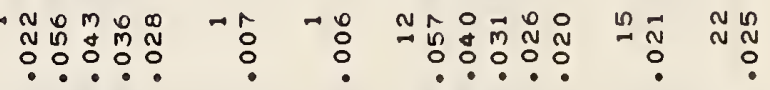

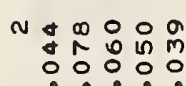

$n+\infty 000$ ค ?.? n $\prod_{0}^{0} \stackrel{0}{0}$

กิ

ㄴํำ

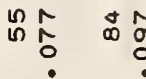

n $n$

m 0

ลีํำ

으의

\section{ที丶⿳一由𠃌 \\ ?.?}

- $m$ in 0

in 0 ?

\section{$\pi+4 m \infty$}

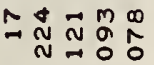
เ? กำด

$\begin{array}{llllll}n & 0 & 0 & 0 & 0 & 0 \\ 0 & N & 0 & 0 & 0 & 0 \\ 0 & 0\end{array}$

$m$

$\stackrel{m}{a}$

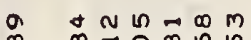
궁ㅇㅇㅇำ

a N

งกำ

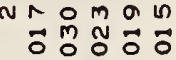

mิ

$\stackrel{\infty}{2}:$

r 0 in $\cos$

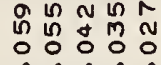

ํํํ

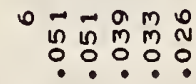

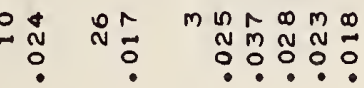

$\begin{array}{ll}a & n \\ N & n \\ m & 0\end{array}$

NM

$\rightarrow \frac{0}{2}$

$=\frac{10}{8}$

으응

n $N$

0
ก
-0
-0.0
0
0
0

$\vec{\sim} \stackrel{\infty}{N}_{m}^{a} \pm \stackrel{0}{a}$

m. $\div$ ?

000000

응응ㅇㅇㅇㅇㅇㅇㅇㅇㅇ

- 0 은

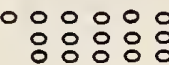

na mn

$\because \overrightarrow{0} \hat{0}$ o

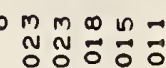

เ. ?

$\infty$

ํำ

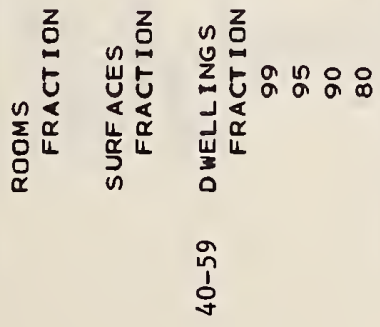

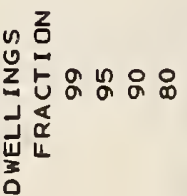

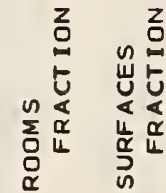




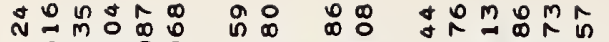

$m: \div: 0$

a $m: 0$

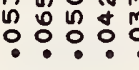

$m=0 \circ 0$

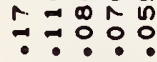

m n 0 on

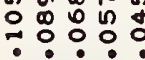

*

章

*

a d N 0
0
0 n $\frac{1}{0}$

ก

2

:

20ํำ

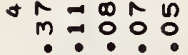

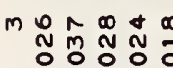

ํํํำ ำ

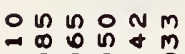

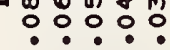

คำ

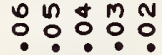

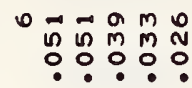

ดิ ถี

$+$

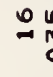

$m$

$r$

$\stackrel{n}{\circ} \quad a$

D. N N

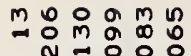

N NOMOD

mo:

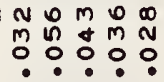

N

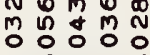

․:.․․․

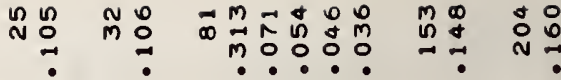

N

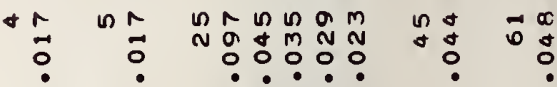

+ก ด N N

$\pi 00$ is

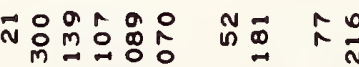

monON $\because \div:$ :

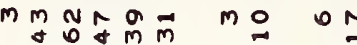

$\div \div: 0.0$

$\vec{m} \doteq$

$n \rightarrow a=r$

$\circ$

$\pm \stackrel{+}{\infty}$

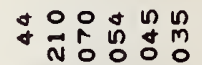

Mำ $\stackrel{m}{m} \stackrel{\infty}{n}$

n

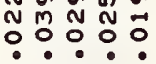

$m=$

$+\frac{2}{2}$

0 응ㅇㅇ웅

요을

资

$\circ m:-\infty m \quad m \circ=$

$m M$ m

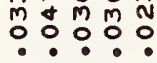

0

กั

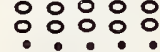

$\circ$

0 :

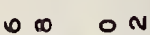
$\because \div \therefore$ ․․․

ำ

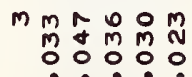

0

웅

$-N D^{m} \infty$

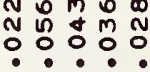

$+\infty$

0

$\pm \hat{0} \stackrel{M}{m}_{m}^{m} \stackrel{\infty}{N} \vec{N}$

:ํํํำ

mio

* 品

คํํํํํํำ

NNOO

ํํำ

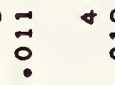

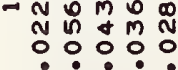

$+$

0

일

离离

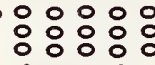

$\circ$

$: \circ:$

am

ผ

On $+\infty$ n

N

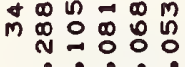

$\stackrel{+}{\stackrel{2}{a}} \vec{\infty}$

$0+m \sim a g$

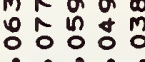

no

$\stackrel{m}{\circ}=$

$n \infty \pi \cap N m$

mån:

$\operatorname{mot}$

000000 00 00 000000

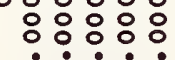

$\therefore: \therefore::$

$\circ:$

$\circ:$

N

은유:

胥

ปกำกำ

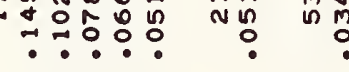

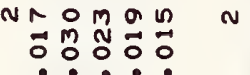

N $m$

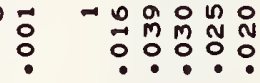

N

$\wedge$

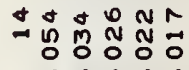

no $m m$

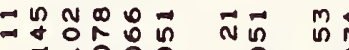

nT mOn nm

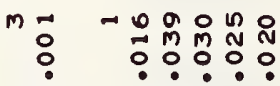

N

$\sim$

*

萃

ํ: : : :

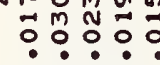

NorDOm RN ON

0000000

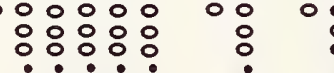

○े

응응:응

$\circ:$

:응

웅

N

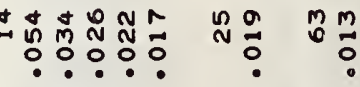

造

* 岩
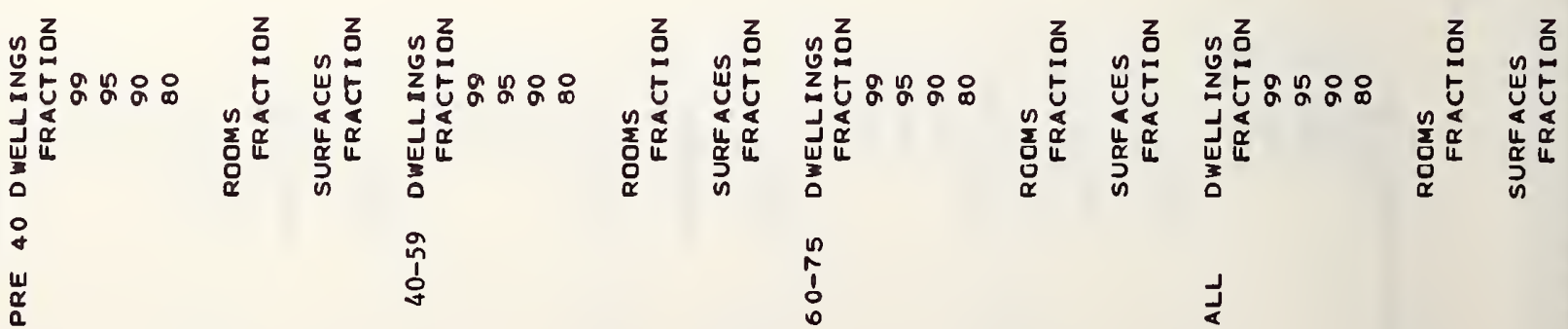
Hat

ตั

manmon an OONROMO

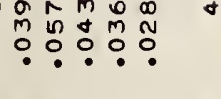

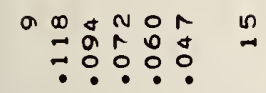

$\ln \frac{0}{2} \sqrt{n}$

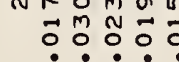

:

\%

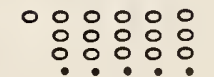

18

$\circ$
$\circ$

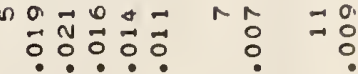

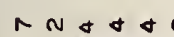

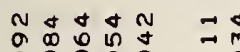

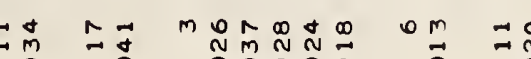

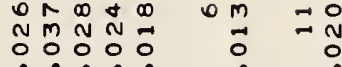

$00: 0000$

0

$\circ$

1.0 .0

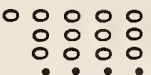
$\begin{array}{lllll}0 & 0 \\ 0 & 0 & 0 & 0 \\ 0 & n & 0 & 0\end{array}$

$\infty \stackrel{\substack{n \\ 0}}{\infty}=$

n

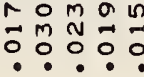

m

in 9

$\begin{array}{llll}0 & 0 & 0 & 0 \\ 0 & 0 & 0 & 0 \\ 0 & 0 & 0 & 0\end{array}$

?ㅇ?

0

\section{0}

$n \in N \ln \pi$

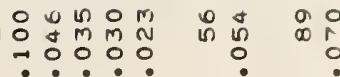

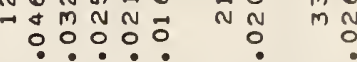

•.?

$(10)$

$\sim n \rightarrow \ln \rightarrow n$

$\rightarrow \because$ in $\Rightarrow$

$\hat{N}$

o $m \overrightarrow{0}$ ON Nmm

? 0 :

ลี

$\begin{array}{rlll}-\pi & 0 & 0 & 0 \\ N & 0 & m & 0 \\ 0 & 0 & 0\end{array}$

$\rightarrow$ o

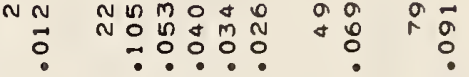

N

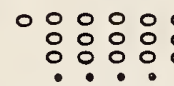

$0 \%$

$\circ$

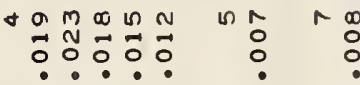

$\infty+\pi+2$

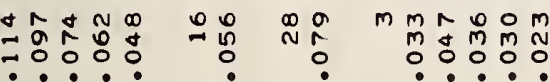

$\underset{N}{N} \circ \stackrel{0}{0}$

$-\begin{array}{rrrr}0 & 0 \\ N & 0 & m & 0 \\ 0 & 0 \\ 0 & 0\end{array}$

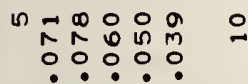

$\circ \prod_{0}^{n} \stackrel{\infty}{\square}$

M M

$0 \underset{N}{N} 2$

- NOMOM

$\begin{array}{lllll}n & 0 & 0 & 0 & 0\end{array}$

- $\bullet$ ・

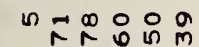

คิํํำ

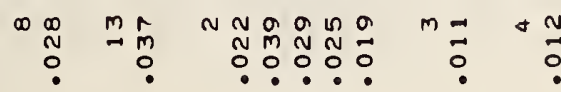

응ㅇㅇㅇ응

$\begin{array}{lllll}0 & 0 & 0 & 0 & 0 \\ 0 & \circ & \circ & \circ & 0 \\ 0 & 0 & 0 & 0 & 0\end{array}$

ㅇํㄴ?

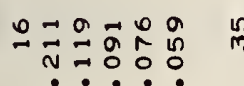

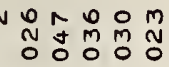

- $\cdot$.

$\infty$ in a $\infty$

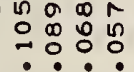

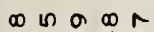

ํ.

noroOm n

n $\mathrm{m}$

: $00 \%$ :

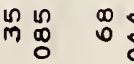

n $\mathrm{N}$ a

$\begin{aligned} & 0 \\ & 0\end{aligned}: \circ: 0$

$m 2$

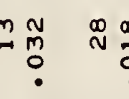

$\stackrel{\infty}{0}$

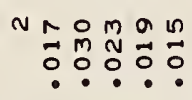

$\stackrel{M}{N} \stackrel{\infty}{N} \frac{\infty}{0}$

NAOMan

$\begin{array}{lll}n & m & 0 \\ 0 & N & 0 \\ 0 & 0 & 0\end{array}$

เก

$\circ:$
$\circ:$

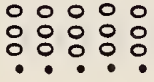

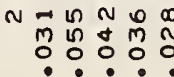

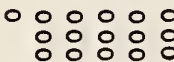

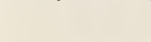

$\begin{array}{lll}0 & 0 & n \\ 0 & 0 \\ 0 & m & N \\ 0 & 0\end{array}$

0
-0
0
0
0
0

000000

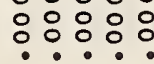

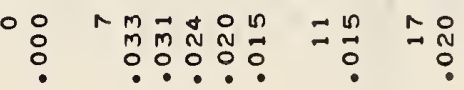

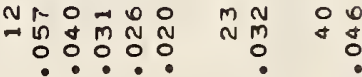

amLNNN No O $\begin{array}{llll}0 & 0 \\ 0 & 0 & 0 & 0 \\ 0 & 0 & 0\end{array}$
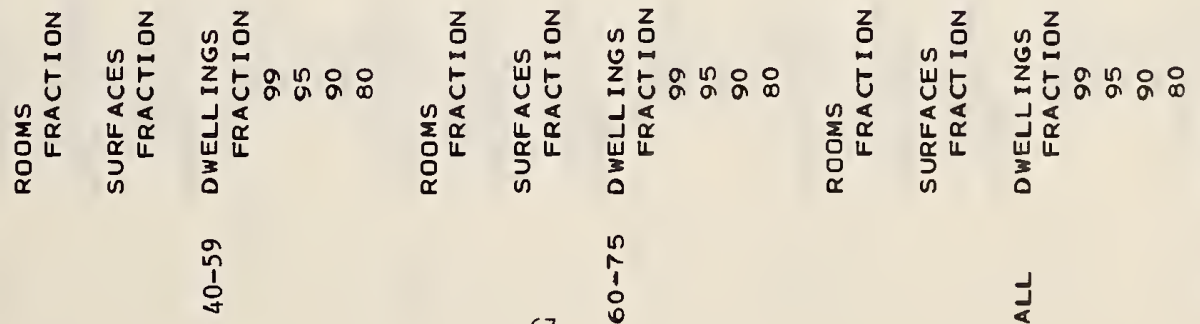

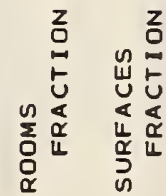

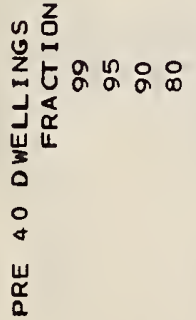

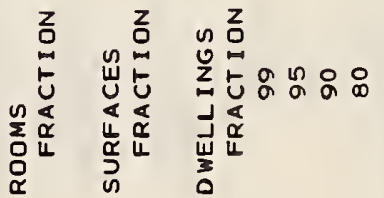

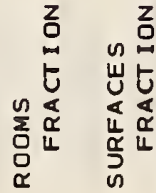
i⿱ 
* 400 N N N

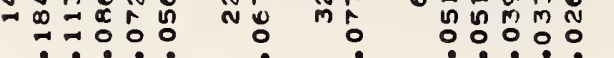

ñ mo

: : : : :

a d a n o r

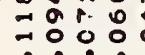

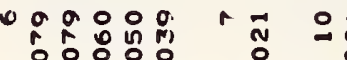

*

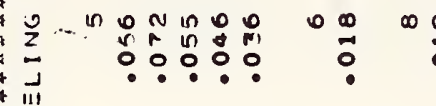

$\stackrel{0}{\circ}$

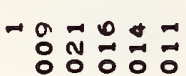

$: 000$

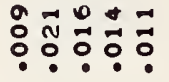

N

:

용 용ㅇㅇㅇㅇㅇ

응ㅇㅇㅇㅇㅇㅇㅇㅇ

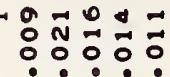

N

$+$

응ㅇㅇㅇㅇㅇ

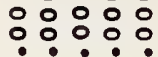

$\because \overrightarrow{0} \frac{0}{0} \overrightarrow{0} \overrightarrow{0} \quad N$

N

응ㅇㅇㅇㅇㅇㅇ

No

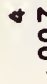

응ㅇㅇㅇㅇㅇㅇ

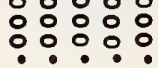

0

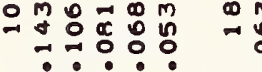

$m$
0

No

OONMm-

$\begin{array}{llll}0 & N & m & m \\ 0 & 0 & 0 & 0 \\ 0 & 0 & 0\end{array}$

$\infty$

N

N NGOHa m

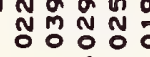

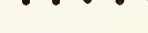

$m m p o O m$

: 0 :

단

z

* 8

*

*

*

*

•คี

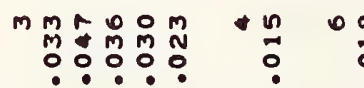

Noaga $m$

n

ํํำํำ

응ㅇㅇㅇㅇㅇㅇㅇㅇㅇ

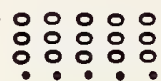

$\circ 8$

$\therefore$

:

으의

gำ

min in $\begin{aligned} & m \\ & 0\end{aligned}$

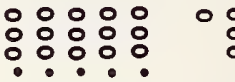

$\circ:$

$\operatorname{tam} x \operatorname{mn}$

$\overrightarrow{0} \stackrel{0}{0} \overrightarrow{0} \overrightarrow{0}$

n $\wedge \sim \infty$

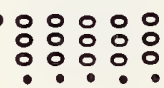

$\circ$ :

ㅇㅇㅇㅇㅇㅇㅇ

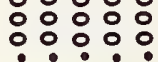

$\circ$

: $0: 0$

:
*

*

$m=0 \notin 0$

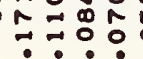
*

*

N O

Nํํํำ

n 0 n

足

곤

ㅇํㅇㅇํㅇ

N

$\stackrel{\sim}{*}=$

ก 0 ก ก

*

*

* 2

NORDOM MN

No:

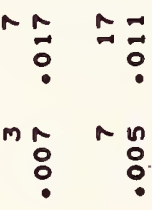

* a

ํ.

$\stackrel{n}{0} \quad \vec{m}$

m

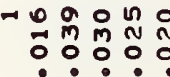

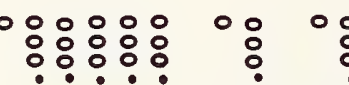

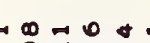

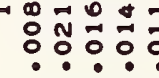

$\rightarrow$

$\because: \stackrel{0}{:}: \stackrel{0}{\circ}:$

0 웅ㅇㅇ웅

$\therefore::::$

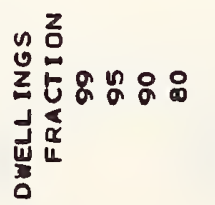

$\frac{2}{3}$
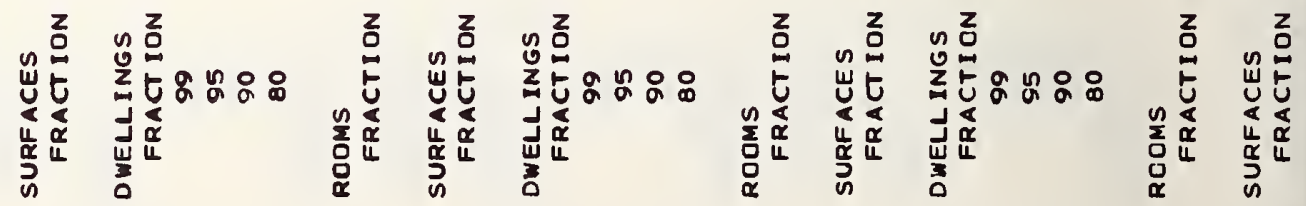

:

on

m

ํํำ

$\sim m \rightarrow \forall 0 n$

mํํำ

?

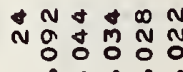

N

-

O 0 O

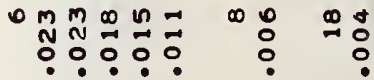

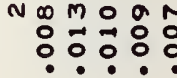

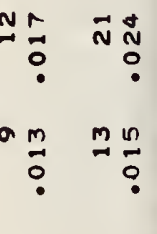

: 
ง

융ㅇㅇㅇㅇㅇㅇㅠ

in 웅

$\div:$

드옹ㅇㅇㅇㅇㅇ

요 $\frac{7}{4} \div$

웅ㅇㅇㅇㅇㅇㅇ

용 90

ํ.?ㄴ.?

a 0 ar $0 \mathrm{~m}-1$

-

N

-

M:은?

a: 1 -

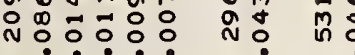

ㅇำ

ก ก

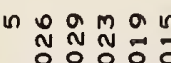

$0 \mathrm{~N}$

Oก

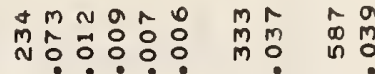

$m_{m} a n+$ $\begin{array}{lll}m & m & n \\ 0 & 0 & 0 \\ 0 & 0 & 0\end{array}$

n-a 2 a n

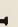
?: : ?

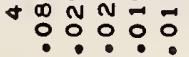

$\wedge$

\begin{tabular}{cc}
$n$ & $a$ \\
$\circ$ & $m$ \\
\hdashline & 0
\end{tabular}

은 $=10$

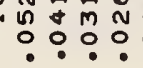

M

बूल

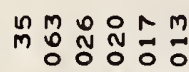

เก

$\ln _{\infty}$

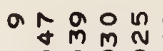

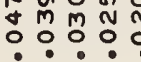

ㅇํㅇํำ

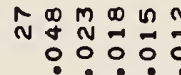

$\begin{array}{ccc}N & 0 & N \\ +N & N & N \\ 0 & 0\end{array}$

$0=\operatorname{mon}-1$

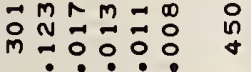

?은?

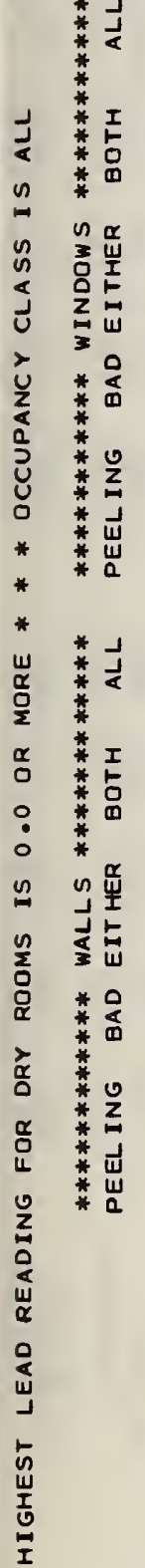

N

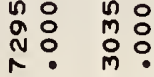

늉ㅇㅇㅇㅇㅇㅇㅇㅇㅇㅇ

$\rightarrow 0$

No 0

$\circ: 00000$ 응ㅇㅇㅇㅇㅇ

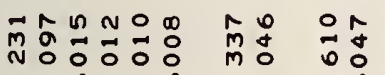

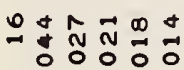

n

$\rightarrow$

$\infty \wedge 00$

$m$ m
$m$ N
O
0

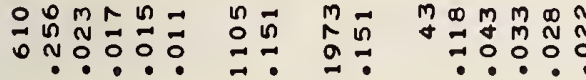

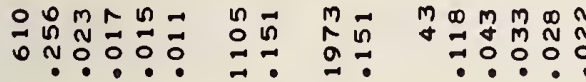

$\operatorname{mon}\left(\frac{1}{2}\right.$

?

은



N:? ? ?

n $\begin{array}{ll}0 & 0 \\ 0 & 0\end{array}$

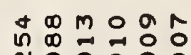

No:?

응 mo

io: in:

NM $m$ o

m̊ำ

$\begin{array}{llll}\infty & n & \infty & 0 \\ 0 & 0 & 0 & 0 \\ 0 & 0\end{array}$

$\infty$ in $0 \circ \mathrm{n}$

ㅇํㅇํำ

$\exists$

n?

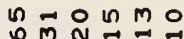

$+\infty N a$ N : $00 \%$ a

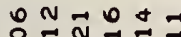

N

$\prod_{0}^{n} \stackrel{n}{n}$

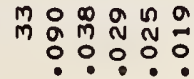

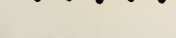

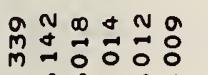

N

in $M$

ำเำ

N

융

$\frac{0}{0}$

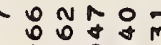
ㅇํㅇㅇํㅇ

$\stackrel{9}{9}$

2
2
2

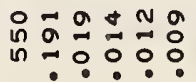

$\vec{a} \doteq \quad 0$

\begin{abstract}
잉
\end{abstract}
ถั

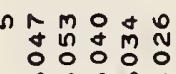

0

an

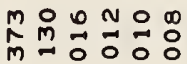

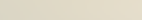

mooooo no a n 000000

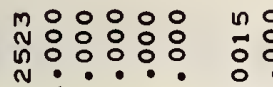

용요

กิ: ㅇ:ㅇㅇㅇ

No

a 0

aㅇㅇㅇㅇㅇㅇㅇ

N

용ㅇㅇㅇㅇㅇ

$\dot{\sim} \cdot \bullet \cdot$

ลิ

in $v$

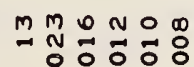

mo $\circ$

$\infty \frac{1}{0}$

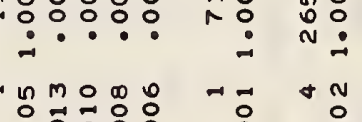

응ㅇㅇㅇㅇㅇ

$+\frac{1}{8}$

용

$\checkmark \circ$ in 90

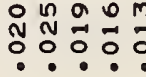

$\sim$

$\stackrel{\infty}{N=}$

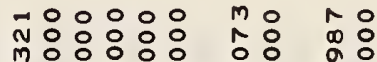

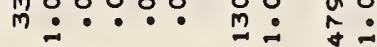

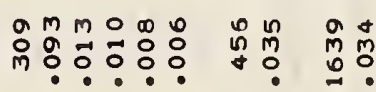

$\stackrel{N}{N}$

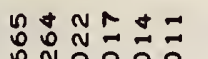

$\stackrel{0}{m}=m$

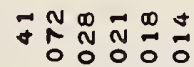

$\mathscr{N}_{0}^{\infty} \stackrel{\infty}{N} \stackrel{n}{N}$

$m$ n N N

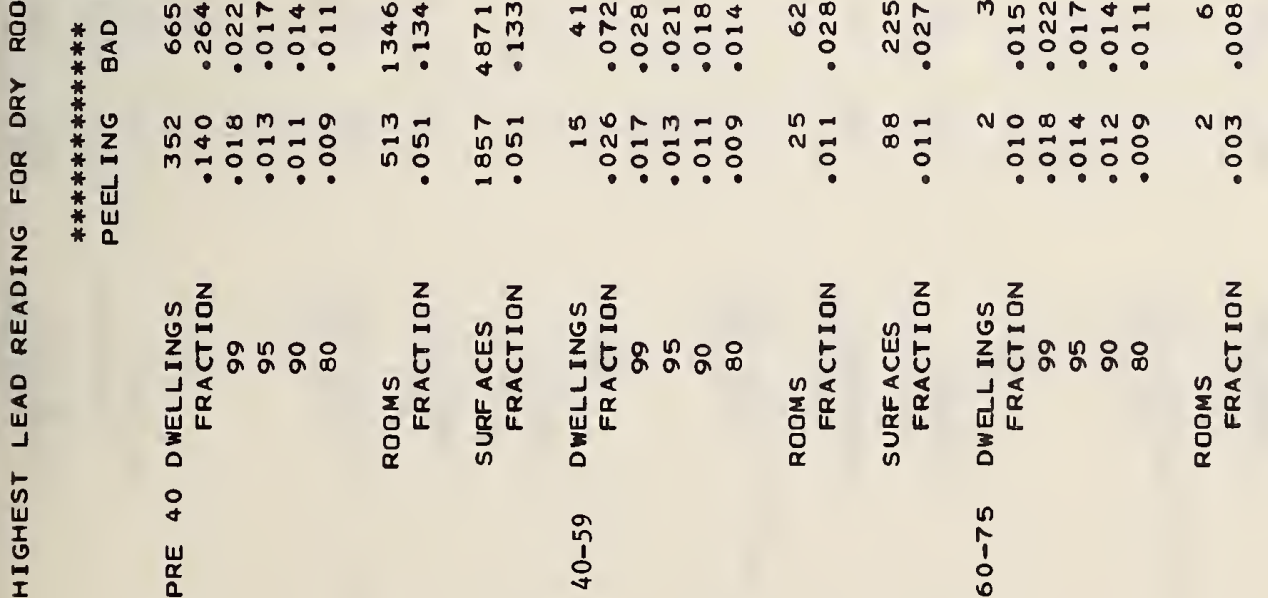

0

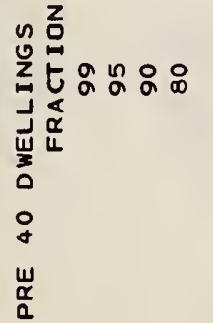

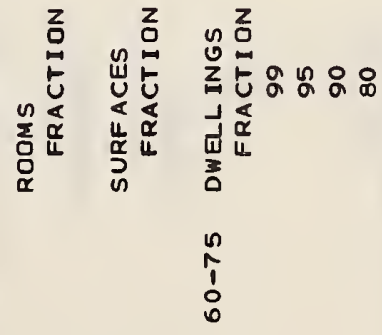

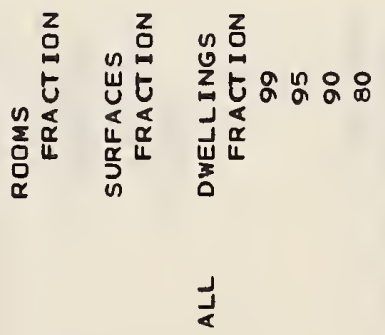


ta a o 0

mpm

min

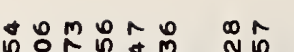

$\operatorname{lom}_{0} \infty$

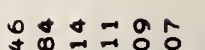

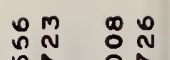
ล : : : : : जः: D?

ondoar

政

웅

$n \wedge \wedge m=$

-

(

-

m:

! $\quad \stackrel{\infty}{\infty}:$ : : : :

in?

$\therefore ?$

$\rightarrow: 0$ :응

$\frac{0.000}{20}$

ำกํํㅁำ

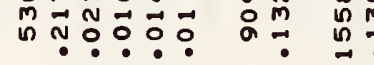

$\infty \infty \sim \rightarrow r$

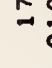

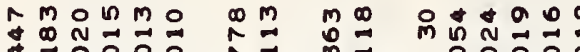
$0: 0.00$

in

กั

-

$\because$ ก

$\rightarrow-n+m+\infty$

$\infty \circ \infty n$

N:ํํ유 N

ํ.:ำ:

$+2$

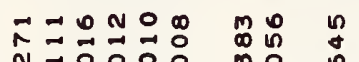

盘

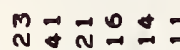

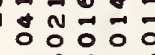

in

$\stackrel{\infty}{=} \div \frac{n}{0}$

a

$m 0 \quad a \circ r m=9$

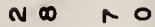
$\begin{array}{ccc}M & N & N \\ 0 & 0 & 0\end{array}$

:

ถิ $\rightarrow 5050$

$\stackrel{\infty}{0} \div 00$

$\div$ ?

*

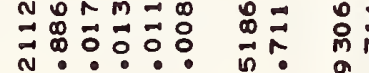

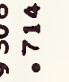

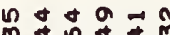

$\vec{\wedge} \ddot{0} \quad \stackrel{0}{0}$

号

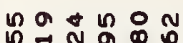

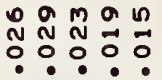

0

N in

$\rightarrow 0 N$ 웅 $\underset{7}{1} \div \frac{0}{0} \div 0$

ติํํㅇํำ

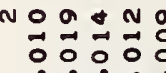

N

N

ammon

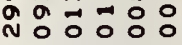

$\overrightarrow{0} 0 \quad \infty 0$ $+000$

\section{0

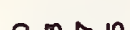

思品

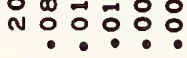

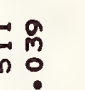

nֻ.

$\sin \frac{2}{9}$

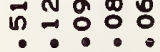

$\stackrel{n}{m} \stackrel{n}{N}$

กับ

ma ๑ 00 ก - $\begin{gathered}0 \\ \circ \\ 0\end{gathered}$

$\rightarrow \div$

$m$
0
0

-

N N 0

$: \stackrel{N}{0}$

ma

$m$

a d

$m \infty=N D=$

N

$+\frac{10}{0}$

n 6

in $\overrightarrow{0 A n N o}$

○? O :

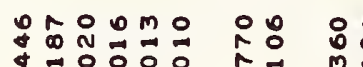

$\sum_{m:}^{0}:$

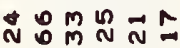

Oํ.?口:

min $\underset{m}{N}$ in

$M \mathscr{N}=\mathbb{N} \stackrel{0}{N}$

ํ.

$+10$

ก $2 \quad 0$ ก

$\vec{\circ} \div \overrightarrow{0} \overrightarrow{0} 0$

$\vec{\omega}$

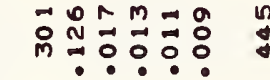

$\stackrel{\overrightarrow{0}}{\overrightarrow{0}}$

$\rightarrow \begin{aligned} & N \\ & 0 \\ & 0\end{aligned}$

ลี

กับ

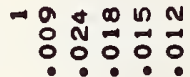

$\rightarrow$

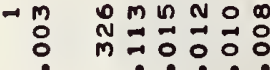

:

年

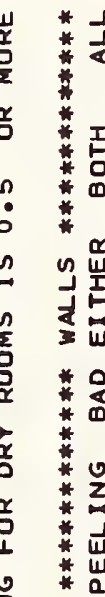

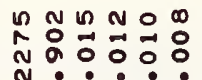

$m$ o

N $\infty$ n mo

\section{ก $m$}

$\infty+a \circ=0$

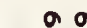

$a$

n

ט月d

$m \infty \sim N$ $\infty$ ○ n 0 人

N m: N

-

$\pi$ ก

- ․ㅇㅇㅇㅠ.

$m \div$

0 N

禹둥ㅇㅇㅇ

10

àm

$\vec{m} \vec{\nabla} \quad \stackrel{0}{0}$

$\infty \circ m \circ \infty$

ñ

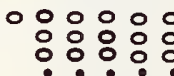

0 :

$\circ$ :

$\operatorname{an}=0 \hat{0}$

ํㅗㅇㅁㅇㅇㅇㅇㅇ

a n $2 n$

a $900 \pi$

\section{or}

0 N $9=0 m$

\section{$x^{2}$}

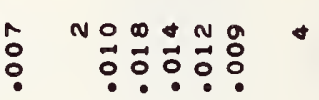

0 0

ט

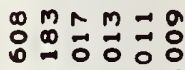

mo

$\approx \overrightarrow{0}$ กำ:

부유ํำ

ก

$\overrightarrow{0} \quad \overrightarrow{0} 0$ m :

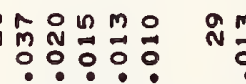

$\underset{0}{m} \quad m:$

- $\because m: 0 \%$

$m$

$m=$

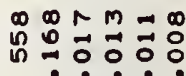

å

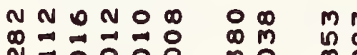

a omogr 드응ㅇㅇㅇ

0

No

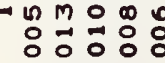

$\rightarrow$

: $m$

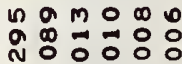

$\infty m a=$

ㅇํㅇ

品

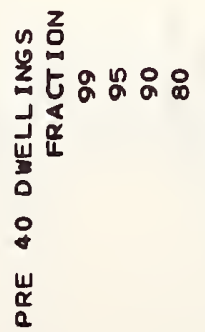

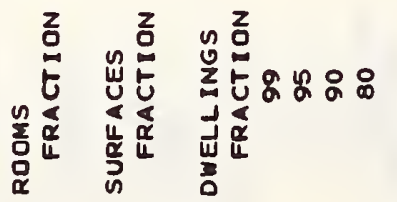

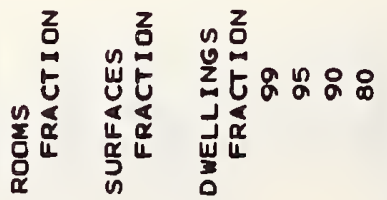

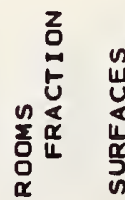

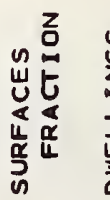

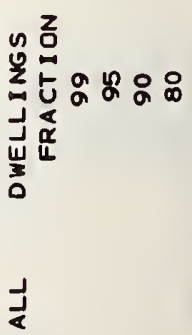




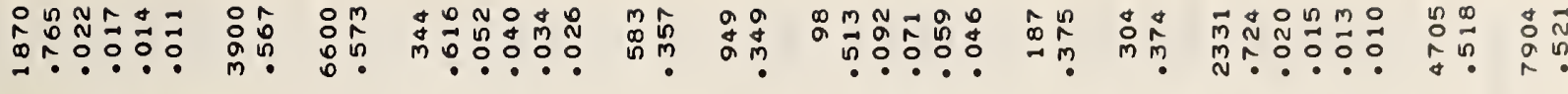
*

+0 o

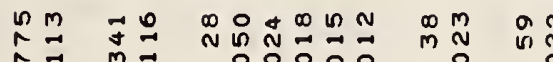

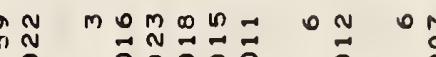

$\overrightarrow{0} \begin{array}{lllll}N & \overrightarrow{0} & \overrightarrow{0} & 0 & 0\end{array}$

$: 0$

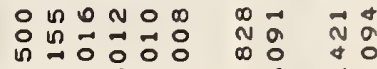

吕吉

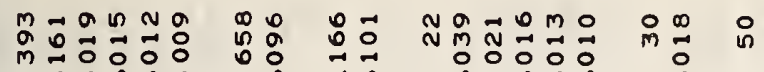

$\infty \quad n \circ a+n$

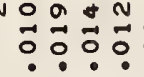

n: $\frac{0}{0}:$

N

กิ

N

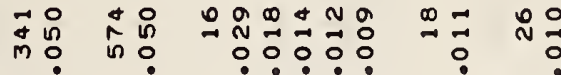

nod a d a

다융

w

N

in

+0 응

non

$\overrightarrow{O N} \underset{0}{N} \overrightarrow{0} \overrightarrow{0}$

$\infty \infty \circ N \rightarrow 0$ ก

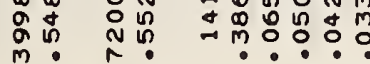

$\sim a$

$a+\pi \ln \theta 0$

$\hat{n} \fallingdotseq 0$

$\stackrel{9}{\circ}$

$\vec{m} \quad m$

A $M r A=$

สำํำ

$00+9$

๓

à

$m$

ด ำ ำกำ

$\rightarrow=$

$\stackrel{a}{a}$

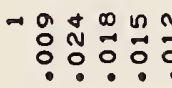

$\rightarrow$

$\rightarrow$

$\vec{m} \quad 0$

u $\alpha$ 요

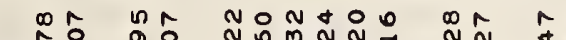

n:0.:

naำ mo no na madnad

o 00

\& $\because$ : ० :

000

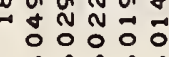

虫

in

na\& on

0 :

$m$

$+\stackrel{2}{2}$

$\rightarrow \circ: \circ: \circ$ ก

$\rightarrow \circ 0 \mathrm{No}$

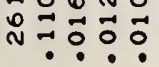

O $\rightarrow$ ON M

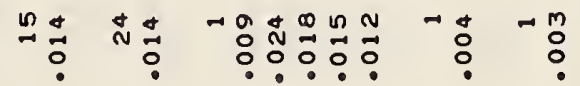

กิ๊

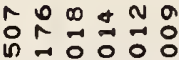

0\%

natown

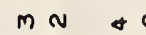

N

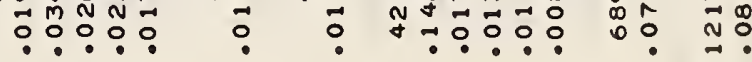

ตำ 0 :

?: ?

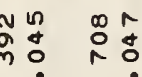

- 0 in a o

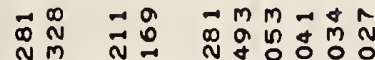

in o- ar o 0 n

$m$

$\infty_{\substack{\infty \\ \sim}}^{0}$

0 a o n

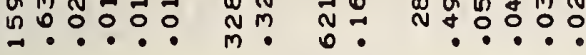

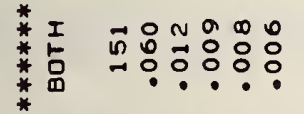

कू

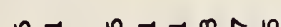

$0+\frac{1}{2}$

mั?

$\rightarrow \rightarrow$ ?

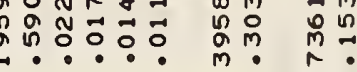

$\sum_{m \rightarrow 0}^{0} \underset{0}{0} \div \frac{0}{0}$

$\ln 10$

$\rightarrow$ ? $\rightarrow 0$

000000

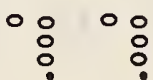

กำ กั

$\vec{m} \stackrel{n}{m} \underset{m}{m} \stackrel{m}{0}=$

ñ 0 n

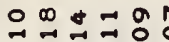

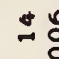

ผ

$\therefore \circ: \circ$

$\begin{array}{ll}\pi \\ n \\ \infty & 0\end{array}$

$\begin{array}{llll}n & m & 0 & \infty \\ \circ & \overrightarrow{1} & 0 & 0 \\ 0 & 0 & 0\end{array}$

$\because \%$ ㅇ?

$\rightarrow \vec{\circ} \quad N$

๓ू๊

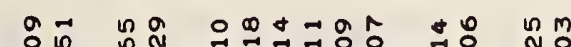

$\circ: \%: 0$

$\therefore: \because:: 0$

$\circ$

$\circ 8$

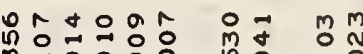

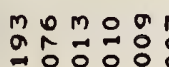

$0+\alpha+0 \rightarrow-\infty$

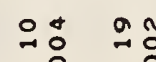

$\sim m 0$
$\circ 00: 0$
$\circ: 0$

$\rightarrow$

N
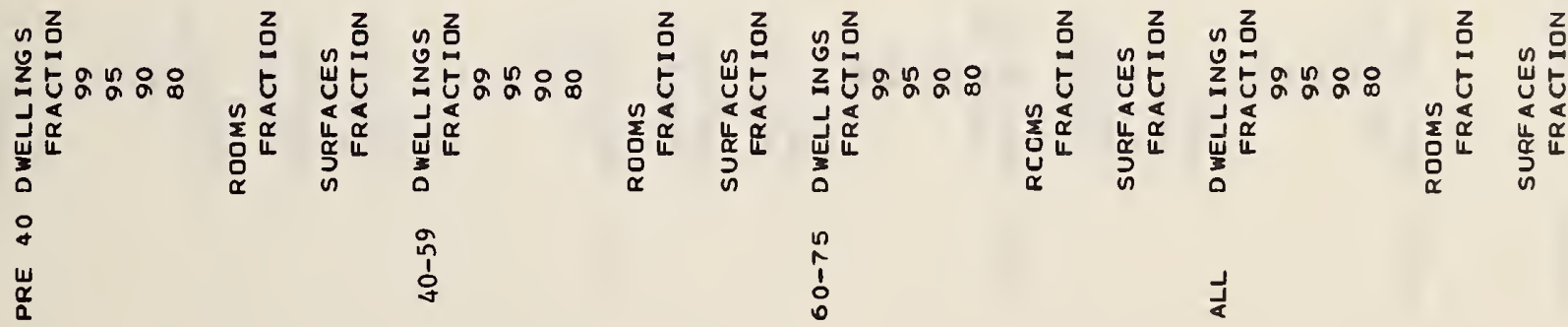


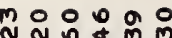
N

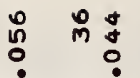

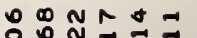

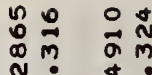
* * I 0 N

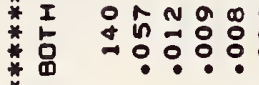
$\infty_{\infty}^{\infty}$ No

0 $=9 \hat{0}$ 0 \&

몸

- $0 a+n o$ a n in 응응 m $\because$ : $0: 0$

○

man n no N

$m \quad m$

- $n$ moar 응 0 용유

$-8$

?:? :

N $\$ m=$

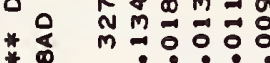

*

* J

in

not $\rightarrow$ a की
$M$
$N$

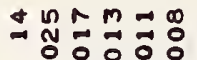

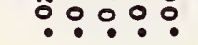

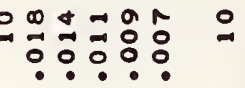

$\stackrel{D}{\Rightarrow} \quad \stackrel{N}{m} \stackrel{N}{0}$

$\because n$

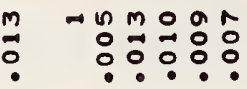

$\stackrel{1}{0}$

:
- $10 m 09$

:응ㅇㅇㅇ

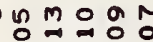
mกำ ㅇํㅇำ

N은 nO요

落

\section{ma}

ֻ

ֻ

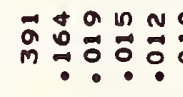

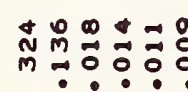

- $9 n=0$

तี

$\begin{array}{ll}n & \Xi \\ 0 & 0\end{array}$

nี

$\approx$

옹

:

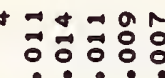

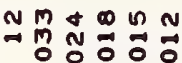

om na

: $: \overrightarrow{0}: \overrightarrow{0}$

ma

n

?

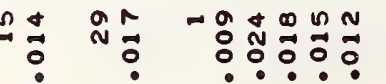

?

$\pm$

$n \in 0 N O \infty$

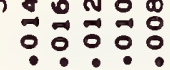

Dก ํ.

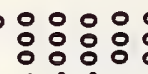

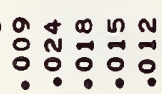

응ㅇㅇㅇㅇㅇ $m$

$\circ$

N

$\mathbf{N}$

N

$\circ$ :

\section{$\frac{0}{2} \quad N$}

N

$\sim$ $\rightarrow$ ก : ? ?

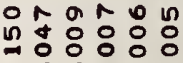

等 nn $n=0$ a เ

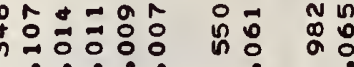

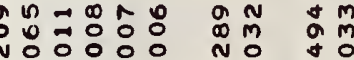
N

* $\lrcorner 0 \infty m \infty n N$

* I *

u

齿

* * *

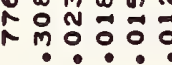

$m \vec{m}$

in:

$n \pm 40 N$ 0
0
0

\section{$\exists \ddot{0}$}

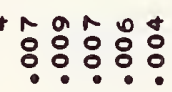

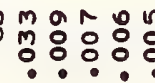

$\stackrel{m}{\circ} \underset{0}{0}$

空:

ำㅇำ

ㅇํ유유:

$M M M O D N \quad \rightarrow$

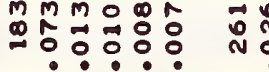

N

$\stackrel{\leftrightarrow}{0} \frac{1}{0}$

000001

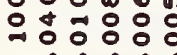

$\stackrel{M}{N}$ ก ก
ก ก กำ

ㅇํㅇㅇํ

ค 옹유:
:

$\div$

\section{웅}

ㅇํㅇ n

$\sim \stackrel{m}{N} \underset{0}{0}$

$n$

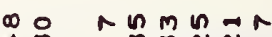
m $m$ N

\section{0}

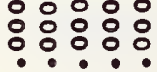

$\circ \circ$

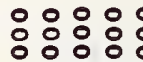

•. •.

용ㅇㅇㅇㅇㅇ

:

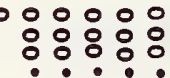

$\pm$

$\circ$

:

0 :

$\circ:$

: 0

$\circ \circ 00$

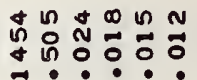

ㅇำ

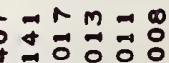
・・・・

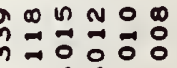

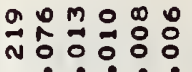
융ํㅇ

90 in

岕 范

* n mo

N N กี้ nू:

$\rightarrow \sim \hat{m}$ ำ *

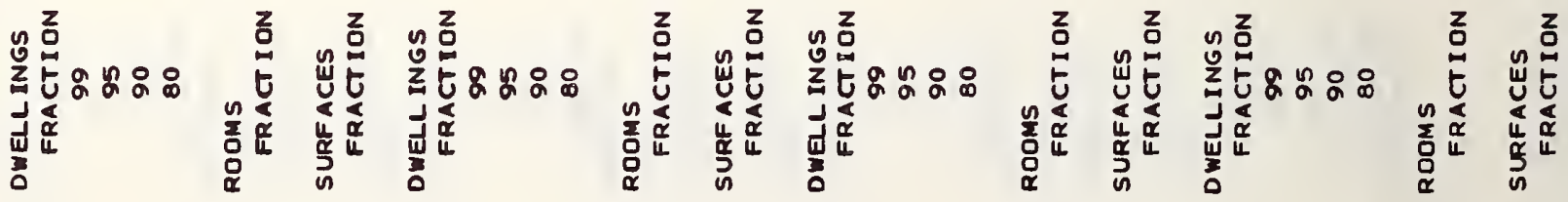

i⿱宀

$n$
1
0
0 
$m o$ on

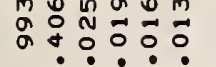

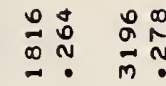

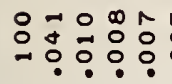

等

ต

$\infty \infty \wedge m=\infty$

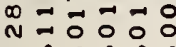

m

0
0
0

A $m$ Na

NMONO an

ํํ응ํㅇำ

人 0 a

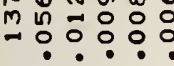

à

A $M N O$

$\begin{array}{lll}M & 0 \\ 0 & 0 & 0 \\ 0 & 0 & 0\end{array}$

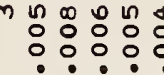

N $\vec{N}$ ก $N$

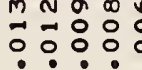

No

$\infty$

$\stackrel{\infty}{\rightarrow}$

$\circ$
$\circ$
$\circ$

m

$\sim$

$\circ$
0

$+\infty$

n :

$m \underset{N}{m} \vec{N}=m 0$

$\begin{array}{ccccc}M & N & N & 0 & 0 \\ 0 & M & 0 & 0 & 0\end{array}$

ñ

เ. .

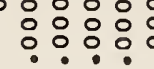

$\circ \%$

$\circ 8$

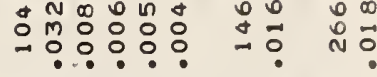

$\therefore \circ: \circ: \circ$

$\circ$

$\circ 8$

N $m \circ \infty \circ m a+n$

n.

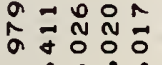

\section{$\sin 0.00$}

N $m=\infty \sim m$

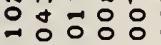

. : :

i

an $\operatorname{man} \infty+\infty$

$\begin{array}{lllll}0 & N & \infty & 0 \\ \infty & m & N & N & N \\ 0 & 0 & 0 & 0 & 0\end{array}$

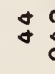

$\stackrel{0}{r}$

+

mo

$\stackrel{N}{M} \quad \stackrel{0}{m} \stackrel{\infty}{N}$

m

$m m$

in

ㅇㅇㅇㅇㅇ

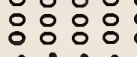

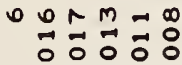

$\infty \stackrel{\infty}{0} \pm 0$

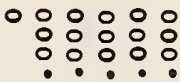

$\circ$

$\stackrel{n}{0}$

a

a

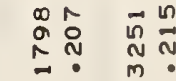

0.000

m:응

N웅ㄹㅇ

m

r

10

$\infty \infty$

$\pm$

0
0

$\circ$

\begin{tabular}{lllll}
$\infty$ & $m$ & 0 & 0 & 0 \\
\multirow{4}{*}{0} & 0 & 0 & 0 & 0 \\
0 & 0 & 0 & 0 & 0
\end{tabular}

ดั

$n$
$N$
$O$ $m m$

n

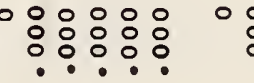

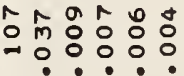

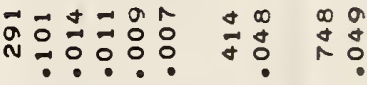

ㄴำ

ㄴํㅇํㅇ

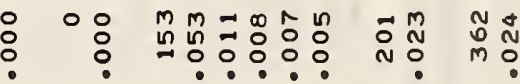

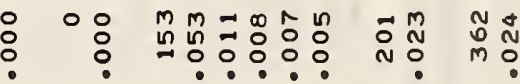

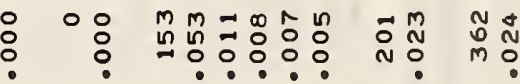

$0 \%$
(

둥유

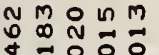

$\because 0 \% 0$

\begin{tabular}{llll}
$\infty$ & 0 & 0 & 0 \\
\multirow{2}{*}{0} & 0 & 0 & 0
\end{tabular}

$a-m \infty n$

\section{a}

$a m-n m o \infty$

$N \mathrm{~m}$

웅유

\&

N

$\infty 00 N \circ \infty$

กั กิ

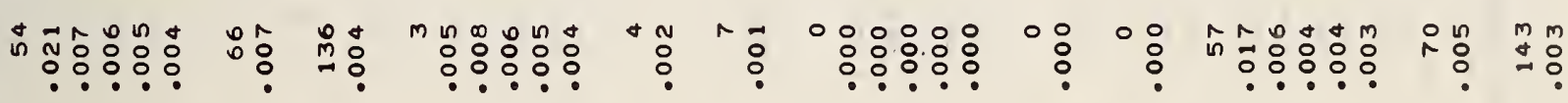

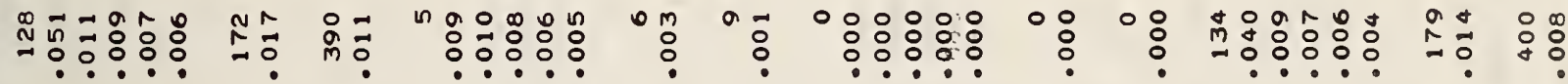

n.

0

a

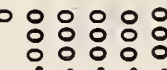

0

$\circ \stackrel{\circ}{\circ}$

iิ

+ $m \circ \infty$

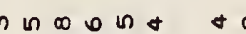

^

$\circ$ \%

$\circ:$

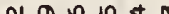

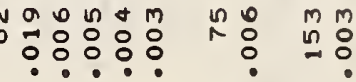

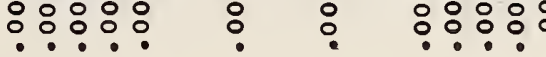
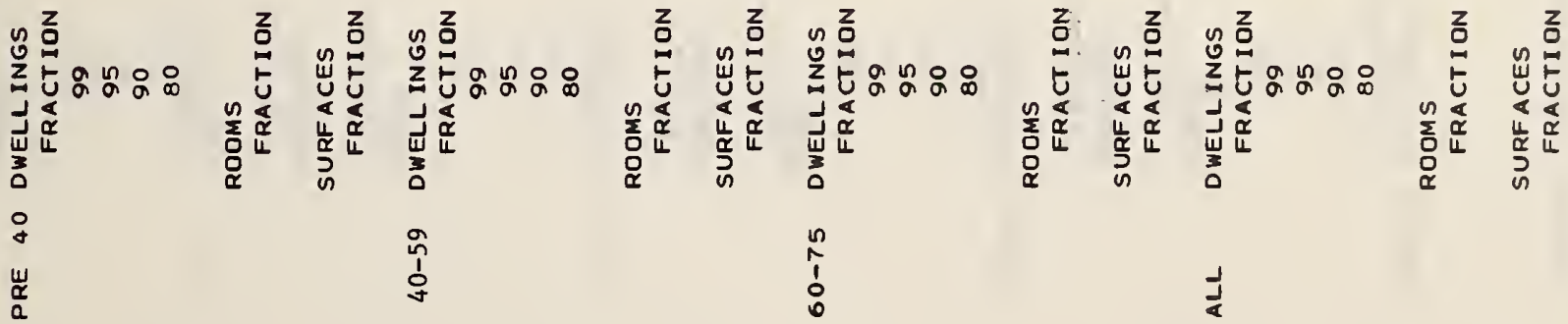


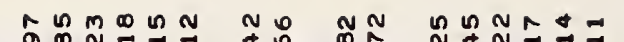

a $\infty \quad n \rightarrow-1$

0 ก? 0

n $=$ a

ำ

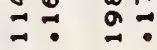

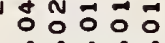

$n+0 \ln +m$

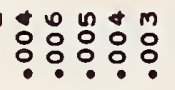

8

$\operatorname{lng} 0+20$ ㅇํㅇㅇㅠ:

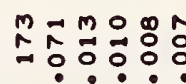

ก

: $m$

+คำㅇํㅇ

ํํㅇ 웅

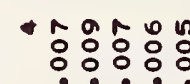

舟母

- 응ㅇㅇㅇ

$\underset{m}{0} \stackrel{\infty}{N} N$

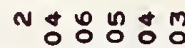

+0
$\circ$
$\circ$

no+ 0 n

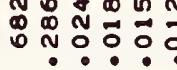

$\infty_{0}^{0}$

응

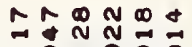

N

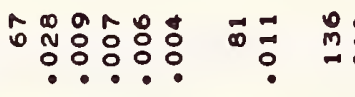

$\stackrel{0}{0}:$

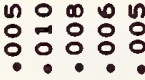

N

$\exists \pm \Rightarrow g r$

告

$N 0 \div 9$

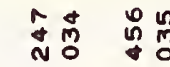

응ㅇㅇㅇㅇㅇㅇ

$m \neq m \circ \infty$

\section{0}

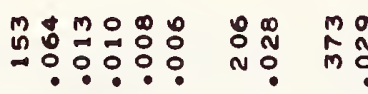

*

- $\overrightarrow{0}=90$

응ㅇㅇㅇ

$\stackrel{N=}{N=}$ N

N

ㅇํㅇㅇํㅇํㅇ

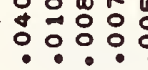

*

(.)

(1)

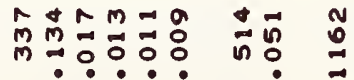

*

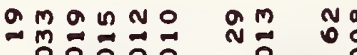
* *

m

?

잉

$\circ \circ$
$\circ$

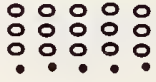

응ㅇㅇㅇㅇㅇㅇ

8

:

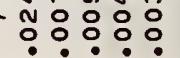

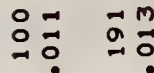

$\left.+\frac{1}{0}\right)$

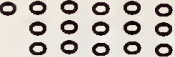

$\circ$

$\circ 8$

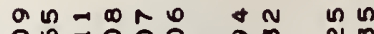

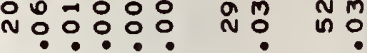

+

$+000000$

응ㅇㅇㅇㅇㅇㅇㅇㅇ

$\circ$ :

8

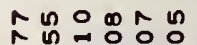

in

no

ㅇํㅇㅇํㅇ

$N=0$
$\circ$
0

$\circ$

0

$0+\infty 0 n+$

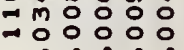

$\pm$

象

No *

a

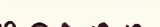

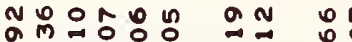

m n้

융요

$m: 0$

웅

.

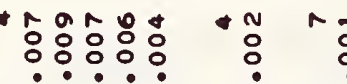

응ㅇㅇㅇㅇㅇㅇㅇㅇ

$\circ$

잉

:

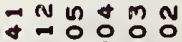

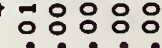

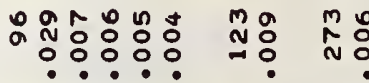

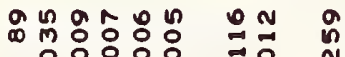

*

*

*

*

-10 0 영

m

응ㅇㅇㅇㅇㅇ
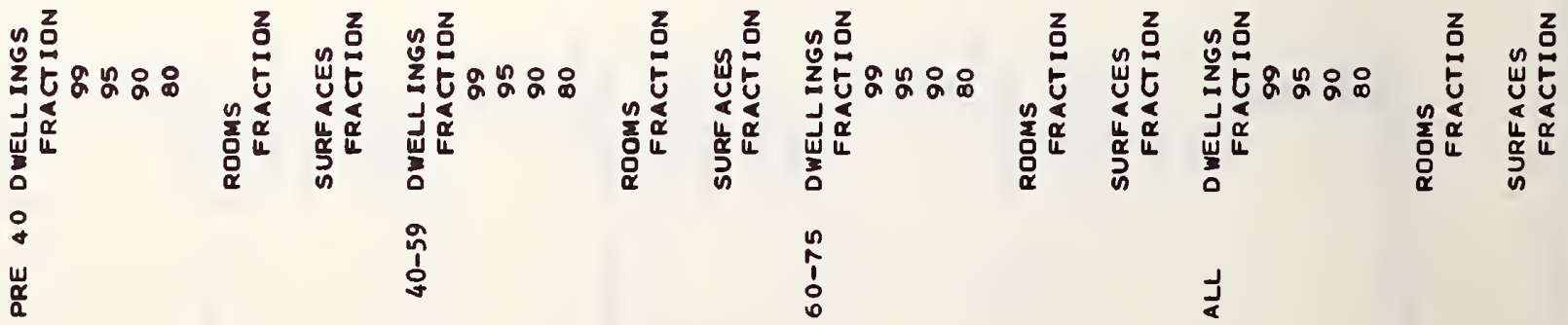
ํㅇㅇㅇㅇㅛ

4

-

$m$ n $N$

mก

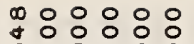

ก!ํํㄴ?

긍ㅇㅇ

$\rightarrow m$

에

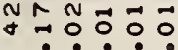

$M \vec{\theta}$

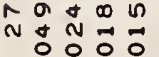

$\rightarrow$

$n \div \infty$

ㅇํ의

m

mo의

mำ: 0000

$\vec{N}$

ก

$M N N N$

ㅇํㅇำ

$\vec{m}$

$\infty+00$

凡

ํำ

$\stackrel{\infty}{N}$

+ or $m=$

0
0

(1)
*

000000

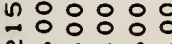

N:

ํㅕㅇ영응

- 응ㅇㅇㅇ

$\infty+N A+$

$\vec{a} \mathbb{N} \overrightarrow{0}$

จ $M=0 M$

$\hat{m} \cong \mathbb{N}: \overrightarrow{0}$

ơ

咅:

승ㅇㅇㅇㅇㅇㅇ

m:ㅇํㅇ

$\stackrel{0}{0}$

NN

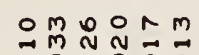

M

$\prod_{n=0}^{\infty} 0 \overrightarrow{0}$

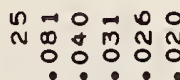

0
$\infty$
0

용

$m n^{\infty} n$

$\rightarrow 0000$

$\stackrel{\infty}{0}$

ma

0

N 0 m $\stackrel{\infty}{N} \stackrel{m}{N}:$

N

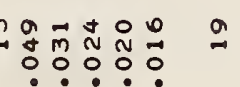

a



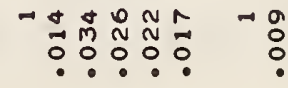

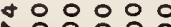

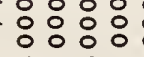
-

A d 0 N

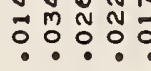

$m=$ a n

คำ

$m=0$ \% $\infty$

ง $\stackrel{\circ}{*}$ m -

$-\infty$

:

n $\begin{gathered}\infty \\ m \\ 0\end{gathered}$

(1)

inำ

$+\overrightarrow{0} \overrightarrow{0} \overrightarrow{0} \overrightarrow{0}$

in $\infty$

a

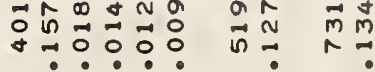

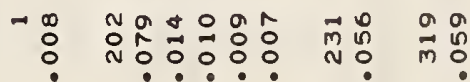

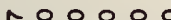

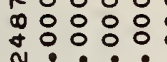

$\begin{array}{ll}\infty & 0 \\ 0 & 0 \\ 0 & 0 \\ 0\end{array}$

\&:

700000

9000

000000 n $0: 0 \%$

$\stackrel{0}{=}: \stackrel{0}{0}$

ㅇํㅇㅇㅠ:

o

ำ

$\infty+m \circ \infty$

$\Rightarrow 0$

ㅇํㅇ요용ํㅇ

$n_{\infty}^{0} 0 \quad 09$

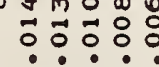

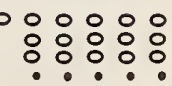

$\circ:$

N

m:? : : : N

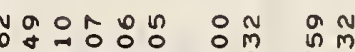

응ㅇำ

oa o in mo

$+N$

min

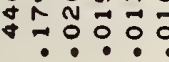

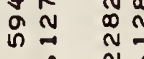

ก 00700

$m_{0}^{n} \pm n$

$\stackrel{a m}{0}$

n) $m$ n

ㅇํㅇำ

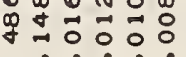

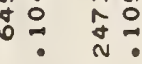

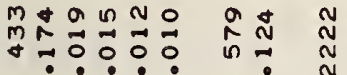

$\wedge \infty m \infty N \quad n$

M

$m \backsim N \cong A$

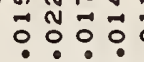

$n$

$2 \frac{m}{0}$

No 0

\section{- -1}

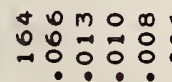

ㅇำ

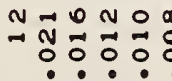

$\stackrel{n}{\sim} \quad \stackrel{0}{0}$

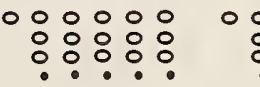

00

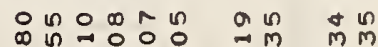

๓

选
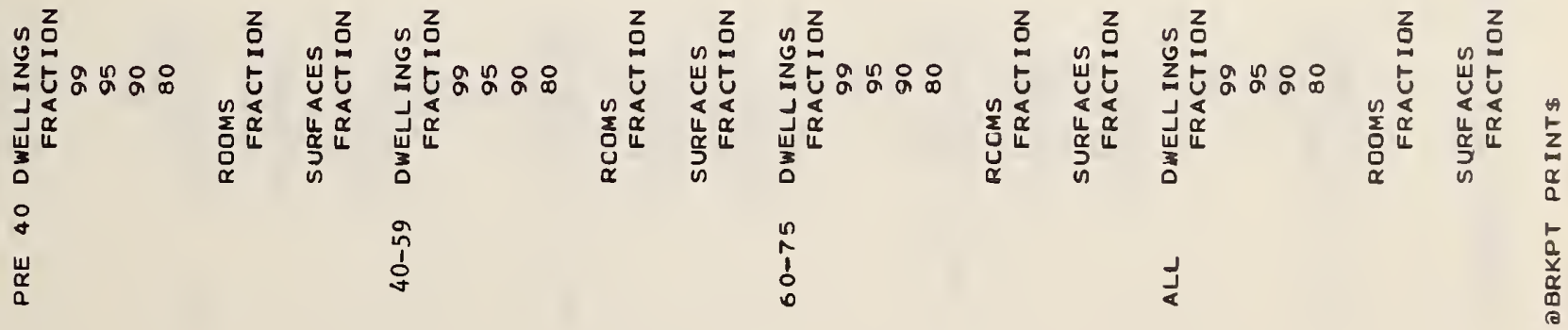
लि口 i

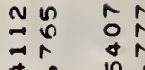

\section{으뭉 nm n}

o n n mo

-

훙ㅇㅇㅇ

a $9 \div 0$

$\rightarrow$ n $m$

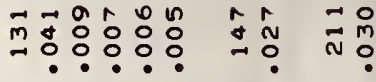

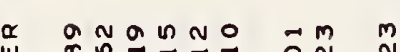

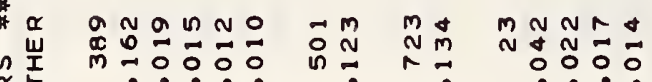

品吉

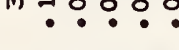

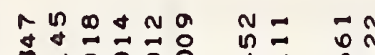

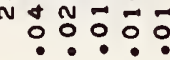

$\hat{N} \stackrel{\infty}{N} \quad M$

$: 00: 0$

$=m-m$

$\stackrel{m}{\circ}$

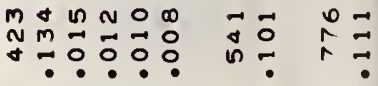

m? 웅

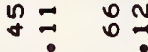

$\begin{array}{ccc}n & 0 \\ m & m & m \\ 0 & 0 & 0 \\ 0 & 0 & 0\end{array}$

n

NกMำก \&

웅유 范 n

$\infty \quad n N 0 N O$

$\stackrel{N}{N} \stackrel{N}{N} \underset{0}{0} \frac{0}{0}$

$\stackrel{m}{0} \div$

$\stackrel{0}{0}$

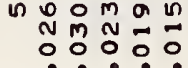

o

a 2 ก

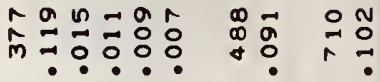

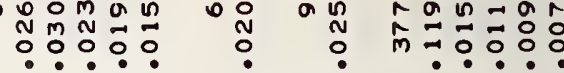

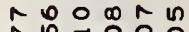

$\rightarrow 000000$

or no

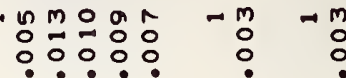

:0:0. no no

*

을

\section{更}

*

*

*

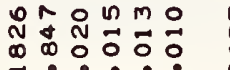

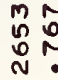

$\frac{\infty}{0}$

m $\rightarrow$ N $n$ No

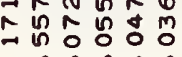

$\stackrel{N}{N}$

Ni

$\overrightarrow{0}$

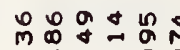

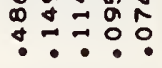

$\underset{d}{\infty}$

in

n웅 $n$

華

$\circ \stackrel{\infty}{\sim} \vec{m}$

ลั.

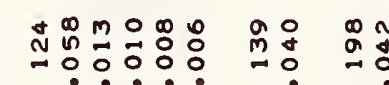

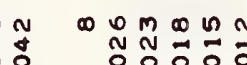

N

$a x \stackrel{N}{N} \underset{0}{0}$

응ㅇㅇㅇㅇㅇㅇ

0

$\circ$ :

ก n

กี

…

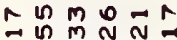

In

ํํำ $\stackrel{n}{N}$

N

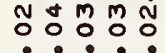

$m \stackrel{\circ}{N}$

$+9$

an

$\vec{a}$ i⿱

i.

กN

$m \div$ : ? : + ! $:$

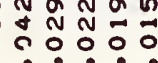

in

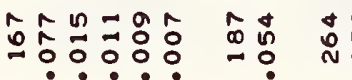

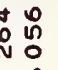

$\cos 2 \pi \cos ^{2}$

- M $\pm \stackrel{\substack{0 \\ 0}}{0}$
ㅇํํ

Nลั

:ㅇํㅇ

$\cong \stackrel{9}{0}$ $\circ \circ$
0 $m$

$\rightarrow$

$\circ$ on $n \rightarrow a$

$\dot{m}=\overrightarrow{0} \overrightarrow{0} 0$

$\rightarrow+100$

$\stackrel{\infty}{\sim} \stackrel{m}{*} 0 \stackrel{0}{0}:$ in

N

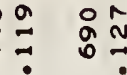

$\because \frac{0}{0}=$

이 $m$

요 ${ }^{1} \quad \infty$ in
*

을

*

*

*

.

*

*
Nै N.・.・

m.m.

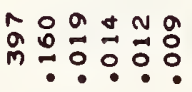

in

$a_{\infty}^{\infty} \infty$

N N NOMOO

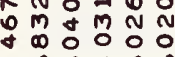

a

a $\quad m$

D NONMN m?

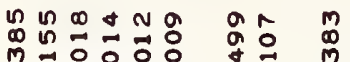

0

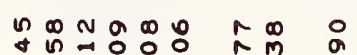

- ํํㅇㅇㅠ:

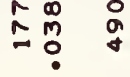

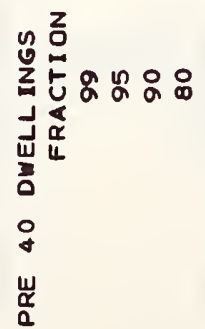

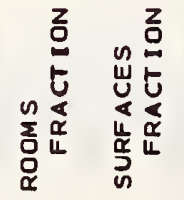
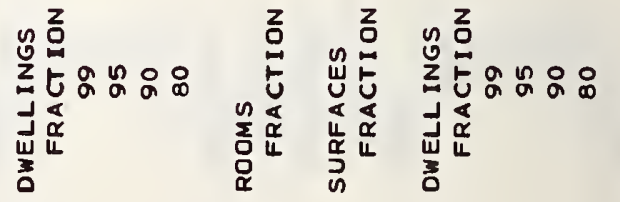

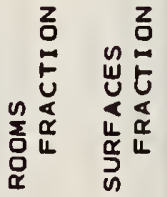


in $\vec{n} M+N$ 的

n

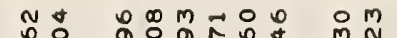

$\stackrel{\infty}{\rightarrow}$ ? ? : ?

$\stackrel{N}{N}$ :

\title{
$\stackrel{\circ}{\rightarrow}$
}

$00=0 \wedge$

\section{풍}

$\infty m$

?.

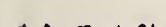

\section{$\ln$}

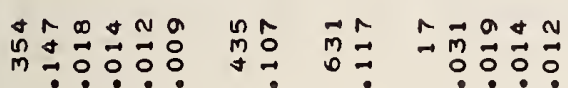

$\overrightarrow{\overrightarrow{0}} \overrightarrow{0} \mathrm{9}$

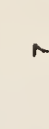

品

$\Delta \vec{m} m \vec{m}=0$

\begin{abstract}
a
\end{abstract}
No n

$\because \stackrel{\sim}{\circ} \stackrel{\infty}{\circ} \overrightarrow{0}=$

O NMOD

m。

in

$\rightarrow 0 \overrightarrow{0} \overrightarrow{0}:$

$\hat{0}$

ins

$\infty \sim m \circ \infty$

뭉ㅇㅇㅇ

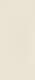

$\vec{n} \underset{0}{n} \stackrel{n}{n}$

a d 음

$\begin{aligned} & 9 \\ & 8\end{aligned}=0$

\section{a}

$\stackrel{m}{N}$

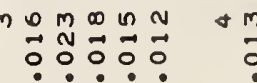

$m \circ m \infty$ เn

$\stackrel{2}{M} \stackrel{\infty}{\circ} \overrightarrow{0} \stackrel{0}{\circ} \overrightarrow{0}$

$\checkmark m$

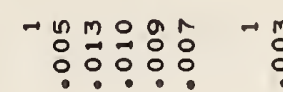

in $\stackrel{9}{N}$

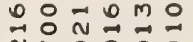

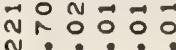

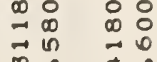

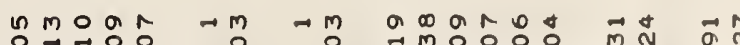

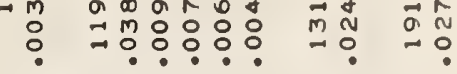

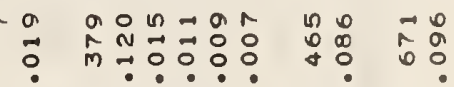

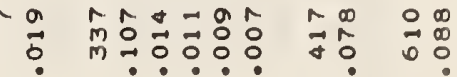

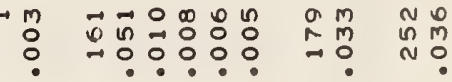

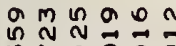

$\hat{N} \stackrel{0}{n} \quad \infty$

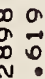

$\infty$ nom in $n$

ᄂก? : ? :

moㅇํำ

$\min _{\rightarrow \rightarrow} \rightarrow$

$\stackrel{m}{n}$

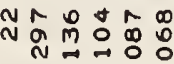

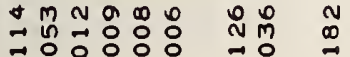

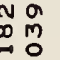

$\circ$ 우 ㄴ $\mathrm{m} \circ$

N $\begin{array}{llll}N & \overrightarrow{0} & \overrightarrow{0} & \overrightarrow{0}\end{array}$

$\sim \stackrel{\circ}{\circ} \div$

N

요

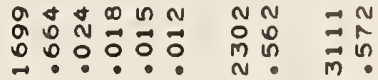

$\cdots$

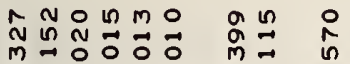

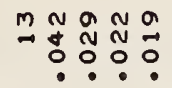

$\cong$

$\infty m$

$\rightarrow 0 \wedge A$

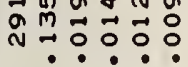

mo

$\vec{n}$

M N

$m$

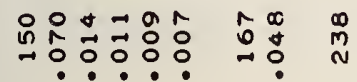

$\stackrel{\infty}{m} \stackrel{\vec{n}}{\mathrm{n}}$

$\infty 0 m \infty$ in

$\stackrel{N}{N} \stackrel{M}{\circ} \overrightarrow{0} \overrightarrow{0}$

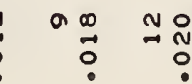

ㅇํํ

응ㅇㅇㅇㅇㅇㅇ

$\circ:$

$\circ 8$

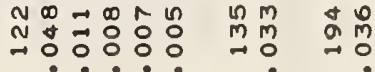

N $\begin{gathered}n \\ \text { N } \\ 0\end{gathered}$

$m \stackrel{2}{0}$

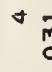

o $n \wedge m \rightarrow$

a $n o a$

$\infty \vec{N} \quad N \hat{m} \vec{m}$

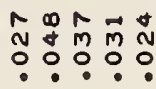

$m \stackrel{0}{0}$

$+$

$\circ: \circ \mathrm{m} \rightarrow$

$m \rightarrow \overrightarrow{0} \overrightarrow{0} \overrightarrow{0}$

L 2

00000000

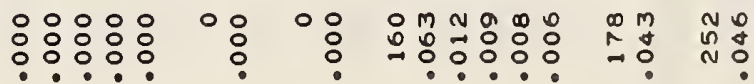

$\circ M N a \infty D \quad \infty M N D$

$\min m$ in $\rightarrow$

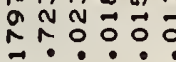

$\begin{array}{ll}0 & \infty \\ 0 & \text { in } \\ 0 & \text { in }\end{array}$

\begin{tabular}{lll}
+ & $m$ \\
0 & 0 \\
\hline & $m$ & 0
\end{tabular}

$\therefore$ 足 $\rightarrow+4$

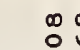

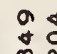

o OR O OM

Na

N

a $\infty \rightarrow 0 m 0$

$$
\text { (n) }
$$

I

n m O n

$\sin 2$

เ?

.

ก) m.?

ก

$\vec{N} \stackrel{\infty}{0}: \overrightarrow{0} \overrightarrow{0}:$

N N N N

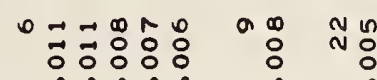

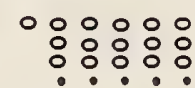

$\circ$ :

$\circ$

○ $\infty$

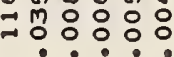

$m \sim m$ เ

$\rightarrow$ 응요

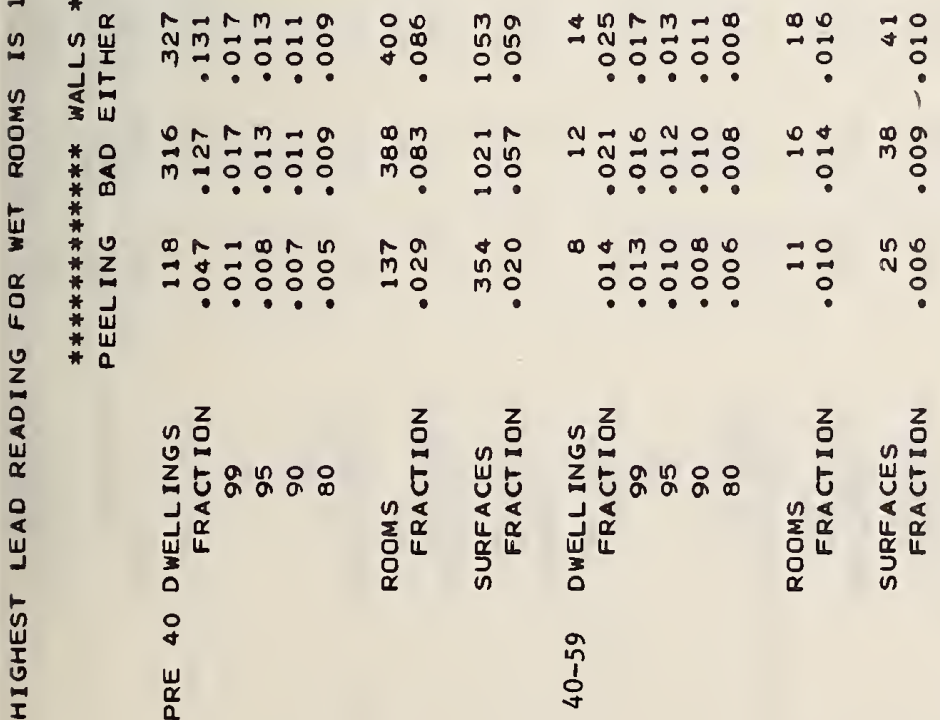

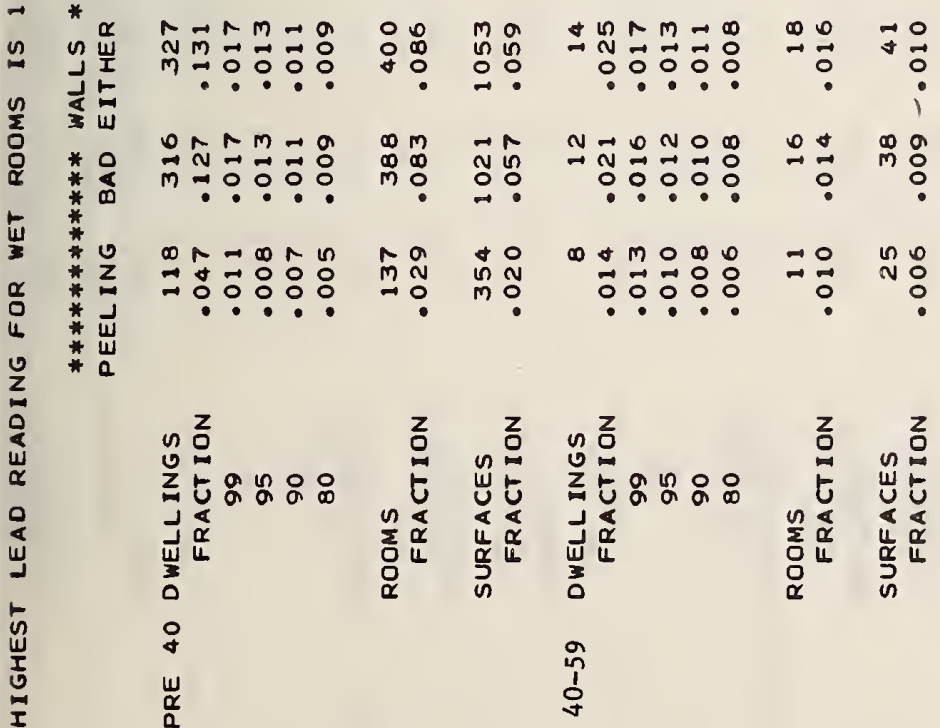

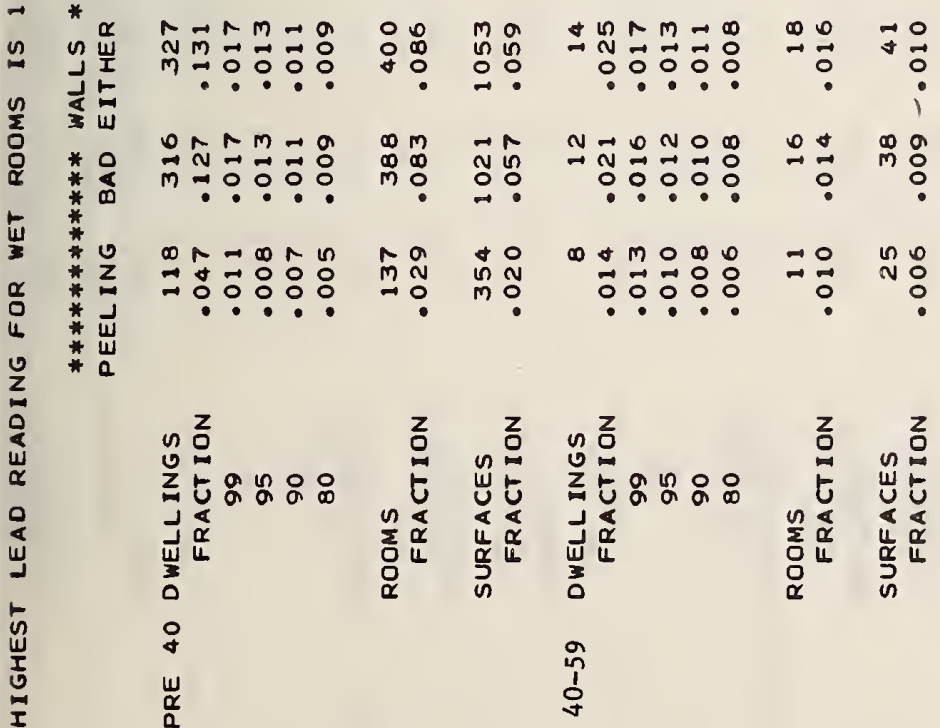

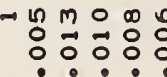

พ

$\infty$ ย

$\ln 200$

m으유유

No $\Rightarrow$

$\stackrel{\sim}{N} \cong M=$

$\operatorname{lin} 0$

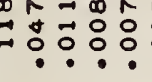

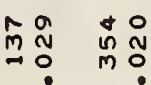

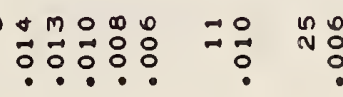

- $\because \frac{m}{\circ} \stackrel{0}{\circ} \stackrel{\infty}{\circ}:$

nin

NลMำ

\begin{abstract}
00000000
\end{abstract}

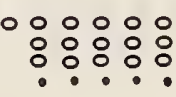

$\circ 0$

$\circ$

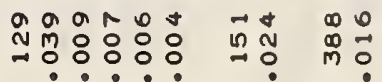

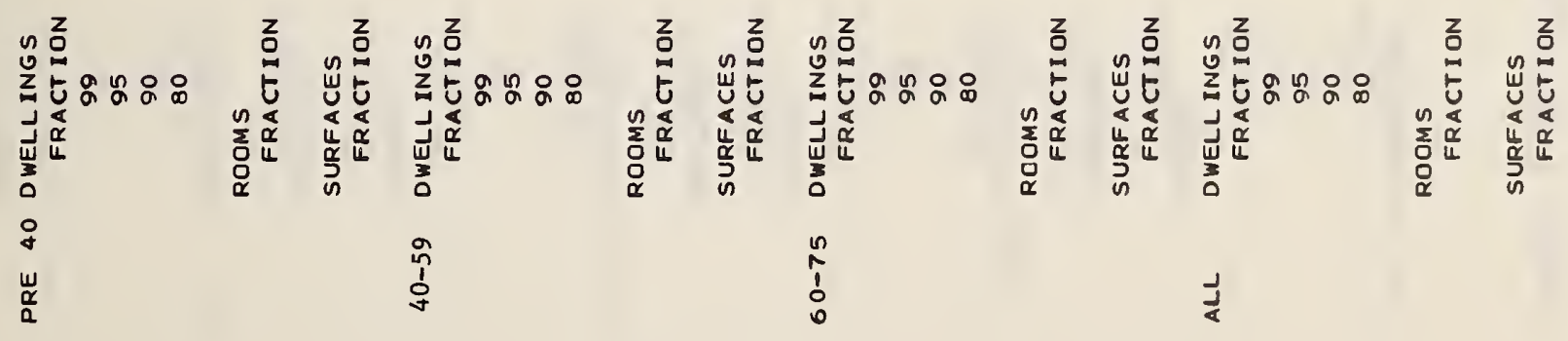


$\vec{N} \stackrel{N}{N}$ m n? ? ?

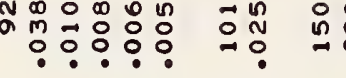

$\infty$ n 00 舟

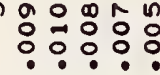

$n N r m \rightarrow a$

$\stackrel{N}{N} \underset{0}{0} \overrightarrow{0}=$

in 0

$n$
in

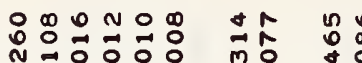

0

*

*

*

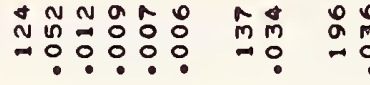

$\rightarrow 050 \%$

$0 \infty n=9$

$\overrightarrow{0} \overrightarrow{0} 0$ :

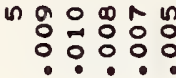

$0 \infty n=9$ $\rightarrow$

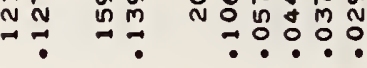

\author{
$00 \infty$
}

\section{웅}

의 $m 00$

$m+\infty$

* J $m \infty \sim-\infty$ N

in

n

\section{$\infty_{0}=0$}

$N=1$
$\infty$
$\infty$
0

ma

$\stackrel{N}{N} \stackrel{\infty}{N}$

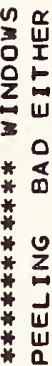

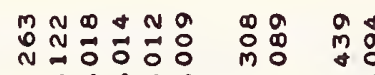

i. -

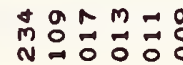

* J monotm

$=$ ตั

$\begin{array}{llll}0 & 0 & 0 \\ N & \infty & 0 \\ N & 0 & 0 \\ N & 0\end{array}$

垈

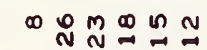

ำ

$\stackrel{n}{n} \equiv$

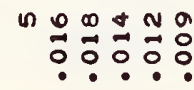

$\overrightarrow{0} \overrightarrow{0} \overrightarrow{0} \overrightarrow{0}$ ?

\section{$\stackrel{0}{0}$}

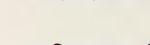




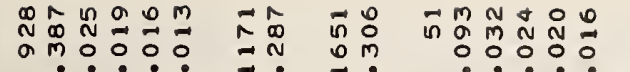

\section{$\log 0.0$}

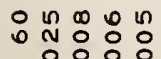

$\therefore: \therefore: 0$

เ

$\infty \pi n=2$

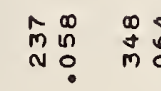

nू

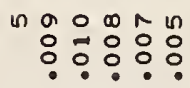

$\infty$ in $m \circ \infty$

둥유.

$\infty$ In mOD

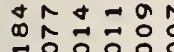

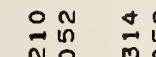

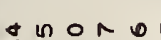

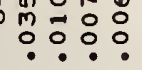

$\vec{O} \underset{N}{N} \stackrel{O}{N} \stackrel{0}{\circ}$

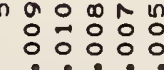

ก:

$\infty \stackrel{\infty}{\circ}$

$: \%$

$\infty$ ?

$\begin{array}{ll}n & \text { n } \\ 0 & 0\end{array}$

$\stackrel{\infty}{\infty} \quad$

$\approx 0$

응ㅇㅇㅇ

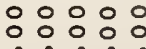

$\simeq 0$

000000

$\therefore: ㅇ: 0$

…을

응유 n in 0 in $0: 0: 0: 0$

옹

$\infty \underset{N}{N}$

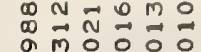

응웅ㅇㅇㅇ

$\circ$

$\circ$

\%

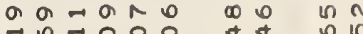

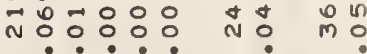

$n n \rightarrow \infty n \pi) \quad \rightarrow \quad \rightarrow \infty$

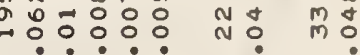

‥

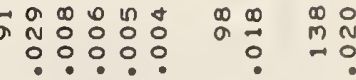

$\circ$

$\circ \%$
กูก

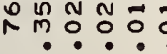

m.

กิ

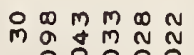

Un N $N$

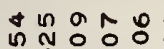

กิำ $\stackrel{\infty}{\wedge}$

$\stackrel{0}{\circ} \div \div \div$

웅

o

:용유

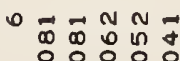

ㅇํㅇ

M $N \underset{0}{N} \pm$

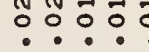

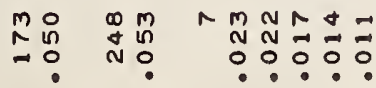

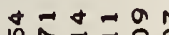

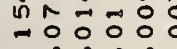

×

$\exists$

NANA

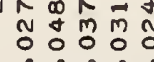

$\infty \stackrel{0}{0}$

$=$

$N \hat{n})$
N
0
0

ON

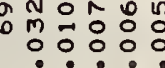

in․ 00

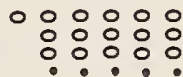

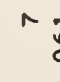

$\circ$

N $m$

$\stackrel{M}{N}$

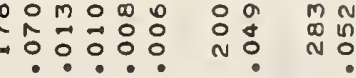

N

$m m$

$m+n a m m$ n $N$

ํํํ유

$\circ \stackrel{0}{\circ}$

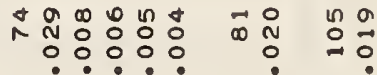

กั

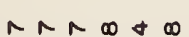

mo: 0.00

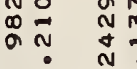

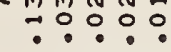

aㅁำ

an

nก $\infty \pi n \infty$

N

เก

$\infty$ in

ติํํㅇำ

क

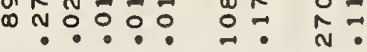

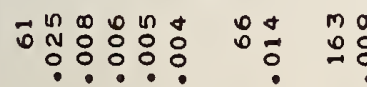

5
$+\therefore: 0$
0
0

0 แn

$\circ: \therefore: \therefore:$

$\circ$ :

$\circ:$

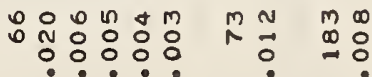

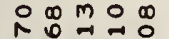

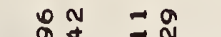

$\overrightarrow{0}=0$
00

a :

ำ

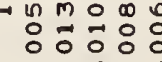

$\rightarrow$

No

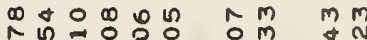

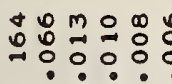

욱

n

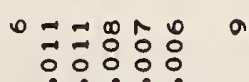

N

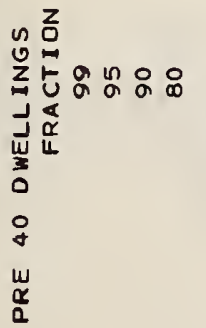

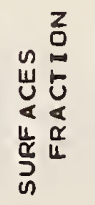

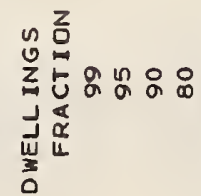

ồ<smiles>O[Hg]</smiles>

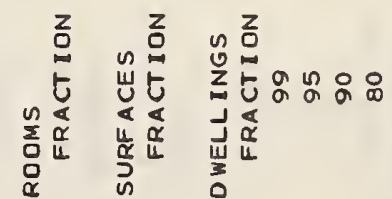

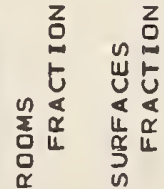

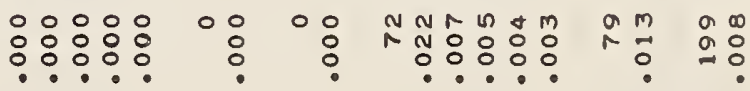




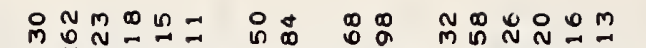

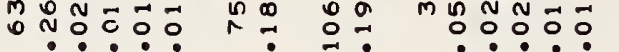

$m_{i} \underset{0}{\infty}$ in

-

잉

$+m$

$+$

กํㅇำ

ก

$\therefore N$

\begin{tabular}{|c|c|c|c|c|c|c|c|c|c|c|}
\hline 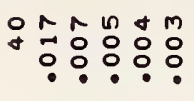 & $\stackrel{m}{+0}$ & $\frac{n}{0} \frac{11}{0}$ & 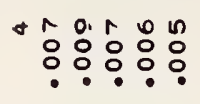 & \&: & n: & $\begin{array}{r}\circ \circ \\
\circ\end{array}$ & $\because:$ & 음 & 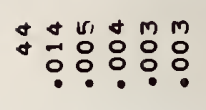 & $\sigma$ \\
\hline 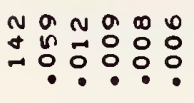 & 足离 & $\stackrel{m}{N}$ : & 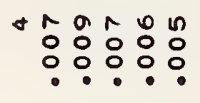 & + & ம) & $\begin{array}{r}\circ \circ \\
\circ:\end{array}$ & ०: & ㅇ: & 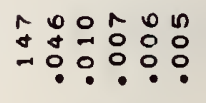 & 0 \\
\hline $\begin{array}{l}n \\
N \\
\end{array}$ & & : & * & + & n: & $\begin{array}{r}\circ \circ \\
\circ\end{array}$ & $\circ:$ & ㅇ: & 응응 & \\
\hline î & 4 & $\stackrel{\infty}{\infty} \stackrel{0}{0}$ & 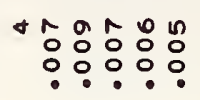 & + & n: & 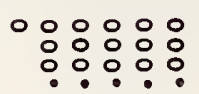 & $\circ:$ & ㅇ: & $\overrightarrow{0} \frac{a}{0}$ & \\
\hline
\end{tabular}

* J m m

$\min \left(\frac{1}{2}\right)$ ก ก.:

in $\rightarrow$ a

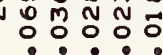

$\stackrel{m}{\text { Noj }} \stackrel{\infty}{\infty} \stackrel{\infty}{d}$

nR

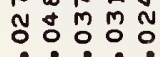
n n

소요요

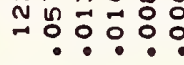

\section{i⿱宀}

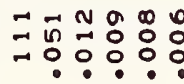

\section{N}

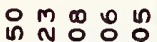

능유:

กำ
然管

N

竞

$\frac{0}{0}$

동ํㅇㅇㅇㅇ

$m:$

$m \div$

응ㅇㅇㅇㅇㅇㅇㅇㅇㅇ

no 00

응응응융영

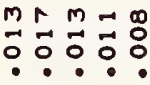

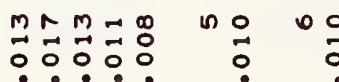

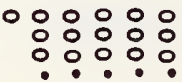

웅형ㅎㅇ용

$m$

$m$ in

응ㅇㅇㅇㅇㅇㅇㅇㅇ

:

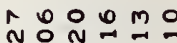

กูกับ กัํํำ

Non 0

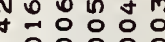

믕ㅇㅇㅇ

우요

N告

in $\operatorname{to}$ 요

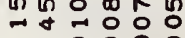

- ㅇํㅇ응

mㄱำ

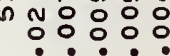

$M N$ No

$\div \div$

$\frac{0}{0}=\frac{0}{0}$

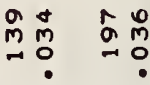

N $m$

$\stackrel{M}{M} \stackrel{0}{m}$

ถึ. a

n * *

N

$n$

n e

뭉

w

:

品

$0 *$

* *

艉

*

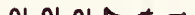

*

*

* *

on in

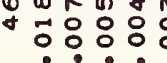

(

空 N

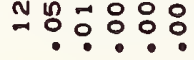

ํํํํํㅇㅇํㅇㅇํㅇ 응응
只告 $=$ 舟

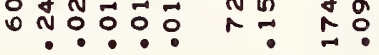
ํ.ำำำ

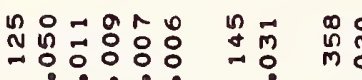

in $: \stackrel{9}{=}$

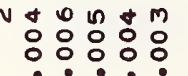

$\stackrel{\substack{\infty \\ 0}}{0}$

0

ํํำำ $m$ ?

:

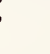

8

$\stackrel{0}{\circ}$

$\stackrel{0}{\circ}$

$m \stackrel{m}{\circ}$

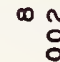

mIN

응ํำ

100

웅융융ㅇㅇㅇ

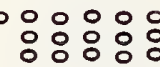

$\because: 0 \%:$

0000

응용ㅇㅇㅇㅇㅇ

○。 000 $\therefore: ㅇ: \div$

m 응

00

웅

$\circ \circ 0$

응

$\circ$

$\circ$ 웅
$+$

$0 m \infty+\cdots$

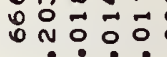

$\hat{\alpha}$

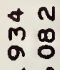

ann 눙융ํำ

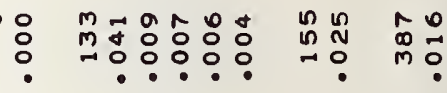

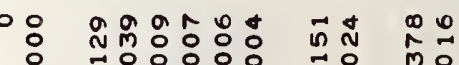
유: 음 in
管总

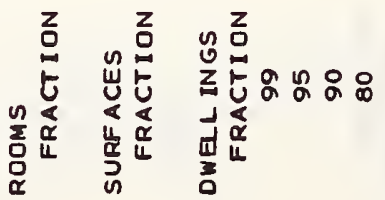

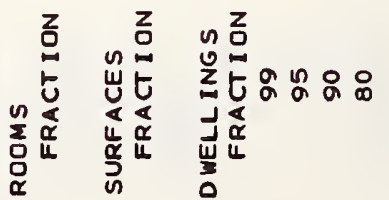

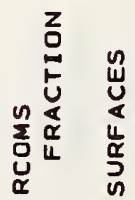

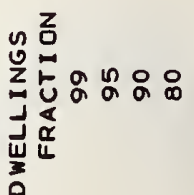

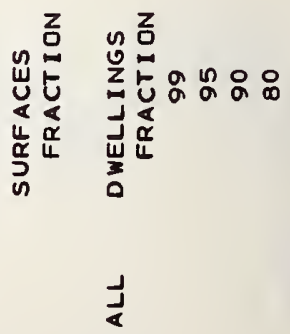

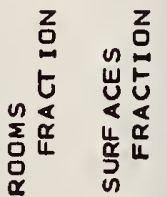


นึ.

N กำ

$\operatorname{com} m-$ 0 ก
$\infty$

N

นกㄴ

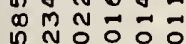

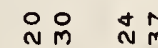

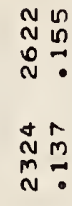

$\pi \infty \infty+n$

$\hat{m} \pm \overrightarrow{0} 0 \overrightarrow{0} 0$

离

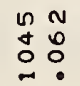

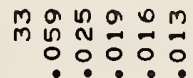

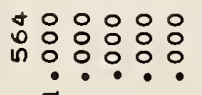

N $M \vec{M} \sim M O$

N

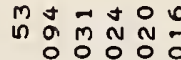

$N+\infty N \infty$

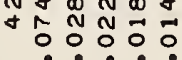

$\infty$

售

N

$: \begin{array}{ll}m \\ 0 \\ 0\end{array}$

a no

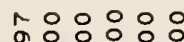

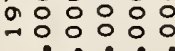

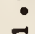

no 000 m: 0000

กั:

ํํㅇํㅇㅇำ

용

웅ㅇㅇㅇ응

ํํㅇㅇㅇㅇㅇ

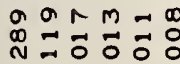

NA

-・?

年

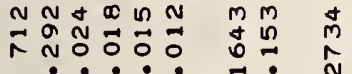

กิ

$+4 \infty$

$+\frac{0}{0} \pi 000$

$\min 2 \pi \pm$

$\begin{array}{llllll}0 & \text { n } & 0 & 0 & 0 & 0 \\ 0 & 0 & 0\end{array}$

$\hat{\circ} \vec{m} \quad \vec{N} \vec{m}$

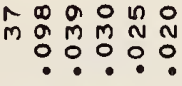

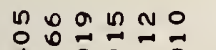

\& $\because \overrightarrow{0}: \overrightarrow{0}: \overrightarrow{0}$

$\stackrel{n}{N} \underset{0}{\infty}$

0

$\begin{array}{ccccc}N & 0 & 0 & m & \infty \\ M & M & N & N & 0 \\ 0 & 0 & 0 & 0\end{array}$

in

in $000000 \quad m \circ \quad m \circ$

ñ

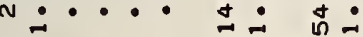

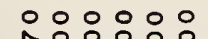

in :ㅇㅇㅇㅇㅛ

70 a $\div$ ?

m. $m$ ?

000000

$\begin{array}{llll}0 & 0 & 0 \\ M & M & N & 0 \\ 0 & 0 & 0 & 0 \\ 0 & 0\end{array}$

$\stackrel{0}{\circ}$

$m \circ$

ํㅇㅇㅇㅇㅇㅇㅇㅇ

in: $\mathrm{M}:$

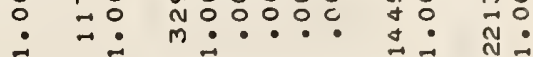

$\frac{0}{2}$

r $r$ r

$\infty \circ m \circ \infty 0$

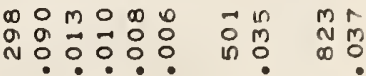

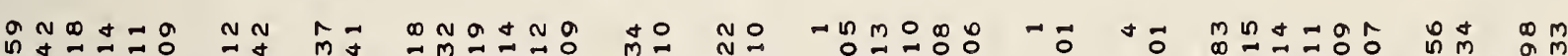

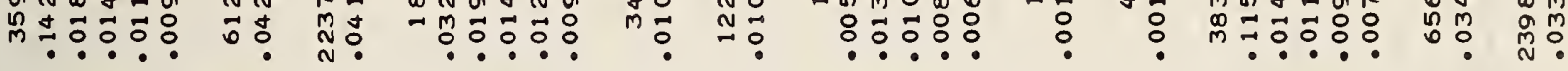

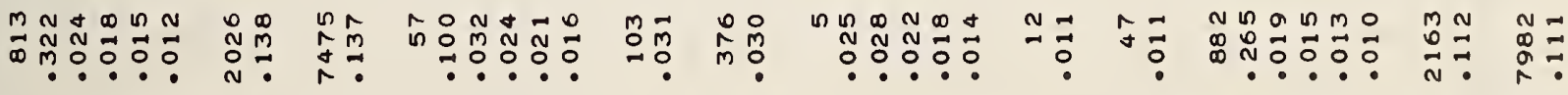

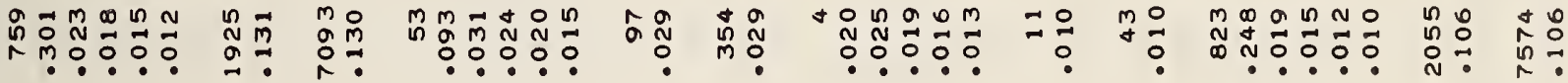

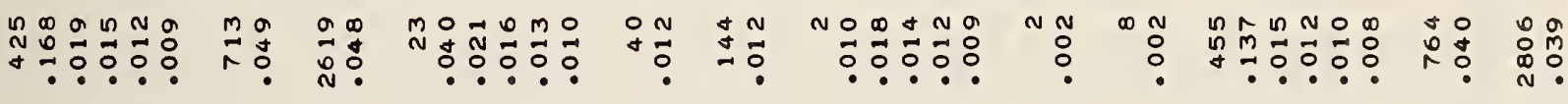

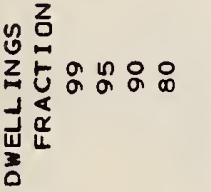

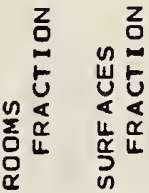

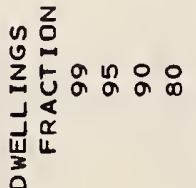

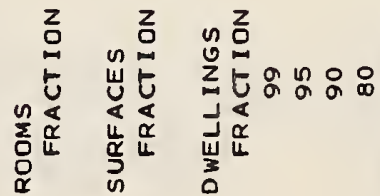

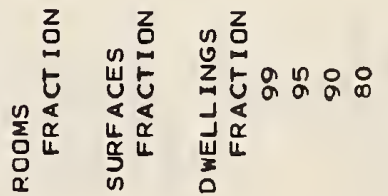

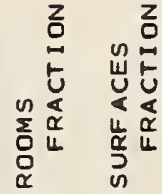

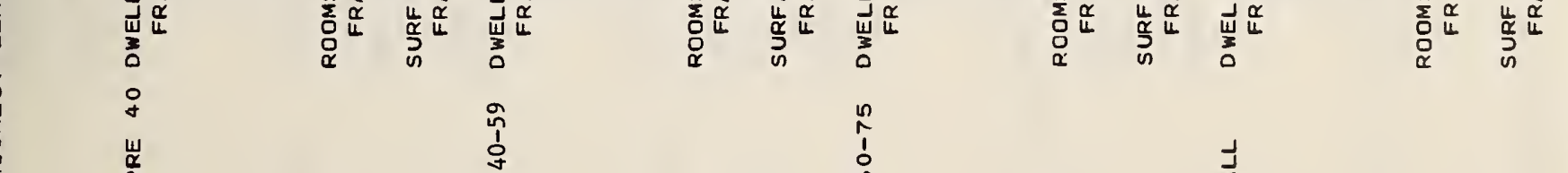




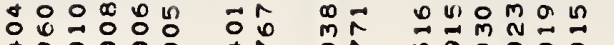
每

\section{0 n}

กั:

$\begin{array}{lll}n & n \\ m & m & m \\ m & 0 & 0\end{array}$

盀

舟舀

an $n \pi d=$

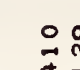

on

号宫

○ \&a-0m-

*

*

กิ่

$\infty n \pi m=0$

$m m=m=a \quad \hat{n}$

$m \div 0$ 뭉ㅇํㅇ
$\stackrel{0}{\stackrel{D}{N}}$

ํำ

กั

Nom $m_{0}^{0.0000}$

n noana n

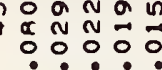

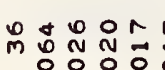

...

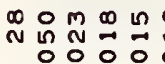

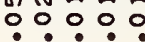

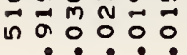

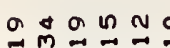

$\circ n$ 蕰 $0 \frac{0}{0} \div \frac{0}{0} \div$
Nิ

N

a

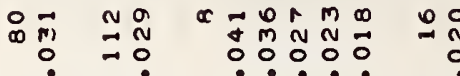

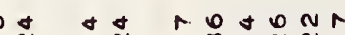

ก a

ma in

: n

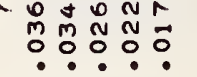

m n $n$ ก 0.0000
$n=0$
$2 \frac{0}{0}$

$m$ :

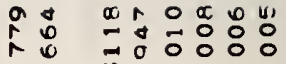

$\cos 2$

N

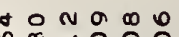

N

na $a n m+n a$

年: $0: 00$

(4)

mino a

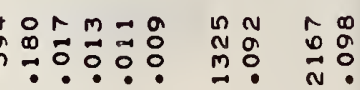

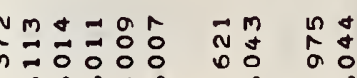

*

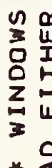

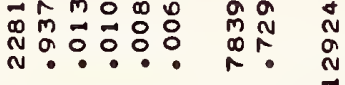

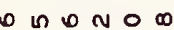

กำ 0000

No

$\rightarrow m m r n=$

คำกำ

$\stackrel{\substack{\infty \\ 0}}{\substack{n \\ m}}$

N $\mathcal{N}^{M} \vec{N}+0$

ถูก N

$\stackrel{m}{\infty}=$

*

*

* 嵌

$\rightarrow \infty \infty+n a$

m.

min
웅용

$+0$

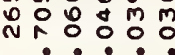

的 $\underset{N}{0}$

Mํ.:ำ:

$\vec{m}$

$\vec{m}$

nimo요

ตำํํำ

o m 0

$0: 0000$
0.000

nom n

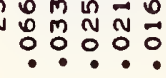

m

\&

N

in

n

$\stackrel{\infty}{+}$

䑤

$F \stackrel{N}{N}$ in

mั

ดั ข
0

ก

?

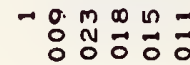

品

0
+0
$m$
$m$
0
0
0

- o n n

0
0
0
0

- $\begin{array}{r}m \\ \vdots\end{array} \stackrel{0}{0}: \stackrel{0}{0}=$
ก

$\begin{array}{ll}\infty & m \\ \infty & m \\ m & 9 \\ 0 & 0\end{array}$

罚

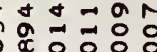

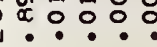

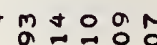

ㅇํㅇํㅇㅇㅇㅇ

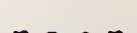

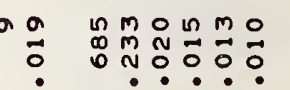

$\sum \stackrel{2}{=}$

ก

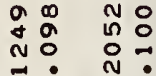

$-m$
$\vdots$
0

$-\frac{1}{0}$

ำกำำ웅

ì 2000

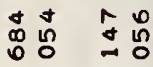

*

$+0 \infty 010$

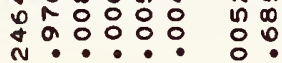

$\infty 0$

\&

$a \infty \quad 0 \rightarrow \Rightarrow n m a=N$

in

㖞

Aี -

aㅇํ의. a

‥・…

ํํㅇํㅇ ํํㅇ

$\circ: \circ: \circ: \circ$

$\circ:$

जे:?:ㅇ

*

mำำํำ

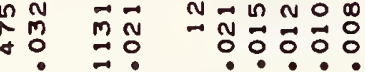

:

…

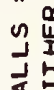

$m \sim m n n=$

\section{$\infty=0$}

N N

$\stackrel{+}{\circ}$

品

mion

$\overrightarrow{0} \stackrel{\infty}{\stackrel{0}{N}}$

m n N N

$\vec{\circ}$ 응 $\overrightarrow{0} \overrightarrow{0}$

$\sim:$

n.

in

กำ

$\stackrel{\infty}{0}$

og an

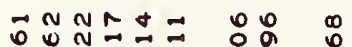

*

บับ

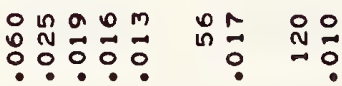

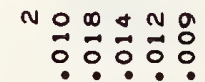

0 เก

$\stackrel{n}{m}$

$n=\infty \neq n a$

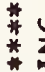

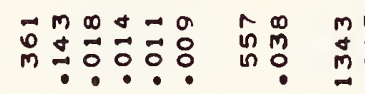

용 N No

- $\begin{array}{rlll}n & 0 & 0 & 0 \\ \circ & 0 & 0 & 0\end{array}$

$m$

亏

ก

ON $\sim \sim \overrightarrow{0} \overrightarrow{0} \overrightarrow{0}:$

?

N n

$\div 0: 0: \circ$
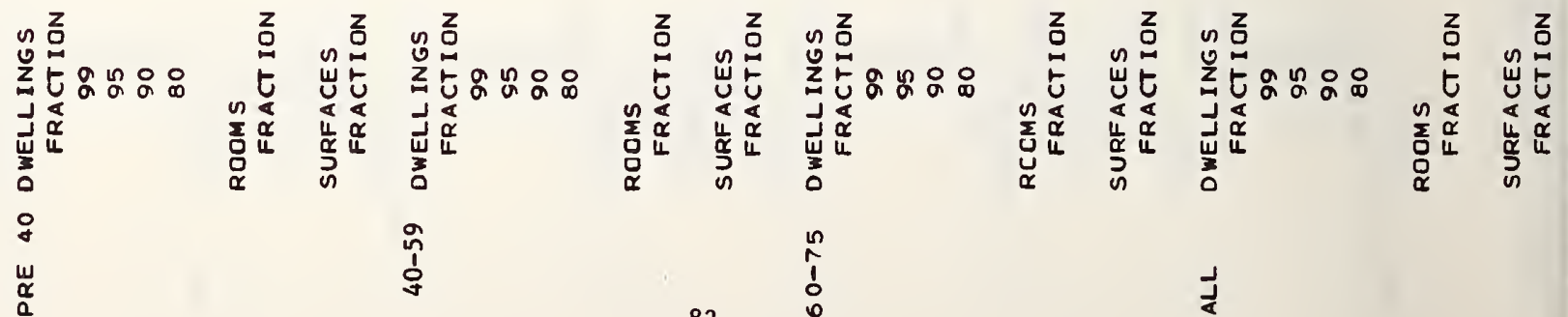


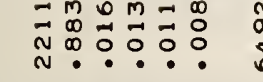

$\begin{array}{lll}N & m & n \\ 0 & 0 & m \\ 0 & n & 0\end{array}$

not o o a

กี $=0$

욤ำ

$\approx m$

in a

a $=0 N O \infty$

m $\vec{c}$

$\begin{array}{lll}0 & 0 \\ 0 & 0 \\ 2 & 0\end{array}$

000 a

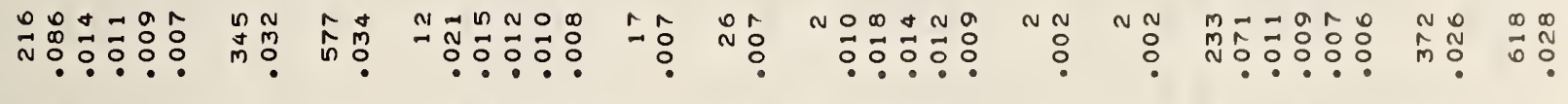

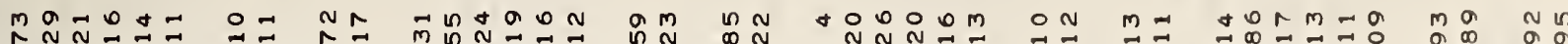

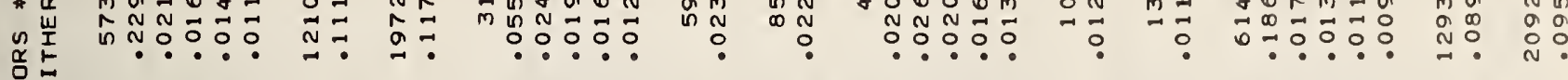

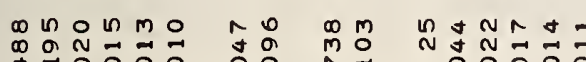

a a $a$

$m$ ก $N$ ก

$a \rightarrow \quad n$

o $\quad N \infty \in N O \infty$

ar $\quad$ a

$\overrightarrow{0} 0 \overrightarrow{0} \cdot \overrightarrow{0} 0 \quad \overrightarrow{0}-\overrightarrow{0}$ กㄴ.

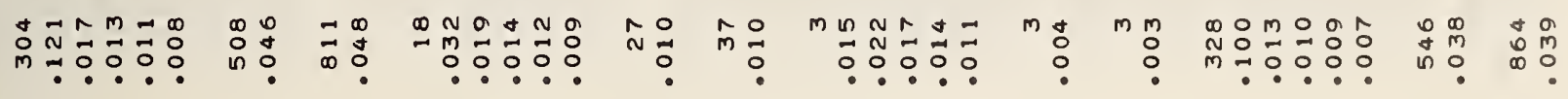

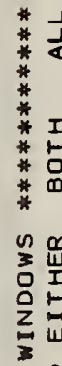

ํํ워ำ N

Mี

må

$\rightarrow a n N+$

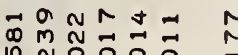

-. 의

ตั

กำ

$0 \rightarrow n \rightarrow 0$

$\stackrel{m}{m} \div \overrightarrow{0}=0$

กิ:

in
I

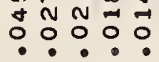

$\hat{m} \stackrel{\infty}{0}$

$\begin{array}{llll}0 & 0 & 0 & 0 \\ N & N & 0 & 0\end{array}$

N

OOO O

Nี

m

$\stackrel{\infty}{0}$

$\stackrel{0}{N} \stackrel{a}{N}$

$\stackrel{\infty}{N} \hat{0}$

隹

máo no

N ${ }^{m}$ M $\mathrm{N} N$

mia o n o

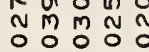

ㅇํ으

$\stackrel{+}{\sim} \frac{0}{0}$ $\ddot{m}=$

? $\begin{array}{rrrrr}9 & m & \infty & n \\ 0 & N & 0 & -1 & = \\ 0 & 0 & 0 & 0 & 0\end{array}$ no

ก

$\infty$

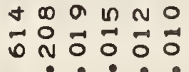

$\infty N$

0

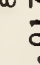

$\ln \infty \infty \pi \infty$

NN

$\begin{array}{rr}-m & r \\ 0 & -1 \\ 0 & 0 \\ 0 & 0\end{array}$

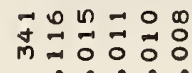

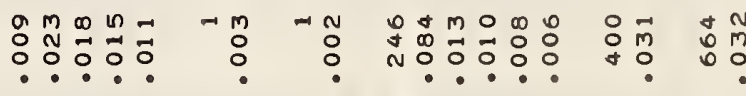

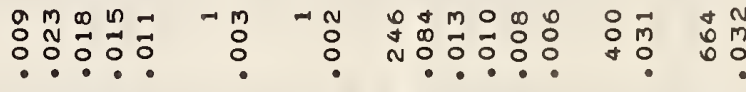

a a 0 in $\stackrel{0}{0} \stackrel{n}{N}$

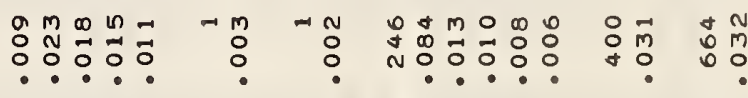

$\begin{array}{lll}n & 0 & n \\ m & 0 & 0 \\ N & 0 & 0\end{array}$

n $m$ in

○

苟吕

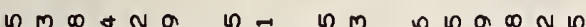

nin $m$ ลง

nी

ㄴ 0 \%

Na

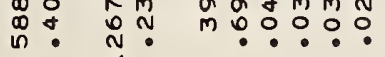

ma

$\stackrel{\substack{n \\ 0}}{\ln 2}$

웅

in

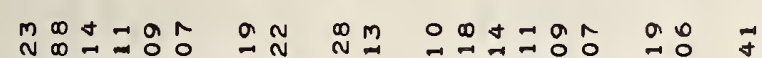

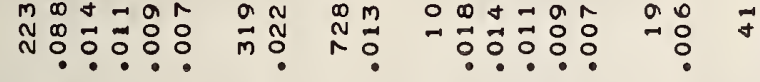

$\cos \overrightarrow{0}+\overrightarrow{0}$

on

n n $\quad 0-1$ o n 0 in mo

N

म००००

$\stackrel{\circ}{\infty}$

$\stackrel{\infty}{m}$

$0 m \infty m-a$

กิ $m \infty$

ㅇㅇㅇㅇㅇㅇㅇ

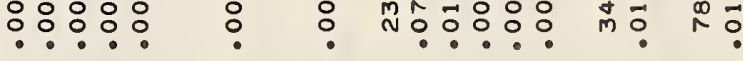

ก

مึ: N N

N $\left.\begin{array}{c}0 \\ 0\end{array}\right)$

no $\infty+n d a$

$m m$

웅 중

$00 \wedge m-1$

N $\mathrm{n}$

뭉

กำ

ㅇ. MO

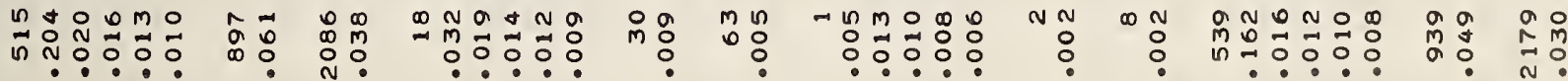

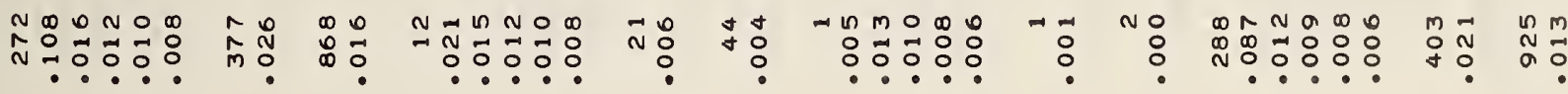
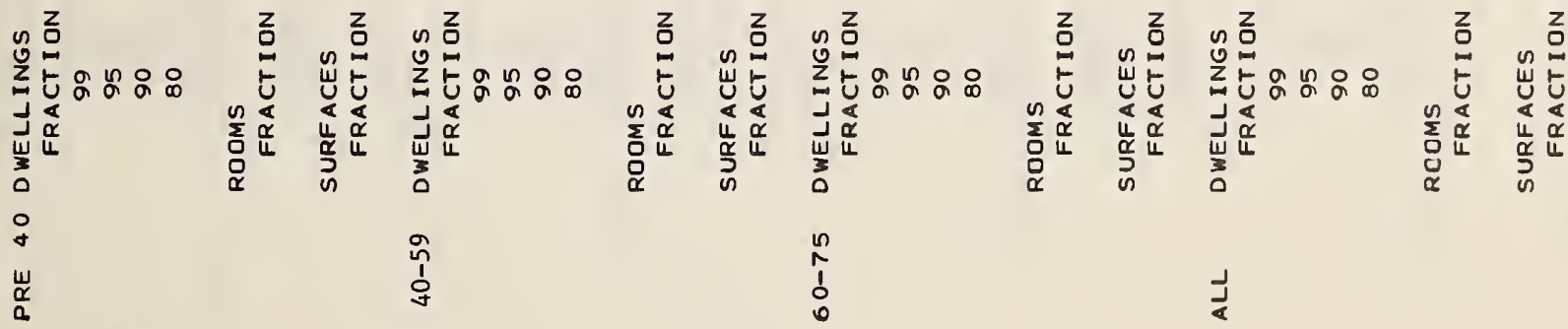


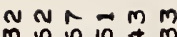

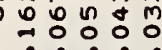

ก

$\approx \overline{0}$

$\vec{m} \mathscr{D}^{\circ} \mathrm{N} \cong \pm$

$a m$

$\stackrel{n}{2}$

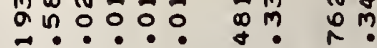

* a ingor a na nanamo nu an nomano

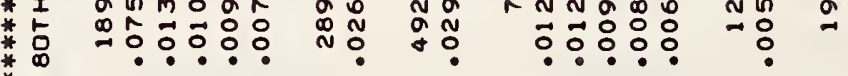

$\alpha \quad \infty$

: $\overrightarrow{0}: 0$ :

N

N

$\overrightarrow{0}-\infty r n$

은 $\quad n$

กั ญั

$\stackrel{u}{I}$

$\stackrel{\infty}{\rightarrow} \stackrel{0}{0}: 0$

\section{in}

in

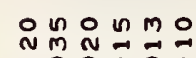

ñ

nOD

뭉ㅇㅇ

$+$

in

on

a. $\quad$ in

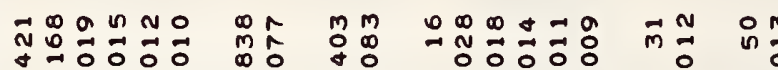

*

*

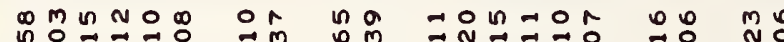

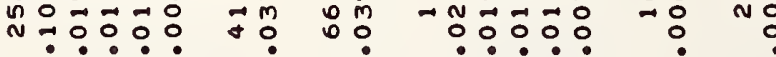

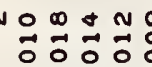

$\because 0 \%$ :

$+$

n

nom $⿻$

$\overrightarrow{0} \overrightarrow{0} \overrightarrow{0} \overrightarrow{0}$ :

N

N

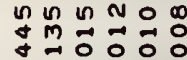

$\underset{\infty}{\infty}$

nก

?. ㅇ․

\&?

* J

ํ.

d $m$ n $N$ in

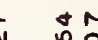

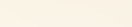

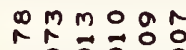

$\rightarrow$ : ००:

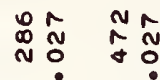

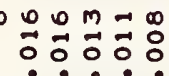

을

no $00 \mathrm{mo}$

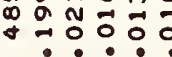

No

or

$n$
$\infty$
0

$m$ m $\infty$ in $N$

$\infty \infty a n n$

$\infty_{0}^{\infty} 00 \cdots 0 \quad \infty$

$*$

+ : : ?

$\stackrel{\infty}{\infty}$

å

$\stackrel{0}{2}$

?은?

a $00 \mathrm{nos}$

$\rightarrow$

*

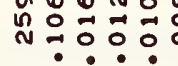

N

$\stackrel{m}{2} \stackrel{0}{0}$

n $m \infty n$

: $: \overrightarrow{0}: \overrightarrow{0}:$

$\rightarrow$

N

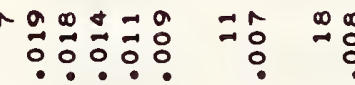

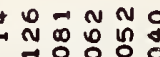

ำ

$\stackrel{\infty}{N}$

nn

ก $ヘ M \cap n=$

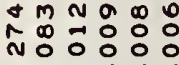

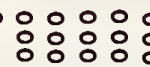

ㅇ?ㅇ

0

$\circ$

- $m=a \hat{0}$

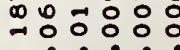

Non n n

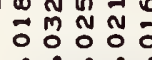

n m n

$m=\infty \neq 0$

0.00

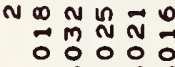

$\frac{m}{0} \quad r$

$\underset{N}{\infty} \stackrel{0}{0}=0$

雍:

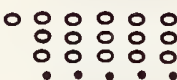

$\circ$

$\circ:$

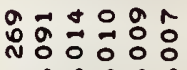

$\underset{m}{a}$

$\stackrel{a}{m} \stackrel{m}{m}$

*

*

我

起吉

*

*

*

*

* Z

ar n a 0

กี

$\ln \pi 0^{2}$

$+1$

N

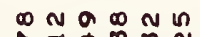
(n)

a.0.0.0.

N

Nm a

กั

in

a

$\stackrel{0}{0}$

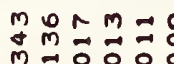

in

anmon

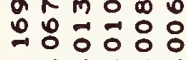

ลู o

n

$\underset{N}{n}$.

$\rightarrow$

iño

ก
$\operatorname{M}_{0}^{0} \stackrel{0}{0} \div 0$

등ㅇㅇㅇㅇ

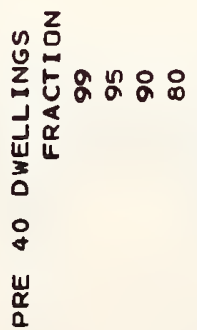

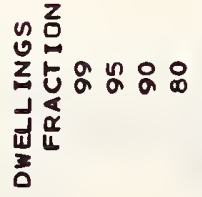

오ำ

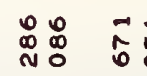

年 。

ก บ

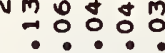

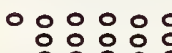

ㅇㅇㅇㅇㅇㅇㅇㅇ

$\stackrel{\circ}{\circ} \vec{m}$

M̂

N

M̊ \&

ก。ำ

+̊ $\rightarrow$ in $m \circ \infty$

잉

-

웅ㅇㅇㅇㅇ

000000

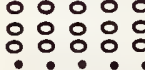

mo $\begin{gathered}\infty \\ m \\ 0\end{gathered}$

N

A $m$ in

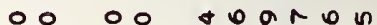

$\circ: 0$

in

NNAN $N$

ก

N

응

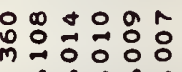

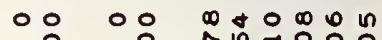

응ํㅇㅇㅇㅇㅠ.

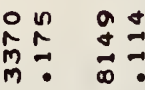

a $=a r$

능 융

$\rightarrow \infty$

in:

$\rightarrow \underset{n}{m} \rightarrow$

응

$\infty N+\infty$

No in:

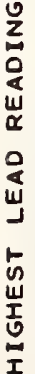
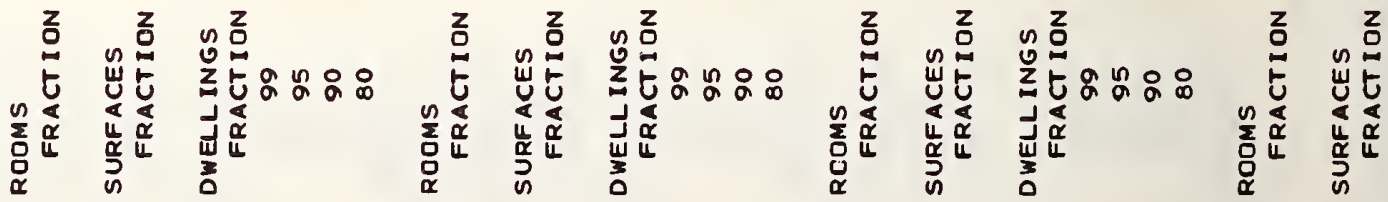

n 

m N $N=$ m ! ? : ?

ก mำำ

웅

$n \sim \infty+n a$

$\infty$ in $\rightarrow \pi 0$ กิ

$\underset{\pi}{-1}$

$\sum_{m}^{m} m \underset{m}{m}=0$

\section{0
$\infty$
0}

$\infty$ n $m \circ 0$

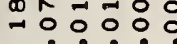

$\infty$

$\stackrel{\infty}{\infty} \stackrel{0}{0}$ $\stackrel{\infty}{\infty}$ in ? $\overrightarrow{0} \overline{0} 0$ 웅ㅇㅇㅇ

\section{$0 x+a$} $\begin{aligned} & \infty \\ & 0 \\ & 0\end{aligned} \overrightarrow{0}: 0$ $\stackrel{0}{\circ}$

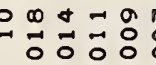

\section{$0=-\infty \sim n$}

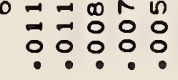

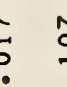

ㄷำ

品

ก)

N

$\stackrel{0}{0}$

$m=$

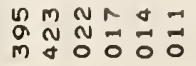

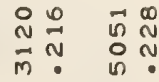

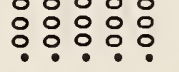

$\circ$

$\circ \circ$

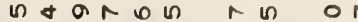

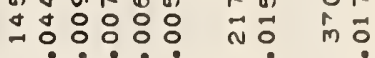

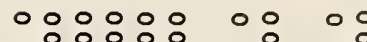

$\therefore$

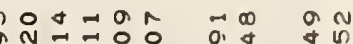

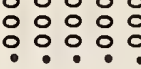

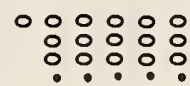

$\circ:$

$\circ 8$

m

in

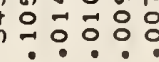

$\begin{array}{lll}0 & 0 \\ 0 & 0 & 0\end{array}$

$\infty m m m 000000$

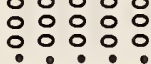

$\circ$ :
$0000 N$ L

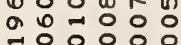

$\circ \circ$ $a \rightarrow a n$

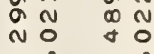

*

\section{r O O O M}

กำ

竞

$\stackrel{0}{0}$

ก ก

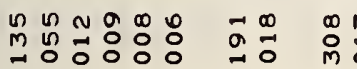

- ㅇํㅇํ․

$m_{i}^{\infty} \infty \pm N$

भก๊

๙ุ

$m+r=a$

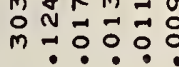

in

กิtâ

๓

N n $m$

ñ

ㅇํㅇ

㖞

?

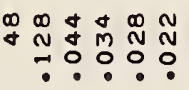

nm $\begin{gathered}\text { n } \\ -10 \\ 0\end{gathered}$

a d

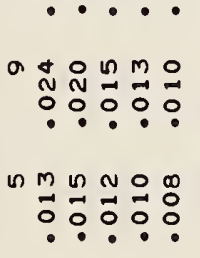

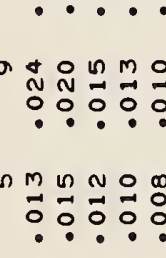

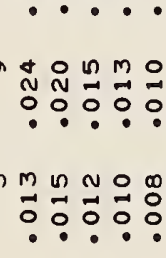

No $\cos 0$

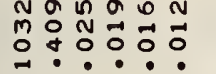

$\stackrel{\circ}{m=} \stackrel{0}{=}$

윰ำ

ตㄴ.

ก NOD n

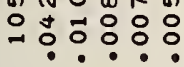

m.ำ

ก

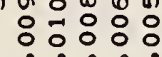

$\infty+m 00$

$\begin{array}{lll}\infty & n & -1 \\ 0 & \stackrel{0}{N} & 0 \\ 0 & 0 & 0\end{array}$

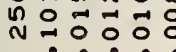

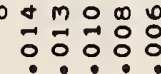

$\infty+m \circ \infty$

둥ㅇㅇㅇㅇㅇ

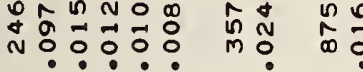

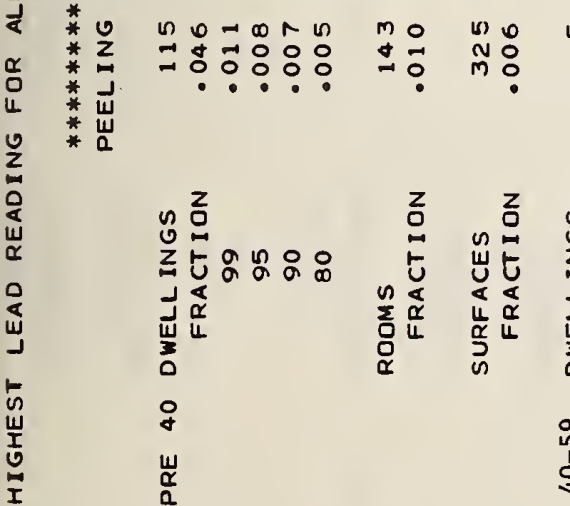

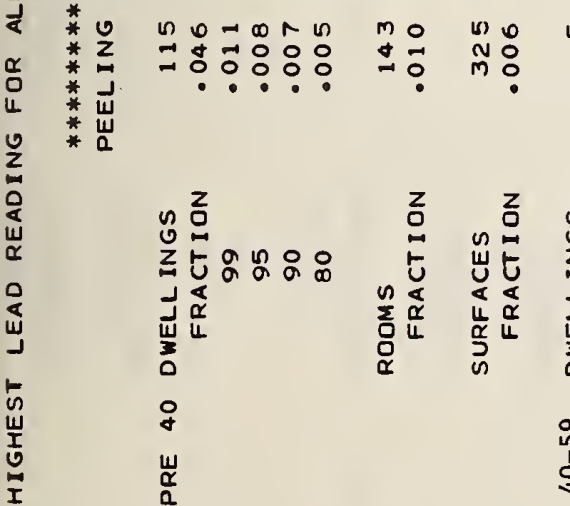

$\stackrel{2}{2}$

$\infty$

00

-

$\stackrel{0}{\circ} \underset{0}{0}$

$\infty$

: $=$ $\infty$

ำ

$=0$

:

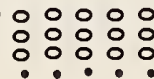

N $\frac{1}{0}$

$N \infty N \underset{M}{N} \mathbb{N}$

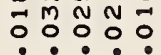

Non $n \rightarrow 0$

$\begin{array}{lllll}\infty & N & n & 1 & 0 \\ 0 & 0 & 0 & 0 & 0\end{array}$

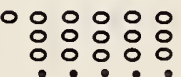

$=\stackrel{0}{0}=\mathrm{m}$

$\therefore \circ:$

$w$ n $m$

N in

$m$

(n)

$\vec{m} \circ \vec{\circ}=00$

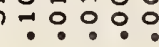

$\circ:$

$\circ \circ$ $m N m \infty n n$

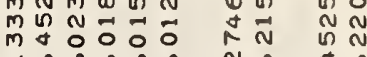

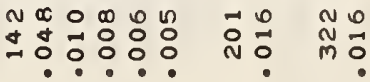

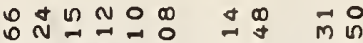

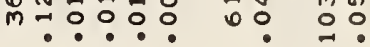

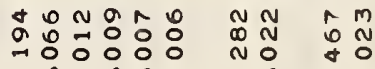

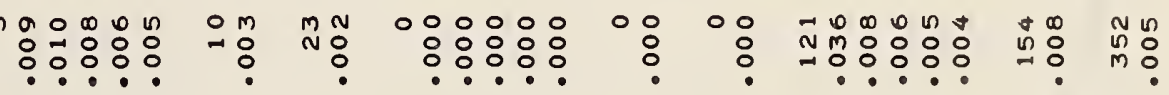
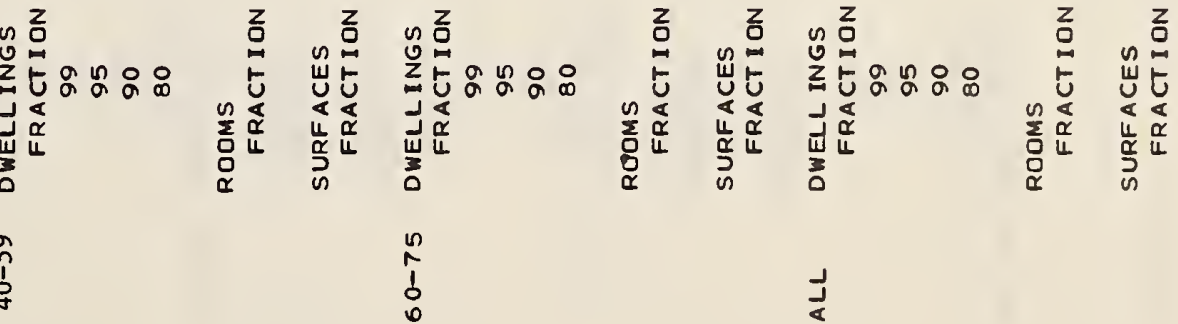
g:

*

\#

*

*

*

*

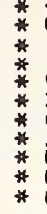

\section{* J o n n a}

*

ब年:

N $\infty \circ \infty$

mo용

$n$
3
0
2
2
3
$*$

กํํํำ

$\Rightarrow r=0$

กั

*

U

* $\quad$ * $\quad$ *

岃

음

*

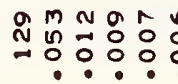

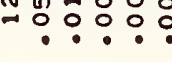

RัM

*

*

คํํㅇㅇㅇㅇㅇ

긍ㅇㅇㅇㅇㅇㅇ

(

n

n

*

*

*

$\stackrel{\alpha}{\alpha} \quad *$

z

造

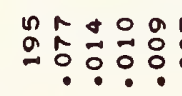

ㅇํㅇ?

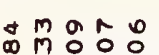

끙ㅇㅇㅇㅇㅇㅇ
政 $\div \div$

$+\frac{m}{2} \stackrel{n}{n}$

\section{?}

+

$-10$

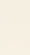

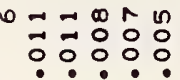

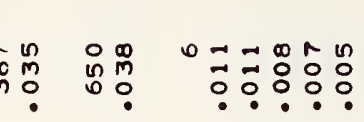

$\begin{array}{lll}\circ & \infty & 0 \\ \text { No } & 0 & m \\ m & 0\end{array}$

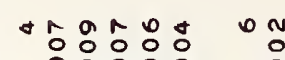

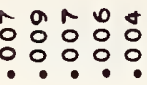

冓

:

$\infty m=$

$\infty m$

$m \cong$

-

$\circ 0: 08$

융ㅇํㅇ

$=0$

ำำㅇำ웅 : $00 \%$

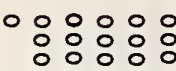

000000

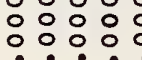

융유 0 :
N N

N

?

-응

ำ

$\exists \frac{0}{0} \quad \overrightarrow{0} \frac{N}{0}$

O O O nNNON0

舟 $\rightarrow 00$ กी

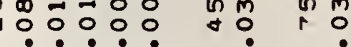

\section{$\circ 0$}

dandaro

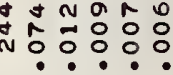

a $a+0$ a

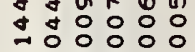

on

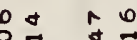
No mo

$\begin{array}{llll}0 & 0 \\ 0 & 0 & 0 & 0 \\ 0 & 0 & 0\end{array}$

N

$\underset{\sim}{\infty}$

$\vec{m} \vec{n}$

$\mathfrak{m}_{0}^{N} m$

ma응

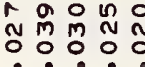

$m$

$m$

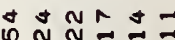

의

n $n \hat{m}$

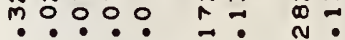

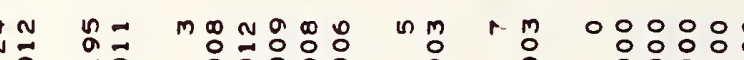

0
0

$\begin{array}{rr}\circ & 0 \\ : & :\end{array}$

مू

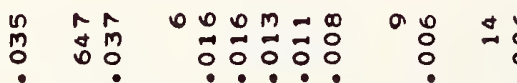

$\circ \circ \circ: \circ \circ$

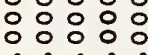

0

$\circ 8$

$M \circ M \circ \infty$

$\circ \rightarrow-n$

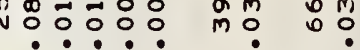

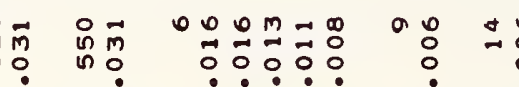

$000 \% 0 ㅇ$

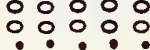

$\circ 8$

$\circ:$

Non O. D

กั N

$m o$

กำ

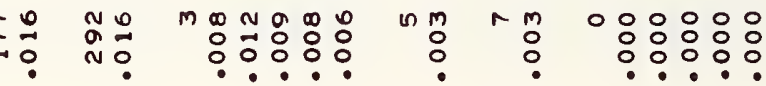

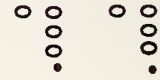

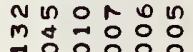

Non

ㅇํㅇㅇㅠ

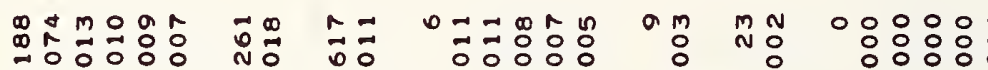

mm

$+2 d$

융.

Im 0 O

$m_{0}^{m} \underset{N}{\infty}$

ing

$\operatorname{m\in N} N \pm=$

$m m$

$\overrightarrow{0}$

nogn no

$\operatorname{lin}^{2} 00000$

MON No

on

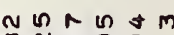

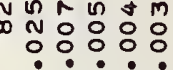

$\infty n+m$

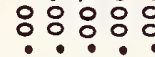

ㅇㅇㅇㅇㅇㅇㅇㅇ

$\because$

$n-m$ m n

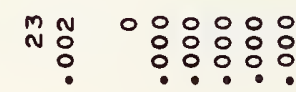

0

$\circ:$

N: $00: 0$

$\infty+00$

ㅇㅇㅇ응

$\circ$

$\circ:$

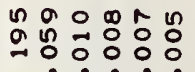

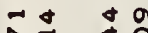

$\begin{array}{ll}\infty & 0 \\ 0 & n\end{array}$

+

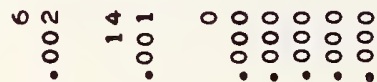

$0 \%$

$\circ:$

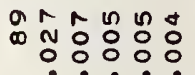

n $n$

응 $m$ ?

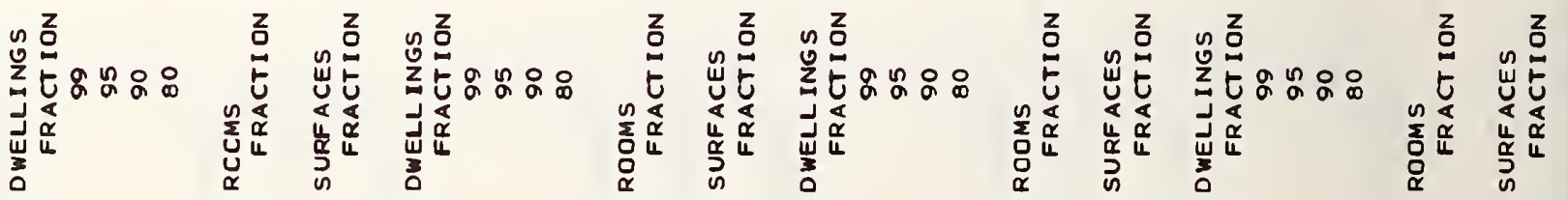




\section{APPENDIX B}

Set "B" Tables

Table of Contents

Occupancy Class

SINGLE-FAMILY

ALL

Room Type

DRY

WET

DRY

WET
Pages

88-99

100-111

112-123

124-135 


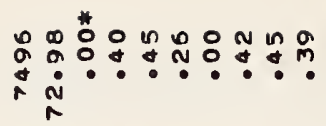

商 * 莫

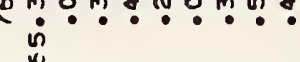

m $\dot{m}$

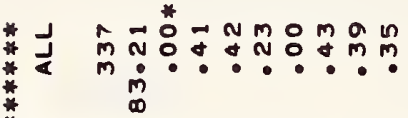
占是 * 品

* * * in an

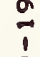
ó \%ㅇ * *

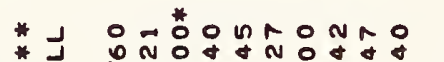
. o

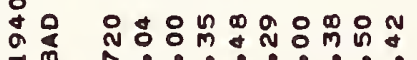
u 㟧 采

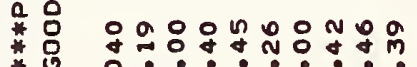
* nn in

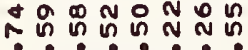
$\dot{\sim} \dot{m} \dot{\sim} \dot{N} \dot{m} \dot{\circ}$ $\dot{1}=\dot{n} \dot{0}$

a.

ヘ

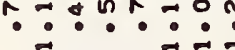

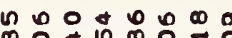

๓⿴囗十

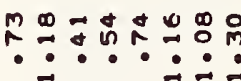
$\because \because \bullet \because \div \dot{-}$

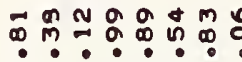

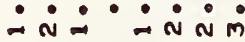

NMmD禹舟

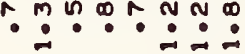

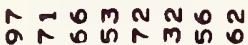

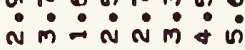

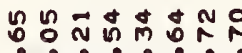

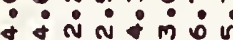

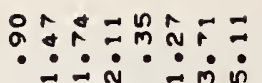

ถึำำำ a

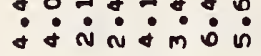

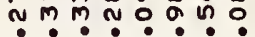

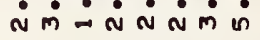

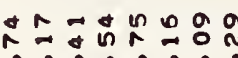

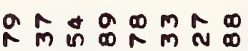

N กาำกำ m

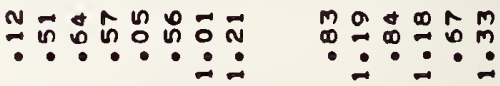

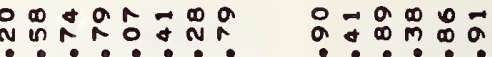

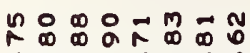
・・・・・

$m_{0}^{\circ} g$ a ー

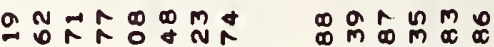

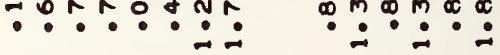

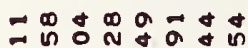
西

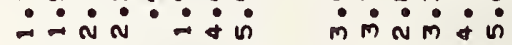

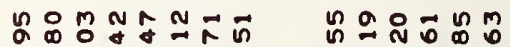

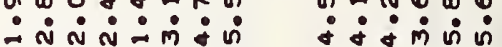

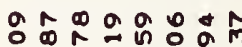
$\because \dot{\sim} \dot{\sim} \dot{n} \dot{m} \dot{m}$

$m$ móm D. N

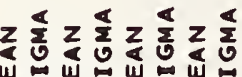

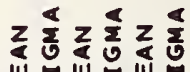

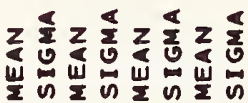

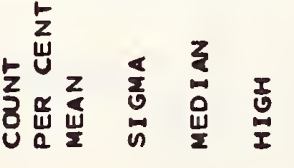

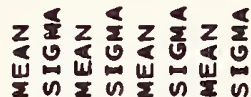

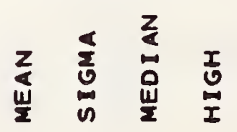




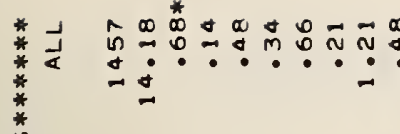

嵌是

当号

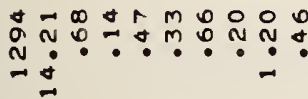

$*$
$*$
$*$
$*$
$*$
0
0
0
0
1
0
0
0
$*$
$*$
$*$
$*$
$*$
$*$

年

$\ddot{-}$

no -

$\rightarrow$

再

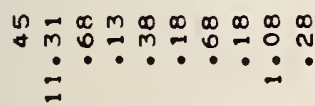

m

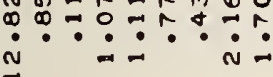

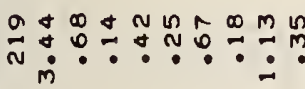

*

个)

$\dot{\sim} \dot{\mathrm{N}} \dot{\mathrm{N}} \dot{\mathrm{m}} \dot{\mathrm{a}} \dot{\mathrm{n}}$

Oํ.

$\dot{\sim} \dot{m} \dot{m} \dot{m} \dot{m} \dot{0}$

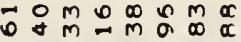

$\dot{N} \dot{m} \dot{\sim} \dot{N} \dot{N} \dot{M} \dot{\sigma}$

$\operatorname{cotag} \theta$

$\infty+\frac{0}{0}, \div$

$m=0 \infty m \infty q$

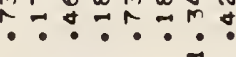

。ㅁ $\because-\infty m \infty \infty \infty \infty \infty \infty$

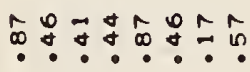

응응응응응응

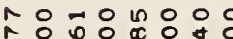

ํำ.ำ.

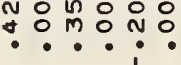

$+\infty$ n $q M \stackrel{m}{\sim} \stackrel{m}{m}$

$\because \cdots \div$

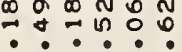

+ $n m a=0 \circ m$ $\because \because$ เก $\because \because \because \div$

Na

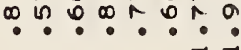

n?

n $⿻$ n

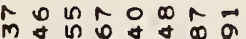

i: $\dot{n} \dot{i}=$

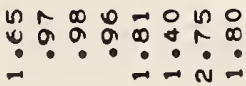

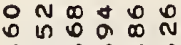

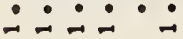

웡ำ $\therefore \div \div \div$

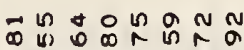

Normm

-.

$\therefore: \frac{0}{0}$

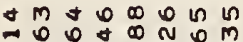
$\dot{m} \dot{m} \dot{-} \dot{N} \dot{m} \dot{0}$

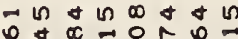
$\because \dot{-} \dot{i} \dot{i} \dot{j} \dot{j}$

n $m-A n d$

$\forall 0$ N

$\dot{\sim} \dot{m} \dot{N} \dot{m} \dot{0}$

Nลa

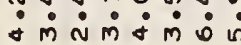

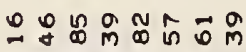
$\dot{v} \dot{v} \dot{\sim} \dot{\sim} \dot{\sim} \dot{0}$

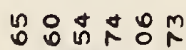
$\dot{m} \dot{m} \dot{m} \dot{m} \dot{n}$

$m \infty+\infty N$ in ค.

กิ: i $\dot{m} \dot{\sim} \dot{m} \dot{m}$ i

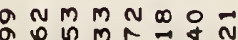
Ni் $\dot{i} \dot{N} \dot{m} \dot{\sigma}$

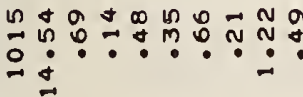
$N M=N N m+i n$

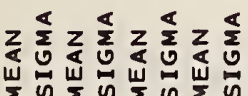
$z$

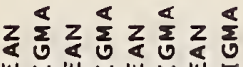
嵅的崖的崖的崖的

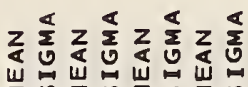

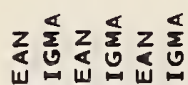
崖的崖的崖的崖崖的崖的崖的 


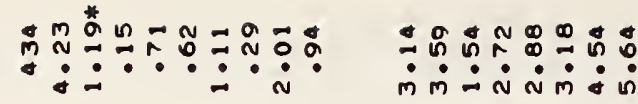

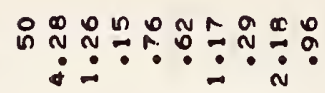

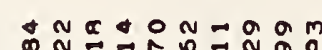

Mก:

\lrcorner ค

*

*

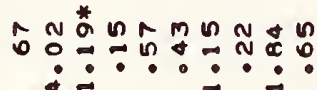

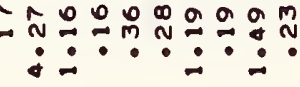

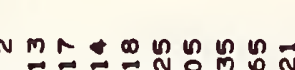

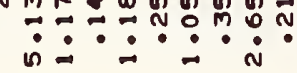

8

å o

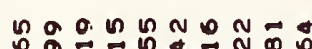

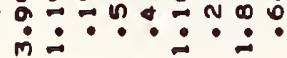

*

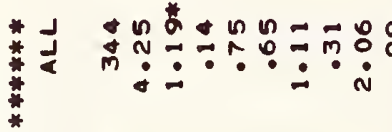

方卤

ำกำ

宸

章吕

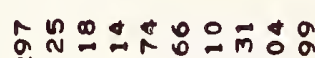

ลำ:ำ: $\dot{m} \dot{m} \dot{0} \dot{m} \dot{0}:$

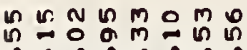

$\dot{m} \dot{m} \dot{\sim} \dot{\sim} \dot{m} \dot{m} \dot{0} \dot{0}$

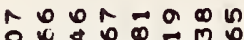

$\because \dot{m} \dot{0} \dot{0} \dot{0} \dot{0}$

$m m-N$ N $m \dot{*}$

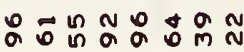

$\dot{-} \cdot \dot{i} \dot{i} \dot{N}$

:ㅇ:ㅇ:융

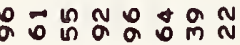

$\ddot{-} \dot{\sim} \dot{\sim} \dot{N}$

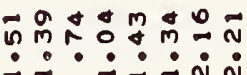

ตัด

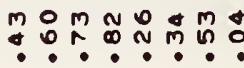

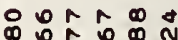

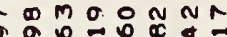

$\dot{-1} \dot{\mathbf{n}} \dot{0} \dot{0}$

$\dot{-\cdots} \dot{i} \dot{\alpha}$

$\because: \because: \because::: \quad: \because::::$

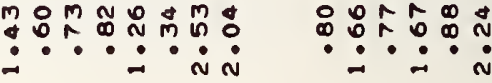

ํํำำ

nmonhOn-

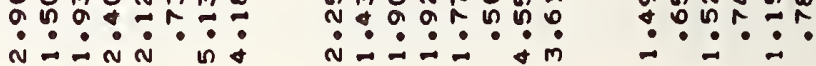

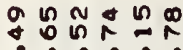

m $\dot{0}: \dot{\sim} \dot{n}$

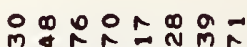

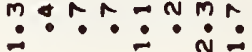

and Rn

-

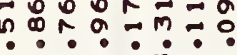

$\dot{m} \dot{m}=\dot{\sim} \dot{m} \dot{m} \dot{0}$

$\therefore$ in

$\dot{N} \dot{\sim} \dot{N} \dot{N} \dot{\sim} \dot{N} \dot{n}$

Noำ

$\dot{\sim} \dot{m} \dot{\sim} \dot{m} \dot{m}$

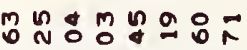
๓ं

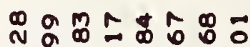

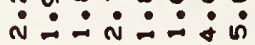

กำ

$\dot{m} \dot{\circ} \dot{m} \dot{\therefore} \dot{0} \dot{0}$

gกำ nMmo

nOำล

$=0 \hat{\infty} 0$

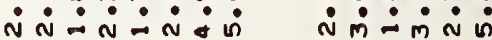

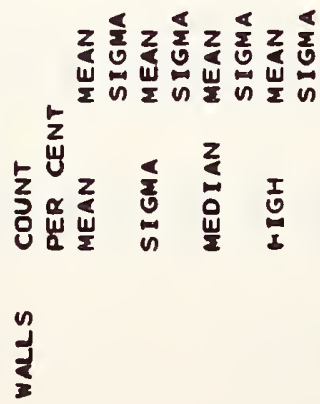

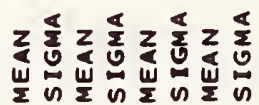

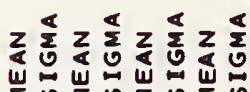

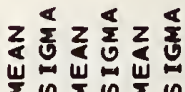

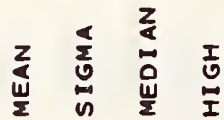

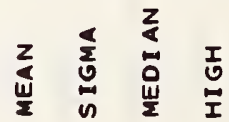

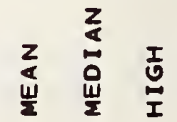

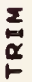

I 


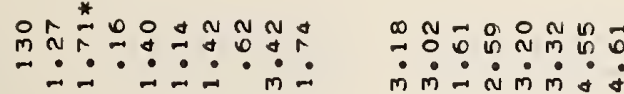

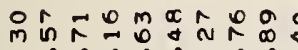

ก:

우

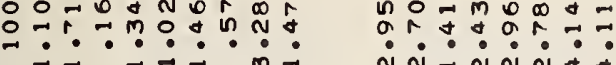

$\dot{n} \dot{n} \dot{n} \dot{a}$

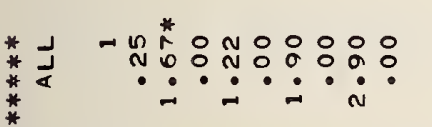

0
0
0

응ㅇㅇㅇㅇㅇㅇㅇㅇㅇ

1

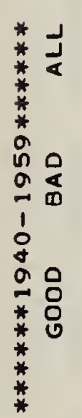

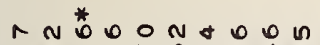

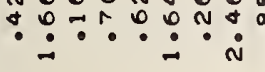

Da

ㄴ.

A n

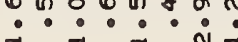

nma $=00$

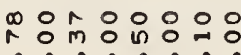
$\dot{n} \dot{n} \cdot \dot{n}$

N $\dot{m}: \div \cdot ?$

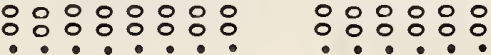

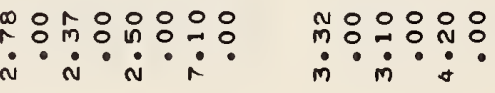

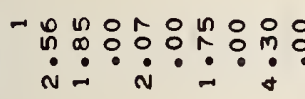

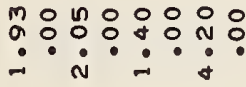

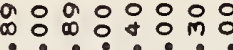

$\dot{-} \div \dot{-}: \dot{0}$

$\stackrel{\infty}{0}: m: \frac{n}{0}: \div:$

טิ

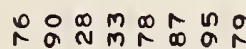

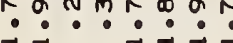

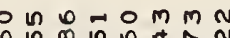

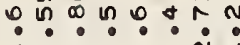

NoT

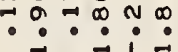

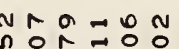

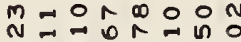

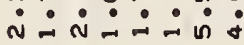

$\therefore \dot{m} \dot{\sim} \dot{m} \dot{\sim}$

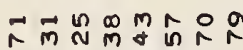
กับ

a. $\because \infty ? \div$ ํ.-ำ

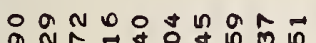
$\dot{-1} \dot{-} \dot{-} \dot{0} \dot{m}$

ดิ $\dot{N} \dot{N} \dot{N} \dot{N} \dot{j}$

OM $\overrightarrow{0} M O A$

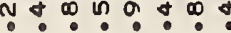
N

$n-n=\ln a$

m? $99 ?$

$-\dot{N}-\dot{N}$ a

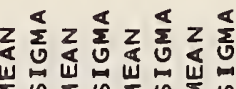

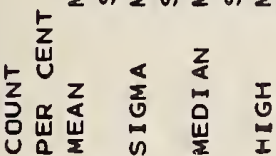




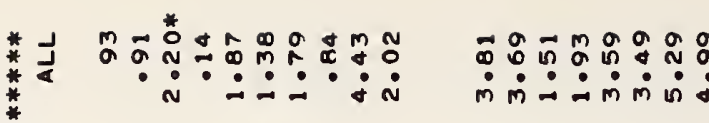

in

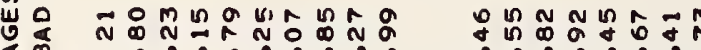

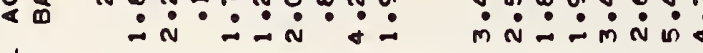

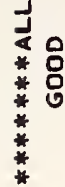

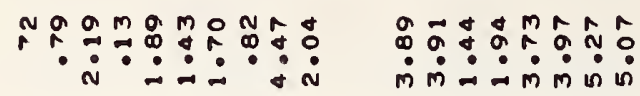

* د 00 草 000000

*

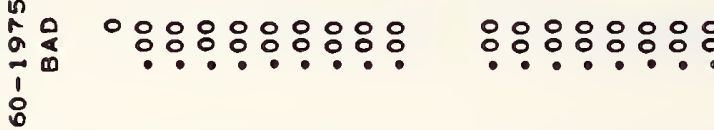

*

$\sum_{\substack{0 \\ 0}}^{n}$

है

응ㅇㅇㅇㅇㅇㅇㅇㅇ

융ㅇ:ㅇ:웅

ㅇㅇㅇㅇㅇㅇㅇㅇㅇㅇ

* •

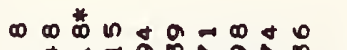

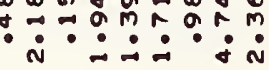

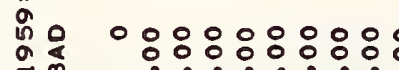

1

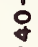

$\stackrel{n}{2}$

r *

*

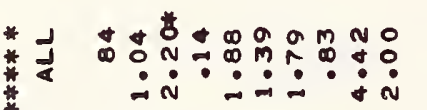

moma : ? : $:$ : $:$ :

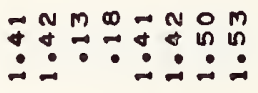

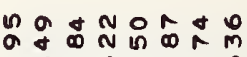

$\because \dot{\sim} \dot{-} \dot{i} \dot{N}$

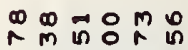

$i \div i \dot{N}:$

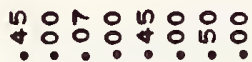

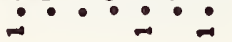

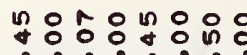

$\dot{-} \cdot \dot{-} \cdot$

웅웡ㅇㅇ in in

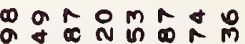

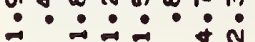

คัm

$\dot{-} \cdot \dot{a} \dot{-1}$

*

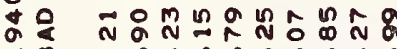

$\rightarrow \infty \quad \dot{n}: \dot{n} \cdot \dot{0}$

岩

$\frac{1}{a}$

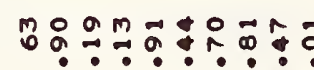

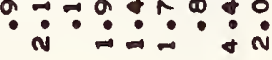

måğ

ㅇํำด ต

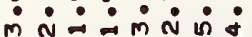

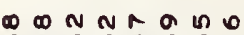

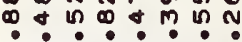
$\dot{N} \dot{\sim} \dot{\sim} \dot{\sim} \dot{0} \dot{0} \dot{8}$

$m$ m $m$ m $\because ?: \div \frac{1}{0}$ $\dot{\sim} \dot{m} \dot{\sim} \dot{m} \dot{\theta}$

뭉웡ㅇㅇㅇㅠ $\dot{N} \dot{\sim} \dot{\sim} \dot{\sim} \dot{\sim} \dot{m}$

$\vec{N}: 9 N N$ N $\dot{N} \dot{\dot{\theta}} \dot{\mathrm{m}} \dot{\mathrm{n}} \dot{\mathrm{n}}$

요요

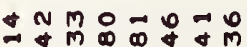
$\because \div 0^{\infty}$ $\dot{0} \dot{m} \dot{-\dot{m} \dot{m}}$ in

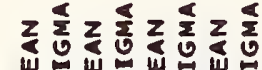

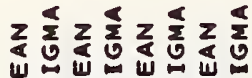

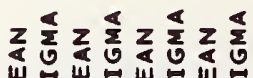

$z \frac{\pi}{2} z \frac{\pi}{0} z \frac{5}{0}$

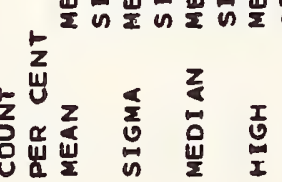

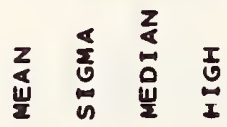

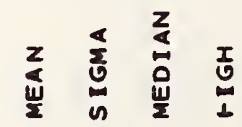

z $\frac{2}{2} \frac{I}{2}$

a)

$\underset{\substack{\alpha \\ \infty}}{\sum}$ 


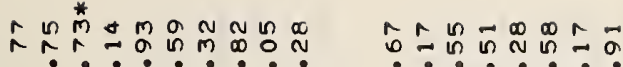
$\ddot{n}-\dot{i} \dot{\mathrm{N}} \dot{\mathrm{n}}$

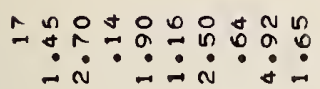

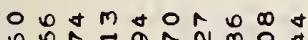

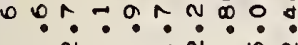

$\dot{m} \dot{\circ} \dot{i} \dot{m} \dot{m}$ in

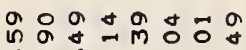

$\dot{m} \dot{1} \dot{-} \dot{m} \dot{m} \dot{0}$

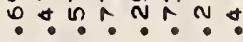

$m \circ \rightarrow$ in m i ar $\left\{\begin{array}{l}m \\ m\end{array}\right.$
웅ำ

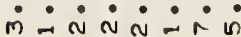

$\rightarrow$ moำ ำ $\dot{m} \dot{\sim} \dot{\sim} \dot{\sim} \dot{\sim} \dot{\sim}$

$\infty a m \infty a n$ $\because \because 0,0,0.0$ $m \rightarrow n N N-0$ in åำ

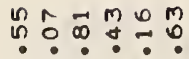
$\dot{m} \cdot \dot{m} i \dot{0}$

$\because a \div 090$ $\dot{-} \dot{\sim} \dot{m} \dot{0}$

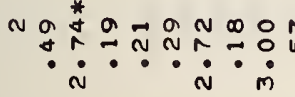

ㅇㅇㅇㅇㅇㅇㅇㅇㅇㅇㅇㅇ응

ㅇํㅇํㅇ

ํำ

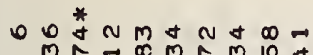

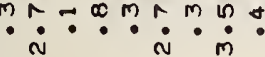

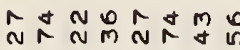

………?

응ㅇㅇㅇㅇㅇㅇㅇㅇㅇㅇ

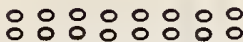

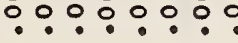

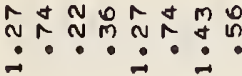

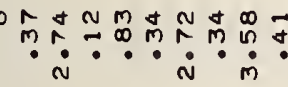

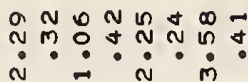

ก.

\&

$\dot{i} \cdot \vec{i}$

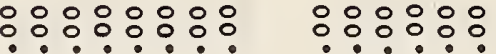

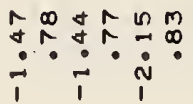

$\infty \ln n \rightarrow\{\infty$ un

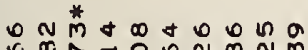

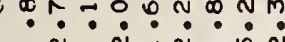

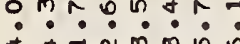

T.

$\dot{m} \dot{i} \dot{N} \dot{N} \dot{i}$

onr

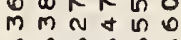

$\dot{0} \dot{\sim} \dot{m} \dot{0}$

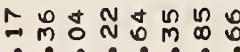

$\dot{m} \rightarrow \dot{N} \rightarrow \dot{v} \rightarrow \dot{i}$

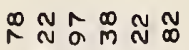

คิดั

$\dot{m} \dot{N} \dot{-} \dot{m} \dot{N} \dot{m}$

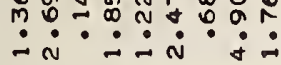

ก

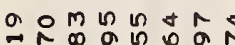
$\because \div \infty .0 \%$

$\checkmark \circ m m \rightarrow m \cup N$

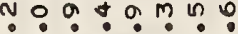
mas $v$ an $\quad \dot{0} \dot{0} \dot{0}$

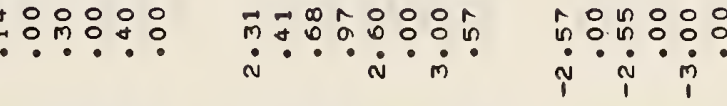

in 0 in:0:

$m \cdot \dot{m} \dot{0}$ $a, n \in N \ln \pi \infty \rightarrow$

\section{$z \sum_{0}^{\infty} z \sum_{0}^{\infty} z \sum_{0}^{\infty} z \sum_{0}^{\infty}$}

$z \sum_{0}^{\infty} z \sum_{0}^{\infty} z \sum_{0}^{\infty} z \sum_{0}^{\frac{\pi}{5}}$

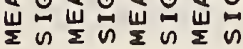
々

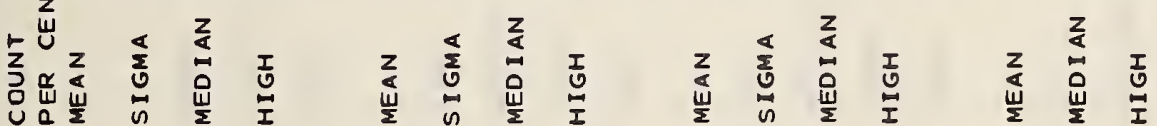

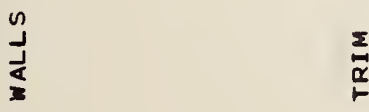

$\underset{\frac{2}{\alpha}}{\stackrel{2}{\leftarrow}}$

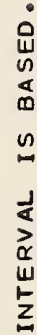

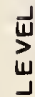

足

$\underset{I}{W}$

$\underset{⿱ ㇒}{I}$

$\frac{3}{0}$

嵌

ֻ

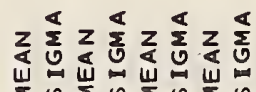

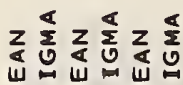

प्u

U

㞾

造 
พัพ

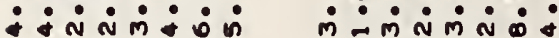

ํำำำํํำำำ

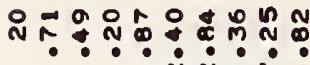

$\therefore \dot{m} \dot{N} \dot{N} \dot{N} \dot{\sim} \dot{m}$

คํํำำคำำํำ

m

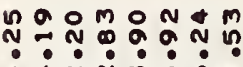

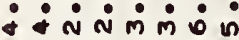

*

*

ำ $0: 08: ㅇ ㅡ ㅇ$

定

!

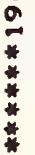

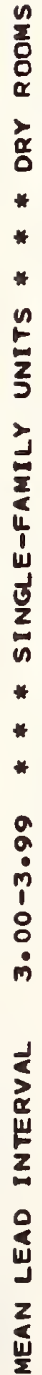

ค

: *.

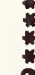

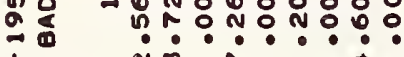

!

8

a 8

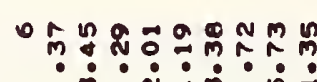

m

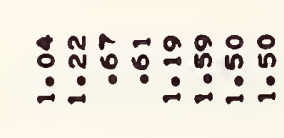

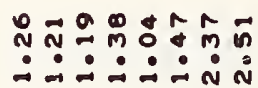

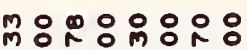

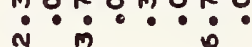

o $a g a+a n m$

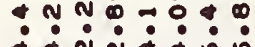
$\because \dot{N} \div \dot{N} \dot{m}: \because \dot{m}$

*

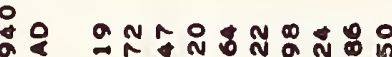

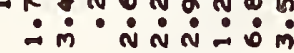

宸

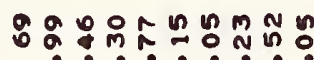

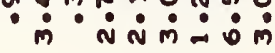

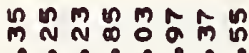

- $\dot{\alpha} \dot{N} \dot{0} \dot{m} \dot{0}$

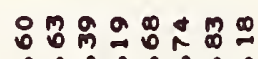

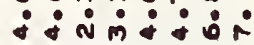

กิㄱ -

m:

$\dot{m} \dot{\sim} \dot{m} \dot{N} \dot{N} \dot{0}$

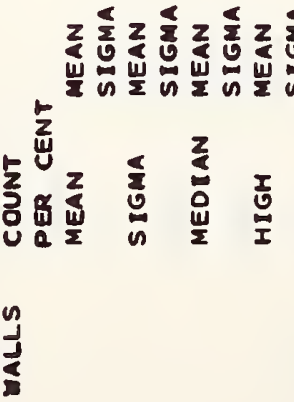

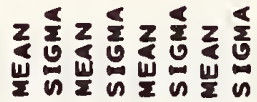

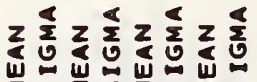

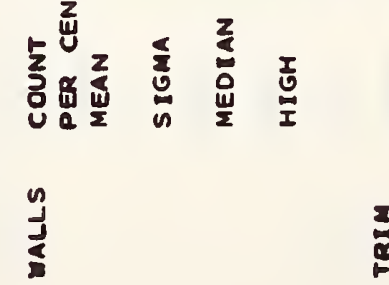

$\underset{2}{2}$ mㅇำ

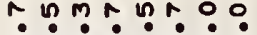

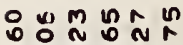

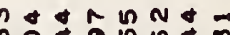

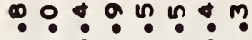
MNMーMND. :กั

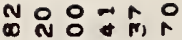

$\because \dot{j} \dot{i}$ i

NA $N M$ $\because 00 \%$ in:

$m+i$ in

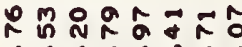
$\dot{m} \dot{\sim} \dot{m} \dot{N} \dot{\sim} \dot{0} \dot{0}$

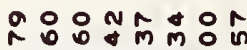
$\dot{N} \dot{N} \dot{\sim} \dot{n} \dot{R}$

$\vec{N} \rightarrow 0 M$

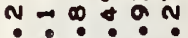
$\dot{N} \dot{1} \dot{1} \dot{i}$

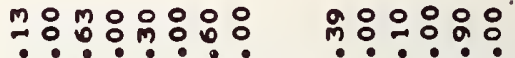
in $\dot{0} \cdot \cdots$

นดำกับ $\dot{N} \dot{N} \cdot \dot{n} \dot{0} \dot{0}$

$\sim \circ \infty \circ M \rightarrow a \infty$ $\neg$ เก $\rightarrow \Rightarrow n$ $\dot{m} \dot{0} \dot{\mathrm{N}} \dot{\mathrm{N}} \dot{0}:$

$-\cos N$

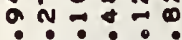
$\div \div \dot{0}$ in

ำกำกำ $\therefore$ ín $\dot{0} \cdot \dot{0}$

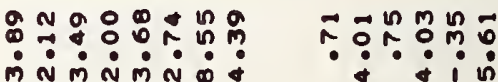

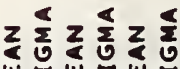

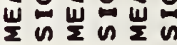

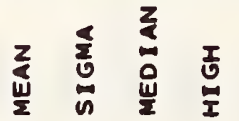

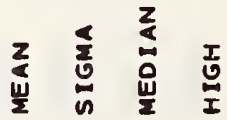

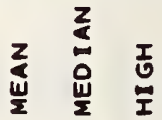




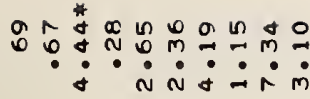

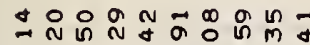

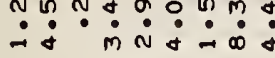

n๐ำ $m \propto n N N \infty$ ⿰纟:ำ - nía

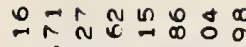
$\dot{0} \dot{N} \dot{N} \dot{a} \dot{m} \dot{0}$

ñ o. of of o o v \& $\dot{m} \dot{N} \dot{a} \dot{a} \dot{r} \dot{r}$

$\infty \infty N A R r$ 000 ก $\dot{m} \dot{m} \dot{N} \dot{q} \dot{m}$.
ง $\dot{+} \dot{m} \dot{0} \dot{0} \dot{0} \dot{m}$

m n

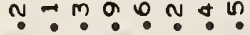
o $\dot{N} \dot{N} \dot{m} \dot{x}$ in

$m a b a r-m a$ m.? $0 . \div$ ด $\dot{\sigma} \rightarrow \mathrm{N} \rightarrow+\infty m$
N imi $\dot{0} \dot{i}$

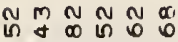
$\dot{m} \dot{m} \dot{m} i \dot{0}$ $\because ? \div 0: r$

imi

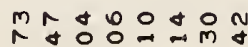

$\approx m \tilde{n} \stackrel{n}{n} m \dot{m}$

Na

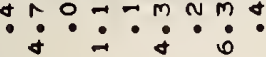

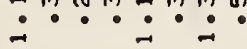

$\dot{m}: \dot{a}: \dot{0}$

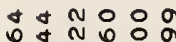

$\dot{p} \dot{p} \quad \dot{p}$

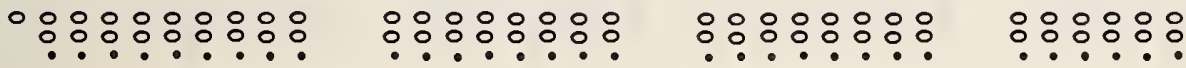

Noำ

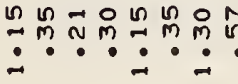

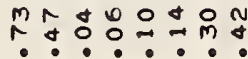

A 2000

$\dot{a}: \dot{a} \cdot \dot{0}$

$\dot{m} \dot{N} \dot{\alpha} \dot{0}$

$\dot{i} \dot{m} \dot{i}$

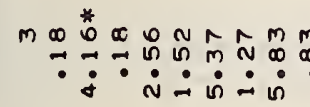

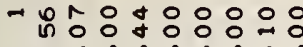
$\dot{\alpha} \dot{m} \dot{m} \dot{0}$

nNOA no n m O m

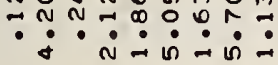

d

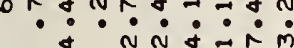

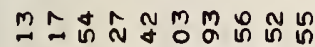
$\because \dot{m} \dot{m} \dot{m} \dot{0} \dot{0} \dot{0}$

㟧

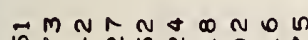

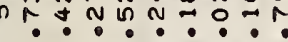
$\dot{\sim} \dot{N} \dot{0} \dot{n}$ må. $\dot{m} \dot{a} \cdot \dot{\sim} \dot{0} \dot{0} \dot{0}$

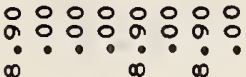

$+\infty a \infty \circ n$ $\because \frac{7}{0.5}: \frac{1}{0}$

$a n=0$ n $m a$. กT. \& $\dot{m} \dot{N} \dot{N} \dot{m} \dot{0}$ in

ดิ

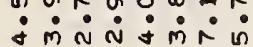

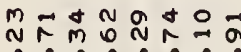
ก - $\dot{m} \dot{N} \dot{N} \dot{m} \dot{0}$
^⿻上丨丶 $\dot{m} \dot{\sim} \dot{m} \dot{N} \dot{0}$

$0 \infty w=m$ n $\dot{i} \dot{\sim} \dot{1} \dot{1} \dot{m}$ N웅융ㅇㅇㅇ $\dot{n} \dot{m} \dot{0} \dot{0} \quad \dot{*} \dot{n}$

กิำ

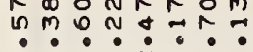
กำ $\dot{p}$ iि $\dot{m} \dot{m}$

ก

- $a+m a r a m$ $\because m m \infty \div \div: 0$ $\rightarrow \frac{1}{4} \div 0 \div 0$ $1 \dot{m} \dot{m} \div 0$ M a $\dot{\sim} \dot{N} \dot{m} \dot{m} \dot{N} \dot{0} \quad \dot{m} \dot{m} \dot{\sim} \dot{0}$

N

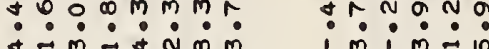
i $m$ i $m \div$ in

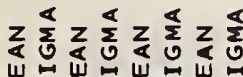
崖出崖出崖的崖的

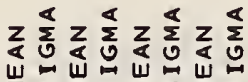

$z \sum_{0}^{\infty} z \sum_{0}^{\infty} z \sum_{0}^{\infty} z \sum_{0}^{\frac{\pi}{0}}$

$z \frac{\alpha}{2} z \frac{\pi}{2} z \frac{\pi}{2}$

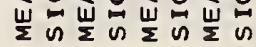

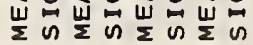
崖崩崖出崖菂

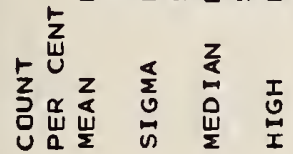

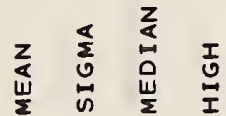

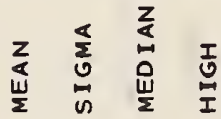
$\begin{array}{lll} & z & \\ z & 0 & \frac{1}{0} \\ \frac{\pi}{\Sigma} & \frac{0}{2} & \frac{1}{I}\end{array}$
$\frac{1}{\alpha}$
$\frac{3}{1}$

$\underset{⿱ 乛 龰}{2}$ 


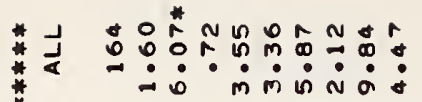

mNำ

$\dot{0} \dot{\mathrm{n}} \dot{\mathrm{n}} \dot{0} \dot{0} \dot{0}$

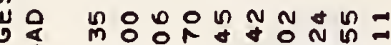

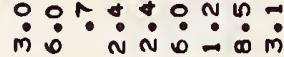

」

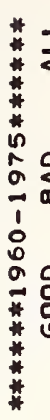

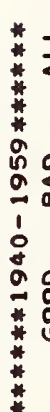

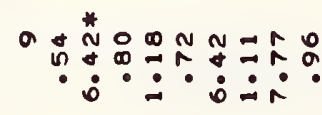

$m$
$\Re: 0$
$m: 0$
$m$

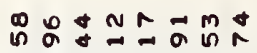

$\dot{\therefore} \dot{\mathrm{N}} \dot{\mathrm{i}} \dot{\mathrm{O}} \dot{\mathrm{a}} \dot{\mathrm{N}}$

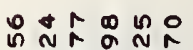
$\dot{N} \dot{\sim} \dot{\sim} \dot{m} \dot{0}$

ㅇ:ㅇ:ㅇ:웅

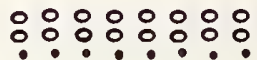

::::웅

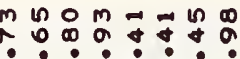

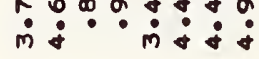

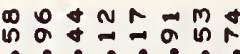
$\dot{n}=\dot{\sim} \dot{-} \dot{0} \dot{\sim} \dot{\omega}$

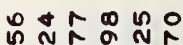

i $\dot{1} \dot{m} \dot{m}$

$0=m-\infty$

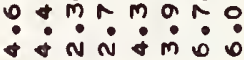

n๓nm $0-\infty 0$

กิ

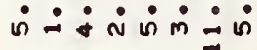

$n+a=0$

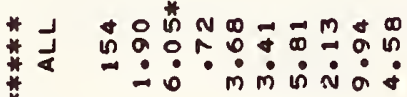

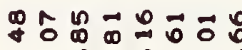
$\dot{\circ} \dot{\sim} \dot{\sim} \dot{0} \dot{0} \dot{0} \dot{0}$

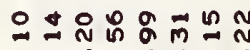

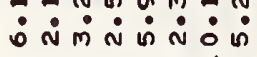

$\dot{1} \dot{1} \dot{\circ} \dot{i}$

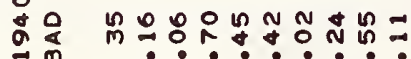
mín 嶎

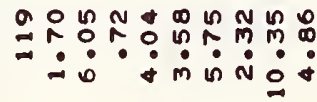

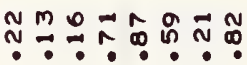

กัดัง

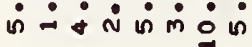

NONMNO

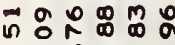
$i$ in $i$ io $\dot{\sigma} \dot{\alpha} \dot{N} \dot{m} \dot{m} \dot{0}$

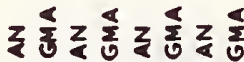

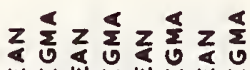

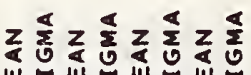

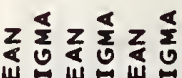

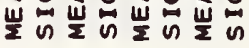

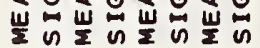

$\underset{\breve{u}}{\stackrel{u}{u}}$

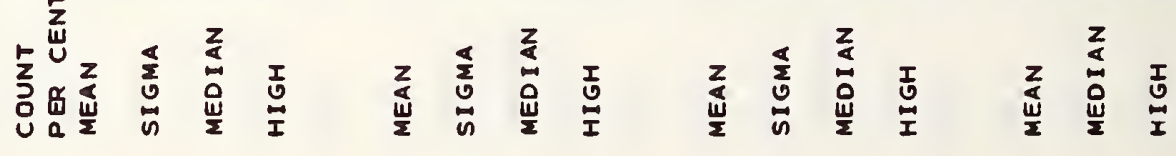

$\stackrel{n}{3}$

$\underset{⿱ 乛 龰}{\alpha}$

J

$\frac{3}{1}$ 


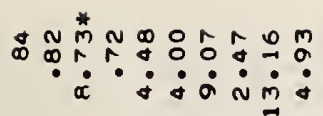

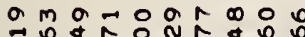
$\dot{a} \dot{a} \dot{\sigma} \dot{\alpha} \dot{\alpha} \dot{m}$ in

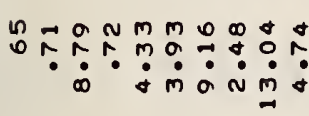

00 \% 000000

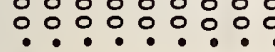

응용용ㅇㅇ웅 응응용ㅇㅇㅇ응 ำำำ

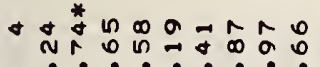
- $\dot{\infty} \dot{\sim} \dot{\alpha} \dot{0}$

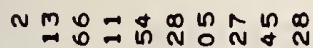
$\because: \because \frac{\pi}{\pi}, \frac{5}{2}$

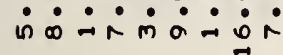

nNNONAN NO -

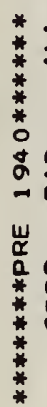

운 $m^{\infty}$ n

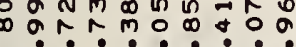

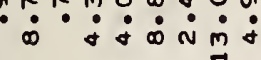

= $\because ? \infty \infty \infty$ \&

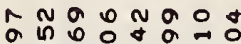
$\dot{m} \dot{\sim} \dot{N} \dot{m} \dot{\sim} \dot{0}$

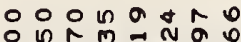

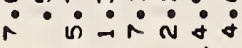

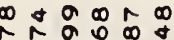
$\dot{1} \dot{1} \dot{1} \dot{i} \dot{0}$

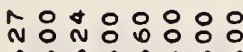
$\dot{n} \dot{m} \dot{m} \dot{a}$

음 0 只 n $\because ล \div \div$ $\dot{\infty} \dot{\sim} \dot{r} \dot{m} \dot{\infty} \dot{0} \dot{r}$

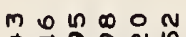
$+\because g, \frac{9}{0}$ $\dot{m} \div \dot{\sim} \dot{0} \dot{0}$ in

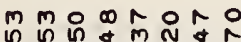
กำ

กn mo man

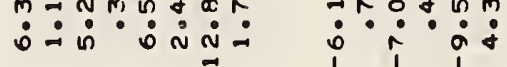

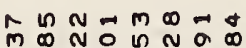

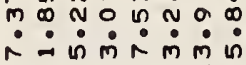

a 00 m in $900 \mathrm{~mm}$ $\dot{p} \dot{p} \dot{p}$ in $\dot{p}$

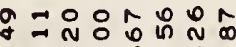
ம் $\dot{\sim} \dot{\sim} \dot{m} \dot{0}$

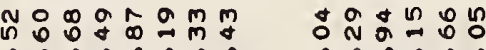

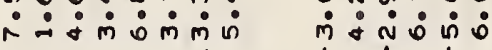

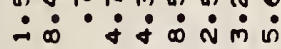

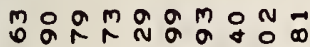
$\dot{\infty} \because \dot{m} \dot{\infty} \dot{N} \dot{m} \dot{\sigma}$

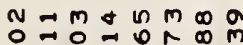

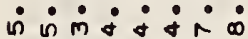

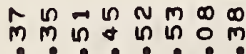

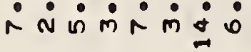

以กำ ำ.ำ. 1 in in

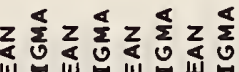

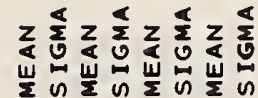

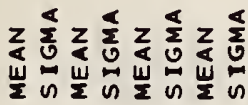

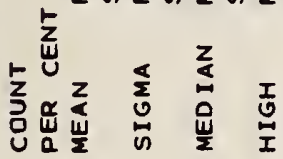

$\underset{2}{\underline{\alpha}}$

」

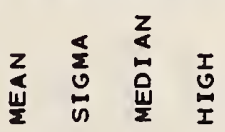




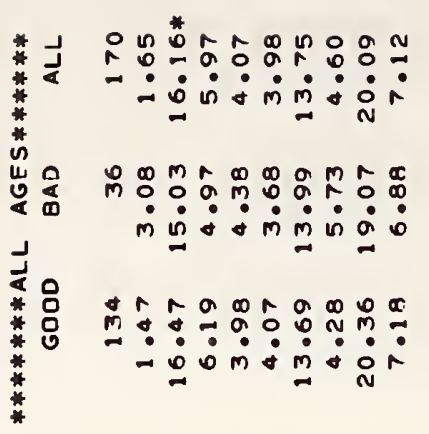

$*$
$*$
$*$
$*$
0
0
0
0
$\vdots$
$\vdots$
0
0
0
7
7
$*$
$*$
$*$
$*$

$00 * 0000000$ 응ㅇㅇㅇㅇㅇㅇㅡ.

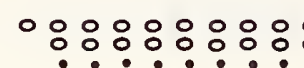

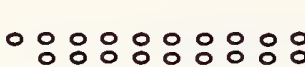

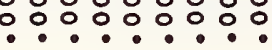

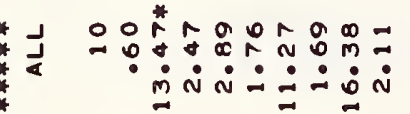

in

要 8

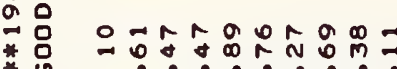

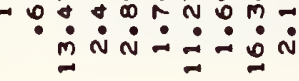
*

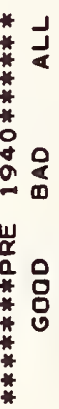

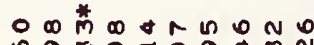

o

$\therefore \dot{0} \dot{0} \dot{i} \dot{0} \dot{N}$

mํำ

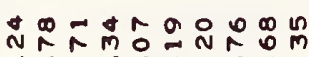

$\because \dot{0} \dot{0} \dot{\circ} \dot{m} \dot{0} \dot{0}$

$\frac{1}{2}$

:

$\circ$

$\frac{1}{2}$

물

z

แำ

$\frac{2}{2}$

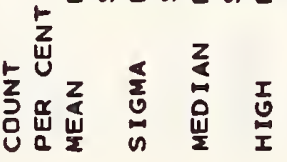

$\frac{n}{3}$ $\dot{m} \dot{i} \dot{+} \dot{m} \dot{\sim} \dot{m} \dot{0}$

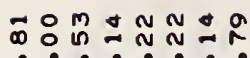

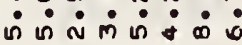

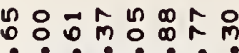
$\dot{0} \dot{0} \dot{m} \dot{\phi} \dot{r} \dot{m}=\dot{\infty}$

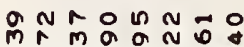

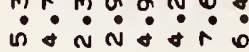

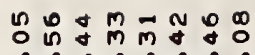
$\dot{N} \dot{\circ} \dot{0} \dot{m} \dot{0} \dot{0} \dot{r}$

O nNOMOn : $: 0 \% \div$ $\dot{m} \dot{0} \dot{m}=\dot{0} \dot{0}$

N $m m \propto \sim q \infty m$ $=$ iि $\dot{0} \dot{a} \dot{0} \dot{a}$

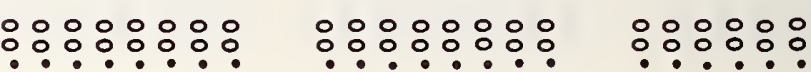

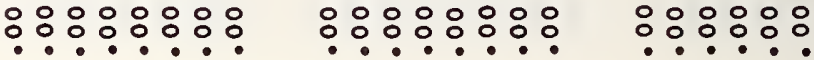

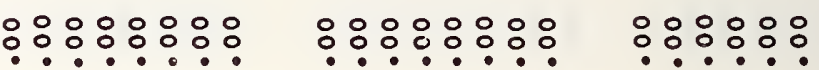

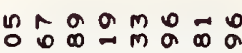
$\dot{0} \dot{0} \dot{m}$ in $\dot{0} \dot{0} \dot{0}$

$m a 0 \%=\ln m$ $\because \dot{\sim} \dot{\sim} \dot{\sim} \dot{\mathrm{N}} \dot{\mathrm{N}}$

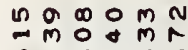
iि

응응ㅇㅇㅇㅇㅇㅇ응

00000000 ㅇㅇㅇㅇㅇㅇ

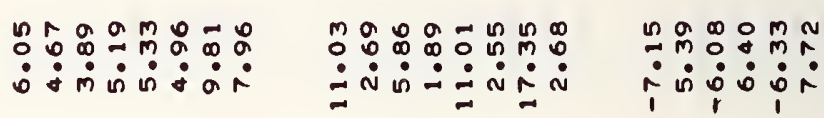

a m n n

n $⿻ \infty \infty^{\infty}=\operatorname{m}$ $\because 0$ ก m :

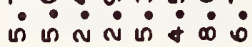
i $\dot{\sim} \dot{m} \dot{0} \dot{\mathrm{o}}$ กี $\dot{0} \dot{\sim} \dot{0} \dot{\sim} \dot{\infty}$

ตㅇํㅁำํํํำ $\dot{\infty} \dot{0} \dot{m} \dot{\sim} \dot{q} \dot{\infty}$

ำ $\dot{m} \dot{\circ} \dot{0} \dot{0} \dot{m} \dot{0}$

m̊ñ i $\dot{i} \dot{i} \dot{*} \dot{0}$

- $\infty \wedge n \circ N \infty \infty$ m. $=$ ம் $\dot{0} \dot{\infty} \dot{m} \dot{0}$

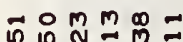
$\ddot{1} \dot{0} \dot{0} \dot{0} \dot{0}$

- $\sim a n$ - งดน

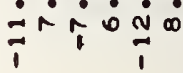

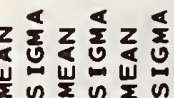

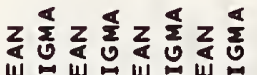

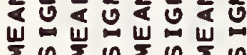

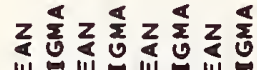
崖的崖的崖的

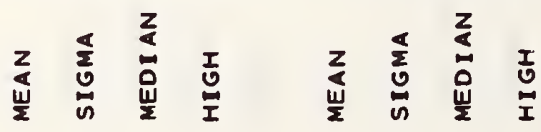

$\begin{array}{lll} & z & \\ z & 0 & I \\ \frac{\alpha}{2} & 0 & 0 \\ \frac{\omega}{2} & \frac{1}{2} & I\end{array}$

$\underset{z}{z}$

J

I 


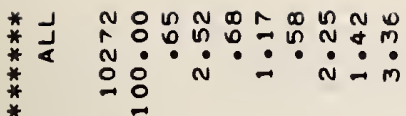

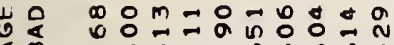
$\Rightarrow \dot{\circ} \div \dot{m} \because \dot{m} \dot{n}$ -

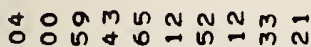

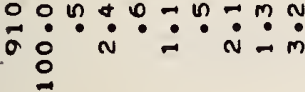

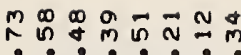

N $\dot{m}-\dot{N} \dot{m} \dot{0}$

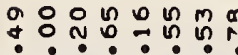
$\dot{\alpha} \dot{\alpha} \dot{\alpha} \dot{x} \dot{m} \dot{0}$ in

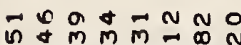

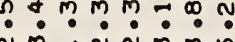

NMन N NMM

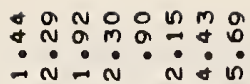

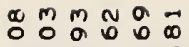
$\therefore \because \because 0$

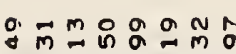

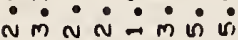

mㅇํำ ํ. $-\dot{N}-\dot{N}$ im

ก⿻上丨丶 $\because \div \bullet \div \div$

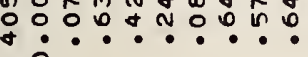
$\therefore$

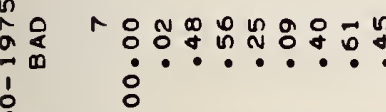

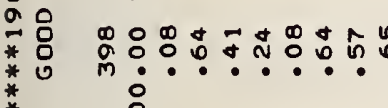
:

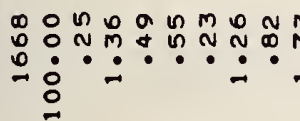

a: a a mon n N

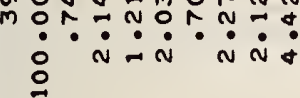

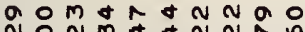

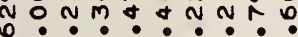
음

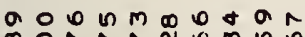

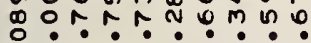

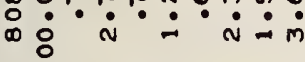

ำ

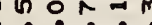
$=\dot{0} \dot{0} \dot{m} \dot{-} \dot{\sim} \dot{N} \dot{\sigma}$

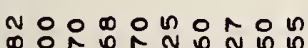

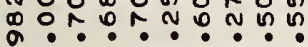
o: $\dot{a} \dot{\sim} \dot{n} \dot{m}$

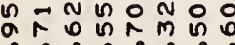
$\dot{N} \dot{m} \dot{\sim} \dot{N} \dot{m} \dot{0}$

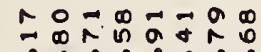
$\dot{m} \dot{m} \dot{a} \dot{0}$

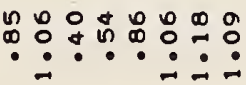
ֻู

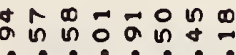
$\because \because \dot{\div} \dot{-} \dot{0}$

งัง $\dot{\sim} \dot{N} \dot{-} \dot{\sim} \dot{\sim} \dot{m}$

응

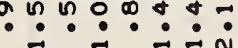

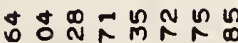
$\dot{\alpha} \dot{\alpha} \dot{\alpha} \dot{\circ} \dot{m} \dot{0}$

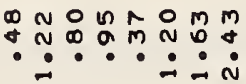

$\because \div .000 .0 \%$

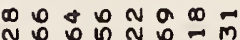

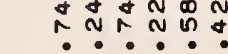

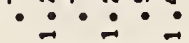

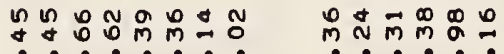

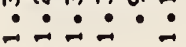

DOMNOON mmMn

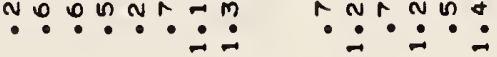

กำดัดต

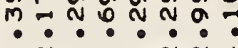

+ 2 n

$-n-\pi-n$ n.

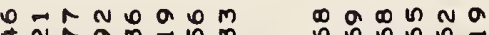
: ․․․․ำ:

$\operatorname{son} n \sin \pi$ ก.ำ.ำ.

NคD ก Mก N

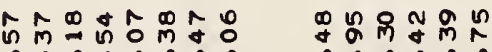

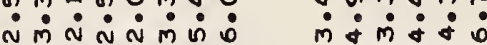

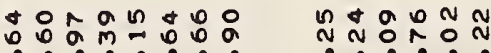
-

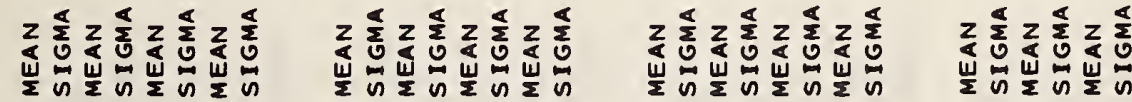

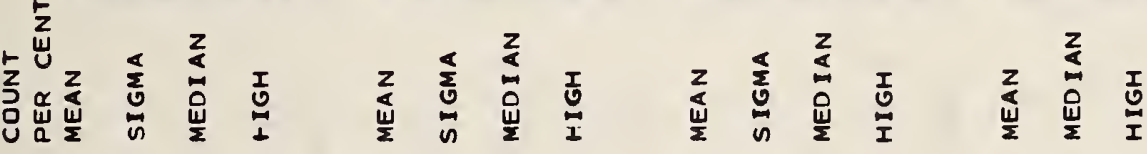

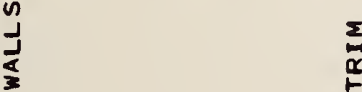




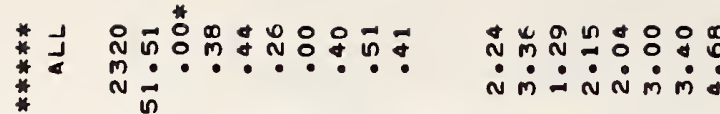

苟

出赵

过 *

*

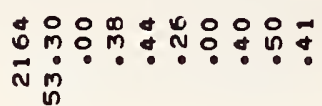

*

文先

28

* 0 *

\begin{tabular}{l}
$\sum_{2}^{n}$ \\
0 \\
0 \\
$\alpha$ \\
5 \\
\hline \\
$*$ \\
$*$ \\
$*$ \\
2 \\
\hline
\end{tabular}

* J

ẫ nDOOOMONND

ดั

!

के

N

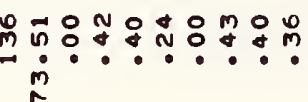

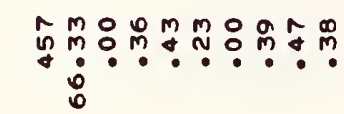

*

* لَ

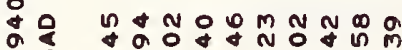

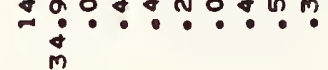

宸品

maำ

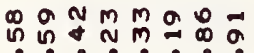
तिं $\dot{\sim} \dot{N} \dot{m} \dot{m}$

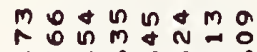

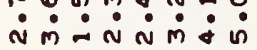

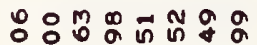
$\dot{\alpha} \dot{N} \dot{N} \dot{m} \dot{m} \dot{0}$

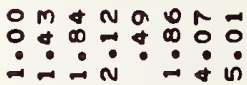

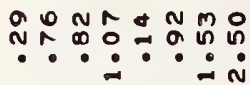

ㅇํำำ

$\because \dot{0} \dot{-1} \dot{\sim}$

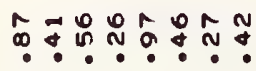

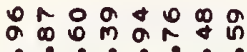

- ํㅡㄴ

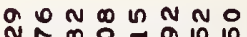

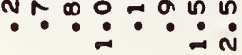

응ํำํำ

$\therefore \dot{\sim} \dot{\sim}$

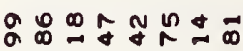

- $\dot{\sim} \dot{\sim} \dot{\sim} \dot{\sim}$ i

4m noำ

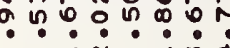

- $\dot{\sim} \dot{\sim} \dot{m}$

욤ำ

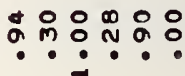

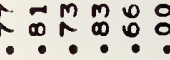

- 
in $\dot{i} \dot{0} \dot{0} \cdot \dot{0}$

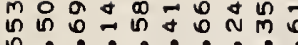
$\pm$

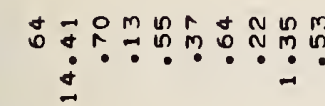

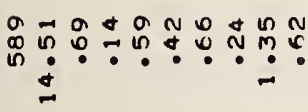

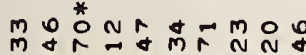

$\dot{a} \cdot-\cdots$

응ㅇㅇㅇㅇㅇㅇㅇㅇ응

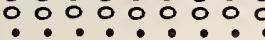

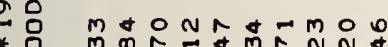

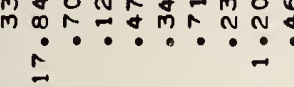

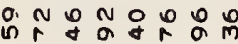

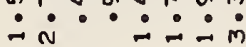

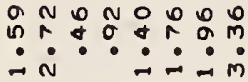

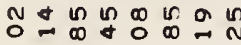

$\dot{-} \dot{-} \dot{-} \dot{\sim} \dot{m}$

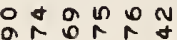

$\dot{n} \dot{m} \dot{m}$

응응ㅇㅇㅇㅇㅇㅇ응

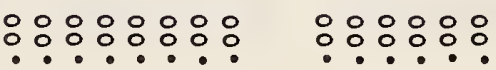

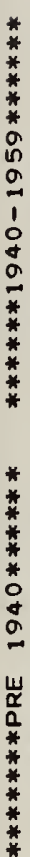

$0 m \infty \infty m+n N$

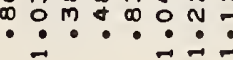

$\therefore: \because: \because: \because$

$\infty m_{\infty}^{\infty} m+\frac{1}{m} N$

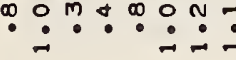

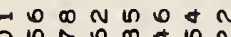

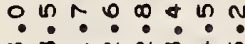
$\dot{m} \dot{m} \dot{\sim} \dot{N} \dot{N} \dot{m} \dot{0}$

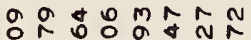
$\dot{\sigma} \dot{m} \dot{\sim} \dot{m} \dot{m} \dot{0} \dot{0}$

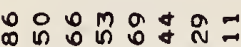
ím $\dot{\mathrm{N}} \dot{\mathrm{N}} \dot{\mathrm{m}} \dot{\mathrm{d}} \dot{\mathrm{n}}$

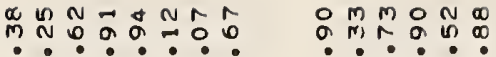
$\dot{m}-\infty \dot{N}$

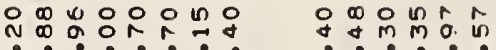

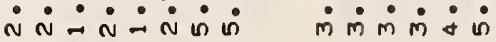

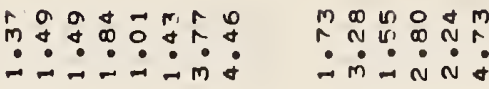

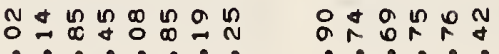
$\therefore \dot{\sim} \dot{\sim} \dot{\sim} \dot{m} \quad \dot{N} \dot{m}$

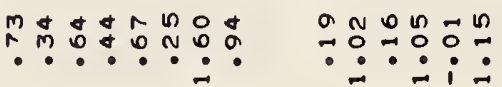

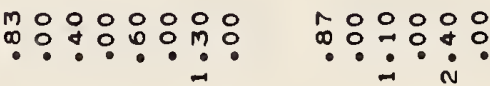

ํำ $\because \div: \div$

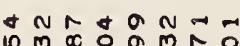

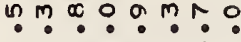

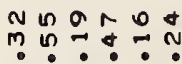

N $\dot{m} \dot{N} \dot{m} \dot{m}$ in

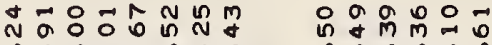
ஸं

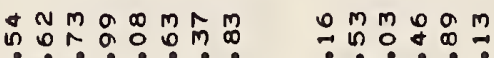
$\ddot{-1} \dot{-1} \dot{+} \dot{\alpha} \quad \dot{m} \dot{N} \dot{m} \dot{N}$

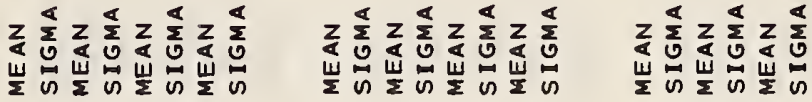

ป

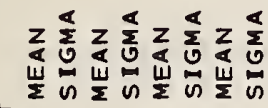

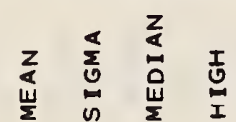

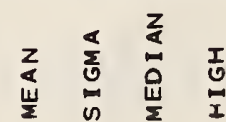

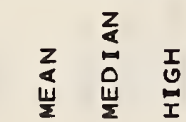

لj

1 
음

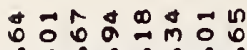

m

$\dot{0}-\dot{0} \cdot \dot{\sim}$

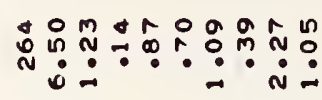

a 900 ก

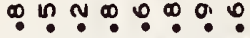

NM $\sim N$ N

n

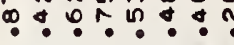

न - -

กํํㅁำ

$\because \because \frac{1}{n}:$

$\because \div \because 0 \div \div \div$

$\ddot{m} \because \because M \div M ! M$

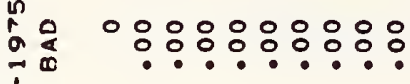

!

$* 0$
$*$
$*$
$*$
$*$

$0+0+\infty N-N \circ$ ก $\dot{m} \div \div ! ?$

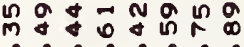
$\therefore \dot{-} \dot{-} \dot{-} \dot{ }$

응ㅇㅇㅇㅇㅇㅇㅇㅇㅇㅇㅇㅇ

高

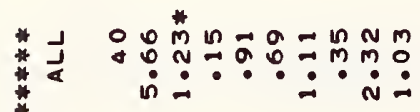
*

กำ

$\stackrel{0}{\infty}$

b

å

- $N$ N

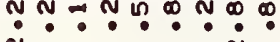
$\dot{N} \dot{\sim} \cdot \dot{i}$

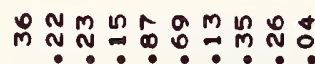
in: $:-i:$

* *

* J ñ ñ

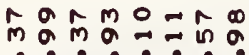

กิ $\because \dot{N} \dot{-} \dot{\sim} \dot{N}$

ดั ง

$\therefore$ : $i$ i.

음

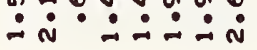

$\dot{m} \dot{m} \dot{-} \dot{m} \dot{m} \dot{0}$

$n=n \infty a+0 n$

$00 m+\infty$

กิ

ㄴ.?ำ

N

$\therefore \bullet \dot{-} \dot{\sim} \dot{N}$

N

- $\dot{n} \cdot i$ i

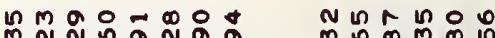

m.

$\because \div \cdot \dot{\text { n }}$

$\therefore \dot{\because} \dot{\sim} \dot{\mathrm{N}}$

ח $\dot{0}$ ம் $-\dot{0} \dot{0} \dot{0}$

$\underset{\alpha}{\alpha}$

सू

$00 N N=M M N$

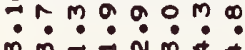

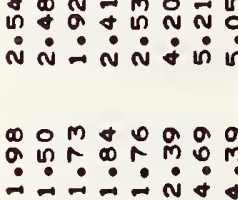

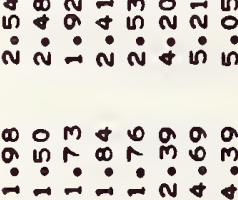

$00_{0}=0$

- $\dot{m} \dot{\sim} \dot{m} \dot{\text { o }}$

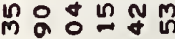

$\dot{m} \dot{\dot{m}} \dot{m} \dot{m}$ i

$$
\text { in }
$$

a

óa

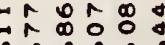

m * N m

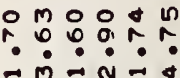

ำ ำํํํำ

응ㅇㅇㅇ응

i $\dot{1} \dot{0} \dot{0}$ $\dot{m} \dot{m} \dot{\sim} \dot{N} \dot{N} \dot{0} \dot{0}$

ㄴำ

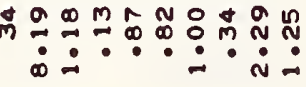
$\dot{m} \dot{m} \dot{N} \dot{j}$

I

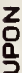

யั

\&

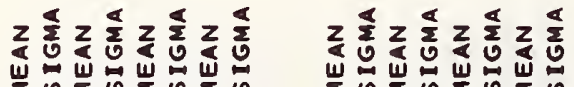

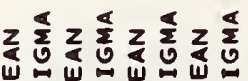

$z \frac{\pi}{2} z \frac{\pi}{2} z \frac{5}{0}$ ๖ 岁

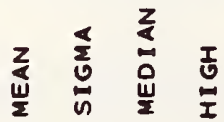

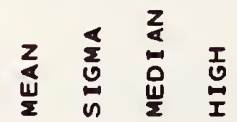




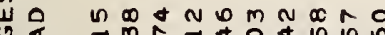
m.

ำกัน : เด ?ะ?

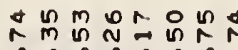

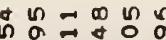
$\dot{m}=\dot{-} \dot{m}$ in $\dot{m} \dot{n} \dot{a} \dot{n}$

$\dot{m} \dot{m} \dot{N} \dot{m} \dot{N} \dot{0}$

$\operatorname{Nana.00}$

ก กับัง

ก $N m-n n m$ $\because \div \because \infty \div \div \div$

$\infty m \backsim r \infty m r$

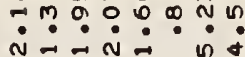

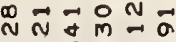
N $M-N N \dot{m}+0$

arang na

$\dot{m} \dot{m} \dot{m} \dot{-}$

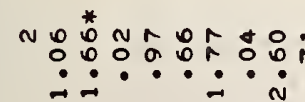

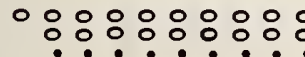

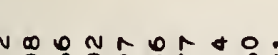

$\div: 0 \% ?: 0 \%$

a d 은

i : ? ? ?

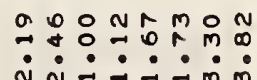

น

$\because \%$ m

$m \infty 000=$

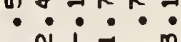

응ㅇㅇㅇㅇㅇㅇㅇㅇ

응ㅇㅇㅇㅇㅇㅇㅇㅇ응

ด้ำ

$\therefore: i \dot{i} \dot{0}$

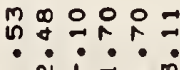

年

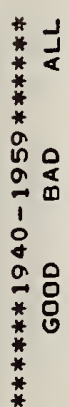

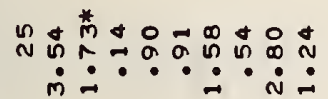

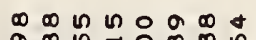

$\because \% ?: \infty \mathrm{m} !$

$9+m m n=0 m$

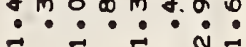

?

융ㅇㅇㅇㅇㅇㅇㅇ

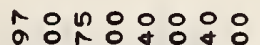

คำำㅇํำㅇํำ

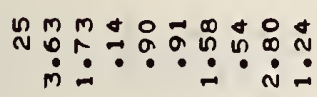

åำ

$\circ \& N M m 0 m$

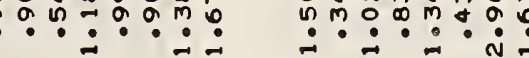

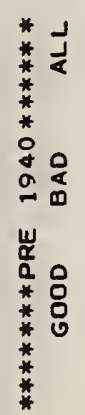

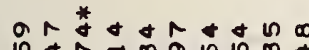

n?:?

$\infty N \infty O \hat{m} \backsim \vec{m}$

$\Rightarrow 0000 n \mathrm{mN}$

ก.?

$\dot{m} \dot{m}=\dot{m} \dot{m}$ in

N $\dot{\sim} \dot{\sim} \rightarrow \dot{0} \dot{0}$

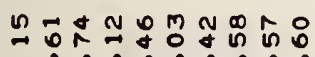
$\dot{m} \div \dot{-} \dot{-} \cdot \dot{m}=$

กับ

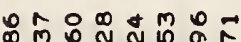

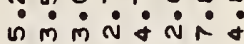
$\dot{m} \dot{m} \dot{N} \dot{m} \dot{N} \dot{0}$

。

N $m M N O \infty$

$\dot{m} \dot{m} \dot{m} \dot{m} \dot{m} \dot{0}$

$\vec{m}+N O N \hat{O}$

กับ? $\div$

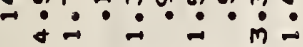

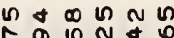

$i \cdot \dot{i} \dot{i} \dot{-}$

응ㅇㅇㅇㅇ

n $+\infty$ n $N$ in

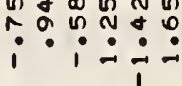

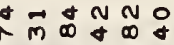

$\because \dot{m} \dot{\sim} \dot{m} \dot{\sim}$

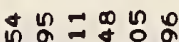

กำ

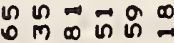

$\because \dot{0} \dot{0} \dot{0}$

是 


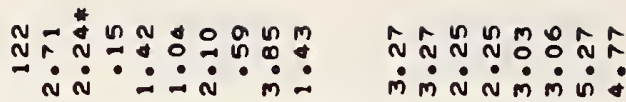

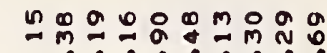
$\because \div \div \div \div \frac{1}{4}$

* nomonma

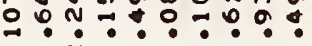
N $\dot{*} \dot{\mathrm{m}}$

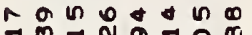
$\because M \div$ ก $\because \div \propto$

m̊ mi $\dot{N} \dot{m} \dot{0}:$ m

Nด *

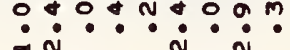

MNM

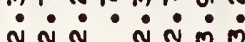

$\infty \circ \infty m \circ \infty \circ N$

$m a+\pi n n k$

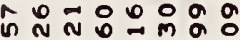
$\dot{N}-\dot{n} \dot{n} \dot{n} \dot{0}$

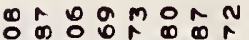
$\dot{m} \dot{i} \dot{\sim} \dot{\sim} \dot{\sim} \dot{m}$

$\because \frac{m}{0} 0_{0}^{0}$ i $\dot{n} \dot{-1}$ 응ㅇㅇㅇㅇㅇㅇㅇㅇㅇㅇㅇㅇ

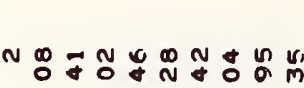
$\therefore \dot{n} \cdot \dot{n} \cdot$

$\operatorname{man}$ N $\dot{N} \dot{N} \dot{N} \dot{m} \dot{m}$ 응ㅇㅇㅇㅇㅇㅇㅇㅇㅇㅇㅇㅇ

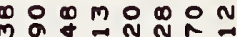
M: $: ? !$

ำำกัด in i $\dot{m}$

*

o - * n m mo n $\because \dot{a} \div \div \div \div$

เง

ニ ลี่

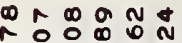
$\dot{N} \dot{\sim} \dot{\sim} \dot{\sim} \dot{0} \dot{m}$

- $\dot{m} \dot{i} \dot{0}$

응응응응응웅

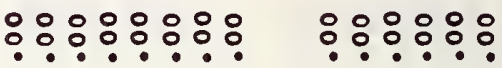

aㅇํㅇำn

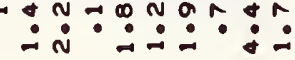

\& $\rightarrow$ ก

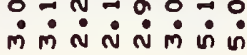

A ำ

$\cos 0$ $\because \ddot{m}: \dot{N}: \dot{n}$

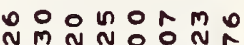
NM N NOO N $\dot{m} \dot{m} \dot{N} \dot{m} \dot{m} \dot{0}$

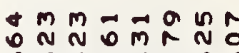
i.

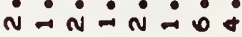

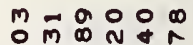

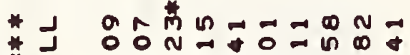
*

울 Ð $\dot{m} \dot{m}: \stackrel{a}{*}: \dot{m} \bar{m}$

กิ $\dot{m} \dot{\mathrm{N}} \dot{\mathrm{m}} \dot{\mathrm{i}} \dot{\mathrm{m}}$

mong m: $: \div \div$ :

ํํํ유물 宸

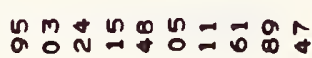
$\dot{m} \dot{n}: \dot{i} \dot{m} \dot{m}$

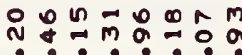

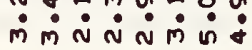

ก i $\dot{*} \dot{n} \dot{\sim} \dot{0}$

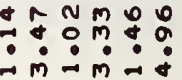

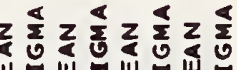
$z \sum_{0} z \sum_{0}^{\mathbb{2}} z \sum_{0}^{5} z \frac{5}{0}$

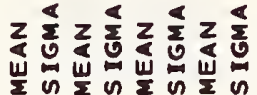

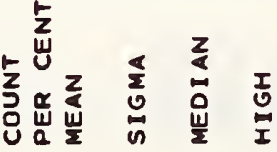
崖的崖的崖的崖的

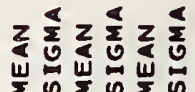




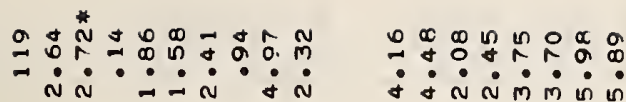

จำำกี $\dot{m} \dot{N} \ddot{i} \dot{n} \bullet \dot{0}$

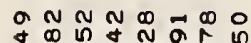
a $\infty$ ก

$00 m+n d a$

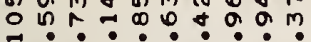

- $\dot{n} \dot{n}-\dot{n} \cdot \dot{a}$

$\begin{array}{lllllll}0 & 0 & 0 & m & n & n & n \\ 0 & 9 & 0 & 0 & 0 & 0 & 0\end{array}$

+ $\dot{-} \dot{i} \dot{m} \dot{m}$ i

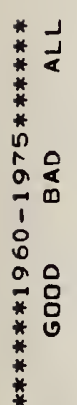

+ $\because \because \because ?$ ?

nNonn n n

ก:

$\operatorname{nan} n-n$

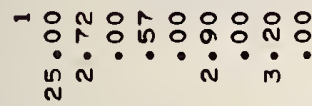

$\hat{\alpha}:$
$\dot{N}:$
$\dot{0}$

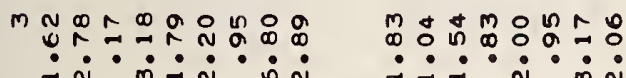

$\dot{-i}: \dot{N} \dot{m} \dot{N}$

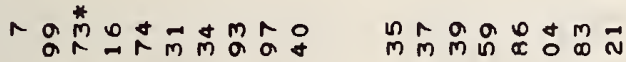

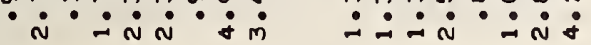

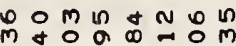

i $\dot{n} \dot{-} \dot{-} \dot{m}$

mㅇำ $\because \dot{1} \dot{1} \dot{N} \dot{n}$

ถู ด̊ำ

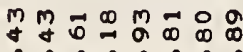
$\dot{\text { i }} \dot{\mathrm{i}} \dot{-} \dot{i} \dot{0}$

$i$ i $-\dot{m}-$

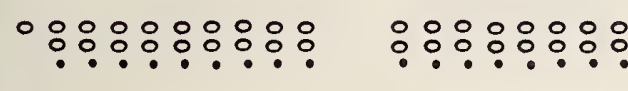

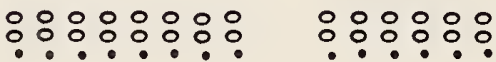

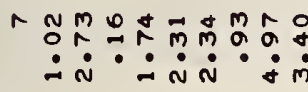

ทิ

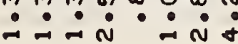

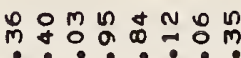
$\dot{N} \dot{N} \dot{-1} \dot{-} \dot{m}$

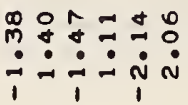

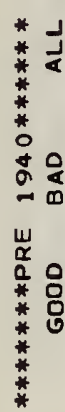

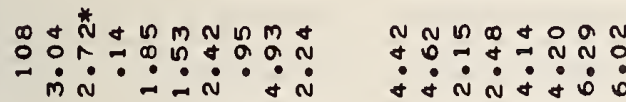

$\infty 02 n \cos 20$

$m \wedge \infty \rightarrow 0 \rightarrow \infty 0$

$\dot{m} \dot{-i} \dot{N} \dot{m} \dot{m} \dot{0}$

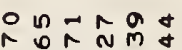

$\because \dot{a} \dot{a} \dot{0}$

in $\dot{*} \dot{\mathrm{N}} \dot{\mathrm{N}} \dot{\mathrm{m}} \dot{0} \dot{0}$

a d $\dot{m} \dot{i} \dot{i} \dot{m} \dot{i} \dot{m}$

ก๊

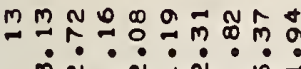
$\tan 0$

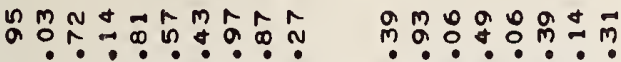
$\dot{m} \dot{n} \dot{a} \dot{a} \dot{a} \quad \dot{a} \dot{a} \dot{n} \dot{a} \dot{0} \dot{0}$

กตั

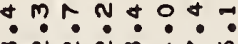

\& $\dot{m} \dot{N} \dot{\sim} \dot{m} \dot{0}$

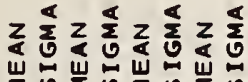

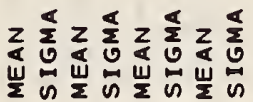

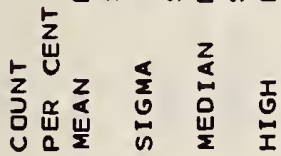

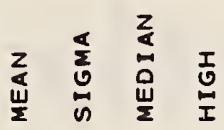

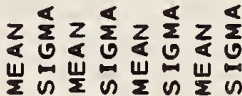

$z \sum_{0}^{\infty} z \frac{\pi}{0} z \frac{\pi}{0}$ 崖的崖的崖的 $\begin{array}{lll}z & z & \\ \frac{a}{2} & 0 & \frac{1}{0} \\ \frac{U}{2} & \frac{U}{2} & \frac{1}{2}\end{array}$

$\frac{1}{3}$

$\underset{⿱ 亠 䒑}{\alpha}$

J

$\stackrel{1}{1}$

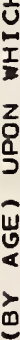

嵒 


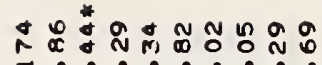

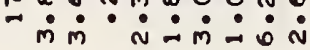

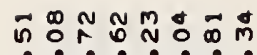

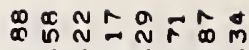

○ニ N

$\dot{\sigma} \dot{N} \dot{m} \dot{\sigma} \dot{0} \dot{0}$

$\dot{m} \dot{\dot{m}} \dot{\sim} \dot{m} \dot{0}$

$\ddot{\circ} \dot{0} \dot{0}$

กำ

mơ

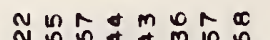

- chan

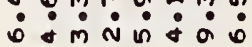

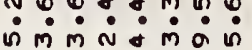

ค.

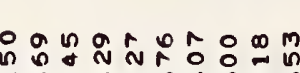

mín

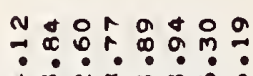

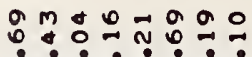

Mำ

*

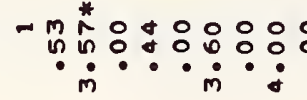

영ㅇㅇㅇㅇㅇㅇㅇㅇㅇㅇㅇㅇ

은

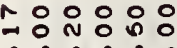

$\dot{m} \cdot \dot{m} \cdot \dot{q}$

$\frac{1}{4} i$

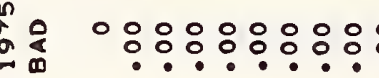

웅응응응응

응응응ㅇㅇㅇㅇㅇㅇ

응ㅇㅇㅇㅇㅇ

* J $\quad \Rightarrow \begin{aligned} & m \\ & \text { * }\end{aligned}$

in in

ษ

O M N Or $\dot{N} \dot{N} \dot{\sim} \dot{N} \dot{N} \dot{m} \dot{0}$

$\dot{m} \dot{N} \dot{\sim} \dot{\sim} \dot{\sim} \dot{m}$

$i$
$\dot{n}$

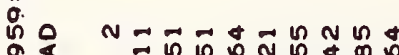

응응ㅇㅇㅇㅇㅇ

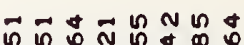

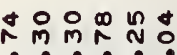
$\dot{=m} \dot{m} \dot{m} \dot{0}$

b

o

बे 0

?

$\dot{N} \dot{N} \cdot \dot{m} \dot{m}$

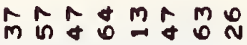

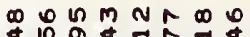

$\because \& Q \hat{0} 0$ in

$\dot{N} \dot{N} \dot{\sim} \dot{N} \dot{N} \dot{m}$

$\dot{m} \dot{\sim} \dot{N} \dot{m} \dot{\sim} \dot{\sim} \dot{m}$

i

* J

AN $\because \because 00 \div \div$

*

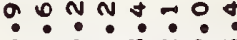

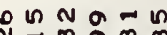
$\because \ddot{m}: \dot{m} \cdot \stackrel{\infty}{0}$

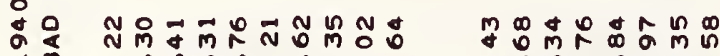
m-máñ

$-\dot{*} m \dot{m}$

in $\dot{m} \dot{N} \dot{N} \dot{\sim} \dot{m}$

$\dot{0} \dot{\dot{m}} \dot{\mathrm{N}} \dot{\boldsymbol{n}} \dot{m} \dot{a} \dot{0}$

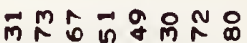

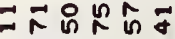

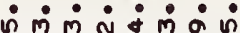

- $\dot{m} \dot{\sim} \dot{m}$ i

$\underset{\alpha}{\alpha}$

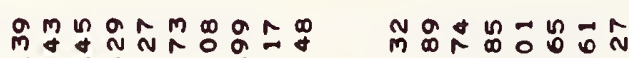
$\dot{m} \dot{m} \dot{m} \dot{m}: \dot{m} \dot{0}:$

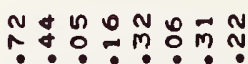

N $N$ N $\because \dot{A}: \dot{i}$ 


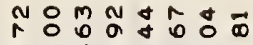

$\dot{\alpha} \dot{\sim} \dot{\sim} \dot{\alpha} \dot{m} \dot{0}$

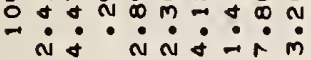

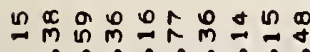

$\dot{m} \dot{\alpha} \dot{m} \dot{\sim} \dot{\alpha} \dot{\alpha} \dot{\infty} \dot{N}$

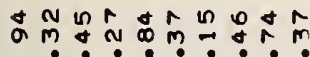

N $\dot{\alpha} \dot{N} \dot{\alpha} \dot{A} \dot{m}$

กะั

$\because \because \because \dot{m} \dot{m} \dot{\alpha}: \dot{\infty} \dot{m}$

N $\dot{m} \dot{-} \dot{0} \dot{m} \dot{m}$

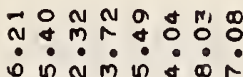

N $\bar{D} M N M m \infty$ -

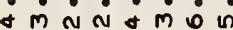

一 ํㅇㅇำ in $\dot{\alpha} \cdot \dot{q} \cdot \dot{0}$

-

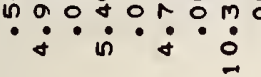

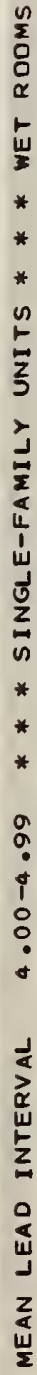

\begin{tabular}{l}
$*$ \\
$*$ \\
$*$ \\
$*$ \\
$*$ \\
$*$ \\
0 \\
0 \\
0 \\
$\vdots$ \\
$\vdots$ \\
$\vdots$ \\
0 \\
\hdashline \\
$*$ \\
$*$ \\
$*$ \\
$*$ \\
$*$ \\
$*$
\end{tabular}

○

ํำ

ก

ำ

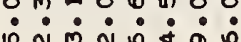

$m_{m}^{m}+m \infty$

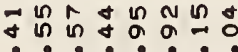

$\dot{\alpha} \dot{\alpha} \dot{m} \dot{0} \dot{m}$

Nㅗㄴ $\stackrel{m}{N} \unrhd$

$\because \dot{i}: \dot{\sim} \dot{m}$

응ㅇㅇㅇㅇㅇㅇ

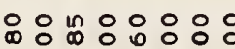

$\stackrel{\circ}{\circ}: \because \because: \div$

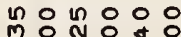

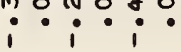

$m$
$\dot{N}:$
$\dot{N}$
$\dot{n}$

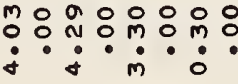

응읭ㅇㅇㅇㅇㅇㅇ

$\dot{i} \dot{i} \dot{i}$

응용ㅇㅇㅇㅇㅇ

응융ㅇㅇㅇㅠ

응융ㅇㅇㅇ

응유유

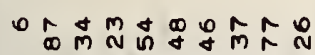

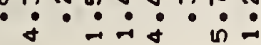

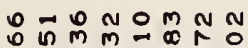
เ? $m: ? \div ?$ $\dot{0} \dot{0} \dot{\sim} \dot{N} \dot{0} \dot{0} \dot{0}$

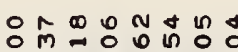
ம் $\dot{1} \dot{m}: \dot{0}: 00$

N $m+m n \infty$

m. ำ?

mog 0 n iे

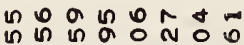
+ $\dot{m} \dot{m} \dot{\alpha} \dot{0} \dot{0}$

ㄴำ $m$

- $\dot{m} \dot{*} \dot{0}$

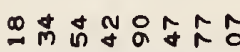
ம் $\dot{m} \dot{\alpha} \dot{\alpha} \dot{0} \dot{0}$

ลัด จ

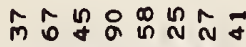
$\dot{0} \dot{\mathrm{N}} \dot{\mathrm{m}} \dot{\mathrm{S}} \dot{\mathrm{O}} \dot{\mathrm{R}}$

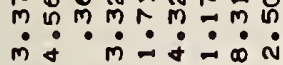

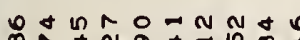
ํ.

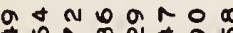
ใด

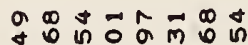

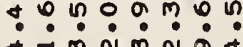
$\rightarrow$ in

$m \neq \sim \infty m$

$\because ? \div: ․$

$m+\div 0$

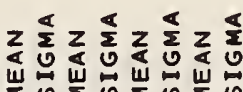

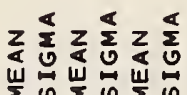
占

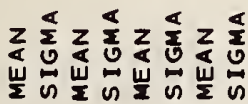

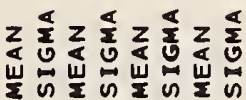

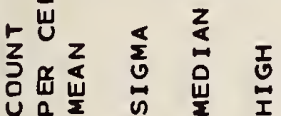

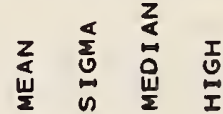

ذ্

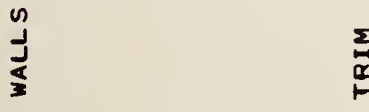

$\underset{+2}{\stackrel{2}{*}}$ 


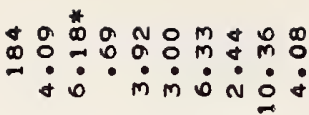

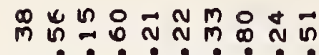
$\dot{\infty} \dot{0} \dot{m} \dot{\sim} \dot{0} \dot{0} \dot{\sigma} \dot{N}$

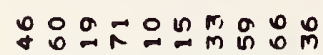
- $\dot{m} \dot{0}: \dot{0} \dot{0} \dot{0}: \dot{0}$

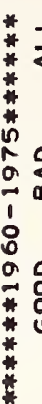

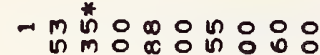
in

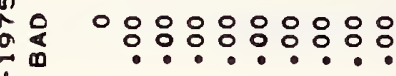

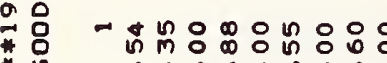

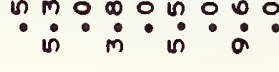

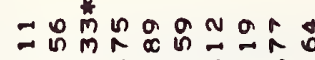
$\therefore \dot{0} \dot{m} \dot{m} \dot{\sim} \dot{0} \dot{0}$

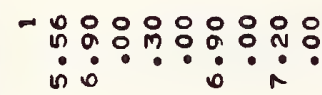

$\circ$ ำ $\therefore \because \because \dot{m} \dot{\sim} \dot{m} \dot{0}$ a mino

Q $\dot{\infty} \dot{0} \dot{m} \dot{N} \dot{0} \dot{\sim} \dot{O} \dot{\sim}$

m

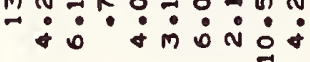

๓ \& $\dot{\sim} \dot{\alpha} \dot{0} \dot{0} \dot{0}$

ำ

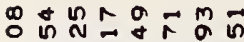
$\dot{\gamma} \dot{\circ} \dot{m} \dot{m} \dot{0} \dot{m} \dot{0}$

กำ

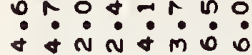

$\therefore: \stackrel{\infty}{\sim}: \circ: \circ: ㅇ$ $\dot{n} \ddot{r} \cdot \dot{m}$ 응ㅇㅇㅇㅇㅠ

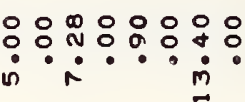

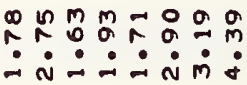

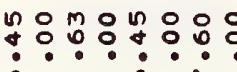

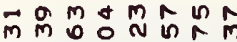

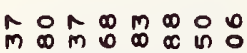

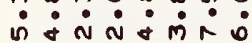

응웅요요

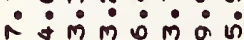

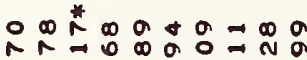

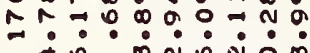

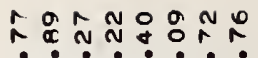

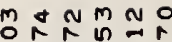

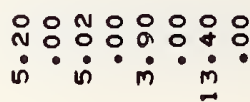

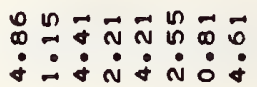

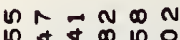

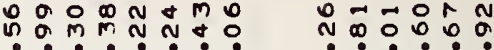

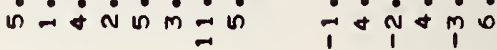

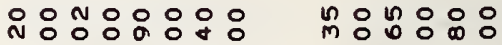
$\dot{n} \dot{n} \cdot \dot{m} \dot{m} \quad i \dot{q}^{\dot{m}}$

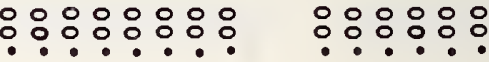

N

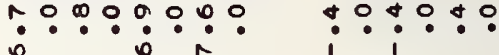
$\therefore \dot{\sim} \dot{\sim} \dot{\sim} \dot{\sim} \dot{N} \dot{0}$

S.

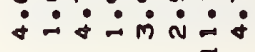

음 i $\dot{1} \dot{0} \dot{0}$;

$\because \because m-\pi m$ m n

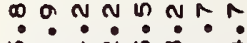

$\rightarrow \infty \infty N O N$ $D \cap N+\infty$

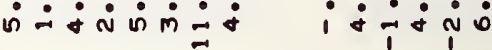

ำ ำ $\dot{0} \dot{\mathrm{N}} \dot{\mathrm{N}} \dot{0} \dot{\mathrm{N}} \dot{-} \dot{+}$

$\therefore \approx$ 每 $i \& i \& i$ in

ำง ம்

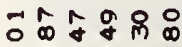
$\dot{1} \dot{\dot{1}} \dot{0} \dot{0} \dot{0}$

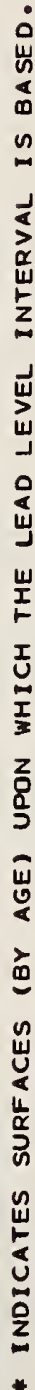

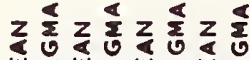

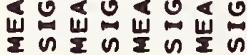

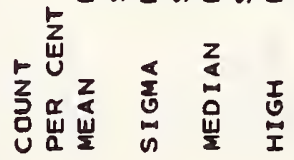

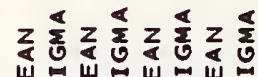

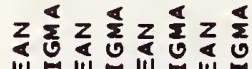

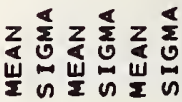
崖出崖的崖的崖的

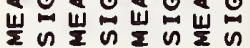

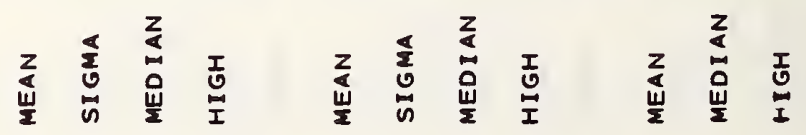

$\underset{\alpha}{\alpha}$
J 


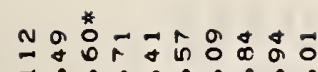

- $\dot{\sim} \dot{\infty} \dot{\dot{m}} \dot{\sigma} \dot{\sim} \dot{N} \dot{\sigma}$

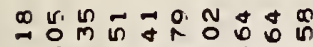
$\because \dot{0} \dot{0} \dot{0} \dot{0}$ -

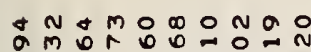
N $\dot{0} \dot{0} \dot{0} \dot{0} \dot{m} \dot{0}$

유융ㅇㅇㅇㅇㅇㅇ 응유:웅

$\circ: \because: \because: \because:$ $\circ: \because: \because::::$

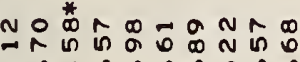
$\dot{\sim} \dot{\infty} \dot{m} \dot{m} \dot{N} \dot{\sim} \dot{m}$

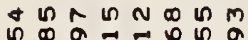

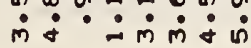

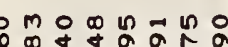

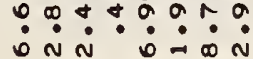

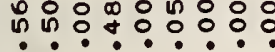
$\dot{n} \dot{0}: \dot{0}:$

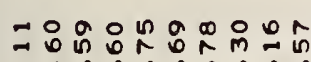
$\therefore \dot{\infty} \dot{m} \dot{m} \dot{\infty} \dot{\sim} \dot{m}$

$m m \infty m n=n$

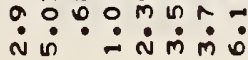

:잉요

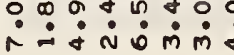

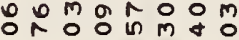
$\ddot{\sim} \dot{\sim} \dot{\sim} \dot{0} \dot{m} \dot{m} \dot{0}$

คต+

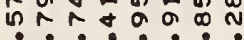
$\dot{0} \dot{m} \dot{0} \dot{0} \dot{a} \dot{\sim}$

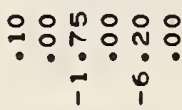

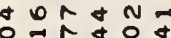

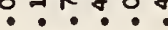
in in $\dot{1} \dot{0}$

6) iि

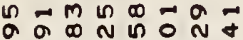
$\dot{0} \dot{\alpha} \dot{\sim} \dot{m} \dot{\sigma} \dot{0} \dot{0}$

$m \infty+0 \wedge n \infty$ นด. $0.0 \%$ $\ddot{\sim} \dot{\sim} \dot{\alpha} \dot{\sim} \dot{m} \dot{0}$

คก $\because: 00 \%$ i กั

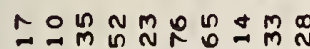
$\because \dot{m} \dot{m} \dot{0} \dot{\sim}$

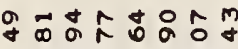
ம் $\dot{\sim} \dot{\sim} \dot{\mathrm{N}} \dot{\mathrm{r}} \dot{\mathrm{m}}$

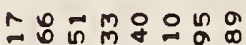

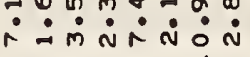

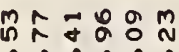
$\dot{N} \dot{N} \dot{N} \dot{0} \dot{+}$ $\dot{N} \dot{\infty} \dot{q} \dot{m} \dot{\infty} \dot{m}$

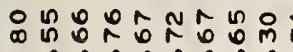

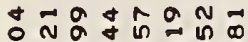

$\dot{0} \dot{\sim} \dot{\sim} \dot{m} \dot{\alpha} \dot{\infty} \dot{0}$

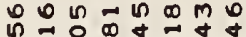
í $\dot{0} \dot{\mathrm{n}} \dot{0} \dot{0}$

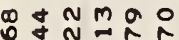

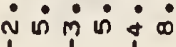

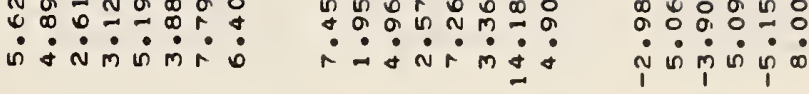

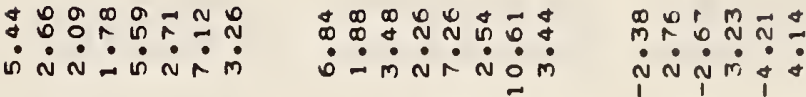

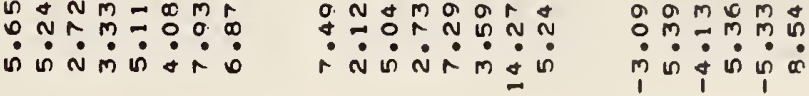

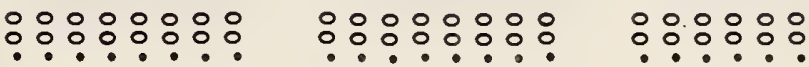

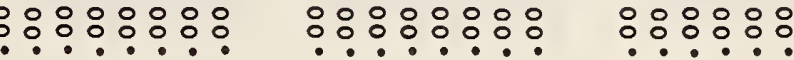




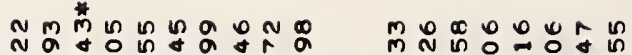
N $\dot{\dot{n}} \dot{0} \dot{\sigma} \dot{\alpha} \dot{N} \dot{m} \dot{0} \dot{0}$ $\ddot{\sim} \dot{0} \dot{m} \dot{0} \dot{0} \dot{0} \dot{0} \dot{\infty}$

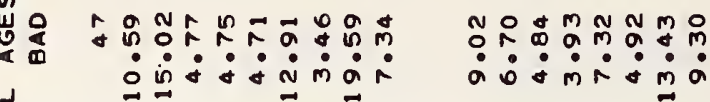

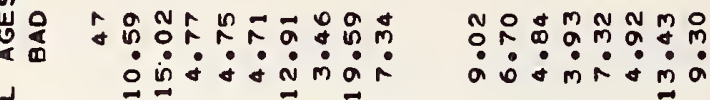
J 0
*
*
*
*

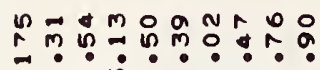
$\dot{0} \ddot{0} \dot{\sigma} \dot{\alpha} \dot{m} \dot{m} \dot{a} \dot{0}$

* ل *

กิำ 000000000 a

8 * *

ง n.ก. $\dot{m} \dot{m} \dot{N} \dot{N} \dot{N} \dot{0} \dot{0} \dot{m}$

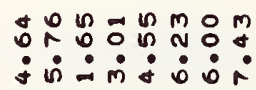

Nลㅇำ

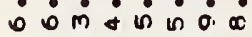
응ㅇㅇㅇㅇㅇㅇㅇ

ชกล์

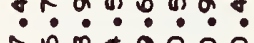

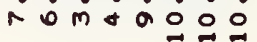

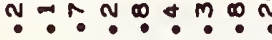
N $\sim \dot{N}=\dot{0} \dot{m}$

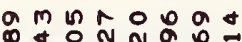

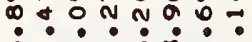

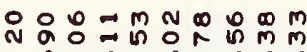
$\dot{N} \dot{\circ} \dot{m} \dot{N} \dot{N}: \dot{0} \dot{0}$ m的穴的的递

$N a m+\infty=\infty a$

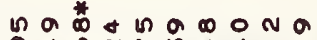

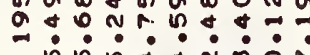

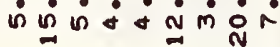

กับ

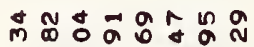
$\dot{0} \dot{0} \dot{m} \dot{r} \dot{m} \dot{0}$ $\dot{0} \dot{0} \dot{\circ} \dot{0} \dot{m} \dot{m} \dot{0}$

mก⿻上丨 nะ: $\dot{0} \dot{0} \dot{0} \dot{\alpha} \dot{m} \dot{0} \dot{T}$

$m \sim ⿻ n$

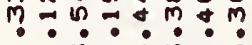

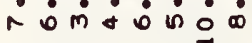

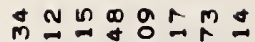
$\dot{\sim} \dot{\sim} \dot{m} \dot{0} \dot{m} \dot{\mathrm{r}}$

N $N$ N ir $\dot{0} \dot{0} \dot{0}$

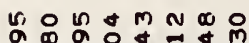
ด. ஸि

จำำำ ำ ค. 1
1

$N \propto M \cap \infty$ n $0 m$ $\because$ ํ. N 0 momar

$\infty+m \circ a 0$ $\because \approx \infty m: 0$ ir io io

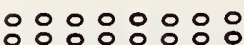

융ㅇㅇㅇㅇ

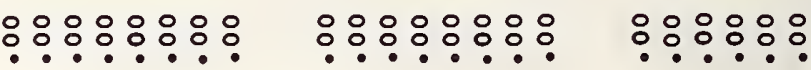

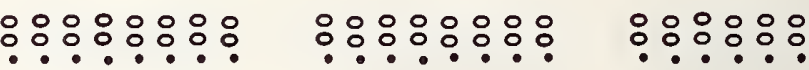

mo요요의

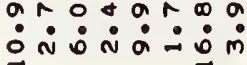

ค indogod

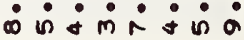

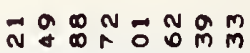

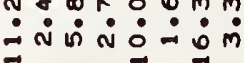

$-0+\infty 0 \infty$ $\because a m n+0$ $\dot{i} \dot{0} \dot{0} \dot{0} \dot{0} \dot{r}$

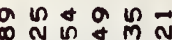
i $\dot{0}=\dot{m} \dot{0}$ กึก $\therefore \dot{0} \dot{m}=\dot{0}$

우요 กำ

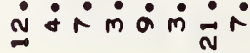

$9 \infty N M O 0$ $\therefore ? \infty \infty \div$ iि pí:

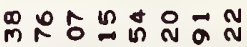

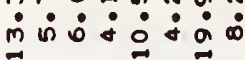

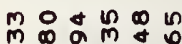

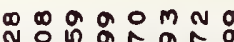
งำดำด.ด Nómágí
으응ํㅇำ i 0 in

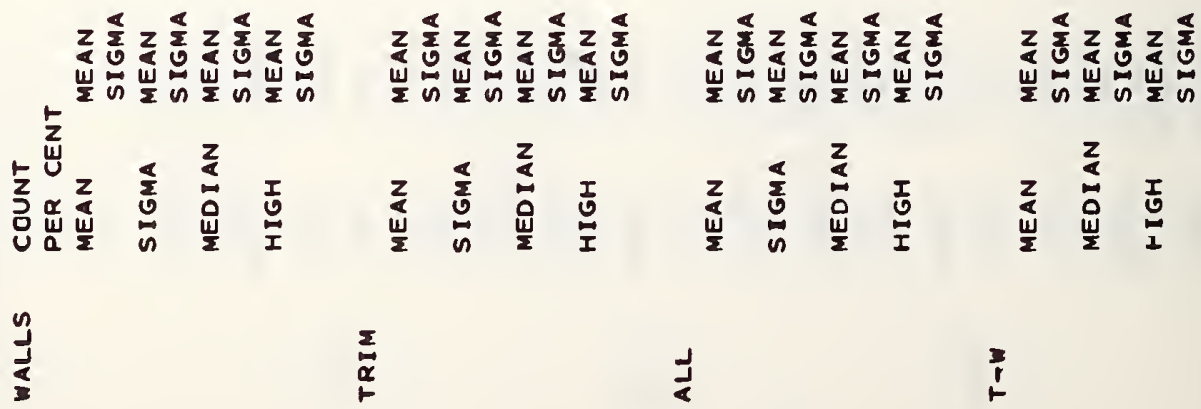




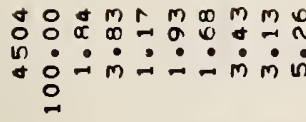

ㅇํำ

$\dot{0} \dot{m} \dot{0} \dot{\sim} \dot{N} \dot{\alpha} \dot{0}$

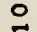

$0000 n \hat{0} \infty m$

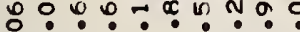
+ $0-m--m$ N

* J a o n n n n

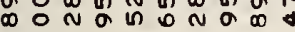

-

$\rightarrow$

- or ñ $\dot{0} \dot{a} \cdot \dot{a} \dot{n} \dot{N}$ 웅

W

:

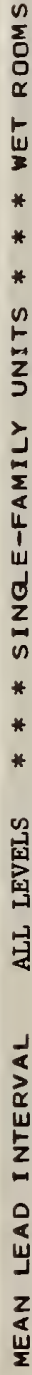

- 0 。 $\dot{0} \dot{\sim} \dot{\sim} \dot{i} \dot{m}$ $\circ$

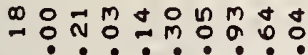
$\dot{0} \dot{a} \dot{n} \dot{a} \dot{a} \dot{0}$ ㅇ.

Oำ

: $\dot{0} \cdot \dot{\sim} \dot{\sim} \dot{m}$

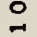

n。 nกำ $m: \dot{N} \dot{0}-\dot{N} \dot{m} \dot{m}$ 웅

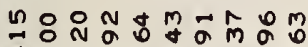
- $\dot{0} \dot{0} \dot{a} \dot{a} \dot{0}$ 음

宸

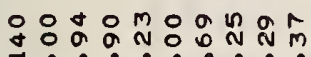
$\vec{m} \dot{0} \dot{\sim} \dot{m} \dot{N} \dot{m} \dot{m} \dot{n}$ 인 a

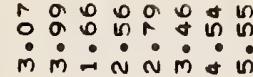

ㄷำ

iि $\dot{\alpha} \dot{m} \dot{\dot{m}} \dot{\sim} \dot{0}$

onn $n$

$\infty \infty$ ?

NMAN $N \dot{m} \dot{\theta}$

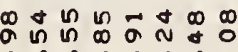

$\because \div . \div \dot{0}$

$\infty m a g=0$

\&? ? ? ? ?

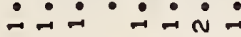

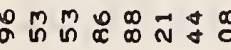

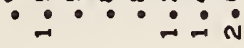

in

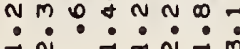

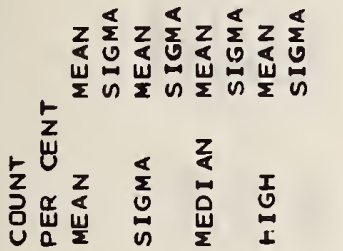

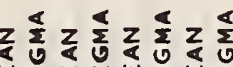

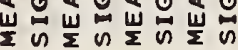

กิำ $\dot{m} \dot{0} \dot{N} \dot{m} \dot{m}$ in

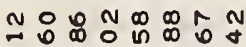
i் $\dot{0} \dot{m}+\dot{m} \dot{0}$

N $\infty$ n m:? : : : mंन NMm 0 i

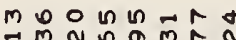
$\because m \sim 0 \% m A N$

$\infty N 009$

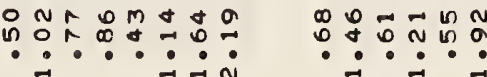

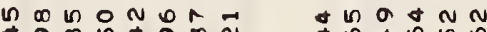
: ?

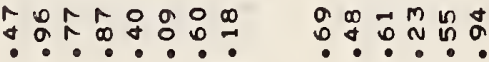
$\operatorname{nan} n \dot{n}$

Nㅇำ

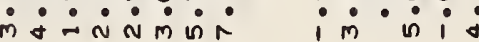

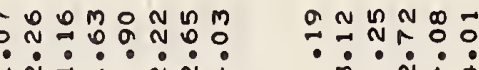
$\dot{\sim} \dot{\sim} \dot{\sim} \dot{N} \dot{N} \dot{m} \dot{m} \dot{i}$

$\rightarrow$ In NOM $\rightarrow$ OM nO $0 \mathrm{~m} \div 0$ $\dot{N} \dot{N} \dot{N} \dot{N} \dot{M} \dot{0}$

$n N \infty \& N m$ o m $m$ n $\dot{a} \dot{0} \dot{0} \dot{0}$

ำดั

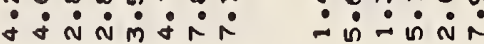

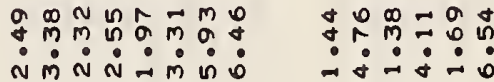
的的致的 


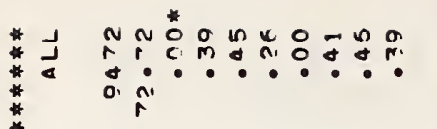

*

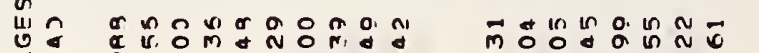

de a

过

*

*

*

ơ

$\frac{1}{0} \quad \stackrel{i r}{i}$

ơ

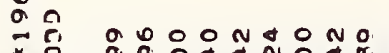

*

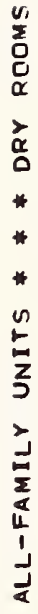

*

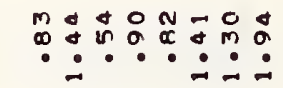

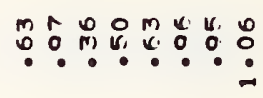

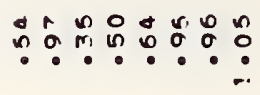

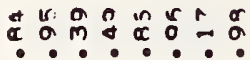

-・.・早

in

$\alpha+-0 a \operatorname{mox} x$

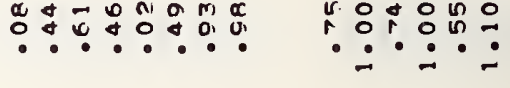

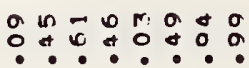

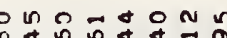

$\therefore ?$

岁是

$\rightarrow \infty$

!

à

*

* J a

*

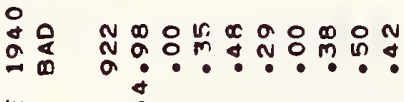

山

崩

*

$\overrightarrow{1} \vec{a}$
:

*

mín

manou $0=\mathrm{m}$

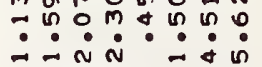

m M N n

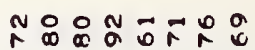

$\because \dot{*} \cdot \dot{i} \dot{n}$

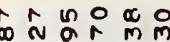

$\therefore \dot{\sim} \dot{\sim} \dot{N} \dot{m}$

ODNOONH

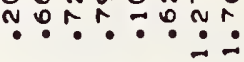

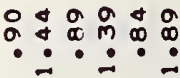

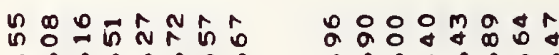

$\dot{\sigma} \dot{\alpha} \dot{N} \dot{\sigma} \dot{m} \dot{0} \quad \dot{\sim} \dot{N} \dot{N} \dot{N} \dot{N} \dot{\sigma}$

กำ ำกำำ

$\dot{\alpha} \dot{0} \dot{m} \dot{0}$

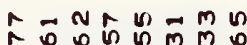

$=0$ N.

$\because \dot{0} \dot{0} \dot{0} \dot{m}$

๑

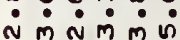

$\dot{\sim} \dot{\sim} \dot{\sim} \dot{\sim} \dot{\sim} \dot{\sim ⿻}$

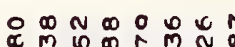

- narmano $\dot{N} \dot{m} \dot{\sim} \dot{N} \dot{m} \dot{\circ}$

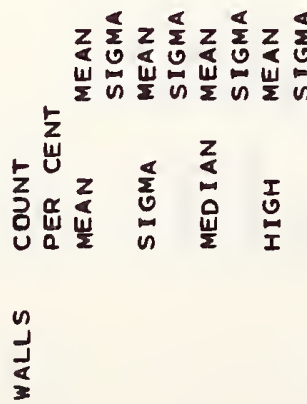

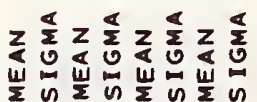

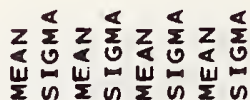

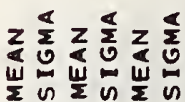

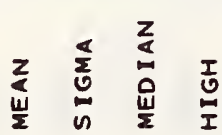

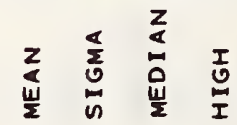

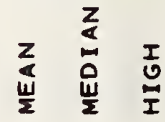

$\underset{\substack{\alpha \\ r}}{\sum}$

Ј

$\stackrel{3}{1}$ 


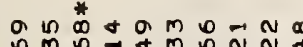
๑ัก \&

夜 a

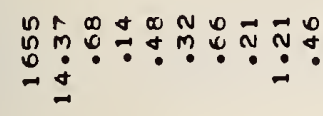

* J

$\hat{n}=m 0 r a \infty$

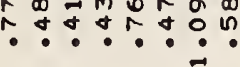

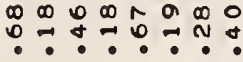

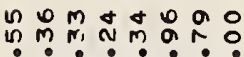
NMAN N N $m$ $\dot{0} \div$

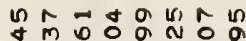

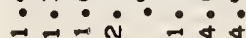

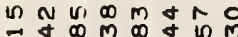

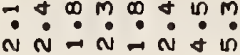

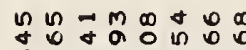

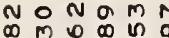

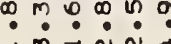

영유유:

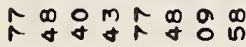
ก.....

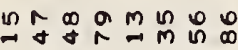

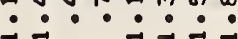

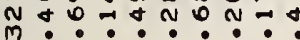
mi

ดก

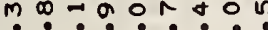
$\dot{a} \cdot \dot{i} \cdot \dot{0}$

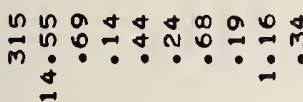

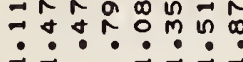

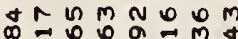

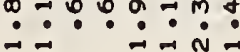
$\therefore \dot{-} \div \dot{-} \check{-}$

a $m \infty m \vec{n}=n$

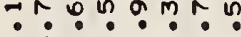
$\dot{m} \dot{m} \dot{\sim} \dot{N} \dot{m} \dot{0}$

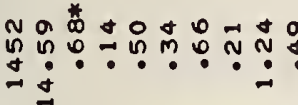

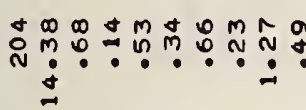

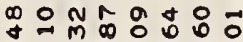
$\dot{\sigma} \dot{\sim} \dot{N} \dot{\alpha} \dot{m} \dot{0} \dot{0}$

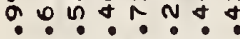
$\dot{N} \dot{m} \dot{\sim} \dot{N} \dot{m} \dot{\sigma} \dot{n}$ $a_{0 \rightarrow \infty}^{0}$

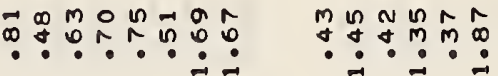

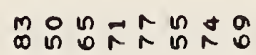
๑ேำ?

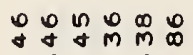
・・・・

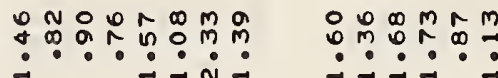

$\rightarrow-\infty$

$m a n \rightarrow \infty n a n$

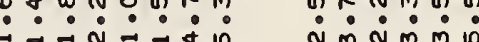

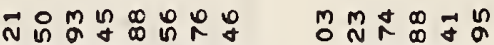

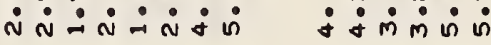

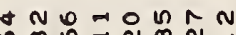

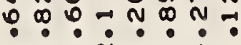
응 noก.บำ N的的的

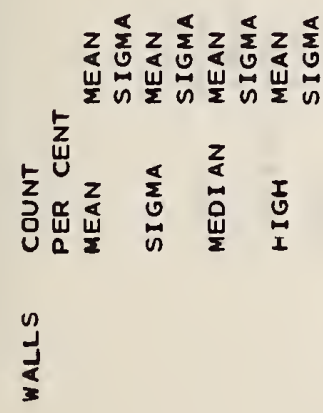

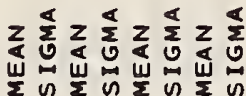

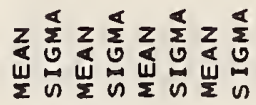

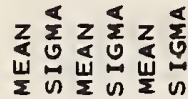

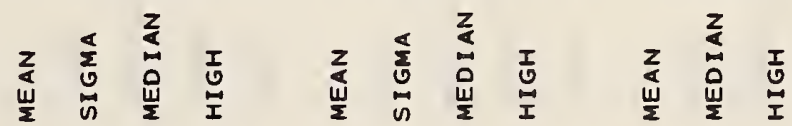

$\underset{\Sigma}{\Sigma}$

$\frac{1}{4}$

$\frac{3}{1}$ 


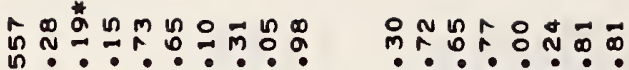

田:

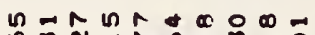

mล:

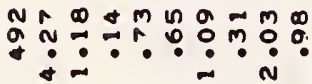

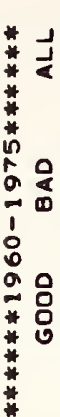

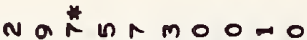

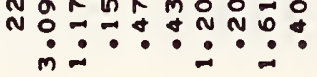

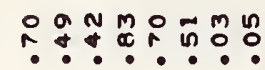

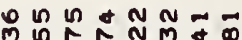

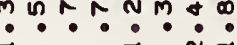

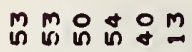

$\therefore \cdot \frac{i}{4}$

\section{$\circ::: ㅇ: ㅇ:$}

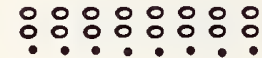

กับ

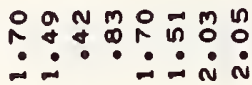

ำกำ

m.5.?ำ.

응유:유:

$\therefore: ㅇ:$

*

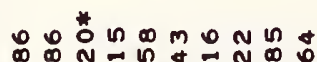

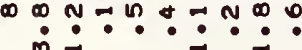

:

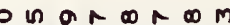

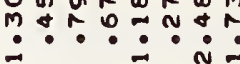

D.

Mmo\&om

ใ?

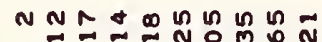

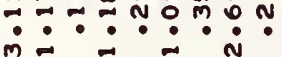

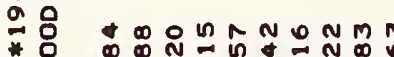

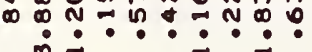

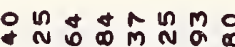

$\because \because \because \%$ ก

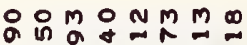

$\dot{n} \dot{-} \dot{\sim} \dot{n} \dot{0}$

ดำํำ

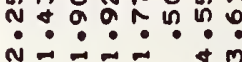

9

$\dot{\square}: \because$

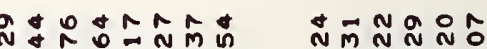

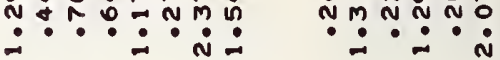

$m \infty$ m $n$ N $\dot{m} \dot{m} \dot{m} \dot{m} \dot{m} \dot{0} \dot{0}$

nี⿻一𠃋十

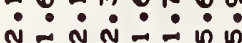

- $N$ n $\infty$ n Q a

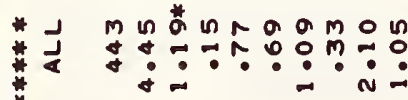
*

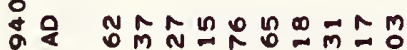

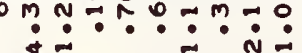

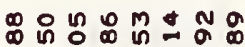

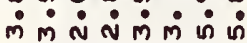

\& $\dot{N} \dot{N} \dot{\sim} \dot{\sim} \dot{\sim} \dot{0}$

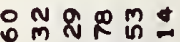
$\dot{m} \dot{0} \dot{m} \dot{m} \dot{0} \dot{0}$

"

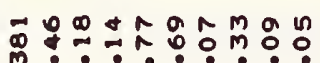

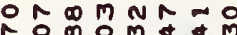
$\ddot{m} \dot{0} \dot{m} \dot{m} \dot{m} \dot{0}$

แ 20 ก त.

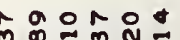

$\dot{0} \dot{n} \dot{0} \dot{0}$
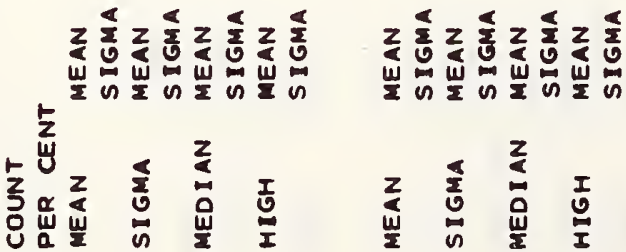

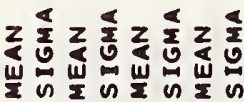

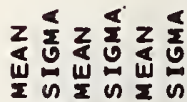




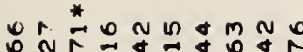

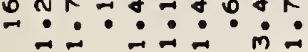

M $\dot{N}: \because \dot{-} \dot{m} \dot{\sim}$

H。 $m \div \div \div m: 8$ in

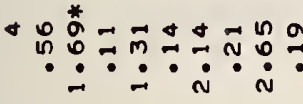

$\circ:::::$

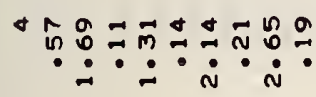

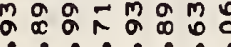

$\dot{N} \dot{-} \dot{\sim} \dot{N} \dot{m} \dot{m}$

กับ

$\dot{m} \dot{m} \dot{\sim} \dot{m} \dot{N} \dot{0}$

måg

$\dot{N} \dot{\sim} \dot{\sim} \dot{\sim} \dot{m} \dot{m}$

$\therefore::::$

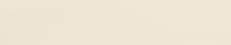

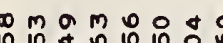

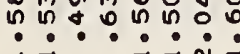

a으.

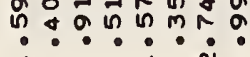

요요

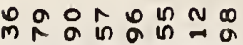

$\dot{\sim} \dot{\sim} \dot{\sim} \dot{\sim} \dot{\sim} \dot{\boldsymbol{m}}$

nom $m$

$\because \div: \because: 0$

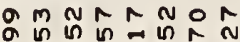

ก⿻

$\because: \because:: \because:: \quad:: \because:::$

ดิ

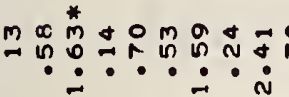

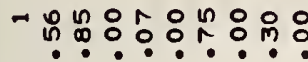

$\therefore \cdot \dot{a}$

N $N$ m

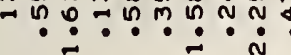

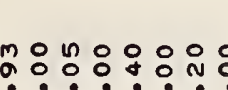

$\dot{a} \dot{0} \cdot \dot{0}$

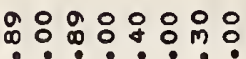

$\because \div \div \dot{4}$

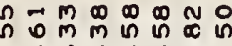

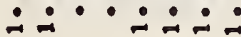

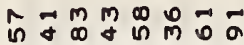

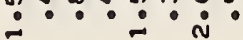

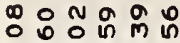

$i \dot{i}: i \dot{0}$

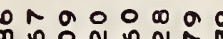

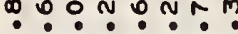

$\dot{m} \dot{m} \dot{\sim} \dot{m} \dot{m} \dot{m} \dot{0}$

$\ln n N+\infty$

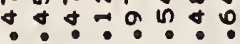

$+n N a+\infty$

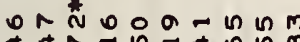

$\pm \div \div ? \div . ? ! ?$

$\dot{N} \dot{\sim} \dot{N} \dot{\sim} \div \dot{0}$

ज的的实

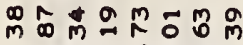

$\dot{0} \dot{\sigma} \dot{m} \dot{\alpha} \dot{\alpha} \dot{\sigma} \dot{\infty}$

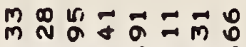
$\dot{m} \dot{m} \dot{\sim} \dot{m} \dot{\sim} \dot{m} \dot{R}$

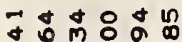

$\dot{m} \dot{\circ} \dot{m} \dot{\sigma} \dot{\sim} \dot{0}$

บำ

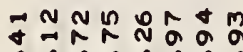
$\dot{m} \dot{m} \dot{\sim} \dot{m} \dot{m} \dot{+}$

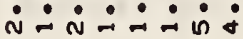

$\infty \infty \circ \pm 0$

ก บำด

$\dot{\sim} \dot{m} \dot{-} \dot{m} \dot{0}$ 
ํํำำกำำำำำำ

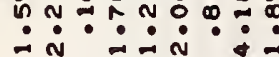

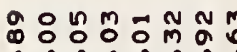

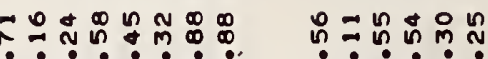
$\dot{m} \dot{m} \dot{\alpha} \dot{\alpha} \dot{m} \dot{m}:$

$\dot{\sim} \dot{\sim} \dot{\sim} \dot{\sim} \dot{\sim} \dot{\sim} \dot{m}$

$\dot{N} \dot{\alpha} \dot{N} \dot{N}$ in

晏

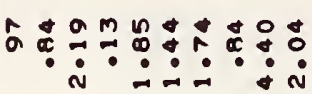

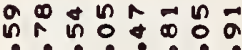

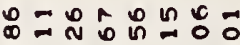

ง $\operatorname{00000}$ mं

i n

$\because \dot{m} \dot{m} \dot{0}:$

$00 \% 0000$

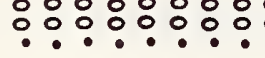

$\circ:::::::: \circ$

8

* 융유유:융

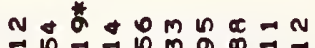

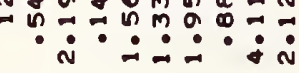

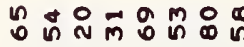

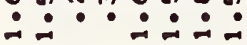

जิ 0000000000

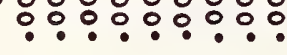
1 8

은 $n \pi+0 m n \infty=N$

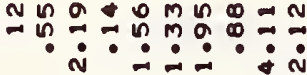

:ㅇ:유:응

웅융ㅇㅇㅇㅇㅇㅇ

응ㅇ:웅

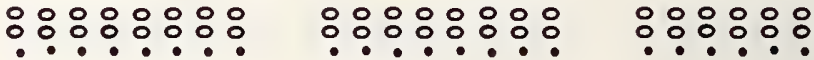

$: \because:: 8:: 8$

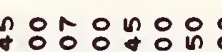

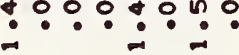

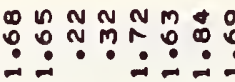

N N ODOONM

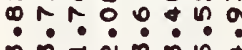

$\log 20$

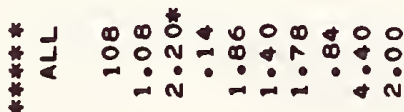

$\dot{m} \dot{m} \dot{\sim} \dot{\sim} \dot{m} \dot{m}:$

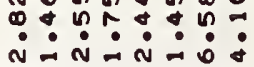

$m=-\infty 00$

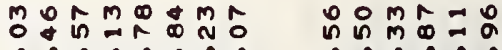
$\dot{\sim} \dot{\sim} \dot{*} \because \dot{N} \quad i \dot{a} \dot{\sim} \dot{1}$

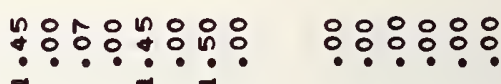

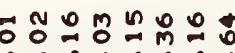
$\therefore \dot{m} \dot{N} \dot{*} \dot{m} \dot{0}$ $\therefore \dot{m} \dot{m} \dot{0}$

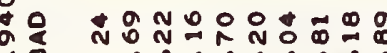

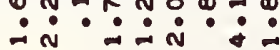

एँ.

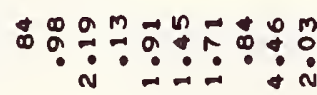

ํํํำำำำ

$\dot{m} \dot{m}=\dot{\sim} \dot{m} \dot{m}$ in

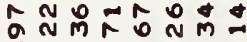
N $\dot{N} \dot{N} \dot{N} \dot{0}:$

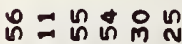

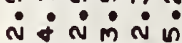

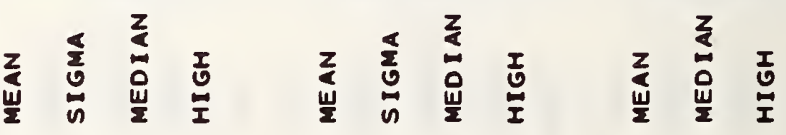

$\underset{\frac{2}{\alpha}}{\underline{\alpha}}$

」

1 


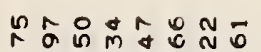
$\dot{m} \dot{m} \dot{\sim} \dot{x} \dot{m} \dot{m} \dot{0}$

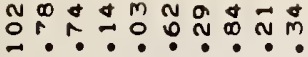
$\because \dot{n} \cdot \dot{n} \cdot \dot{n}$

ง $\dot{-i} \dot{i} \dot{i} \dot{v} \dot{0}$

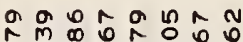
$\because \dot{m}: \because: 0$

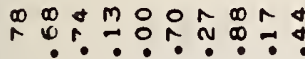
in $\dot{a} \dot{a}$ in

\& 0 응ำ $\dot{m} \dot{a} \dot{i} \dot{m} \dot{m} \dot{0}$

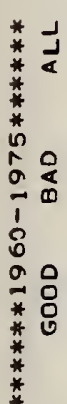

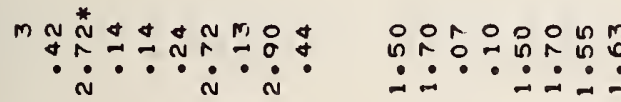

응ㅇㅇㅇㅇㅇㅇㅇㅇㅇㅇㅇㅇ

응응ㅇㅇㅇㅇㅇㅇㅇㅇㅇㅇㅇㅇ

ㅇํㅇํㅇ

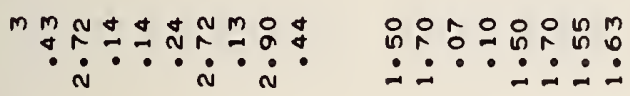

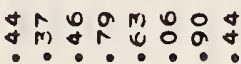
i $\cdot \dot{n}$ i

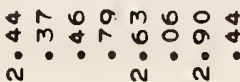

antoon $\frac{1}{1} \div-\frac{1}{1} \div$

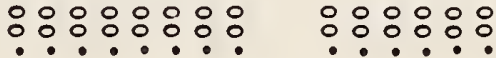

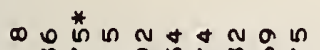

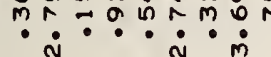

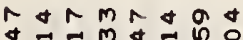
$\because \div \div ! \div \div !$

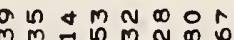
$\dot{m} \cdot \dot{n} \cdot \dot{m}$

man $\dot{1}-\dot{1}-\dot{1} \dot{0}$

$\circ: 0 \%: 0 \% 0 \%$

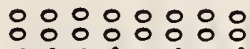

응ㅇㅇㅇㅇㅇ응

응: : : :

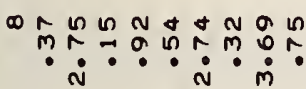

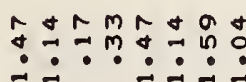

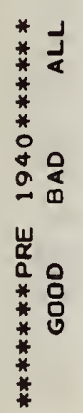

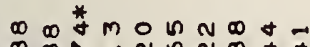

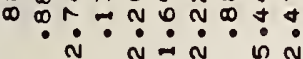

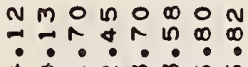

웅 $n \mathrm{~m}$ เ $\mathrm{m}$

กร.ำรใด

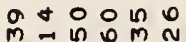

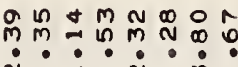

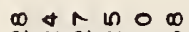

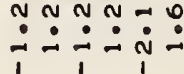

N $\because \dot{N} \cdot \dot{a} \dot{a} \cdot \dot{m}$

Oัd $\dot{q} \dot{m} \dot{-} \dot{q} \dot{m} \dot{0} \dot{q}$

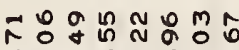
$\dot{m} \dot{N} \dot{N} \dot{m} \dot{m} \dot{i} \dot{m}$

ํํำ

$\dot{m} \dot{m} \dot{m}$

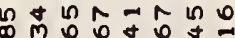

ำษ

i $: \dot{a}: \dot{v}: \dot{0}$

iो $\dot{0} \dot{0} \dot{m} \dot{0}$

N $\vec{m} \vec{m}+0$ $\dot{m} \dot{\sim} \dot{\sim} \dot{\sim} \dot{\sim} \dot{0}$

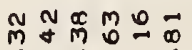

$\dot{m} \dot{\sim} \dot{N} \dot{N} \dot{N} \dot{\sim} \dot{-} \dot{+} \dot{\sim} \dot{m}$

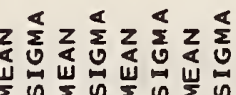

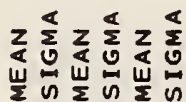

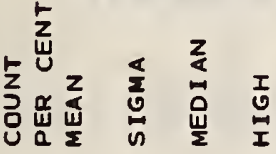

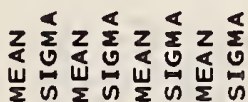

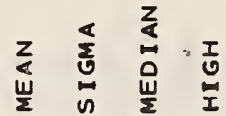

$\frac{1}{2}$

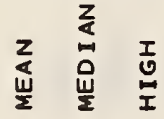

1 $\frac{1}{3}$

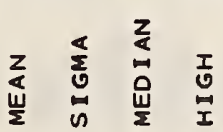

$\underset{\substack{x \\ \leftarrow}}{x}$ 


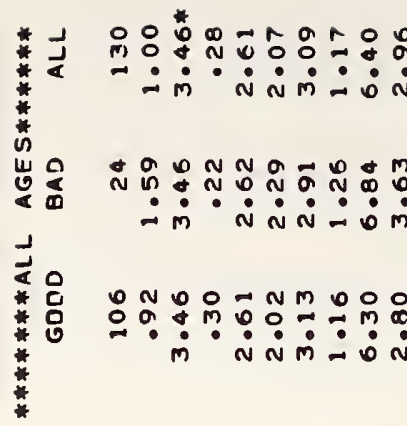

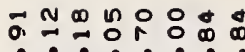

oxo의 웅

ก으ำ

$\dot{m} \dot{\sim} \dot{m} \dot{0} \dot{0}$

-.....

$\because \div: ?$ ?

ก.

ที ค \&

$\dot{m} \dot{\sim} \dot{m} \dot{m} \dot{N} \dot{0}$

oิ

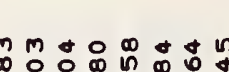

$\infty \circ: 0$.

om $m+a$

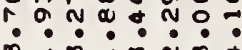

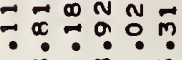

$m+\dot{N} \dot{m} \dot{m}$ 的

$m-m-m N \infty$.

$\dot{m} \dot{m} \dot{i}$

"2.

$00 \% 000000$

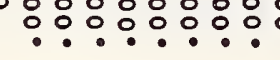

응ㅇㅇㅇㅇㅇㅇㅇㅇㅇㅇㅇㅇㅇㅇㅇ

응ㅇㅇㅇㅇㅇㅇㅇㅇㅇ

응ㅇㅇㅇㅇㅇㅇ

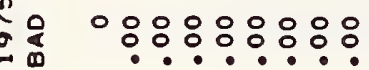

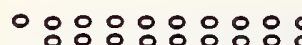

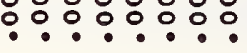

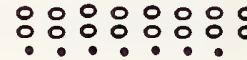

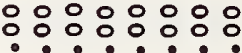

응ㅇㅇ웅

* J

ผ

i ก

$\underset{0}{0}=0$ N

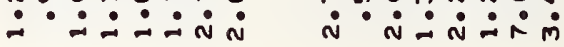

$\dot{i}=\dot{i}-\dot{i}$

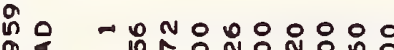

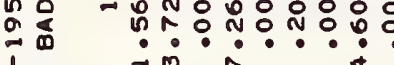

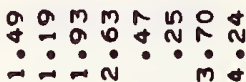

:

옵ㅇㅇㅇ응ㅇㅇㅇㅇㅇ

H Nm $\mathrm{Na}$

8

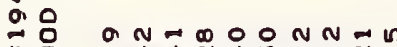

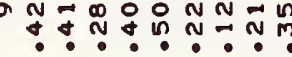

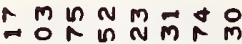

R

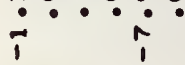

$\therefore \dot{-} \div \dot{-1}$

$\dot{N} \dot{N} \cdot \dot{N} \dot{0} \dot{N}$

mad $\dot{n} \div \dot{n} \dot{i} \dot{i}$

*

or

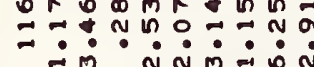

ค ำ

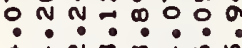

nก $N+\infty$ n 00

$\sim \infty 00 \infty$ Mே:

in

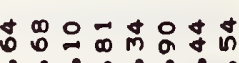

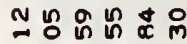

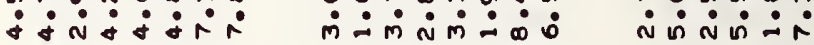

คิ $\dot{m} \dot{\dot{\alpha}} \dot{\mathrm{N}} \dot{\mathrm{m}} \dot{\mathrm{m}} \boldsymbol{\mathrm { r }} \dot{\mathrm{n}}$

ก N

岀

maㅇํำ $\because \dot{m} \dot{N} \dot{N} \dot{M} \dot{0} \dot{N}$

ลำ 幺กำ - ? ? :? $\dot{m} \dot{N} \dot{m} \dot{m} \dot{N} \dot{0} \dot{0}$ 


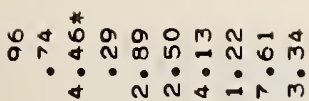

m 0 $\therefore \dot{\sim} \dot{m} \dot{\sim} \dot{\sigma} \dot{\sim} \dot{m}$

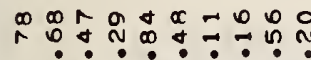

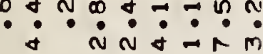

ํㅓㅁํำ $\dot{\sigma} \dot{m} \dot{\sim} \dot{\sim} \dot{\alpha} \dot{m} \dot{0} \dot{n}$

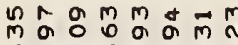

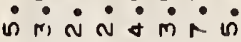

ํำ $\dot{\sigma} \dot{m} \dot{m} \dot{m} \dot{m} \dot{0}$

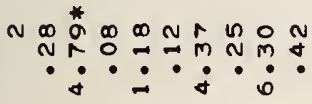

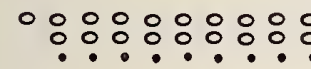

ตำ

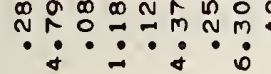

*

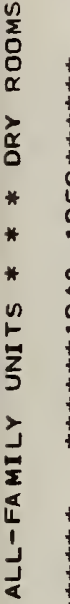

nm.

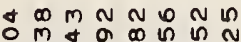

$\dot{\sim} \dot{m} \cdot \dot{\sim} \dot{m} \dot{m}$

A. mormon $\dot{m} \dot{\sim} \dot{\sim} \dot{m} \dot{0} \dot{0}$

t $\dot{-} \dot{m} \dot{N} \dot{\alpha} \dot{N} \dot{\alpha}$

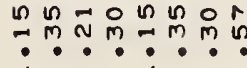

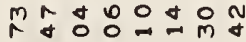

$\dot{m} \dot{\sim} \dot{a} \dot{0}$

응ㅇㅇㅇㅇㅇ

응유:응웅

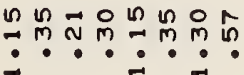

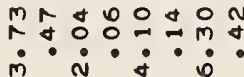

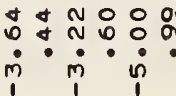

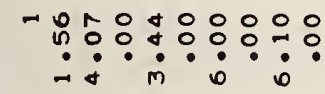

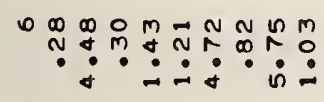

응유융요

송웅ㅇㅇㅇㅇㅇㅇㅇㅇㅇ

i่ $\dot{m}: \dot{0}:$

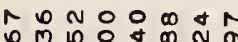

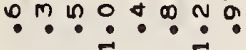

N

$\dot{m}: \dot{m} \cdot \dot{m}=\dot{m}$

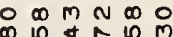

mं

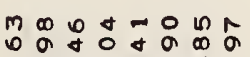
- $\dot{m} \dot{\mathrm{n}} \dot{\mathrm{a}} \dot{\mathrm{m}} \dot{0} \dot{0}$

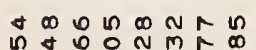

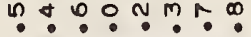

moำ $\because \because \because 9:$

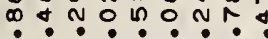
$\because: \dot{m} \dot{0} \dot{0}$

ㅇำ

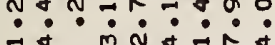
宸

ดี a $\dot{m} \dot{i} \dot{a} \dot{A} \dot{m}$

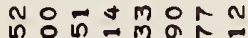
$\dot{\alpha} \dot{\alpha} \dot{m} \dot{\circ} \dot{0}:$

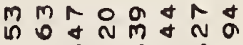

= $\dot{0} \dot{m} \dot{\sim} \dot{\alpha} \dot{0} \dot{0}$

a $m \sim \infty \infty \infty \infty$ $2 \pi$

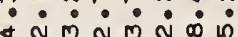

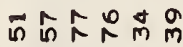
$\therefore \dot{m} \dot{0} \dot{0}$

กำ 응ำ $i \dot{0} \dot{i}$

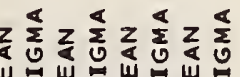

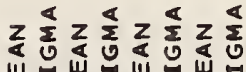

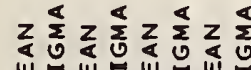
崖的崖的崖的崖尚

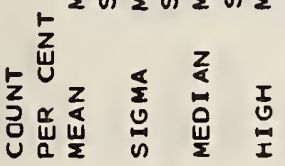

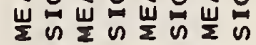

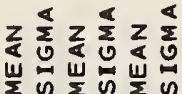

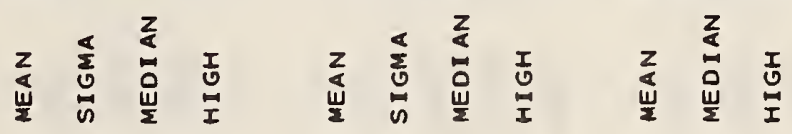

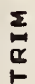

J

$\frac{3}{1}$ 


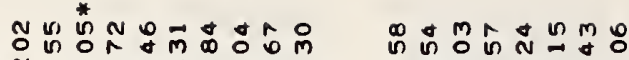

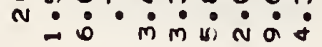
*

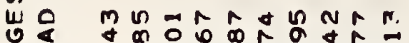

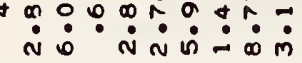

$J$
J
*
*
*
*
$*$
$*$

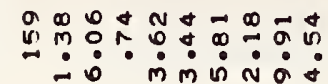

*

$\dot{\alpha} \dot{\alpha} \dot{N} \dot{\alpha} \dot{0} \dot{0}$

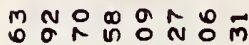
$\dot{0} \dot{i} \dot{N} \dot{0} \dot{0} \dot{0}$

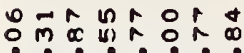
- $\dot{0} \dot{\mathrm{s}} \dot{0} \dot{0}$

$00 \% 0000000$ ㅇㅇㅇㅇㅇㅇㅇㅇㅇำ

ㅇㅇㅇㅇㅇㅇㅇㅇㅇ

응ㅇㅇㅇㅇㅇㅇㅇㅇㅇㅇㅇㅇㅇ

응ㅇㅇㅇㅇㅇㅇ

응ㅇㅇㅇㅇㅇ응

응ㅇㅇㅇㅇㅇㅇㅇ
*

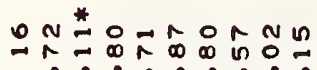

$\because \dot{0} \dot{0} \dot{0} \dot{0} \dot{0}$

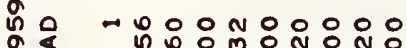

a

$\therefore \dot{0} \dot{0} \dot{0} \dot{0}$

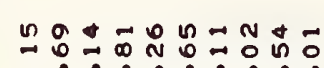

$\because \because \div: \because: \dot{0}:$

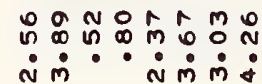

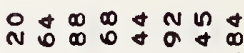

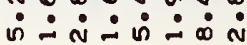

응ㅇㅇㅇㅇㅇㅇㅇㅇ

응ㅇㅇㅇㅇㅇㅇㅇㅠ

。 $\dot{\circ} \dot{\sim} \dot{m} \dot{N} \dot{\sim} \dot{\mathrm{O}} \dot{\mathrm{n}}$

ลิ a ம் $\dot{m}$ i $\dot{\mathrm{N}} \dot{0}$ i

ค - ก. $\dot{1}+\dot{1} \dot{0} 0$

응융ㅇㅇㅇㅇㅇ

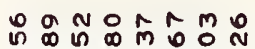
i $\dot{m} \cdot$ is $\dot{m} \dot{m} \dot{+}$

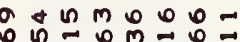

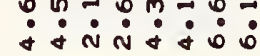

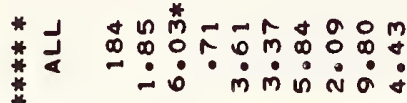

$\ln \infty N=0 n+n$ i.

NN⿴囗十心 $\dot{+} \dot{i} \dot{0} \dot{0}$

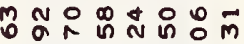
$\dot{0} \dot{\sim} \dot{\mathrm{N}} \dot{0} \dot{a} \dot{0}$

次早 $\dot{N} \dot{0} \dot{N} \dot{N} \dot{0} \dot{\infty} \dot{m}$

岩

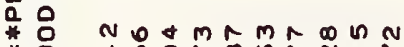
$\pm: 0 ?$ ก ? $\therefore \dot{0} \dot{m} \dot{m} \dot{0} \dot{0} \dot{+}$

n웅

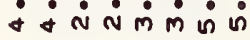

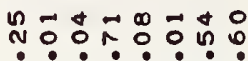
iि $\dot{\mathrm{a}} \dot{\mathrm{N}} \dot{\mathrm{m}} \dot{0} \dot{\mathrm{O}}$

กำ

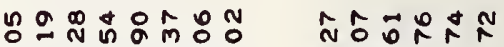

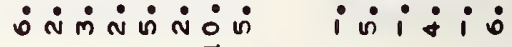

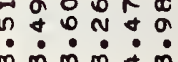
$m m m+m$

:ㅇ:::요

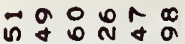
$\ddot{m} \dot{m} \dot{m} \dot{m} \dot{q}$ *

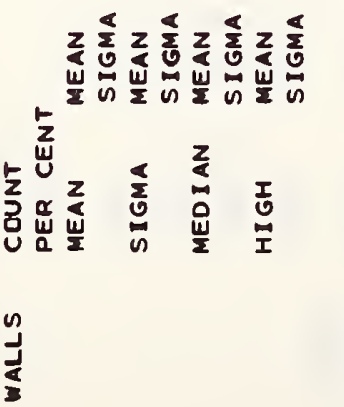

z⿺⿻一𠃋火

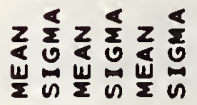

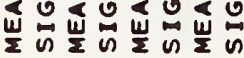

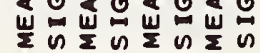

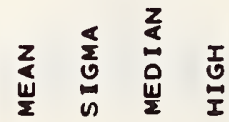

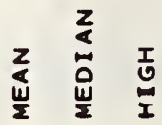

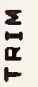

J

1 


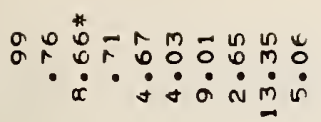

m m n $\because \dot{C} \dot{a} \dot{\alpha} \dot{\infty} \dot{N} \dot{m}$

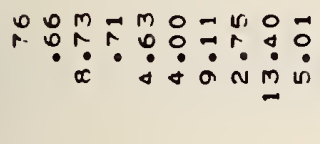

융융ㅇㅇㅇㅇㅇㅇㅇㅇㅇ ㅇํㅇํㅇ

응ㅇㅇㅇㅇㅇㅇㅇㅇㅇㅇㅇㅇ

응ㅇㅇㅇㅇㅇㅇㅇㅇㅇㅇ 응ㅇㅇㅇㅇㅇㅇ응

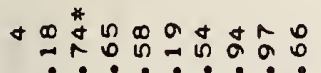
$\because \dot{\infty} \dot{0} \dot{0} \dot{0} \dot{a} \dot{\sigma}$

N $\because \dot{m} \div \dot{\sim} \dot{0} \dot{0} \div \dot{0}$

nat a n n n n n $\because \dot{0}: \because \dot{0} \div \dot{0} \dot{m}$

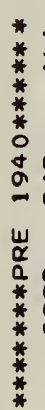

응ㅇㅇㅇㅇㅇㅇ

응ㅇㅇㅇㅇㅇㅇㅇㅇㅇ

ล

$\dot{m} \dot{i} \dot{N} \dot{m} \dot{N} \dot{+}$

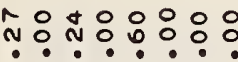

in $\dot{m} \dot{m} \dot{a}$ $\dot{m} \dot{\dot{N}} \dot{\mathrm{N}} \dot{\mathrm{m}} \dot{\mathrm{O}} \dot{0}$

กิด ดิ

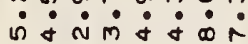
ம் $\dot{m} \dot{N} \dot{m} \dot{m}$

ลี

$m$
$\sim$ $\dot{0} \dot{0} \dot{m} \dot{0} \dot{0} \dot{0} \dot{0}$

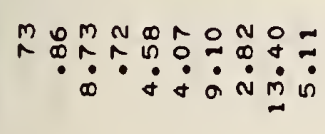

웡 iि $\dot{A} \dot{m} \dot{0} \dot{0}:$

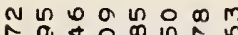
$\dot{0} \dot{\sim} \dot{\sim} \dot{\sim} \dot{m} \dot{m}$

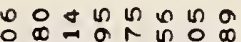
i $\dot{\text { i }} \dot{m} \dot{m} \dot{\alpha} \dot{0} \dot{0}$ mañ

$\because \dot{\sim} \dot{\sim} \dot{R} \dot{\alpha} \dot{\sigma}$

is in

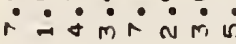

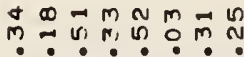

?r.m?

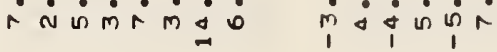

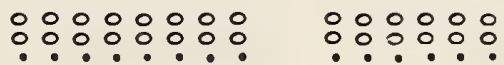

웅 $\dot{r} \dot{i} \dot{i} \dot{a} \dot{a}$

$\cos 0$ $\dot{i}: \dot{i} \cdot \dot{i}$

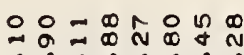

$\dot{a} \dot{i} \dot{i} \dot{0} \dot{\dot{0}} \dot{r}$

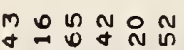
$\dot{m} \dot{i} \dot{0} \dot{0} \dot{0}$

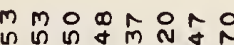

กn

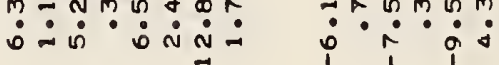

d

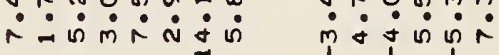

NO요요

Nิน ถูก $\because \div \because \div 0$ a

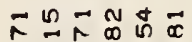

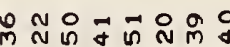
$\dot{\sim} \dot{\mathrm{N}} \dot{\mathrm{m}} \dot{\mathrm{r}} \dot{\mathrm{m}} \dot{\mathrm{j}} \dot{0}$

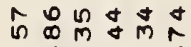

iो $\dot{i}$ i i

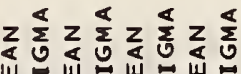

$z \sum_{0}^{\infty} z \sum_{0} z \sum_{0}^{\infty}$

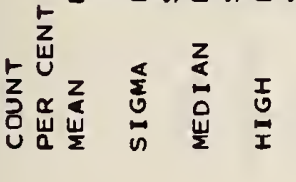

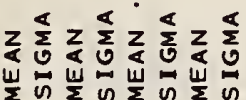

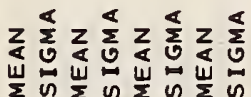

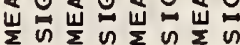

崖崖的崖的

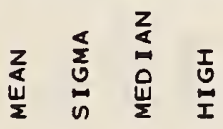

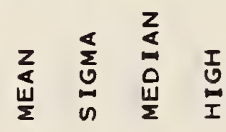

$\begin{array}{lll} & z & \\ z & 0 & I \\ \frac{U}{\Sigma} & \frac{1}{\Sigma} & I\end{array}$

$\underset{\sim}{\Sigma}$

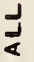

1 
च ำ

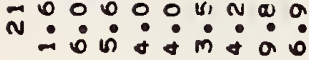

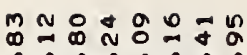

ம்

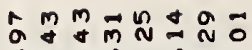

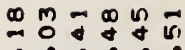

$\dot{a} \dot{\sim} \dot{m} \dot{0} \dot{0} \dot{0}$

$\therefore \dot{i} \dot{i}=\dot{\infty}$

ㅇำำำ

$\dot{m} \dot{0} \dot{\sigma} \dot{\sigma} \dot{m} \dot{m} \dot{0} \dot{0} \dot{0}$

ง

的向它: $\dot{0}$

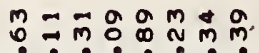

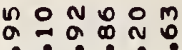

누 $\dot{0} \dot{0} \dot{0} \dot{\infty}$

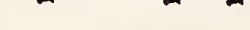

ำํำำ

: $\dot{0} \dot{0} \dot{0} \dot{0} \dot{m} \dot{0}$

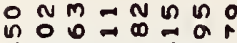

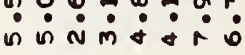

-

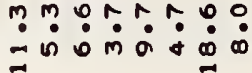

ir no

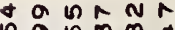

ด.ด ตั. $\approx$

11

$00 \% 0000000$

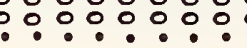

응ㅇㅇㅇㅇㅇ응

$\therefore: \because: \because::: \quad::::::$

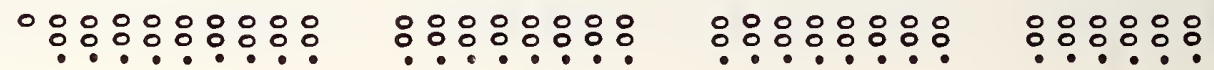

$\circ: 80: 0: 0$

$\circ: 0: 0: 08$

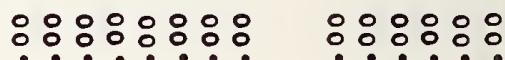

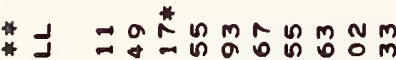

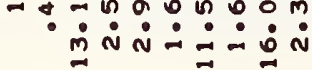

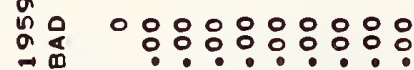
o

。ํำ

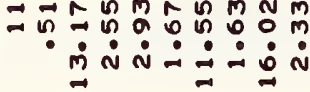

* J a a a

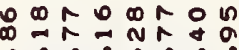

ம் $\dot{0} \dot{m} \dot{0} \dot{0} \dot{0}$

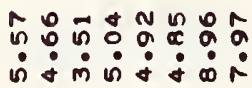

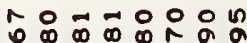

$\dot{\circ} \dot{0} \dot{0} \dot{0} \dot{0} \dot{0}$

$N=N=M$

in

ம் $\dot{\theta} \dot{m} \dot{\theta} \dot{+} \dot{0}$

ㅇ:ㅇ:ㅇㅇㅠ

응응ㅇㅇㅇㅇㅇ응

웅ㅇㅇㅇㅇㅡ.

웅ㅇㅇㅇㅇㅇㅇ

まำ \&

-

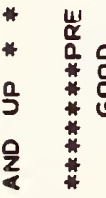

은

$\rightarrow \because \dot{0} \dot{0} \dot{0} \dot{m} \dot{0}:$

O 0 \& $m+o^{N}$

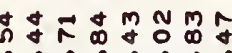

$\dot{\sim} \dot{m} \dot{m} \dot{0} \dot{0} \dot{0}$

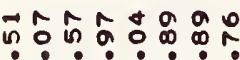

in

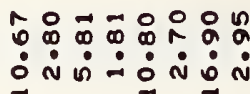

กㅇำ

$\dot{\alpha} \dot{*} \dot{m} \dot{a} \dot{0}$

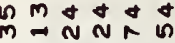

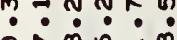

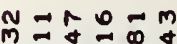

$\dot{1} \dot{0} \dot{0} \dot{0} \dot{0}: \dot{0}$

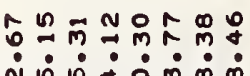

$N \backsim n+0 m \infty$

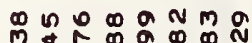

$\because$ ம

$\Rightarrow \curvearrowleft 0 m \infty m \infty \infty$

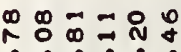

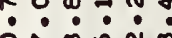

$\stackrel{\circ}{i} \dot{0} \underset{i}{N}$

$:$

$\stackrel{1}{\lessgtr}$

$\stackrel{⿱ 亠 乂}{\rightleftarrows}$

z

$\stackrel{0}{4}$

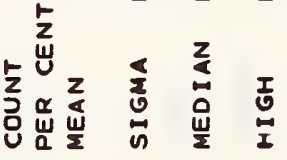

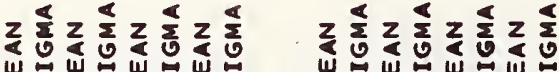

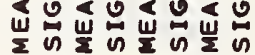

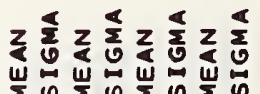

$z \sum_{0} z \sum_{0} z \frac{5}{0}$

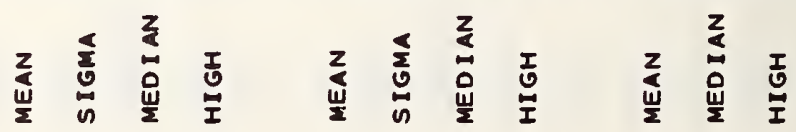

$\frac{n}{3}$

$\underset{\substack{\alpha \\ r}}{\underline{\alpha}}$

d

i 


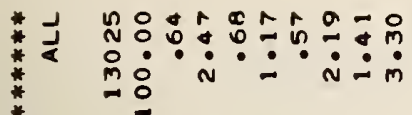
约

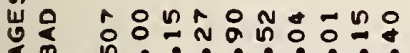

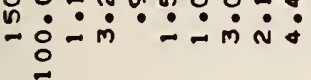

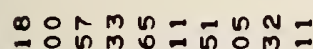

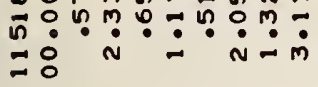

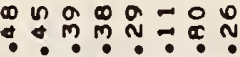

Ni் $\dot{N} \dot{N} \dot{m} \dot{m}$

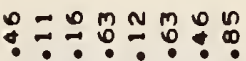

- $\dot{N} \dot{N} \dot{m} \dot{0}$

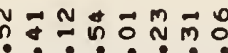

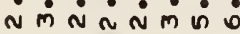

웅요

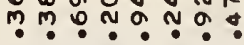

$-\dot{N}-\dot{N} \dot{m} \dot{v}$

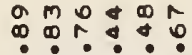

$\dot{m} \dot{m} \dot{m}$ i

으무요

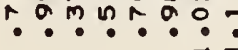

N

Noㅇำ

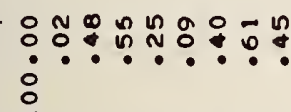

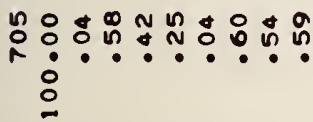

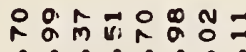

?.?.?:?:

N Doก

ก!กำดัด

?ำ

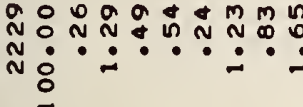

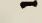

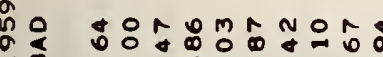
$\dot{\circ}: \dot{:}: \dot{0} \div \dot{m}$ $\circ$

แกำ กค

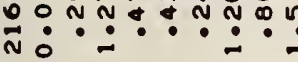
우

+ 0 r $m+0000$ ă

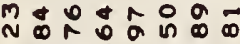
$\dot{m} \dot{m}-\dot{N} \dot{N} \dot{m} \dot{0}$

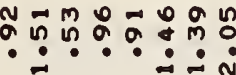

ก๊ํำ $\dot{i} \dot{i} \dot{i} \dot{n} \dot{m}$

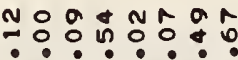

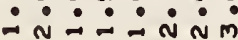

ํำ $\dot{\sim} \dot{N} \dot{n}-\dot{m}$

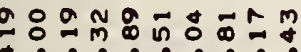

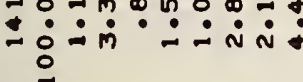

* $\dot{+} \dot{i} \dot{N} \dot{+} \dot{m} \dot{0}$

nㅇํㅇำ

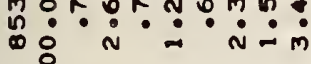

a N $\dot{m} \dot{\text { n }} \dot{m} \dot{0}$

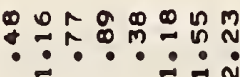

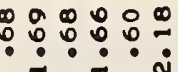

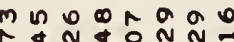

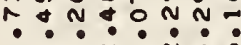

웅용ㅇㅇㅇㅛ :m m $m:$ - Niñ

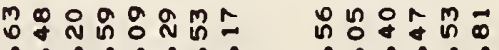

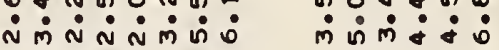

:ㅇำกำ

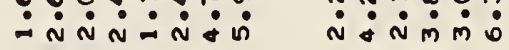
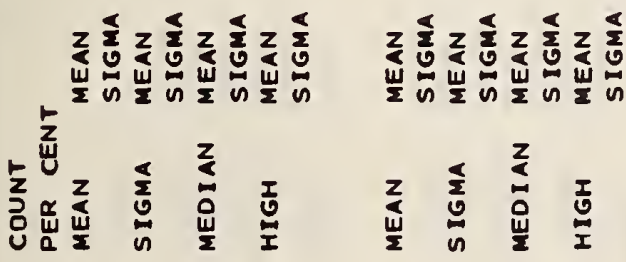

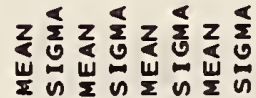

$z \frac{\pi}{2} z \frac{\pi}{2} z \frac{\pi}{0}$

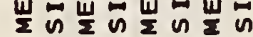
㞫药㞱的部

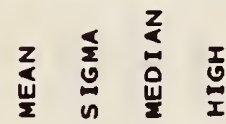

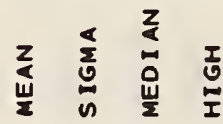

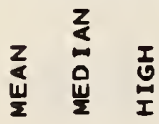

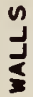

$\underset{\frac{2}{\alpha}}{\underline{\alpha}}$

J<smiles>[3H][3H]</smiles> 


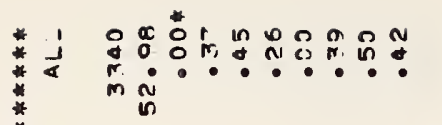

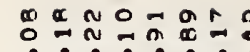

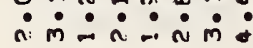

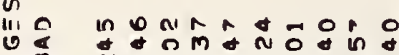

\& 0 N in

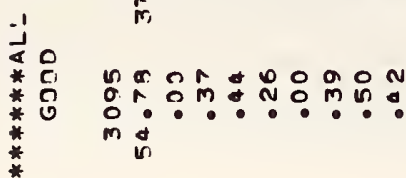

* 」

* J

ấ mooo-

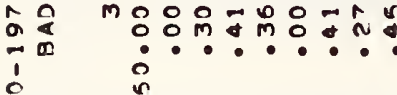

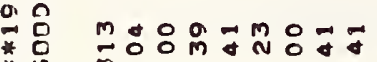

*

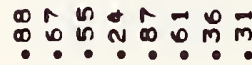

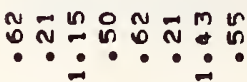

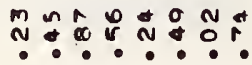

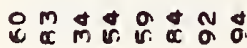

?..? ? ma

ir $-n 0 m-00000$

욤

i is

aㅇํㅇ

กำ $\ddot{0}$

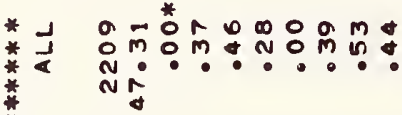

D

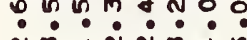

Mัn $\ddot{N}-\dot{N}-\dot{N} \dot{M}$

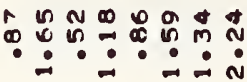

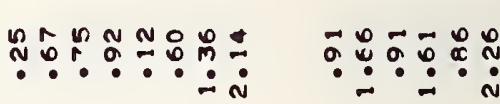

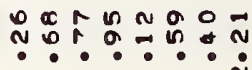

$N \infty N M \infty N$ $\because \because \because \because !$

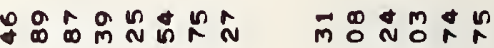
...?

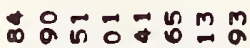
mं $\dot{m} \dot{m} \dot{m} \dot{0} \dot{0}$

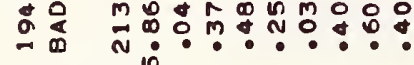

$\stackrel{41}{\alpha}$

品

*

0
0
0
0

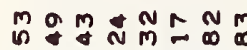

?:

NMANNMM

ดั

$\because \div \div \div \div:$

ำ ํำ

जिंिंm:

•:

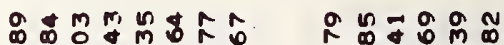
$\therefore \dot{N} \dot{N} \dot{\sim} \dot{N} \dot{0} \quad \dot{m} \dot{m} \dot{m} \dot{m} \dot{n}$

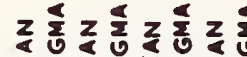

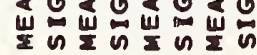

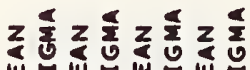

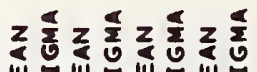

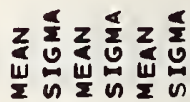

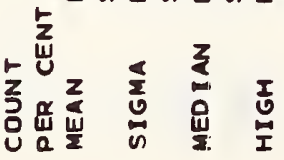

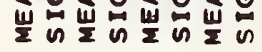

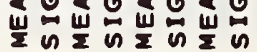

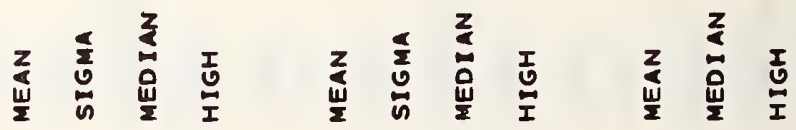

ב

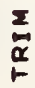




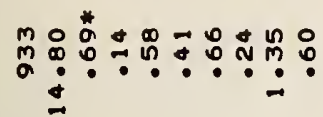

maำำำ

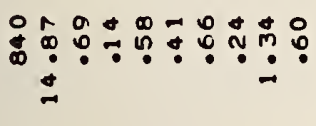

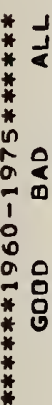

우ำm

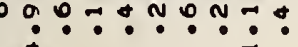

$\pm$

$\circ:::::: ㅇ$

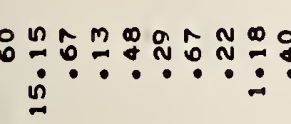

告

这 $\div$

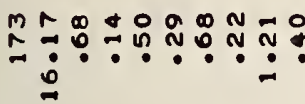

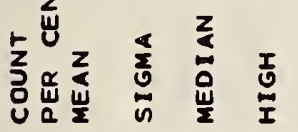

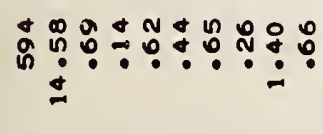
占

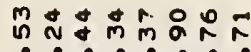
N $\dot{m} \dot{\sim} \dot{N} \dot{N} \dot{m}:$

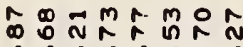
$\dot{m} \dot{m} \dot{N} \dot{\sim} \dot{m} \dot{m}$ in

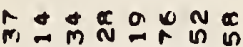

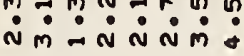

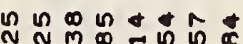

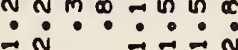

:

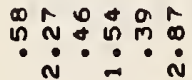

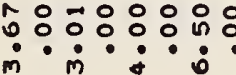

ํำ $\therefore \dot{n}$

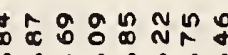

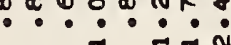

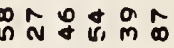
$\ddot{\sim} \cdot \dot{i}$

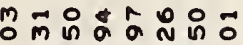
$\dot{-1} \because \because \dot{0}$

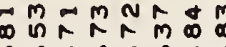
$\because \cdots .$.

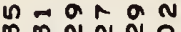
- יㅜㅁ

ลํํํำㅇำ $\therefore \dot{m}=\dot{0} \dot{0}$

ธี

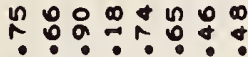
i $\dot{m} \because \dot{\sim} \dot{m} \dot{m}$

ก⿻上丨⿻上丨

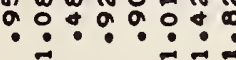

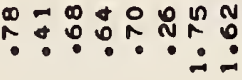

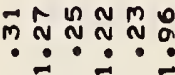

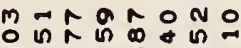
मिं

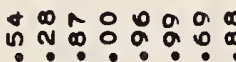

$m-n=m=$ -

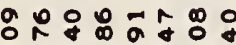

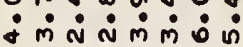

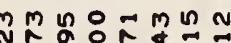

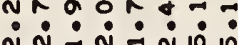

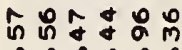
$\dot{m} \dot{x} \dot{m} \dot{m} \dot{8}$

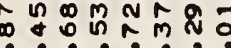

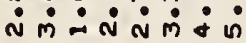

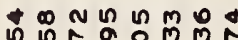

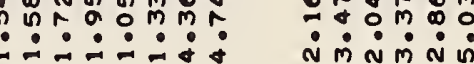

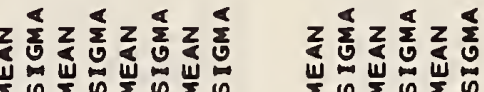

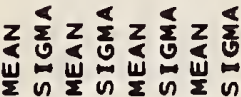

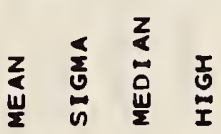

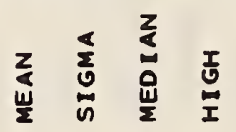

$\underset{⿱ 乛 龰}{\stackrel{\alpha}{r}}$

d

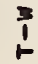


ธำ

d

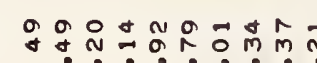

+

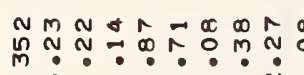

$\dot{0} \cdot \cdot \dot{a} \dot{0}$

$*$
$*$
$*$
$*$
$*$
$*$
0
0
0
0
7
0
0
0
0
0
$*$
$*$
$*$
$*$
$*$
$*$
$*$

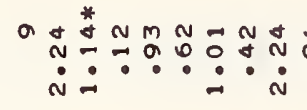

응응ㅇㅇㅇㅇㅇㅇ

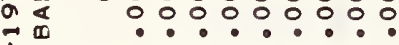

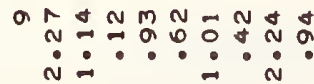

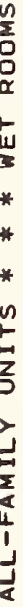

*

in

กั

!

品

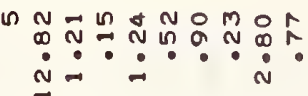

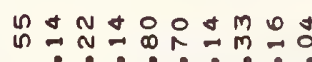

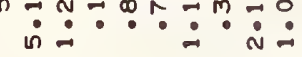

*

*

*

mํำ:

के

$*$

$\therefore$

年

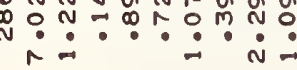

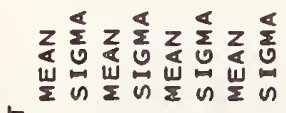

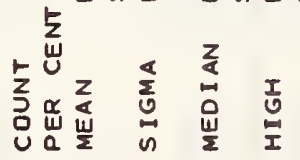

$\frac{1}{3}$

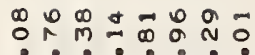

$\dot{m} \dot{m} \dot{\sim} \dot{\sim} \dot{\sim} \dot{0}$

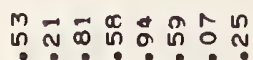

$\dot{\sigma} \dot{\sim} \dot{\sim} \dot{\sim} \dot{m} \dot{m} \dot{0} \dot{0}$

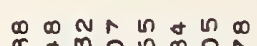

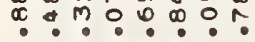

a man na a
ด แก

ด.

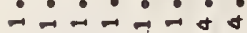

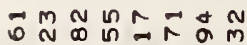

$\dot{N} \dot{m} \dot{a} \dot{N} \dot{N} \dot{0}$

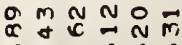

$\dot{N} \dot{\alpha} \dot{N} \dot{m} \dot{m}$ in

x

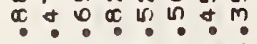

न- न न न व

$F m=a m n$

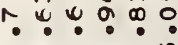

$\rightarrow m-n-n$

$\wedge \infty \infty \propto m \sim-\infty$

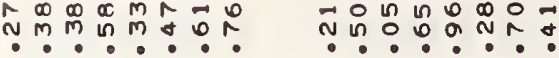

$m=0000$

$\left.\because: \frac{\pi}{4}\right)$

10

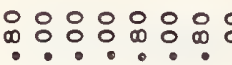

응응응요유

:ㅇ:웅

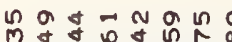

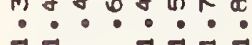

$\rightarrow-$

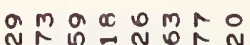

$\because \dot{-} \dot{0} \dot{0}$

Naนn

ง..ด

คํำ

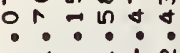

M テ N

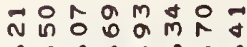

-..ำ

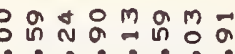

- -1 .

ดํํำ

ㅇํㅇํํํํํํํํㄹ

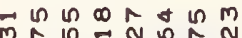

ง

ค n N m m

$\ddot{-} \dot{-} \dot{-} \dot{\sim} \dot{\sim}$

-. :

$\because \because \because$ i

น n 000

$\dot{m} \dot{m}: \dot{N} \dot{m} \dot{m}: \dot{0}$

ก

ำดัด กับ

$N 0+a \sigma 0$

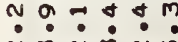

N $\mathrm{M} N \dot{m} \mathrm{~N}$ i

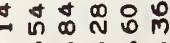

$\dot{m} \dot{\sigma} \dot{\sim} \dot{m} \dot{m}$ in

\&

品

$\dot{\sim} \dot{m} \dot{\sim} \dot{\sim} \dot{N} \dot{\sim}$

กดบก ก

$: 00$ ㅇ․․?

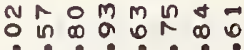

$\dot{\sim} \dot{-} \dot{-} \dot{-} \dot{\dot{\alpha}} \dot{0}$

ति $\dot{m} \dot{\sim} \dot{m} \dot{\sim}$

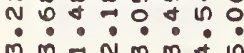

$m M-N M m \& n$

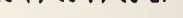

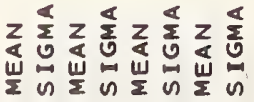

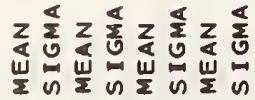

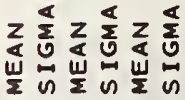

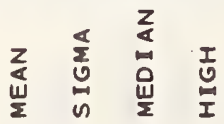

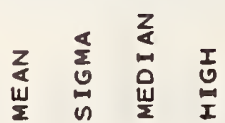

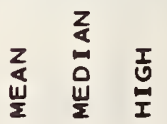

$\underset{\sim}{\alpha}$

J

$\frac{3}{1}$ 


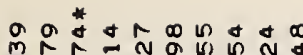

min.

ำ

$\dot{m} \dot{m} \dot{\sim} \dot{N} \dot{m} \dot{m} \dot{0} \dot{0}$

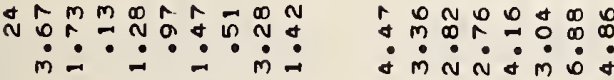

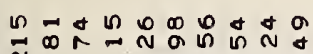

N

d

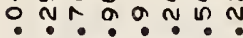

$\dot{m} \dot{m} \dot{\sim} \dot{N} \dot{N} \dot{m} \dot{\sigma}$

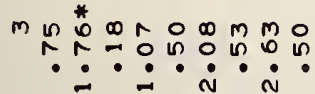

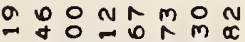

$\dot{N} \dot{N} \dot{\sim} \dot{\sim} \dot{\sim} \dot{m} \dot{m}$

응ㅇㅇㅇㅇㅇㅇㅇㅇ응

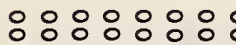

ํ.ㅇำ웅

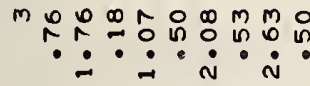

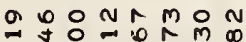

$\dot{N} \dot{N} \dot{-} \dot{\sim} \dot{m} \dot{m}$
ำㅇำ

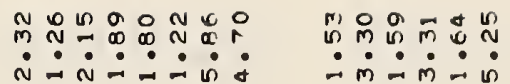

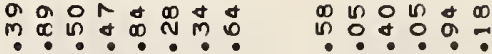

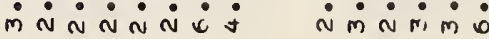

N

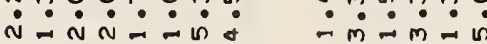

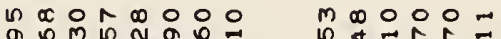
$\because \because \dot{n} \dot{i} \dot{N} \dot{i} \dot{i} \dot{m}$

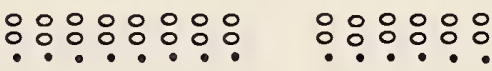

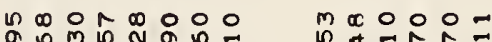

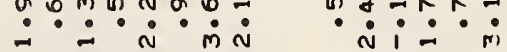

\section{ล $\dot{n} \cdot \cdots \dot{a}$}

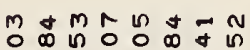

$\therefore: \div: \dot{0}$

onamo n

กำ

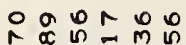

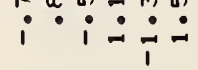

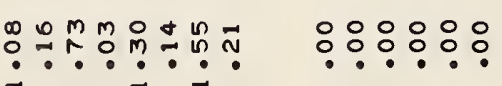

$\circ: \because: \because:: ㅇ ㅛ$

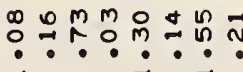

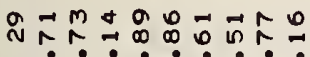

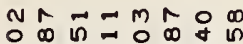

$\therefore: \div: 0$.

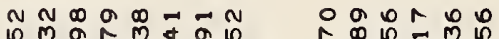

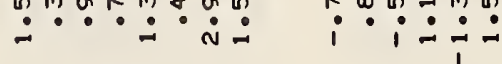

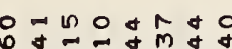

$m \emptyset \stackrel{a}{*}+m a+n m m$

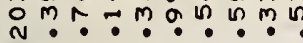

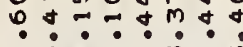

ก

m. m. m m m

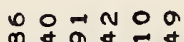

$m$ m $\mathrm{N}$ m $\mathrm{m}$ m in

oำ

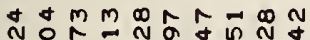
$\dot{a}: \dot{0}: \dot{m}$ $\dot{\sim} \dot{m} \dot{N} \dot{\alpha} \dot{\sim} \dot{\sim}$

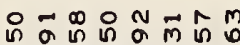
$\dot{m} \dot{N} \dot{N} \dot{N} \dot{N} \dot{0}$

แึก $\dot{\sim} \dot{m} \dot{N} \dot{\sim} \dot{m}$

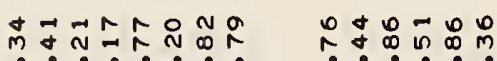

m

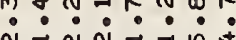

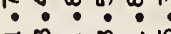
$\dot{m} \dot{m} \dot{\dot{m}} \dot{m} \dot{m} \dot{0} \dot{0}$ 
* J *

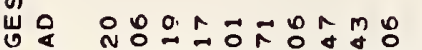

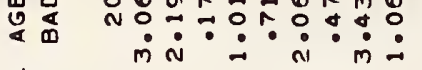

\lrcorner
」 0
$* 0$
$* 0$
$*$
$*$
$*$

*

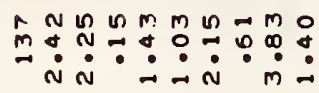

N N $\rightarrow$ n $m-$

no

i.

ros

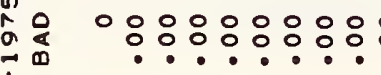

8

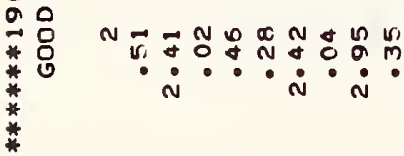

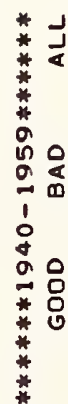

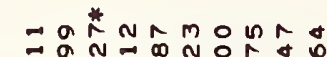

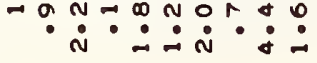

응응 응응응응응

๑. : : : : :

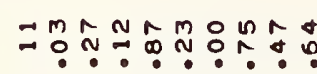

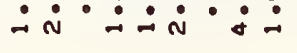

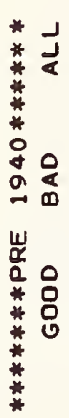

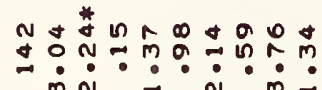

$\dot{m} \dot{N} \dot{N} \dot{m}=$

aㅇำ

$\dot{m} \dot{n} \cdot \dot{i} \cdot \dot{m}-$

$m$ m n $n-10000$

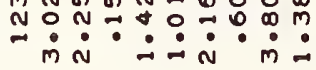

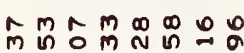

$\dot{m} \dot{\mathrm{v}} \dot{\mathrm{m}} \dot{0}$

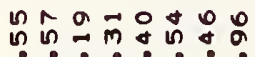

m $\dot{m} \dot{N} \dot{m} \dot{m}$ o

กำ

- $\dot{m} \dot{N} \dot{\sim} \dot{m} \dot{0}$

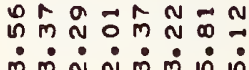

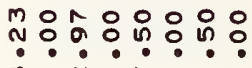

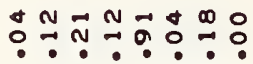

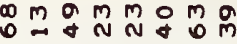

i $\dot{\sim} \dot{\sim} \dot{\mathrm{N}} \dot{0} \dot{\mathrm{m}}$

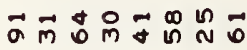

$\dot{N} \dot{i} \dot{\sim} \dot{a} \dot{\sim}$

N

$\therefore \dot{m} \dot{0}$

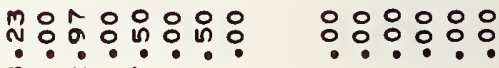

$\infty \quad \dot{r}=$

舟윰

$\because \dot{m}: \dot{a}: \dot{m}$

NMM $m$ M

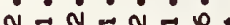

$\vec{m}$ ดิ

$\because \dot{m}: \dot{m}: 0$

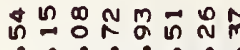

$\dot{m} \dot{m} \dot{N} \dot{\sim} \dot{N} \dot{0} \dot{0}$

ก

i :

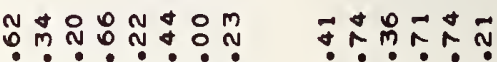

$\dot{N} \dot{i} \dot{N} \dot{i} \dot{0} \dot{0} \quad \dot{m} \dot{m} \dot{m} \dot{m} \dot{\sim}$

minmm no

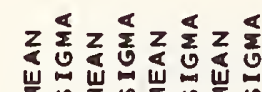

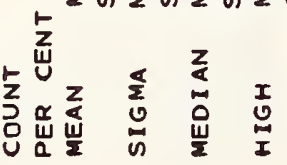

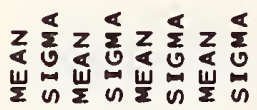

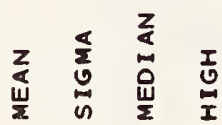

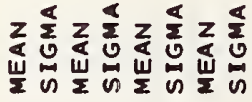

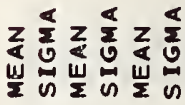

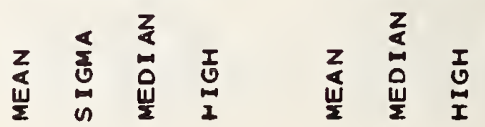




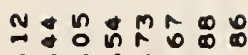

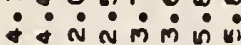

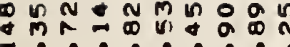

$\dot{n} \dot{N} \dot{\sim} \dot{\alpha} \because \dot{\alpha}$

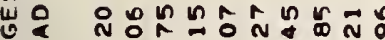

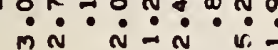

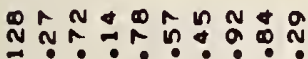

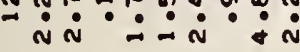

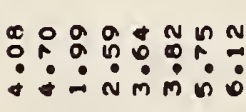

ติำำ ำำกำ

- $\dot{N} \dot{N}: \dot{N}:$

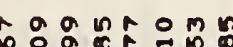

○ ค

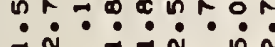

$\therefore: \because \because \div \dot{0}$

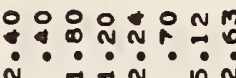

mะคำ

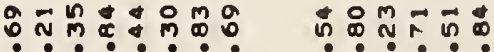

$\dot{m} \dot{N} \dot{N} \dot{N} \dot{0} \quad \dot{N} \dot{N} \dot{N} \dot{N}$

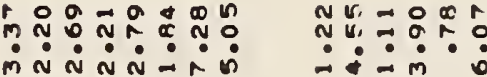

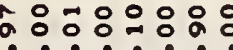

$\dot{\sim} \dot{\sim} \dot{m} \dot{m}$

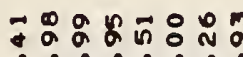

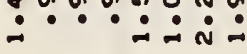

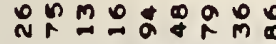

$\ddot{\sim} \dot{\sim} \dot{\sim} \dot{\sim} \dot{\sim} \dot{\sim}$

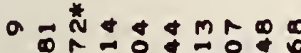

- $\dot{n} \cdot \dot{n} \dot{n} \dot{0}$

N

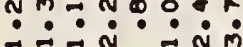

mะ⿻ำ

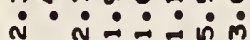

ㅇำ

Mำํํํํำ ํํํํํํํำ

$\dot{n} \cdot \dot{m} \dot{m}$

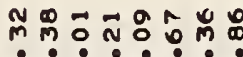

$\dot{\sim} \dot{\sim} \dot{\sim} \dot{\sim} \dot{\sim}$

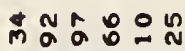

$\because i \div \dot{i}$

$\circ: \because: \because::::$

$:::::: 8$

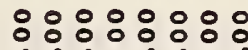

$\dot{i} \dot{\sim} \dot{\sim} \dot{m}$

怘。

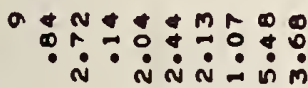

N 009 n $0 \mathrm{~m}$

ก:

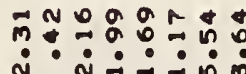

$: \because:::$ :

N N $\sim \dot{0} \dot{m}$

의 $m+n$

$\because \dot{-1}: \dot{m}$

m

$\therefore 000000$

:ำ

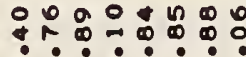

요요요

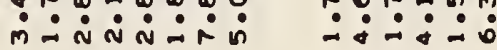

\&

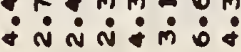

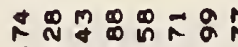

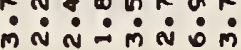

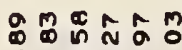

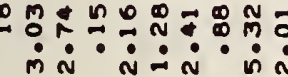

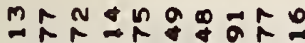

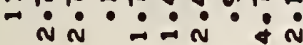

กี $\dot{\sigma} \dot{\sim} \dot{N} \dot{\phi} \dot{0} \dot{0}$

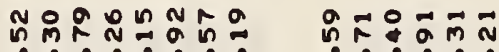
$\dot{\sim} \dot{m} \dot{m}$ in

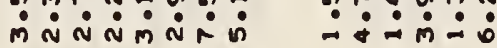




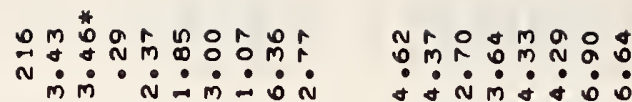

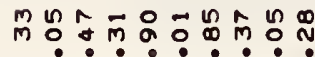

in $\dot{m} \dot{i} \dot{i} \dot{\sim} \dot{m}$

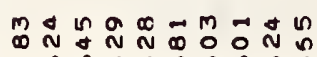

$\dot{m} \dot{m} \dot{N} \dot{m} \dot{\dot{0}} \dot{\mathrm{N}}$

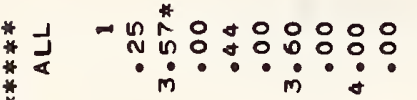

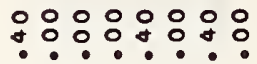

$m \sim 0 \%$

$\dot{\theta} \dot{0} \dot{m} \dot{\theta} \dot{0} \dot{0}$

$\dot{-} \cdot \dot{-} \dot{\sim}$

年。

!

$\stackrel{a}{*}$

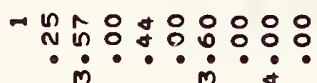

*

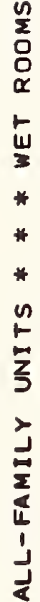

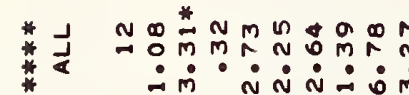

*

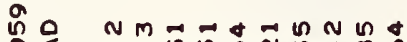

点里

!

o

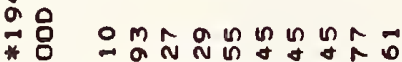

- $\dot{m} \dot{N} \dot{N} \dot{0} \dot{m}$

*

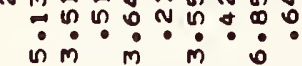

$\ddot{0}$

n $n$ m $m \& m m$

$\dot{m} \dot{m} \dot{N} \dot{m} \dot{N} \dot{m} \dot{0}$

웅융ㅇㅇ융

N

$\dot{m} \dot{m} \dot{N} \dot{m} \dot{N} \dot{m} \dot{0}$

응ㅇㅇㅇㅇㅇㅇㅇㅇㅇㅇ

minmig

*

a

å

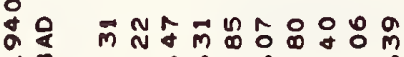

*

* $\underset{\alpha}{\alpha}$

*

a *

*

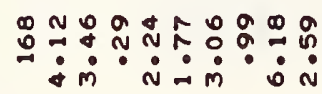

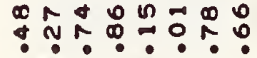

RMR

$\dot{\theta} \dot{i} \dot{m} \dot{*} \dot{0}$

華

$\ddot{m} \dot{-1} \dot{m} \dot{m} \dot{N} \dot{0}$ i

긍 웅요

กิด $\dot{m} \dot{m} \dot{m} \dot{N} \dot{\infty} \dot{\infty}$

ดิ $\dot{0} \dot{0} \dot{\mathrm{N}} \dot{\mathrm{m}} \dot{\mathrm{m}} \dot{0} \dot{0}$

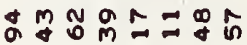
- $\dot{m} \dot{m} \dot{N} \dot{0} \dot{0} \dot{0}$

ㅇํㅇㅇํ용ำ $\dot{0} \dot{-1} \dot{0} \dot{0}$

ค̂̉

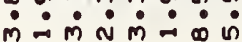

$m m=m a N$

ง m m m :

$+\dot{N} \dot{m} \dot{\sigma} \dot{0} \dot{0}$

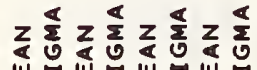

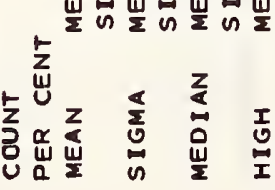

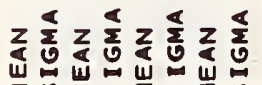

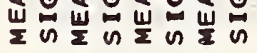

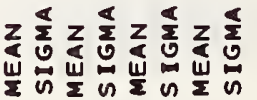

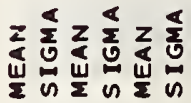

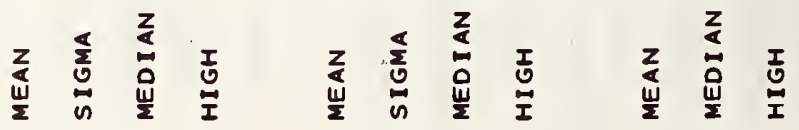

$\frac{3}{3}$

$\underset{\substack{\alpha \\ t}}{\Sigma}$

J

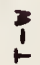


2000 Im:

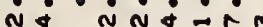

ถิด . - $\dot{m} \dot{N} \dot{N} \dot{\circ} \dot{0} \dot{0}$

กี้ำ

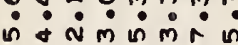

N $\dot{m} \dot{0} \dot{m} \dot{N} \dot{0} \dot{i} \dot{m}$

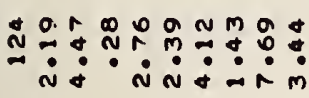

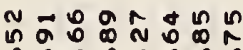

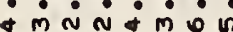

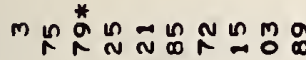

$\because \dot{n} \dot{a} \dot{a} \dot{i}$

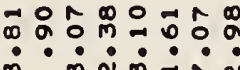

$\dot{m} \dot{m} \dot{N} \dot{m} \dot{A} \dot{N}$

$: ㅇ: ㅇ::$

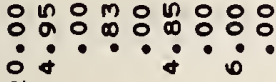
n

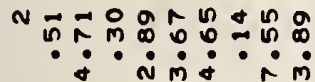

พ $\dot{m} \dot{*} \dot{N} \dot{i} \dot{m}$

แ $\because \because ? \div \dot{m} \cdot \stackrel{\circ}{\circ}$

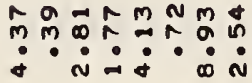

DNNOM $i \div-1$ in

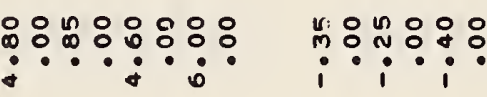

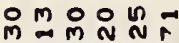
$\dot{i}: \dot{1}:-i$

n m * i⿺辶,

ผ 的定安的:

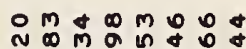

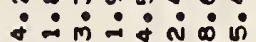

$\stackrel{\infty}{N} \doteq m_{m}^{m} \overrightarrow{0}=\stackrel{m}{\sim}$ n.

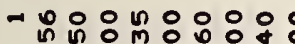
กำำ:ำ:ำ

$: \because: \because::::$

ํํㅇํํํ유:웅

$\dot{a}: \dot{i}$

$=m-70^{\infty} N$ $\therefore: \operatorname{and:0}$

Nี $\dot{m} \dot{0} \dot{0} \dot{\sim} \dot{\dot{m}} \dot{0}$

ํำ - $\dot{\sim} \dot{m} \dot{N} \dot{\alpha} \dot{0} \dot{0}$

ํํㅇํำ:

$=\mathscr{0}$ ini:

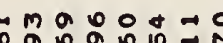
$\therefore .90 .0 \% 7$

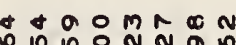

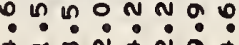

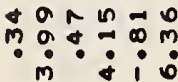
i:

ี กูก N

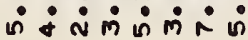

Mำ

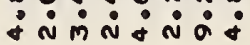

은ำำำ $\dot{m} \dot{\circ} \dot{m} \dot{N} \dot{\sigma} \dot{-\infty} \dot{m}$

㟧

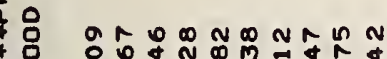

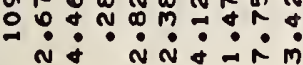

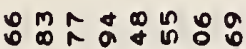

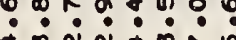

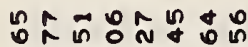
$\dot{-1} \dot{m} \dot{\alpha} \dot{0} \dot{0} \dot{0}$

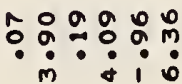

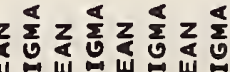

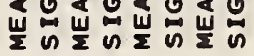

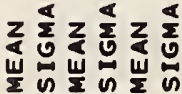

点

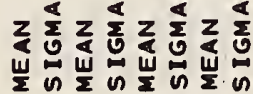

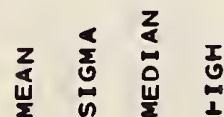

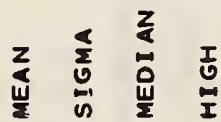

$\begin{array}{lll} & z & \\ z & 0 & \frac{1}{2} \\ \frac{1}{2} & \frac{0}{2} & \end{array}$

$\underset{\sim}{\underline{\alpha}}$ 


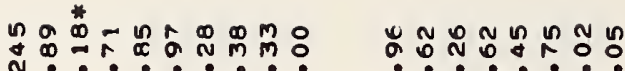

N

\section{m}

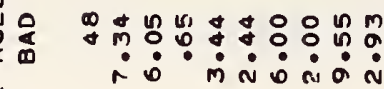

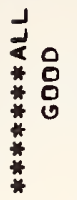

คำ

蓓

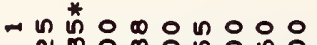

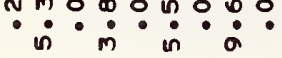

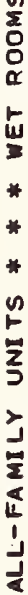

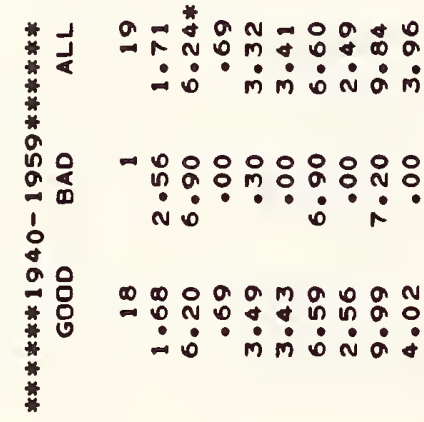

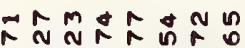

$\therefore \dot{N} \dot{\sim} \dot{\sim} \dot{N} \dot{m}$

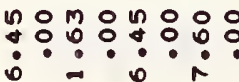

- o o a a n n

+ on

$\dot{\sim} \dot{N} \dot{\sim} \dot{\sim} \dot{N} \dot{m}$

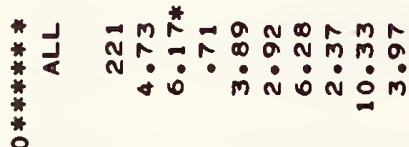

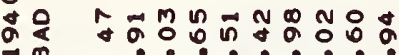

*

$*$

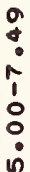

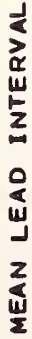

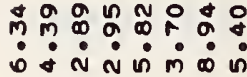

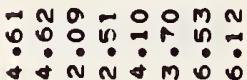

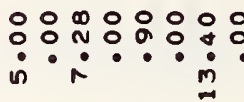

응응ㅇㅇㅇㅇㅇ

$\therefore: \stackrel{\circ}{\circ}: \stackrel{\circ}{\circ}: \stackrel{\circ}{:}:$ $\because \because \frac{0}{0}:$ ?

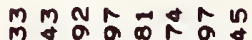
$\dot{0} \dot{\sim} \dot{\sim} \dot{m} \dot{0}$ 的

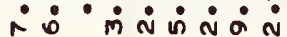

ำกำกำำำำำ - $\dot{0} \dot{m} \dot{m} \dot{0} \dot{0}:$

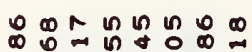
$\dot{a} \dot{\alpha} \dot{4} \dot{0} \dot{0} \dot{0}$

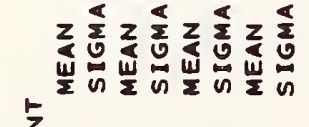

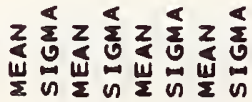

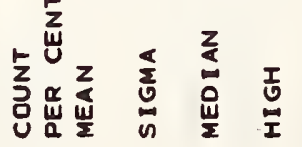

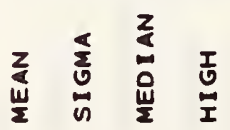

$\stackrel{n}{3}$

$\underset{\substack{\alpha \\ \alpha}}{\underline{\alpha}}$

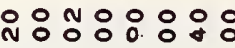

in

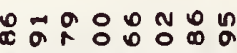

$\dot{\dot{m}} \dot{\mathrm{N}} \dot{\mathrm{s}} \dot{0} \dot{m}$

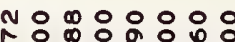

$\therefore: \div:: 0: 0$

$0 ี ⿻ m m \circ$

- $\dot{m} \dot{0} \dot{0} \dot{0}$

mog

ヘิ กำ

OMNNM

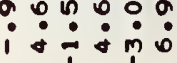

二的男品是品品

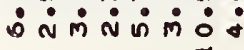

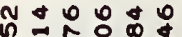
i\&i: $\dot{1}$

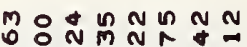

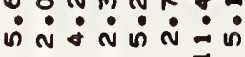

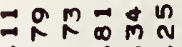
$\because \dot{-1} \dot{0} \dot{0}$

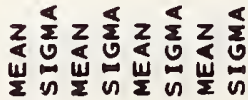

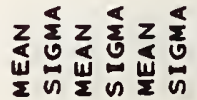<smiles>CCOCCOC</smiles><smiles>CO[As]</smiles> 
*

计 $\dot{N} \dot{N}$ i

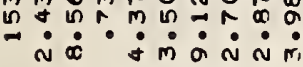

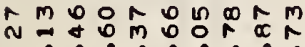
$\dot{\sigma} \dot{\alpha} \dot{m} \dot{\sim} \dot{\sigma} \dot{\sim} \dot{\sim}$

$0 m \infty n \propto m+r \infty \infty$

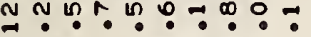
$\dot{N} \dot{a} \dot{m} \dot{a} \dot{m} \dot{y}$

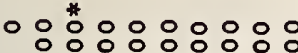
ㅇ․ㅇ․․ㅇำ

응ㅇㅇㅇㅇㅇㅇㅇㅇㅇ 은응은 응요응응

$\therefore:::$

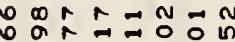

:

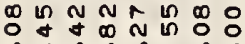
$\dot{0} \dot{N} \dot{0} \dot{N} \dot{x} \dot{m}$

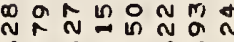
$\because \dot{\sim} \dot{\mathrm{N}} \dot{\mathrm{N}} \dot{\mathrm{O}}$ ? N $\dot{N} \dot{m} \dot{m}$

mºn

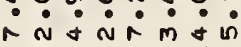

am $m$ Nó $\stackrel{1}{0} \div: 0$. is

$\therefore: ㅇ: ㅇ:$

응응ㅇㅇㅇㅇㅇㅇㅇ

응ㅇㅇㅇㅇㅠ

$\therefore::: ㅇ$ ํํำ

응융ㅇㅇ

응ㅇㅇㅇㅇㅇㅡ.

ม⿻ $\because \dot{\infty} \because \dot{m} \dot{\sigma} \dot{\sim} \dot{m} \dot{m}$

ลัง $\dot{m} \dot{q} \dot{\sim} \dot{\sim} \dot{\mathrm{m}} \dot{\mathrm{q}} \dot{\mathrm{n}}$

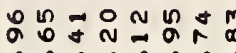
$\dot{\circ} \dot{\sim} \dot{\mathrm{N}} \dot{\mathrm{N}} \dot{m} \dot{m}$

ลีคะ

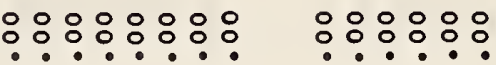

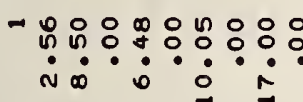

잉ํำ

$\dot{0} \dot{N} \dot{N} \ddot{0} \dot{\infty} \dot{\sim}$

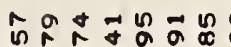

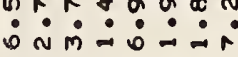

ㅇํำำำำ

$\vec{i}$

$m=\infty-\infty m a n N n$

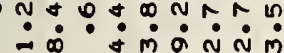

$=0 N=m$ N نं

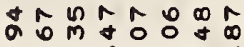
$\dot{0} \dot{\sim} \dot{\mathrm{N}} \dot{0} \dot{m} \dot{m}$

N $N O M O$ மिं $\dot{1} \dot{i} \dot{0}$

$000 \% 9 \infty$ $\because \because 0 \div \%$ $\dot{0} \dot{\alpha} \dot{m} \dot{m} \dot{m} \dot{0}$

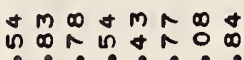

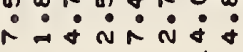

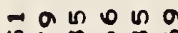
กำตำ -

พำ $\dot{+\infty} \dot{\infty} \dot{m} \dot{\sim} \dot{\sim} \dot{\sim}$

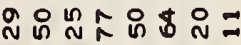
$\dot{\sim} \dot{\sim} \dot{\sim} \dot{\sim} \dot{\sim} \dot{\infty} \dot{m}$

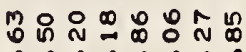
$\dot{\sim} \dot{\sim} \dot{m} \dot{N} \dot{N} \dot{\sim} \dot{N}$ i $4 m^{1}+a$

ถึก๊ $\dot{N} \dot{\sim} \dot{N} \dot{m} \dot{m}$

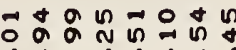
$\dot{0} \dot{\alpha} \dot{m}$ in $\dot{0} \dot{0}$

$\operatorname{dim}_{0} m \infty$

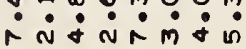

ำำ

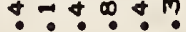

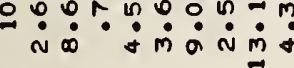
in

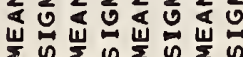

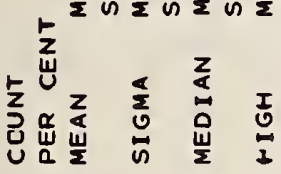

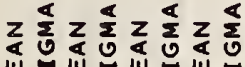

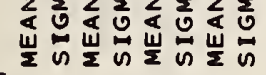

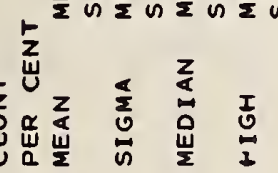

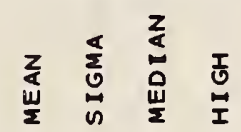

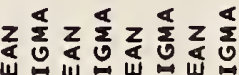

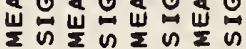

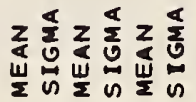

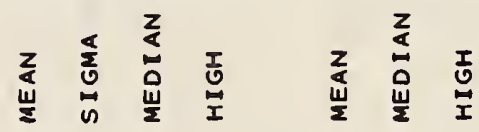




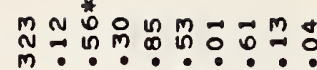

in $\dot{n} \dot{n} \dot{\sigma} \dot{\sigma} \dot{m} \dot{m} \dot{g} \dot{r}$

웅ํㅇํํำ

$\dot{0} \dot{0} \dot{0} \dot{0} \dot{m} \dot{m} \dot{0}$

$m \infty+000+a+n$

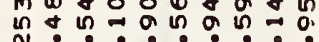

on

$*$
$*$
$*$
$*$
$*$
5
0
0
0
1
0
0
0
$*$
$*$
$*$
$*$
$*$
$*$

$\circ: \stackrel{*}{\circ}: \circ: 0: 0: 0$

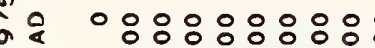

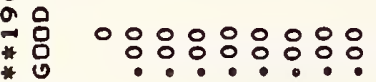

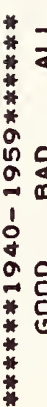

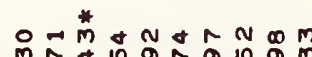

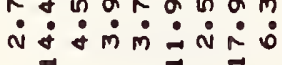

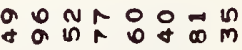

เேี

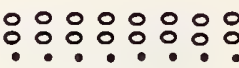

$\because: \because: \because:::$

$: \because::: ㅇ$

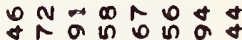

$\therefore \therefore \dot{0} \dot{0} \dot{0}$

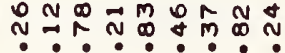

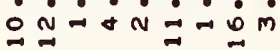

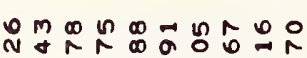

$\dot{\sim} \dot{q} \dot{\sigma} \dot{m} \dot{m} \dot{\sim} \dot{N} \dot{0} \dot{0}$

ㅇํ요

$\because \div 0: 0$

का

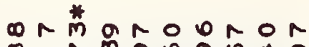

* * *

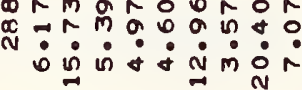

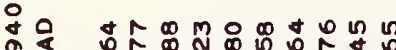

oน D N D $\dot{\circ} \dot{\sim} \dot{0} \dot{\sigma} \dot{\dot{m}} \dot{m} \dot{\mathrm{N}}$

w

ง

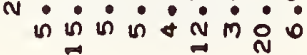

$\alpha \infty 0+0 n \infty \pi$

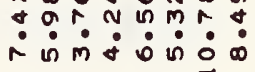

$\exists \Omega$ ลักับ $\dot{0} \dot{0} \dot{\sim} \dot{\sim} \dot{\sim} \dot{\mathrm{N}}$

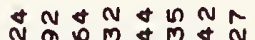
$\dot{0} \dot{0} \dot{0} \dot{0}$

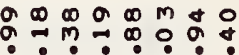

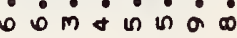

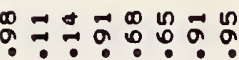

$\ddot{\sim} \dot{0} \dot{0} \dot{0} \dot{q}=\dot{0}$

$\leftarrow 00 \% 000$

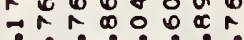
a omomar

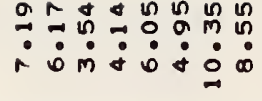

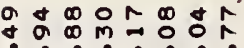
त

DO수에 iि $\dot{i} \dot{0} \dot{0}$

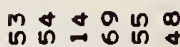
किमिक्ष

tonman $m$ in $\div \div 0$ ir í⿴囗十

$\because: \because::: ㅇ:::$

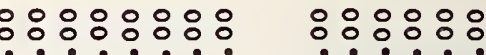

m

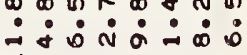

$m=0$

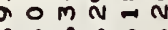

$\dot{i} \dot{\sim} \dot{i} \dot{0} \dot{\sim}$

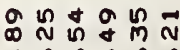

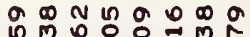

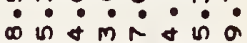

$\dot{\sim} \dot{0} \dot{\sim} \dot{m} \dot{\infty}$

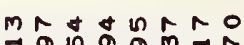

$\dot{\sim} \dot{0} \dot{0} \dot{\sim} \dot{0} \dot{0} \dot{0} \dot{0}$

$\infty \curvearrowleft \infty-\infty$

ก?

iे $\dot{0} \dot{0} \dot{\sim} \dot{0}$

กำ ๒

$\dot{\sim} \dot{\sigma} \dot{\sim} \dot{m} \dot{0} \dot{\sim} \dot{\sim}$

$m \sim=\infty D=$

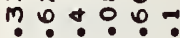

ir i o

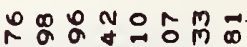
ลं

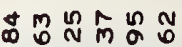

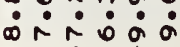

$m \backsim 0 \bullet \bullet \Rightarrow \infty m$

N $\dot{\sim} \dot{\sigma} \dot{0} \dot{m} \dot{m} \dot{R} \dot{r}$

๑ $N$ ำ வिं

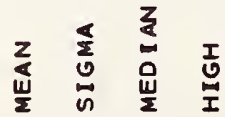

$\underset{\frac{\alpha}{2}}{\frac{\alpha}{1}}$

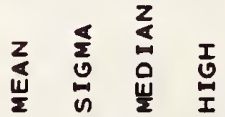

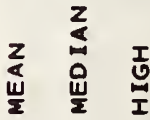

I

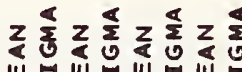

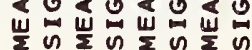

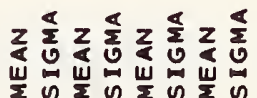

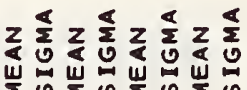

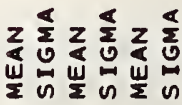

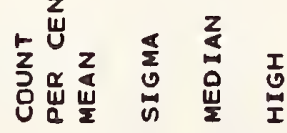




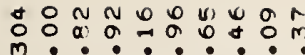

w $\dot{0} \dot{0} \dot{m} \dot{-} \dot{-} \dot{m} \dot{m}$ 的

7

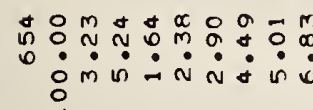

o on $000-a+a m$

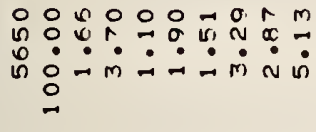

$*$
$*$
$*$
$*$
$*$
$*$
0
0
0
1
$\vdots$
0
0
0
$*$
$*$
$*$
$*$
$*$
$*$
$*$

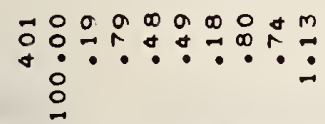

$-$

足

$*$
$*$
$*$
$*$
$*$
$*$
0
0
0
0
0
$\vdots$
$\vdots$
0
$\vdots$
0
0
$*$
$*$
$*$
$*$
$*$
$*$
$*$

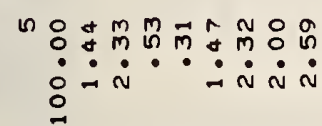

o orndatomo

旁:

$-$

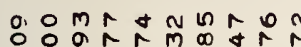

$\exists \dot{\circ} \dot{\sim} \cdot \dot{\sim} \dot{\sim} \dot{m}$

aㅇำ $2 \pi$

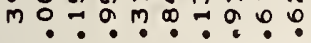

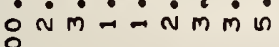

$\circ$

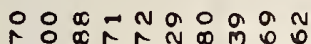

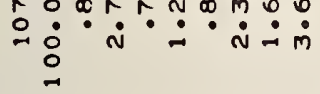

*

$*$
$*$
$*$
$*$
$*$
$*$
$*$
0
0
0
0
0
0
0
0
0
0
0
0
$*$
$*$
$*$
$*$
$*$
$*$
$*$
$*$
$*$
$*$

aOg nNMR GNo

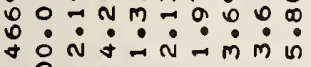

-

事是

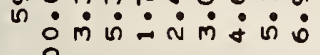
$\therefore$

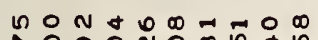

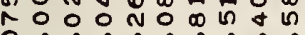

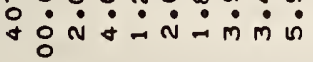

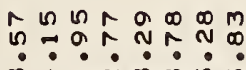

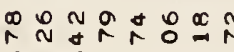

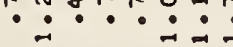

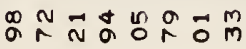

$\because \dot{\sim} \dot{\sim} \dot{\sim} \dot{m} \dot{\sim}$

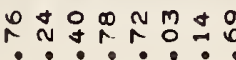

$\because \because \frac{a}{0}$

a m

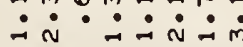

×:

$\dot{\sim} \dot{m} \dot{\sim} \dot{\sim} \dot{m} \dot{0} \dot{0}$

ㄸำ

$\therefore \dot{\sim} \dot{\sim} \dot{\sim} \dot{\sim}$

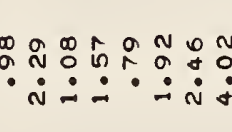

† $\begin{gathered}m \\ +\infty\end{gathered}$

$\therefore \dot{-} \dot{-} \dot{\sim} \dot{\sim}$

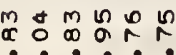

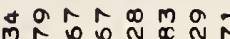

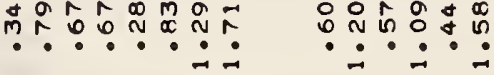

N

$\therefore \dot{\alpha} \dot{-} \dot{\sim} \dot{\alpha}$

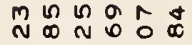

$\because \dot{\sim} \cdot \dot{i} \dot{m}$

음

$\because ? \mathfrak{0}^{\infty} \cdot 0 \div$

$\cos \cos ^{0}$

$\because: 90$

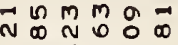

- $\dot{\sim} \dot{\sim} \dot{m}$

$\infty \operatorname{con} 0-0$ n

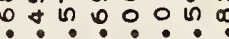

$\dot{\sim} \dot{m} \dot{\sim} \dot{\sim} \dot{m} \dot{0}$

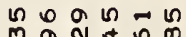

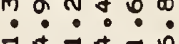

ㅎํํำ $\dot{\sigma} \dot{\sim} \dot{\sim} \dot{m} \dot{m} \dot{r}$

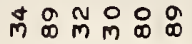
$\dot{\sim} \dot{\sim} \dot{\sim} \dot{\boldsymbol{n}} \dot{\boldsymbol{r}}$

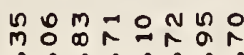

ถูกับ

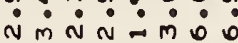

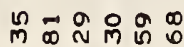

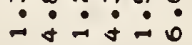

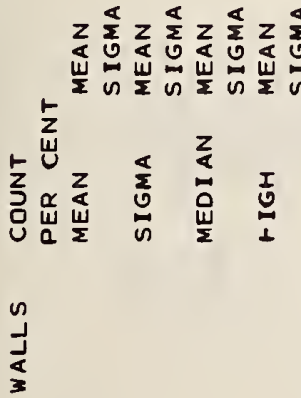

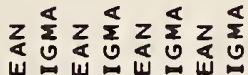

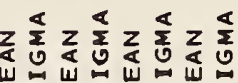

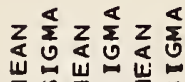

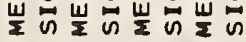

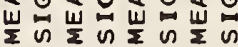

崖岕崖的崖出

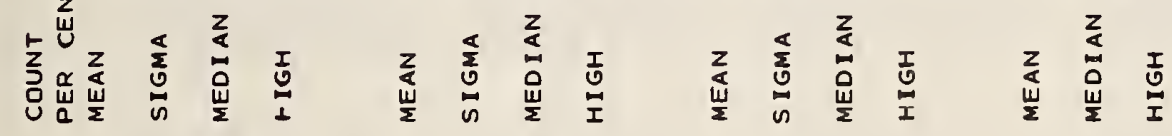

$\stackrel{n}{3}$

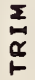

\ّ

$\stackrel{3}{1}$ 


\section{APPENDIX C}

Set "C" Tables

Table of Contents

Occupancy Class

SINGLE-FAMILY

ALL
Room Type

DRY

WET

DRY

WET
Pages

137-148

149-160

161-172

173-184 


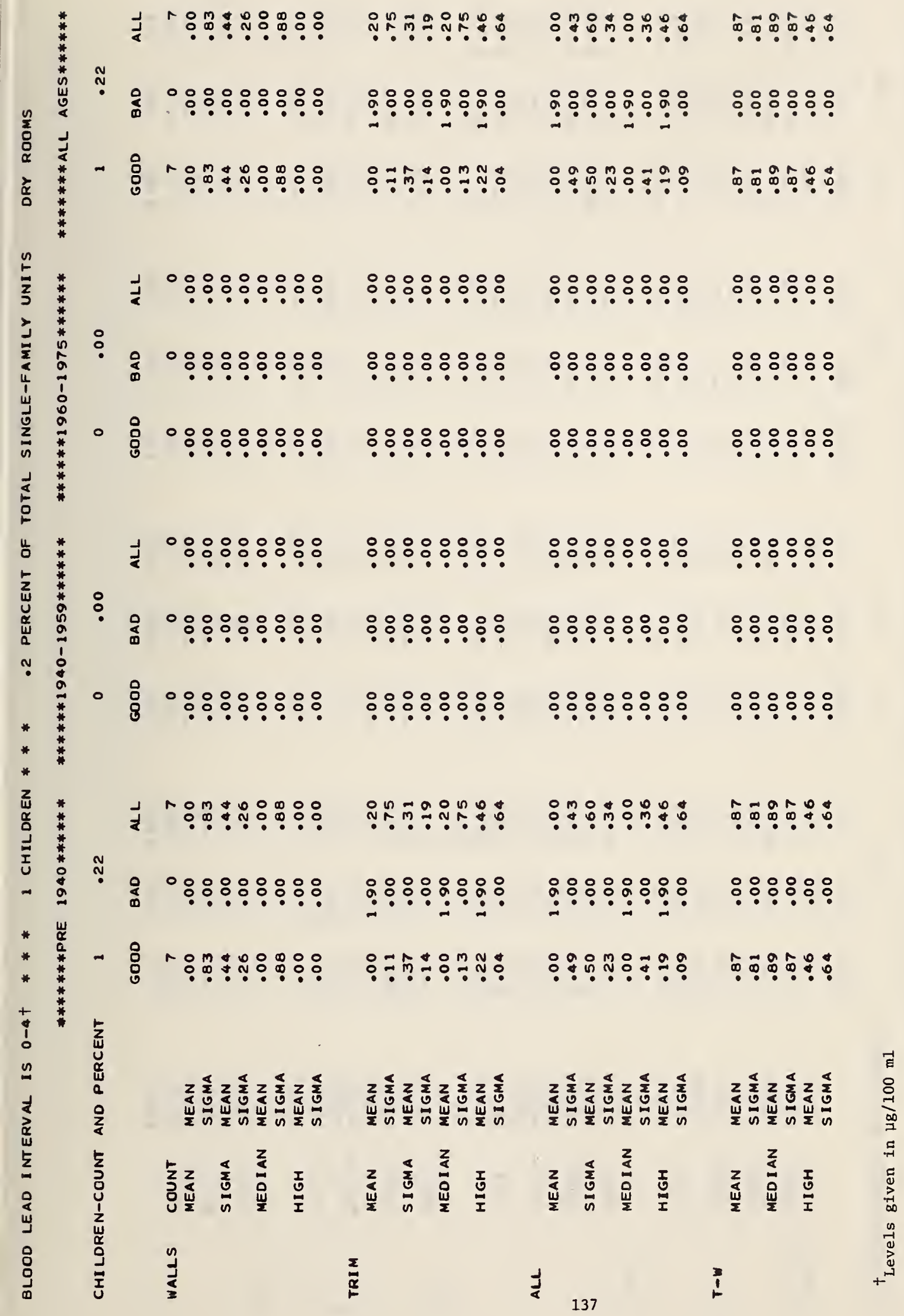




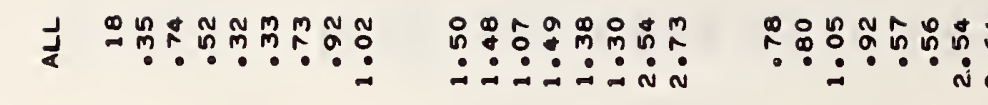

$\because$

-

m 8

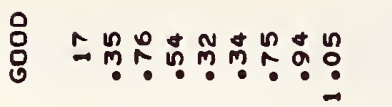

$\dot{m} \dot{i} \dot{n} \dot{n} \dot{0}$

ถ

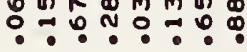

ดำ $\overrightarrow{0}$ กิกำㅇำ

$\dot{n} \dot{i} \dot{n} \dot{n} \dot{0}$

กีคํำ

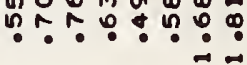

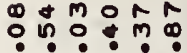

$\because \dot{-1} \dot{\sim} \dot{N}$

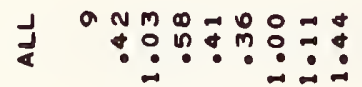

$\stackrel{0}{?}$

ำ

\%

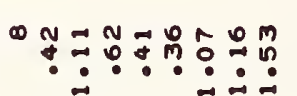

เ $m \propto m$ m $m$ ำ $: *$ ? ? :

a n ㅇำㄴ? : :

$N=\infty \circ \sim \circ$

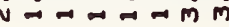

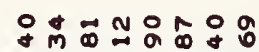
$\dot{m} \dot{i} \dot{\sim} \dot{N} \dot{0}$

ดำ $\dot{N} \dot{a} \dot{a} \dot{a} \dot{0}$

ำㅇํㅇㅇㅇㅇㅇㅇ $\dot{n} \cdot \dot{n} \cdot \dot{0}$

m.mo

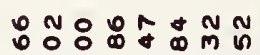

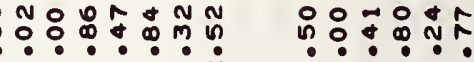

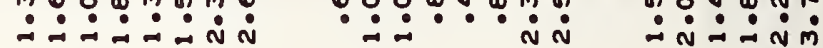




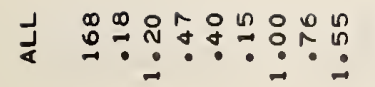

$\stackrel{0}{i}$

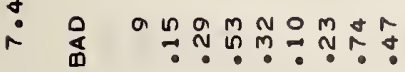

m

号

$\stackrel{0}{\circ} \div$
กิ $⿻$ ก

$\dot{N} \dot{m} \dot{i} \dot{N} \dot{m} \dot{m} \dot{j}$

ดิ คั

$\dot{N} \dot{N} \dot{-} \dot{N} \dot{m} \dot{m}$

ก

Nim $\dot{\sim} \dot{N} \dot{m} \dot{m}$

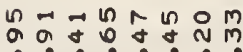

-

$\rightarrow \rightarrow-\vec{m}$

a

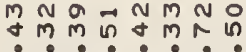

군

$\because$

$\stackrel{8}{0}$

응ㅇㅇㅇㅇㅇㅇ응

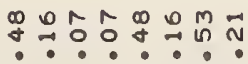

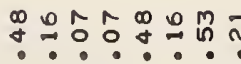

ㅇㅇㅇㅇㅇ응

$m$

ㅇํำำง ำ ำำ

จำㅇํำ

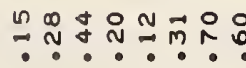

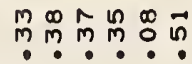

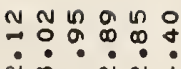

$\dot{\sim} \dot{m} \dot{\sim} \dot{N} \dot{s}$

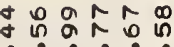
$\dot{\sigma} \dot{\dot{m}} \dot{m} \dot{\mathrm{n}} \dot{\mathrm{n}}$ $\rightarrow \dot{N} \dot{\sim} \dot{N} \dot{N}$

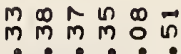

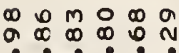

ํํㅇำ

กิ

OND nOMm

$\hat{a} \rightarrow \circ M \infty N$

ำ.?ำ?

m

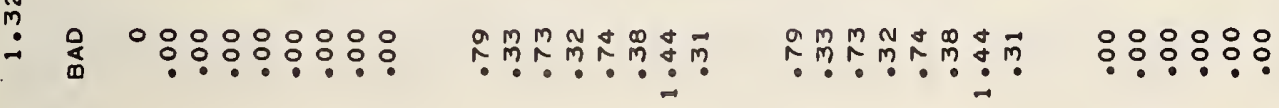

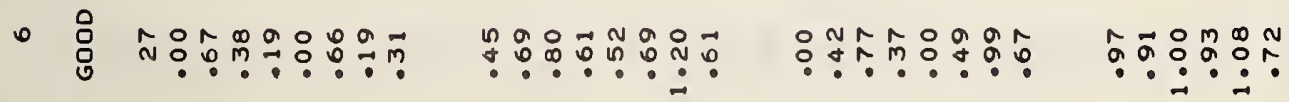

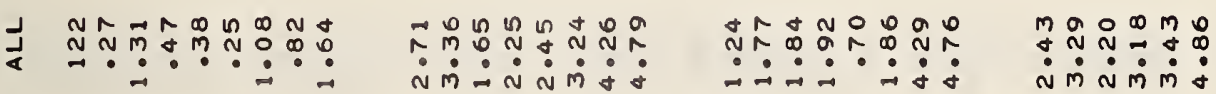

$\stackrel{\bullet}{?}$

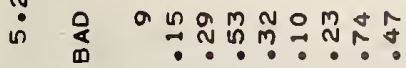

g $\dot{m} \dot{m} \dot{-} \dot{-\dot{m}} \dot{\boldsymbol{n}} \dot{m}$

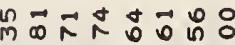

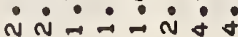

+ $\dot{\circ} \dot{m} \dot{m} \dot{0} \dot{0}$

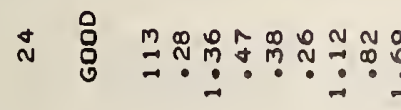

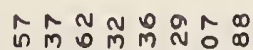
ก.?

N้ำ $\rightarrow$ i $\rightarrow: \dot{i} \dot{0}$

ํำ Nं்

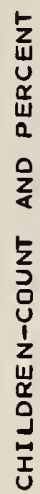

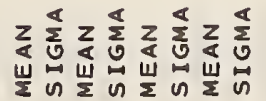

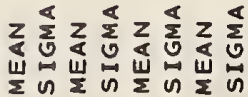

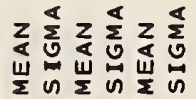

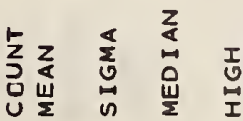

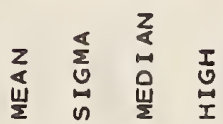

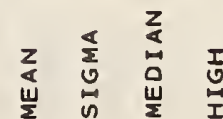

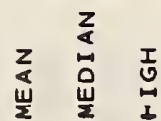

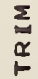




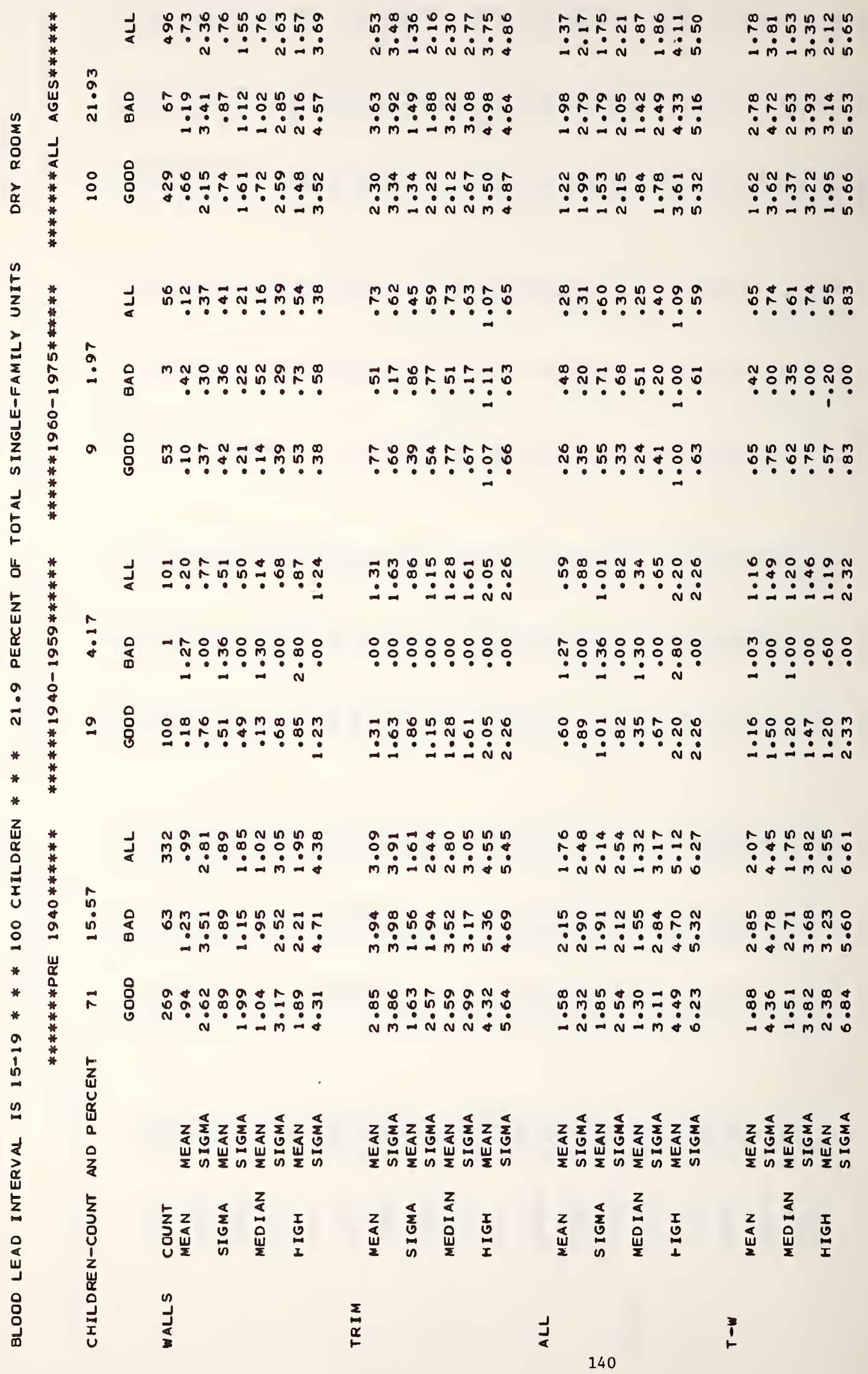




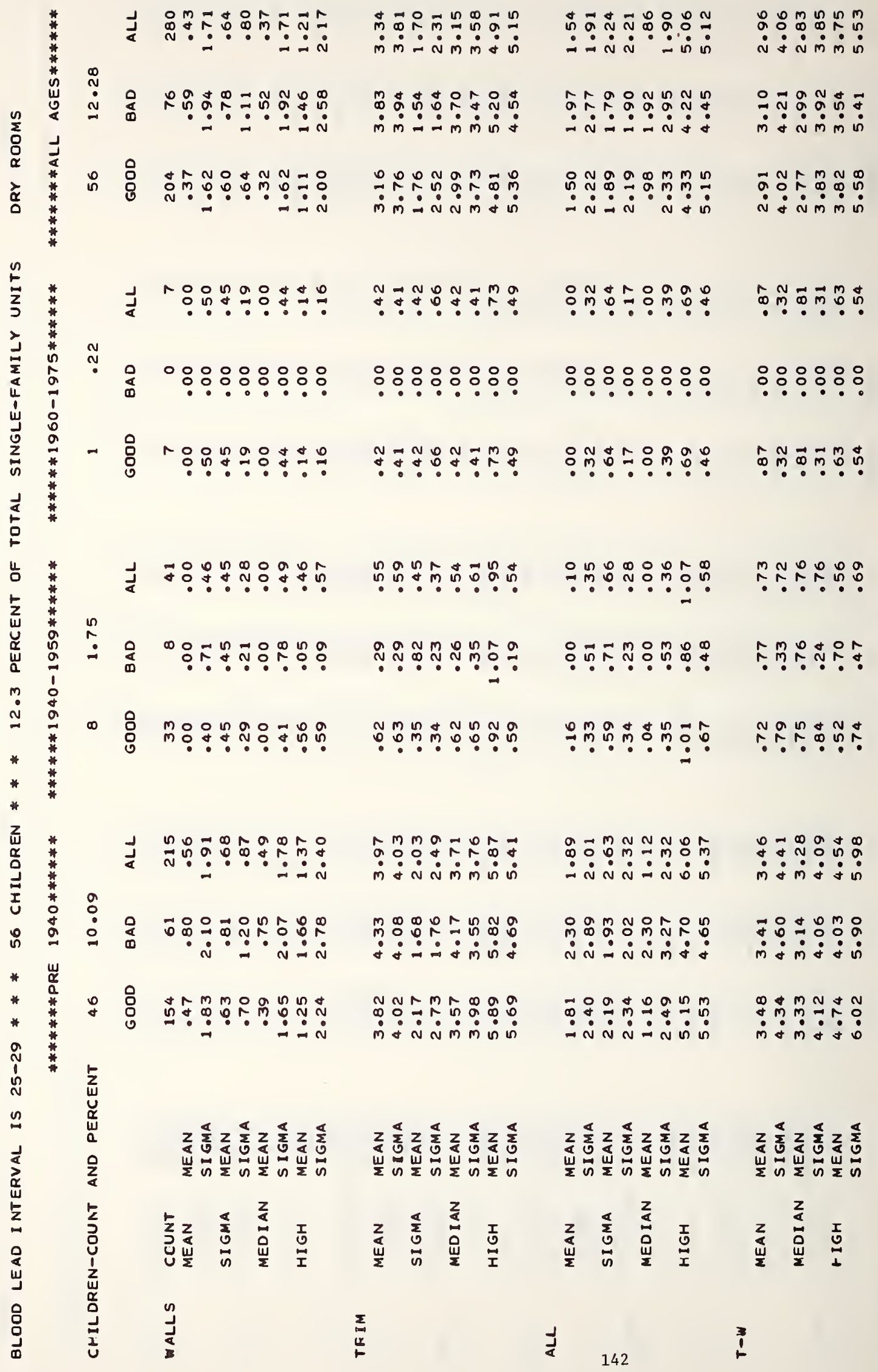




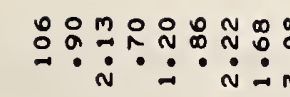

$\overrightarrow{0}$

요용

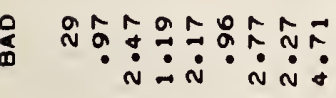

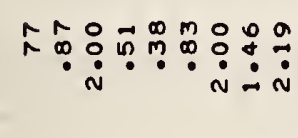

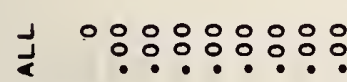

웅ㅇㅇㅇㅇㅇㅇㅇ

응융ㅇㅇㅇㅠ

:

\&

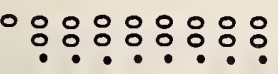

응ㅇㅇㅇㅇㅇㅇㅇㅇㅇㅇㅇㅇ

-

응ㅇㅇㅇㅇㅇㅇㅇ

응ㅇㅇㅇㅇㅇㅇㅇㅇㅇ

응용ㅇㅇㅇㅇㅇㅇㅇ

응ㅇㅇㅇㅇㅇㅇㅇㅇㅇㅇㅇ

웅ㅇㅇㅇㅇㅇㅇㅇ

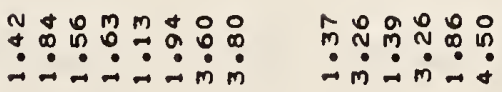

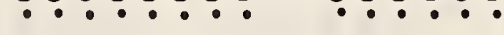

$*$
$*$
$*$
$*$
$*$
0
0
$a$
1
0
0
$a$
$*$
$*$
$*$
$*$
$*$

لُ

○ $\ddot{N} \dot{m} \dot{N} \dot{m} \ddot{\dot{m}} \dot{\dot{\phi}}$

\& a n

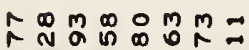
$\dot{4} \dot{\mathrm{n}} \dot{\mathrm{n}}: \dot{\mathrm{n}}$

no $\infty m \hat{m} \overrightarrow{0}$ N.

$\stackrel{N}{\text { N }}$

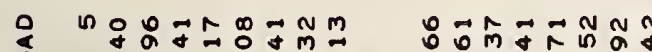

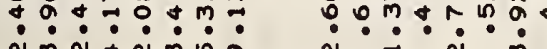
Nmín

- $\quad$ 品

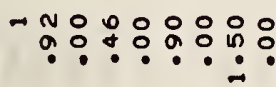

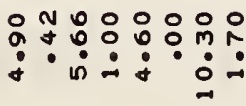

in $\bar{m}$ ŏ $0 \hat{m} \pm \hat{m} N$

$\dot{m} \dot{\sim} \dot{m} \dot{m} \dot{N} \dot{R}$

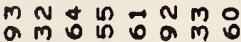
$\dot{0} \dot{n} \dot{n} \dot{0}$

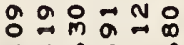
$\dot{a} \dot{m} \dot{\mathrm{N}} \dot{0} \dot{0}$

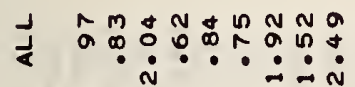

o n 2 nata กㄴ? niman

\&

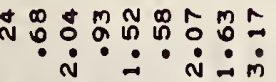

ชิ Nัa $\dot{m} \dot{m} \dot{\sim} \dot{m} \dot{\mathrm{N}} \dot{0}$

$a \infty m \infty=\infty \Rightarrow N$ ก) $\rightarrow-\dot{-1} \dot{-1}$

- N $\rightarrow$ NOD $\therefore \dot{0} \dot{0}:$

m N ผ

용ำ $\dot{m} \dot{\circ} \dot{m} \dot{m} \dot{0}$

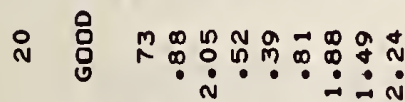

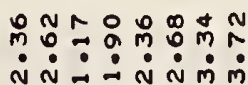

ก m. เ? แ? : ด ? ?

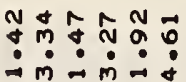

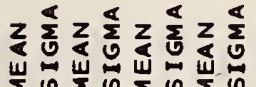

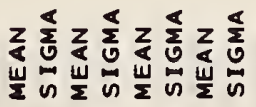

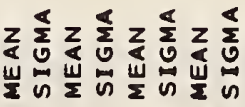

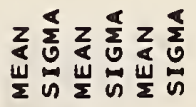

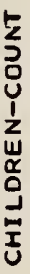

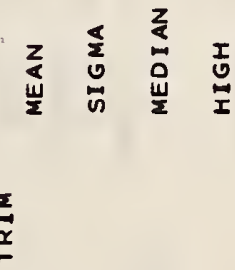

$\frac{1}{8}$

143 


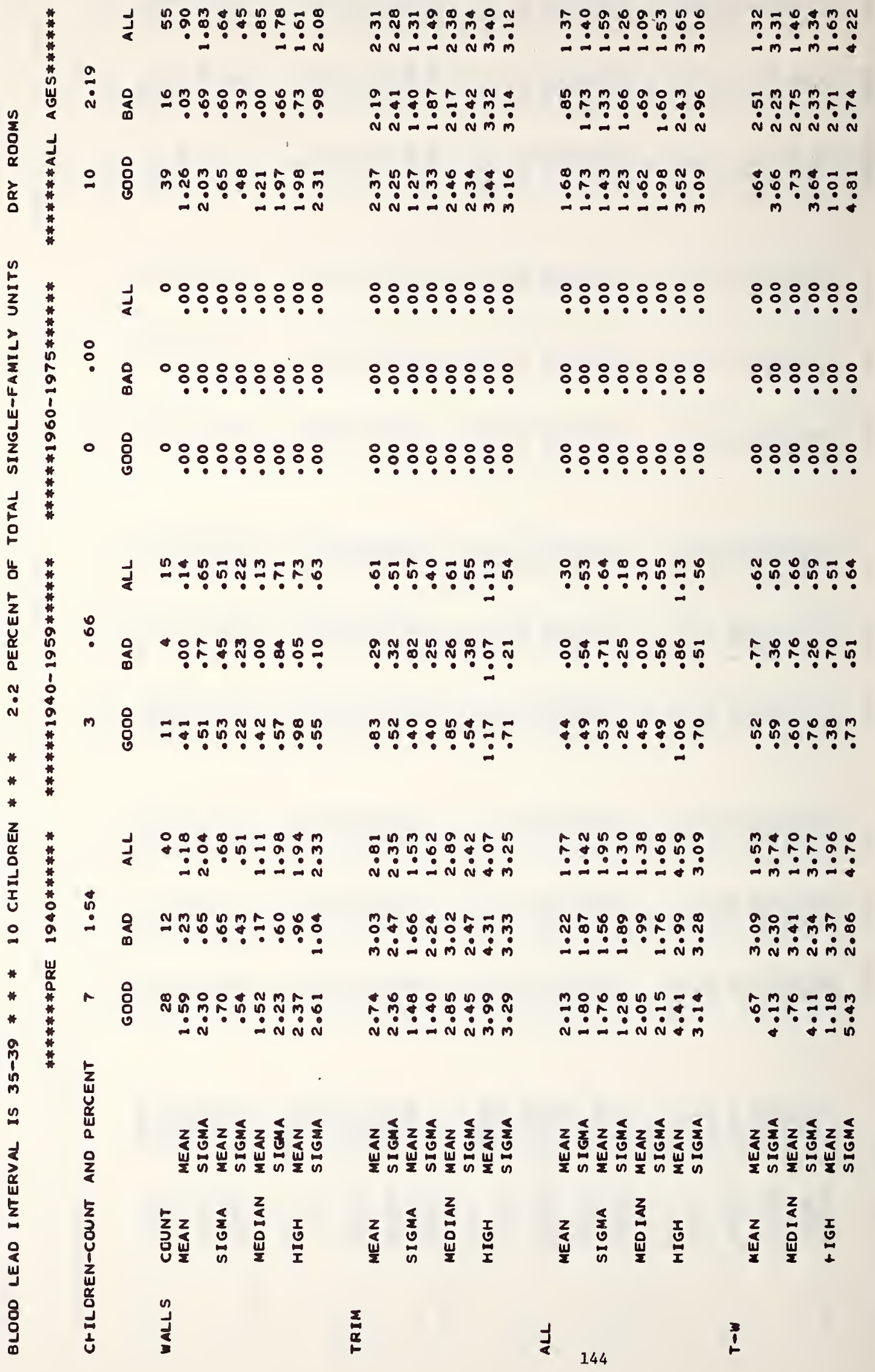


J

$\stackrel{4}{*}$

-

\&

- $\dot{m} \dot{v} \dot{v}=\dot{m} \dot{m}$

N

ㅇํㅇำ

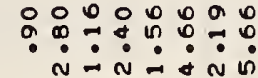

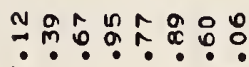

$\dot{0} \dot{\sim} \dot{0}$

J. 0 응ㅇㅇㅇㅇㅇㅇㅇㅇ

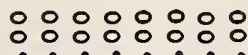

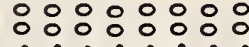

คัด

$\dot{N} \dot{m} \dot{m} \dot{m} \dot{0}$

ON N $\tilde{m}_{m}^{n}$

ம் $\dot{0} \dot{0} \dot{0} \dot{m}$

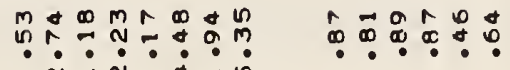
a $-n-d-1$

$\underset{\infty}{N}$ in $N$ N $\dot{0} \dot{0} \dot{0} \dot{0}$ $\div: 0 \%: 0$

$\stackrel{0}{\circ}$

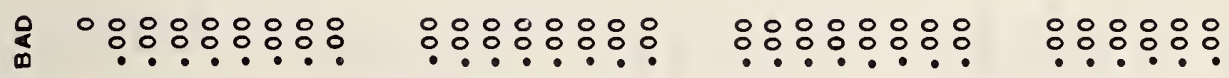

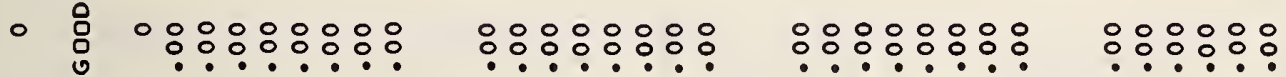

:

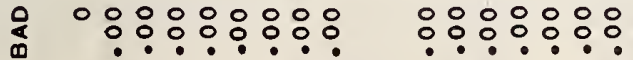

$\circ$

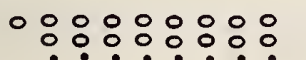
ํํㅇํㅇำ

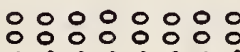

$\because: 0 \%: 0: ?$

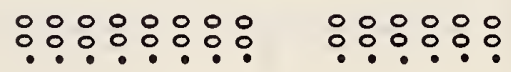

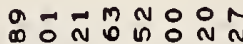
$\rightarrow \infty n O N O$ $\dot{N} \dot{N} \dot{N} \dot{0} \quad \dot{m} \dot{a} \dot{N} \dot{N}$

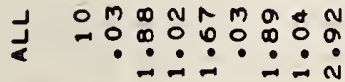

$\stackrel{+}{?}$

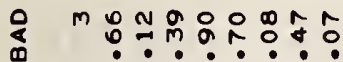
$\because \dot{m} \dot{N} \dot{\sim} \dot{m} \dot{m}$

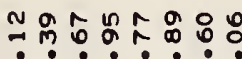
$\because \sim ?: \dot{0} \dot{0}$

N

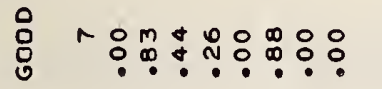

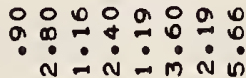

ำ i $\dot{\sim} \dot{m} \dot{m} \dot{\mathrm{N}} \dot{0} \dot{0}$

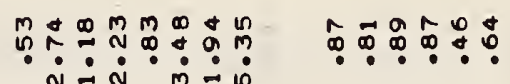

O iि $\dot{0} \dot{0} \dot{0} \dot{m}$

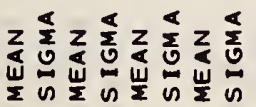

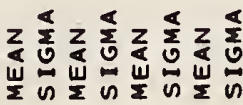

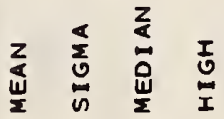

$\underset{⿱ 亠 䒑}{2}$

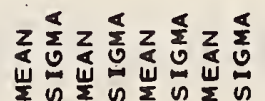

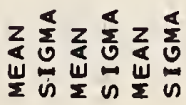

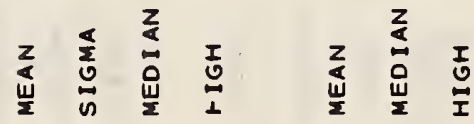




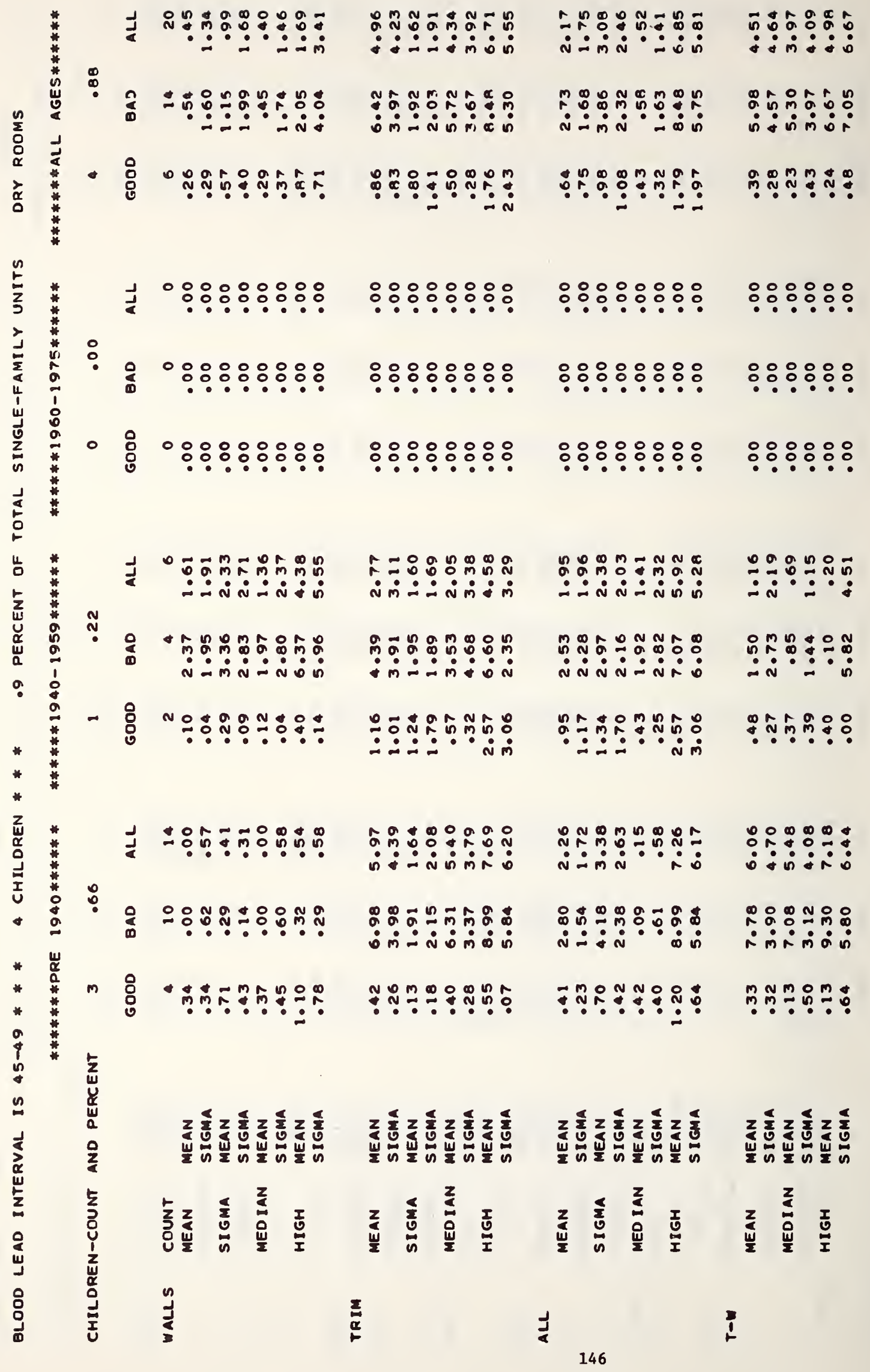




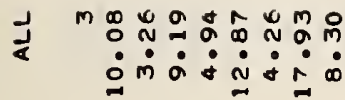

$\stackrel{N}{\sim}$

¿

$-8$

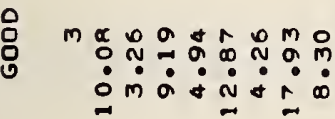

」 용:::::은

$:$

유::::유

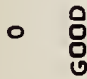

$\circ:: 8:::::$

응응ㅇㅇㅇㅠ

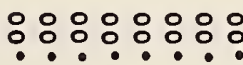

응응ㅇㅇㅇㅇㅇㅇ응

․ำ ํำㅇำ

응ํำ

$\dot{m} \dot{0} \dot{m} \cdot \dot{0}$

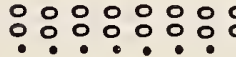

응응응응웅

ํำ:ํ.

응응ㅇㅇㅇ

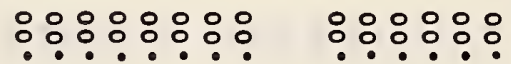

ำ
J

응유:응응

$:$

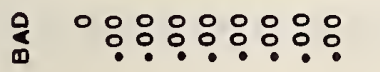

융ㅇ:유:

- 8

융

응응ㅇㅇㅇㅇㅇ

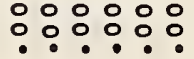

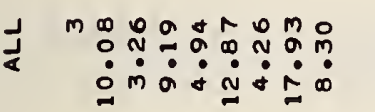

ㅇํㅇำ

约:

min

$:: ㅇ: ㅇ:$

¿

$-8$

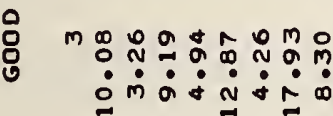

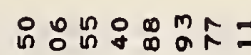

$\dot{m} \dot{N} \dot{-} \dot{m}: \dot{0}$

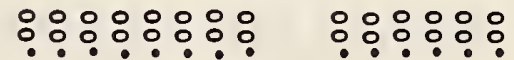

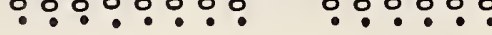

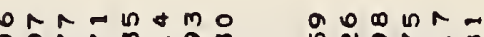

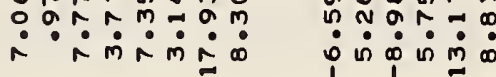

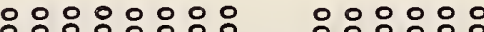

응용ㅇㅇ

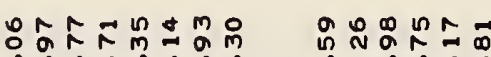

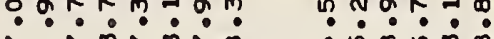
iि $\dot{0} \dot{1} \dot{m} \dot{\infty}$

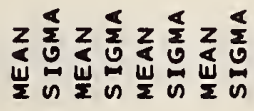

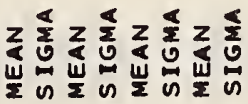

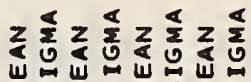

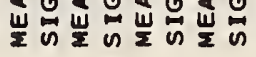

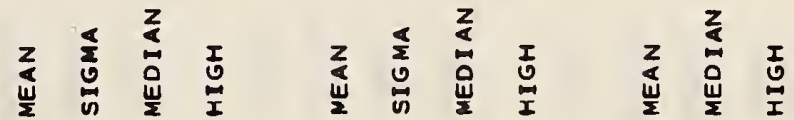

$\underset{\alpha}{a}$

$\frac{1}{4}$

147 $z \frac{4}{2} z \frac{\pi}{2} z \frac{\pi}{2}$

敋的崖会 


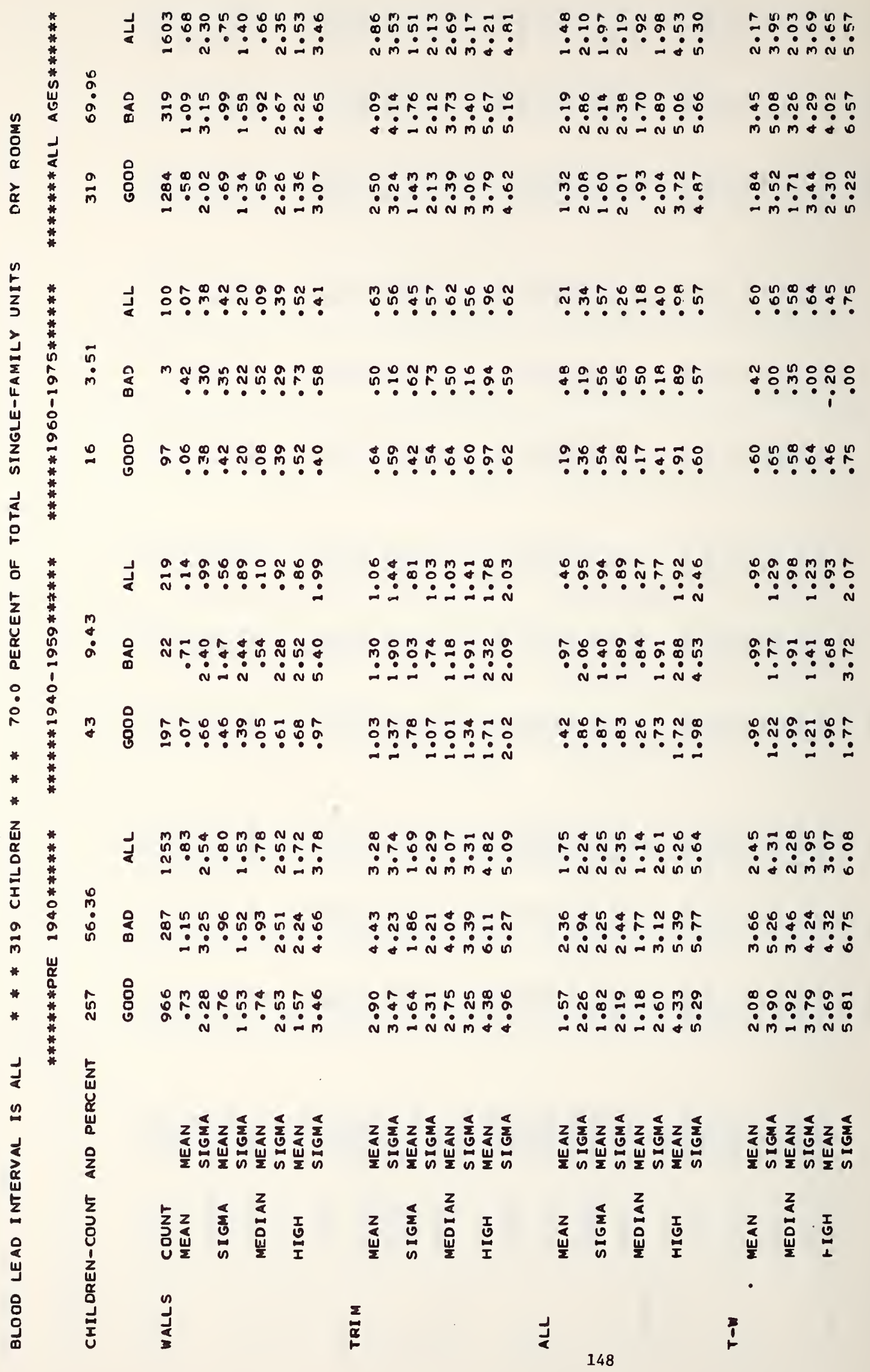




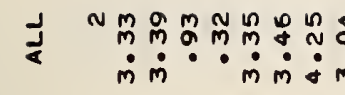
กี้

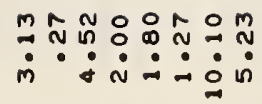
뜬유ำ
$\dot{m} \dot{0} \dot{0} \dot{0} \dot{m} \dot{\alpha} \dot{\sim} \dot{a}$
$\dot{\infty} \cdot \dot{\infty} \dot{n} \stackrel{\dot{n}}{n}$
$\stackrel{N}{\sim}$

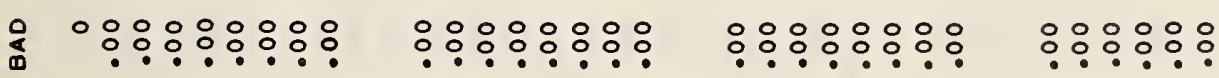
- 号

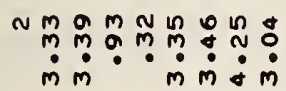

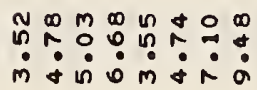

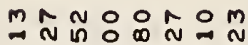
$\infty \infty \circ \circ n$

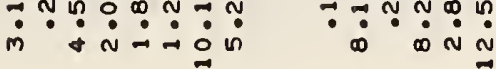

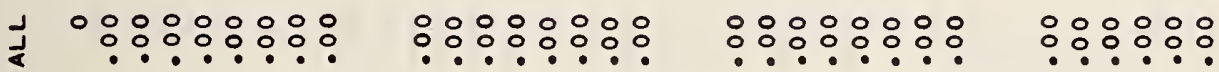
:

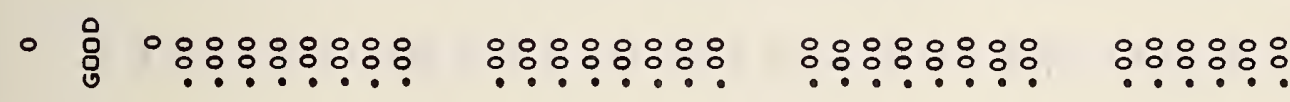

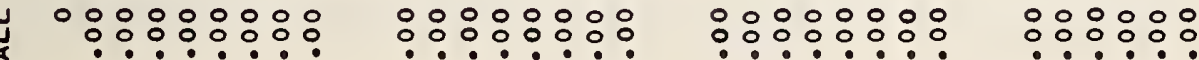

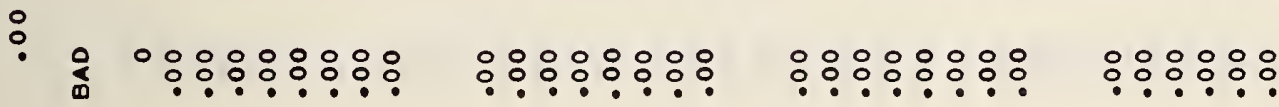

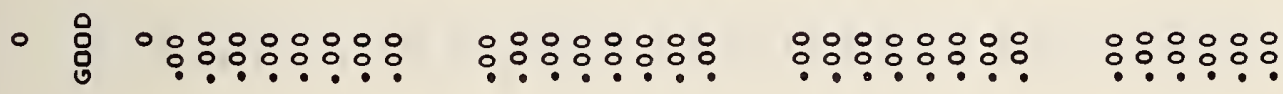

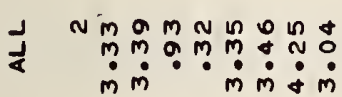
n m m m in
MNNOONOM
$\dot{m} \dot{0} \dot{0} \dot{0} \dot{m} \dot{0} \dot{0}$

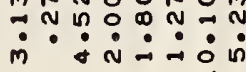

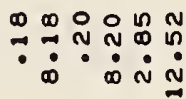
$\stackrel{N}{\mathfrak{N}}$

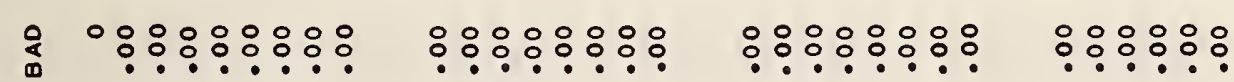

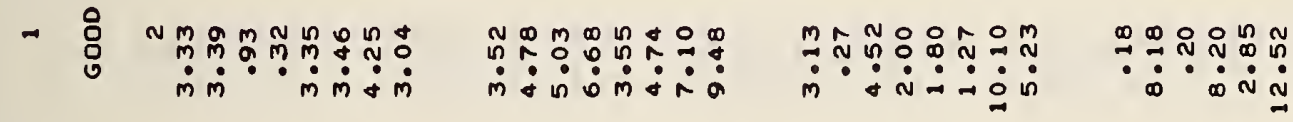

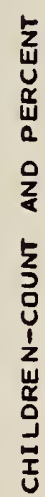

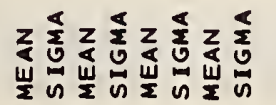

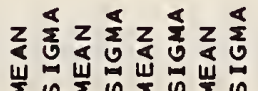

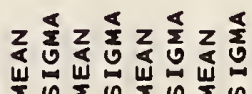

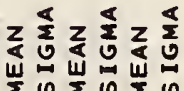

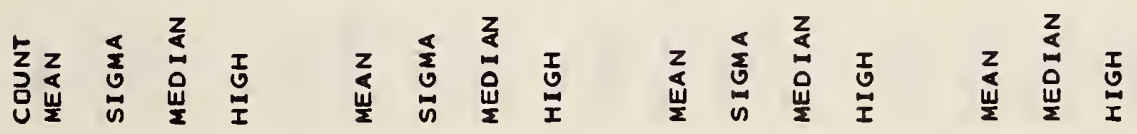
בُ
$\underset{n}{x}$
๖ 149 
」

:

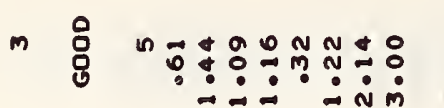

ลัะด ำกำ

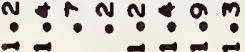

$\therefore-\div \div \dot{-1}$

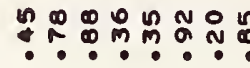

」N응을응운

$N$

$\stackrel{N}{*}$

응 용ㅇㅇㅇㅇㅇㅇ

- 吕

N응은응영엉

๒゚

양ㅇㅇㅇㅇㅇㅇㅇㅇ

응ㅇㅇㅇㅇㅇㅇㅇㅇㅇㅇ

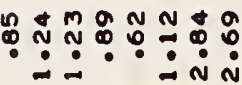

คำดั

$\because: \because i$ i

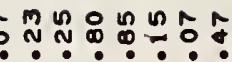

Sำก

คำ?

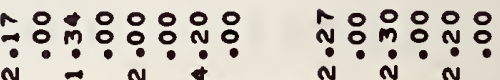

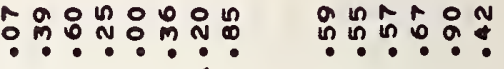

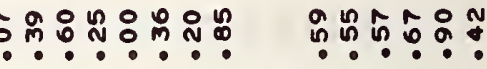

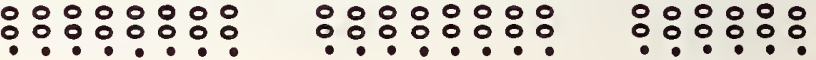

:

각ㅇㅇㅇㅇㅇㅇㅇㅇㅇㅇ

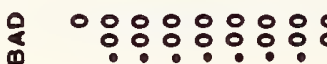

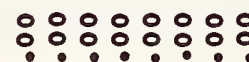

웅ㅇㅇㅇㅇㅇㅇㅇㅇㅇ

:ㅇ:ㅇ:ㅇ:

- 응 양ㅇㅇㅇㅇㅇㅇ

ㅇ:ㅇ:ㅇ::용

응ㅇㅇㅇㅇㅇㅇㅇㅇㅇㅇ

:ㅇ:ㅇㅇ:웅 J

ก้นกำ

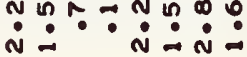

ถูก 的

N $⿻$ n $\sum_{\infty}^{0}$

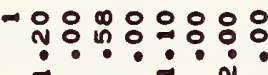
능웅ㅇㅇㅇㅇㅇㅇㅠ $\because \dot{-} \dot{\sim} \dot{\sim} \dot{\alpha}$ $\therefore \div \div$

N 吕

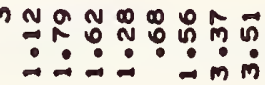
แ ตํั

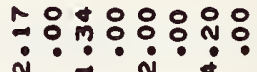
ㄴํำㅇํㅇํำ

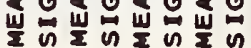

$z \leqslant z \leqslant \frac{2}{2} \leq \frac{1}{0}$

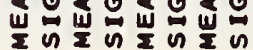

$\mathfrak{m} \neq 9: 90$

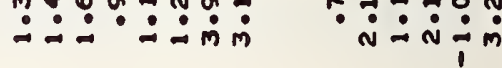


$\stackrel{0}{\stackrel{0}{0}}$

\& n ำล งด ด

ป in $\dot{m} \dot{m}$ in

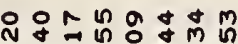
N $\dot{N} \dot{-} \dot{v} \dot{m} \dot{m}$
ง 品 ง

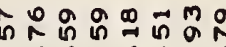

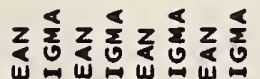

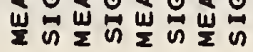

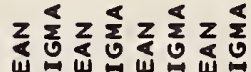

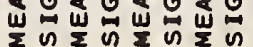

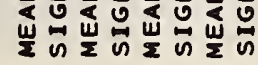

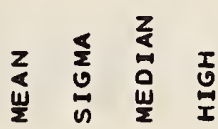

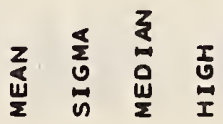

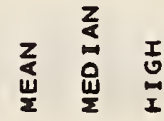

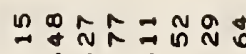
$\dot{\sim} \dot{N} \dot{-} \dot{\mathrm{N}} \dot{\mathrm{m}} \dot{\mathrm{m}}$

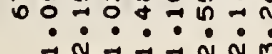

ก ำ:

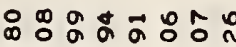
$\dot{0} \dot{\sim} \dot{v} \dot{0} \dot{0}$

m 응

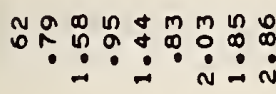

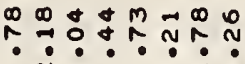
$\because \dot{n}-\dot{a} \dot{n} \dot{m}$

กับ

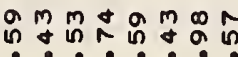

中

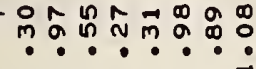

0

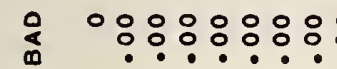

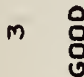

ヘํำ ถึกิ …・・

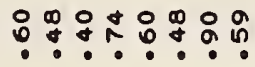

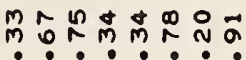

m:?.?冂卄.

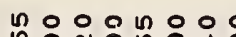

กำ

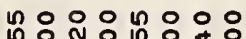

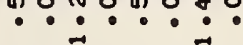

:ㅇ:유

눙ำ

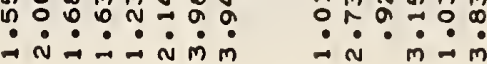
ํำ $\dot{-} \dot{-\dot{m}} \dot{\boldsymbol{m}} \dot{\mathrm{m}}$

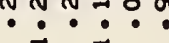

ân

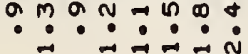

응ㅇㅇㅇㅇㅇㅇㅇㅇㅇ
융ㅇㅇㅇㅇㅇ

nำ

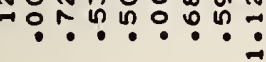

-

$\rightarrow$

$\checkmark$
:ำ

m

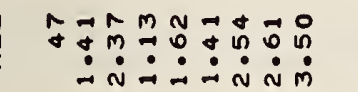

คด w $\dot{*} \dot{i} \dot{N} \dot{*} \dot{m}$

ดิตัตั

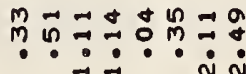

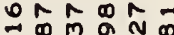
$\because \because: \div$ $\dot{N} \dot{N}$ $\because \dot{-} \dot{-} \dot{i}$

융ㅇㅇㅇ

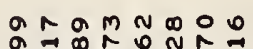

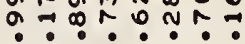
$\because \dot{n} \dot{A} \dot{\sim} \dot{4} \dot{4}$

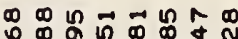
:

ำํำ $\div \div: \div \div \dot{0}$

magma $\because 90 \div \%$ ? i $\dot{0} \dot{\sim} \dot{N}$ i

mี 


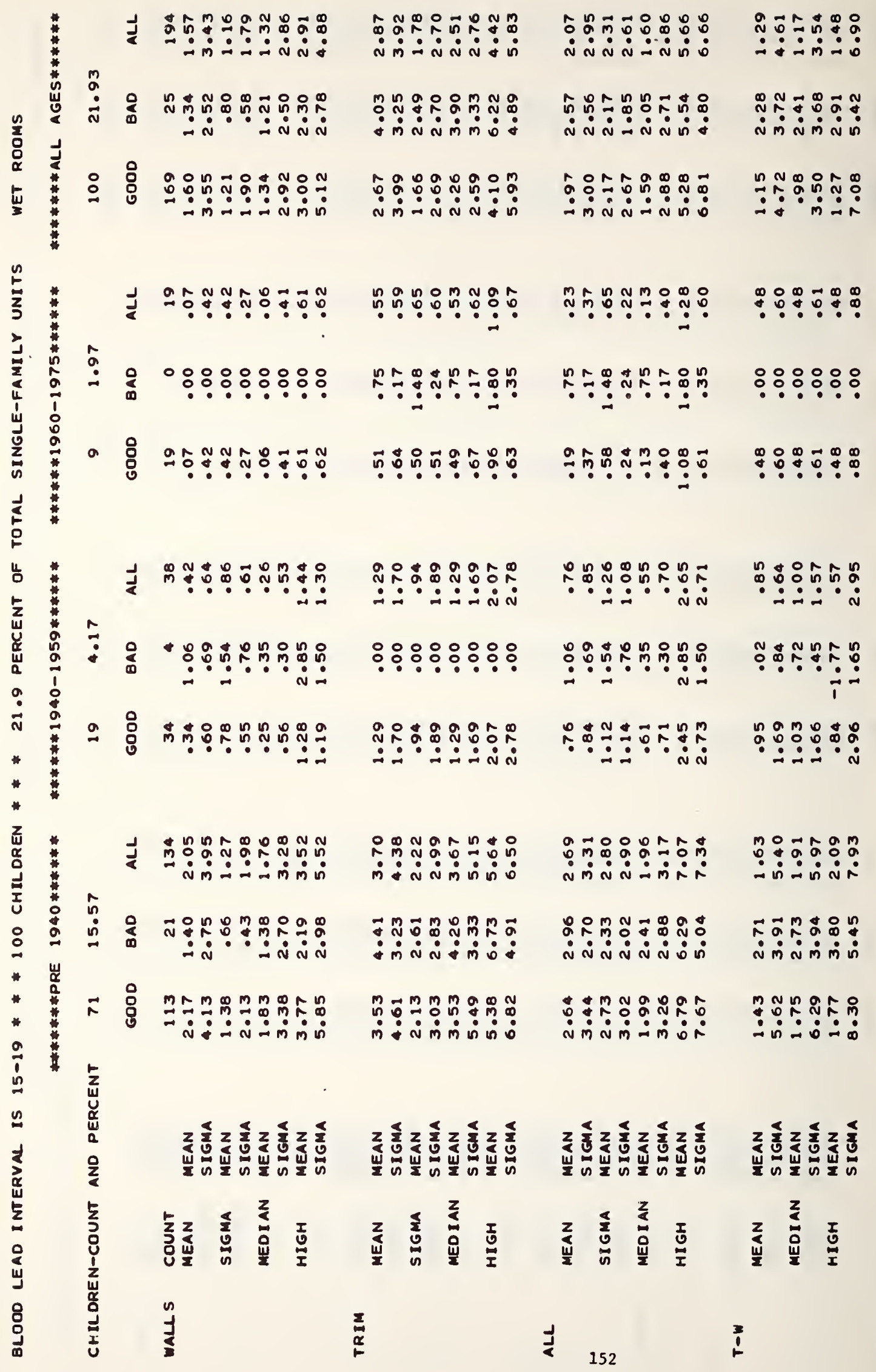




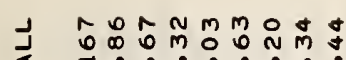

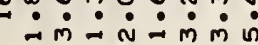

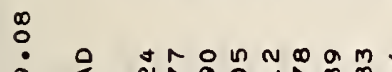

$\dot{\sim} \dot{m} \dot{-} \dot{N} \dot{m} \dot{m} \dot{\sigma}$

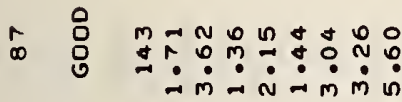

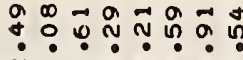

-

$\dot{m} \dot{m} \dot{\sim} \dot{N} \dot{m} \dot{q}$

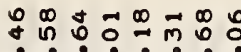

$\dot{0} \dot{m} \dot{\sim} \dot{0} \dot{0}$

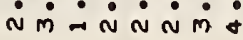

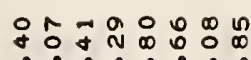

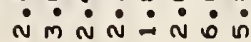

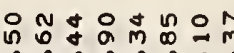

ดั

MñAma

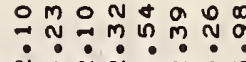

$\dot{\sim} \dot{m} \dot{\sim} \dot{N} \dot{\sim} \dot{\sim} \dot{0}$

m.ำ.ำ

$\because \dot{\sim} \dot{\sim} \dot{0}:$
J

$\stackrel{8}{\circ}$

象

응ㅇㅇㅇㅇㅇㅇㅇ

N

응

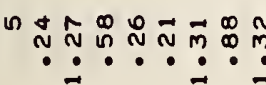

กิ

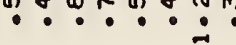

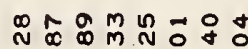

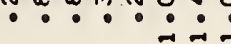

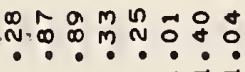

ก 2 a

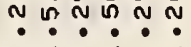

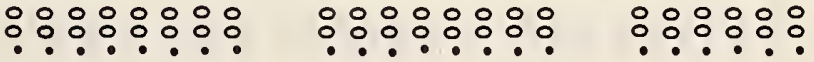

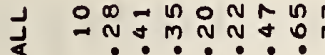

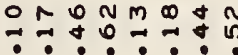

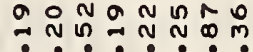

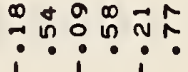

$\because$

용ㅇㅇㅇㅇㅇㅇ

n 웅

ㅇำำ

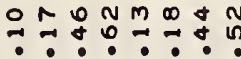

a O N

๑ก กับ N N

$\stackrel{\infty}{*} \stackrel{0}{0}$ 品

\

\& $\dot{m} \dot{m} \dot{\mathrm{N}} \dot{m} \dot{\mathrm{n}}$

i $\dot{m} \dot{N} \dot{N} \dot{m} \dot{0}$

จำ $\dot{N} \dot{m} \dot{\sim} \dot{\sim} \dot{m} \dot{m}$ ம

$\stackrel{+}{\because}$

ำำำำ

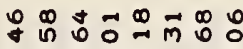

i

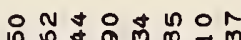

?:

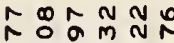
i $\dot{m} \dot{-} \dot{\sim} \dot{m} \dot{m} \dot{d}$

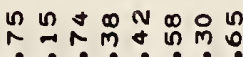

N $\dot{m} \ddot{-} \dot{N} \dot{N} \dot{0}$

$0 \div 007 N N N$

m? M $\dot{\sim} \dot{m} \dot{N} \dot{\sim} \dot{N} \dot{0}$

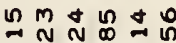

$\because \dot{-} \dot{m} \dot{-} \dot{0}$

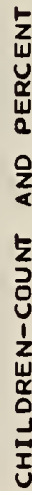

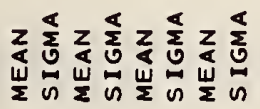

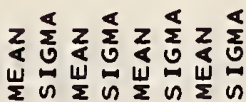

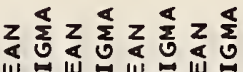

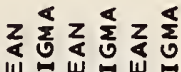
崖的致的崖的 崖的崖虹的
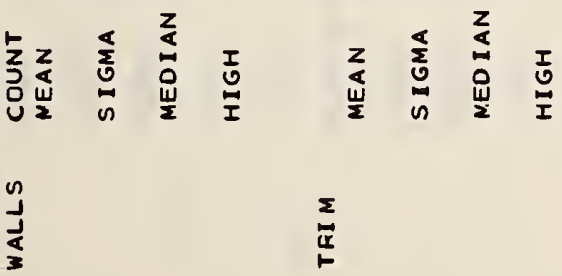

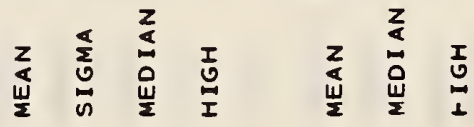




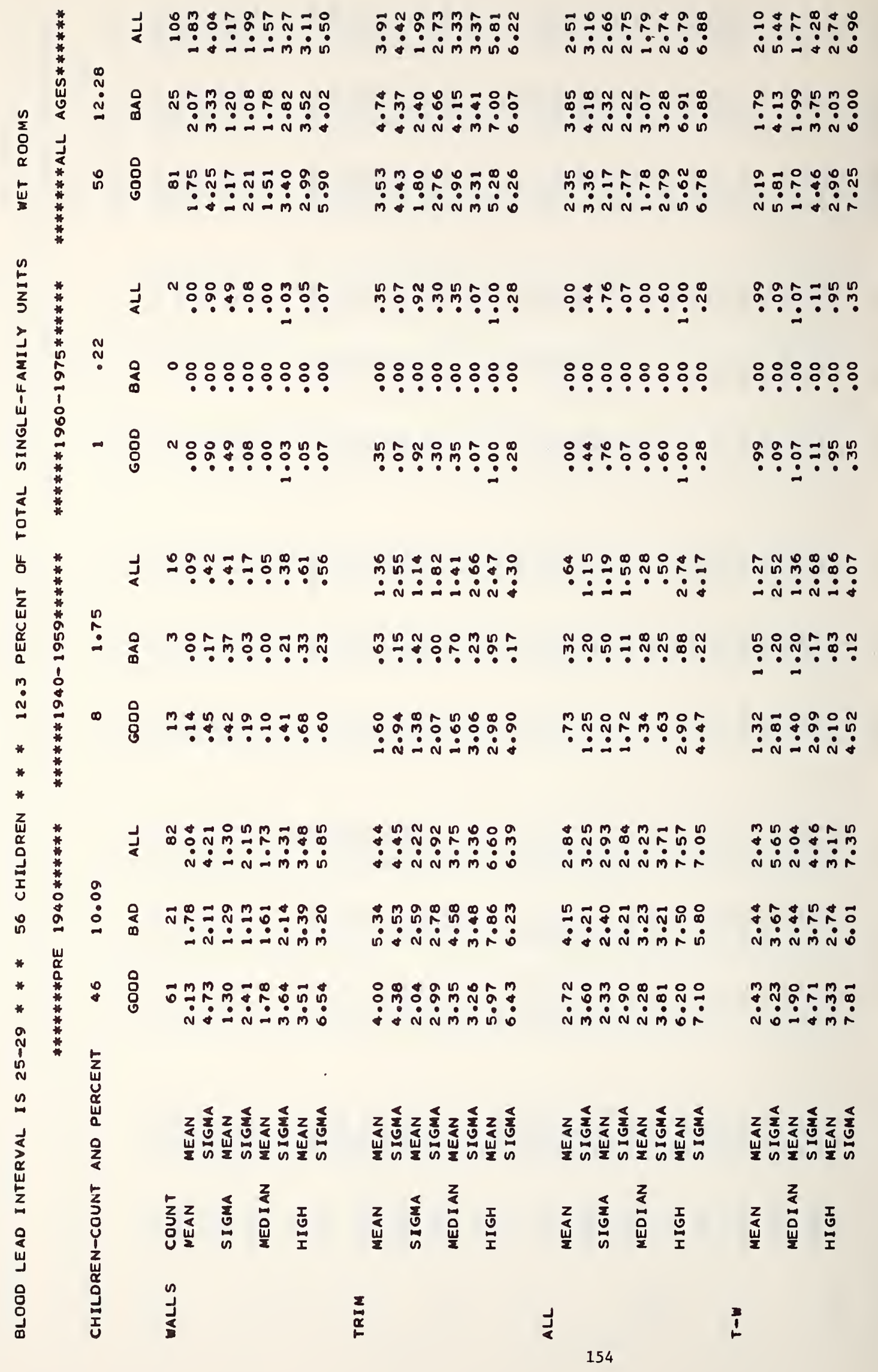




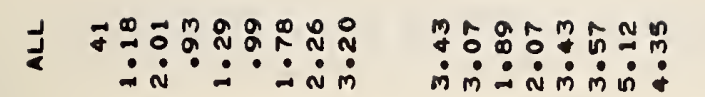

¿

응

\section{J}

$:$

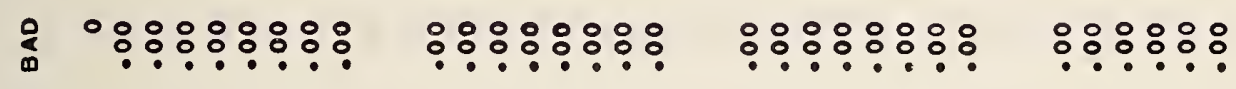

$\circ 8$

ğ

$\because::::::::$

:::::유:

::ㅇ:::유:

:ㅇ:ㅇ:웅

M M

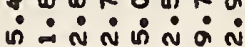

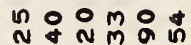
N พับำ: जिं் $\dot{N} \dot{\sim} \dot{\sim}$

N NDU

$\dot{0} \dot{+} \dot{0} \dot{0} \dot{\alpha}$

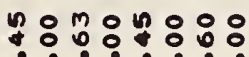

०: ::: : :

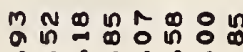

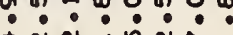

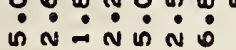

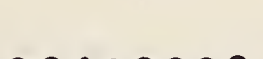

$-8$

ระ

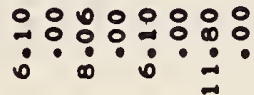

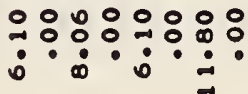

:ㅇ:ㅇ:웅

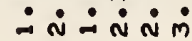

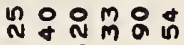
- $\dot{N} \dot{i} \dot{N} \dot{m}$ 」

:

药

M้ ํํำ ถู

$\dot{\dot{m}} \dot{\mathrm{N}} \dot{\dot{\theta}} \dot{\mathrm{m}} \dot{0} \dot{m}$

ลㅇํำ

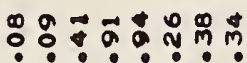

mi்
Mำ

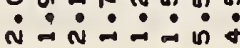

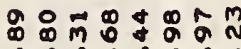

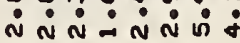

TNNODONM

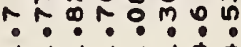
พัดี $\dot{N} \dot{N} \dot{m} \dot{m} \dot{0}$

ลิ $\dot{\sim} \dot{\sim} \dot{\sim} \dot{m}$ 


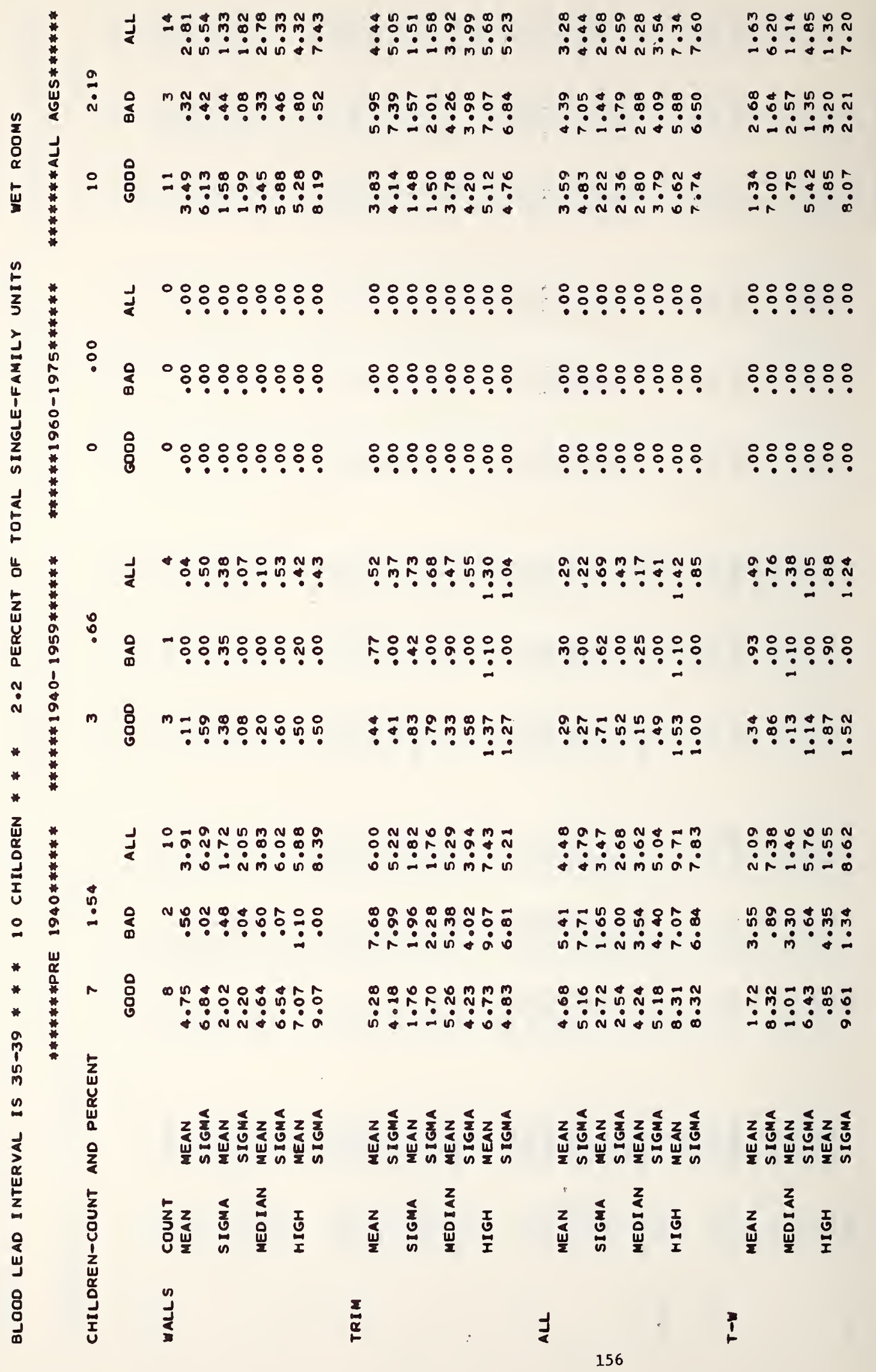




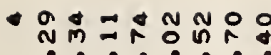
i $\dot{n} \dot{i} \dot{n} \dot{m}$

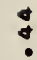

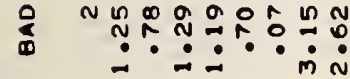

N 吕

号 N

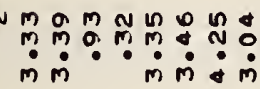

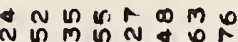

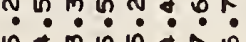

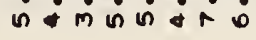

๓กำ

- $\dot{m} \dot{m} \dot{0} \dot{0} \dot{0} \dot{m}$

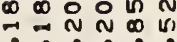

- $\dot{\infty} \dot{\infty} \dot{\sim} \dot{\sim}$

J

응유:유:웅

응유:융유

응응ㅇㅇㅇㅇㅇㅇ

응응ㅇㅇㅇ

$:$

๑

$\circ \stackrel{8}{8}$

วั้

$\therefore:: 8:: 8:$

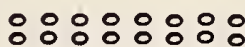

$\because: \circ: \circ: 0$

$\because \therefore: ㅇ:$

」

$\therefore$

๓

- $\quad$ 의

J

ดลNMUดN

$\because: 00: 00: 0$

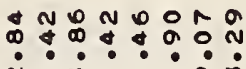

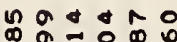

$\stackrel{\infty}{0}: 0 \stackrel{0}{0}:$

$\stackrel{8}{\square}$

๑

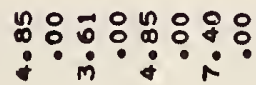

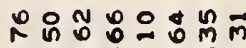

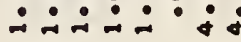

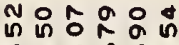

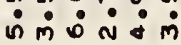

N

兽

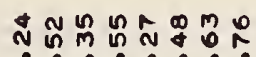

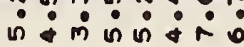

m

ดำำดำ

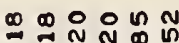

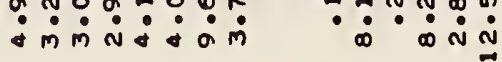




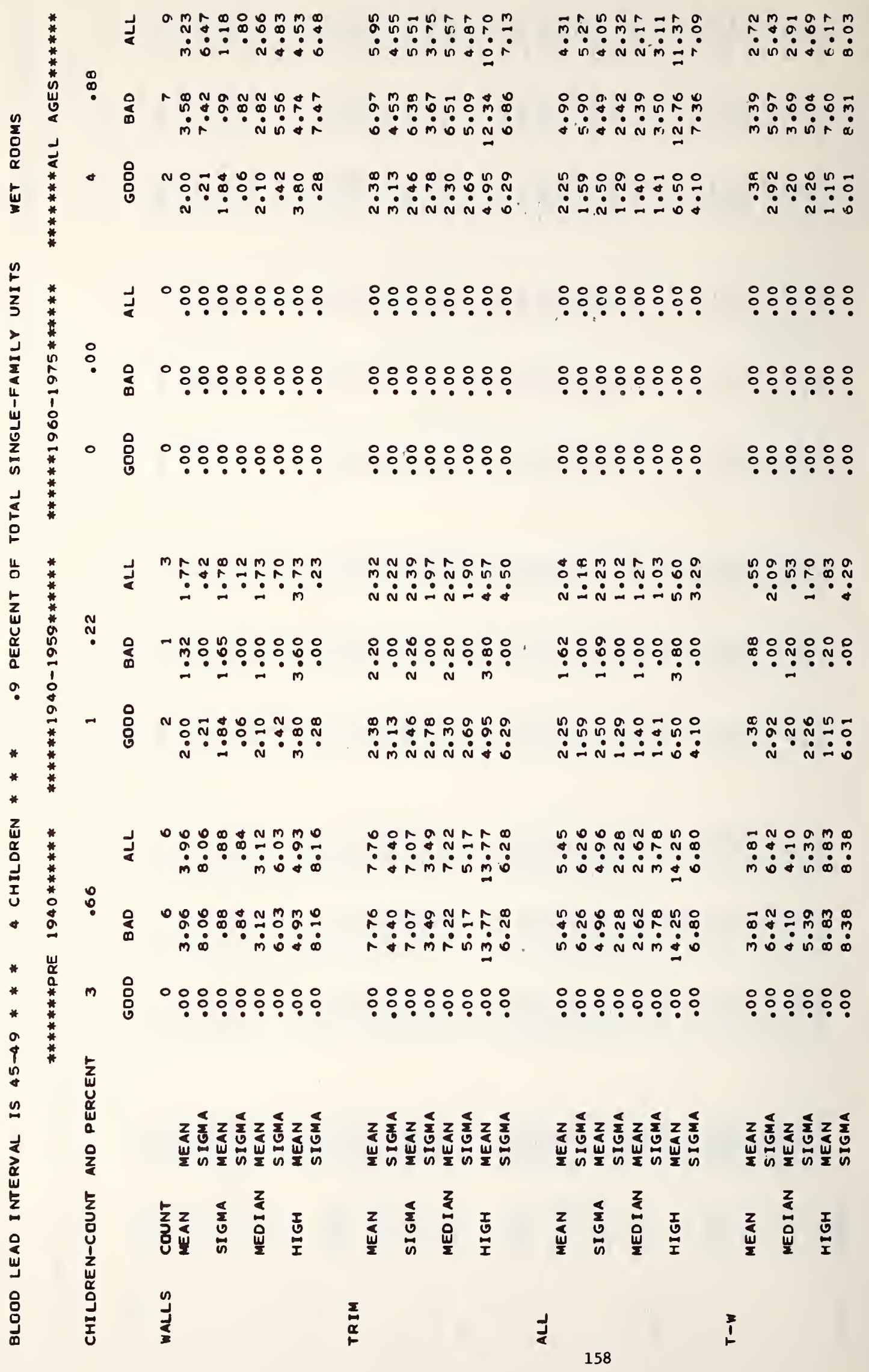




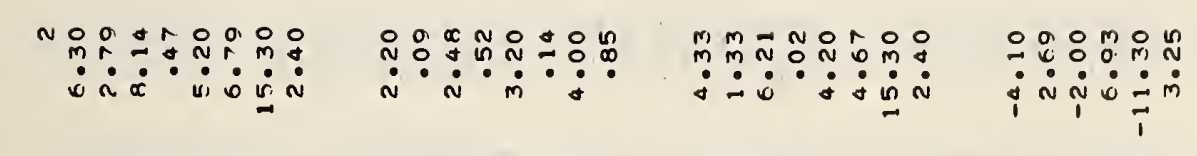

․

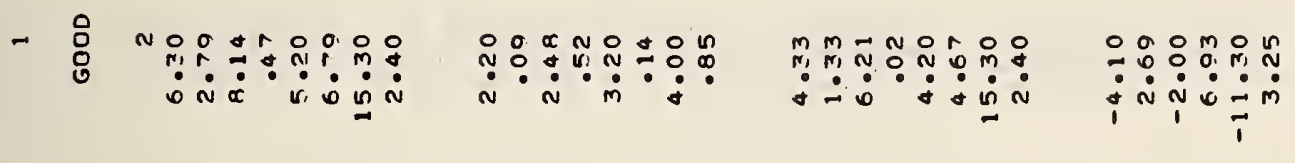

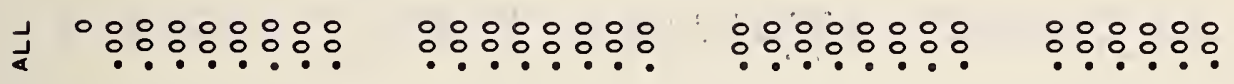

:

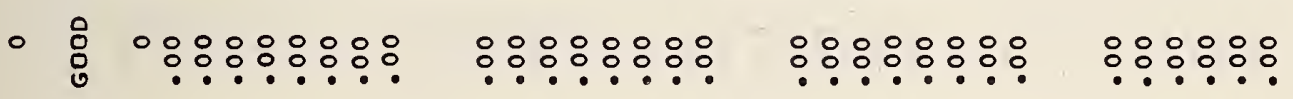

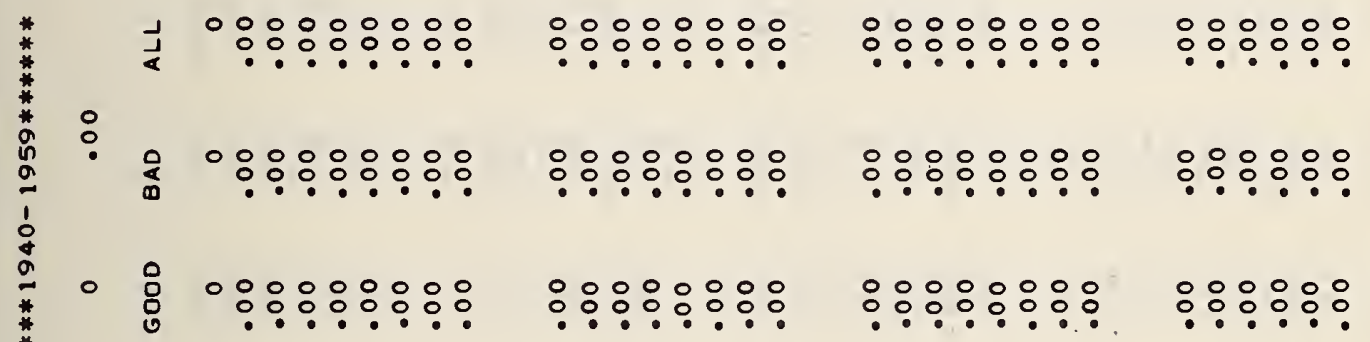

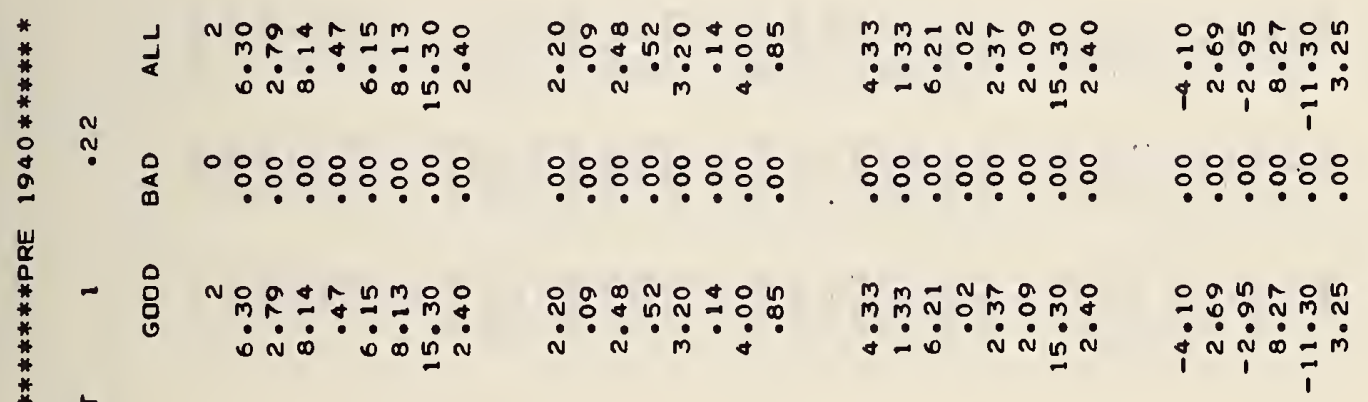

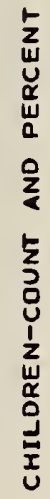
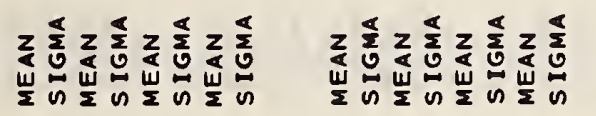

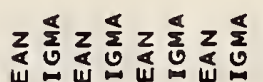

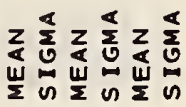

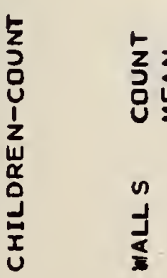

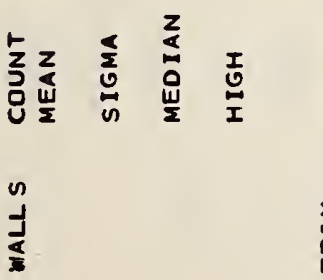

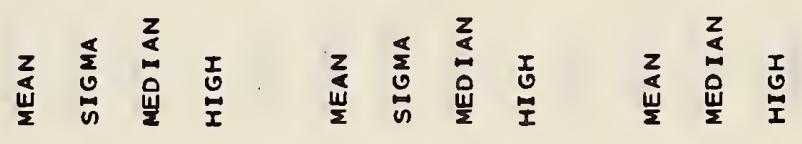

$\underset{\Sigma}{\Sigma}$

$\stackrel{\jmath}{\&} 159$

$\stackrel{1}{\vdash}$ 


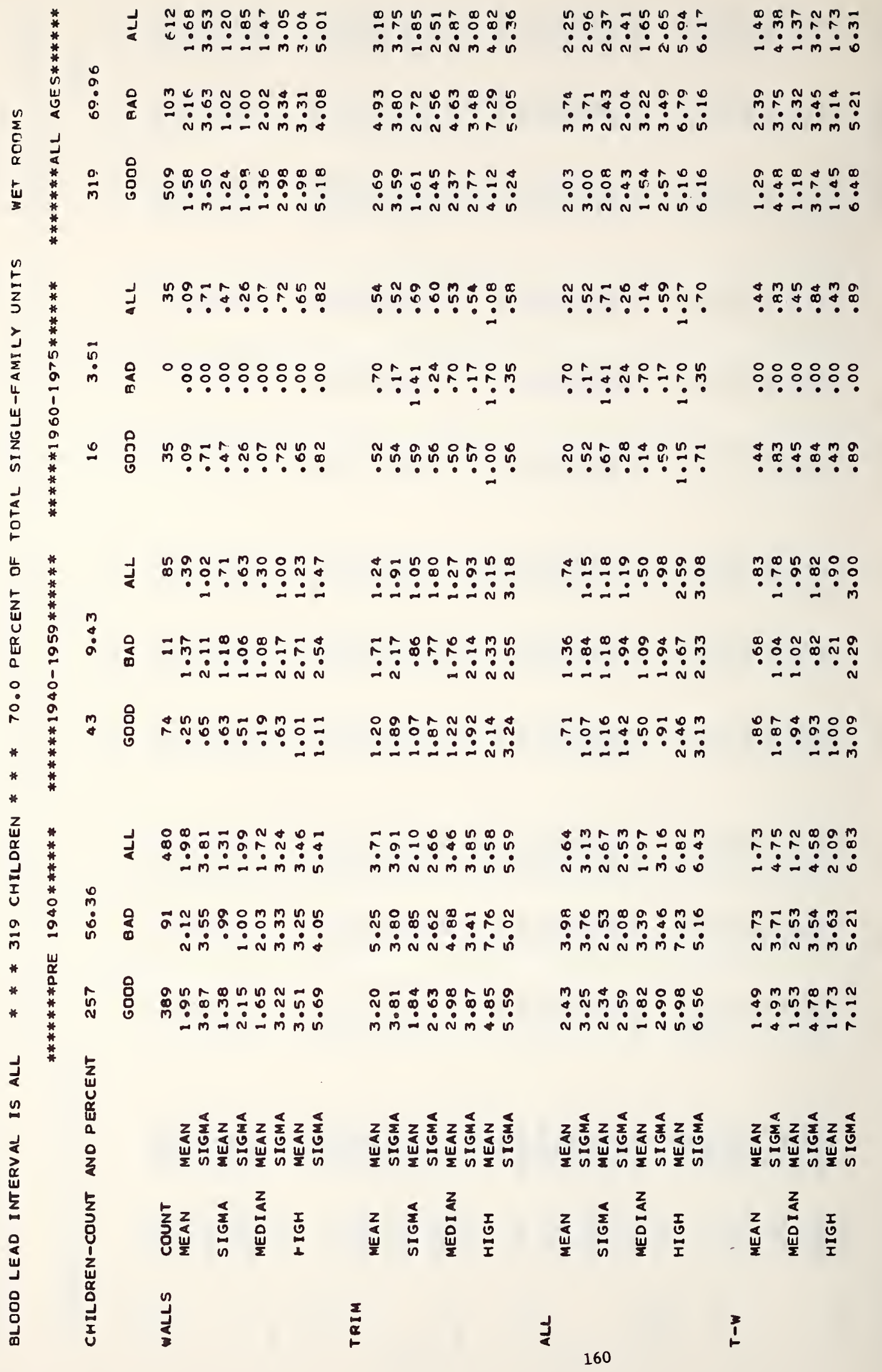




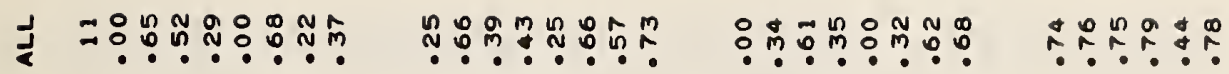

$\vdots$

ه

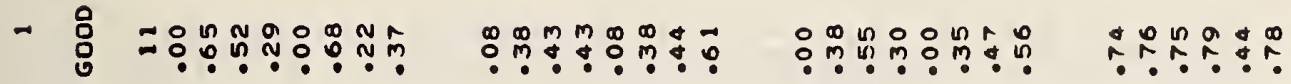

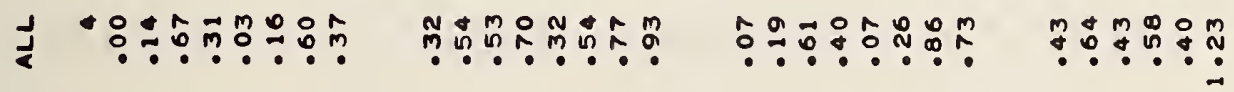

ก :

- 亭

ل

:

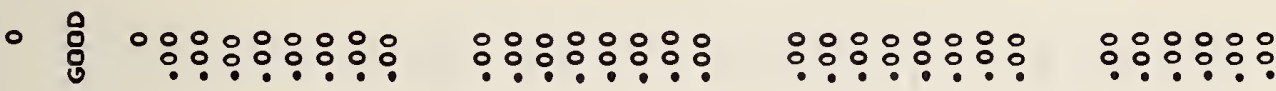

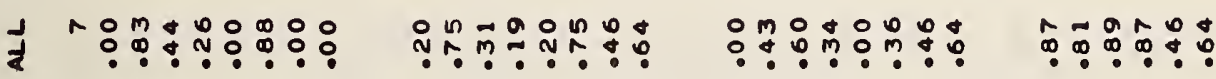

ง

๕

- 立

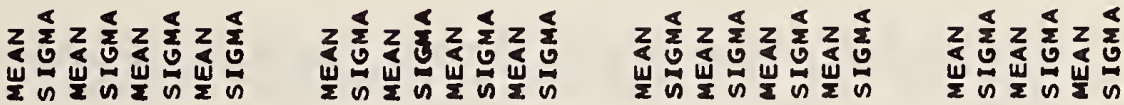

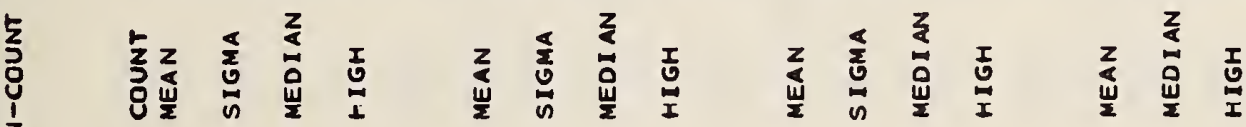

$\frac{1}{3}$

$\underset{1}{\mathbf{n}}$

」

1 


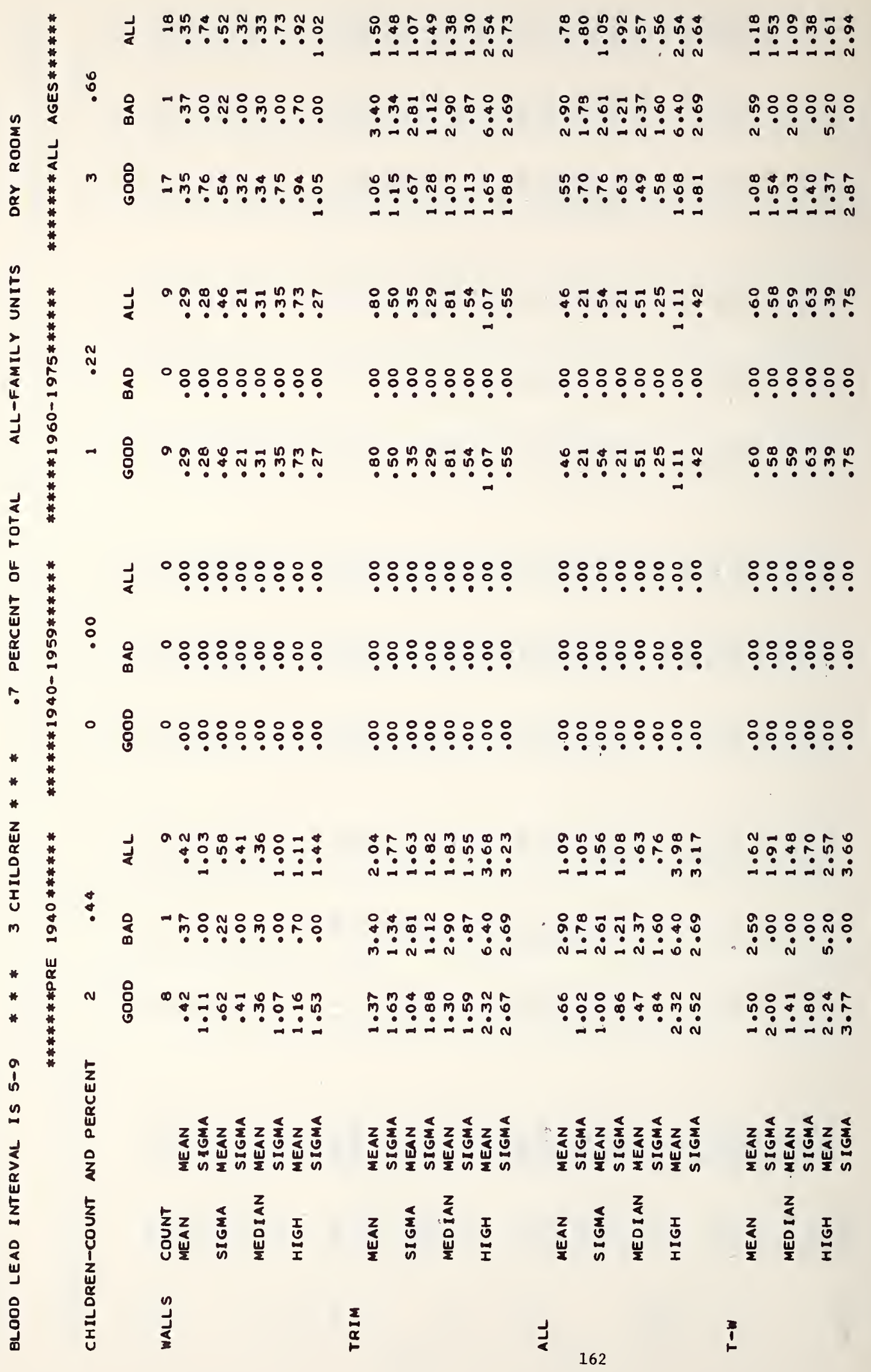




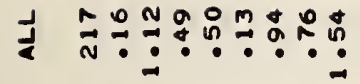

ำ

m

\section{لٌ}

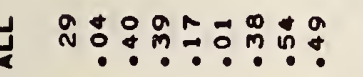

?ำ?

$\stackrel{\circ}{\circ}$

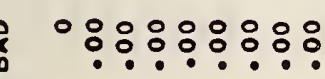

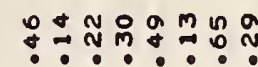

$m 8$

品

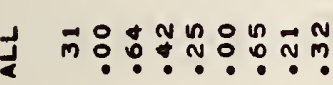

ำ

$\stackrel{n}{n}$

-

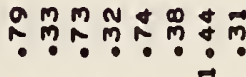

-

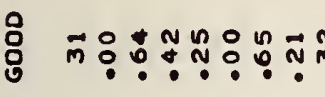

$: \because \frac{0}{0}: \stackrel{2}{\circ}: \div$

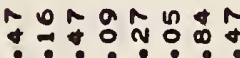
Nim $\dot{n} \dot{N} \dot{m} \dot{ }$

J

:

品

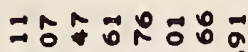
$\dot{m} \dot{m} \dot{a} \dot{\mathrm{i}} \dot{\mathrm{m}} \dot{\mathrm{m}}$

* $0_{0}^{\circ}$ m

药 $\dot{N} \ddot{m} \dot{\sim} \dot{N} \dot{m} \dot{m}$ ำตำำ
คี

$\infty_{\infty}^{\infty} \prod_{\infty} \sim M ⿻$ $\dot{i} \dot{\sim} \dot{N} \dot{N} \dot{0}$

운 $\rightarrow \dot{i}:-\dot{i} \dot{m}$

क्ष。

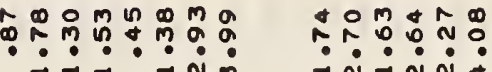

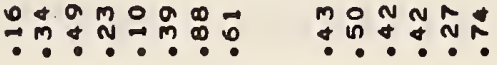

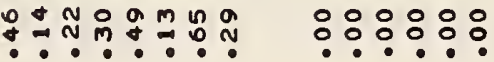

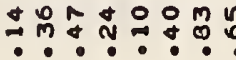

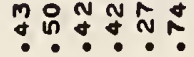

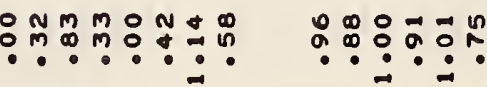

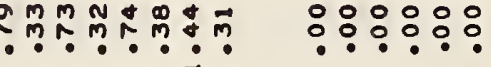
ㅇํำํํำ告?

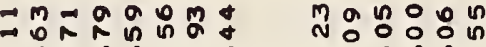

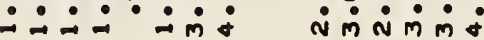

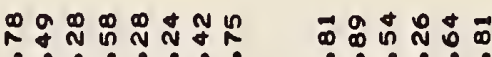

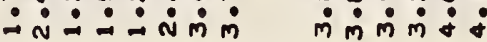

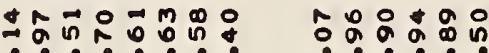

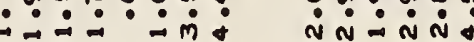

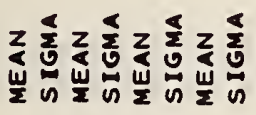

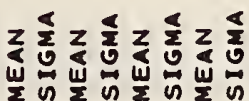

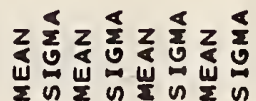

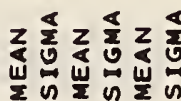

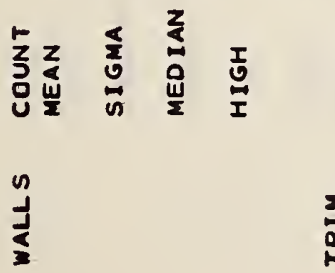

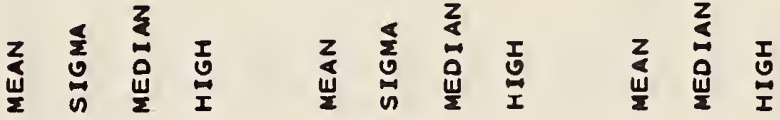

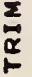

1 


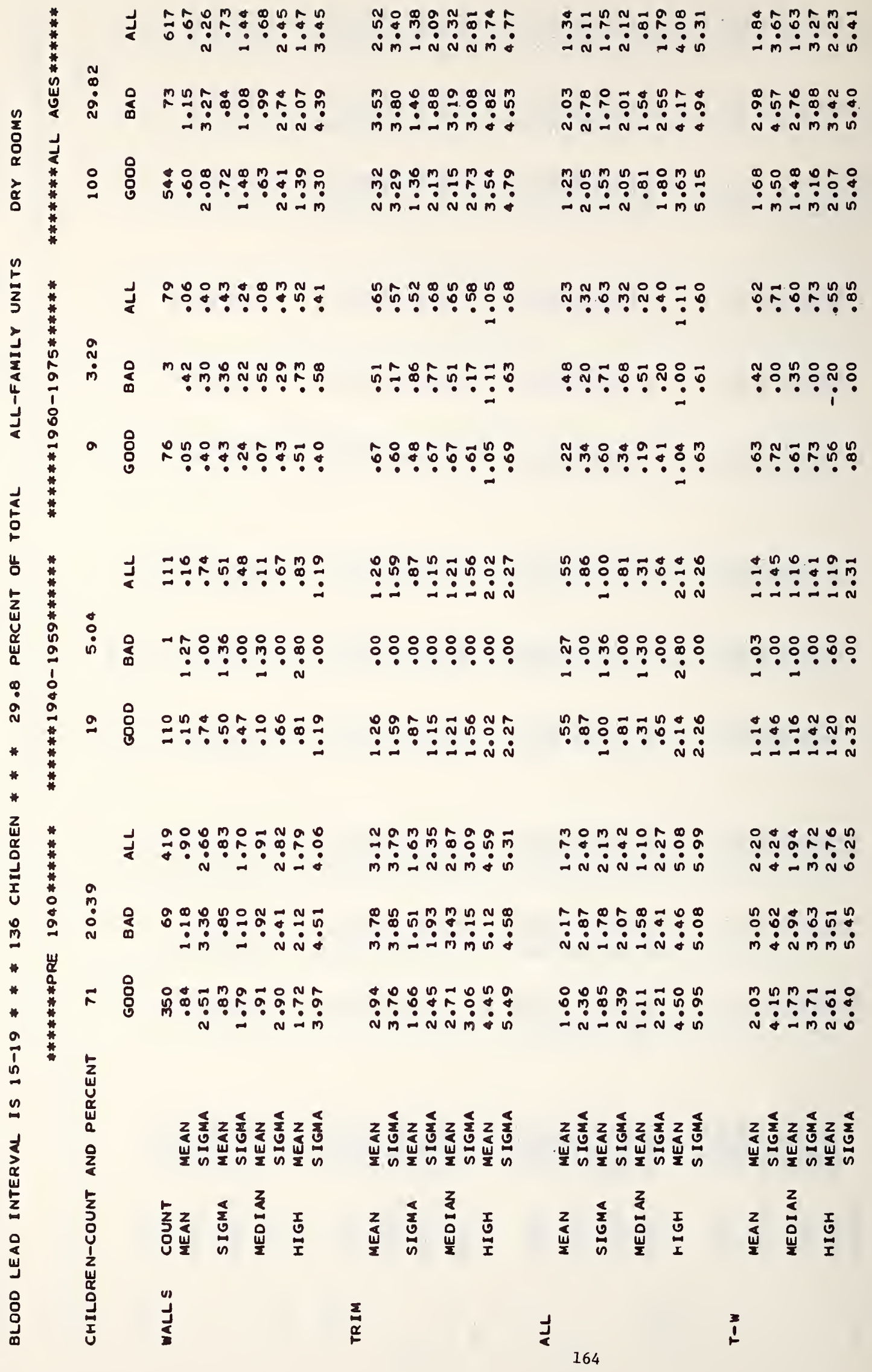




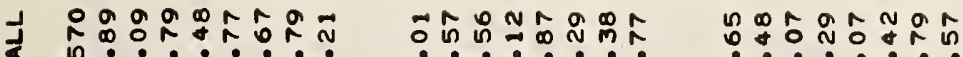

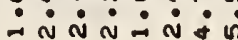

온 $\dot{m} \dot{m} \dot{\sim} \dot{\sim} \dot{N} \dot{m} \dot{\alpha}$ $\dot{\sim} \dot{\alpha} \dot{\sim} \dot{m} \dot{\sim}$

$\stackrel{n}{:}$

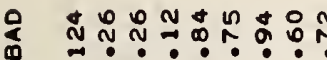

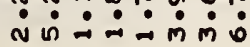

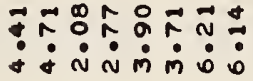

^

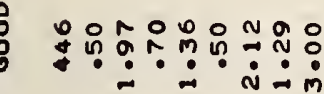

พ $m \infty=0 n$

ทด ตัต

NNANMm:

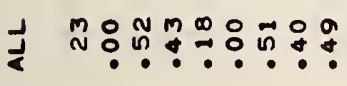

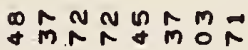

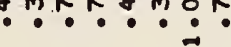

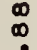

8

$\circ:::::$

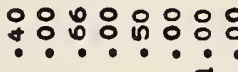

N

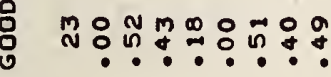

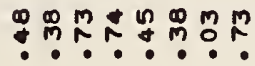

Mำ

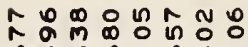
ति $\dot{\sim} \dot{\sim} \dot{\sim} \dot{0} \dot{R}$

ต Nm $n$

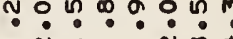

- Nन N

ำ

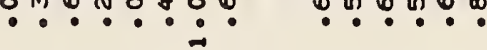

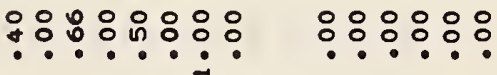

กูก

吊

$\dot{\sim}$

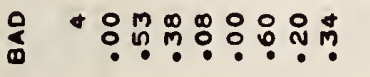

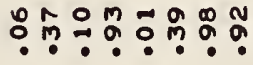

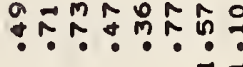

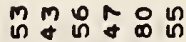

$\because \because \div \dot{\div} \div$

(n)

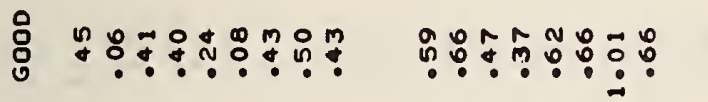

nNa nanton

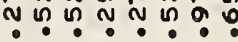

ำกำ

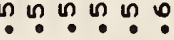

Jُ

ì

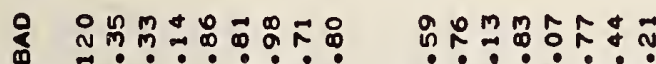

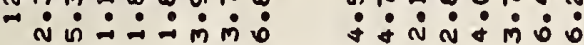

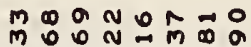
$\dot{m} \dot{m} \dot{0} \dot{m} \dot{0}:$

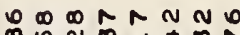
๑n $-\dot{N} \dot{N} \dot{N} \dot{\sim}$ 的

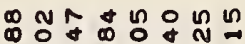

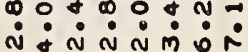

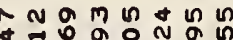

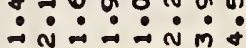

ㅇํㅇำ N N พกำ

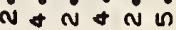

๑ักํำ $\dot{m} \dot{0} \dot{m} \dot{0} \dot{0}$

:

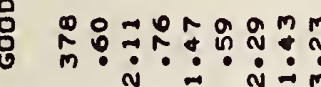

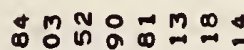

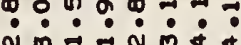

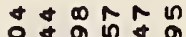
im $\dot{\sim} \dot{m} \dot{0}:$ 


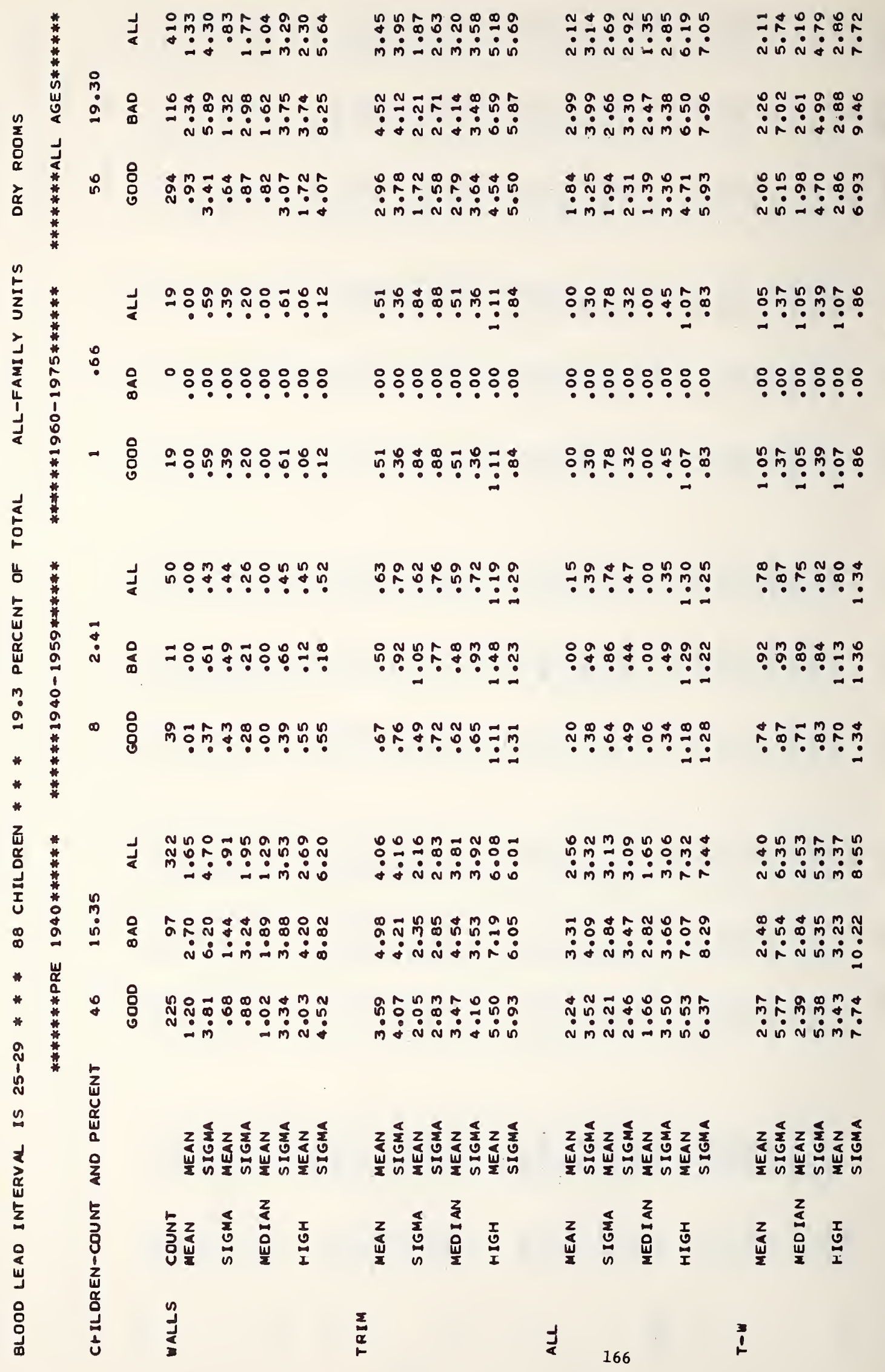




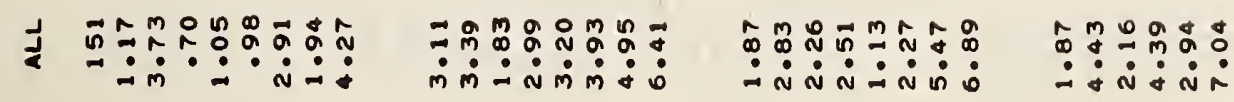

$\mathfrak{l}_{0}^{\infty}$

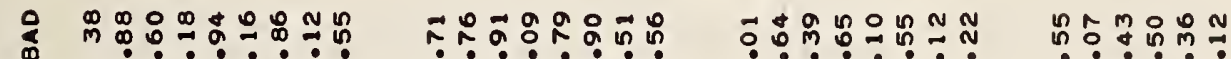

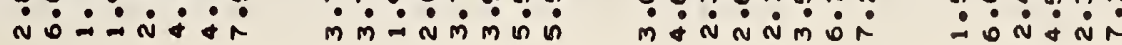

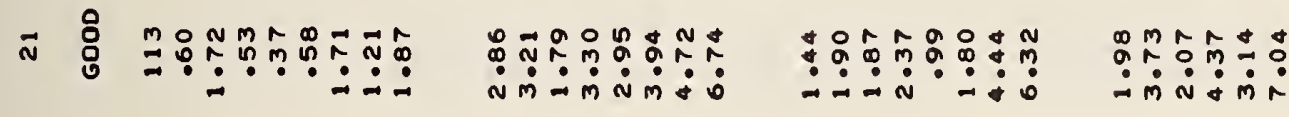

ل

:

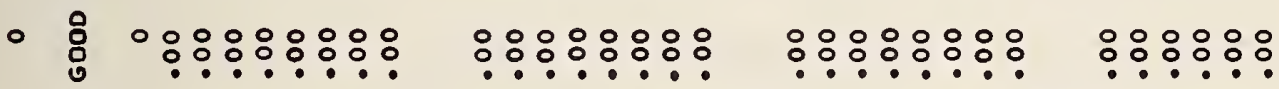

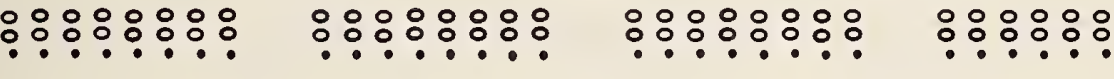

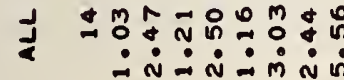

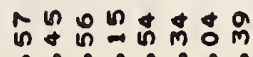

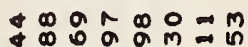
눙 $n=N$

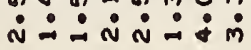
$\therefore \dot{0} \dot{0}: \dot{0} \dot{0}$
$\because \dot{N}: \dot{n}: \dot{0}$

$\stackrel{0}{0}$

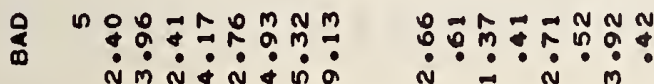

m

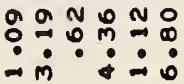

$\dot{N} \dot{m} \dot{i} \dot{m} \dot{0} \dot{0}$

$-\quad 8$

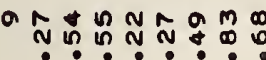

กิ

N

ONONOO $\dot{N} \dot{i} \dot{\sim} \dot{N} \dot{q} \dot{q}$

$\therefore \dot{0} \dot{0} \dot{0} \dot{m}$

$\therefore \div \div \div \div$

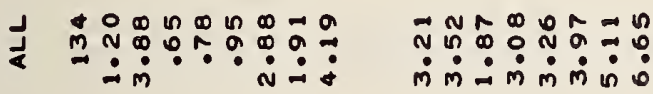

$\stackrel{\circ}{\infty}$

\&

옹

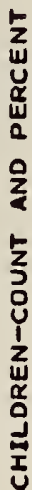
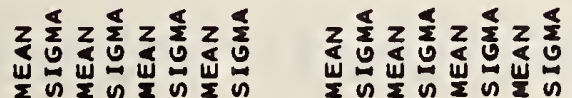

ดูก๊ำ N $\dot{m} \dot{-1} \dot{m} \dot{m} \dot{+} \dot{0}$

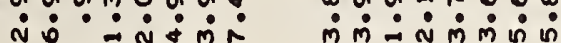

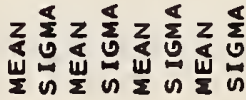

$z \frac{\pi}{2} z \frac{\pi}{2} z \frac{5}{0}$

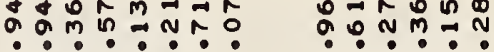

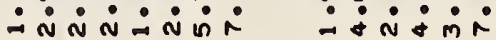

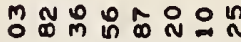
$\dot{m} \dot{\alpha} \dot{\sim} \dot{\sim} \dot{m} \dot{0}$

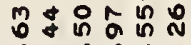
$\therefore \dot{\sim} \dot{m} \dot{\sim}$

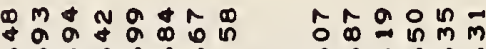

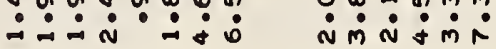

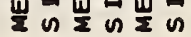

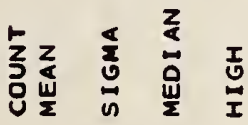

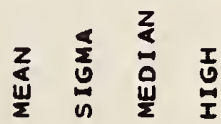

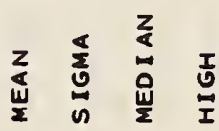

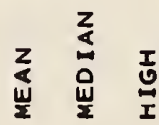

$\underset{\substack{\alpha \\ \mathfrak{\alpha}}}{\sum}$

J 


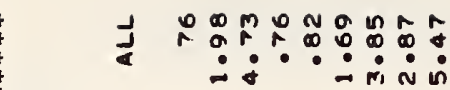

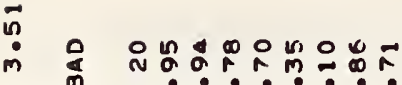

$m$ 更

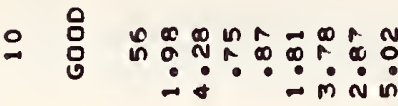

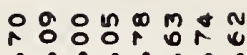

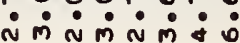

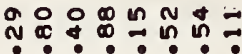

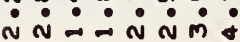

๓

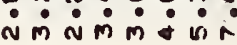

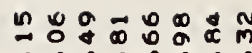

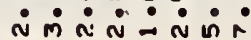

ดีดัด $\because \dot{m} \dot{\sim} \dot{\alpha} \dot{\sim} \dot{m} \dot{0}$

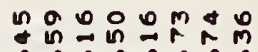
जिए人

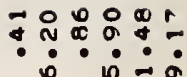

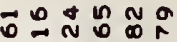
$\because \div: \div: 0$

$\sum^{N} \stackrel{x}{\sim}$ mะ. กำ. $\dot{0}: \dot{0}$

거 응응응ㅇ:

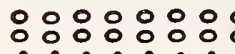

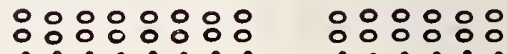
응

-

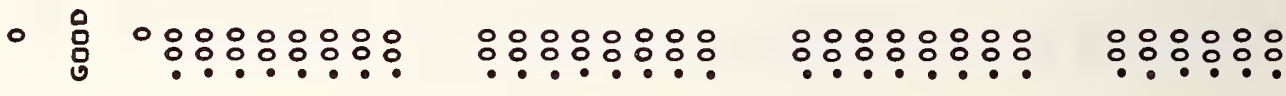

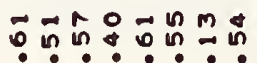

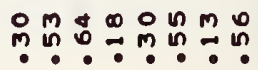

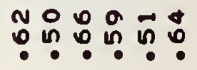

$\because$

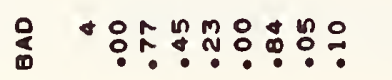

บิ พัN

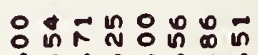

ำำำำำ

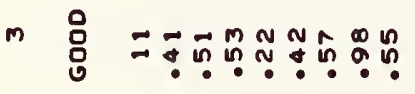

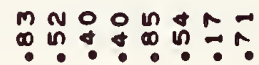

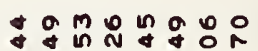

noO $00 \mathrm{~m}$

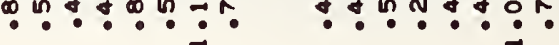

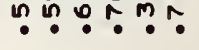

J

ลำ

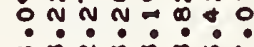

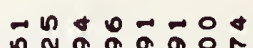
ำดำำ

nog 00

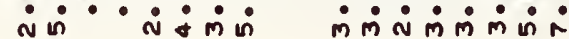

NMN

$\because \div: \div$

品

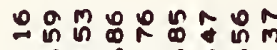

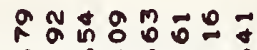

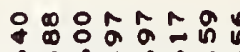
$\dot{\sim} \dot{0} \dot{\circ} \dot{\dot{m}} \dot{\mathrm{r}}$ $\dot{N} \dot{N} \dot{\sim} \dot{N} \dot{N} \dot{8}$ $\dot{\sim} \dot{m} \dot{N} \dot{N} \dot{\sim} \dot{m} \dot{0}$

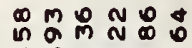
$\dot{0} \dot{\sim} \dot{0} \dot{\boldsymbol{i}}$

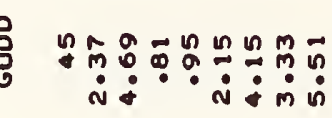

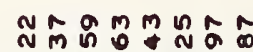

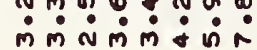

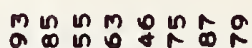

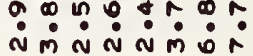

จำำ $\because \because: \frac{0}{0}$ 


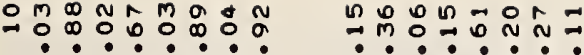

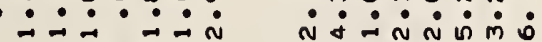

$\stackrel{\circ}{\circ}$

ه m

N

号

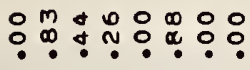

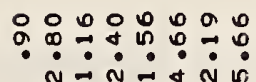

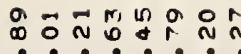

inis.

$\cong \notin$ is $\underset{\sim}{\sim} \stackrel{m}{\sim}:$

Nं $\dot{\sim}$ in $\dot{\sim}$

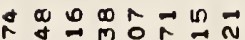
त $\dot{1} \dot{m} \dot{m} \dot{m} \dot{0} \dot{0}$

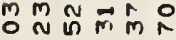
जि $\dot{0} \dot{0} \dot{0}$

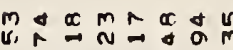
$\dot{\sim} \dot{\sim} \dot{\sim}: \dot{a}$

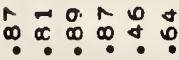

J

$\circ: 0: 0: 0::$

응응응ㅇㅇ응

:

\&

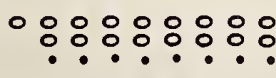

응ㅇ:ㅇㅇㅇ

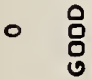

응ㅇㅇㅇㅇㅇㅇㅠ

응융ㅇㅇ응

응응ㅇㅇ응

응응응응웅

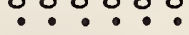

응응ㅇㅇㅇ

응응응ㅇㅇㅇ

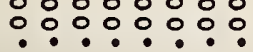

ํํํำ

응유:응응

응응ㅇㅇ응

:

유

$\circ$

: $\circ$

$\because: \because: \because: \because:$

$\because:: \circ:: ㅇ$

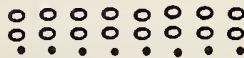

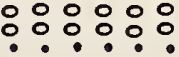

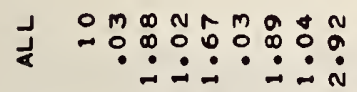

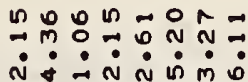

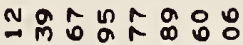

$\dot{\sim} \cdot \dot{0} \dot{0} \dot{0}$

乎

N

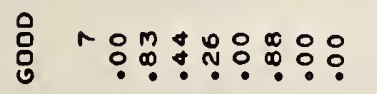

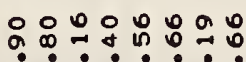

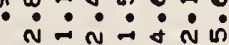

ดิ

$\cong$

i $\dot{\sim} \dot{\sim} \dot{0}$

จับับ $\dot{N} \dot{N} \dot{m} \dot{m} \dot{m} \dot{N} \dot{0}$

$m+\infty m \sim \infty d$

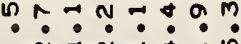

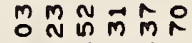
ம் $\dot{0} 0 \dot{0}$

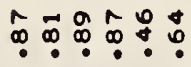

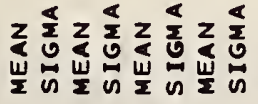

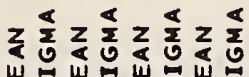

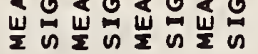

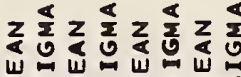
㞫出岂的崖的岂出

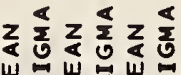
岂药崖的崖的

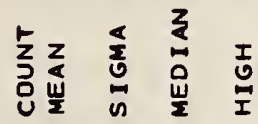

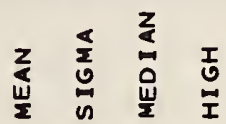

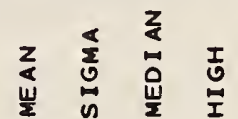
$\begin{array}{lll}z & z & \\ z & \frac{I}{0} \\ \frac{w}{2} & \frac{1}{2}\end{array}$ 


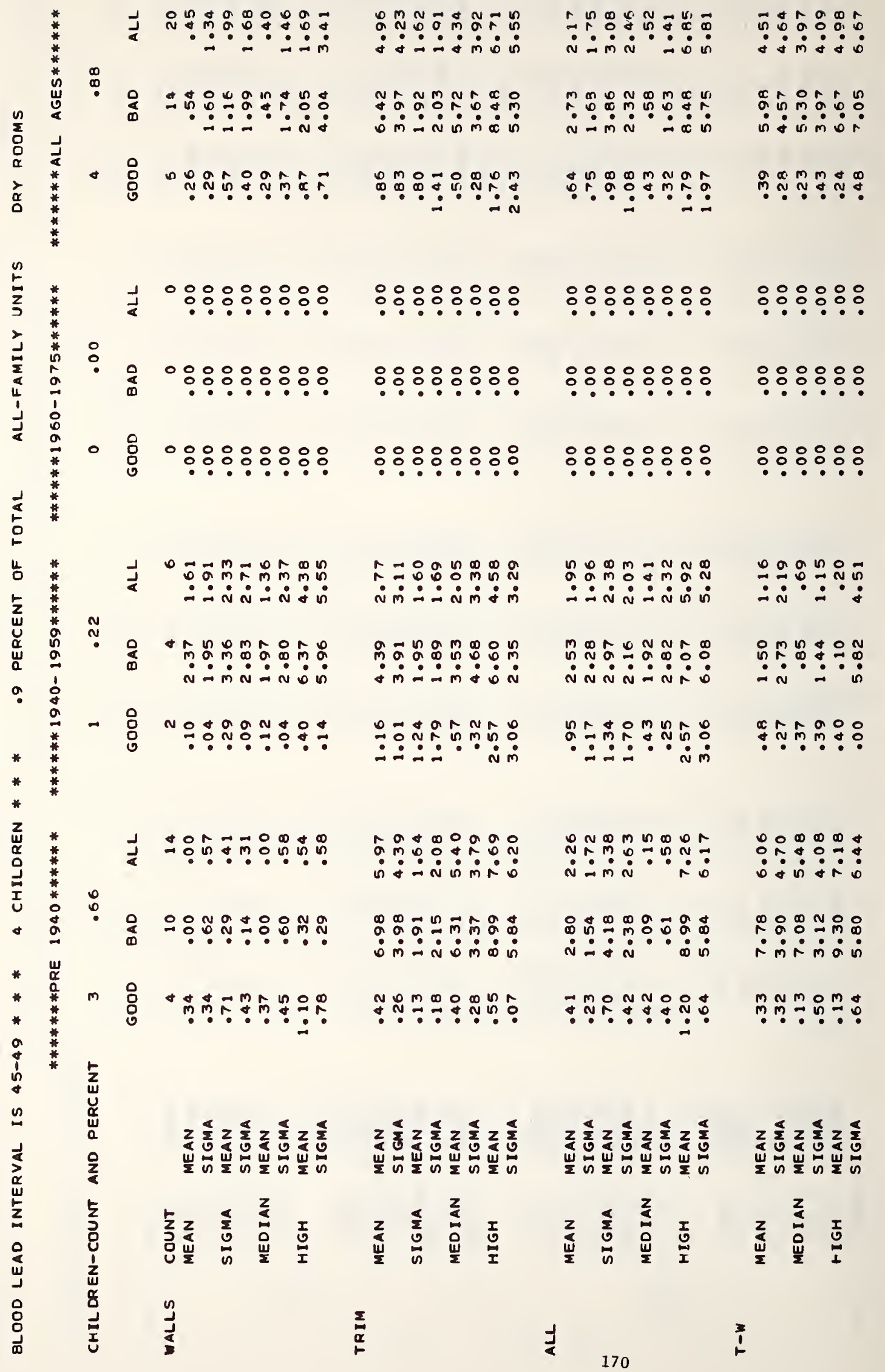




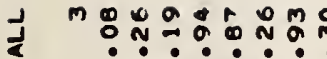

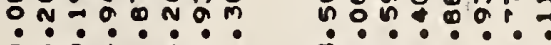

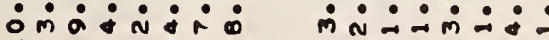

$\stackrel{N}{N}$

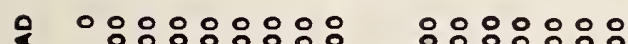

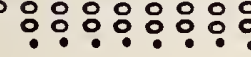

- 8

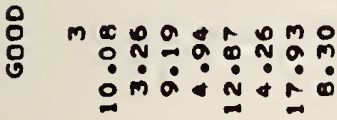

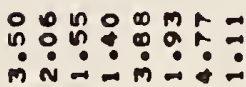

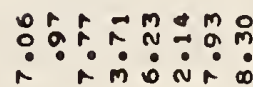

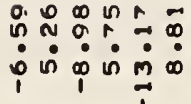

J

ㅇ:ㅇ:ㅇ:ㅇㅇㅇ

ㅇㅇㅇㅇㅇㅇㅇㅇㅇ

ㅇㅇㅇㅇㅇㅇㅇㅇㅇ

웅ㅇㅇㅇㅇㅇ

8

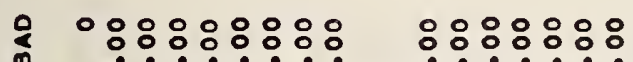

- 吕

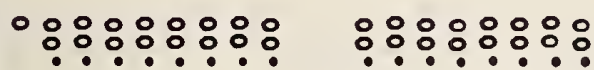

J $0: \%: \%: \%::$

:ㅇ: : : : : :

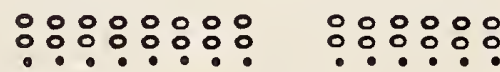

:

$\because$

i

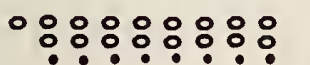

ㅇ:ㅇㅇㅇㅇㅇㅇ

:ㅇ:ㅇㅇ:웅

$: \because::::$

-

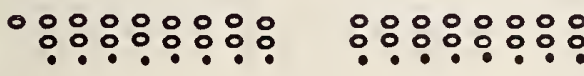

웅ㅇㅇㅇㅇㅇㅇㅇㅇ

ㅇํㅇํ:?

융ㅇㅇㅇㅠ

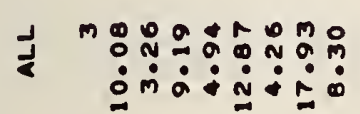

ำ요

n:?:巳:?:

$\stackrel{N}{\mathfrak{N}}$

\& $0: \%: 8:: 8:$

ㅇㅇㅇㅇㅇㅇㅇㅇㅇㅇ

$\begin{array}{r}-\quad 8 \\ \hline\end{array}$

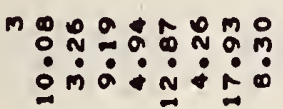

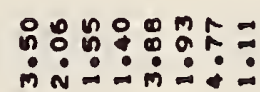

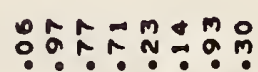

$\therefore$ 前:

용ㅇㅇㅇㅇㅇ

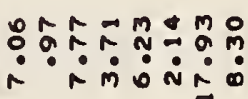

ดั

กับดท:

in:

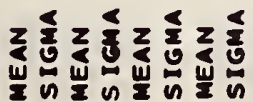

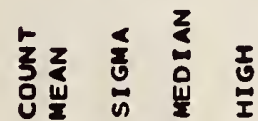

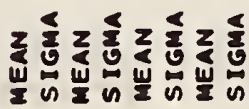

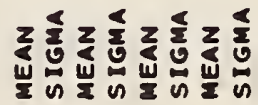

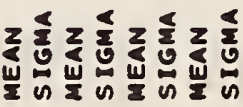

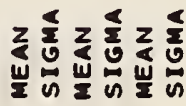

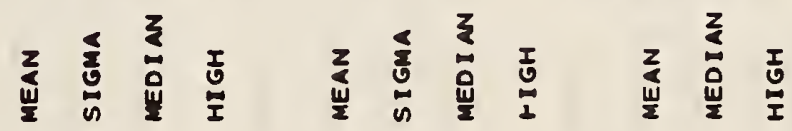

$\underset{2}{2}$
I 


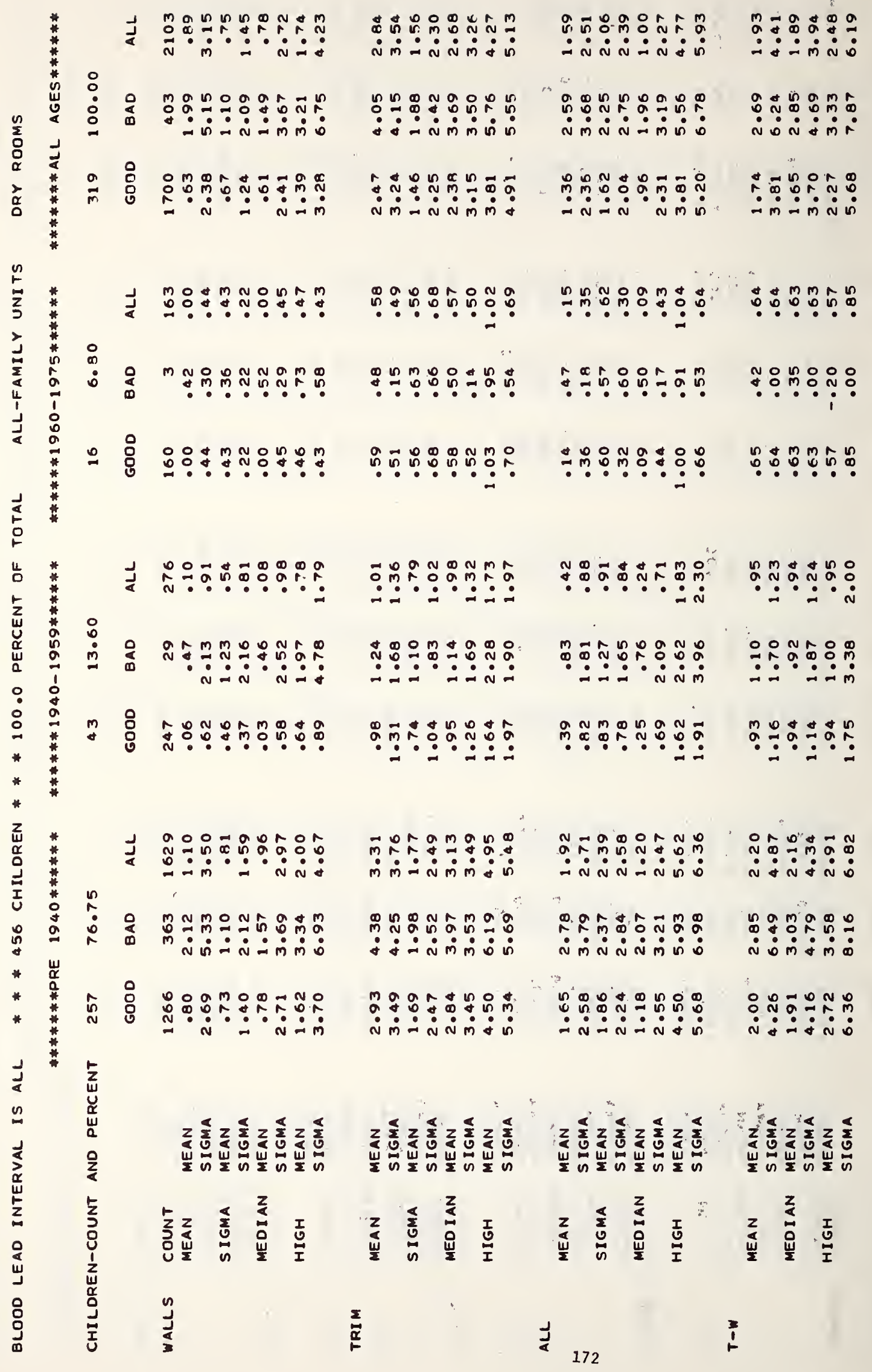




\section{J}

$\stackrel{8}{8}$

ه

- 总 +

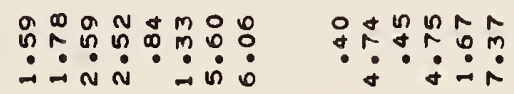

-

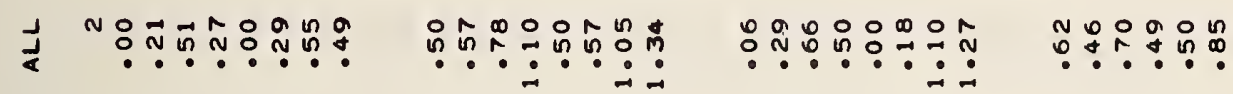

ก

- 品

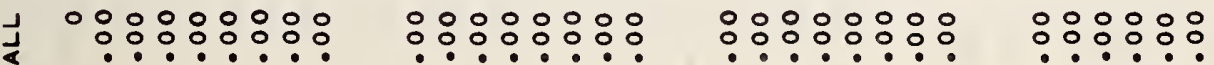

:

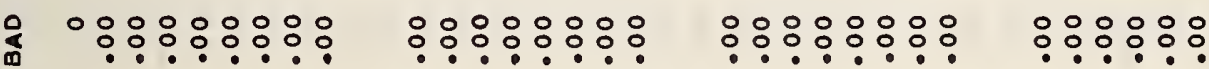

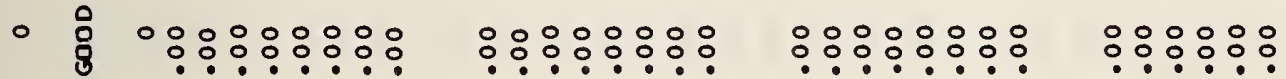

\begin{tabular}{|c|c|c|c|c|c|}
\hline & $\frac{d}{2}$ & 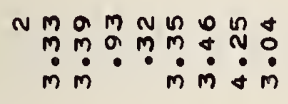 & 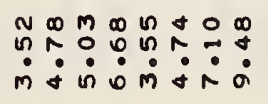 & 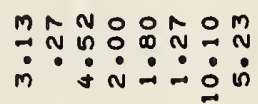 & 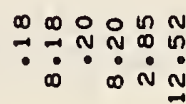 \\
\hline$\stackrel{\mathrm{N}}{-}$ & 是 & 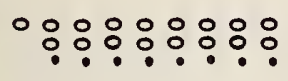 & ㅇㅇㅇ: : : : : : : & :ㅇ:ㅇ:ㅇ: : : & :::ㅇ: \\
\hline - & 吕 & 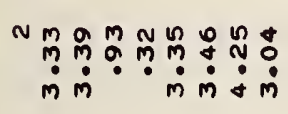 & 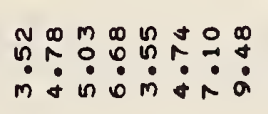 & $\begin{array}{l}m \sim N \\
\stackrel{N}{N}:\end{array}$ & $\stackrel{\infty}{\longrightarrow} \underset{\infty}{\infty} \stackrel{\circ}{\stackrel{\circ}{N} \stackrel{\circ}{\sim}}$ \\
\hline
\end{tabular}

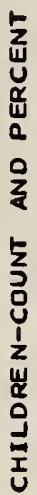

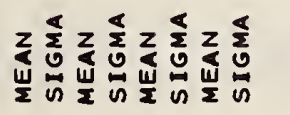

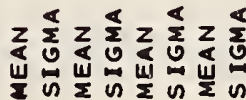

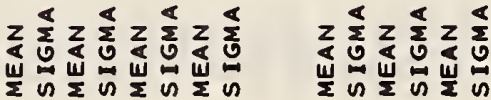

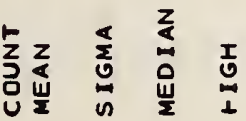

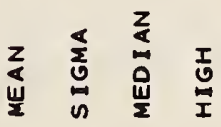

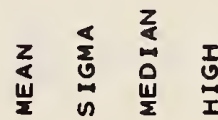

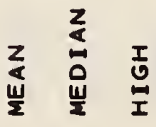

$\frac{n}{3}$

$\underset{\substack{\alpha \\ ⺊}}{\sum}$

$\stackrel{\jmath}{\&}$ 


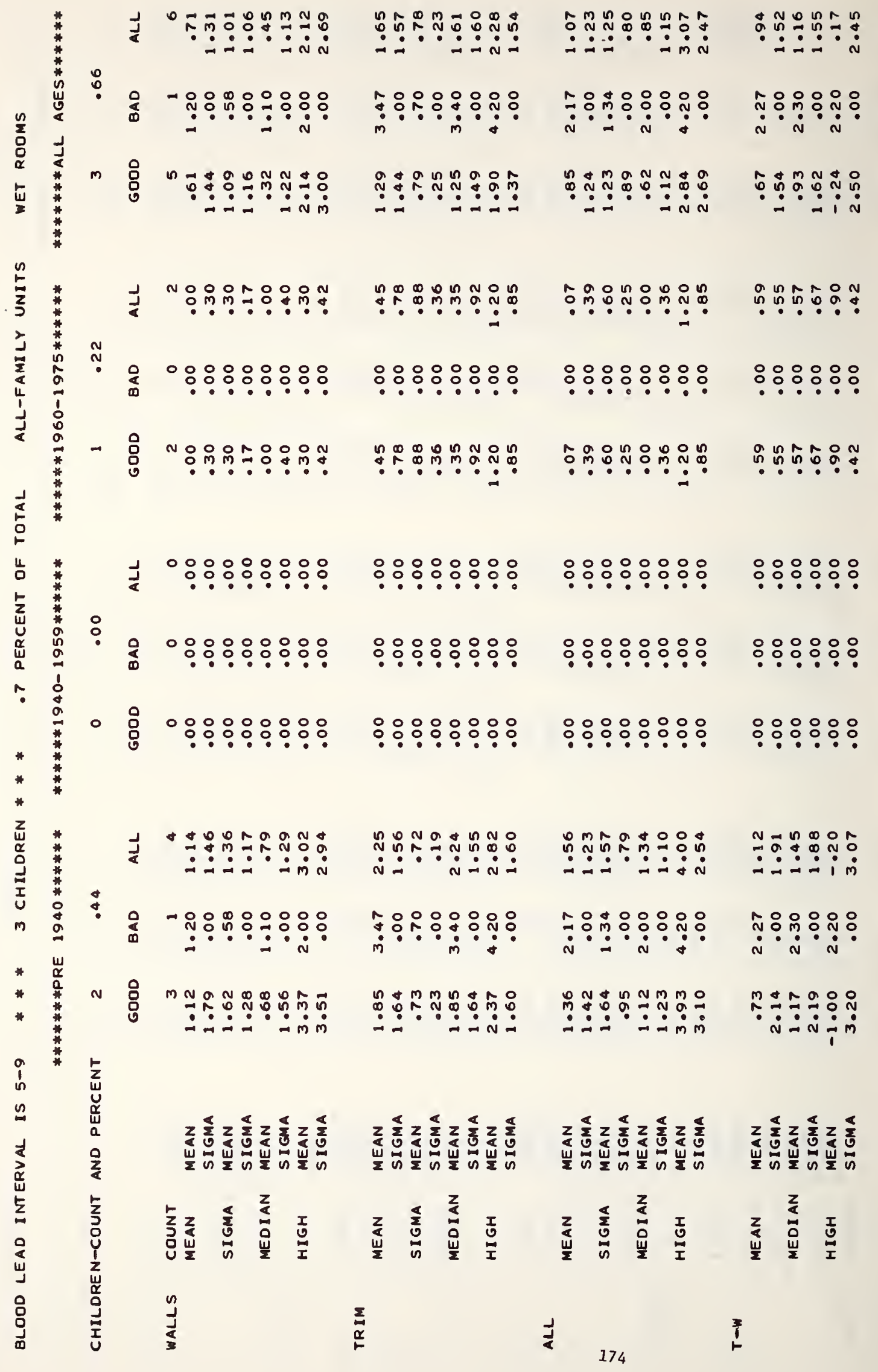


」

in

ก

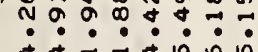

m

임

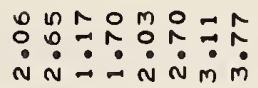

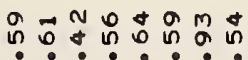

ำกำกับำกำ

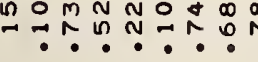

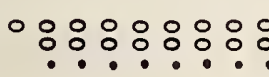

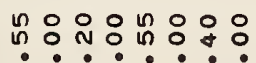

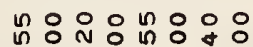

․․…

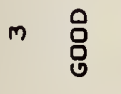

$\ln 0, \frac{5}{n} n \sin ^{2}$

$\cos 0.50$

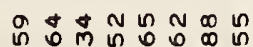

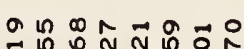

ำกำกำ

․ำ.ำ.

ป

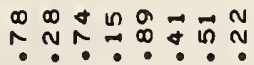

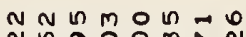

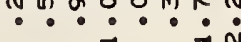

$\stackrel{n}{?}$

这

융ㅇ:ㅇ:웅

유:유:웅

ㅇㅇㅇㅇㅇㅇㅇㅇㅇㅇㅇㅇ

- 8

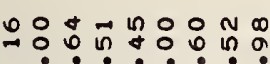

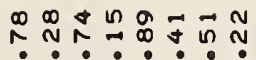

N $⿻$ ก

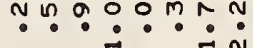

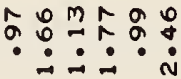

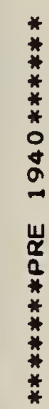

*

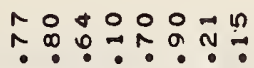

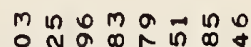

ㄴ.

$\dot{N} \dot{N} \dot{\sim} \dot{\sim} \dot{N} \dot{0}$

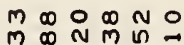

- $\dot{\mathrm{N}} \dot{-} \dot{-} \dot{\mathrm{N}} \dot{\mathrm{N}} \dot{\mathrm{m}}$

ผ $\dot{N} \dot{N} \dot{N} \dot{0}$

$\stackrel{0}{\infty}:$

n

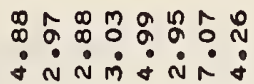

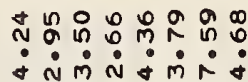

NOMNO

ดำำ

노ำดั

N $\dot{0} \dot{\sim} \dot{\sim}$ i

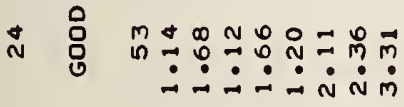

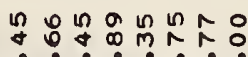

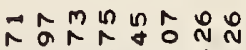

ํำ-

N

$\dot{\sim} \dot{N}=\dot{N} \dot{\sim} \dot{j}$

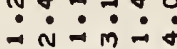

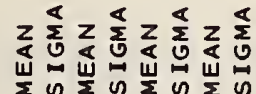

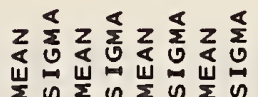

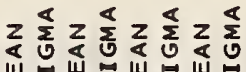

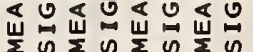

\section{int}

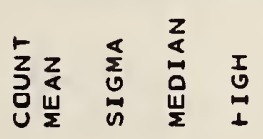

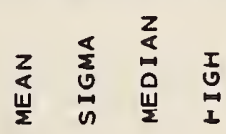

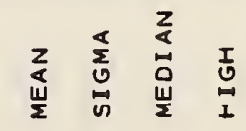

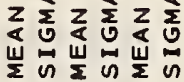

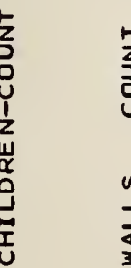

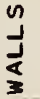

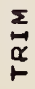

J

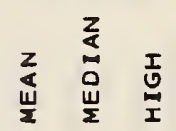




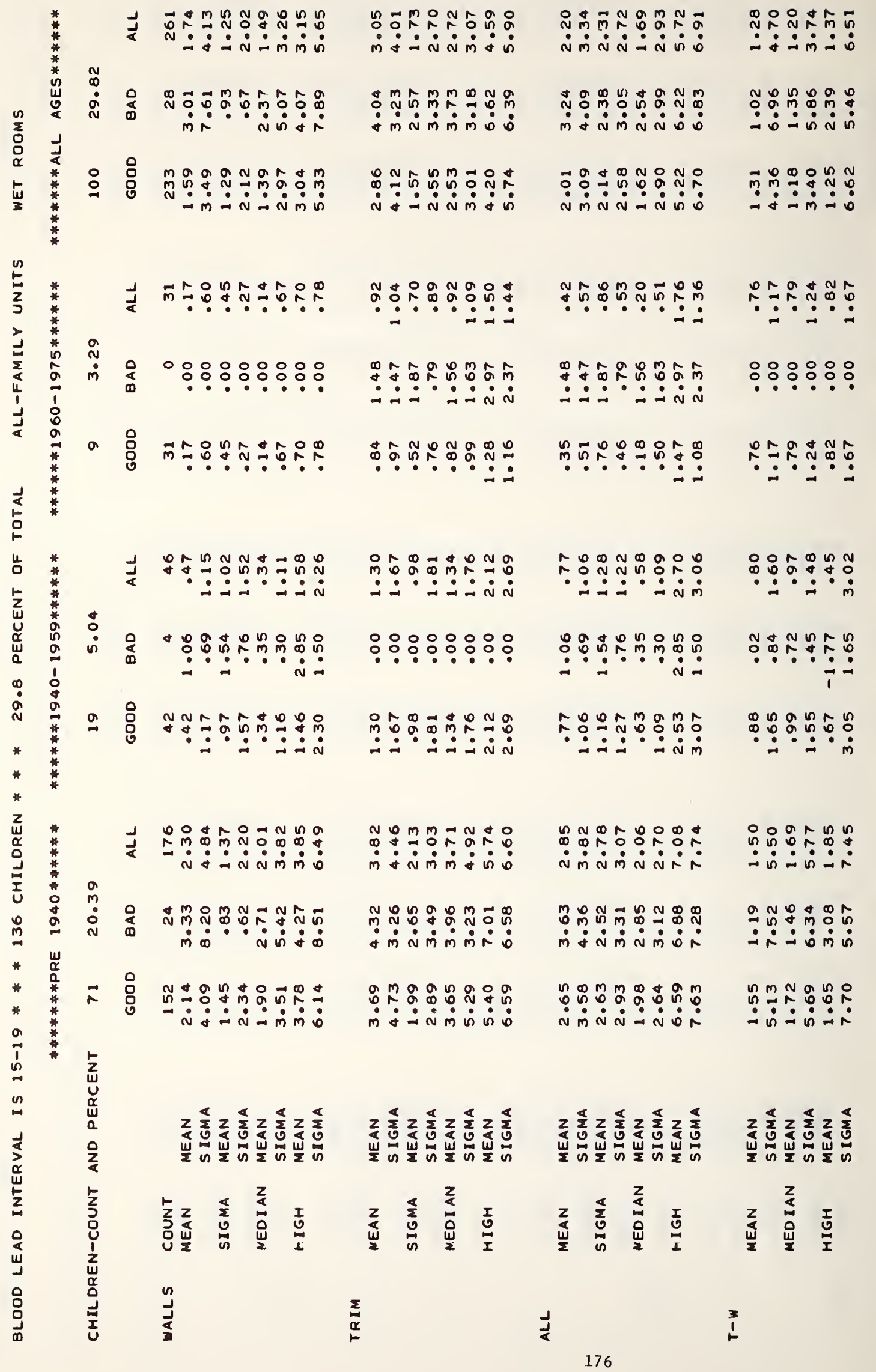




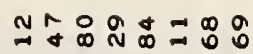

$\dot{m} \dot{m} \dot{\sim} \dot{N} \dot{m} \dot{q} \dot{q}$

$\stackrel{n}{\sim}$

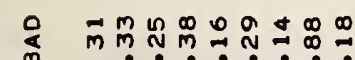

$\dot{m} \dot{0} \dot{-} \dot{m} \dot{0} \dot{0}$

$\hat{\infty}$

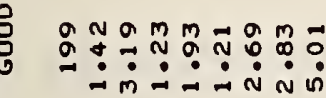

Ј

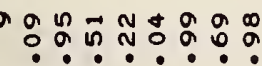

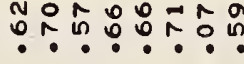

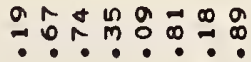

○ำ

in $\dot{m} \dot{N} \dot{m} \dot{m}$

ที กิ ที ัง

$\dot{N} \dot{m} \dot{i} \dot{N} \dot{N} \dot{+}$

品前

$-\dot{n}-\dot{N}-\dot{n} \dot{0}$

$m \infty \dot{m} \overrightarrow{0} \dot{m}$

$\dot{i} \dot{m} \dot{m} \dot{m} \dot{n}$

$\stackrel{\infty}{\infty}$

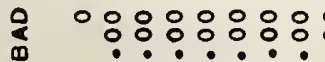

ㅇㅇㅇㅇㅇㅇㅇㅇㅇㅇㅇ

웅ㅇㅇㅇㅇㅇㅇㅇ

$0 \% 0 \%: 0$

N

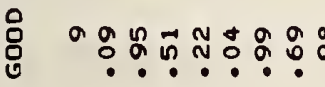

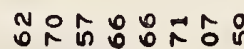

?ㄴ?

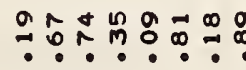

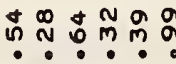

ง

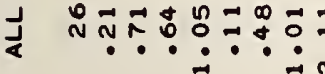

$\stackrel{\infty}{\infty}$

ผ

n 응

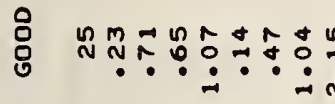

ำ

응ㅇㅇㅇㅇㅇㅇㅇㅇ

웅ㅇㅇㅇㅇㅇㅇㅇㅇㅇㅇㅇ

ํ.:ํ.

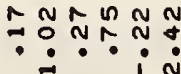

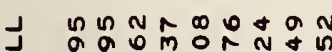
$\because \dot{m} \dot{\sim} \dot{\sim} \dot{m} \dot{m}$

( )

กก ก

$\dot{m} \dot{m} \dot{N} \dot{m} \dot{m} \dot{0} \dot{\sigma}$

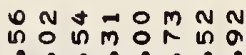

N $\dot{m} \dot{N} \dot{N} \dot{0}$

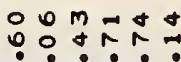

$\dot{a} \dot{0} \dot{m} \dot{0} \dot{0}$

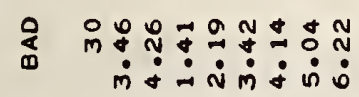

$0_{0}^{\infty}+\stackrel{\infty}{+} \hat{0} \hat{N} \stackrel{\infty}{\$} 0$

m

-min $\dot{m} \dot{m} \dot{0}$

a

$\dot{0} \dot{0}: \dot{N} \dot{0}$

0
0
0

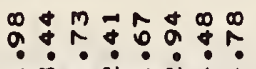

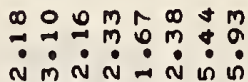

$M \cap N 0 \backsim N$

? $\div \div ?$

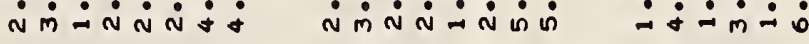




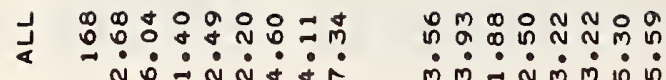

$\stackrel{\circ}{m}$

a \&

in

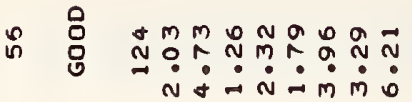

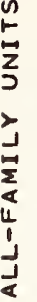

站

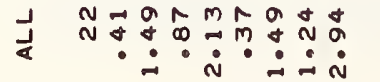

$\vec{a}$

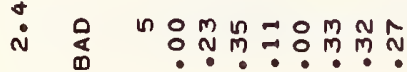

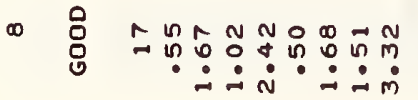

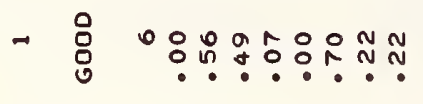

J

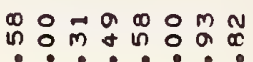

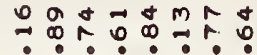

$\dot{m} \dot{m} \dot{\sim} \dot{N} \dot{m} \dot{\sigma}$

药

$\dot{\circ} \dot{m} \dot{N} \dot{m} \dot{m} \dot{0}$

- $\bullet \cdot \bullet \cdot$

응ㅇㅇㅇㅇㅇㅇㅇㅇㅇ

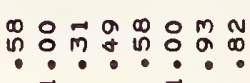

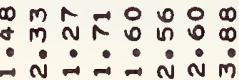

क

$\ddot{-} \dot{-} \dot{-} \dot{m} \dot{j}$

ก) ก पर

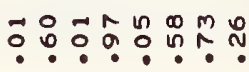

요 $\dot{\sim} \dot{\sim} \dot{\sim} \dot{i} \dot{\mathrm{N}} \dot{\mathrm{j}}$

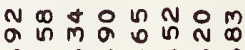
$\because \dot{-} \dot{-} \dot{m} \dot{\sigma}$

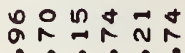
i $\dot{a} \dot{a} \dot{0}$

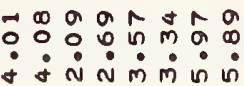

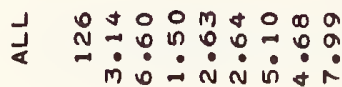

$\checkmark O+D N a 0$

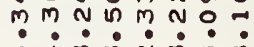

$N$ n o $m+\infty$ $\dot{m} \dot{m} \dot{m} \sim \dot{m} \infty$

$\therefore:$ i

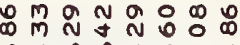

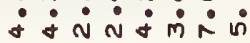

மá $\dot{0} \dot{0} \dot{0} \dot{0}$

+ ดิ

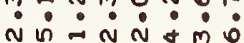

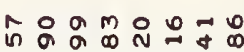
ம் $\dot{m} \dot{\sim} \dot{m} \dot{m}$ in

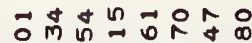
ம் $\dot{m} \dot{\circ} \dot{m} \dot{0} \dot{0}$

madanma :? N $\dot{m} \dot{N} \dot{N} \dot{N} \dot{R}$
กับ ia $\dot{0} i:$

$m \vec{m}=\mathbb{a}$ ? $\dot{0} \dot{a} \dot{0} \dot{0}$
N $N=$ ⿰ก $N$

$\because \frac{0}{4} \div \div$

ดำำำ i:iri $-\dot{0} \dot{0} \dot{\mathrm{N}}$

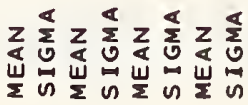

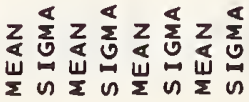

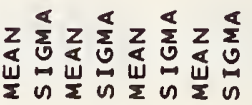

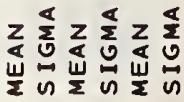

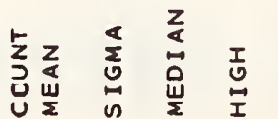

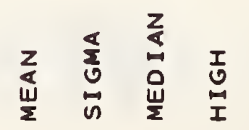

$\underset{5}{\Sigma}$

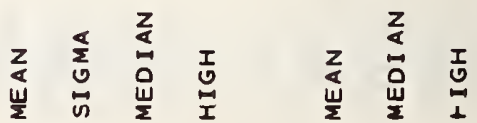


বㄴ.

$\stackrel{\infty}{\circ}$

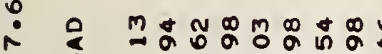
N

」 $0: \because::::::$

$\because: \because: \because: \because:$

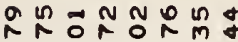

N

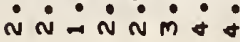

ดั

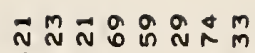

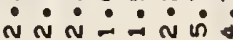

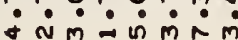

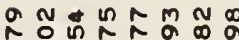
$\dot{m} \dot{m} \dot{N} \dot{\sim} \dot{m} \dot{m} \dot{0} \dot{m}$

ㅇำ ก.

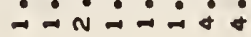

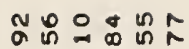
- $\dot{m} \dot{\sim} \dot{m} \dot{N} \dot{8}$

\& $\because \dot{N} \dot{N} \dot{N}$

og in o n m n

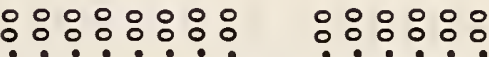

웅

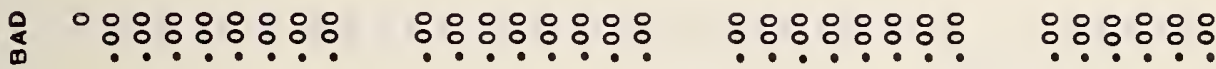

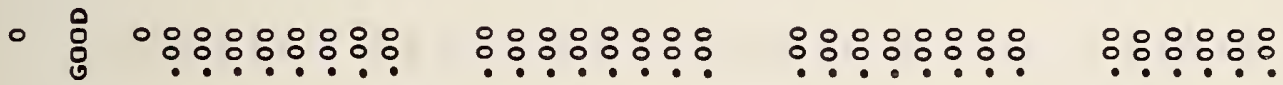

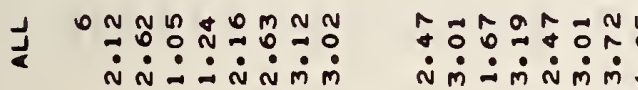

$\because$

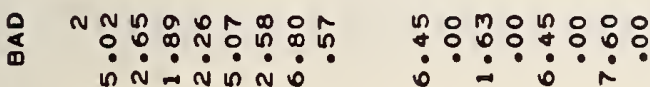

$-\quad$ og

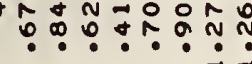

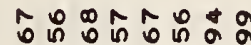
$\dot{i} \dot{i} \dot{m} \dot{\sim} \dot{\alpha}$

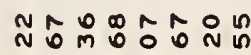

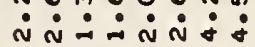

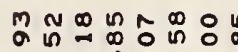
$\dot{\sim} \dot{N} \dot{\sim} \dot{\sim} \dot{\sim}$

$\Rightarrow M N M \sim 0 N+$ $\because \dot{\mathrm{v}} \dot{\mathrm{m}} \dot{\mathrm{v}} \dot{\mathrm{m}}$ mे

$\because \dot{\sim}$

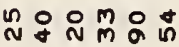

$\Rightarrow n+n$ n $\because: \div 9$ ? $\therefore \dot{0} \cdot \dot{0}$

ก๊

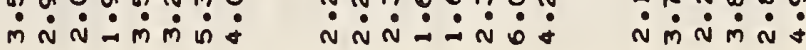

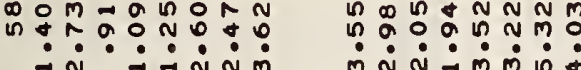

กิ๊ $\therefore \dot{m}: \dot{m} \dot{m}$

ก $\dot{m} \dot{0} \dot{0} \dot{0}:$

น $\dot{N} \dot{m} \cdot \dot{n} \dot{m}$

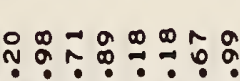

চ 

ตีดัดัตั $\dot{N} \dot{m} \dot{N} \dot{\alpha} \dot{N}$

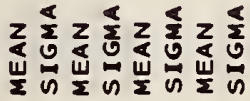

z $\frac{1}{2} z \frac{5}{2} z \frac{5}{2} z \frac{5}{0}$

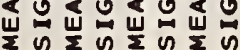

z $\frac{5}{2} z \frac{5}{2} z \frac{\pi}{2} z \frac{5}{2}$

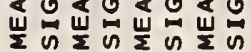

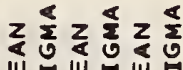
崖出崖出崖的

年

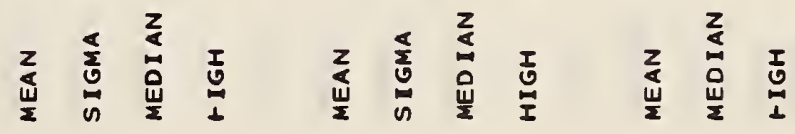

J 


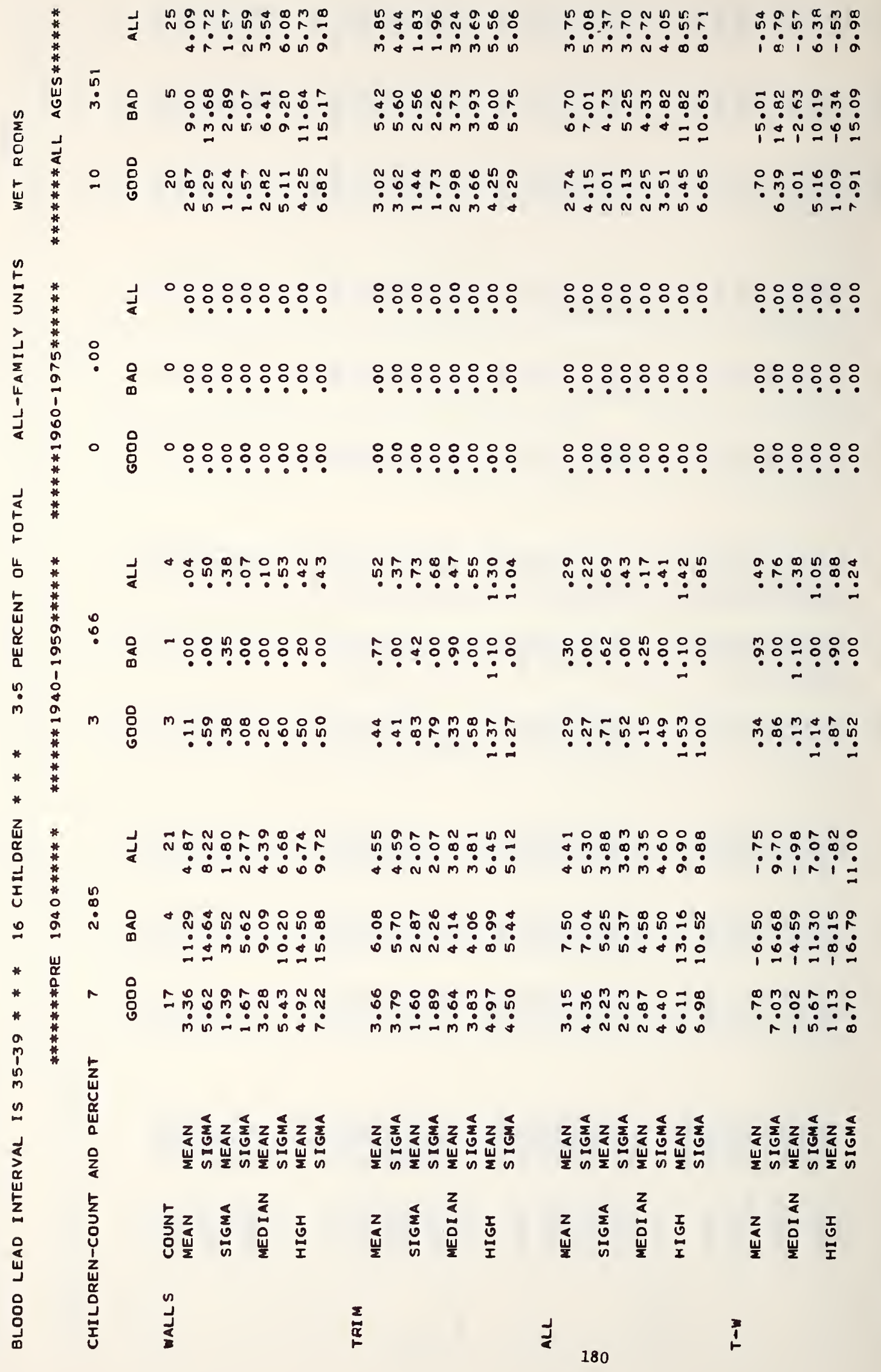




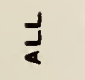

$\because \dot{v} \div \dot{v}$

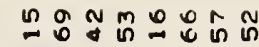
$\dot{0} \dot{0} \dot{0} \dot{0}$

N.

$\dot{m} \cdot \dot{0} \dot{m}$

(1)

N $\sin \dot{0} \dot{m}$ r

$\dot{8}$

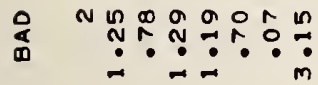

N

$N m$
$m$
$m$
$\dot{m}$
$\dot{m}$

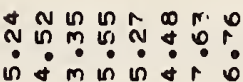

ดัN

$\dot{\circ} \dot{m} \dot{m} \dot{\alpha} \dot{\sigma} \dot{\sigma} \dot{m}$

$\infty$ 웅요

$\because \ddot{\infty} \dot{\infty} \dot{\infty}$

응요용

응ㅇㅇㅇㅇㅇㅇㅇ

응ㅇㅇㅇㅇㅇㅇㅇㅇ

:ㅇ:ㅇㅇㅇ

:

응ㅇㅇㅇㅇㅇㅇㅇㅇ

응ㅇㅇㅇㅇㅇ응

응응ㅇㅇㅇㅇㅇㅇ은

::ㅇ:웅

- 品

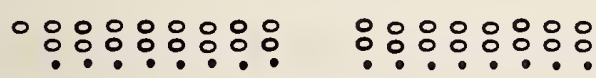

응응응응응

๑คำํ.

$\therefore: 0: 0:$

$\frac{1}{2}$

00000000 응유:유:

응융ㅇㅇㅇㅇㅇ

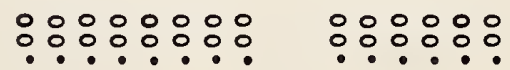

$\therefore$



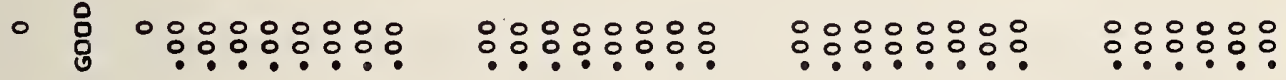

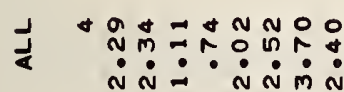

กด NMด ค N $\because: 00,0$ in iि $\dot{m} \dot{m} \dot{\circ} \dot{0} \dot{m} \dot{\sim}$

\& $N$ N $\infty \div \infty \div 0 \div$

ก

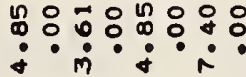

ํํํํํํํำำำ.ำ.

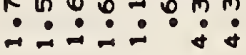

จ

N

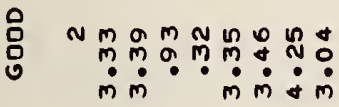

ง

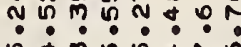

赵

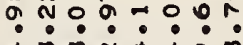
- mm
กีํํำำㅇํㅇ

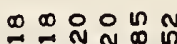

$\dot{\sim} \dot{m} \dot{0} \dot{m}$

$\dot{n} \dot{m} \dot{0} \dot{N} \dot{m}$

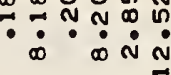

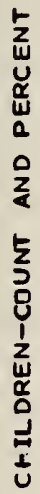

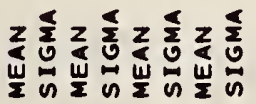

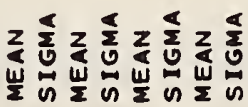

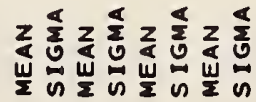

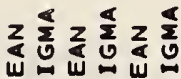
崖出崖的崖出崖出

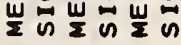




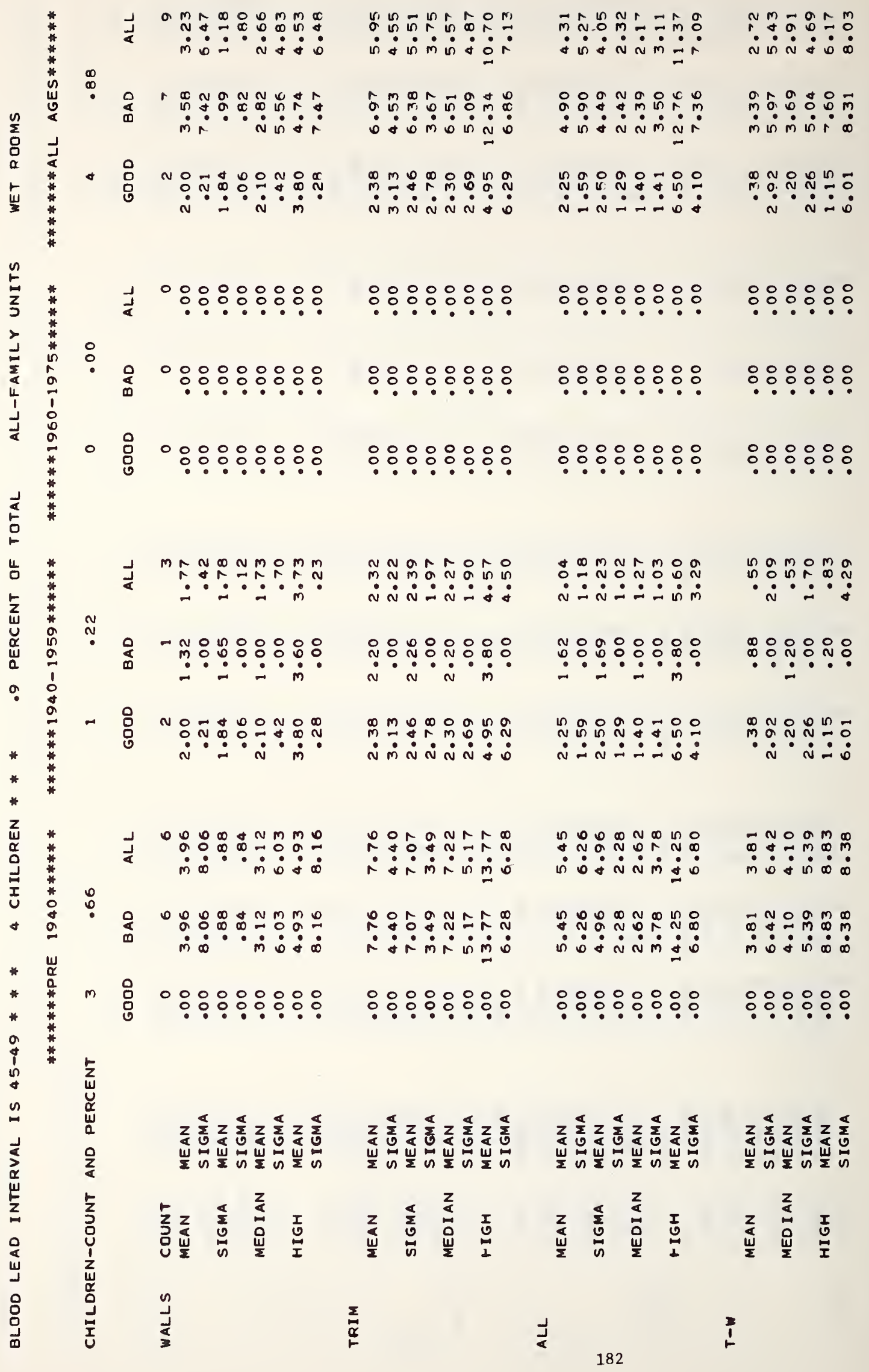




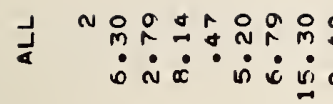

这

유:ㅇ::요

- 吕

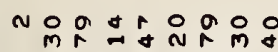
$\dot{0} \dot{N} \dot{x} \dot{0} \dot{0} \dot{n}$

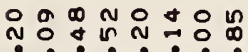

n $\dot{n} \cdot \dot{m} \cdot$

mM $m$ N

$\dot{a} \dot{0} \dot{0} \dot{0}$ in

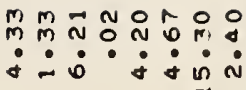

영요의

$\dot{\alpha} \dot{n} \dot{0} \dot{m}$

$\dot{N} \dot{\sim} \dot{m}:$

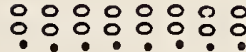

응융ㅇㅇ응

응유융

$\circ: \because:: 0:: \circ$

응ㅇㅇㅇㅇㅇㅇㅇ

:ㄴํำำ

응응ㅇㅇㅇㅠ

$\therefore: ㅇ ㅇ ㅇ$

:

$\underset{\infty}{\infty}$

$\circ:::: ㅇ$

유:유:웅

응ㅇ:웅

융ㅇㅇㅇㅠ

$\circ 8$ $\circ: \because: \because::: \circ$

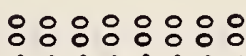

$\therefore::: ㅇ:$

$\therefore: ㅇ: 8$

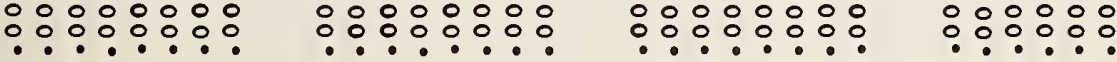

:

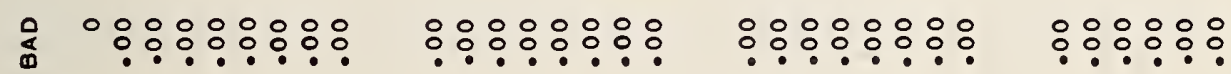

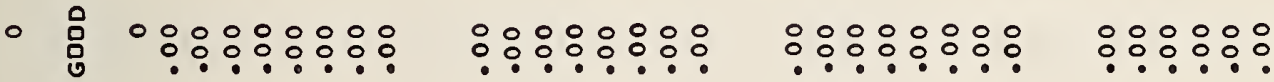

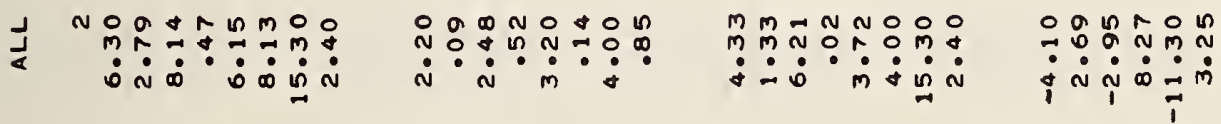

৫

总 N

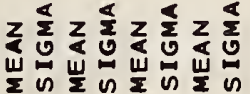

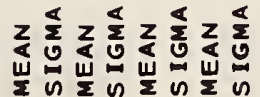

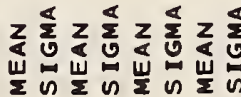

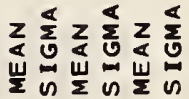




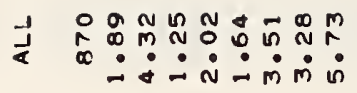

:

a $\begin{aligned} & \text { a } \\ & \text { m }\end{aligned}$

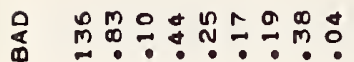

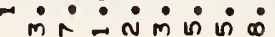

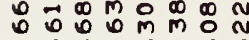

$\dot{i} \dot{m} \dot{N} \dot{i} \dot{i} \dot{n}$

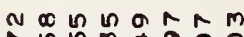

$\because$ ก ก

N

J

$\infty$

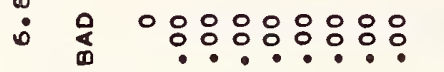

- ㅇํำ

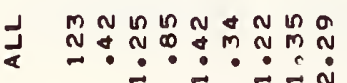

0

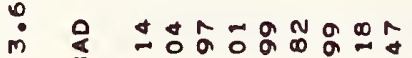

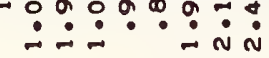

응 ำ $\rightarrow-\dot{-} \dot{\sim}$

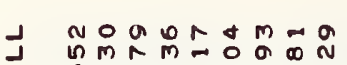

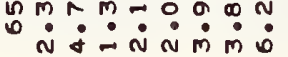

$n$

ก

$r \quad \infty \quad \dot{a} \dot{\sim} \dot{m} \dot{0} \dot{0}$

in

品

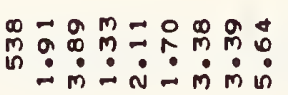

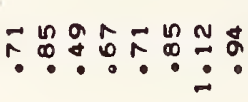

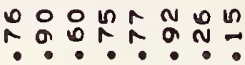

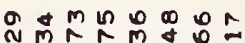

$\therefore \dot{-} \div \dot{0} \dot{\mathrm{w}}$

$M N \&: 00 \%$

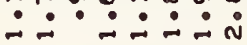

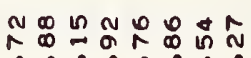

$\ddot{-} \dot{-} \dot{-} \dot{n}$

aำ $m-N$

$\because \div: \div \div \div \div$

^⿻日禸

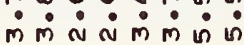

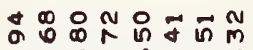

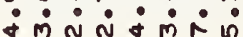

ค $m^{m} m_{0} 0 m^{m} N$ N. $\infty$ : $\dot{m} \dot{m} \dot{\sim} \dot{\mathrm{m}} \dot{m} \dot{\dot{\theta}}$

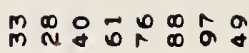
i $\dot{m} \dot{N} \dot{\sim} \dot{\sim} \dot{\sim} \dot{0}$

N $\therefore:-\dot{4}-\dot{0}$

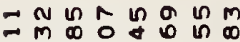
$\because \dot{0} \dot{m} \dot{0}$

$\stackrel{\infty}{m} \underset{N}{N}$ กิ $\because \because \dot{i}-\dot{R}$

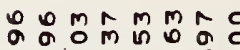

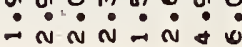

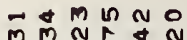
m. $m ? \frac{9}{4}$ $\because \dot{\theta}-\dot{m}-0$

N in

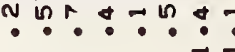

$\circ \infty 0 M \notin N$ $\because \because \div$ :

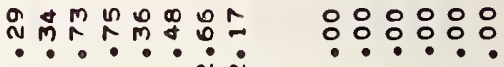
$\dot{\sim} \dot{-} \dot{\sim} \dot{\mathbf{N}}$

Nmmorog $m$ on

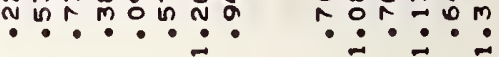

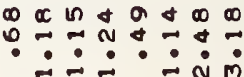

0 t $\because \div: \dot{m}$

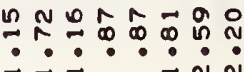

$\infty_{0}^{\infty} \overrightarrow{0}=\mathbb{N} N \stackrel{\infty}{N}$

: $: ?$

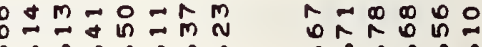

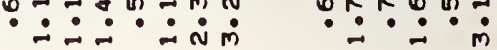

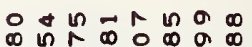
$\infty \ln \pi \circ \infty$

moㅇำ i $\dot{m} \dot{N} \dot{N} \dot{N} \dot{0} \dot{\sim} \dot{0} \dot{\sim} \dot{\sim} \dot{\sim}$

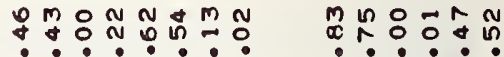

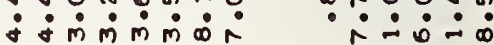

m

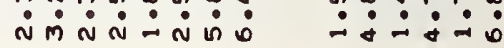

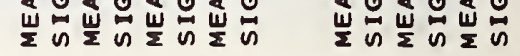

$z \frac{\pi}{2} z \frac{\pi}{0} z \frac{5}{0}$

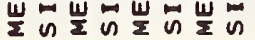

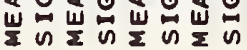

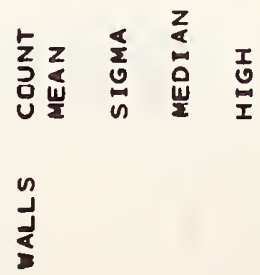

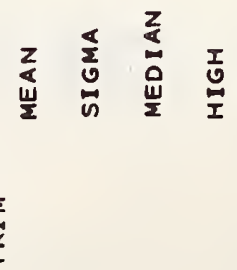

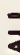

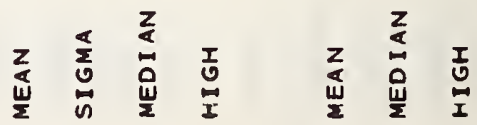


APPENDIX D

Data Collection Form for Housing Inspections 


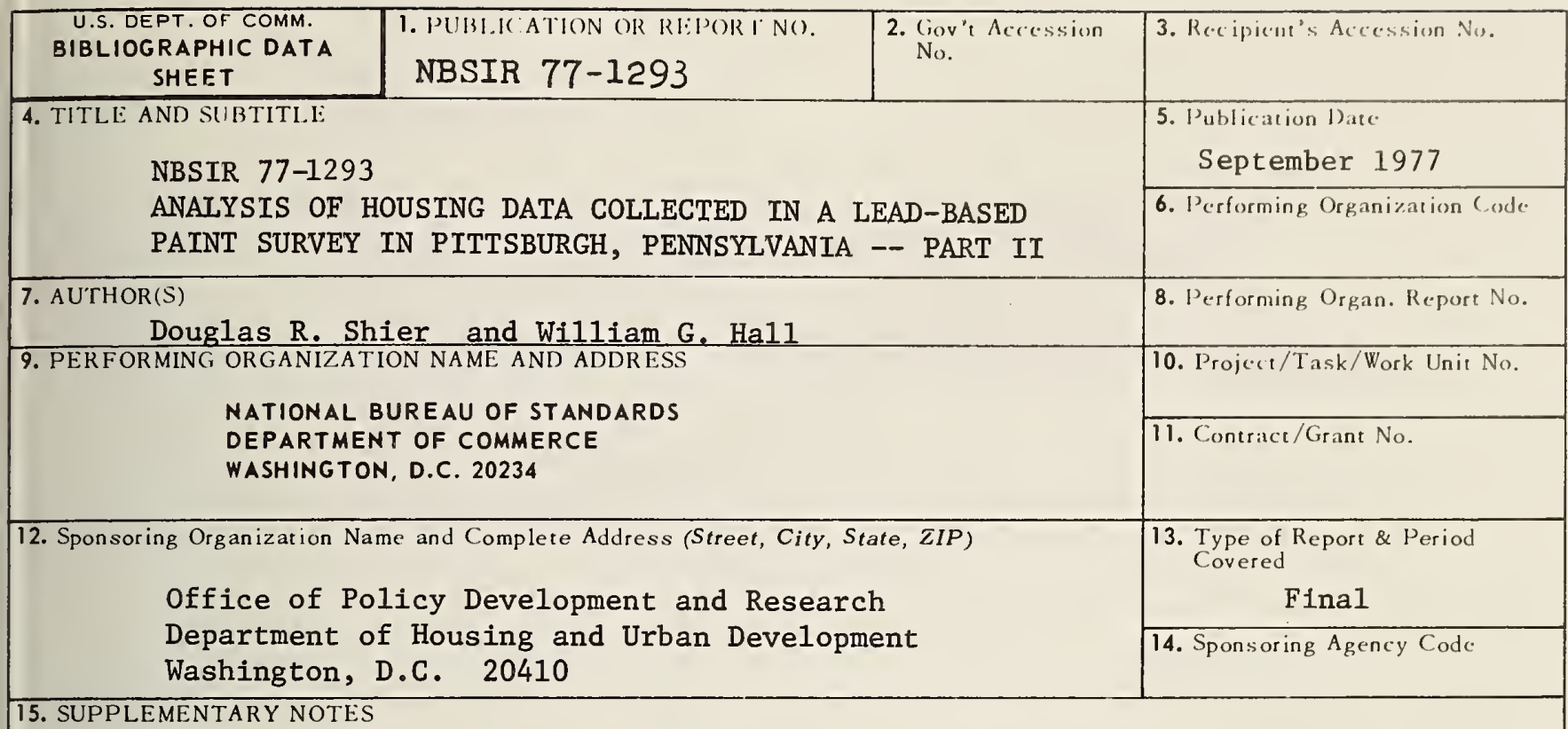

16. ABSTRACT (A 200-word or less factual summary of most significant information. If document includes a significant bibliography or literature survey, mention it here.)

This report represents Part II of a two-part document describing a lead-based paint survey conducted in Pittsburgh, Pennsylvania.

Part I of this document (NBSIR 77-1250) presented details of the design, methodology and findings of this survey. The current report (Part

II) presents and describes a series of tables summarizing the Pittsburgh survey data base, which includes measured lead levels of various surfaces in dwelling units and blood lead levels of children resident in those units. Most of the data analyses conducted in Part I derive from these tables. Thus, the tabulations given in this report provide not only the basis for those analyses but also a potential source for additional data analyses.

17. KEY WORDS (six to twelve entries; alphabetical order; capitalize only the first letter of the first key word unless a proper name; separated by semicolons)

Key Works: Blood lead; children; data analysis; housing; lead paint; lead poisoning; surveys.

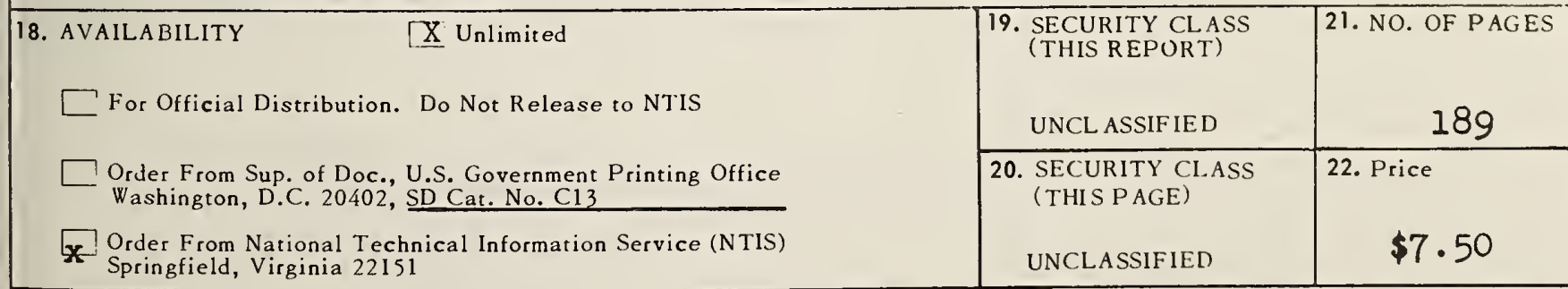




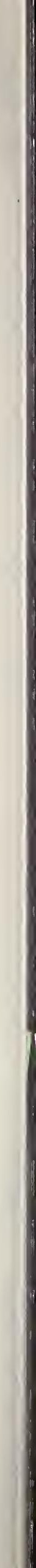

